\title{
Fracture Mechanics Analyses of Partial Crack Closure in Shell Structures
}

\author{
By \\ Jun Zhao \\ Master of Applied Science \\ A thesis submitted to the Faculty of Graduate Studies and Research in partial fulfillment \\ of the requirements for the degree of
}

\section{Doctor of Philosophy}

Department of Mechanical and Aerospace Engineering

Ottawa-Carleton Institute for

Mechanical and Aerospace Engineering

\author{
Carleton University \\ Ottawa, Ontario \\ Canada \\ May 2007 \\ (C) Copyright \\ 2007, Jun Zhao
}




$\begin{array}{ll}\begin{array}{l}\text { Library and } \\ \text { Archives Canada }\end{array} & \begin{array}{l}\text { Bibliothèque et } \\ \text { Archives Canada }\end{array} \\ \begin{array}{l}\text { Published Heritage } \\ \text { Branch }\end{array} & \begin{array}{l}\text { Direction du } \\ \text { Patrimoine de l'édition }\end{array} \\ \begin{array}{l}\text { 395 Wellington Street } \\ \text { Ottawa ON K1A ON4 }\end{array} & \begin{array}{l}\text { 395, rue Wellington } \\ \text { Ottawa ON K1A ON4 } \\ \text { Canada }\end{array}\end{array}$

Your file Votre référence ISBN: 978-0-494-33518-5 Our file Notre référence ISBN: 978-0-494-33518-5

NOTICE:

The author has granted a nonexclusive license allowing Library and Archives Canada to reproduce, publish, archive, preserve, conserve, communicate to the public by telecommunication or on the Internet, loan, distribute and sell theses worldwide, for commercial or noncommercial purposes, in microform, paper, electronic and/or any other formats.

The author retains copyright ownership and moral rights in this thesis. Neither the thesis nor substantial extracts from it may be printed or otherwise reproduced without the author's permission.
AVIS:

L'auteur a accordé une licence non exclusive permettant à la Bibliothèque et Archives Canada de reproduire, publier, archiver, sauvegarder, conserver, transmettre au public par télécommunication ou par l'Internet, prêter, distribuer et vendre des thèses partout dans le monde, à des fins commerciales ou autres, sur support microforme, papier, électronique et/ou autres formats.

L'auteur conserve la propriété du droit d'auteur et des droits moraux qui protège cette thèse. $\mathrm{Ni}$ la thèse ni des extraits substantiels de celle-ci ne doivent être imprimés ou autrement reproduits sans son autorisation.
In compliance with the Canadian

Privacy Act some supporting forms may have been removed from this thesis.

While these forms may be included in the document page count, their removal does not represent any loss of content from the thesis.
Conformément à la loi canadienne sur la protection de la vie privée, quelques formulaires secondaires ont été enlevés de cette thèse.

Bien que ces formulaires aient inclus dans la pagination, il n'y aura aucun contenu manquant.

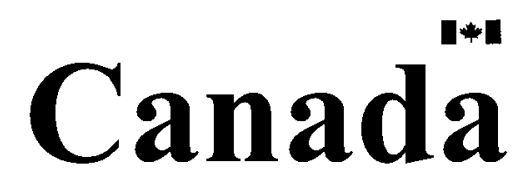




\begin{abstract}
This thesis presents the theoretical and finite element analyses of crack-face closure behavior in shells and its effect on the stress intensity factor under a bending load condition. Various shell geometries, such as spherical shell, cylindrical shell containing an axial crack, cylindrical shell containing a circumferential crack and shell with double curvatures, are all studied. In addition, the influence of material orthotropy on the crack closure effect in shells is also considered. The theoretical formulation is developed based on the shallow shell theory of Delale and Erdogan, incorporating the effect of crack-face closure at the compressive edges. The line-contact assumption, simulating the crack-face closure at the compressive edges, is employed so that the contact force at the closure edges is introduced, which can be translated to the mid-plane of the shell, accompanied by an additional distributed bending moment. The unknown contact force is computed by solving a mixed-boundary value problem iteratively, that is, along the crack length, either the normal displacement of the crack face at the compressive edges is equal to zero or the contact pressure is equal to zero. It is found that due to the curvature effects crack closure may not always occur on the entire length of the crack, depending on the direction of the bending load and the geometry of the shell. The crack-face closure influences significantly the magnitude of the stress intensity factors; it increases the membrane component but decreases the bending component. The maximum stress intensity factor is reduced by the crack-face closure. The significant influence of geometry and material orthotropy on rack closure behavior in shells is also predicted based on the analytical solutions.
\end{abstract}


Three-dimensional FEA is performed to validate the theoretical solutions. It demonstrates that the crack face closure occurs actually over an area, not on a line, but the theoretical solutions of the stress intensity factor and the FEA solutions are in good agreement, because the contact area is very small compared with the shell thickness. 


\section{Acknowledgements}

I wish to express my sincere gratitude to my thesis supervisors, Professor R. Liu and Dr. X.J. Wu, for their guidance in this research.

I would also like to thank Dr. T. Zhang, and Dr. Z. Zhang for their assistance in solving the mathematics problems and constructing the finite element models. 


\section{Table of Contents}

Page

Abstract $\quad$ iii

Acknowledgements 2 v

Table of Contents

List of Tables $\quad$ xi

List of Figures $\quad$ xii

Nomenclature $\quad$ xxii

1 Introduction $\quad 1$

1.1 Background 1

1.1.1 Application of shells 1

1.1.2 Existing fracture mechanics of shell 2

1.1.3 The crack-closure theory 4

1.2 Objectives, Methodologies and Significance $\quad 5$

$\begin{array}{ll}\text { 1.2.1 Theoretical solution } & 5\end{array}$

1.2.2 A case study $\quad 8$

1.2.3 Finite element solution 9

$\begin{array}{ll}1.3 \text { Organization of the Thesis } & 10\end{array}$

2 Literature Review $\quad 13$

$\begin{array}{lc}\text { 2.1 Fundamentals of Elastic Shells } & 13\end{array}$

2.1.1 Geometric definition of shells 13 
2.1.2 Stress analysis in shells

2.1.3 Mathematical theory of shallow shells 20

$\begin{array}{ll}2.2 \text { Development of elastic shell theories } & 21\end{array}$

2.2.1 Kirchhoff-Love hypothesis 21

$\begin{array}{ll}\text { 2.2.2 Classical shell theory } & 24\end{array}$

2.2.3 Transverse shear deformation theory 25

$\begin{array}{ll}2.3 \text { Fracture Mechanics of shells } & 26\end{array}$

$\begin{array}{ll}\text { 2.3.1 Crack problems in shells } & 26\end{array}$

2.3.2 Application of shell theories in fracture analysis 27

2.4 Crack Closure Theory

$\begin{array}{ll}\text { 2.4.1 Crack closure in plates } & 32\end{array}$

$\begin{array}{ll}2.4 .2 \text { Crack closure in shells } & 37\end{array}$

2.5 Finite Element Methods for Shell Problems $\quad 40$

2.5.1 Applications in fracture mechanics $\quad 40$

2.5.2 Modeling of shell problems $\quad 42$

$\begin{array}{ll}\text { 2.5.3 Current challenges } & 46\end{array}$

2.5.4 Determination of stress intensity factors $\quad 49$

2.6 Composite Shells $\quad 51$

2.6.1 Composite materials for shells $\quad 51$

$\begin{array}{ll}\text { 2.6.2 Material orthotropy } & 51\end{array}$

2.6.3 Laminated shells $\quad 55$

3 Theoretical Analysis $\quad 60$ 
3.1 Specially Orthotropic Shell with Double Curvature

3.1.1 Formulation of the problem 60

3.1.2 Modeling of crack-face closure 63

3.1.3 Determination of crack closure regions 67

3.1.4 Solution of stress intensity factor 72

$\begin{array}{ll}\text { 3.1.5 Analytical results } & 74\end{array}$

3.2 Specially Orthotropic Cylindrical Shell 83

3.2.1 Definition of an axial crack and a circumferential crack in a specially orthotropic cylindrical shell $\quad 83$

3.2.2 Crack closure in a specially orthotropic cylindrical shell containing an axial $\begin{array}{lr}\text { crack } & 85\end{array}$

3.2.3 Crack closure in a specially orthotropic cylindrical shell containing a $\begin{array}{ll}\text { circumferential crack } & 94\end{array}$

3.3 Specially Orthotropic Spherical Shell 102

3.3.1 Definition of a crack in a specially orthotropic spherical shell $\quad 102$

3.3.2 Crack closure in a specially orthotropic spherical shell 104

3.4 Isotropic Shells 113

$\begin{array}{ll}\text { 3.4.1 Problem formulation } & 113\end{array}$

$\begin{array}{ll}\text { 3.4.2 Double-curvature shells } & 115\end{array}$

3.4.3 Cylindrical shells 120

3.5 Summary of the Results 127

$\begin{array}{ll}\text { 3.5.1 Crack closure behaviors } & 127\end{array}$

$\begin{array}{ll}\text { 3.5.2 Stress intensity factors } & 129\end{array}$ 
4.1 A Pressurized Cylinder Containing an Axial Crack 135

$\begin{array}{ll}\text { 4.2 Determination of Bending Stress } & 135\end{array}$

$\begin{array}{ll}\text { 4.3 Solution of Stress Intensity Factors } & 139\end{array}$

4.3.1 Determination of crack closure regions 139

$\begin{array}{ll}\text { 4.3.2 Results of stress intensity factors } & 140\end{array}$

5 Finite Element Analysis $\quad 143$

$\begin{array}{ll}5.1 \text { Significance and Objectives } & 143\end{array}$

$\begin{array}{ll}5.2 \text { Construction of the Models } & 144\end{array}$

$\begin{array}{ll}\text { 5.2.1 Modeling crack problems in shells } & 144\end{array}$

$\begin{array}{ll}\text { 5.2.2 Simulation of crack-face closure } & 146\end{array}$

$\begin{array}{ll}\text { 5.2.3 Selection of shell parameters } & 147\end{array}$

$\begin{array}{ll}\text { 5.2.4 Boundary conditions of the FEA models } & 149\end{array}$

$\begin{array}{ll}\text { 5.2.5 Meshing of the models } & 153\end{array}$

$\begin{array}{ll}5.3 \text { FEA Solutions } & 155\end{array}$

$\begin{array}{ll}\text { 5.3.1 Data processing } & 155\end{array}$

$\begin{array}{ll}\text { 5.3.2 Spherical shell } & 156\end{array}$

$\begin{array}{ll}\text { 5.3.3 Cylindrical shell containing an axial crack } & 165\end{array}$

$\begin{array}{ll}\text { 5.3.4 Cylindrical shell containing a circumferential crack } & 172\end{array}$

$\begin{array}{ll}\text { 5.3.5 Shell with double curvature } & 178\end{array}$

$\begin{array}{ll}6 \text { Discussion } & 185\end{array}$ 
6.1 Effects of Crack Closure on Stress Intensity Factors

7 Conclusions

References

Appendices

Appendix A

206

Appendix B 209

Appendix C 210

Appendix D

211 


\section{List of Tables}

Table 3.1 Ratios of specially orthotropic material constants

Table 3.2 Critical $\lambda$ values for partial closure under positive bending

Table 3.3 Closure solutions of stress intensity factor ratios $\left(F_{m b}=F_{b b}\right)$ for the mildly orthotropic material (titanium), $\varphi=0.725$ and $\varphi=1.38, \sigma_{b}>0$

Table 3.4 Closure solutions of stress intensity factor ratios $\left(F_{b b}, F_{m b}=-F_{b b}\right)$ for the mildly orthotropic material (titanium), $\varphi=0.725$ and $\varphi=1.38, \sigma_{\mathrm{b}}<0$

Table 3.5 Closure solutions of stress intensity factor ratios $\left(F_{m b}=F_{b b}\right)$ for the strongly orthotropic material (graphite-epoxy), $\varphi=0.037$ and $\varphi=26.67, \sigma_{\mathrm{b}}>0$

Table 3.6 Closure solutions of stress intensity factor ratios $\left(F_{b b}, F_{m b}=-F_{b b}\right)$ for the strongly orthotropic material (graphite-epoxy), $\varphi=0.037$ and $\varphi=26.67, \sigma_{\mathrm{b}}<0$

Table 3.7 Closure solutions of stress intensity factor ratios $\left(F_{m b}=F_{b b}\right)$ for isotropic materials, $\varphi=1, \sigma_{\mathrm{b}}>0$

Table 3.8 Closure solutions of stress intensity factor ratios $\left(F_{b b}, F_{m b}=-F_{b b}\right)$ for isotropic materials, $\varphi=1, \sigma_{\mathrm{b}}>0$

Table 5.1 Geometrical parameters of cylindrical and spherical shells

Table 5.2 Geometrical parameters of shells with double curvature 


\section{List of Figures}

Figure 1.1 Demonstration of shell shallowness

Figure 2.1 General form of shell structures with double curvatures

Figure 2.2 Definition of stresses in an infinitesimal element of a shell

Figure 2.3 Membrane action of a shell

Figure 2.4 Bending condition of a shell

Figure 2.5 Stress resultants and stress couples in a shell

Figure 2.6 Schematic drawings of crack closure and non-closure in

(a) plates and (b) shells

Figure 2.7 Modeling of crack-face closure of a plate under bending:

(a) crack opening without closure,

(b) crack opening with closure and

(c) equivalent force and moment at the mid-plane of the plate

Figure 2.8 Distributions of the contact force along the crack for isotropic spherical shells under positive bending (closure at inner surface) assuming full-length closure

Figure 2.9 Comparison between closure and non-closure solutions for isotropic spherical shells under bending:

(a) stress intensity factor ratios and

(b) maximum stress intensity factor ratios

Figure 2.10 Cylindrical coordinate system at the crack tip

Figure 2.11 Laminate construction 
Figure 2.12 A laminate made up of laminae with different fiber orientations

Figure 3.1 Geometry and coordinates for a through-the-thickness crack in a shell with double curvature

Figure 3.2 Illustration of crack-face opening displacement and crack- face rotation

Figure 3.3 Bending loads: (a) positive bending and (b) negative bending

Figure 3.4 Contact force and equivalent bending moment representing the crack-face closure:

(a) Contact force at inner surface (positive bending) and

(b) Contact force at outer surface (negative bending)

Figure 3.5 Contact force distributions assuming full-length closure for a specially orthotropic shell having double curvature under positive bending with $a / h=10$ :

(a) $\varphi=0.725$ and $\psi=0.25$; (b) $\varphi=0.725$ and $\psi=4$;

(c) $\varphi=1.38$ and $\psi=0.25$; (d) $\varphi=1.38$ and $\psi=4$

Figure 3.6 Illustration of closure regions and open region of the crack face

Figure 3.7 Correct distribution of the contact force in partial closure of a specially orthotropic shell having double curvature under positive bending with $a / h=10, \varphi=0.725$ and $\psi=0.25$, under positive bending

Figure 3.8 Comparison between closure and non-closure solutions of stress intensity factor ratio for a specially orthotropic shell having double curvature with $a / h=10 \quad$ ( closure and ----.-non-closure):

(a) $\varphi=0.725$ and $\psi=0.25$; (b) $\varphi=0.725$ and $\psi=4$;

(c) $\varphi=1.38$ and $\psi=0.25 ;$ (d) $\varphi=1.38$ and $\psi=4$

Figure 3.9 Comparison between closure and non-closure solutions of the maximum stress intensity factor ratio for a specially orthotropic shell having double curvature with $a / h=10$ : 

(a) $\varphi=0.725$ and $\psi=0.25$; (b) $\varphi=0.725$ and $\psi=4$;
(c) $\varphi=1.38$ and $\psi=0.25 ;$ (d) $\varphi=1.38$ and $\psi=4$

Figure 3.10 Effects of material orthotropy and shell curvature on the reduction of the maximum stress intensity factor ratio for a specially orthotropic shell having double curvature under positive bending with $a / h=10$ :

(a) $\psi=0.25$ and (b) $\psi=4$

Figure 3.11 Closure solutions of the stress intensity factor ratios for a specially orthotropic shell having double curvature:
(a) $\varphi=0.725$ and $\psi=0.25$; (b) $\varphi=0.725$ and $\psi=4$;
(c) $\varphi=1.38$ and $\psi=0.25$; (d) $\varphi=1.38$ and $\psi=4$

Figure 3.12 Geometry and coordinates for a through-the-thickness axial crack in a cylindrical shell (a) top view and (b) side view

Figure 3.13 Geometry and coordinates for a through-the-thickness circumferential crack in a specially orthotropic cylindrical shell (a) top view and (b) side view

Figure 3.14 Contact force distributions assuming full-length closure for a specially orthotropic cylindrical shell containing an axial crack under positive bending with $a / h=10$ :
(a) $E_{1} / E_{2}=0.725$
(b) $E_{1} / E_{2}=1.38$;
(c) $E_{1} / E_{2}=0.037$ and
(d) $E_{1} / E_{2}=26.67$

Figure 3.15 Comparison between closure and non-closure stress intensity factor ratios for a specially orthotropic cylindrical shell containing an axial crack with $a / h=10(\longrightarrow$ closure and - - - non-closure $):$
(a) $E_{1} / E_{2}=0.725$; (b) $E_{1} / E_{2}=1.38$;
(c) $E_{1} / E_{2}=0.037$ and (d) $E_{1} / E_{2}=26.67$ 
Figure 3.16 Comparison between closure solutions and non-closure solutions of the maximum stress intensity factor ratios for a specially orthotropic cylindrical shell containing an axial crack with $a / h=10$ :

(a) $E_{1} / E_{2}=0.037$ and (b) $E_{1} / E_{2}=26.67$

Figure 3.17 Reductions of the maximum stress intensity factor ratios due to the crack closure effect for a specially orthotropic cylindrical shell containing an axial crack with $a / h=10$ :

(a) under positive bending and (b) under negative bending

Figure 3.18 Contact force distributions assuming full-length closure for a specially orthotropic cylindrical shell containing a circumferential crack under positive with $a / h=10$ :

(a) $E_{1} / E_{2}=0.725 ;$ (b) $E_{1} / E_{2}=1.38$;

(c) $E_{1} / E_{2}=0.037$ and (d) $E_{1} / E_{2}=26.67$

Figure 3.19 Comparison between closure and non-closure stress intensity factor ratios for a specially orthotropic cylindrical shell containing a circumferential crack with $a / h=10$ ( $\longrightarrow$ closure and ----non-closure):

(a) $E_{1} / E_{2}=0.725 ;$ (b) $E_{1} / E_{2}=1.38$;

(c) $E_{1} / E_{2}=0.037$ and (d) $E_{1} / E_{2}=26.67$

Figure 3.20 Comparison between closure solutions and non-closure solutions of the maximum stress intensity factor ratio for a specially orthotropic cylindrical shell containing a circumferential crack with $a / h=10$ :

(a) $E_{1} / E_{2}=0.037$ and (b) $E_{1} / E_{2}=26.67$ 
Figure 3.21 Reductions of the maximum stress intensity factor ratios due to the crack closure effect for a specially orthotropic cylindrical shell containing a circumferential crack with $a / h=10$ :

(a) under positive bending and (b) under negative bending

Figure 3.22 Geometry and coordinates for a through-the-thickness crack in a spherical shell: (a) top view and (b) side view

Figure 3.23 Contact force distributions assuming full-length closure for a specially orthotropic spherical shell under positive bending with $a / h=10$ :
(a) $E_{1} / E_{2}=0.725$;
(b) $E_{1} / E_{2}=1.38$;
(c) $E_{1} / E_{2}=0.037$ and
(d) $E_{1} / E_{2}=26.67$

Figure 3.24 Comparison between closure and non-closure stress intensity factor ratios for a specially orthotropic spherical shell with $a / h=10$

( - closure and ---- non-closure):
(a) $E_{1} / E_{2}=0.725$; (b) $E_{1} / E_{2}=1.38$;
(c) $E_{1} / E_{2}=0.037$ and (d) $E_{1} / E_{2}=26.67$

Figure 3.25 Comparison between closure solutions and non-closure solutions of the maximum stress intensity factor ratio for a specially orthotropic spherical shell with $a / h=10$ :

(a) $E_{1} / E_{2}=0.725 ;$ (b) $E_{1} / E_{2}=1.38$;

(c) $E_{1} / E_{2}=0.037$ and (d) $E_{1} / E_{2}=26.67$

Figure 3.26 Reductions of the maximum stress intensity factor ratios due to the crack closure effect for a specially orthotropic spherical shell with $a / h=10$ :

(a) under positive bending and (b) under negative bending

Figure 3.27 Distributions of the contact force assuming full-length closure for an isotropic shell having double curvature with $a / h=10$ : 
(a) $R_{1} / R_{2}=0.25$ and (b) $R_{1} / R_{2}=4$

Figure 3.28 Comparison between closure and non-closure stress intensity factor ratios for an isotropic shell having double curvature with $a / h=10$ ( $\longrightarrow$ closure and-- - non-closure): (a) $R_{1} / R_{2}=0.25$ and (b) $R_{1} / R_{2}=4$

Figure 3.29 Comparison between closure solutions and non-closure solutions of the maximum stress intensity factor ratio for an isotropic shell having double curvature with $a / h=10:$ (a) $R_{1} / R_{2}=0.25$ and (b) $R_{1} / R_{2}=4$

Figure 3.30 Closure solutions of the stress intensity factor ratios for an isotropic shell having double curvature: (a) $R_{1} / R_{2}=0.25$ and (b) $R_{1} / R_{2}=4$

Figure 3.31 Distributions of the contact force assuming full-length closure for an isotropic cylindrical shell containing an axial crack with $a / h=10$ :

(a) under positive bending and (b) under negative bending

Figure 3.32 Distributions of the contact force assuming full-length closure for an isotropic cylindrical shell containing a circumferential crack with $a / h=10$ :

(a) under positive bending and (b) under negative bending

Figure 3.33 Comparison between closure and non-closure stress intensity factor ratios for an isotropic cylindrical shell with $a / h=10$ (--- closure and - non-closure):

(a) containing an axial crack and (b) containing a circumferential crack

Figure 3.34 Comparison between closure solutions and non-closure solutions of the maximum stress intensity factor ratio for an isotropic cylindrical shell with $a / h=10:$ 
(a) containing an axial crack and (b) containing a circumferential crack

Figure 3.35 Closure solutions of the stress intensity factor ratios for an isotropic cylindrical shell containing an axial crack:

(a) under positive bending and (b) under negative bending

Figure 3.36 Closure solutions of the stress intensity factor ratios for an isotropic cylindrical shell containing a circumferential crack:

(a) under positive bending and (b) under negative bending

Figure 3.37 Effects of material orthotropy and shell geometry on crack closure behavior of shells under positive bending

Figure 4.1 A thick-walled cylinder subjected to internal pressure and external pressure

Figure 4.2 Circumferential stresses in a cylinder subject to internal pressure and external pressure:

(a) stresses due to internal pressure and external pressure respectively and

(b) resultant stress due to concurrent internal pressure and external pressure

Figure 4.3 Contact force distributions along the crack length of the cylinder containing an axial crack under internal and external pressure

Figure 4.4 Stress intensity factor ratios for the axially cracked cylindrical shell subjected to internal and external pressure

Figure 4.5 Stress intensity factors for the axially cracked cylindrical shell subjected to internal and external pressure: (a) in non-closure case and (b) in closure case

Figure 5.1 A cracked shell subjected to (a) membrane force and (b) bending moment 
Figure 5.2 A pressurized cylinder contains a through-the-thickness axial crack

Figure 5.3 Crack closure modeling in FEA

Figure 5.4 Physical unreality due to unreasonable $\lambda$ values

Figure 5.5 Modeling of pure bending load on the crack surface:

(a) positive bending and (b) negative bending

Figure 5.6 Crack surface under Neumann boundary condition

Figure 5.7 Resultant tensile force and resultant compressive force on the crack surface:

(a) under positive bending and (b) under negative bending

Figure 5.8 Definition of boundary conditions of FEA model

Figure 5.9 Mesh patterns of FEA models:

(a) spherical shell

(b) cylindrical shell with an axial crack

(c) cylindrical shell with a circumferential crack

(d) shell with double curvature $R_{1} / R_{2}=4$ and

(e) shell with double curvature $R_{1} / R_{2}=0.25$

Figure 5.10 CODs of the spherical shell with $\lambda=2$ at a distance of $a / 2$ from the crack tip:

(a) positive bending and (b) negative bending

Figure 5.11 COD contours on the crack face of the spherical shell with $\lambda=2$ :

(a) positive bending and (b) negative bending

Figure 5.12 Variations of SIF through the shell wall of the spherical shell with $\lambda=2$ :

(a) positive bending and (b) negative bending

Figure 5.13 CODs of the spherical shell with $\lambda=4$ at a distance of $a / 2$ from the crack tip:

(a) positive bending and (b) negative bending

Figure 5.14 COD contours on the crack face of the spherical shell with $\lambda=4$ : 
(a) positive bending and (b) negative bending

Figure 5.15 Variations of SIF through the shell wall of the spherical shell with $\lambda=4$ :

(a) positive bending and (b) negative bending

Figure 5.16 CODs of the axially cracked cylindrical shell with $\lambda=2$ at a distance of $a / 2$ from the crack tip: (a) positive bending and (b) negative bending

Figure 5.17 COD contours on the crack face of the axially cracked cylindrical shell with $\lambda=2$ : (a) positive bending and (b) negative bending

Figure 5.18 Variations of SIF through the shell wall of the axially cracked cylindrical shell with $\lambda=2$ : (a) positive bending and (b) negative bending

Figure 5.19 CODs of the axially cracked cylindrical shell with $\lambda=4$ at a distance of $a / 2$ from the crack tip: (a) positive bending and (b) negative bending

Figure 5.20 COD contours on the crack face of the axially cracked cylindrical shell with $\lambda=4:$ (a) positive bending and (b) negative bending

Figure 5.21 Variations of SIF through the shell wall of the axially cracked cylindrical shell with $\lambda=4$ : (a) positive bending and (b) negative bending

Figure 5.22 CODs of the circumferentially cracked cylindrical shell with $\lambda=2$ at a distance of $a / 2$ from the crack tip:

(a) positive bending and (b) negative bending

Figure 5.23 COD contours on the crack face of the circumferentially cracked cylindrical shell with $\lambda=2$ : (a) positive bending and (b) negative bending

Figure 5.24 Variations of SIF through the shell wall of the circumferentially cracked cylindrical shell with $\lambda=2$ : (a) positive bending and (b) negative bending Figure 5.25 CODs of the circumferentially cracked cylindrical shell with $\lambda=4$ at a 
distance of $a / 2$ from the crack tip:

(a) positive bending and (b) negative bending

Figure 5.26 COD contours on the crack face of the circumferentially cracked cylindrical shell with $\lambda=4$ : (a) positive bending and (b) negative bending

Figure 5.27 Variations of SIF through the shell wall of the circumferentially cracked cylindrical shell with $\lambda=4$ : (a) positive bending and (b) negative bending

Figure 5.28 CODs of the shell with $R_{1} / R_{2}=0.25$ and $\lambda=2$ at a distance of $a / 2$ from the crack tip: (a) positive bending and (b) negative bending

Figure 5.29 COD contours on the crack face of the shell with $R_{1} / R_{2}=0.25$ and $\lambda=2$ :

(a) positive bending and (b) negative bending

Figure 5.30 Variations of SIF through the shell wall of the shell with $R_{1} / R_{2}=0.25$ and $\lambda=2$ : (a) positive bending and (b) negative bending

Figure 5.31 CODs of the shell with $R_{1} / R_{2}=4$ and $\lambda=2$ at a distance of $a / 2$ from the crack tip: (a) positive bending and (b) negative bending

Figure 5.32 COD contours on the crack face of the shell with $R_{1} / R_{2}=4$ and $\lambda=2$ :

(a) positive bending and (b) negative bending

Figure 5.33 Variations of SIF through the shell wall of the shell with $R_{1} / R_{2}=4$ and $\lambda=2$ : (a) positive bending and (b) negative bending 


\section{Nomenclature}

a half of crack length

$b \quad$ height of a shell cap

c material constant

$C_{i j} \quad$ material constants

E Equivalent Young's modulus

$E_{1} \quad$ Young's modulus in $x$ direction

$E_{2} \quad$ Young's modulus in $y$ direction

$F \quad$ stress function

$F_{1} \quad$ membrane stress function

$F_{2} \quad$ bending stress function

$F_{b b} \quad$ bending component of stress intensity factor

$F_{m b} \quad$ membrane component of stress intensity factor

$G_{1} \quad$ derivative of crack-face opening displacement

$G_{2} \quad$ derivative of crack-face rotation

$G_{i j} \quad$ shear modulus

$g_{1} \quad$ solution of membrane stress intensity factor

$g_{2} \quad$ solution of bending stress intensity factor

$h \quad$ thickness of shell

$K_{I} \quad$ stress intensity factor

$K_{I b} \quad$ stress intensity factors referring to bending moments

$k_{i j} \quad$ kernels of the coupled singular integral equations

$K_{I s} \quad$ stress intensity factors referring to stress resultants 
$m_{\mathcal{c}} \quad$ equivalent bending moment due to crack closure

$M_{i j} \quad$ stress couples

$N_{i j} \quad$ stress resultants

$p_{i} \quad$ external load components

$p_{1}, p_{2}, q$ the components of the load intensity

$P(x) \quad$ an equivalent contact force along crack closure edge

$r, \theta \quad$ the polar coordinates at crack tip

$P_{i} \quad$ inner pressure of a cylinder

$P_{O} \quad$ outer pressure of a cylinder

$p_{o} \quad$ maximum pressure load on crack surface

$p(r) \quad$ Neumann traction on crack surface

$R \quad$ radius of cylindrical or spherical shell

$R_{i} \quad$ principal curvature radii

$r_{i} \quad$ radius of the inner surface of a cylindrical shell

$r_{o} \quad$ radius of the outer surface of a cylindrical shell

$S \quad$ shallowness of a shell cap

$s \quad$ arc length

$u_{i} \quad x$ or $y$ component displacement

$u \quad$ crack-face opening displacement

$V_{i} \quad$ transverse shear stress resultants

w $\quad z$ component displacement

$x_{\alpha}, y_{\beta}$ orthogonal curvilinear coordinates

$y_{c} \quad$ closure boundary point coordinate

xxiii 
$\beta \quad$ crack-face rotation

$\beta_{i} \quad$ change of the slope of the normal to the middle surface

$\delta_{i j} \quad$ the Kronecker delta

$\varepsilon_{i j} \quad$ strain components

$\eta \quad$ normalized crack -length coordinate

$\kappa \quad$ dimensionless parameter

$\lambda, \lambda_{0}$ isotropic-shell parameter

$\lambda_{1}, \lambda_{2}$ specially-orthotropic-shell parameter

$\sigma_{b} \quad$ bending stress

$\sigma_{i j} \quad$ stress components

$\sigma_{m} \quad$ membrane stress

$\sigma_{\theta} \quad$ circumferential stress induced in the cylinder wall

$\tau \quad$ integral variable

$v \quad$ equivalent Poisson's ratio

$v_{1} \quad$ Poisson's ratio in $x$ direction

$v_{2} \quad$ Poisson's ratio in $y$ direction

$\varphi \quad$ ratio of Young's modulus $E_{l} / E_{2}$

$\psi \quad$ ratio of Shell radii $R_{l} / R_{2}$

$\Omega \quad$ load potential

$\nabla \quad$ vector differential operator

xxiv 


\section{Chapter 1 Introduction}

\subsection{Background}

\subsubsection{Application of shells}

Shells are curved structured members with thin walls. They are often used under pressurized conditions or to transfer bending loads into membrane loads, which maximizes the load carrying capability of the structures. Therefore, the application of shell structures can be found mostly as pressure vessels and pipelines in the chemical and petroleum industries, pressurized tanks or tubes in spacecrafts and airplanes, etc.

To keep in compliance with the diversifying applications of shells, various properties of shell structures have been developed in recent years. For example, to achieve uniform strength, reduce weight, or to carry variable distributed applied loads in different elements of the structure, shells of variable thickness are considered. In addition, with the development of composite materials, laminated composite shells have been widely used as engineering components and structures, owing to their high strength/stiffness-to-weight ratios, advanced corrosion resistance and directionally optimized properties, which also introduces new issues such as material anisotropy into the design and study of shells. Thus, structural analyses of shells become more difficult and cumbersome due to the introduction of these complexities with respect to the geometry, environment and materials. 
Depending on the mechanical behavior of the material, the loading and the environmental conditions of the shell structures, it is often necessary to consider possible failure mechanisms in order to ensure structural integrity and safety of the people who rely on the structure. This requires a fracture mechanics treatment of shell structures containing cracks. Such cracks may exist as initial manufacturing flaws, material imperfections, or damage developed during severe working conditions. These cracks may propagate under fluctuating external loads via fatigue crack propagation, or under sustained loads and sufficiently severe environmental conditions as stress corrosion cracks, or as a combination of both, namely as fatigue corrosion cracks. In using this approach cracks in shells, and the conditions under which they may be deemed either safe or dangerous, hold a perennial interest.

\subsubsection{Existing fracture mechanics of shell}

Theoretical treatments of cracks in shells are often difficult because of the mathematical complexities in dealing with the mechanical behavior of shell structures in three-dimensions. The presence of a curve in a shell changes its behavior from plates, in that stretching loads will induce both extensional and bending stresses, as well bending loads will also result in both types of stresses [1]. The subject of eventual concern therefore is that of the simultaneous stress fields produced in a shell containing a crack; hence it is a problem of how to combine the stress intensity factors (SIFs) in extension and bending to predict failure. In terms of assumptions, there are two shell theories, one is the earlier developed classical shell theory [2] and the other is the later transverse shear theory [1]. Both theories are established in terms of the linearized shallow shell theory [3- 
5], in which a segment is called shallow if the ratio of its height to base diameter is less than 1/8. As shown in Figure 1.1, for a cap formed by a through-the-thickness crack in a shell, the ratio of $b$ to $2 a$, is commonly called "shallowness" of the cracked shell cap, $S$, which takes the form as:

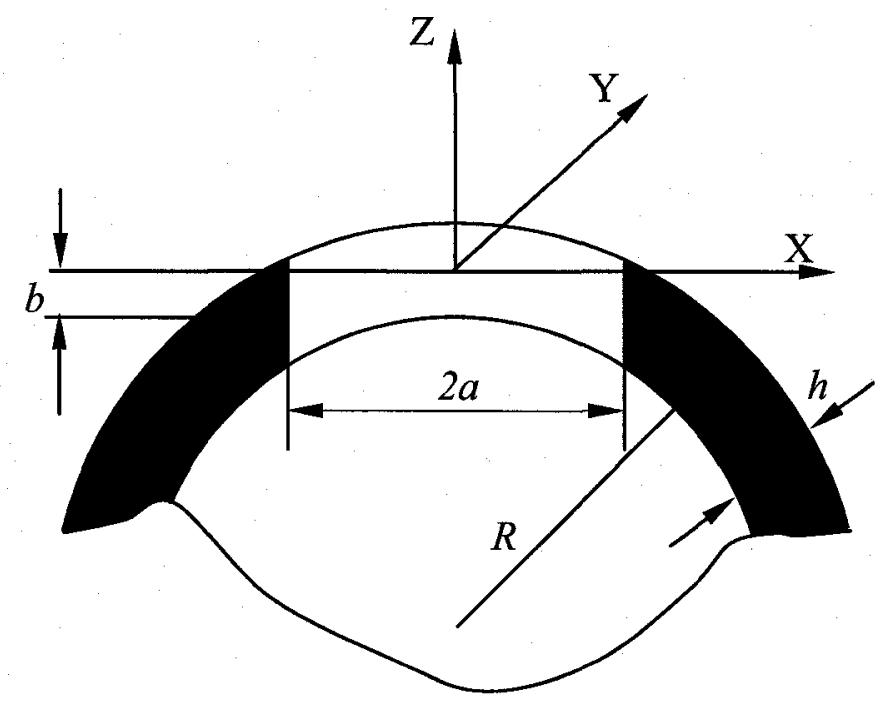

Figure 1.1 Demonstration of shell shallowness

$$
S=\frac{b}{2 a}=\frac{R-\sqrt{R^{2}-a^{2}}}{2 a}
$$

In the classical shell theory, the application of Kirchhoff effective transverse shear resultants [1] results in an unrealistic, hyper-singularity in the transverse shear stress near the crack tip, which makes it impossible to combine the membrane and bending SIFs as a linear sum. The transverse shear theory has overcome this fundamental difficulty by 
developing a higher order system of shell equations that account for the effect of transverse shear. Thus the solution permits the membrane and bending stress fields to be combined as a linear sum. These theories have been employed in solving crack problems in spherical shells [6,7] and cylindrical shells [8-14] as well as shells with double curvatures [15].

\subsubsection{The crack-closure theory}

One deficiency of the above theories is that crack faces are allowed to interpenetrate or overlap at the compressive edges under the bending condition, which is physically unrealistic. In reality, crack-face closure on the compressive edges occurs when a shell or a plate is subjected to a bending load. In recent years, crack-face closure in plates and shells has attracted significant attention, and some of the new solutions have been incorporated into ASME design code. For instance, Young and Sun [16,17] investigated the crack closure behavior in a plate based on the classical plate theory. In the formulation the complete solution was obtained by superposing the membrane components due to the contact forces at the crack face to the non-closure bending components. The distribution of the contact forces was found uniform by considering the contact condition that prevented mutual penetration of the crack faces at the compressive edges. The results indicate that the closure of the crack faces tends to reduce the crack opening displacement at the tension side and, consequently, reduce the stress intensity factor. Liu et al $[18,19]$ studied the crack closure in isotropic spherical shells. By assuming line-contact at the compressive edges, the Delale and Erdogan shell theory [7, 15] was extended to incorporate the effect of crack-face closure. The unknown contact 
force was then computed by solving a mixed-boundary value problem iteratively to ensure that either the normal displacement of the crack face at the compressive edges equals zero or the contact pressure equals zero along the crack length. The results show that due to the curvature effect the closure behavior in shells differs considerably from that in plates. Instead of complete closure over the entire crack length in plates, partial closure may occur in shells when the curvature is large, depending on the geometry of the shell and the bending load. When partial closure occurs, the crack faces at the compressive edges are in contact only in the regions close to the crack tips, leaving the central region of the crack face open. Also the sign of the bending moment, which determines the contact of the crack faces at either the inner surface or at the outer surface of the shell, affects the closure behavior in shells, whereas no such dependence exists in plates. It has been found that crack-face closure influences significantly the SIFs. The bending SIF is always equal to the membrane SIF in magnitude, which is not the case in the non-closure solution. The maximum stress intensity factor of the closure solution is reduced as compared to the closure-free solution.

\subsection{Objectives, Methodologies and Significance}

\subsubsection{Theoretical solution}

In the present research the crack closure theory of a shell under a bending load has been developed for more general cases, that is, for various shell geometries such as shells with arbitrary double curvatures (elliptical shells), cylindrical shells (when one of the curvature radii approaches infinity) with a circumferential crack or an axial crack. 
Material orthotropy has also been considered, representing fibre laminate composites. The formulation of the problem is still based on the linearized shallow shell theory, and extends the shear deformation theory of Dalale and Erdogan $[7,14,15]$ to incorporate the crack-face closure effect. The line-contact assumption, simulating the crack-face closure at the compressive edges, is still employed. Thus the contact forces at the closure edges are introduced, which can be translated to the mid-plane of the shell, accompanied with an additional distributed bending moment. The following steps are taken in obtaining the solutions:

\section{Step 1 Development of integral equations}

In terms of the shear deformation theory, crack problems in shells can be reduced to a pair of coupled singular integral equations. The solution of the equations may be retrieved under a general loading condition, namely, combined membrane and bending loads. However, considering the nature of the crack closure behavior, the crack face closure is a physical behavior directly following the crack face rotation. Thus, the effect of membrane load to the crack faces closure may be omitted because it has no contribution to the crack face rotation. Only the bending load on the crack faces is considered as the driving force of the crack face rotation, and consequently the crack face closure. Thus, it dominates the entire process of the crack closure behavior. To simplify the analytical process in the current research, the membrane force in the stress function of the integral equations is assumed to be zero. For practical problems, however, the general loading condition must be considered in solving the integral equations. 


\section{Step 2 Solution of the kernels}

In order to obtain the asymptotic solution of the kernels in the integral equations, the roots of the characteristic equation of the problem are derived first. The asymptotic solutions of the kernels are then obtained by solving the integrals of an infinite-interval variable.

\section{Step 3 Simulation of crack-face closure}

The crack-face closure effect is characterized by introducing a contact force at the compressive edges of the crack faces. Accordingly the constraint condition of the closure is that the normal displacement of the crack faces at the compressive edge must remain equal or greater than zero, that is, no material penetration or overlapping should occur at the compressive edges of the crack faces. The contact force can be translated to the mid-plane of the shell, accompanied with an additional distributed bending moment. Consequently, the mid-plane contact force and the accompanied moment may be considered membrane force and bending moment respectively and added to the stress functions of the integral equations.

\section{Step 4 Determination of crack closure regions}

Due to the curvature effect, crack closure may not always occur on the entire length of the crack, depending on the shell geometry and the bending load. Therefore, it is necessary to determine the closure regions before solving the integral equations. This can be done by computing the contact forces along the crack length, assuming that fulllength closure occurs. If the value of the contact force appears negative somewhere along 
the crack length, it means that the crack faces are not in contact there, that is, crack closure does not occur in the region. For the closure and non-closure regions, the integral equations are different; in the closure regions, the coupled integral equations are reduce to a single integral equation, accompanied with the unknown contact force. The exact boundaries between the closure and non-closure regions are determined iteratively; convergence is achieved when the mixed conditions are met, i.e., the crack face displacement as well as the contact pressure should be equal to zero or greater than zero at every point along the crack length.

\section{Step 5 Numerical solution of the integral equations}

The coupled integral equations are solved for the unknown functions using Gauss-Chebyshev integration formulas. Since the unknown functions in the integral equations are singular at the crack tips, an equivalent representation of the unknown functions is firstly introduced to facilitate the numerical computation. Then a computer code in FORTRAN 90 is used in processing the numerical computation. The stress intensity factor at the crack tip is finally calculated from the solutions of the unknown stress functions.

\subsubsection{A case study}

To demonstrate the effect of crack-face closure on the SIF of a cracked shell under a bending load, a case study of a pressurized cylinder containing an axial crack has been carried out. Both internal and external pressures are applied on the cylinder, which results in variable circumferential stress through the wall thickness and thus produces a 
bending load in the wall. In this case study, to facilitate the analytical solution, the cylinder parameters and the pressure loads are chosen specially to produce pure bending stresses in the circumferential direction of the wall, that is, membrane stresses equals to zero. The developed crack-closure theory is then applied to the solution of this problem. The SIFs in both closure and non-closure scenarios are determined and compared to reflect the significant influence of crack-face closure on the SIFs.

\subsubsection{Finite element solution}

For complex fracture mechanics problems, certain approximations and reasonable assumptions are necessarily made in analytical solutions. For example, in the crack closure theory developed in this thesis, a line-contact assumption is made to simulate crack-face closure at the compressive edges of a shell subjected to a bending load. To stay in fully consistence with the framework of formulating crack problems in shells (Plane sections remain plane in shell bending), the crack closure can only occur at the compressive edge of the crack face, that is, the contact of the crack faces must occur on a line (edge), instead of in an area. However, in reality, the contact of the crack faces of a shell under bending may occur in an area due to the deformation of the shell material. In order to validate the line-contact assumption, three-dimensional finite element analyses (FEA), using the ABAQUS software package, have been carried out to solve the crack problems in shells under bending loads with consideration of crack-face closure. The simulation of crack-face closure is achieved by placing a rigid plate against the crack face, which is stationary when the crack face deforms under a bending load. The initial contact region of the crack faces would be a line, but it may become an area with the deformation 
of the crack faces under a bending load. The real contact region can be examined after the computation. The opening (normal) displacement of the crack face near the crack tip is obtained from the FEA simulation and thus the SIF is determined with certain mathematical treatment. To justify the line-contact assumption in the analytical formulation, the theoretical solutions are finally compared with the FEA results to evaluate the magnitude of possible errors.

The significance of this research lies in the improvement of fracture mechanics treatment to cracked shell with consideration of partial crack-face closure, which represents the physical reality of a cracked shell under a bending load and thereby allows reasonable and accurate determination of the SIFs. Consequently, the new solution may provide a reliable guideline in fracture and failure analyses of shells for various engineering applications.

\subsection{Organization of the Thesis}

This thesis consists of seven chapters in total, with a list of references and four appendices which contain the detailed formulations of each step involved in the theoretical solutions and the FORTRAN code developed for the numerical solutions of the integral equations.

Chapter 1 is a brief introduction to this thesis. The background, significance and objectives of this research are highlighted. The methodologies adopted in this research to solve crack-face closure problems in shells are outlined. 
Chapter 2 is the literature review, which summarizes previous research in the fracture mechanics of shell structures. The history of shell theories is presented from the initial classical theory to the improved deformation shear theory. The deficiency of the existing shell theories is pointed out and the significance of the present research is thereby stressed.

Chapter 3 describes the theoretical formulation of crack-face closure problems in shells under bending conditions. Various shell geometries are studied. The shell material properties are considered in the formulation. The SIFs are determined in both closure case and non-closure case for comparison.

Chapter 4 presents a case study for the application of the developed crack closure theory in this research. A pressurized cylinder containing an axial crack is taken as an example to demonstrate the effect of crack-face closure on the SIF value.

Chapter 5 describes the FEA modeling of crack-face closure in shells under bending loads. The SIFs for different shell geometries are computed based on the corresponding opening displacements of the crack faces near the crack tip. The contact regions of the crack faces are examined to investigate the real behavior of crack-face closure in shells, thus to discuss the effectiveness of the line-contact assumption made in the analytical solutions. 
The discussion on the analytical results and the FEA results is made in Chapter 6. The variations of crack closure behavior in shells with shell parameters and the bending load are analyzed. The SIFs in closure and non-closure cases are compared to demonstrate the significance of the crack-face closure effect. The theoretical solutions and the FEA solutions are also compared to estimate the errors, thus to validate the line-contact approximation in the theoretical solutions.

The conclusions drawn from this research are presented in Chapter 7. 


\section{Chapter 2 Literature Review}

\subsection{Fundamentals of Elastic Shells}

\subsubsection{Geometric definition of shells}

A shell structure is a thin-walled and curved member. It has two prominent geometric features. Firstly, its thickness is much smaller than the other geometrical dimensions, e.g., at least one-order of magnitude smaller than its radius of curvature, such that two-dimensional approximate theories are reasonably satisfactory to describe the load-bearing capacity of the structure [20]. Secondly, it is bounded by two curved surfaces. A mid-surface, which passes middle way between the two bounding surfaces, is usually referred to as the reference surface. The thickness of the shell may or may not be uniform. The general form of a shell may be characterized with two principal curvatures, i.e., the maximum and the minimum normal curvatures of the reference surface, in two mutually perpendicular directions. Finally, as illustrated in Figure 2.1, such a shell geometry may be fully described by the shape of the mid-surface and the thickness function [20] within a Cartesian coordinate system. The points on the middle surface are located by $x=x_{\alpha}, y=y_{\beta}, z=f\left(x_{\alpha}, y_{\beta}\right)$, in which $x_{\alpha}$ and $y_{\beta}$ are orthogonal curvilinear coordinates coinciding with the orthogonal lines of the two principal curvatures of the shell, and $f\left(x_{\alpha}, y_{\beta}\right)$ is the equation of the middle surface of the shell. In the special cases:

(1) When one of the principal curvature radii $\left(R_{1}\right.$ or $\left.R_{2}\right)$ becomes infinite, a cylindrical shell is formed.

(2) When the two principal curvature radii are equal, $R_{I}=R_{2}=R$, a spherical shell is formed. 
(3) When the two curvature radii become infinite, a plate is formed.

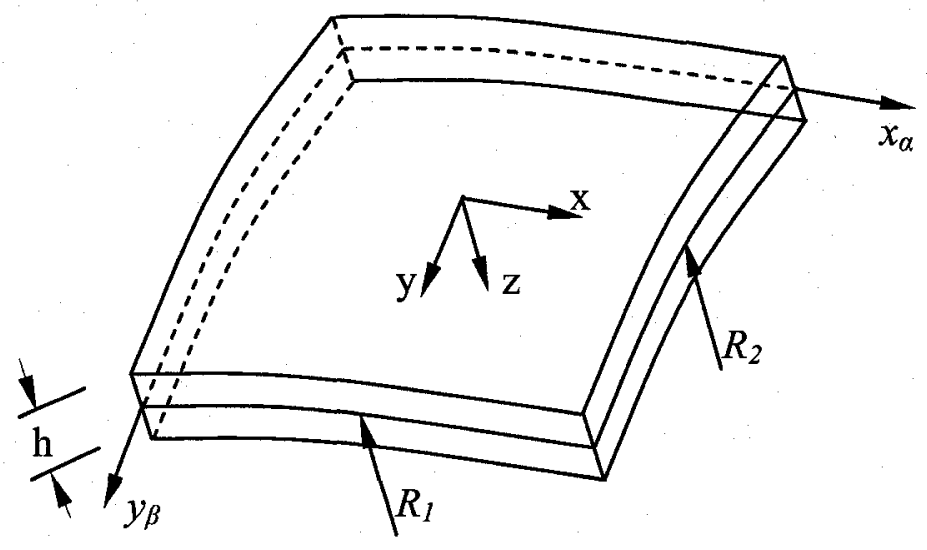

Figure 2.1 General form of shell structures with double curvature

\subsubsection{Stress analysis in shells}

To keep the generality of the theoretical treatment through out this thesis, in dealing with the hypothesis adopted by the different elastic shallow shell theories, homogeneous, isotropic elastic materials and homogeneous orthotropic elastic materials are chosen for discussion. An orthotropic material has three mutually perpendicular planes of elastic symmetry.

The definition of stresses in a shell may follow the same way as in the plane stress case of any elastic body in a Cartesian coordinate system [20], as shown in Figure 2.2. Consider an infinitesimal element with two differential magnitudes of arc lengths, $d s_{x}$ and $d s_{y}$, the element is defined by two pairs of planes which are normal to the mid-surface of the shell. The origin of the Cartesian coordinate system is located at a corner of the 
element with the $x$ and $y$ axes tangent to the lines of principal curvatures, and $z$ perpendicular to the mid-surface. Due to the hypothesis that governs the thin shell theory, i.e., both the stress and strain in the transverse normal direction of the mid-surface, $\sigma_{33}$ and $\varepsilon_{33}$, are null, a plane stress condition is generally valid for thin elastic shell. However, the transverse shear stress and strain do exist, which are taken into account in some high order shallow shell theories.

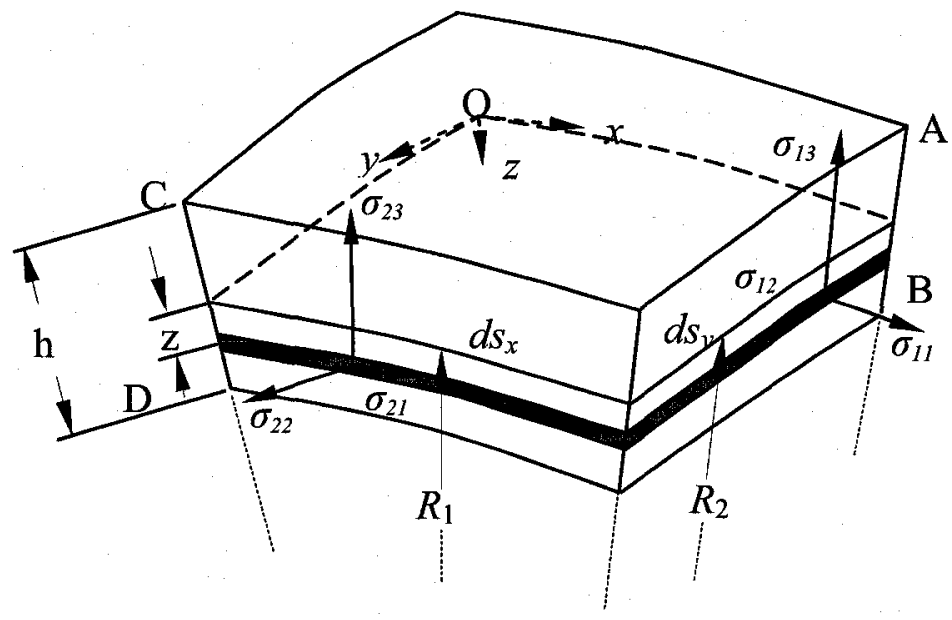

Figure 2.2 Definition of stresses in an infinitesimal element of a shell

The stresses acting on the plane faces of the element and the related strain components are $\sigma_{11}, \sigma_{22}, \sigma_{12}, \sigma_{13}, \sigma_{23}$, and $\varepsilon_{11}, \varepsilon_{22}, \varepsilon_{12}, \varepsilon_{13}, \varepsilon_{23}$ respectively. Without considering thermal stresses, the generalized Hooke's law gives:

$$
\begin{gathered}
\varepsilon_{11}=\frac{\sigma_{11}}{E_{1}}-\frac{v_{12}}{E_{2}} \sigma_{22} \\
\varepsilon_{22}=\frac{\sigma_{22}}{E_{2}}-\frac{v_{21}}{E_{1}} \sigma_{11}
\end{gathered}
$$




$$
\begin{gathered}
\varepsilon_{12}=\frac{\sigma_{12}}{2 G_{12}} \\
\varepsilon_{13}=\frac{\sigma_{13}}{2 G_{13}} \\
\varepsilon_{23}=\frac{\sigma_{23}}{2 G_{23}}
\end{gathered}
$$

In general, two types of actions, namely, membrane action and bending action of a shell structure are distinguished in attempting to understand the stresses induced in a shell under external loads. The membrane action carries the external loads by inducing internal forces in the reference surface of the shell only, whereas the bending action carries the external loads by moments. In shell theories, it is convenient to define the internal forces or moments acting on the sides of a shell element as forces or moments per unit length and these are termed as stress resultants and stress couples respectively. As demonstrated in Figure 2.3, the membrane stress resultants are indicated with respect to the mid-surface, where $N_{11}$ and $N_{22}$ are two such resultants, being forces per unit length which act normally to the sides $\delta y$ and $\delta x$ respectively, while $N_{12}$ and $N_{21}$ are shear forces per unit length acing in the plane of the sides $\delta y$ and $\delta x$, respectively.

However, the membrane stress resultants are insufficient to predict the true elastic behavior of a shell element since of necessity the external load must give rise to flexure or bending of the shell and such bending may only be resisted by internal moments and forces induced in the shell, thus the bending condition of the shell structure results, as demonstrated in Figure 2.4, where the stress couples $M_{11}$ and $M_{22}$ are bending moments, 
while $M_{12}$ and $M_{21}$ are torsional moments respectively. $V_{1}$ and $V_{2}$ are the transverse shear stress resultants.

Although for demonstration purposes, one may isolate the membrane and bending theories as though either membrane or bending action could occur independently, it is incorrect in analyzing the elastic behavior of a shell mathematically. The general description of the reaction of a stressed elastic shell may be demonstrated as in Figure 2.5, where both membrane and bending stress resultants are given with respect to the mid-plane of a shell.

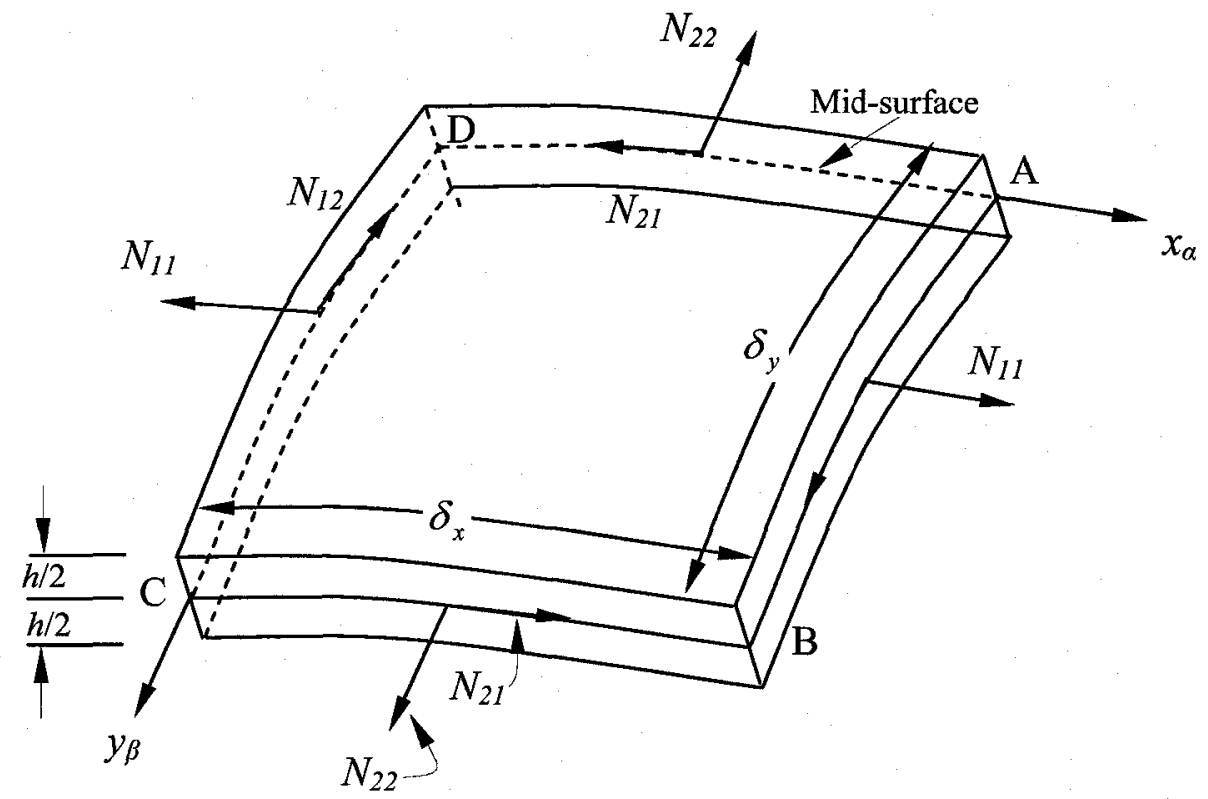

Figure 2.3 Membrane action of a shell 


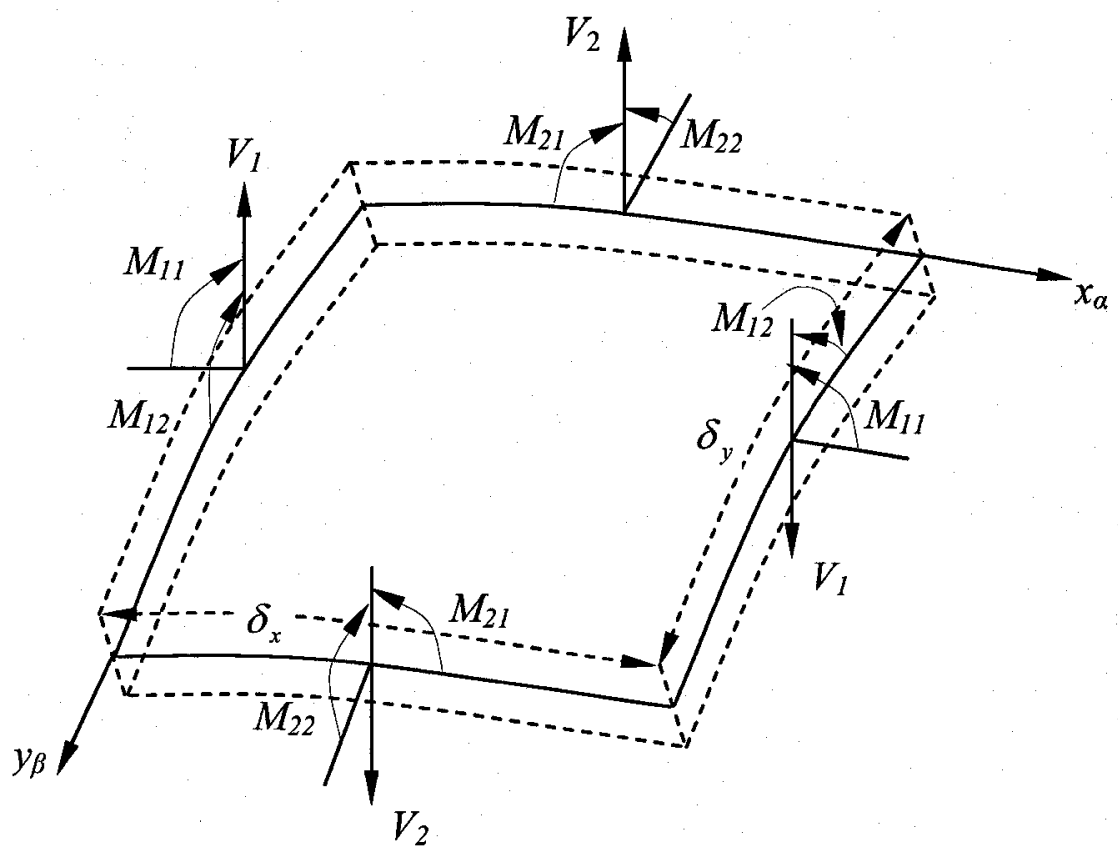

Figure 2.4 Bending condition of a shell

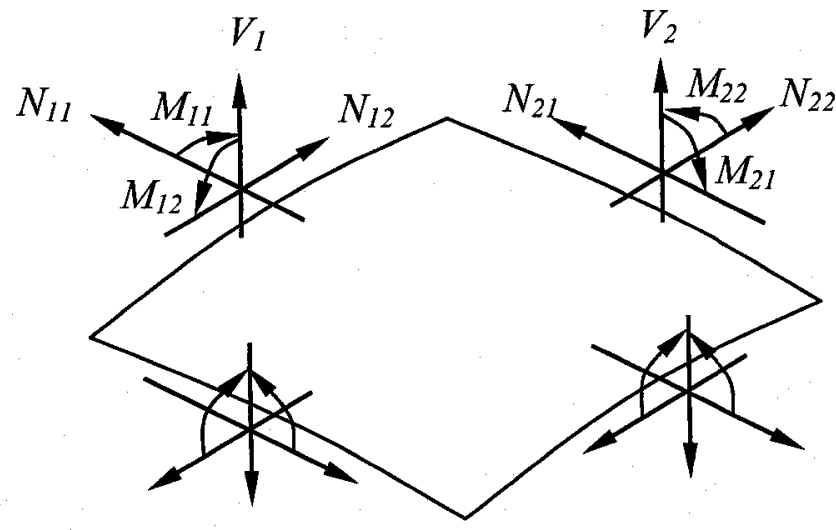

Figure 2.5 Stress resultants and stress couples in a shell 
Since the strains and stresses are predicted linearly distributed across the thickness of a thin elastic shell [20], an expression for the stress resultants in terms of the stresses may be derived by first solving eqn (2.1) for the stresses, and then integrating them through the thickness of the shell. Thus, by eliminating the variations of stresses with respect to the thickness, a two-dimensional theory with only stress resultants and stress couples is obtained. The complete set of stress resultants and stress couples in terms of stress are expressed as [20]

$$
\left\{\begin{array}{c}
N_{11} \\
N_{22} \\
N_{12} \\
N_{21} \\
V_{1} \\
V_{2}
\end{array}\right\}=\int_{-h / 2}^{h / 2}\left\{\begin{array}{l}
\sigma_{11}\left(1-z \kappa_{2}\right) \\
\sigma_{22}\left(1-z \kappa_{1}\right) \\
\sigma_{12}\left(1-z \kappa_{2}\right) \\
\sigma_{21}\left(1-z \kappa_{1}\right) \\
\sigma_{13}\left(1-z \kappa_{2}\right) \\
\sigma_{23}\left(1-z \kappa_{1}\right)
\end{array}\right\} d z
$$

and

$$
\left\{\begin{array}{l}
M_{11} \\
M_{22} \\
M_{12} \\
M_{21}
\end{array}\right\}=\int_{-h / 2}^{h / 2}\left\{\begin{array}{l}
\sigma_{11}\left(1-z \kappa_{2}\right) \\
\sigma_{22}\left(1-z \kappa_{1}\right) \\
\sigma_{12}\left(1-z \kappa_{2}\right) \\
\sigma_{21}\left(1-z \kappa_{1}\right)
\end{array}\right\} z \cdot d z
$$

where $\kappa_{1}=1 / R_{1}$ and $\kappa_{2}=1 / R_{2}$. It is noted that even though $\sigma_{12}=\sigma_{21}$, shearing forces $N_{12}$ and $N_{21}$ are not generally equal, nor are twisting moments $M_{12}$ and $M_{21}$, because in general $R_{1} \neq R_{2}$. For thin shells, however, the shell thickness $h$ is small compared to the curvature radii $R_{1}$ and $R_{2}$, consequently $z / R_{1}$ and $z / R_{2}$ are significantly less than unity and may be neglected. On this basis, from eqns (2.2) and (2.3), one may obtain $N_{12}=N_{21}$ and $M_{12}=M_{21}$. 


\subsubsection{Mathematical theory of shallow shells}

For an elastic shallow shell having double curvature, the mathematical theory basically contains the formulation of the equilibrium equations as well as strain and displacement relations. The differential equations of equilibrium are given as

$$
\begin{aligned}
& N_{i j, i}+p_{i}=0, \\
& V_{i, i}+\left[z_{, j} N_{i j}\right]_{, i}+q=0, \\
& V_{i}=M_{i j, i},
\end{aligned}
$$

where $p_{1}, p_{2}$, and $q$ are the components of the load intensity in $x, y$ and $z$ directions respectively, $N_{i j}, M_{i j}$, and $V_{i j}$ are the stress resultants, stress couples, and transverse stress resultants respectively. Throughout this chapter, the Latin indices $i, j$, and $k$ in all equations take the values of 1 and 2 only. Repeated indices imply summation over all the values of the indices. The comma denotes partial differentiation and $\delta_{i j}$ is the Kronecker delta.

The equilibrium equations are insufficient to determine these resultants uniquely, therefore to achieve a solution additional equations involving strain and displacement have to be considered [21]. Given $w$ as the displacement in the $z$-direction, $u_{i}$ the displacement of the middle surface, and $\beta_{i}$ the change of the slope of the normal to the middle surface along $x$ and $y$ axes respectively, assuming a uniform thickness of the shell, $h$, the stress strain relations take the form as [5]

$$
\varepsilon_{i j}=\frac{1}{2}\left[\left(u_{i, j}+u_{j, i}\right)+\left(z_{, j} w_{, i}+z_{, i} w_{, j}\right)\right]
$$




$$
\begin{aligned}
& =\frac{1}{C}\left[-v N_{k k} \delta_{i j}+(1+v) N_{i j}\right], \\
M_{i j} & =\frac{1}{2} D\left[2 v \beta_{k, k} \delta_{i j}+(1-v)\left(\beta_{i, j}+\beta_{j, i}\right)\right], \\
\beta_{i} & =-w_{, i}+\frac{6}{5 G h} V_{i},
\end{aligned}
$$

where $C=E h, D=E h^{3} /\left[12\left(1-v^{2}\right)\right] ; E, G$, and $v$ are Young's modulus, shear modulus, and Poisson's ratio, respectively.

\subsection{Development of elastic shell theories}

\subsubsection{Kirchhoff-Love hypothesis}

Shell theory originated historically from the special case of elastic plates. The theory traditionally involves the analysis of stress and strain in thin elastic bodies with certain geometric restriction. A rudimentary theory of plates was initially developed by Sophie Germain. It was then corrected and improved by Kirchhoff [22] and now is widely used to analyze thin plates.

Kirchoff developed the strain energy plate bending equation based on two basic assumptions:

(1) The straight lines which are initially perpendicular to the middle plane remain straight in bending, and perpendicular to the middle plane of the bent plate.

(2) The middle plane of the plate does not stretch under transverse load when the plate exhibits a small deflection. 
However, the presence of curvature in a shell causes the load bearing behavior to deviate significantly from that of plates, in that stretching loads will induce both extensional and bending stresses, while bending loads will also lead to both types of stresses [1]. Aron and shortly thereafter Love extended Kirchhoff's idea and laid out the foundations for a thin shell theory [23-26]. Love [27] presented firstly a successful thin shell theory by introducing the approximations that are commonly termed Kirchhoff-Love hypothesis:

(1) The shell is thin, which suggests that the thickness of the shell is everywhere less than one tenth of the curvature radius of the reference surface.

(2) The deflection is small so that quantities containing second and higher-order terms are neglected in comparison with first-order terms in the strain equations.

(3) The transverse normal stress is negligible.

(4) The normal to the reference surface of the shell remains normal to it and also does not change in length during deformation.

As the reflection of Kirchhoff's idea in dealing with thin shell problems, this assumption implies two conditions: firstly, the displacement is linearly distributed across the thickness of the shell; secondly, the transverse normal strain, $\varepsilon_{3}$ and transverse shear strain, $\varepsilon_{13}$ and $\varepsilon_{23}$ are null. In application, the transverse shear stress resultants $V_{n}$ and the twisting moment $M_{n s}$ are combined as Kirchhoff effective transverse shear $V_{n}+\partial M_{n s} / \partial s$, in which $n=1,2$, and the subscript $s$ of twisting moment represents the boundary curve of the reference surface of a shell.

The first three assumptions of the Kirchhoff-Love hypothesis are also held in other shallow elastic shell theories developed in later years. However, the diversity of 
thin elastic shell theories lies in the treatments of the transverse shear strains that are omitted by Love's fourth approximation. Transverse shear deformations are generally considered in stress analysis of moderate or thick plates or shells in order to analyze the problems correctly by using complicated equations of three-dimensional elasticity. To overcome these problems, Reissner $[28,29]$ introduced a theory of plates that takes account of transverse shear deformations in 1944 and 1945. This theory has been used since then to analyze moderately thick plates and provides a reasonable alternative to the full blown equations of three-dimensional elasticity. Following the work by Reissner, several researchers introduced other modified plate theories that include the effect of both the transverse shear stresses and the transverse shear strains [30]. Compared with plate members, the most essential feature of shell structures is the existence of curvature, which subsequently results in complexities not only in the geometrical description, but also in the load distributions. The mechanical properties of a shell element are generally expressed as its resistance to deformation in terms of separable stretching and bending effects. However, loads are carried by a shell in general through a combination of bending and stretching actions. The difficulties in finding a relatively simple way of describing the interaction between the two effects have been troublesome since the beginning of the development of shell theories. It has been proved that this interaction depends mainly on how the transverse shear terms are taken into account in solving shell problems.

Two typical shell theories — the classical theory and the shear deformation theory have been developed and used mainly in engineering design of shell structures. The shear 
deformation theory is not formulated uniquely, depending on the way the transverse shear deformation is treated.

\subsubsection{Classical shell theory}

Love's thin shell theory was later gradually improved and it has been proved in engineering practice as an important tool in the cases where precision is required in regard of strength analysis and economy. Reissner [2-4] finalized the so-called classical shell theory, which was based on a linearized shallow shell assumption, that is, within a certain range of crack shallowness, $S$. A slightly curved shell is often said to be shallow, which means that the middle surface is sufficiently smooth and all points on this surface are close enough to some plane, as demonstrated in Chapter 1 . The solution of the classical shell theory allows satisfaction of only four nature boundary conditions by introducing the Kirchhoff effective shear resultant, instead of discussing the twisting moment and transverse shear items individually.

Reissner's solution of a shell problem results in two simultaneous fourth order differential equations in terms of a stress function $F$ and a load potential $\Omega$ as:

$$
\begin{aligned}
& \nabla^{2} \nabla^{2} F-\frac{h E}{r} \nabla^{2} w=-(1-v) \nabla^{2} \Omega, \\
& D \nabla^{2} \nabla^{2} w+\frac{1}{r} \nabla^{2} F=p-\frac{2 \Omega}{r}
\end{aligned}
$$

where $r$ is the distance from the apex of the shell, and

$$
\nabla^{2} \equiv \frac{\partial^{2}}{\partial r^{2}}+\frac{1}{r} \frac{\partial}{\partial r}+\frac{1}{r^{2}} \frac{\partial^{2}}{\partial \theta^{2}}
$$


$F$ can be obtained from the relevant equation of compatibility and $\Omega$ is expressed in terms of the external load terms $p_{i}$

\subsubsection{Transverse shear deformation theory}

The successful introduction of the transverse shear deformation into plate analysis sheds lights on the research on thin elastic shells. The equivalent theory of transverse shear deformation for plates given by Reissner [31, 32] was applied on thin shells by Naghdi in 1956 [5]. Naghdi's research contributed to a significant progress of thin shell theory by involving transverse shear deformation in his eighth order system of differential equations for elastic shallow shells with small displacements. Naghdi's solution of shell problems yields an eighth order partial differential equation in terms of $w$ alone,

$$
\begin{gathered}
D \nabla^{4} \nabla^{4} w-\left(1-\lambda \nabla^{2}\right)\left[\left(\nabla^{2} z\right) \nabla^{2}-z_{, i j} \frac{\partial^{2}}{\partial x_{i} \partial x_{j}}\right] \\
\left.\left\{C\left\{2 z_{, 12} w_{, 12}-z_{, 11} w_{, 22}-z_{, 22} w_{, 11}\right\}+\left\lfloor\int p_{1} d x_{1}\right\rfloor_{, 22}+\left\lfloor\int p_{2} d x_{2}\right\rfloor_{, 11}-v p_{i, j}\right\}\right\} \\
\left.=\nabla^{4}\left\{\left(1-\lambda \nabla^{2}\right) q-\left\{z_{, 1} \int p_{1} d x_{1}\right\rfloor_{, 1}+\left\lfloor z_{, 2} \int p_{2} d x_{2}\right\rfloor_{, 2}\right\}\right\}
\end{gathered}
$$

Lately, to solve the crack problems in a spherical elastic shell with radius $r$, Sih and Hagendorf [33] developed a tenth-order system of shallow thin shell equations to account for the transverse shear effect. Sih's tenth order theory differs from Naghdi's eighth order theory in that the former takes into consideration the terms $u_{i} / R$ in the expression of transverse shear strains and $V_{i} / R$ in the membrane equilibrium equations, which are neglected in the latter. By involving the refined expression of transverse shear deformation in solving shell problems, Naghdi and Sih's equations satisfy all five 
individual boundary conditions on a free edge of the shell, which differs significantly from the classical shell theory and further improved the fracture analysis in a shell.

\subsection{Fracture Mechanics of Shells}

\subsubsection{Crack problems in shells}

Since flaws or imperfections are inevitable in any manufactured products due to the material manufacturing process or damages in use, in practice, they are generally treated as "cracks", and the design of shell structures requires the treatment of crack problems from the viewpoint of fracture mechanics. Striping the features of engineering objectives and geometry recognition (cylindrical shells, spherical shells, etc.), shell theories are used to deal with a class of idealized mathematic models to analyze the influence of cracks on the strength of a loaded shell structure, which may be evaluated in terms of different character parameters in the vicinity of the crack tip, depending on the nature of deformation mechanisms. Other than crack tip opening displacement (CTOD) and $J$-integral methods for elasto-plastically deformed materials, SIF contributes largely to the application of linear elastic fracture mechanics. The methods in which SIF is determined depend mostly on the complexity of shell geometry and the loading condition. Analytical methods are applicable on relatively simple geometry and the equations may be solved numerically based on satisfaction of the boundary conditions. In engineering problems of complex geometry and with complicated stress systems, FEA or experimental methods are often used. 
Theoretical treatment of crack problems in shells involves much mathematical complexity. Because of the peculiarity of the fracture problems in shell structures, there are analytical limitations in solving the problems based on existing shell theories. If these limitations are disregarded, the crack problems in shells do not seem to be mathematically tractable [1]. Aside from the linearity of the problems, the limitations arise from the geometry and material behavior of the shell. Therefore, a crack is generally assumed to run through the thickness with the orientation along the principal plane of curvatures of the shell. The relative size of the crack is shallow with respect to the radii of curvature and the dimensions of the shell. The material behavior is limited to linear elastic homogeneous materials.

Under these assumptions, the relevant information in the vicinity of a crack in shell structures may be obtained from a local perturbation problem in which the only external loads are the crack surface tractions. Firstly, the membrane and bending resultants at the potential location of the crack may be solved under given external loads based on existing shell theories without considering the crack. Then the membrane and bending forces are inversely applied on the crack surface as the only tractions of the external loads. Finally, the SIFs may be determined by solving the crack problems locally with the crack face boundary conditions.

\subsubsection{Application of shell theories in fracture analysis}

The classical shell theory was first applied in solving stresses in a spherical shell by Ang, Folias and Williams [34], associating the shell problem with the bending of 
plates. Sih and Setzer [35] studied the general character of the crack tip stress field in a shell and pointed out the coupling effects of extension and bending stresses as well as the intensity of the local stress field. Folias [36] solved the crack problems in spherical shells by utilizing a set of singular integral equations which were developed by Knowles and Wang $[37,38]$ for crack problems in plates. Cauchy type kernels in the singular integral equations were approximated for small values of the shell parameter in the Folias [36] formulation. There were other treatments of shells with a crack such as those presented by Erdogan and Kibler [6], Sih and Dobreff [39]. Their results covered a significantly wide range of shell parameters. The crack problems in cylindrical shell were studied by Folias [8, 10], Copley and Sanders [9], and Erdogan and Ratwani [11, 12].

However, the drawback of the solutions obtained in terms of the classical shell theory are recognized to be that the physical boundary conditions of the crack surface in a cracked thin shell structure are only satisfied partially in the Kirchhoff sense. The twisting moment $M_{n s}$ and transverse shear $V_{n}$ on the crack surface were combined as one item, the Kirchhoff effective transverse shear $V_{n}+\partial M_{n s} / \partial S$. This approach resulted in an unrealistic, hyper-singularity in the transverse shear stress near the crack tip. By defining $r$ and $\theta$ as the polar coordinates at the crack tip, and $K_{I s}$ and $K_{I b}$ as the SIFs referring to respective stress resultants and moment fields for symmetrical loading, the membrane stress resultants for small $r$ are:

$$
N_{11}=\frac{K_{L S}}{\sqrt{2 \pi r}} \cos \left(\frac{\theta}{2}\right)\left[1-\sin \left(\frac{\theta}{2}\right) \sin \left(\frac{3 \theta}{2}\right)\right],
$$




$$
\begin{aligned}
& N_{22}=\frac{K_{I s}}{\sqrt{2 \pi r}} \cos \left(\frac{\theta}{2}\right)\left[1+\sin \left(\frac{\theta}{2}\right) \sin \left(\frac{3 \theta}{2}\right)\right], \\
& N_{12}=\frac{K_{I s}}{\sqrt{2 \pi r}} \cos \left(\frac{\theta}{2}\right) \sin \left(\frac{\theta}{2}\right) \sin \left(\frac{3 \theta}{2}\right),
\end{aligned}
$$

and the local bending and twisting moments are given by

$$
\begin{aligned}
& M_{11}=\frac{K_{I b}}{\sqrt{2 \pi r}}(1-v) \cos \left(\frac{\theta}{2}\right)\left[1-\sin \left(\frac{\theta}{2}\right) \sin \left(\frac{3 \theta}{2}\right)\right], \\
& M_{22}=\frac{K_{I b}}{\sqrt{2 \pi r}} \cos \left(\frac{\theta}{2}\right)\left[(3+v)-(1-v) \sin \left(\frac{\theta}{2}\right) \sin \left(\frac{3 \theta}{2}\right)\right], \\
& M_{12}=\frac{K_{I b}}{\sqrt{2 \pi r}} \sin \left(\frac{\theta}{2}\right)\left[2+(1-v) \cos \left(\frac{\theta}{2}\right) \cos \left(\frac{3 \theta}{2}\right)\right] .
\end{aligned}
$$

Since either of stretching or bending on the shell structure may induce stresses in different types, a united SIF, $K_{I}(z)$, which may vary with the shell thickness coordinate $z$, is necessary to represent the combined stress field at the crack tip, and it may facilitate both stress field investigation and further failure analysis. However, the two types of SIFs in eqns (2.8) and (2.9) cannot be summed linearly due to the difference in the $\theta$ dependence of the stress resultants. This makes it difficult to justify the superposition of membrane and bending solutions in fatigue crack propagation and fracture analysis.

This difficulty in the classical shell theory was overcome by the transverse shear deformation theory $[5,7,14,15,33]$. Sih and Hagendorf [33] developed a tenth order system of equations to account for the transverse shear effect in solving the crack problems in a spherical thin elastic shell. Lately, the shear deformation theory of Naghdi 
was applied by Krenk [13], Delale and Erdogan [14] in solving the problem of a cylindrical shell containing an axial or containing a circumferential crack.

In the research of Delale and Erdogan, they demonstrated that the effect of using the Kirchhoff assumption to approximate the crack surface boundary conditions in shells was the same as in the bending of cracked plates, but it resulted in errors in the computed SIFs and difference of the angular distribution of membrane and bending stress resultants. Then they [7, 15] further applied Naghdi's eighth order theory in solving a through-the-thickness crack problem in spherical shells, considering both isotropic and orthotropic material properties. Hence the asymptotic behaviors of membrane and bending stress resultants around the crack tip are identical and furthermore the transverse shear stress has no singularity.

The equations developed by both Naghdi and Sih in the transverse shear deformation theory satisfy all five individual boundary conditions on a free edge of the shell and represent a three dimensional character in the crack front stresses, which are similar to those found by Sih [40] for a through-the-thickness crack in a thick plate. By solving the integral equation of stress resultants and moments, the membrane and bending stress resultants are found in the same form of $\theta$ dependence, as expressed in eqns (2.10) and (2.11) respectively in terms of their SIFs in the crack tip vicinity,

$$
\begin{aligned}
& N_{11}=\frac{K_{I s}}{\sqrt{2 \pi r}} \cos \left(\frac{\theta}{2}\right)\left[1-\sin \left(\frac{\theta}{2}\right) \sin \left(\frac{3 \theta}{2}\right)\right], \\
& N_{22}=\frac{K_{I s}}{\sqrt{2 \pi r}} \cos \left(\frac{\theta}{2}\right)\left[1+\sin \left(\frac{\theta}{2}\right) \sin \left(\frac{3 \theta}{2}\right)\right],
\end{aligned}
$$




$$
N_{12}=\frac{K_{I s}}{\sqrt{2 \pi r}} \cos \left(\frac{\theta}{2}\right) \sin \left(\frac{\theta}{2}\right) \cos \left(\frac{3 \theta}{2}\right)
$$

and

$$
\begin{gathered}
M_{11}=\frac{K_{I b}}{\sqrt{2 \pi r}} \cos \left(\frac{\theta}{2}\right)\left[1-\sin \left(\frac{\theta}{2}\right) \sin \left(\frac{3 \theta}{2}\right)\right], \\
M_{22}=\frac{K_{I b}}{\sqrt{2 \pi r}} \cos \left(\frac{\theta}{2}\right)\left[1+\sin \left(\frac{\theta}{2}\right) \sin \left(\frac{3 \theta}{2}\right)\right], \\
M_{12}=\frac{K_{I b}}{\sqrt{2 \pi r}} \cos \left(\frac{\theta}{2}\right) \sin \left(\frac{\theta}{2}\right) \cos \left(\frac{3 \theta}{2}\right) .
\end{gathered}
$$

This feature guarantees the linear sum of the two types of stress fields. Thus it allows a united SIF $K_{I}(z)$, as expressed in eqn (2.12), which is consistent with the basic concept in fracture mechanics.

$$
\begin{aligned}
& \sigma_{11}=\frac{K_{I}(z)}{\sqrt{2 \pi r}} \cos \left(\frac{\theta}{2}\right)\left[1-\sin \left(\frac{\theta}{2}\right) \sin \left(\frac{3 \theta}{2}\right)\right], \\
& \sigma_{22}=\frac{K_{I}(z)}{\sqrt{2 \pi r}} \cos \left(\frac{\theta}{2}\right)\left[1+\sin \left(\frac{\theta}{2}\right) \sin \left(\frac{3 \theta}{2}\right)\right], \\
& \sigma_{12}=\frac{K_{I}(z)}{\sqrt{2 \pi r}} \cos \left(\frac{\theta}{2}\right) \sin \left(\frac{\theta}{2}\right) \cos \left(\frac{3 \theta}{2}\right) .
\end{aligned}
$$

Sih and Hagendorf [33] solved the governing equations numerically for both extension and bending loads and demonstrated the SIF variations in terms of the ratio of shell thickness to crack length. The difference between the classical and shear deformation theories was compared quantitatively under both loading conditions. Larger values of SIF were predicted based on the shear deformation theory for a cracked shell 
under extension. There was a dramatic departure of the SIF results from those of the classical theory under a bending load, in which opposite trend of the variations of SIFs was found. Erdogan's theory $[7,14,15]$ differs from that of Sih $[1,33]$ in that the former neglects the terms $u_{i} / R$ in the expressions of transverse shear strains and $V_{i} / R$ in the membrane equilibrium equations. However, the results given by both of them for isotropic shells are nearly identical. The research results of Delale and Erdogan on cylindrical and spherical shells $[12,7]$ indicate that due to the curvature effects the SIFs for shells may be considerably higher than those for plates, giving the same shell thickness and crack length. Later, Delale and Erdogan studied the effects of material orthotropy and Poisson's ratio on the SIFs based on Naghdi's eighth order theory. They formulated a more general case of a specially-orthotropic shell with two nonzero curvatures [15]. Two materials, titanium (mildly orthotropic) and graphite (severely orthotropic), were considered in their study to investigate the effect of material property on the SIFs. The numerical solutions of the problem were obtained by using Gauss-Chebyshev integration formulas. The effects of material orthotropy on the SIFs of cylindrical shells, spherical shells and shells with double curvature were investigated.

\subsection{Crack Closure Theory}

\subsubsection{Crack closure in plates}

One deficiency of the classical and the shear deformation shell theories has been noticed. In their solutions material overlap is allowed at the compressive edges when a bending load is involved, which is physically unrealistic. In reality, crack-face closure on 
the compressive edges may occur as crack faces rotate when a shell or a plate is subjected to a bending load, as illustrated in Figure 2.6.
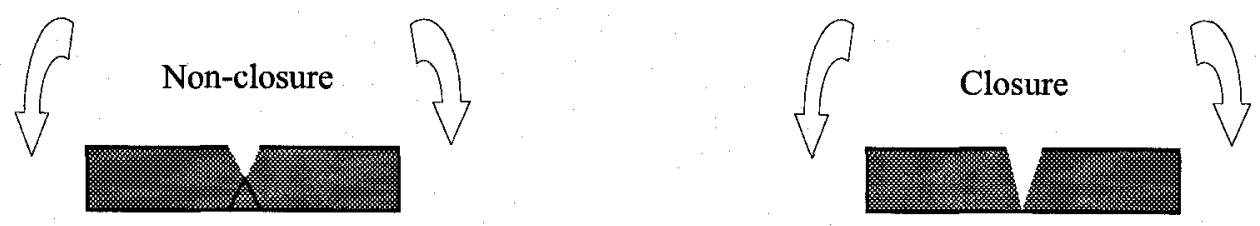

(a)

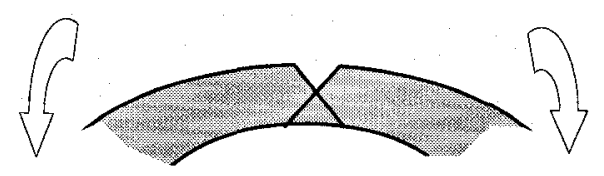

(b)

Figure 2.6 Schematic drawing of crack closure and non-closure in (a) plates and (b) shells

Many loading modes can induce bending stresses in shells. One example is compressing a shell between two plates, as analyzed by Updike and Kalnins [32, 41]. Crack-face closure in plates was first studied by Jones and Swedlow [42], in their numerical solution of the problem of a central crack in a rectangular plate subjected to uniform all around bending. They used a FEA based on Kirchhoff's theory, applying the Kirchhoff's boundary conditions along the crack edge. They modeled the actual physical situation that occurs for the bending problem, that is, as bending occurs, the crack surfaces come together at the compression edges and do not overlap. The crack surface is still modeled as stress free with the same boundary conditions as for the non-closure case, but an additional constraint equation must be added to account for the closure 
phenomenon. This constraint condition takes the form of zero normal displacement at the compression edge of the crack face. Thus, the closure model approximates the three-dimensional contact surface by a two-dimensional contact line at the compression edge of the crack face. This approximation arises due to the inherent assumption that plane sections remain plane in plate bending. Their results show that the effects of crack closure are significant in the problem of bending a plate containing a through-the-thickness crack. They reported a significant increase in crack opening displacement, a redistribution of stresses local to the crack, and an increase in the bending SIF on the tension surface when closure effects were included in the analysis.

Heming [43] improved the solutions of Jones and Swedlow by using the sixth order Mindlin's theory of plate bending later on, which overcame the difficulty in modeling the free surface of the crack with the Kirchhoff's approximation of the boundary conditions. The use of the higher order theory allows for a correct modeling of the stress-free surface of the crack by admitting independent specification of three boundary conditions at any points. Contrary to the conclusion obtained by Jones and Swedlow, his results show a decrease of the crack opening displacement at the tension side. He ascribed the difference to the simplified boundary condition on the crack face inherent in the classical plate theory. Both of their studies on bending a plate assume that crack closure occurs only along the compressive edge of the crack surface, but they did not determine the SIFs.

Alwar and Nambissan [44] conducted a three-dimensional FEA to model crack closure behavior in plates. They determined the SIF by extrapolating the displacement or 
the stresses to the crack tip using near tip expressions and FEA solutions. Their results also show a decrease of crack opening displacement and SIFs. In addition, they examined the exact region of crack closure and demonstrated the influence of crack closure on the SIF. A later experimental investigation by Smith and Smith [45] on crack closure in plates showed that the closure took place over an area and was dominated by the plate geometry.

Young and Sun [16, 17] investigated the crack closure behavior of a through-the-thickness central crack in an infinite plate subjected to all around bending analytically and by FEA. In their analytical solutions, the formulation was based on the classical plate theory and the plane elasticity assumption, and closed form solutions were obtained. By assuming line contact at the compressive edges, the closure condition can be ascertained by applying, along the center line of the crack face, an equivalent uniform force and a uniform moment which is the product of the contact force and half plate thickness, as shown in Figure 2.7. The unknown contact force can be found from the crack closure condition that the resultant normal displacement at the compressive edge due to the equivalent force, the equivalent bending moment and external bending moment is zero.

The whole field solutions for moments and stresses can be obtained by superposing the non-closure solutions and the solutions associated with the equivalent force and moment on the crack surface. The effect of crack closure can be demonstrated by the difference of the crack opening, the moment distribution, and the SIF between the 
closure and non-closure cases. It is shown that the crack opening displacement at the tension edge is reduced because of the closure at the compressive edge and the crack closure reduces the SIF significantly of plates under bending.

In their FEA modeling, the constraint condition of crack-face closure is also that the normal displacement at the compression edge of the crack face is zero. The SIF was obtained by superposing the membrane component and the bending component which were evaluated indirectly from the strain energy release rate using the FEA results of nodal forces and nodal displacements. The FEA results are in good agreement with the analytical solutions.

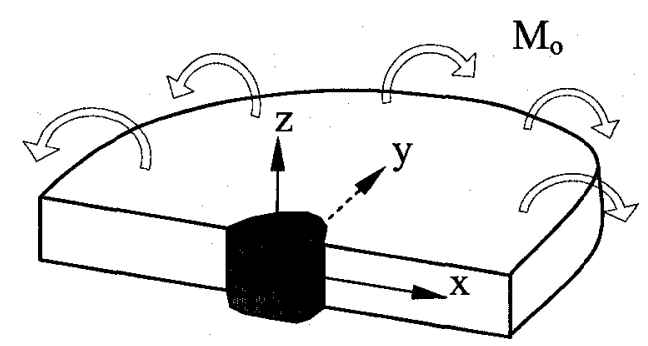

(a)

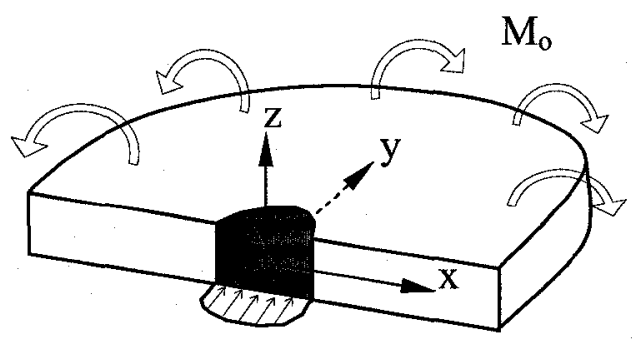

Contact force $P(x)$

(b) 

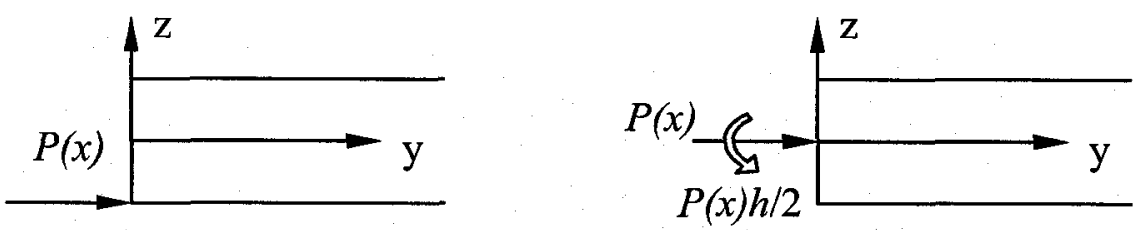

(c)

Figure 2.7 Modeling of crack-face closure of a plate under bending: (a) crack opening without closure, (b) crack opening with closure and (c) equivalent force and moment at the mid-plane of the plate

\subsubsection{Crack closure in shells}

The crack closure behavior in a shell structure differs significantly with what was obtained in a plate. Liu et al $[18,19]$ investigated crack closure behavior in an isotropic spherical shell containing a through-the-thickness crack under a bending load by using the similar ideas of Young and Sun in treating plate bending problems. In their solution, the assumption of line-contact at the compressive edge was still employed in formulating crack closure in shells. However, due to the curvature effect, the assumption of uniform contact force along the crack length of the crack surface which was applied in Young's solution has been modified; instead it was computed as an unknown function of the crack length coordinate by solving the problem with adding the constraint condition of crack-face closure. It was interesting to notice that the contact force was non-uniform along the crack length and crack closure behavior varied with the curvature of the shell. The closure may occur over the full length or only some segments of the crack in the shell (characterized by negative contact force), termed partial crack closure, depending 
on the geometry of the shell and the nature (direction) of applied bending load, as shown in Figure 2.8.

Liu et. al. formulated crack closure problems in shells by extending the shallow shell theories of Delale and Erdogan [7, 15], incorporating the effect of crack-face closure. The solution of SIF was obtained by solving a mixed-boundary value problem iteratively, that is, along the crack length, either the normal displacement of the crack face at the compressive edges is equal to zero or the contact pressure is equal to zero. It was found that crack-face closure influenced significantly the magnitude of the SIFs; it reduced the bending component but increased the membrane component; the maximum SIF was reduced to below the closure-free solution, as seen in Figure 2.9. The controlling parameter $\lambda$ represents curvature of the shell.

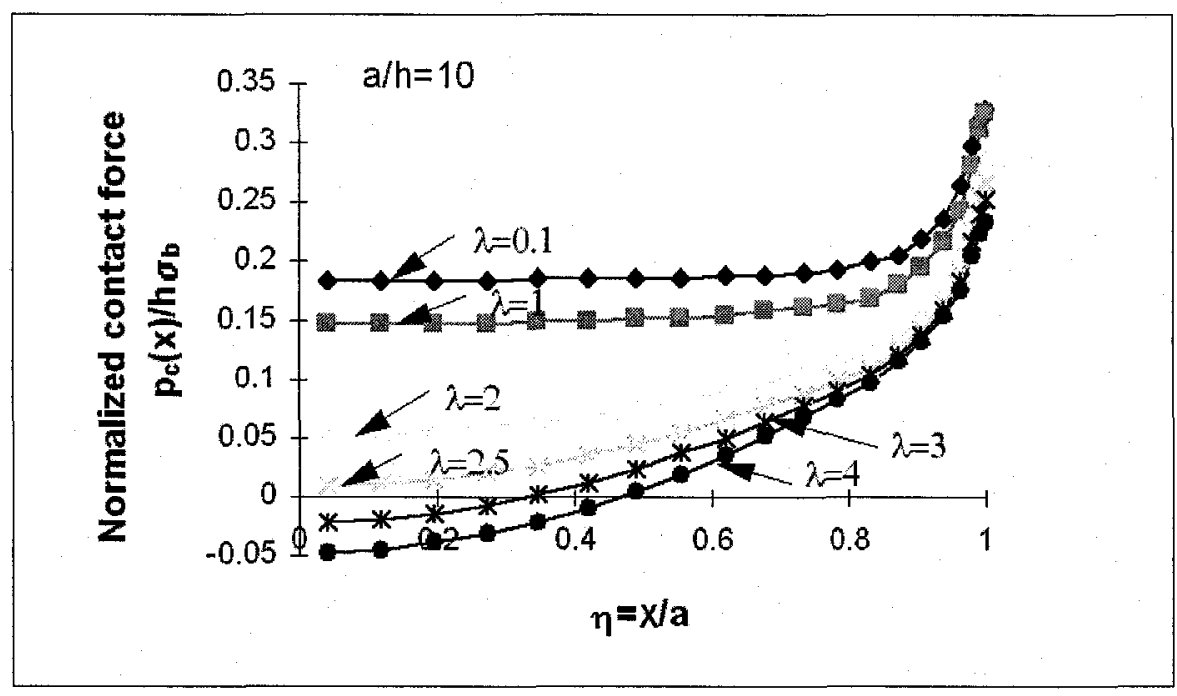

Figure 2.8 Distributions of the contact force along the crack for isotropic spherical shells under positive bending (closure at inner surface) assuming full-length closure 


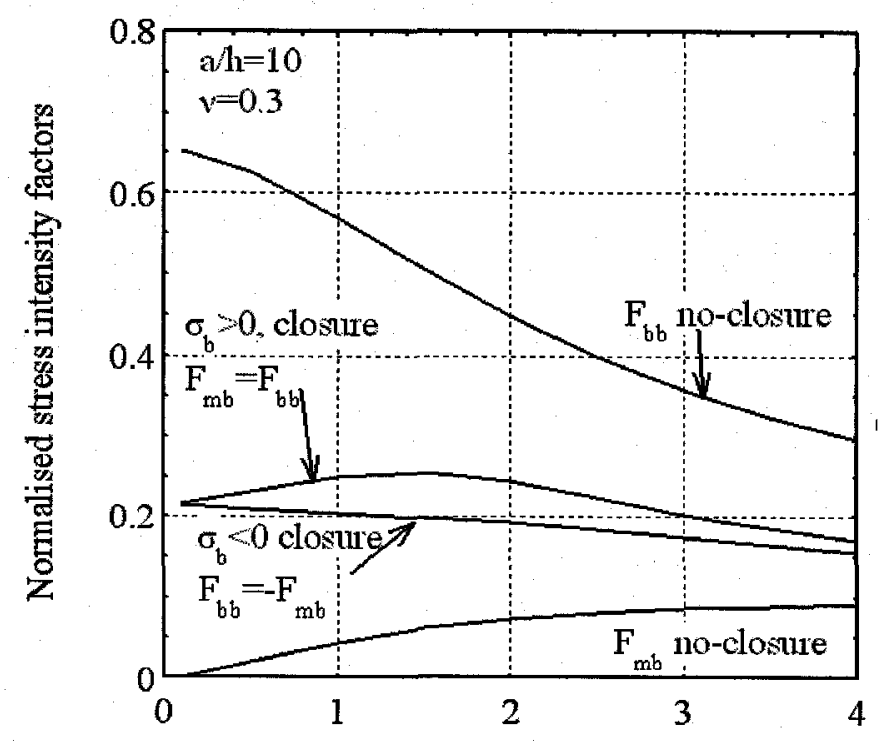

$\lambda$

(a)

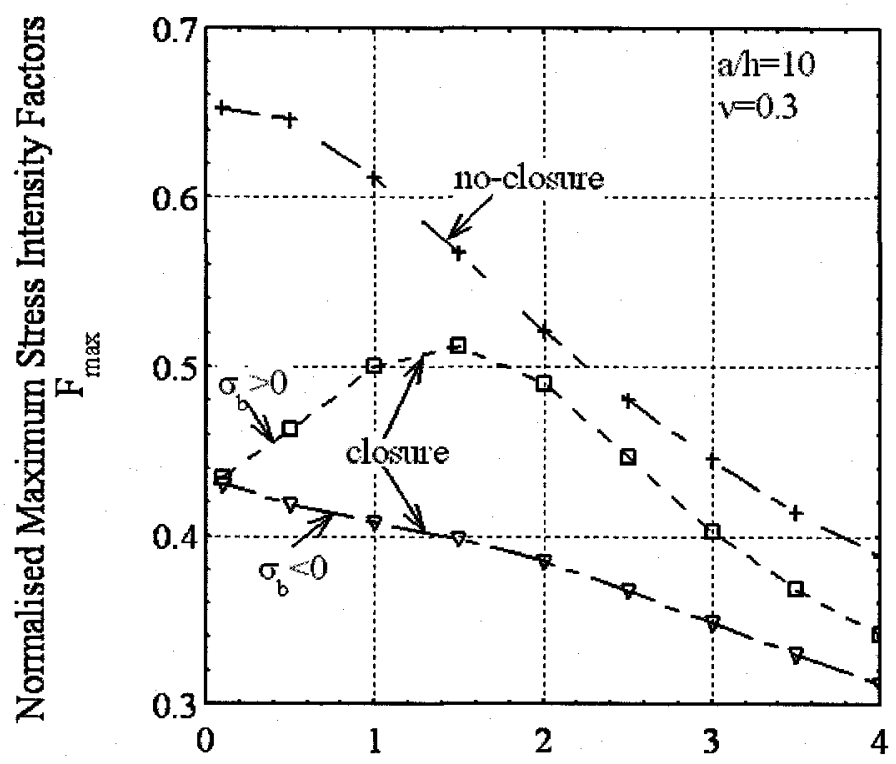

$\lambda$

(b)

Figure 2.9 Comparison between closure and non-closure solutions for isotropic spherical shells under bending: (a) stress intensity factor ratios and (b) maximum stress intensity factor ratios 


\subsection{Finite Element Methods for Shell Problems}

\subsubsection{Application in fracture mechanics}

The solutions of engineering problems usually follow either analytical or numerical methods. In the analytical methods, the governing equations may be solved in a closed-form for simple cases such as bending of beams, or in approximate methods using series solutions to governing differential equations for more complex geometries or more complex loading conditions such as plates and shells under bending.

However, analytical methods are only applicable to relatively simple conditions. Due to the complexity of geometry, loading and boundary conditions of the structure, or material properties, it is most likely that a specific engineering problem may not be solved with an analytical method. The limitation of the analytical solutions of SIF published in text books lies in the hypothesis to facilitate the solution and the difficulties in deciding whether or not the validity of the hypothesis has been beyond, which may subsequently result in the loss of either accuracy or the generalization of the problem.

The inability of analytical solutions of SIF has strongly prompted the application of numerical methods in solving for SIFs. Several numerical methods, for example, boundary element method, finite difference method, and finite element method, have contributed to the solutions of engineering problems. The finite element technique has proved to offer the best alternative to analytical methods due to its capability of solving complex problems with general geometry, loading and boundary conditions. It uses 
matrix algebra to solve the system of simultaneous equations. Thus the FEA method is also known as the matrix method in literature. Given enough computing resources and well defined and meshed models, FEA methods can produce reliable solutions from the engineering point of view.

The FEA method has become a useful technique for solving linear elastic fracture mechanics problems. The finite-element approaches to fracture mechanics can be described as either direct or indirect methods [46]. In the former, the SIFs are calculated directly from the FEA solution, while in the latter, an energy-release rate is calculated and SIFs inferred from it. Direct methods can be used with either conventional (nonsingular) elements or with elements containing a stress singularity at the crack tip. In the former case, it is necessary to curve-fit the stress or displacement away from the crack tip and extrapolate to the crack tip to determine the SIF. In the latter case, it is calculated directly. Shapes of elements include triangular [47], quadrilateral [48] and polygonal [49]. The indirect approach determines the SIF based on computing the change in potential energy for two crack lengths $[50,51]$ or computing a change in compliance for two crack lengths $[52,53]$.

Other approaches are based on evaluating the change in the stiffness matrix due to crack extension and require only one analysis [54-56]. The $J$ integral evaluated for a linear-elastic material, is identical to the energy-release rate and offers a convenient way of evaluating the energy-release rate with a single analysis [57]. 


\subsubsection{Modeling of shell problems}

The FEA technique has become the dominant technique for solving shell problems. The essential motivation of modeling a three-dimensional continuum with specific finite elements for shell structure analysis, also known as shell elements, seems obvious. Firstly, shell elements may provide engineers with reasonable solutions for problems involving shell structures with largely reduced analysis cost. Secondly, the complexity of the analysis procedure in interpretation of the numerical results may also be reduced. These advantages have initiated the research activities of shell elements, and still motivate researchers up to now to challenge largely open problems arising in the modeling of shell structures, especially in the field of non-linear mechanics.

Three basic element types have evolved for general shell analyses [58]: (1) flat shell elements from the superposition of plane stress elements to plate bending elements, (2) curved shell elements formed from inserting assumed displacement fields into the selected thin shell theory, and (3) degenerate elements generated by applying constraints or modifying continuum elements to comply with thin shell assumptions without resorting to a specific shell theory.

Flat elements were the first to be employed as they required no additional development work, simply a superposition. These are primarily low order elements in the form of triangles. Sometimes four triangles are assembled into a quadrilateral element. The major detrimental factor in the use of flat elements is the excess stiffness present in low order triangular elements. Use of higher order elements is needed to overcome this 
excess stiffness, however then the error present in the geometric approximation associated with flat elements has a constraining influence on the minimum number elements that must be used.

Curved elements derived from a selected shell theory were the main focus in the sixties. This activity was motivated to overcome the shortcomings of the flat shell element approach. The early curved elements used shallow shell theory [59]. These shallow elements were superseded later by some elements capable of handling the shells with large curvature [60]. However, this type shell element introduced some new problems; for example, they could not maintain compatibility along inter-element boundaries and generate strain under rigid body motion.

The advent of degenerate shell elements began with the Ahmad's research [61]. This element received reasonable success for moderately thick shells. This model introduced normal rotations as independent unknowns. The process was cast in a Mindlin type theory which includes shear deformations. When applied to thin shells where Kirchhoff type conditions are to prevail, however, the system was not that successful, because of the excess stiffness characteristics, a phenomenon termed membrane and/or shear locking.

Literature review shows that the development of shell elements falls into several chronological stages. Since the first bending element introduced in 1961, development of shell elements was firstly based on Kirchhoff-Love kinematical hypothesis, namely that 
the transverse shear strains are null. Kirchhoff-Love's assumption states that the material fiber orthogonal to the mid-surface in the undeformed configuration remains straight, unstretched and always orthogonal to the mid-surface of the shell during deformation. The designer of Kirchhoff bending elements took the advantage of a simplified mathematical model, unfortunately, which also had limitation in the application of the code [62]. The second stage of the development of shell elements came from another direction, that is, extending isoparametric mapping to shell elements. The first degenerated eight-node isoparametric shell element was published by Ahmad [63] in 1969. The basic idea behind the word "degenerate" is that the six translational degrees of freedom (DOF) at corresponding nodes of the twenty-node continuum element are replaced by three translations and two rotations, then these five new displacement DOF are interpolated from outside nodes to points on the mid-surface of the shell. Due to the precise correspondence of the procedure in degenerating the brick element to the classical plate/shell kinematics, this group of shell elements is also called Mindlin or Reissner-Mindlin shell elements showing their strong dependence on Mindlin kinematical assumptions. Reissner-Mindlin kinematical assumption is less strict than Kirchhoff-Love assumption, which states that the material fibre orthogonal to the mid-surface in the undeformed configuration remains straight, unstretched during deformation, but may not always be orthogonal to the mid-surface. An important advantage of Mindlin shell elements is the inclusion of transverse shear deformations in its model. However, Ahmad element did not show many advantages over the lower order Kirchhoff elements unless "reduced order integration" technique was incorporated [64]. The accuracy of the isoparametric element in shell structure was dramatically improved 
as reduced integration method is applied. However, this technique was also reported to suffer from a spurious zero-energy mode, hourglassing, which leads to rank deficiency and has to be suppressed with specific treatments, for instance, stabilization matrix method or assumed independent strain methods $[65,66]$.

Among all the difficulties appearing in the development of shell elements, numerical lockings, namely shear and membrane lockings, have mostly disturbed FEA modeling of thin shell structures. Shear and membrane lockings obtain their name from the excessive stiffness of the elements. Numerical locking on shell elements may be discussed within the framework of the asymptotic behavior of shell models, which happens when the thickness of the shell becomes small. As the thickness of a shell structure decreases, the behavior of the shell changes dramatically and falls into different categories, namely the membrane-dominated and bending-dominated cases [67]. The asymptotic behavior of the shell model is also decided mostly by the shell geometry and boundary conditions. A seemingly very small change of the boundary conditions or shell geometry can result in a totally different response. Thus, the corresponding finite element approximations tend to dramatically deteriorate when the thickness of the structure decreases. Numerical lockings have attracted wide investigations, and plenty of remedies have been claimed to overcome these locking difficulties [68-70]. However, the present available shell elements all show sensitivity to the asymptotic behavior of the shell. A theoretically well addressed finite element schemes for shell analyses may only work quite well for certain shell problems, but it may have very poor performance for other cases. The knowledge of the actual physical sensitivity of shell structures and the 
numerical unreliability are still insufficient. Thus, an effective finite element scheme that may be applicable to more general cases of shell analysis, that is, independent on the diversity of shell behavior and boundary conditions, is difficult to achieve [71].

FEA methods which characterize element displacements with variable polynomial degree, $P$, were first extended to shell analysis by Szabo and Sahrmann in 1988 [72]. The $P$ element facilitates the implementation of adaptive mesh refinement because it increases the polynomial degree of elements much easier rather than increases the number of element as in the traditional finite methods. Leino et al. claimed that the $P$ element technology might produce significant high accuracy [73].

Up to now, most commercial shell element models rely on one basic simplification of the exact three-dimensional continuum of shell structures, which trade the displacement distribution across the thickness of the structure with some kinematical assumptions. Thus, the discretization in the transverse direction of the shell is generally omitted in current shell elements. However, this method might be incapable for some specific engineering problems, where the transverse deformation has to be considered in a more precise way.

\subsubsection{Current challenges}

One of the inabilities of current shell elements lies in the fundamental kinematics inherent in all shell theories, namely that the straight fibres normal to the mid-plane of the shell do not change their length after deformation. This assumption excludes material 
stretching in the transverse direction of the shell, which leads to only plane stress condition possible. The FEA based on this assumption may provide good solutions in most cases of shell problems. However, sometimes the corresponding transverse shear stresses may play an important role in the structures and the corresponding engineering problems cannot be treated with current shell elements. For instance, the simulation of sheet metal forming in which highly non-linear contact condition is involved, or the analysis of delamination of laminated shell structures, have to provide explicit description of the transverse deformations.

Recently, a considerable amount of research effort has been focused on the finite element techniques in dealing with these non-linear mechanics, in which the application of a three-dimensional continuum-based shell element shows acceptable solutions in this field [74-79]. An essential difference between solid-shell elements and the degenerated-shell elements is that the nodes of the previous one possess only three displacement degrees of freedom, which helps to exempt the laborious task of defining algebraic constraints or introduce solid-to-shell transition elements. Moreover, solid-shell elements provide a complete three-dimensional state of stress, in which an explicit integration through the thickness of the shell is performed. Thus, solid-shell elements are considered suitable for shell analyses involving complex geometrical deformation, for example, sheet metal forming, or when plane-stress condition is difficult to be imposed. To fulfill three-dimensional state of stress in a shell structure, for relatively simple shell models, three-dimensional continuum elements have also been applied in shell fracture mechanics analyses involving crack surface constraints [18, 19]. The application of 
solid-shell elements or continuum solid elements in shell fracture analyses is also bothered by some difficulties [77]. Besides shear and membrane lockings, trapezoidal and thickness lockings must also be alleviated to enhance the reliability of solid-shell elements in solving thin shell problems. Trapezoidal locking happens when lower-order solid-shell elements such as bilinear quadrilaterals are used to construct curved shells. The cross section of the quadrilaterals appears in a trapezoidal shape. Thickness locking is due to the parasitic strain in the thickness direction, which causes the bending of the element too stiff. Thus a false plane strain state is imposed instead of the physical plane-stress state. The negative effect of the above mentioned locking behaviors on the quality of the shell structure analyses with shell elements is crucial. After about two decades of investigation in the locking difficulties, advanced finite element techniques have been developed to overcome these element deficiencies, which include reduced integration, stabilization, assumed strain, hybrid strain or stress methods with respect to different locking behaviors [68]. It is extensively reported that these methodologies have achieved success for many cases of shell analyses.

However, nowadays the challenge in the shell FEA is also due to the shortage of mathematical techniques available in shell structure analyses, which is essential to provide us the complete mathematical solution that is required to design a general-purpose shell element. Thus, the justification of the performance and the reliability of existing shell elements may only be carried by means of classical test problems, which are also called benchmarks in the text. Besides the difficulties of very restricted source of benchmarks of shell analyses, a deep insight of the nature of the exact 
solutions of the test problems is crucial, which are usually unavailable for a certain type of shell problems.

\subsubsection{Determination of stress intensity factors}

To obtain accurate solutions of SIF using FEA, the crack tip singularity has to be modeled adequately. Crack elements or very fine mesh in the crack tip area are generally applied in order to provide a detailed description of crack tip deformation [80]. Various methods of determination of SIFs in FEA may be found in the text, for example, asymptotic methods or energy methods [81]. The essential concept in the asymptotic methods is very straightforward, which is based on the analytical equation of stress and displacement fields around the crack tip where SIFs dominate the expression of the stress field at the vicinity of crack tip. Once the numerical data of stress and displacement fields at the vicinity of crack tip are retrieved from the FEA solution, the crack opening displacement becomes available at any points on the crack surface. The SIFs are then readily to be calculated [82]. Theoretically, SIF at a certain crack tip is defined as a unique value irrespective of the distance from the crack tip, $r$, in a static analysis once the loading and boundary conditions are specified. However, the numeric results computed in the asymptotic method usually predict a curve of SIFs versus $r$ rather than a single exact value of SIF, which generally appears to become linear at the vicinity of the crack tip. To improve the accuracy of the SIF solution, linear extrapolation techniques are normally used in order to find a consistent and precise numerical estimation of SIFs [82]. The SIF is determined from the FEA result using the following displacement-based definition, 


$$
K_{I}(z)=\frac{E}{4\left(1-v^{2}\right)} \frac{u_{\theta}(r, \pi, z) \sqrt{2 \pi}}{\sqrt{r}},
$$

where $u_{\theta}$ denotes the crack face opening displacement along the plane $\theta=\pi$, and $(r, \theta, z)$ are cylindrical coordinates of a generic point in a cylindrical coordinate system attached at the crack tip [83], see Figure 2.10. The stress intensity factor, $K_{I}$, varies with the shell thickness coordinate, $z$.

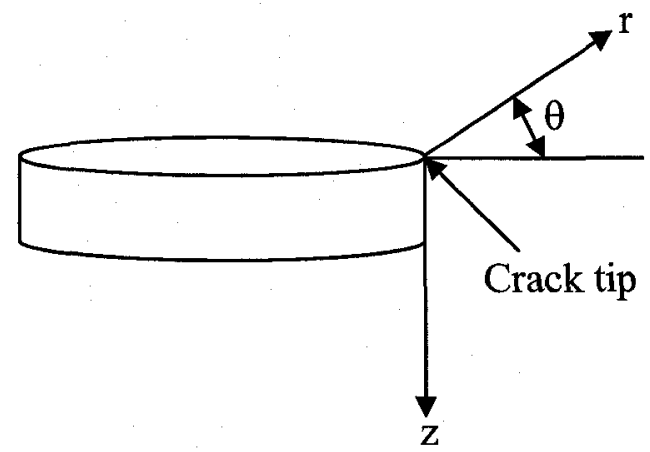

Figure 2.10 Cylindrical coordinate system at the crack tip

Another approach to calculate SIF is based on energy methods, in which the pathindependent integral, $J[57]$, is commonly used. It is well recognized that SIFs satisfy a certain relationship with $J$-integral [82] within the field of linear elastic fracture mechanics. Once the $J$ factors are calculated in FEA, the SIFs are obtained. Due to the unique path-independent character of the specific integral around the crack tip, $J$-integral, this method takes the advantages of a lower cost in FEA compared with other methods because the local knowledge of the deformation in the vicinity of the crack tip is not crucial any more. 


\subsection{Composite Shells}

\subsubsection{Composite materials for shells}

Composite materials are ideal for structures that require high strength-to-weight and stiffness-to-weight ratios. Aircraft and spacecraft are typical weight-sensitive structures in which composite materials are cost-effective. Structures composed of composite materials are now among the most important structures used in modern aerospace industry. Such lightweight and highly reinforced structures are also being increasingly used in mechanical and civil engineering applications. With the advent of composite materials, shell structures are again emerging as an economic alternative to other forms of structure.

There are three groups of composite materials [84]: (1) fibre composites in which the reinforcement is in the form of fibers and the fibers are either continuous or discontinuous in form, chopped fibers, short fibers, etc. Normally, fibers are much stiffer and stronger than the same materials in bulk form because fibers have fewer internal defects. (2) particle composites in which the reinforcement is made of particles and the particles can be either metallic or non-metallic. A particle, in contrast to fibers, does not have a preferred orientation. (3) laminated composites which consist of layers of at least two different materials that are bonded together.

\subsubsection{Material orthotropy}

Among the types of composite materials, laminated composites are most popular in engineering shell structures. A laminate is a collection of laminae stacked to achieve 
the desired stiffness and thickness, as shown in Figure 2.11. Lamination is used to combine the best aspects of the constituent layers in order to achieve a more useful material [84]. The ability to structure and orient material layers in a prescribed sequence leads to several particularly significant advantages of composite materials compared with conventional monolithic materials. The most important among these is the ability to tailor or match the lamina properties and orientations to the prescribed structural loads. The properties that can be emphasized by lamination are strength, stiffness, corrosion resistance, low weight, etc.

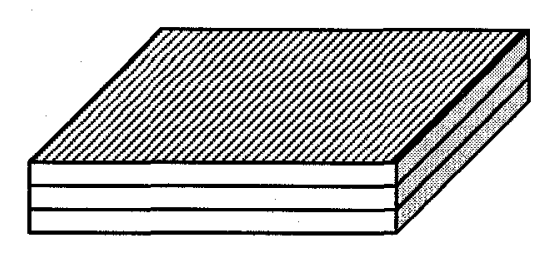

Laminate

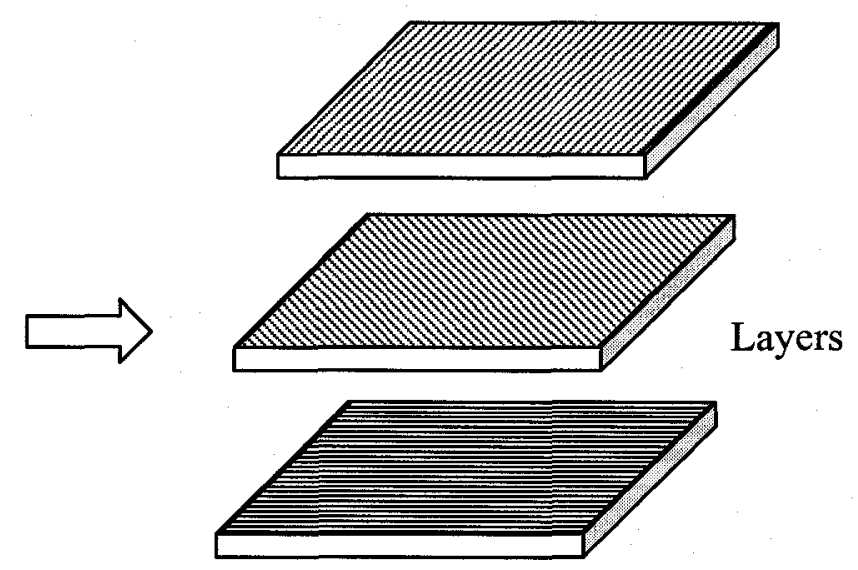

Figure 2.11 Laminate construction

Unidirectional fiber-reinforced laminae can be stacked so that the fibers in each lamina are oriented in the same or different directions [85], as shown in Figure 2.12. The layers are usually bonded together with the same matrix material as that in a lamina. 


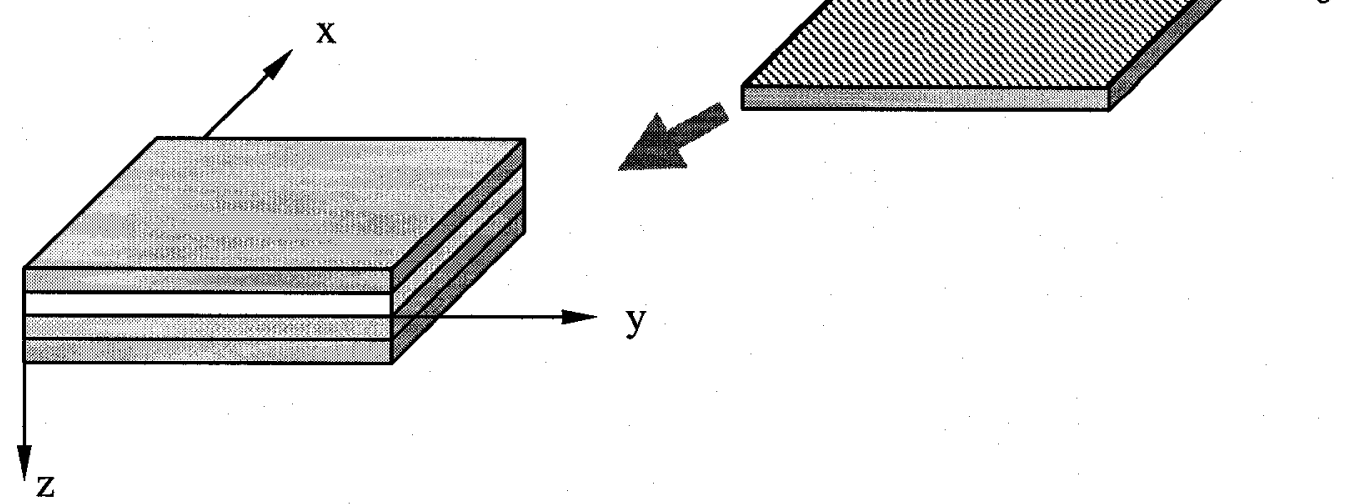

Figure 2.12 A laminate made up of laminae with different fiber orientations

Generally speaking, laminates are made by the stacking of basic layers that are usually plane orthotropic elastic materials. If the layers are stacked with arbitrary orientations of orthotropy axes with respect to a fixed reference frame, the laminate obtained is normally fully anisotropic, with coupled in- and out-of-plane behaviors [86], and the stress - strain relations of the laminate follow the generalized Hook's law, expressed as 


$$
\left\{\begin{array}{l}
\sigma_{11} \\
\sigma_{22} \\
\sigma_{33} \\
\sigma_{23} \\
\sigma_{31} \\
\sigma_{12}
\end{array}\right\}=\left[\begin{array}{llllll}
C_{11} & C_{12} & C_{13} & C_{14} & C_{15} & C_{16} \\
C_{21} & C_{22} & C_{23} & C_{24} & C_{25} & C_{26} \\
C_{31} & C_{32} & C_{33} & C_{34} & C_{35} & C_{36} \\
C_{41} & C_{42} & C_{43} & C_{44} & C_{45} & C_{46} \\
C_{51} & C_{52} & C_{53} & C_{54} & C_{55} & C_{56} \\
C_{61} & C_{62} & C_{63} & C_{64} & C_{65} & C_{66}
\end{array}\right]\left\{\begin{array}{l}
\varepsilon_{11} \\
\varepsilon_{22} \\
\varepsilon_{33} \\
\varepsilon_{23} \\
\varepsilon_{31} \\
\varepsilon_{12}
\end{array}\right\},
$$

where the 36 material constants $C_{i j}(i, j=1,2, \ldots 6)$ are called elastic coefficients. By considering the potential energy of deformation per unit of volume, it is shown that the elastic constant matrix is symmetric, that is, $C_{i j}=C_{j i}$. Thus, only 21 of the elastic constants are actually independent for a most general anisotropic material [84]. If there are two orthogonal planes of material property symmetry, it is shown that symmetry will exist relative to a third mutually orthogonal plane. This form of elastic symmetry is very important because it occurs in many engineering materials used in modern constructions. Due to the symmetry, the stress - strain relations are then reduced to

$$
\left\{\begin{array}{l}
\sigma_{11} \\
\sigma_{22} \\
\sigma_{33} \\
\sigma_{23} \\
\sigma_{31} \\
\sigma_{12}
\end{array}\right\}=\left[\begin{array}{cccccc}
C_{11} & C_{12} & C_{13} & 0 & 0 & 0 \\
C_{12} & C_{22} & C_{23} & 0 & 0 & 0 \\
C_{13} & C_{23} & C_{33} & 0 & 0 & 0 \\
0 & 0 & 0 & C_{44} & 0 & 0 \\
0 & 0 & 0 & 0 & C_{55} & 0 \\
0 & 0 & 0 & 0 & 0 & C_{66}
\end{array}\right]\left\{\begin{array}{c}
\varepsilon_{11} \\
\varepsilon_{22} \\
\varepsilon_{33} \\
\varepsilon_{23} \\
\varepsilon_{31} \\
\varepsilon_{12}
\end{array}\right\}
$$

Materials with the stress - strain relations of eqn (2.15) are referred to as orthotropic. It can be seen that for an orthotropic material there is no interaction between direct stresses and shearing strains as occurs in anisotropic materials. There are 9 independent constants for an orthotropic material. Many engineering materials, such as fiber laminates and reinforced concrete can be treated as orthotropic. 


\subsubsection{Laminated shells}

A number of theories exist for layered anisotropic shells [87-95]. Many of these theories were developed originally for thin shells and are based on the Kirchhoff-Love kinematic hypothesis that straight lines normal to the undeformed mid-surface remain straight and normal to the mid-surface after deformation. The first analysis that incorporated the bending-stretching coupling (owing to unsymmetric lamination in composites) was made by Ambartsumyan [87-89]. In his analysis, it is assumed that the individual orthotropic layers are oriented such that the principal axes of material symmetry coincide with the principal coordinates of the shell reference surface. Thus, Ambartsumyan's work deals with what is today known as laminated orthotropic shells, rather than laminated anisotropic shells. Dong et al. formulated a theory of thin shells laminated of anisotropic material, which is an extension of the theory developed by Stavsky [96] for laminated anisotropic plates to Donnell's shallow shell theory [94]. Cheng and Ho presented an analysis of laminated anisotropic cylindrical shells using Flugge's shell theory [90].

All of the laminated shell theories mentioned above neglect transverse shear deformations. Gulati et al. [97] improved the shell theories by taking into account the effects of transverse shear deformations and transverse isotropy. Whitney and Sun [98] developed a shear deformation theory for laminated cylindrical shells that includes both transverse shear deformation and transverse normal strain. 
The effects of material orthotropy on the failure analyses of laminated shells have also been studied. Elasto-plastic analysis of cylindrically orthotropic composite thick-walled tubes under uniform pressure was conducted by Zhou and Qiu [99]. Stress analysis of orthotropic cylindrical shells subjected to internal and external pressures was given by Verijenko et al. [100]. Exact elasticity solutions were obtained for both open-ended and closed-ended cylinders. Kumosa [101] performed an FEA study on orthotropic cylindrical shells containing circumferential cracks. Two different types of loading boundary conditions were concerned which assume either the application of axial tension or torsion. Particular attention was given to the problem of transverse shear deformations which occur around long circumferential cracks in thin-walled cylindrical shells under the loading conditions assumed. The effect of orthotropy ratio was investigated in a wide range. It was found that the deformation was highly dependent upon the elastic properties, cylinder geometry and loading boundary conditions

For an orthotropic material, with $x_{1}$ and $x_{2}$ as the axes of orthotropy, the engineering material constants are defined through the following stress-strain relations:

$$
\begin{aligned}
& \varepsilon_{11}=\frac{1}{E_{1}}\left(\sigma_{11}-v_{1} \sigma_{22}\right), \\
& \varepsilon_{22}=\frac{1}{E_{2}}\left(\sigma_{22}-v_{2} \sigma_{11}\right), \\
& \varepsilon_{12}=\frac{\sigma_{12}}{2 G_{12}}, \\
& \frac{v_{1}}{E_{1}}=\frac{v_{2}}{E_{2}} .
\end{aligned}
$$


With the four independent material constants the differential operators arising from the formulation of shells do not seem to be factorable and consequently, the analysis becomes intractable. However, it can be shown that if the elastic constants are related through

$$
G_{12}=\frac{\left(E_{1} E_{2}\right)^{\frac{1}{2}}}{2\left[1+\left(v_{1} v_{2}\right)^{\frac{1}{2}}\right]},
$$

then, with a simple coordinate transformation, the orthotropic shell equations may be reduced to essentially those of isotropic shells $[7,15]$, thus the shell has only three independent elastic constants. Such a material in shell form is said to be specially orthotropic. By defining

$$
\begin{aligned}
E & =\left(E_{1} E_{2}\right)^{1 / 2}, \\
v & =\left(v_{1} v_{2}\right)^{1 / 2}, \\
G_{a v} & =E / 2(1+v), \\
c & =\left(E_{1} / E_{2}\right)^{1 / 4},
\end{aligned}
$$

the stress - strain relations become

$$
\begin{aligned}
& \varepsilon_{11}=\frac{1}{E}\left(\frac{\sigma_{11}}{c^{2}}-v \sigma_{22}\right), \\
& \varepsilon_{22}=\frac{1}{E}\left(c^{2} \sigma_{22}-v \sigma_{11}\right), \\
& \varepsilon_{12}=\frac{\sigma_{12}}{2 G_{a v}},
\end{aligned}
$$


where $E$ and $v$ are the effective modulus and Poisson's ratio, respectively, $c$ is the stiffness ratio. From the above equations, it follows that in specially orthotropic materials the measured shear modulus $G_{12}$ is equal to the effective shear modulus $G_{a v}$.

The crack problems in specially orthotropic shells have been studied by Delale and Erdogan $[7,15]$. Their studies considered various shell geometries with material special orthotropy, such as spherical shells, cylindrical shells with an axial crack or with a circumferential crack, and shells with two nonzero curvatures. The through-the-thickness crack is located in one of the principal planes of curvature and the plane of the crack is a plane of symmetry with respect to loading as well as to the geometry of the shell. The axes of material orthotropy coincide with the principal planes of curvature, that is, one of the orthotropic axes is parallel to the crack plane, which represents the practical case that cracking commonly occurs along the fiber because the interface in a composite is always the weak area for fracture. The results show that the SIFs are rather strongly dependent on the degree of material orthotropy. Gulgec and Yahsi [102] analyzed the problem of a specially orthotropic cylindrical shell with a fixed end containing a circumferential through crack under the general loading conditions. The problem was formulated for orthotropic materials using Reissner's shell theory. The primary objective was to study the effect of the end constraining on the SIF, the effects of the shell curvature and thickness, material orthotrophy, and the Poisson's ratio on the SIF. However, crack closure behavior for orthotropic materials has never been considered in any previous research. Yahsi and Erdogan [103] also studied a specially orthotropic cylindrical shell containing an arbitrarily oriented crack. It was assumed that the axes of material 
orthotropy coincided with the crack plane but not the axis of the cylinder. The nonsymmetric problem was solved for arbitrary self-equilibrating crack surface tractions, which, added to an appropriate solution for an uncracked shell, would give the result for a cracked shell under the most general loading conditions. 


\section{Chapter 3 Theoretical Analysis}

\subsection{Specially Orthotropic Shell with Double Curvature}

\subsubsection{Formulation of the problem}

Consider the general case that a thin shallow shell with two nonzero curvatures contains a through-the-thickness crack, as illustrated in Figure 3.1, subjected to a bending load. The shell has a thickness $h$ and curvature radii $R_{1}$ and $R_{2}$ in the $x z$ plane and the $y z$ plane, respectively, containing a crack of length $2 a$. The material of the shell is assumed to be specially orthotropic, which satisfies eqn (2.17). $E_{1}, v_{1}$ and $E_{2}, v_{2}$ are elastic constants of the material in the $x$-direction and $y$-direction respectively, The crack is located in one of the principal planes of curvature, along the $y$-direction, and the axes of material orthotropy coincide with the principal planes of curvature, as seen in Figure 3.1. According to the formulation developed by Delale and Erdogan $[7,15]$ the problem of a cracked shell subjected to membrane force and bending moment can be reduced to a pair of coupled singular integral equations,

$$
\begin{aligned}
& \int_{-\sqrt{c}}^{\sqrt{c}} \frac{G_{1}(\tau)}{\tau-\eta} d \tau+\int_{-\sqrt{c}}^{\sqrt{c}}\left[k_{11}(\eta, \tau) G_{1}(\tau)+k_{12}(\eta, \tau) G_{2}(\tau)\right] d \tau=2 \pi F_{1}(\eta), \\
& \frac{1-v^{2}}{\lambda_{0}^{4}} \int_{-\sqrt{c}}^{\sqrt{c}} \frac{G_{2}(\tau)}{\tau-\eta} d \tau+\int_{-\sqrt{c}}^{\sqrt{c}}\left[k_{21}(\eta, \tau) G_{1}(\tau)+k_{22}(\eta, \tau) G_{2}(\tau)\right] d \tau=2 \pi \frac{h}{a} F_{2}(\eta), \\
& \int_{-\sqrt{c}}^{\sqrt{c}} G_{i}(\tau) d \tau=0, \quad(\mathrm{i}=1,2),
\end{aligned}
$$


where $\eta=y \sqrt{c} / a,-a<y<a, v$ and $c$ are given by eqns (2.19) and (2.21), respectively, $G_{1}$ and $G_{2}$ denote the derivatives of the crack-face opening displacement, $u\left(0^{+}, \eta\right)$, and the crack-face rotation, $\beta\left(0^{+}, \eta\right)$, respectively, expressed as

$$
\begin{aligned}
& G_{1}(\eta)=\frac{\partial}{\partial \eta} u\left(0^{+}, \eta\right) \\
& G_{2}(\eta)=\frac{\partial}{\partial \eta} \beta\left(0^{+}, \eta\right)
\end{aligned}
$$

The crack-face opening displacement and the crack-face rotation are schematically shown in Figure 3.2. The parameter $\lambda_{0}$ is dimensionless, expressed as

$$
\lambda_{0}^{4}=12\left(1-v^{2}\right) a^{2} / h^{2}
$$

which is related to shell material property and shell geometry.

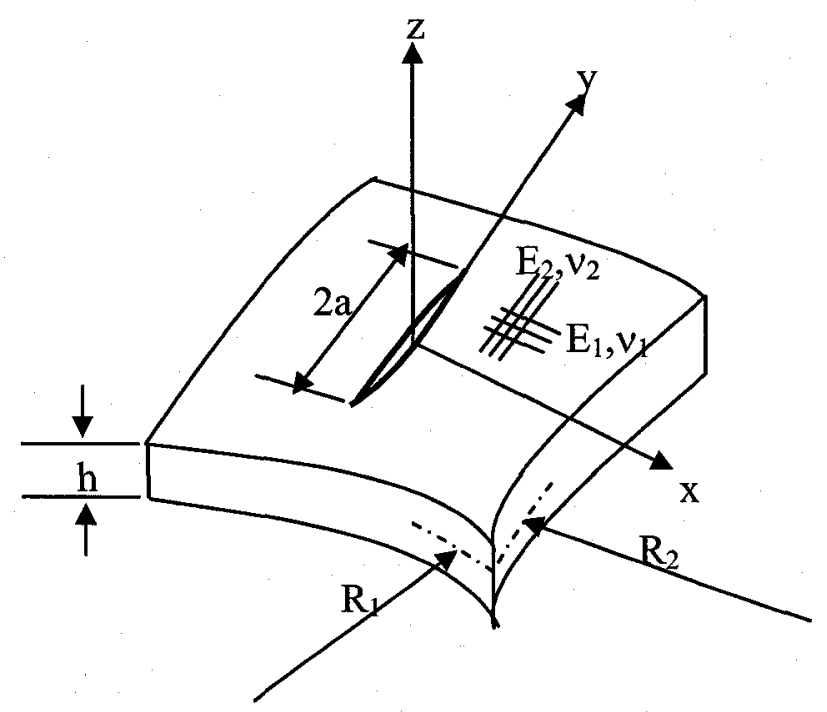

Figure 3.1 Geometry and coordinates for a through-the-thickness crack in a shell with double curvature 


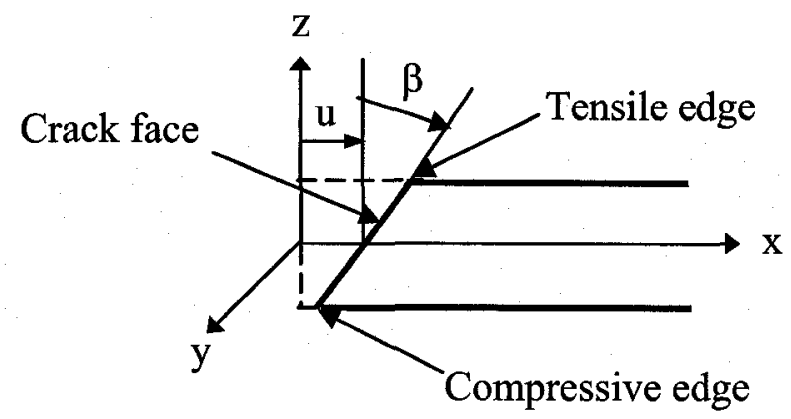

Figure 3.2 Illustration of crack-face opening displacement and crack-face rotation

Within the coordinate system defined in Figure 3.1, according to the shear deformation theory of Delale and Erdogan, the equilibrium equations for the general shell problem can be expressed as $[7,15]$,

$$
\nabla^{4} \nabla^{4} \phi+\nabla_{\lambda}^{2} \nabla_{\lambda}^{2}\left(1-\kappa \nabla^{2}\right) \phi=0
$$

where

$$
\begin{aligned}
& \nabla_{\lambda}^{2}=\lambda_{1}^{2} \frac{\partial^{2}}{\partial y^{2}}+\lambda_{2}^{2} \frac{\partial^{2}}{\partial x^{2}} \\
& \phi=\frac{F}{a^{2} h E}
\end{aligned}
$$

The dimensionless quantities, $\kappa, \lambda_{1}$ and $\lambda_{2}$, are given below:

$$
\begin{aligned}
& \kappa=\frac{12(1+v)}{5 \lambda_{0}^{4}}, \\
& \lambda_{1}^{4}=12\left(1-v^{2}\right) \frac{c^{2} a^{4}}{h^{2} R_{1}^{2}}, \\
& \lambda_{2}^{4}=12\left(1-v^{2}\right) \frac{a^{4}}{c^{2} h^{2} R_{2}^{2}} .
\end{aligned}
$$


Since $\lambda_{1}$ and $\lambda_{2}$ are associated with the curvature radii $R_{1}$ and $R_{2}$, respectively, for a given shell material, shell thickness and crack length, they represent the curvatures of the shell in the two principal axes, respectively. The effective modulus $E$ is given by eqn (2.18). In the integral equations, $F$ is defined as the stress functions, which are expressed by

$$
\begin{gathered}
F_{1}(\eta)=-\sigma_{m} /(c E) \\
\text { and } F_{2}(\eta)=-\sigma_{b} /(6 c E) \text {. }
\end{gathered}
$$

$F_{1}$ and $F_{2}$ are related to the membrane stress $\sigma_{m}$ and bending stress $\sigma_{b}$ at the crack surface, respectively. Since the crack closure behavior considered in this thesis is associated with bending load only, we may take $\sigma_{m}=0$ for simplicity in the present analysis. The determination of the functions $G_{1}$ and $G_{2}$ in eqn (3.1) would complete the solution of the problem, which needs to obtain the solutions of the asymptotic kernels $k_{i j}$ $(i, j=1,2)$ first in the equation. The detailed mathematical procedure for the solutions is given in Appendix A.

\subsubsection{Modeling of crack-face closure}

Taking the same assumption of line contact at the compressive edge of Young and Sun $[16,17]$ for plate bending, the crack closure of a shell subjected to a bending load can be modeled by applying a contact force along the compressive edge of the crack face. Whether the contact would occur at the inner surface or at the outer surface depends on the nature of the bending load applied, that is, the direction of the bending load. In this thesis, the bending load that causes contact of crack faces at the inner surface of the shell is considered as positive bending $\left(\sigma_{b}>0\right)$, whereas it is negative bending $\left(\sigma_{b}<0\right)$, as demonstrated in Figure 3.3. 


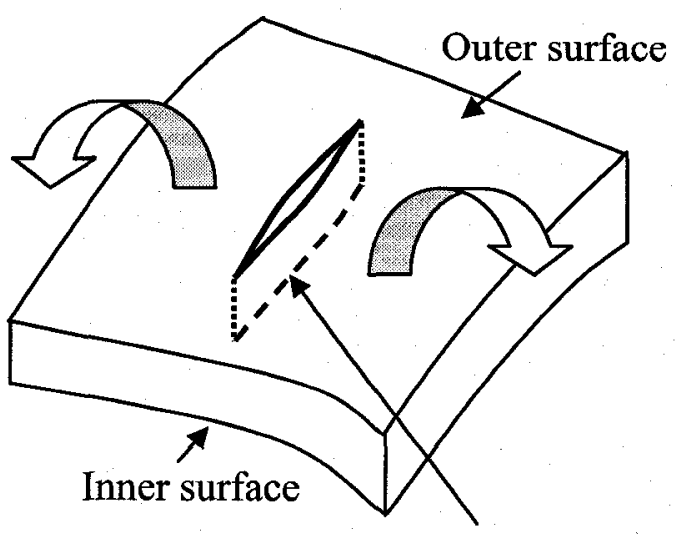

Closure at inner surface

(a)

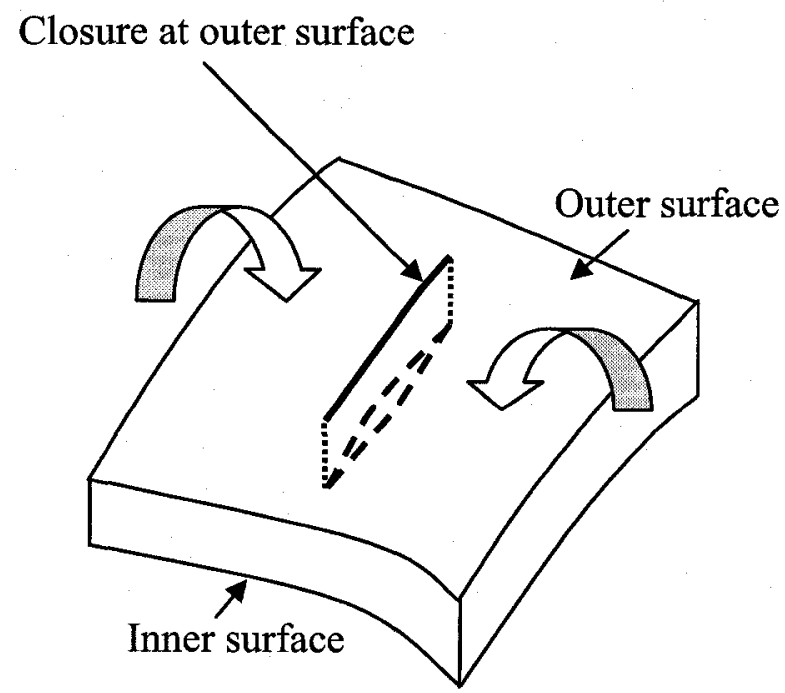

(b)

Figure 3.3 Bending loads: (a) positive bending and (b) negative bending

The line-contact force $p(\eta)$, which may vary along the crack length, can be translated to the mid-plane of the shell, accompanied with an additional distributed bending moment, as shown in Figure 3.4. The additional bending moment is calculated as 


$$
m_{c}(\eta)=\frac{1}{2} h p(\eta)
$$

Thus the stress functions $F_{1}$ and $F_{2}$ in the closure condition are expressed as

$$
\begin{gathered}
F_{1}(\eta)=-\frac{p(\eta) / h}{c E} \\
\text { and } F_{2}(\eta)=-\frac{\sigma_{b}(\eta) \pm 3 p(\eta) / h}{6 c E}
\end{gathered}
$$

where the positive sign ' + ' denotes contact at the edge on the outer surface $(z=h / 2)$ while the negative sign '-' denotes contact at the edge on the inner surface $(z=-h / 2)$. This sign convention is used wherever the sign ' \pm ' appears in this thesis.
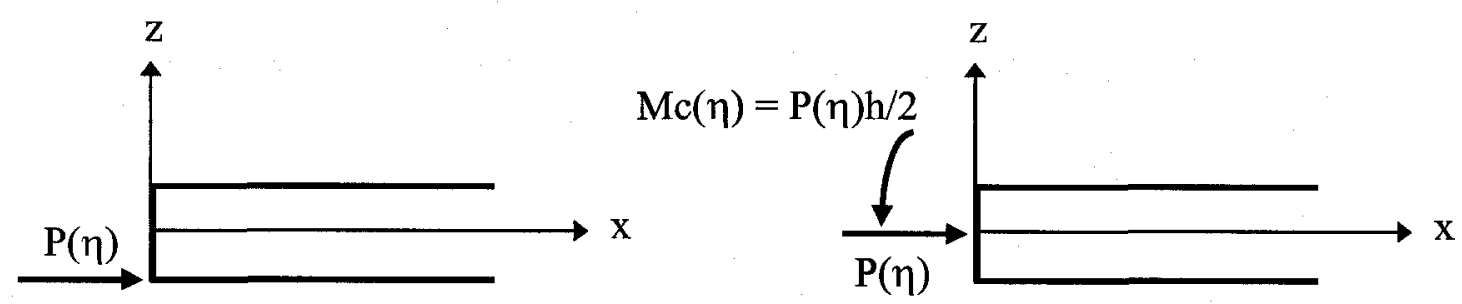

(a)
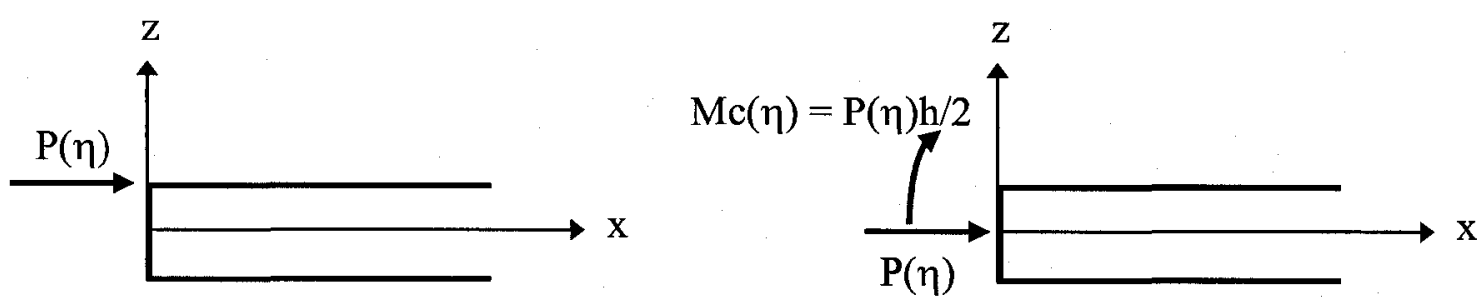

(b)

Figure 3.4 Contact force and equivalent bending moment representing the crack-face closure: (a) contact force at inner surface (positive bending) and (b) contact force at outer surface (negative bending) 
The physical constraint of the crack-face closure is that no material penetration or overlapping would occur at the compressive edge. Accordingly the constraint condition of the closure is that the normal displacement of the crack face at the compressive edge must remain equal or greater than zero, that is,

$$
u\left(0^{+}, \eta\right) \pm \frac{h}{2 a} \beta\left(0^{+}, \eta\right) \geq 0, \quad-\sqrt{c} \leq \eta \leq \sqrt{c}
$$

and noting eqn (3.2) the following expression is derived,

$$
\int_{\eta}^{\sqrt{c}} G_{1}(\tau) d \tau \pm \frac{h}{2 a} \int_{\eta}^{\sqrt{c}} G_{2}(\tau) d \tau \leq 0
$$

Thus the constraint condition can be expressed as

$$
G_{1}(\tau) \pm \frac{h}{2 a} G_{2}(\tau)=0
$$

Substituting eqn (3.15) into the integral eqn (3.1) eliminates $G_{1}$ but in the meanwhile the closure constraint introduces the unknown contact force $p$,

$$
\begin{aligned}
& \pm \frac{h}{2 a} \int_{-\sqrt{c}}^{\sqrt{c}} \frac{G_{2}(\tau)}{\tau-\eta} d \tau+\int_{-\sqrt{c}}^{\sqrt{c}}\left[ \pm \frac{h}{2 a} k_{11}(\eta, \tau) G_{2}(\tau)-k_{12}(\eta, \tau) G_{2}(\tau)\right] d \tau=2 \pi \frac{p(\eta) / h}{c E}, \\
& -\frac{1-v^{2}}{\lambda_{0}^{4}} \int_{-\sqrt{c}}^{\sqrt{c}} \frac{G_{2}(\tau)}{\tau-\eta} d \tau+\int_{-\sqrt{c}}^{\sqrt{c}}\left[ \pm \frac{h}{2 a} k_{21}(\eta, \tau) G_{2}(\tau)-k_{22}(\eta, \tau) G_{2}(\tau)\right] d \tau=\pi \frac{h}{a}\left[\frac{\sigma_{b}(\eta) \pm 3 p(\eta) / h}{3 c E}\right]
\end{aligned}
$$

Thus, in the case of full-length crack closure, that is, $p>0$ everywhere and eqn (3.15) holds, the coupled integral equations (3.1) reduce to a single integral equation for $G_{2}$, by eliminating $p$ in eqns (3.16) and (3.17):

$$
\int_{-\sqrt{c}}^{\sqrt{c}}\left[-\left(\frac{1-v^{2}}{\lambda_{0}^{4}}+\frac{h^{2}}{4 a^{2}}\right) \frac{1}{\tau-\eta}-\frac{h^{2}}{4 a^{2}} k_{11}(\eta, \tau) \pm \frac{h}{2 a}\left(k_{12}(\eta, \tau)+k_{21}(\eta, \tau)\right)-k_{22}(\eta, \tau)\right] G_{2}(\tau) d \tau=\frac{\pi h \sigma_{b}}{3 a c E} .
$$


In this case $G_{1}$ can be readily obtained from (3.15) in terms of $G_{2}$. The contact force $p$ can now be computed from eqn (3.16) as:

$$
p(\eta)= \pm \frac{c E h^{2}}{4 \pi a} \int_{-\sqrt{c}}^{\sqrt{c}} \frac{G_{2}(\tau)}{\tau-\eta} d \tau+\frac{c E h}{2 \pi} \int_{-\sqrt{c}}^{\sqrt{c}}\left[ \pm \frac{h}{2 a} k_{11}(\eta, \tau) G_{2}(\tau)-k_{12}(\eta, \tau) G_{2}(\tau)\right] d \tau
$$

\subsubsection{Determination of crack closure region}

Due to the curvature effect crack closure in shells may not always occur over the entire length of the crack, therefore the first step in the solution is to determine the closure regions along the crack. To do this, one may assume full-length closure first and compute $p(\eta)$ over the entire crack using eqns (3.18) and (3.19). If $p>0$ over the entire length of the crack, it means full-length closure occurs, in this case eqns (3.16) and (3.17) are used to solve the problem. However, if $p<0$ somewhere along the crack, this indicates that the crack faces are not actually in contact at the compressive edges there, that is, partial crack closure would occur. In this case, eqns (3.16) and (3.17) cannot be used in the whole range of the crack, thus it is necessary to determine the closure and non-closure regions. It is worth noting that the zero-crossing point of the contact force is not the actual boundary between the closure and non-closure regions. To find this boundary one may select an approximate position which is around the zero-crossing point of the contact force but closer to the center of the crack. The purpose of doing this is trying to include some region with negative contact force in the next calculation so that the accurate boundary can be determined by moving the selected point towards the crack tip gradually in multi-step calculation of the contact force until its value is positive 
everywhere in the closure region, with the contact force being zero in the open region. It is an iterative process to obtain the accurate closure regions.

Two specially orthotropic materials are selected as examples for current analysis, one is a mildly orthotropic material, titanium, with the Young's modulus of $1.039 \times 10^{5}$ $\mathrm{MPa}$ and $1.434 \times 10^{5} \mathrm{MPa}$ in two perpendicular directions respectively. The Poisson's ratio of titanium are 0.1966 and 0.2714 and the shear modulus is $4.675 \times 10^{4} \mathrm{Mpa}$. The Young's modulus ratio of titanium is calculated as $E_{1} / E_{2}=0.725$ or 1.38 corresponding to different crack orientations. Another is a strongly orthotropic material, graphite-epoxy, with the Young's modulus of $1.034 \times 10^{4} \mathrm{MPa}$ and $2.758 \times 10^{5} \mathrm{MPa}$ in two perpendicular directions respectively and the corresponding Poisson's ratio of 0.0075 and 0.2 as well as the shear modulus of $2.758 \times 10^{4} \mathrm{MPa}$. The four ratios of the Young's modulus and the corresponding four ratios of the Poisson's ratio are calculated and presented in Table 3.1.

Table 3.1 Ratios of specially orthotropic material constants

\begin{tabular}{|c|c|c|c|c|}
\hline & Titanium & Titanium & Graphite-epoxy & Graphite-epoxy \\
\hline$E_{1} / E_{2}$ & 0.725 & 1.38 & 0.037 & 26.67 \\
\hline$v_{1} / v_{2}$ & 0.724 & 1.38 & 0.038 & 26.67 \\
\hline
\end{tabular}

Two curvature ratios, $\psi=R_{1} / R_{2}=0.25$ or 4 , representing the crack orientation with respect to the principal plane with larger curvature radius or that with smaller curvature radius, are concerned. Figure 3.5 shows an example of the contact force distribution along the crack assuming full-length closure, for the cases of the Young's modulus ratio $\varphi=E_{1} / E_{2}=0.725$ and 1.38 , and the curvature ratio, $\psi=0.25$ and 4 
respectively, in positive bending condition. The contact forces are computed for the case $a / h=10$ and the shell parameter $\lambda$ varying from 0.5 to 6 , where $\lambda^{4}=12\left(1-v^{2}\right) a^{4} /\left(h R_{1}\right)^{2}$

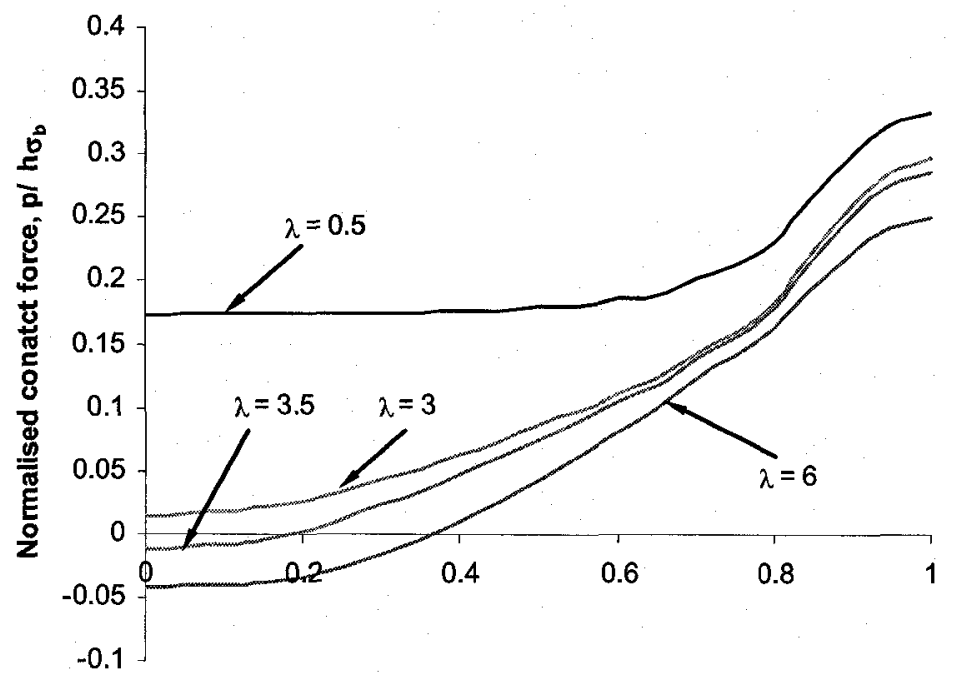

Distance from the centre of the crack, y/a

(a)

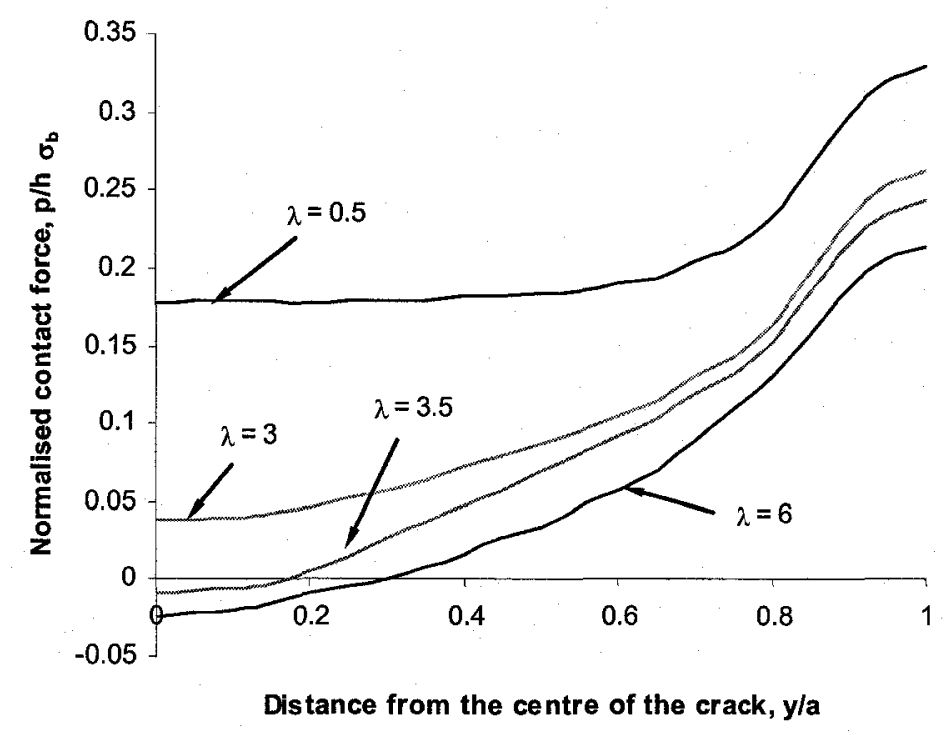

(b) 


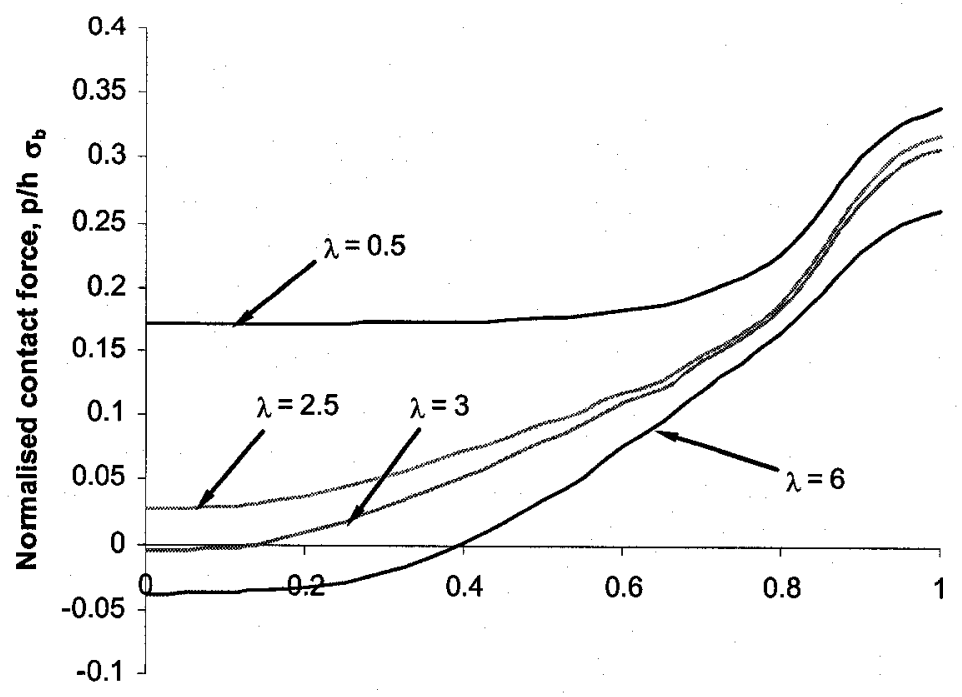

Distance from the centre of the crack, y/a

(c)

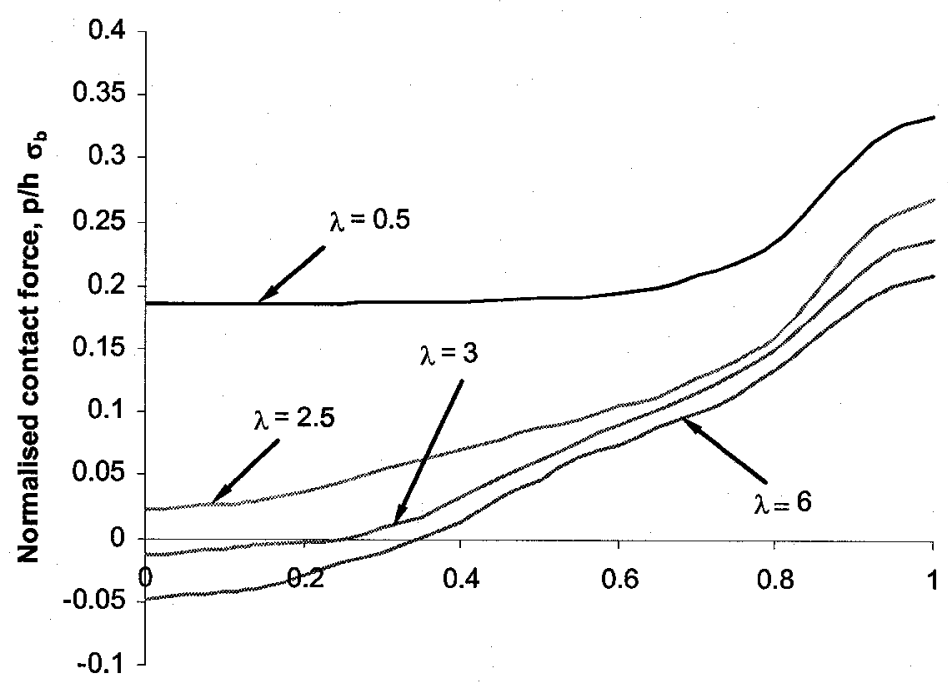

Distance from the centre of the crack, y/a

(d)

Figure 3.5 Contact force distributions assuming full-length closure for a specially orthotropic shell having double curvature under positive bending with $a / h=10:$ (a) $\varphi=0.725$ and $\psi=0.25$; (b) $\varphi=0.725$ and $\psi=4$;

(c) $\varphi=1.38$ and $\psi=0.25$; (d) $\varphi=1.38$ and $\psi=4$ 
For a given material, when the crack length and the shell thickness are set, $\lambda$ varies only with the shell radius $R_{1}$. However, for a given value of $\psi, \lambda$ also varies with $R_{2}$. Therefore $\lambda$ represents the curvatures of a shell in both principal planes. The larger the $\lambda$ value, the larger the curvatures of the shell are, that is, the smaller the curvature radii are.

It is seen in Figure 3.5 that the contact force distributes nonuniformly along the crack. When $\lambda$ is small, the contact force is positive everywhere along the crack. However, with the increase of the $\lambda$ value, the contact force appears negative around the centre of the crack, which indicates that partial closure occurs. When partial closure occurs, there would be two closure boundaries on the crack, which are symmetrical about the centre of the crack. In the region between the closure boundary and the crack tip, that is, the ranges of $-a<y<-y_{c}$ and $y_{c}<y<a$, where $-y_{c}$ and $y_{c}$ represent the coordinates of the closure boundaries along the crack length shown in Figure 3.6, which are the closure regions, eqns (3.16) and (3.17) are used. The integral equations (3.1) are used for the open region, that is, the range of $-y_{c}<y<y_{c}$, which is between the two closure boundaries, see Figure 3.6. In the open region, the stress functions $F_{1}(\eta)=0$ and $F_{2}(\eta)=-\sigma_{b} /(6 c E)$, because there is no contact force. To solve the problem for the entire crack length requires the combination of eqns (3.1), (3.16) and (3.17) for both closure region and non-closure region. With iterative manipulation the accurate closure boundaries can be achieved such that the contact force is positive everywhere in the closure region and zero in the open region, as shown in Figure 3.7. 


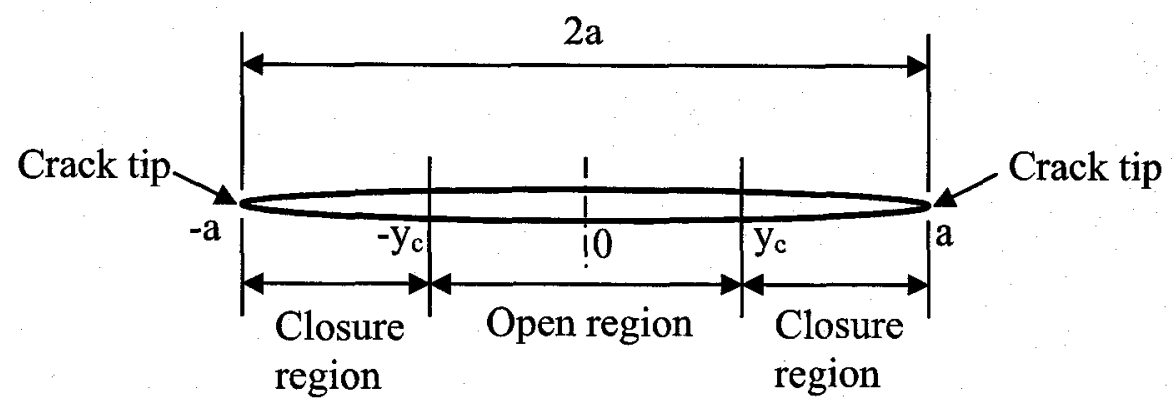

Figure 3.6 Illustration of closure regions and open region of the crack face

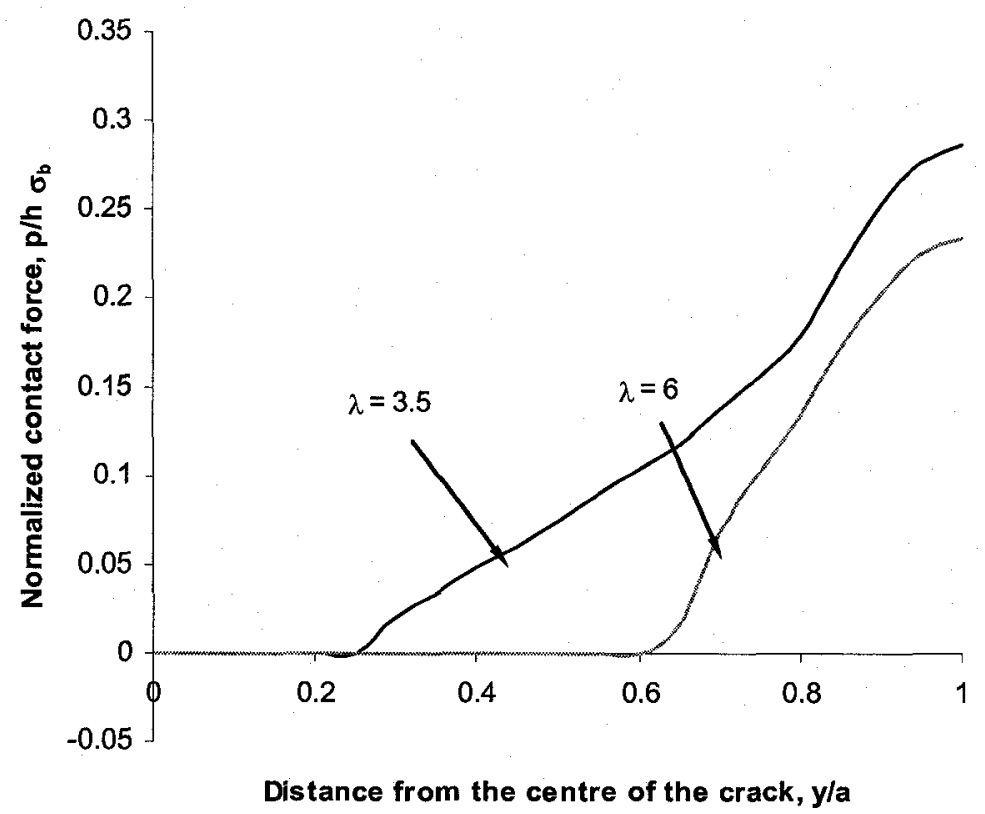

Figure 3.7 Correct distribution of the contact force in partial closure of a specially orthotropic shell having double curvature under positive bending with $a / h=10, \varphi=0.725$ and $\psi=0.25$, under positive bending

\subsubsection{Solution of stress intensity factor}


Let $t=\tau / \sqrt{c}$ and $d=\eta / \sqrt{c}$. Since the unknown functions $G_{1}$ and $G_{2}$ are singular at the crack tips the following representations are introduced to facilitate the numerical computation,

$$
G_{i}(t)=\frac{g_{i}(t)}{\sqrt{1-t^{2}}}, \quad-1 \leq t \leq 1, \quad(i=1,2)
$$

The coupled integral equations (3.1) are then solved using the Gauss-Chebyshev integration formulas [104]; see Appendix B. The numerical solution of the integral equations was implemented through computer programming using FORTRAN 91 language. The selected FORTRAN codes are attached to this thesis in Appendix D.

The SIF at the crack tip is due to the synergetic contribution of membrane stress and bending stress, which is formulated by Delale and Erdogan [7, 15],

$$
k_{I}=-\frac{c E \sqrt{a}}{2}\left[g_{1}(1)+\frac{z}{a} g_{2}(1)\right]
$$

The corresponding stress intensity factor ratios are expressed as

$$
\begin{aligned}
& F_{m b}=-\frac{c E}{2 \sigma_{b}} g_{1}(1) \text { and } \\
& F_{b b}=-\frac{c E}{2 \sigma_{b}} \frac{h}{2 a} g_{2}(1) .
\end{aligned}
$$

Due to the relations in (3.15) the membrane component $F_{m b}$ and bending component $F_{b b}$ of the stress intensity factor are always equal when the closure occurs in the inner surface of the shell while the two components are equal in magnitude but opposite in sign when the closure is in the outer surface. 


\subsubsection{Analytical results}

It is clear from Figure 3.5 that shell curvatures affect the crack closure behavior, complete closure occurs only when the shell curvature is small while partial closure occurs for large curvatures. In the limiting case, $\lambda$ approaching to zero, that is, the shell radii $R_{1}$ and $R_{2}$ approaching infinity, which represents plates, complete crack closure or full-line closure would occur, which can be deduced from Figure 3.5.

The contact force distributions for a shell under negative bending with the different values of $\varphi$ and $\psi$ being discussed were also determined and the results demonstrate that the contact force is positive over the entire crack length for all the $\lambda$ values being considered (up to 6) and for all the $\varphi$ and $\psi$ values being discussed. This means full-length crack closure always occurs under negative bending as long as $\lambda \leq 6$. Certainly, at even higher $\lambda$ values, partial closure may occur.

The stress intensity factor ratios in the closure case and non-closure case for the shells with different $\varphi$ and $\psi$ values under positive bending and negative bending respectively were computed. Figure 3.8 illustrate the results for the mildly orthotropic material in the cases of the radii ratio $\psi=0.25$ and 4, respectively. It is shown that the crack-face closure has significant effects on the SIFs. It reduces the bending component but increases the membrane component in both positive and negative bending cases, for all values of $\varphi$ and $\psi$. Positive bending causes higher SIF than negative bending. It may be easier to show the effect of crack closure on the SIFs by comparing the maximum stress intensity factor ratios. As shown in Figure 3.9, these ratios are reduced by the effect 
of crack-face closure in both positive and negative bending conditions, especially when $\lambda$ is very small $(\lambda<2)$. Again, in the limiting case, $\lambda \rightarrow 0$, representing plates, the reduction of the maximum stress intensity factor ratio has a maximum.

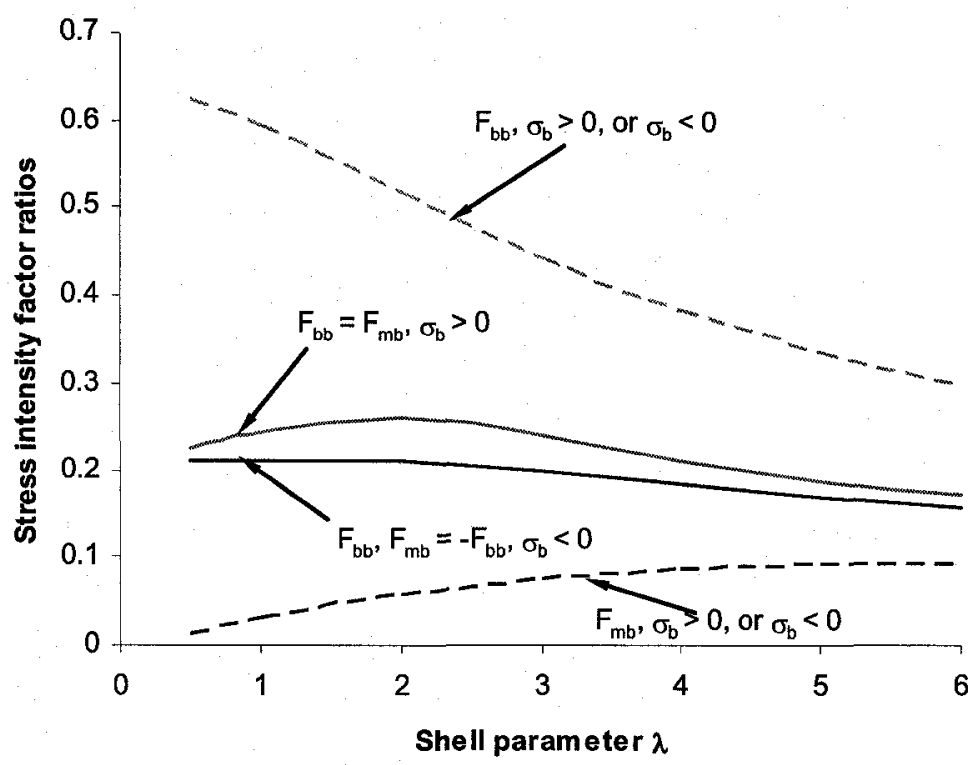

(a)

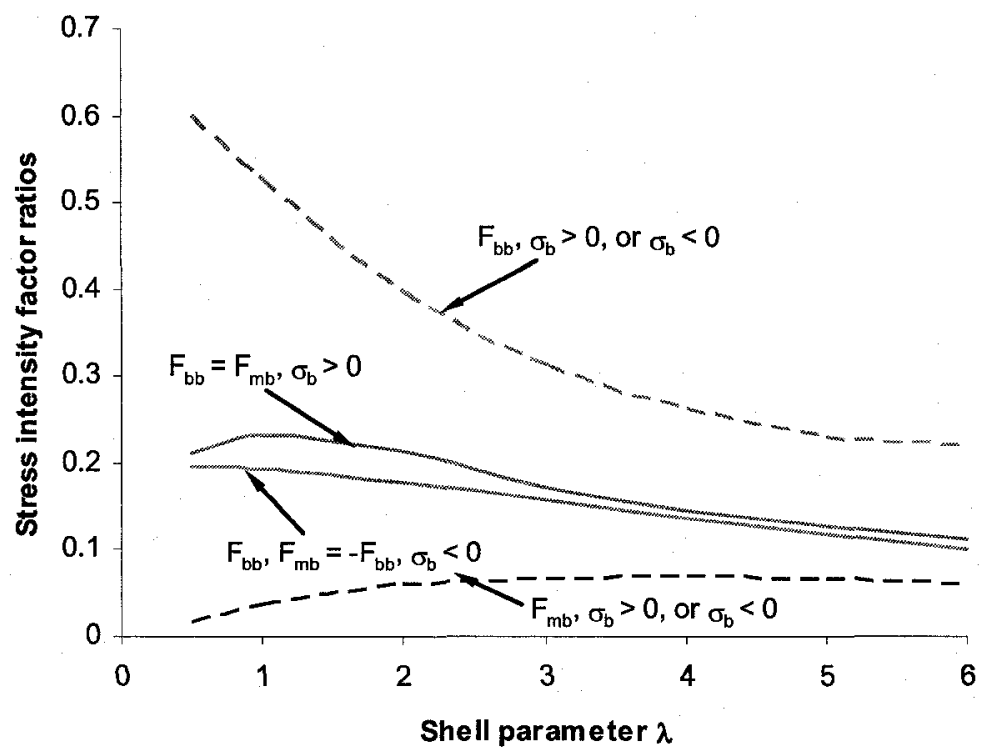

(b) 


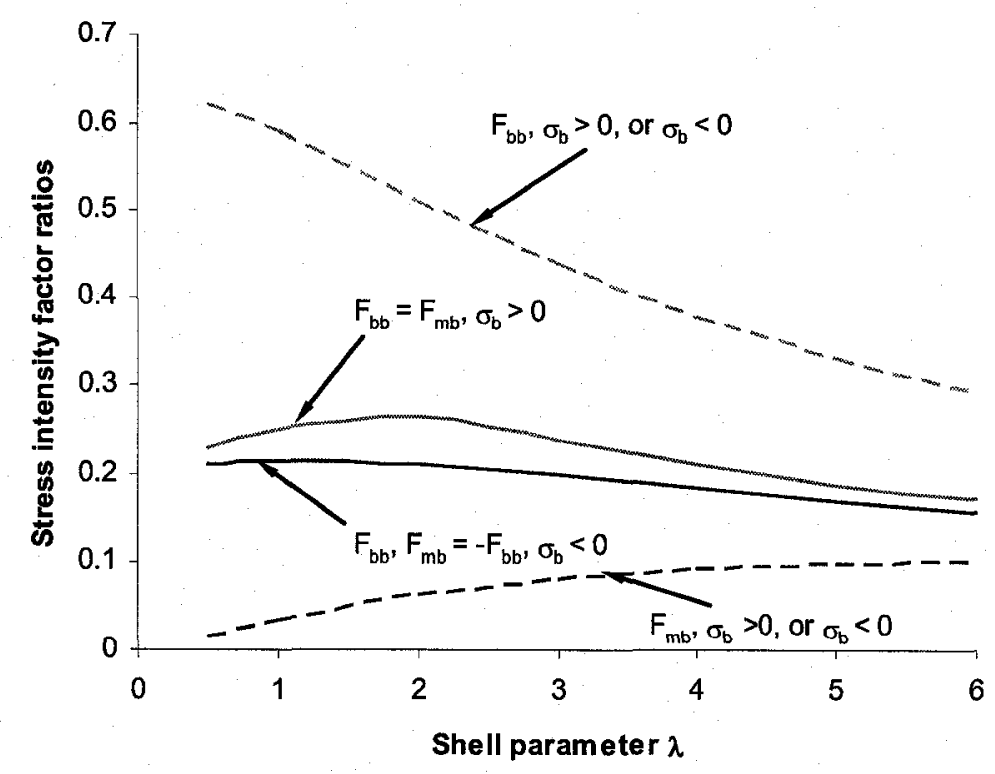

(c)

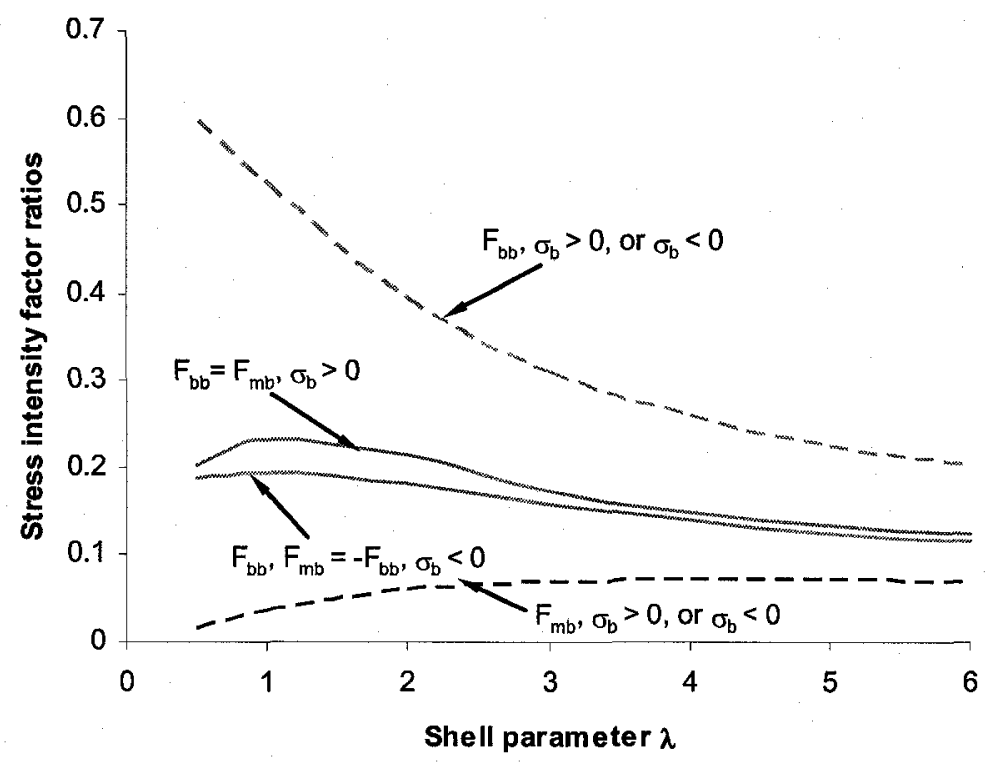

(d)

Figure 3.8 Comparison between closure and non-closure solutions of stress intensity factor ratio for a specially orthotropic shell having double curvature with $a / h=10$ ( $\longrightarrow$ closure and ---.-- non-closure):

(a) $\varphi=0.725$ and $\psi=0.25$; (b) $\varphi=0.725$ and $\psi=4$;

(c) $\varphi=1.38$ and $\psi=0.25 ;$ (d) $\varphi=1.38$ and $\psi=4$ 


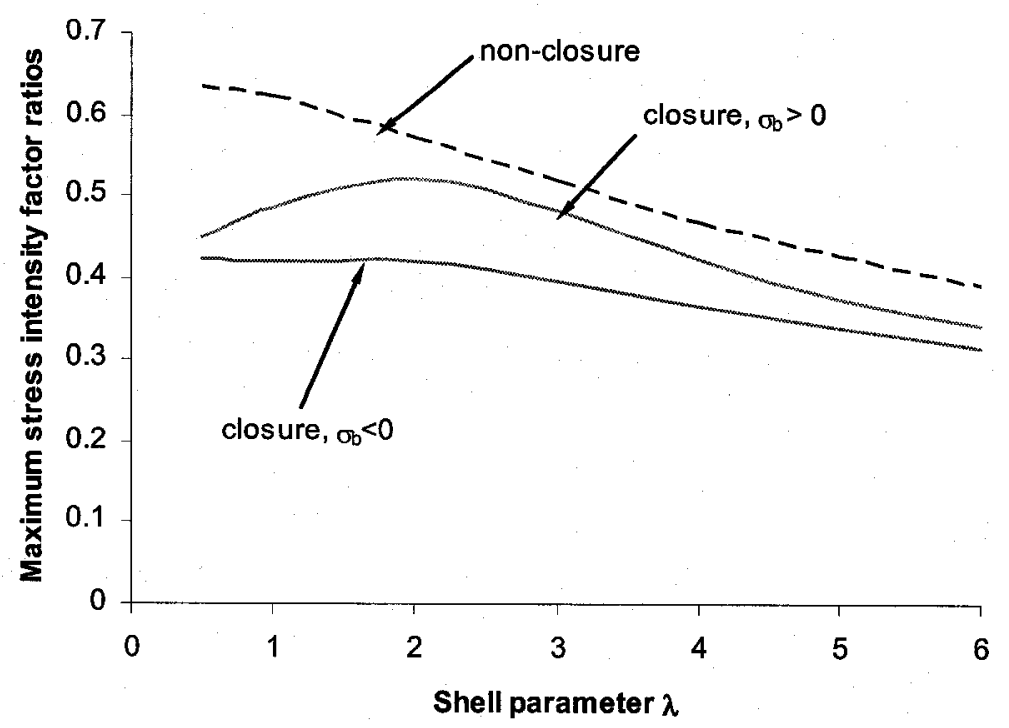

(a)

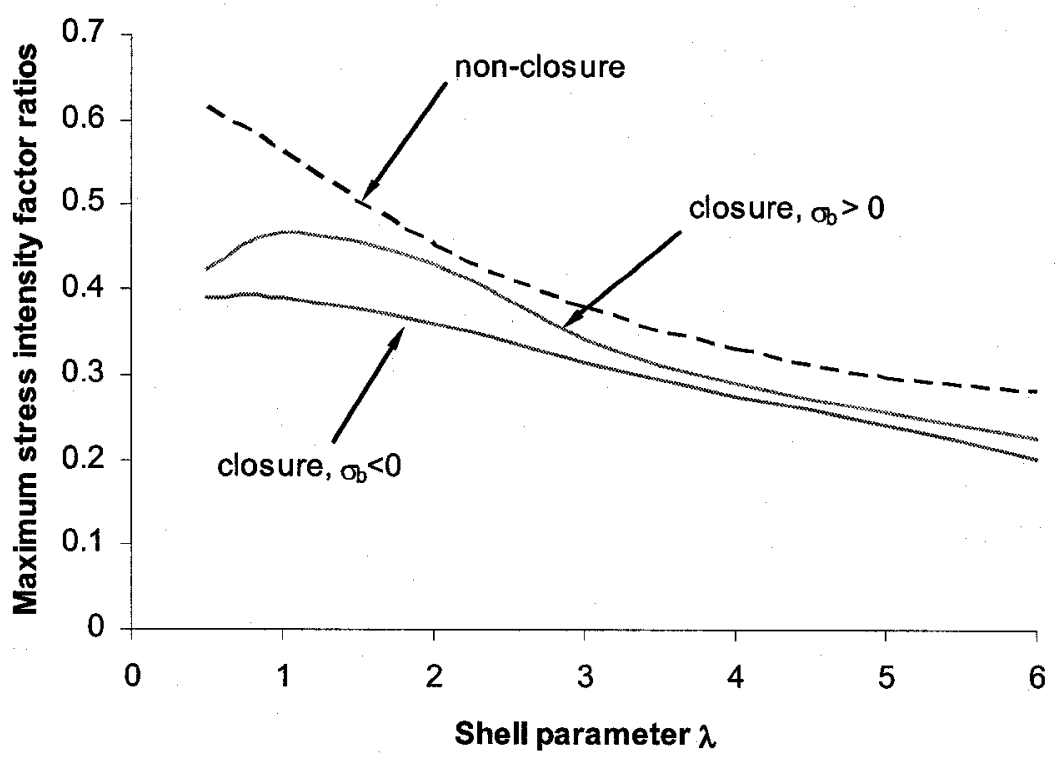

(b) 


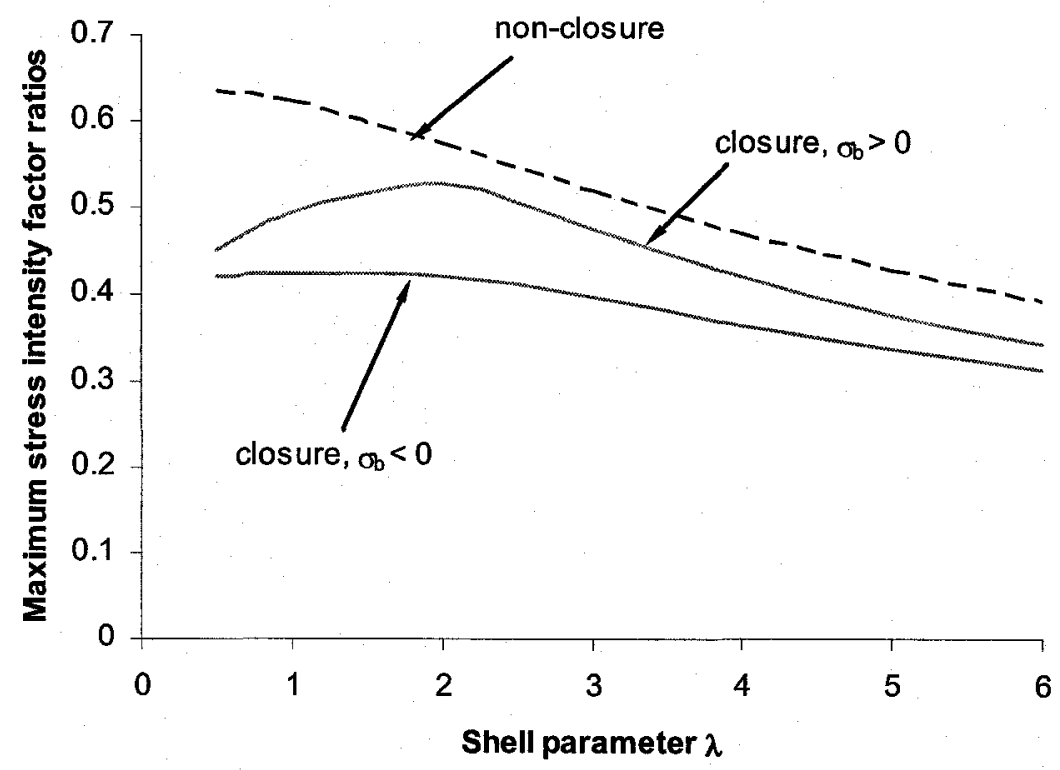

(c)

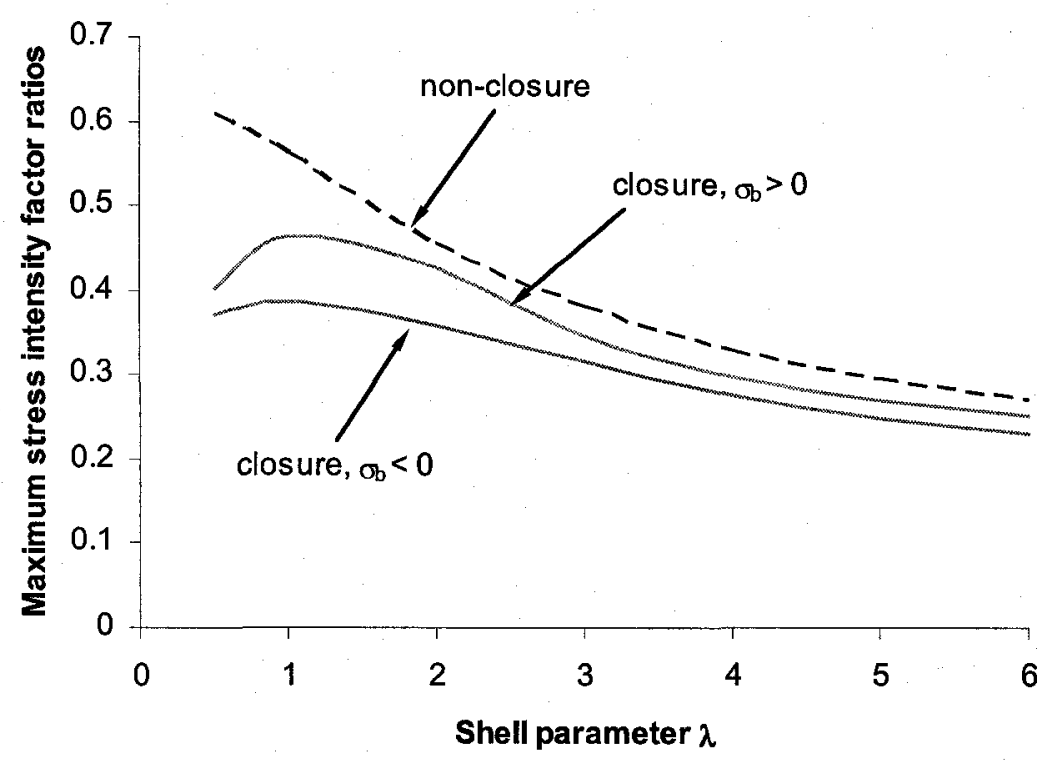

(d)

Figure 3.9 Comparison between closure and non-closure solutions of the maximum stress intensity factor ratio for a specially orthotropic shell having double curvature with $a / h=10$ : (a) $\varphi=0.725$ and $\psi=0.25$; (b) $\varphi=0.725$ and $\psi=4$; (c) $\varphi=1.38$ and $\psi=0.25$; (d) $\varphi=1.38$ and $\psi=4$ 
To investigate the influences of material orthotropy and shell curvature on the closure effect one may compute the difference of the maximum stress intensity factor ratio between the closure case and non-closure case for different values of $\varphi$ and $\psi$. As illustrated in Figure 3.10 for positive bending, the influence of material orthotropy on the crack closure effect varies with $\psi$. For the smaller value of $\psi=0.25$ the reduction of the maximum stress intensity factor ratio by the closure decreases with the increase of $\varphi$ for small $\lambda$ values, but for large $\lambda$ values the variation of the reduction with $\varphi$ is not significant, see Figure 3.10(a). However, for $\psi=4$, there are no apparent relationships between $\varphi$ and the closure effect except that the reduction of the maximum stress intensity factor ratio is the least at $\varphi=26.67$, see Figure 3.10(b). Similar results can be obtained for negative bending, but the reductions are larger than those in positive bending, because the stress intensity factor ratios in negative bending case are lower than those in positive bending case, see Figure 3.8 .

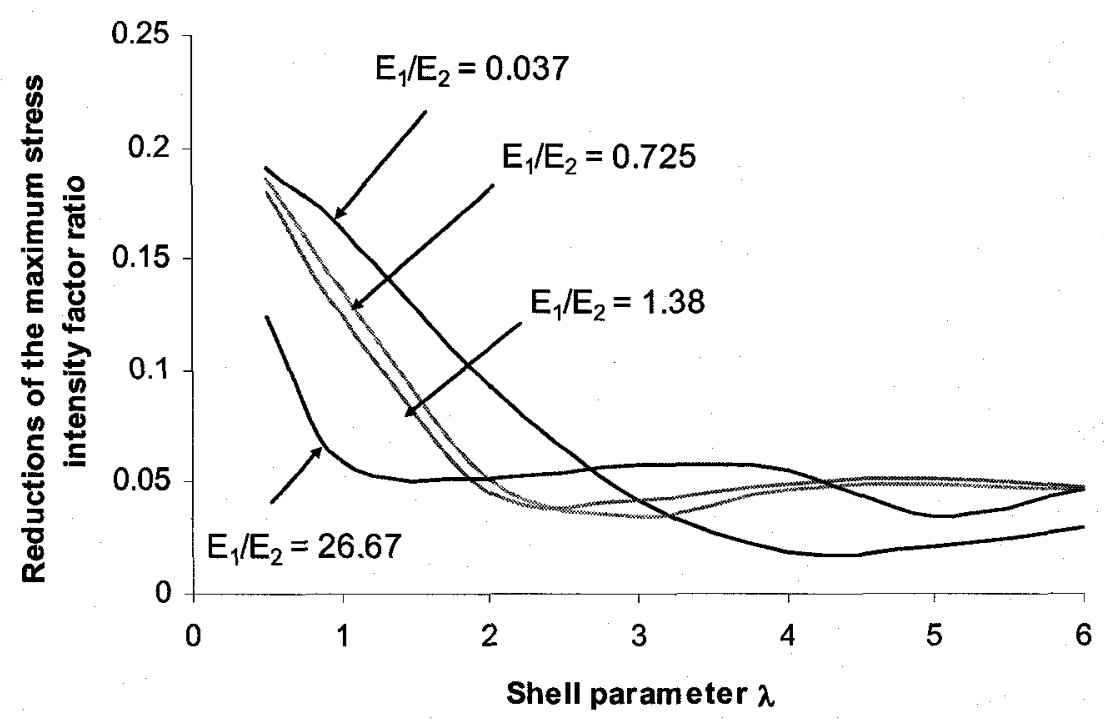

(a) 


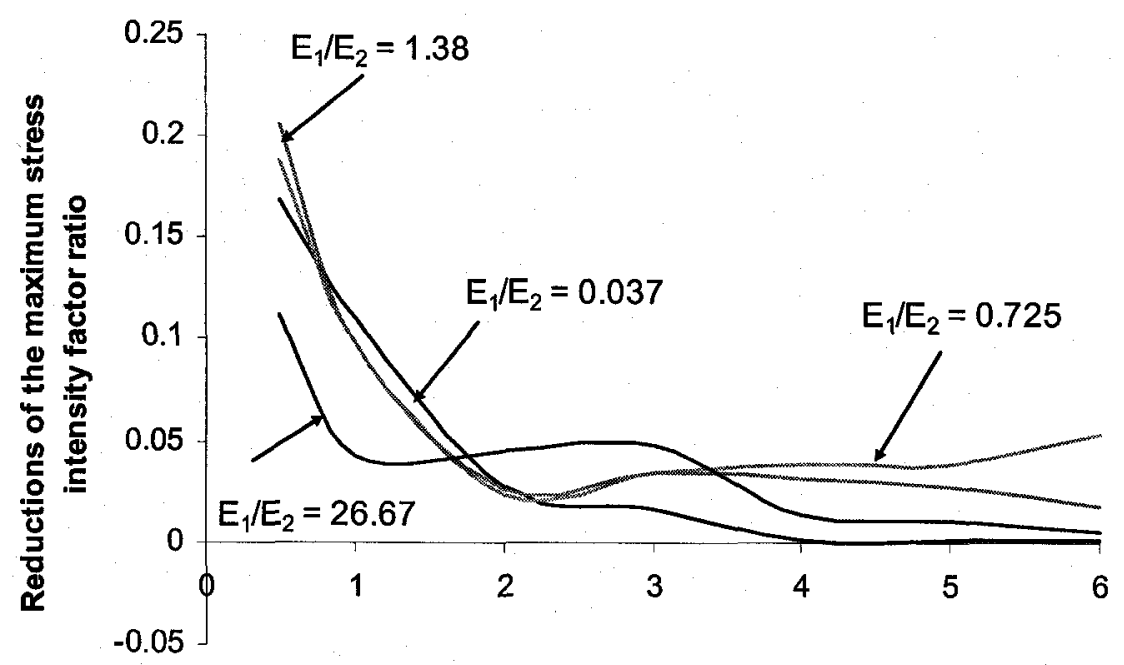

Shell parameter $\lambda$

(b)

Figure 3.10 Effects of material orthotropy and shell curvature on the reduction of the maximum stress intensity factor ratio for a specially orthotropic shell having double curvature under positive bending with $a / h=10$ :

(a) $\psi=0.25$ and (b) $\psi=4$

The closure SIFs for various ratios of $a$ to $h$ of the shell geometries $R_{1} / R_{2}=0.25$ and $R_{1} / R_{2}=4$ with different $E_{1} / E_{2}$ ratios were computed. Figure 3.11 presents the results for $E_{1} / E_{2}=0.725$ and $E_{1} / E_{2}=1.38$. For small values of $a / h$ ratios, for example, $a / h=1$ and $a / h=2$, large values of $\lambda$ (greater then 2) are unreasonable due to the shell geometrical restriction. In consistence with the solutions of non-closure SIF published in the Stress Intensity Factor Handbook, the results presented in this thesis are of $\lambda$ up to 1 for $a / h=1, \lambda$ up to 2 for $a / h=2, \lambda$ up to 5 for $a / h=5$ and $\lambda$ up to 6 for $a / h=10$, as seen in Figure 3.11. 


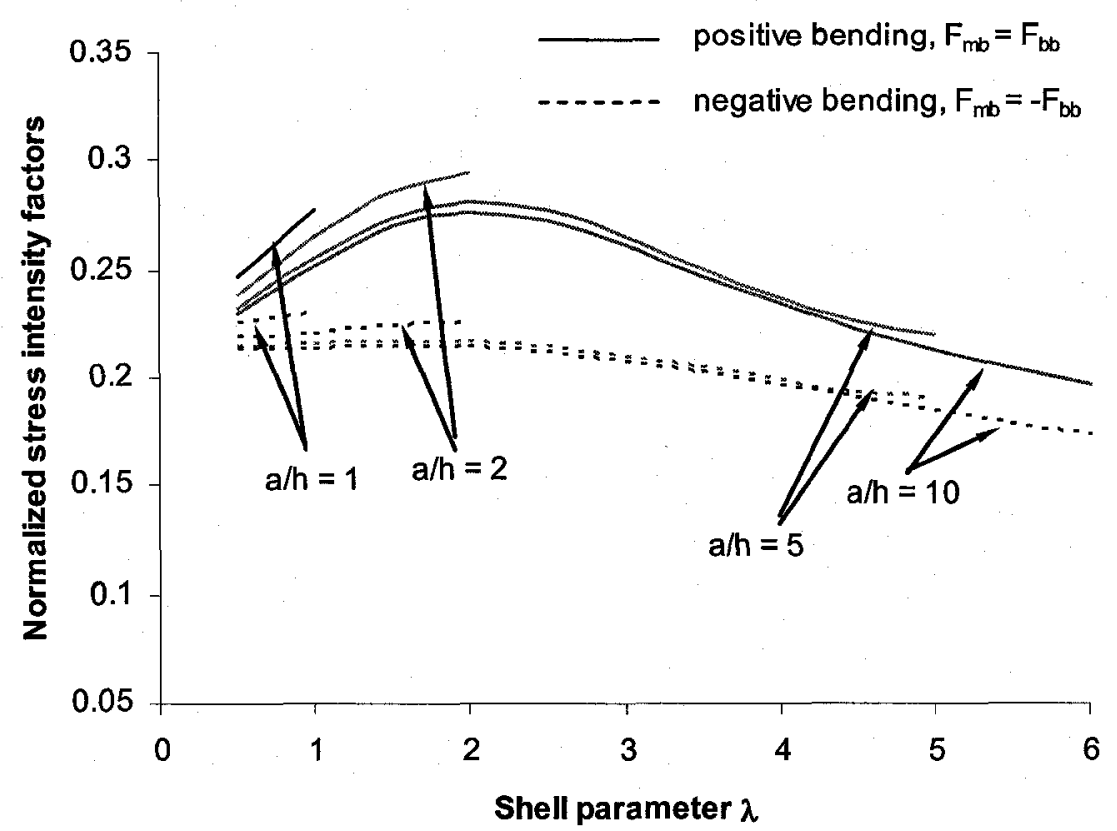

(a)

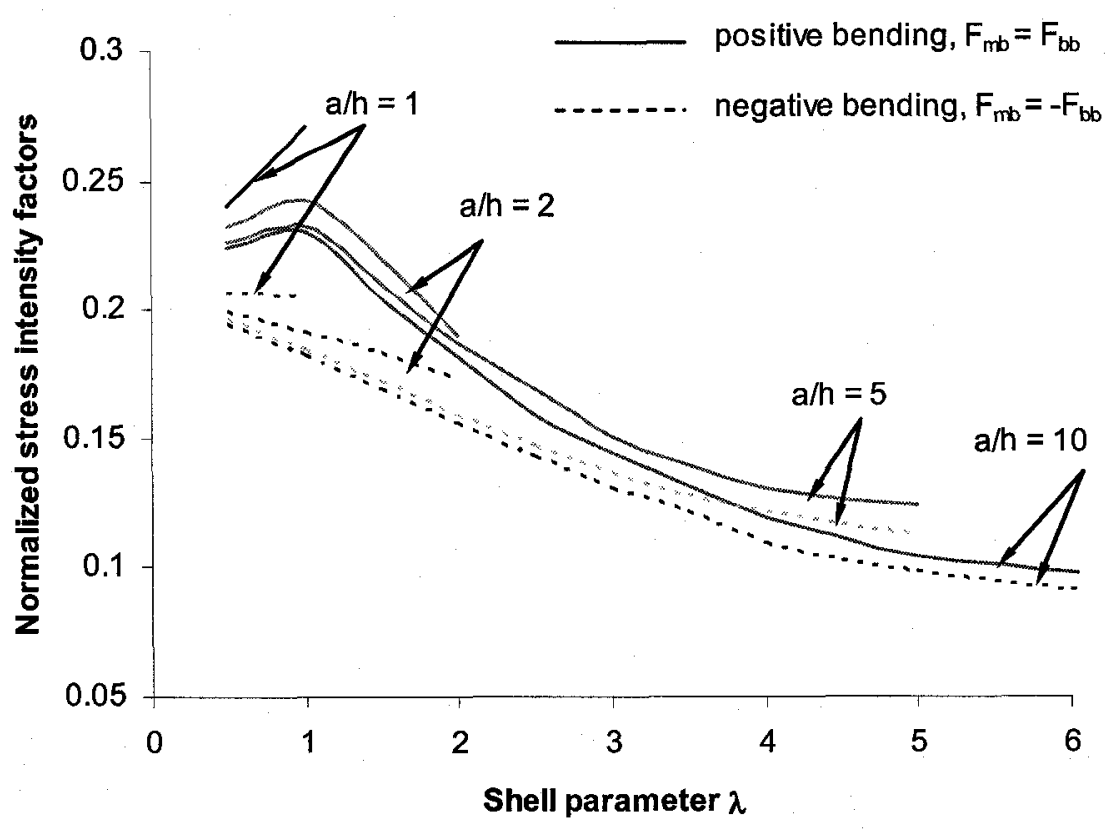

(b) 


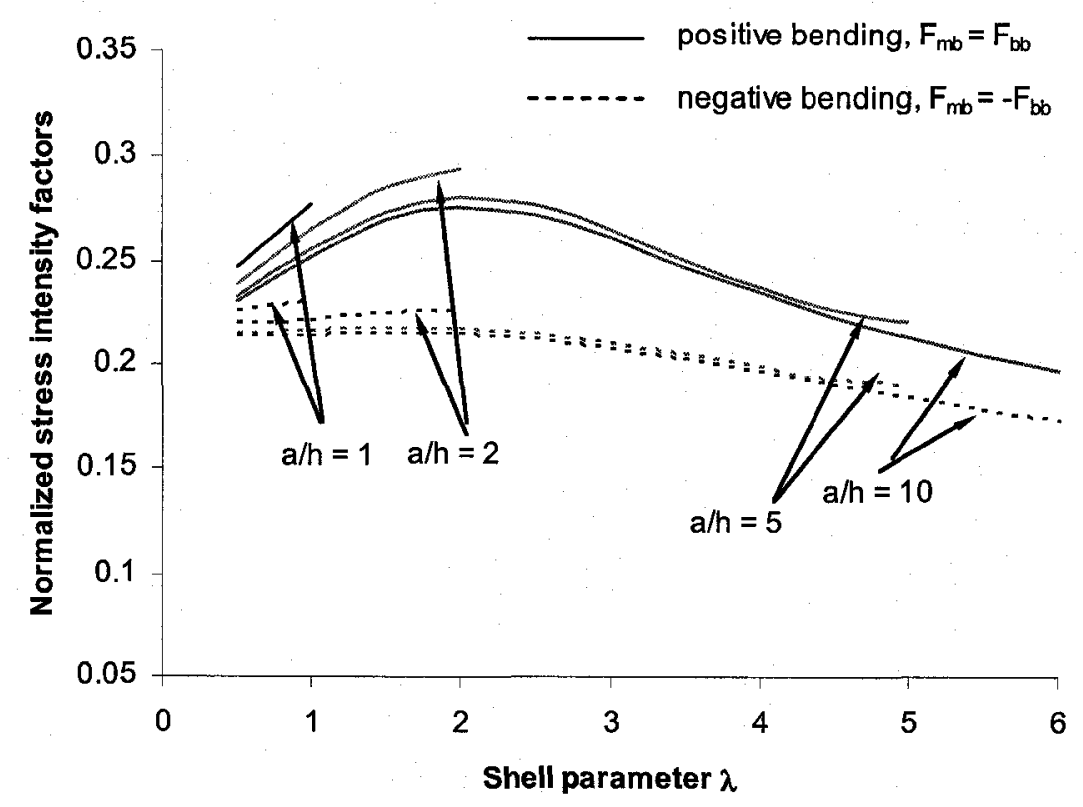

(c)

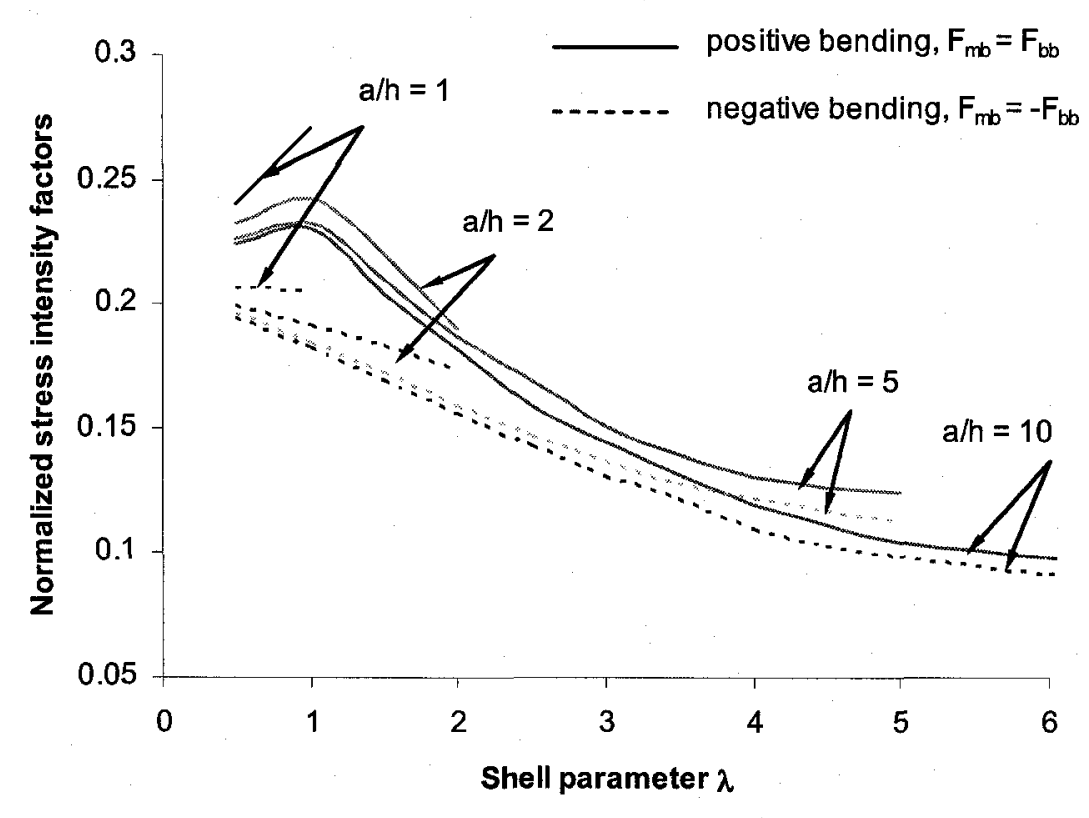

(d)

Figure 3.11 Closure solutions of the stress intensity factor ratios for a specially orthotropic shell having double curvature: (a) $\varphi=0.725$ and $\psi=0.25$; (b) $\varphi=0.725$ and $\psi=4 ;$ (c) $\varphi=1.38$ and $\psi=0.25 ;$ (d) $\varphi=1.38$ and $\psi=4$ 


\subsection{Specially Orthotropic Cylindrical Shell}

\subsubsection{Definition of an axial crack and a circumferential crack in a specially orthotropic cylindrical shell}

For the general case that a specially orthotropic shell has two nonzero curvatures containing a crack in Figure 3.1, if consider a special case that one of the curvature radii approaches to infinite, say $R_{2} \rightarrow \infty$, then the problem becomes a specially orthotropic cylindrical shell containing an axial crack, as seen in Figure 3.12, in this case, $R_{1}=R$, which is the radius of the cylinder. Similarly, in another special case, $R_{1} \rightarrow \infty$, then the problem becomes a specially orthotropic cylindrical shell containing a circumferential crack, as seen in Figure 3.13, in this case, $R_{2}=R$, which is the radius of the cylinder.

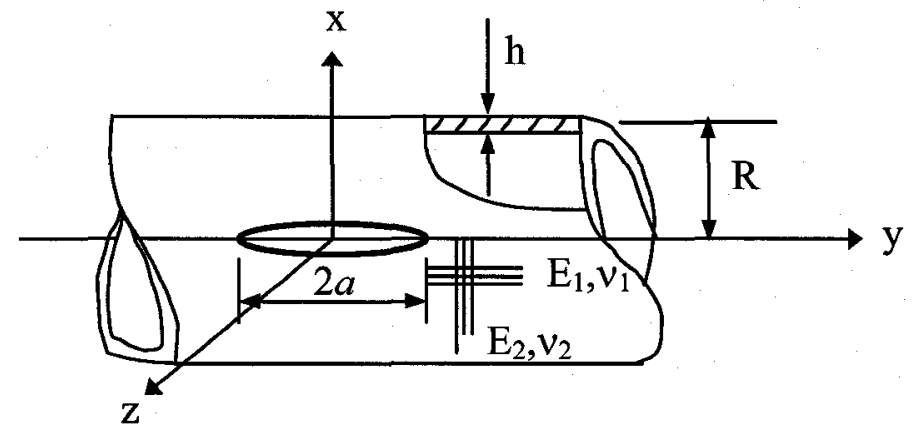

(a)

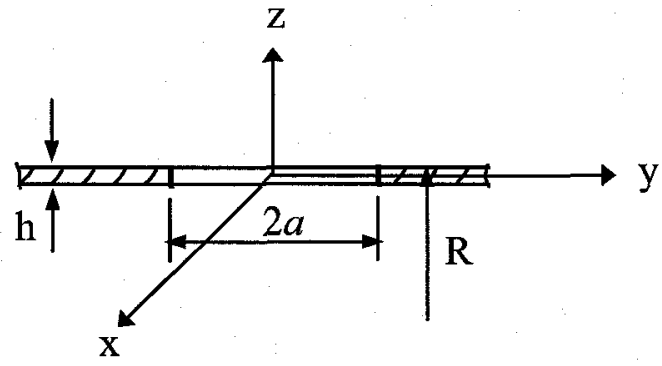

(b)

Figure 3.12 Geometry and coordinates for a through-the-thickness axial crack in a cylindrical shell: (a) top view and (b) side view 


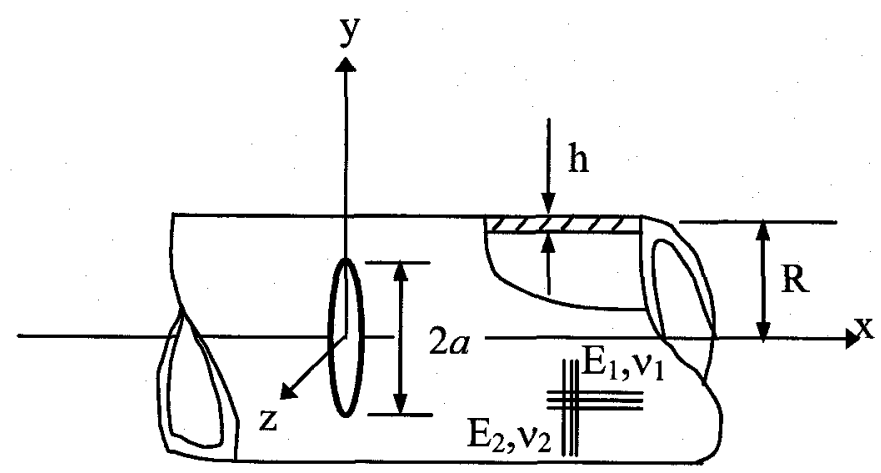

(a)

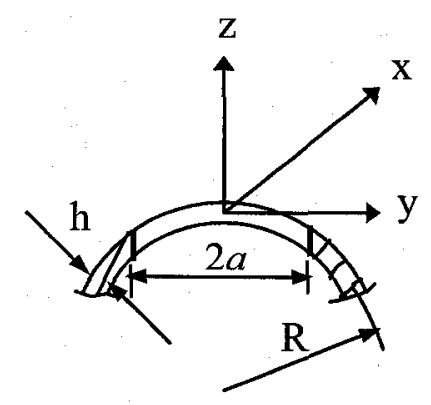

(b)

Figure 3.13 Geometry and coordinates for a through-the-thickness circumferential crack in a specially orthotropic cylindrical shell: (a) top view and (b) side view

The thickness of the shell wall is $h$ and the crack length is $2 a$. The material of the shell is assumed to be specially orthotropic with $E_{1}, v_{1}$ and $E_{2}, v_{2}$ being the elastic constants in the axial and circumferential directions respectively. Within the coordinate systems defined in Figure 3.12 and in Figure 3.13, the equilibrium equations for a cylindrical shell containing an axial and a cylindrical shell containing a circumferential crack can be written in terms of the $z$-component displacement $w[7,13,14]$, 


$$
\nabla^{4} \nabla^{4} w+\lambda_{i}^{4}\left(1-\kappa \nabla^{2}\right) \frac{\partial^{4} w}{\partial x^{4}}=0, \quad i=1,2
$$

where $i=1$ for axial crack and $i=2$ for circumferential crack. $\lambda_{1}$ and $\lambda_{2}$ are expressed as

$$
\begin{aligned}
& \lambda_{1}^{4}=12\left(1-v^{2}\right) \frac{c^{2} a^{4}}{h^{2} R^{2}} \\
& \lambda_{2}{ }^{4}=12\left(1-v^{2}\right) \frac{a^{4}}{c^{2} h^{2} R^{2}}
\end{aligned}
$$

The problems can be solved using Fourier transforms. The characteristic equations of the problems and their four roots, which appear in the expressions of the kernels $k_{i j}$ of the integral equations, are given in Appendix $\mathrm{A}$, where for axial crack $\lambda_{1} \neq 0$ and $\lambda_{2}=0$; for circumferential crack $\lambda_{1}=0$ and $\lambda_{2} \neq 0$.

\subsubsection{Crack closure in a specially orthotropic cylindrical shell containing an axial crack}

Again, the two orthotropic materials which are used in the analysis of shells with double curvature are taken as examples in cylindrical shell study. Figure 3.14 illustrates the contact force distributions along the crack length for a mildly orthotropic (titanium material) cylindrical shell and a strongly orthotropic (graphite-epoxy material) cylindrical shell containing an axial crack under positive bending. It is shown that similar to the shells with two nonzero curvatures the contact force distributes nonuniformly along the crack length for cylindrical shells, as shown in Figure 3.14. For small values of $\lambda$, where $\lambda=\left[12\left(1-v^{2}\right)\right]^{1 / 4} a / \sqrt{R h}$, which represents the curvature of the shell when the crack length, $a$, and shell thickness, $h$, are given, the contact force is positive over the entire crack length. However, with the increase of the $\lambda$ value the contact force becomes 
negative in the region around the center of the crack. This confirms that the shell curvature does influence the crack closure behavior; it may cause partial crack closure. As deduced from Figure 3.14, in the limiting case, at $\lambda$ approaching zero, that is, the cylinder radius $R$ approaching infinity, which represents plates, complete crack closure would occur.

It is also important to report that material orthotropy affects the crack closure behavior in cylindrical shells containing an axial crack. Comparing the contact force distributions for the different $E_{1} / E_{2}$ ratios in Figure 3.14, for the mildly orthotropic shell partial closure occurs when the $\lambda$ value equals or great than 4 for $E_{1} / E_{2}=0.725$ and 3.5 for $E_{1} / E_{2}=1.38$, while for the strongly orthotropic shell partial closure occurs when the $\lambda$ value is up to 8 for $E_{1} / E_{2}=0.037$ and 2 for $E_{1} / E_{2}=26.67$. This manifests that the curvature effect on the closure behavior is enhanced with the increase of the $E_{1} / E_{2}$ ratio.

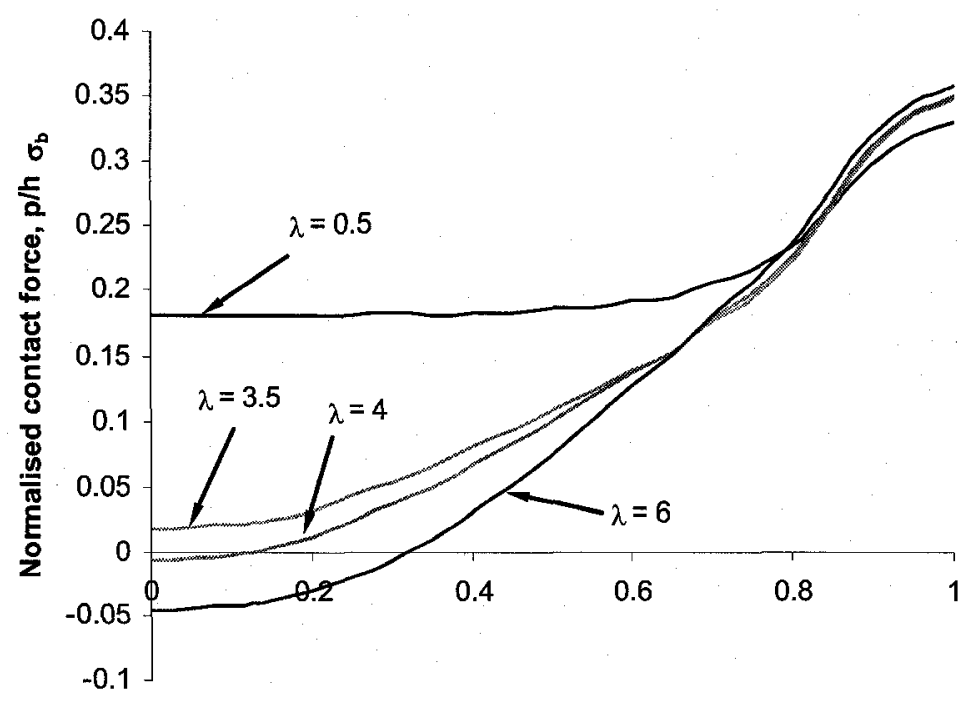

Distance from the center of the crack, y/a

(a) 


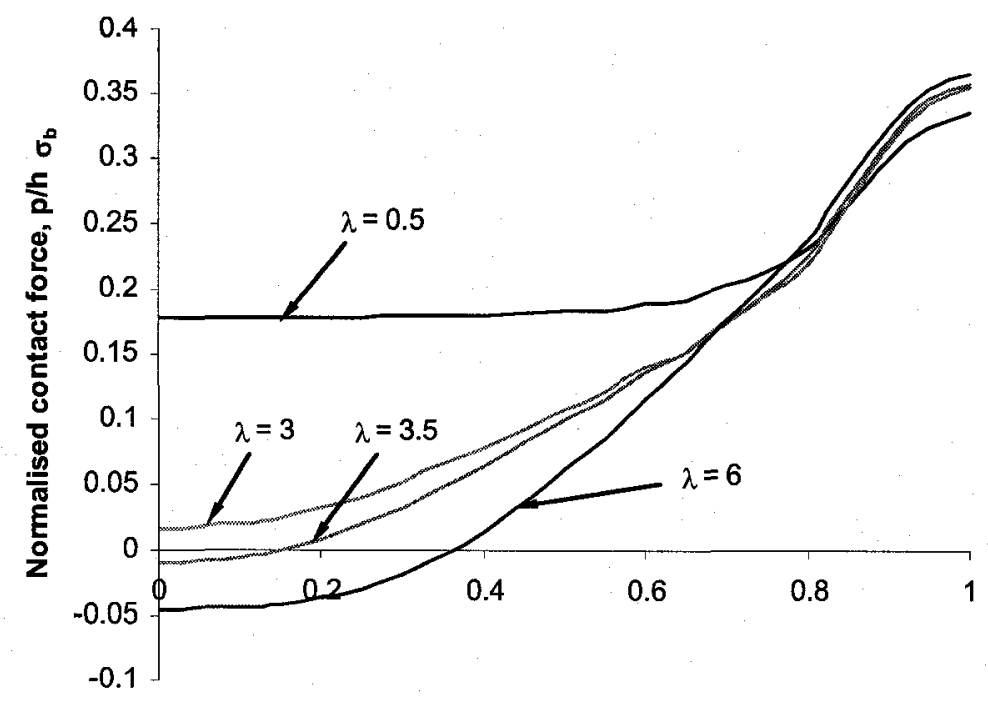

Distance from the center of the crack, y/a

(b)

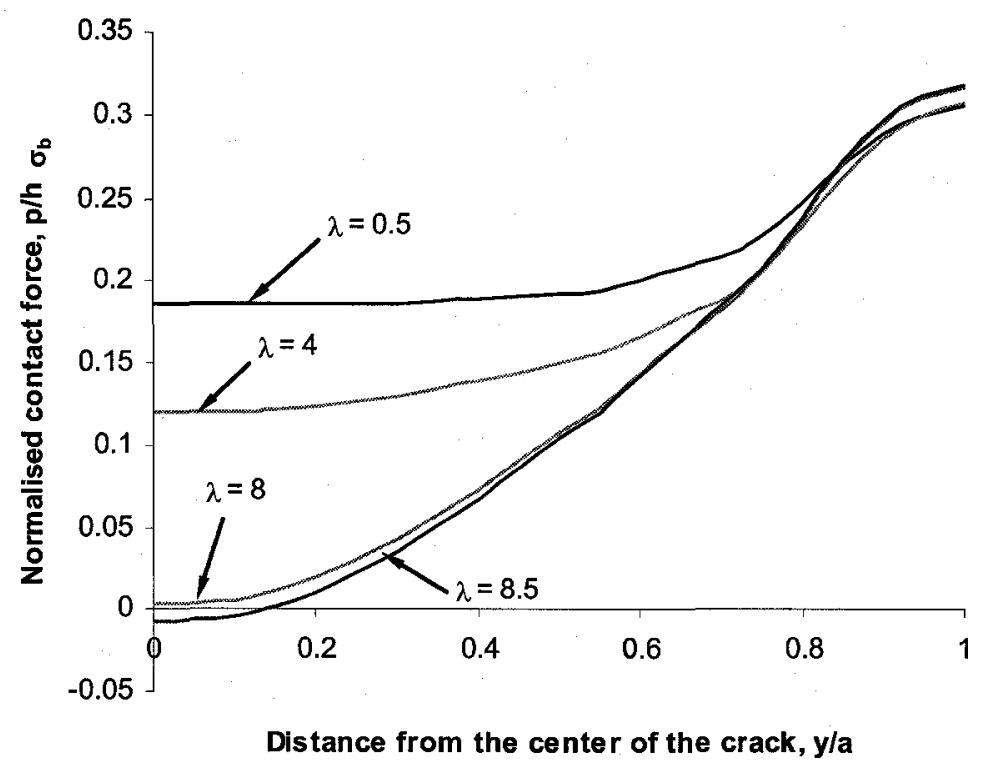

(c) 


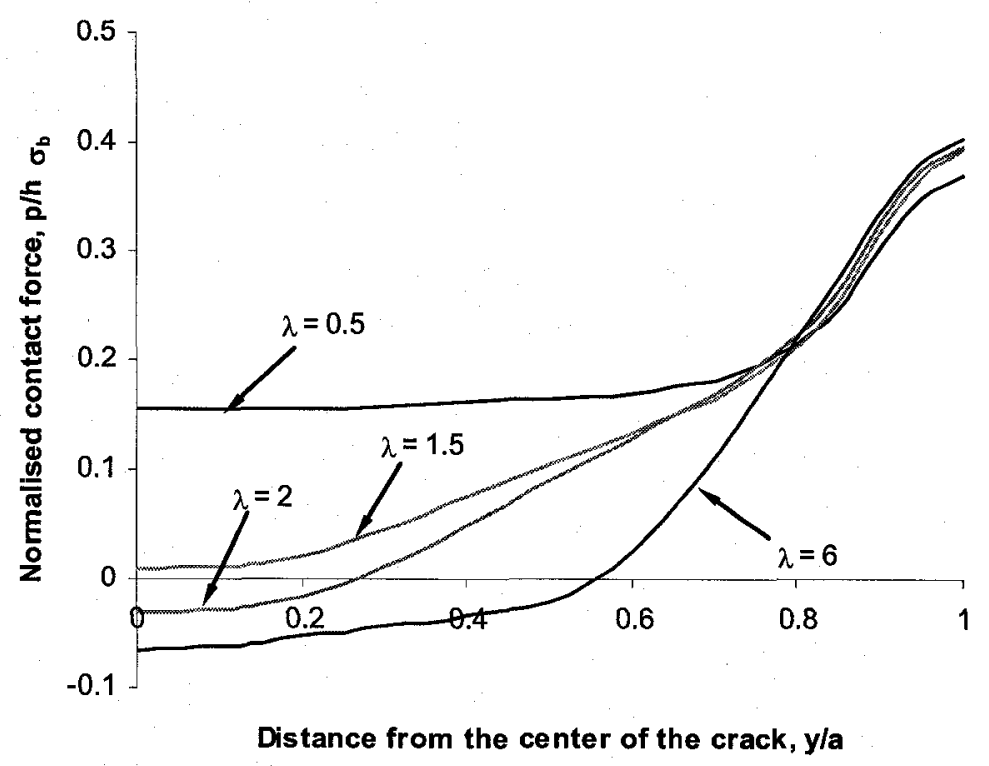

(d)

Figure 3.14 Contact force distributions assuming full-length closure for a specially orthotropic cylindrical shell containing an axial crack under positive bending with $a / h=10$ :

(a) $E_{1} / E_{2}=0.725 ;$ (b) $E_{1} / E_{2}=1.38 ;$ (c) $E_{1} / E_{2}=0.037$ and (d) $E_{1} / E_{2}=26.67$

The crack closure in an axially cracked cylindrical shell under negative bending was also studied. The results show that the contact force is positive over the whole crack length for all the $\lambda$ values being considered (up to 6). This means that entire-length crack closure always occurs in an axially cracked cylindrical shell under negative bending as long as $\lambda \leq 6$. However, it can be expected that for even higher $\lambda$ values, partial closure may occur over the central region of the crack.

Comparing the SIFs in the closure case with those in the non-closure case, as presented in Figure 3.15 for an axially cracked cylindrical shell with different $E_{1} / E_{2}$ 
ratios, it can be seen that the crack-face closure has significant effect on the SIFs. It reduces the bending component but increases the membrane component. Positive bending causes higher stress intensity factor than that of negative bending.

Taking the strongly orthotropic cylindrical shell as an example, Figure 3.16 illustrates the maximum stress intensity factor ratios in both the closure and non-closure cases for an axially cracked cylindrical shell. It is shown that the maximum stress intensity factor ratios are decreased by the effect of crack closure. The reduction of the values increases with the decrease of the $\lambda$ value. In other words, the crack closure effect on the SIFs is enhanced when the $\lambda$ value is small.

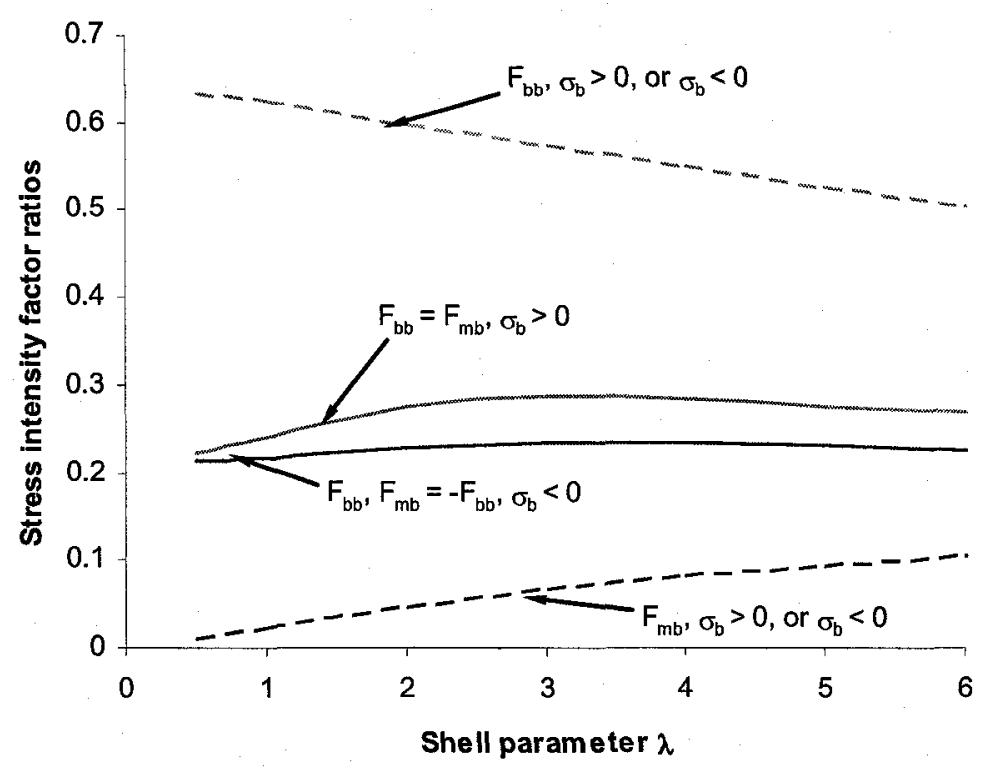

(a) 


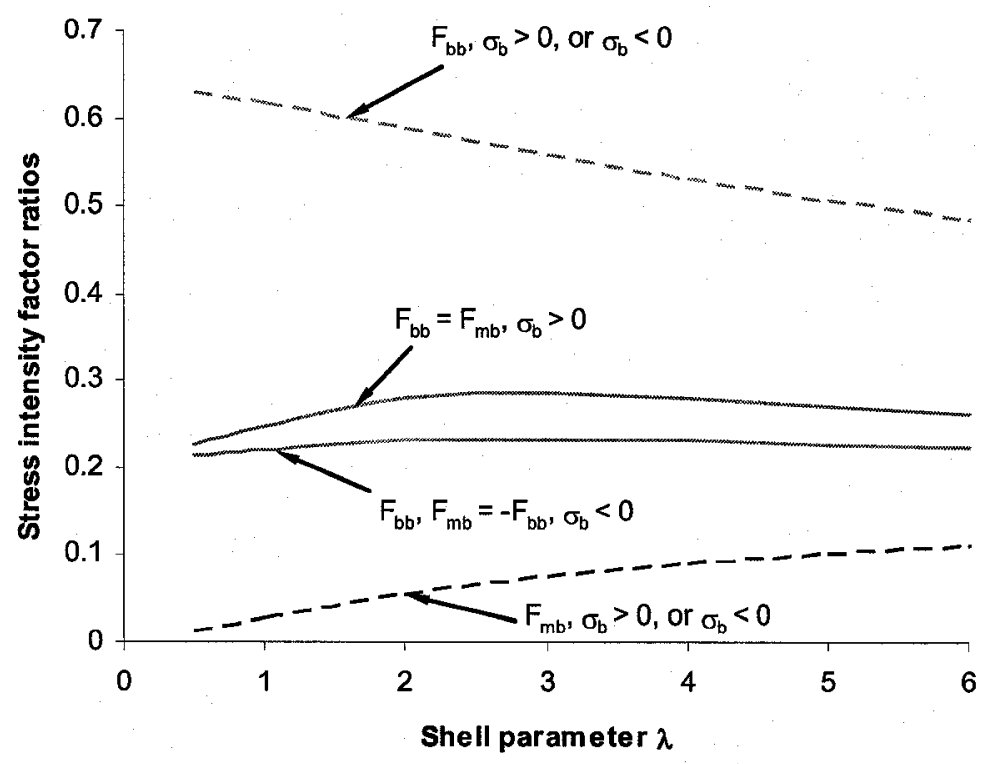

(b)

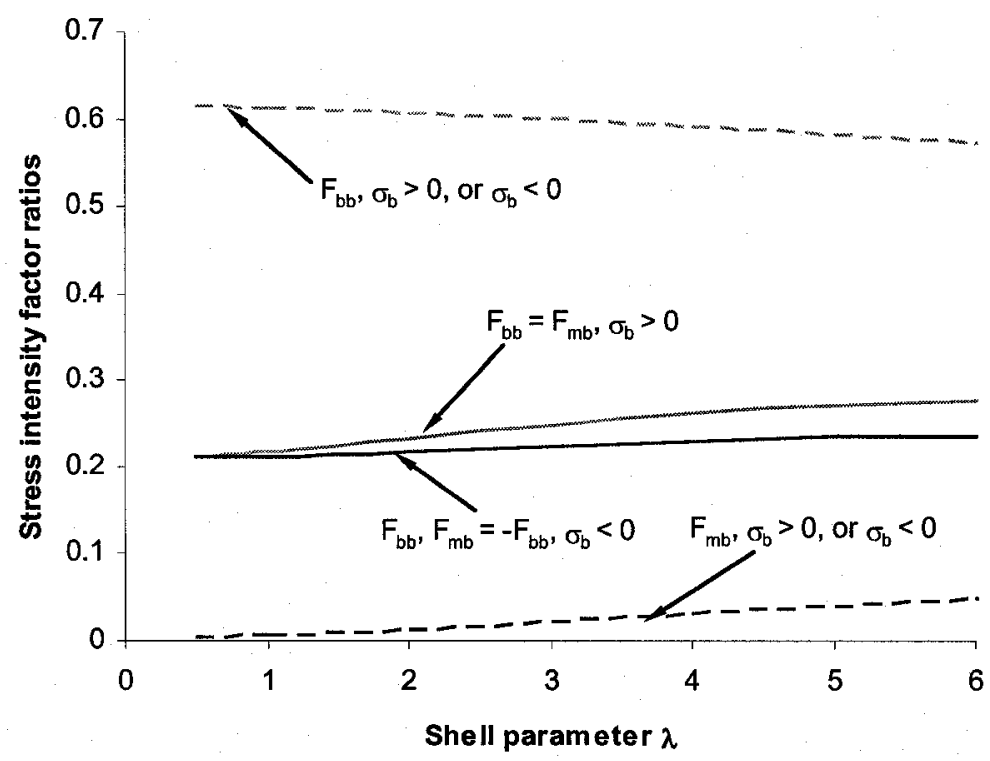

(c) 


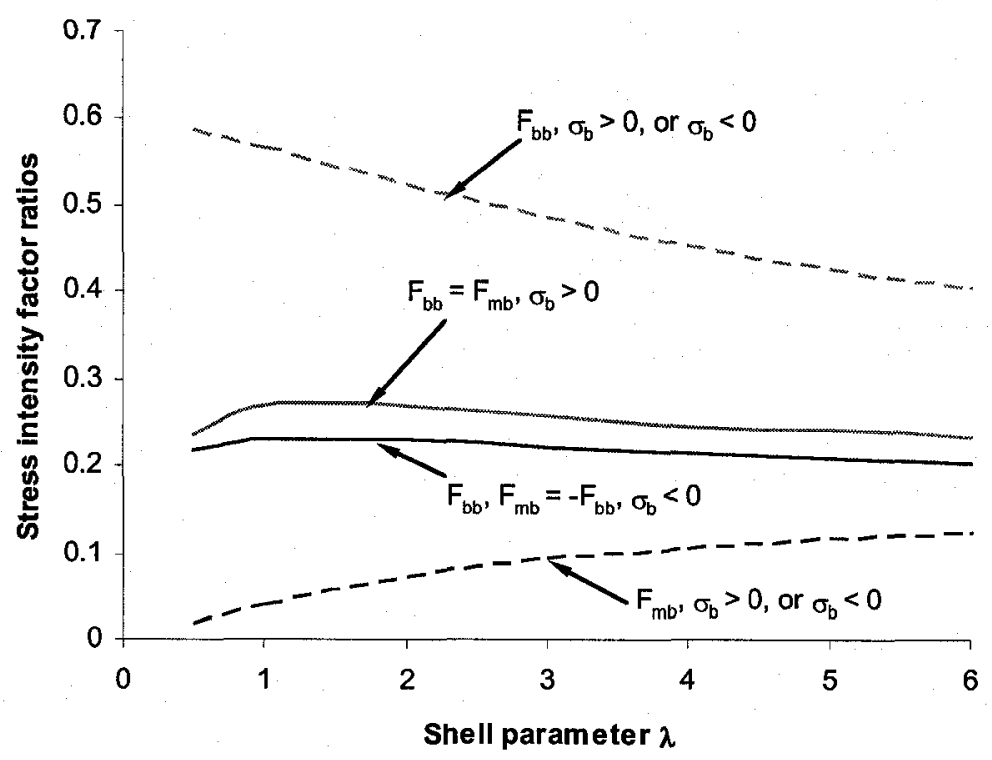

(d)

Figure 3.15 Comparison between closure and non-closure stress intensity factor ratios for a specially orthotropic cylindrical shell containing an axial crack with $a / h=10(\longrightarrow$ closure and - - - non-closure):

(a) $E_{1} / E_{2}=0.725 ;$ (b) $E_{1} / E_{2}=1.38 ;$ (c) $E_{1} / E_{2}=0.037$ and (d) $E_{1} / E_{2}=26.67$

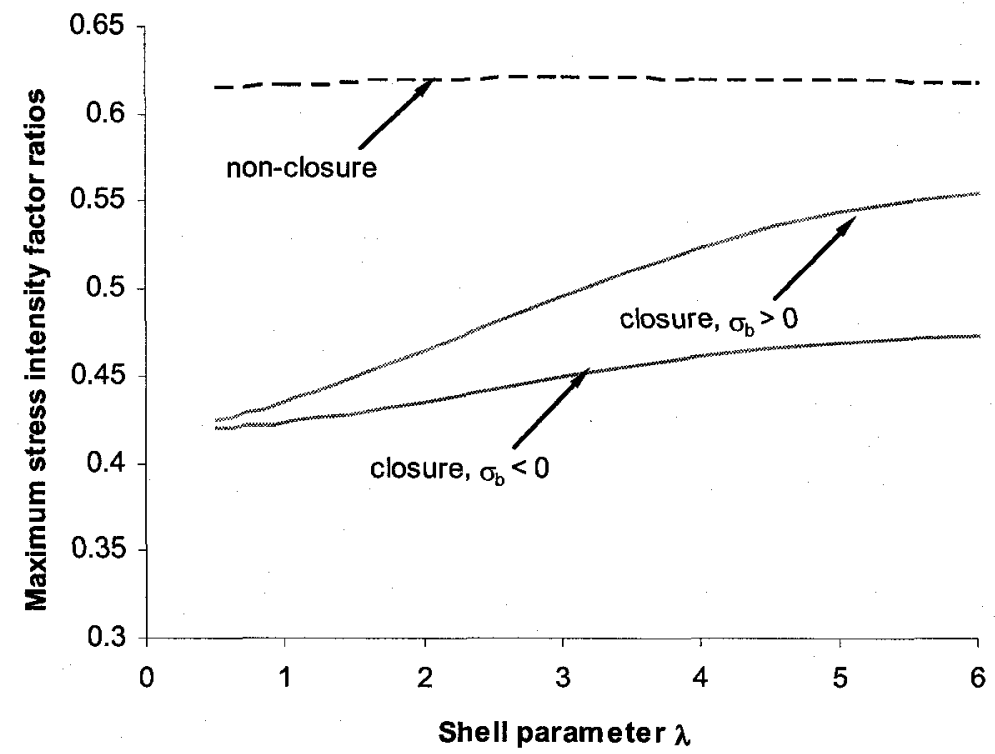

(a) 


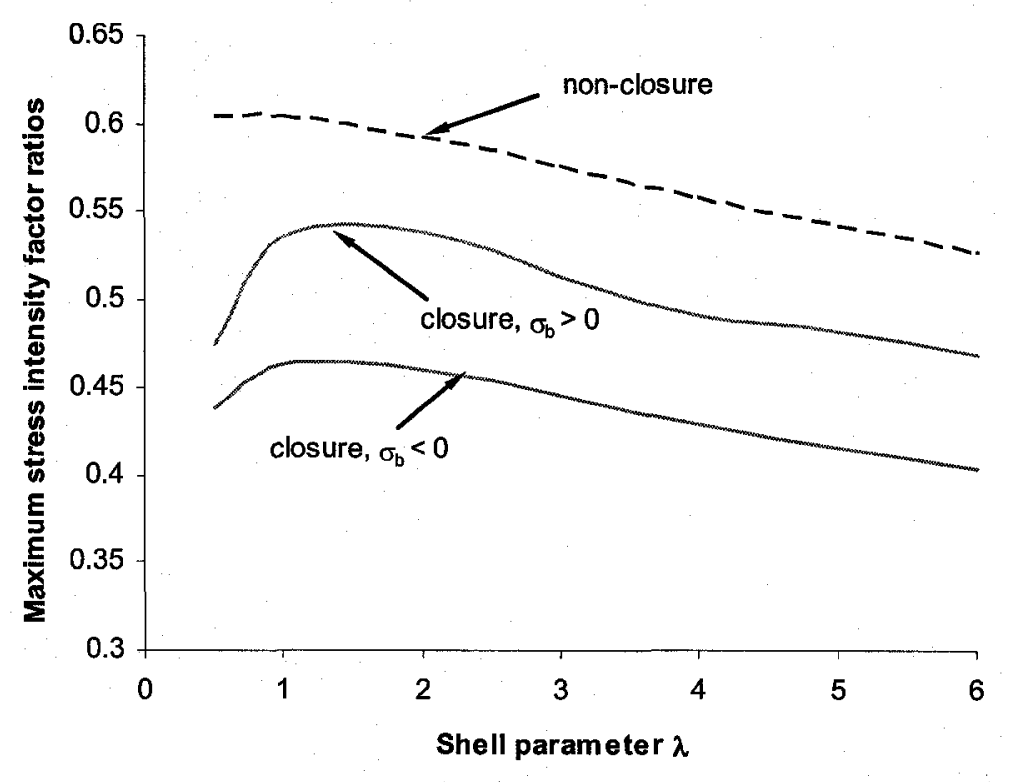

(b)

Figure 3.16 Comparison between closure solutions and non-closure solutions of the maximum stress intensity factor ratios for a specially orthotropic cylindrical shell containing an axial crack with $a / h=10$ :

(a) $E_{1} / E_{2}=0.037$ and (b) $E_{1} / E_{2}=26.67$

As concerns material orthotropy, comparing the maximum stress intensity factor ratios in Figure 3.16, it is found that the maximum stress intensity factor ratio of non-closure is almost constant with the $\lambda$ value when the $E_{1} / E_{2}$ ratio is small $(0.037)$, while it greatly decreases when the $E_{1} / E_{2}$ ratio is large (26.67). In the closure case, the maximum stress intensity factor ratios of positive bending and negative bending all increase with $\lambda$ for small value of the $E_{1} / E_{2}$ ratio but the former is more significant. However, for large value of the $E_{1} / E_{2}$ ratio, the maximum stress intensity factor ratios decrease with $\lambda$ except when $\lambda$ is very small. 
The more evidence to this issue can be obtained from Figure 3.17, which illustrates the reduction of the maximum stress intensity factor ratios due to the crack closure effect for a mildly orthotropic and a strongly orthotropic cylindrical shell containing an axial crack. It is observed that material orthotropy plays an important role in reducing the maximum SIFs by the crack closure effect. The amount of the reduction increases with the decrease of the $E_{1} / E_{2}$ ratio generally, but in positive bending the opposite is observed when $\lambda$ is larger for smaller values of the $E_{1} / E_{2}$ ratio.

It is also observed that small values of $\lambda$ represent large radii of the cylinder. From Figure 3.15 it shows that the difference of the stress intensity factor ratios between the closure solution and the non-closure solution is the maximum when $\lambda$ approaches zero. This indicates that crack closure has stronger influence on the SIFs of plates than shells.

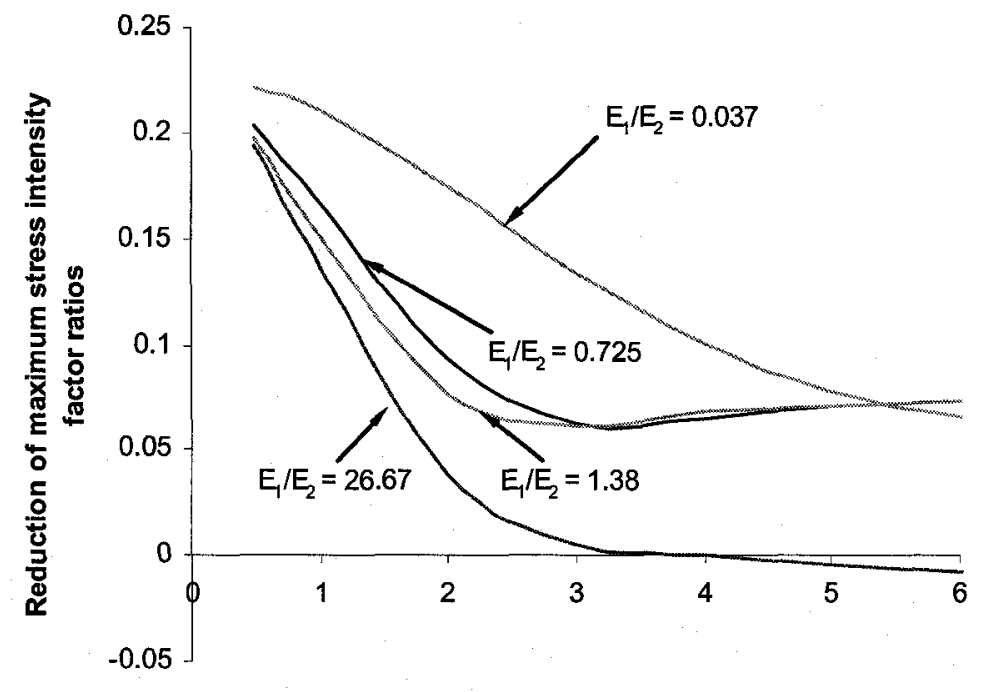

Shell parameter $\lambda$

(a) 


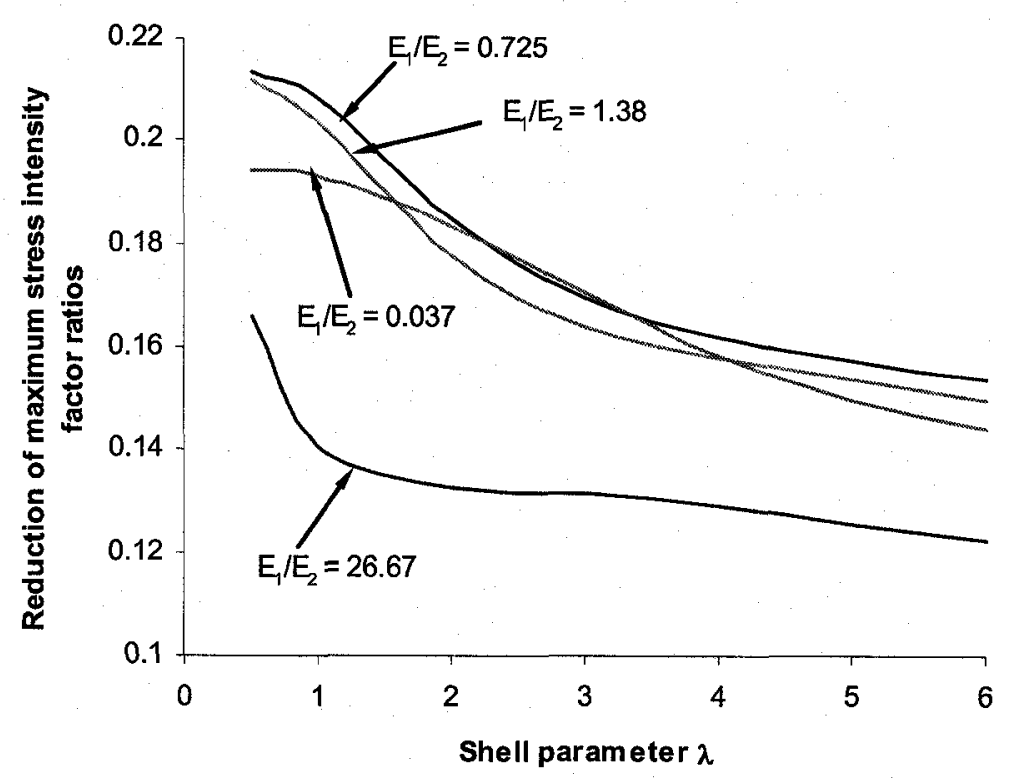

(b)

Figure 3.17 Reductions of the maximum stress intensity factor ratios due to the crack closure for a specially orthotropic cylindrical shell containing an axial crack with $a / h=10:$ (a) under positive bending and (b) under negative bending

Finally, it is shown that the effect of material orthotropy on the SIFs is stronger in a cylindrical shell containing an axial crack than in plates. As seen in Figure 3.17, when the $\lambda$ value is very small, the four curves which represent different $E_{1} / E_{2}$ ratios are very close, but they are divergent with increasing the $\lambda$ value.

\subsubsection{Crack closure in a specially orthotropic cylindrical shell containing a circumferential crack}

Similar analyses for an axially cracked cylindrical shell have been made for a circumferentially cracked cylindrical shell. The contact forces along the crack length 
were computed for the shell with the two orthotropic materials, mildly orthotropic titanium and strongly orthotropic graphite-epoxy, respectively, assuming full-length closure under positive bending. As shown in Figure 3.18, the contact force distributes nonuniformly along the crack length. Also, it is interesting to notice that, different from an axially cracked cylindrical shell, for a circumferentially cracked cylindrical shell, material orthotropy has little effect on the closure behavior. As seen in Figure 3.18, full-length closure always occurs in an orthotropic cylindrical shell containing a circumferential crack under positive bending when $\lambda$ is less than 6.5 regardless of the values of the $E_{1} / E_{2}$ ratio, where $\lambda=\left[12\left(1-v^{2}\right)\right]^{1 / 4} a / \sqrt{R h}$, which represents the shell curvature when the crack length $a$ and shell thickness $h$ are set, because the contact force is positive everywhere along the crack when $\lambda$ is less than 6.5 .

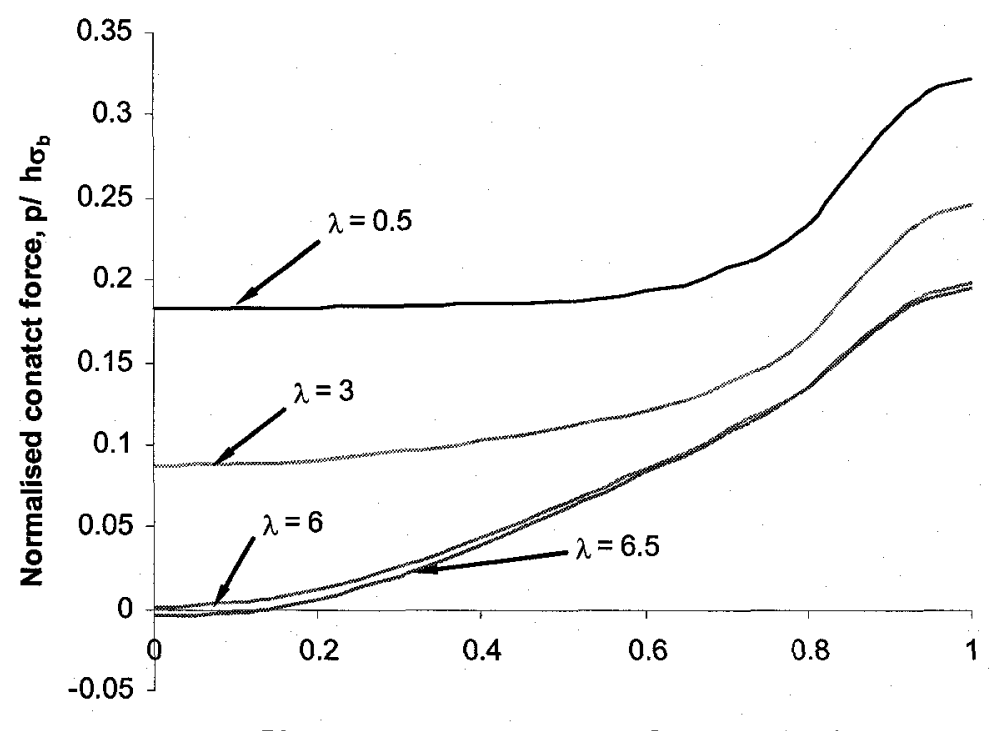

Distance from the center of the crack, y/a

(a) 


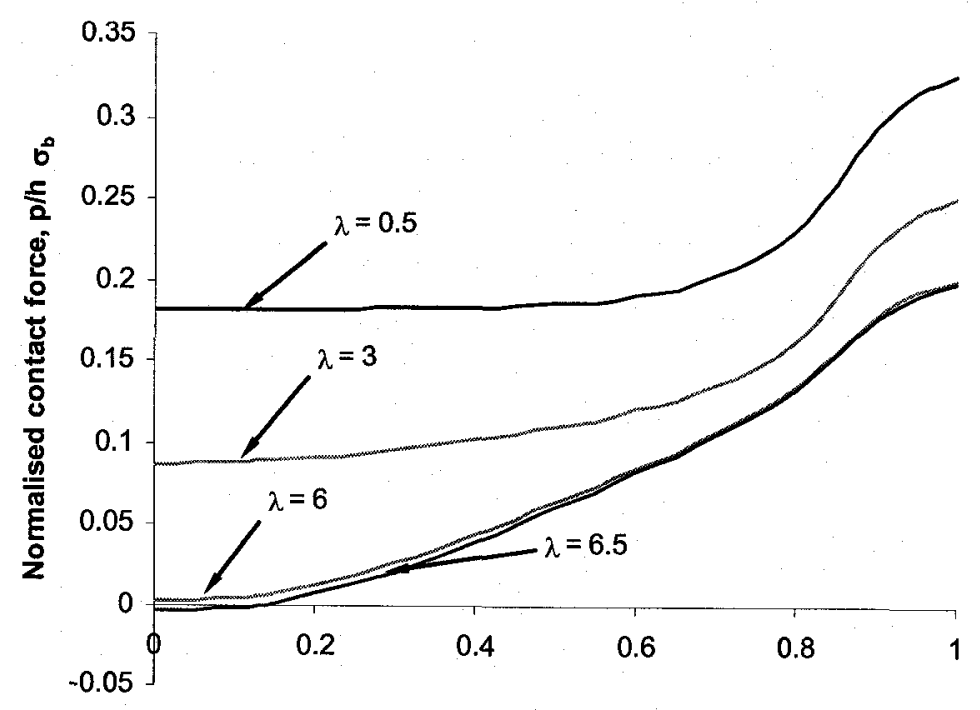

Distance from the center of the crack, y/a

(b)

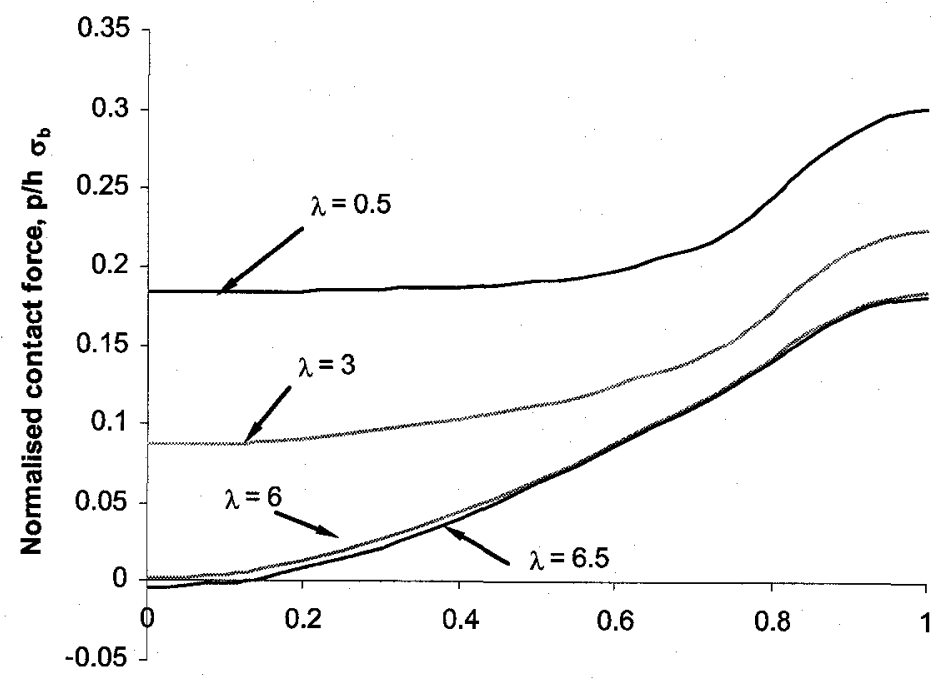

Distance from the center of the crack, y/a

(c) 


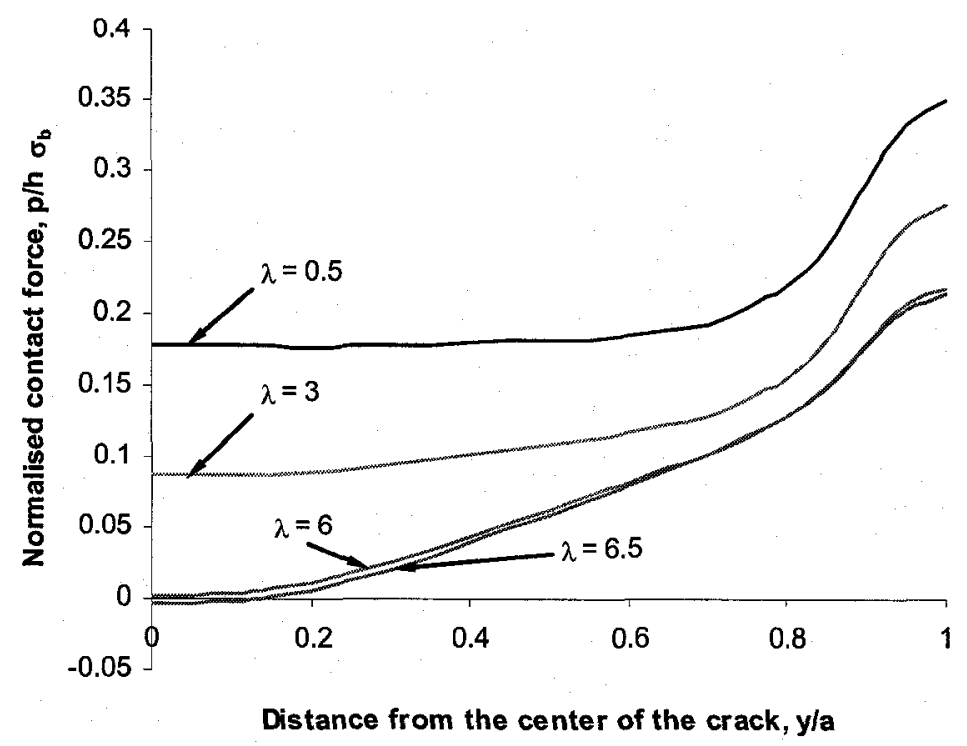

(d)

Figure 3.18 Contact force distributions assuming full-length closure for a specially orthotropic cylindrical shell containing a circumferential crack under positive with $a / h=10$ :

(a) $E_{1} / E_{2}=0.725 ;$ (b) $E_{1} / E_{2}=1.38 ;$ (c) $E_{1} / E_{2}=0.037$ and (d) $E_{1} / E_{2}=26.67$

The same conclusion made on an axially cracked cylindrical shell under negative bending can be also drawn for a circumferentially cracked cylindrical shell.

Figure 3.19 presents the SIFs in the closure case and in the non-closure case for a specially orthotropic cylindrical shell containing a circumferential crack with different $E_{1} / E_{2}$ ratios. Figure 3.20 illustrates the maximum stress intensity factor ratios in the two cases. The same conclusions made for an axially cracked cylindrical shell regarding the effect of crack closure on the SIFs can be also obtained for a circumferentially cracked cylindrical shell, that is, crack-face closure reduces the bending component but increases the membrane component; positive bending causes higher SIF than negative bending; the 
maximum stress intensity factor ratios are decreased by the effect of crack closure; and the reduction of the value increases with the decrease of the $\lambda$ value.

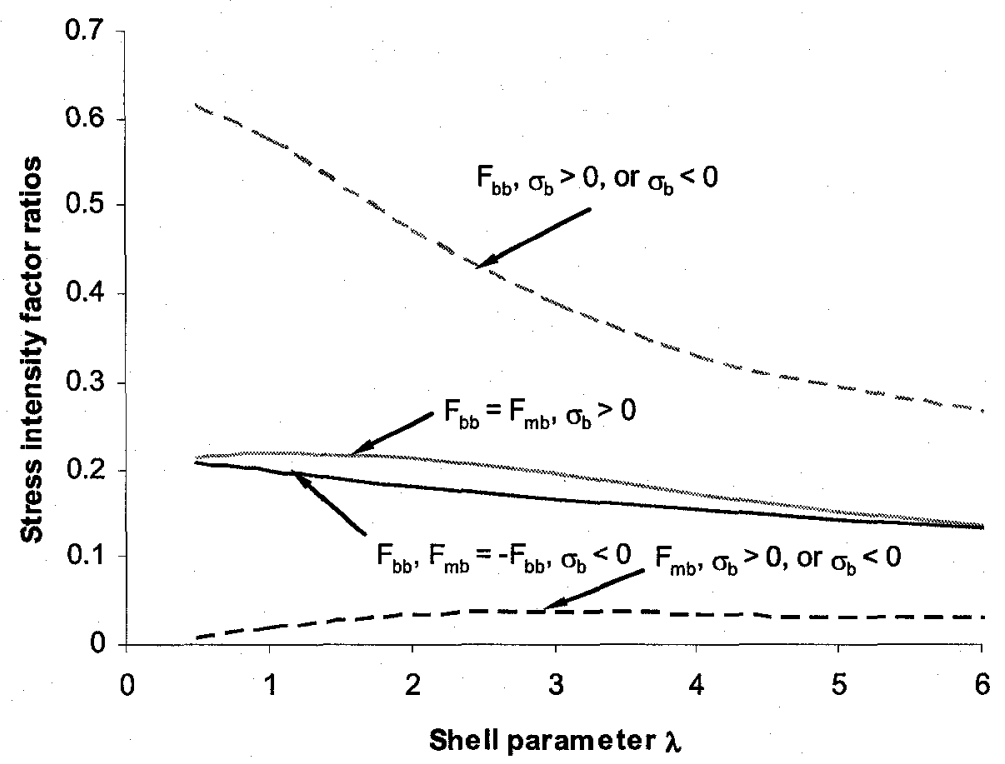

(a)

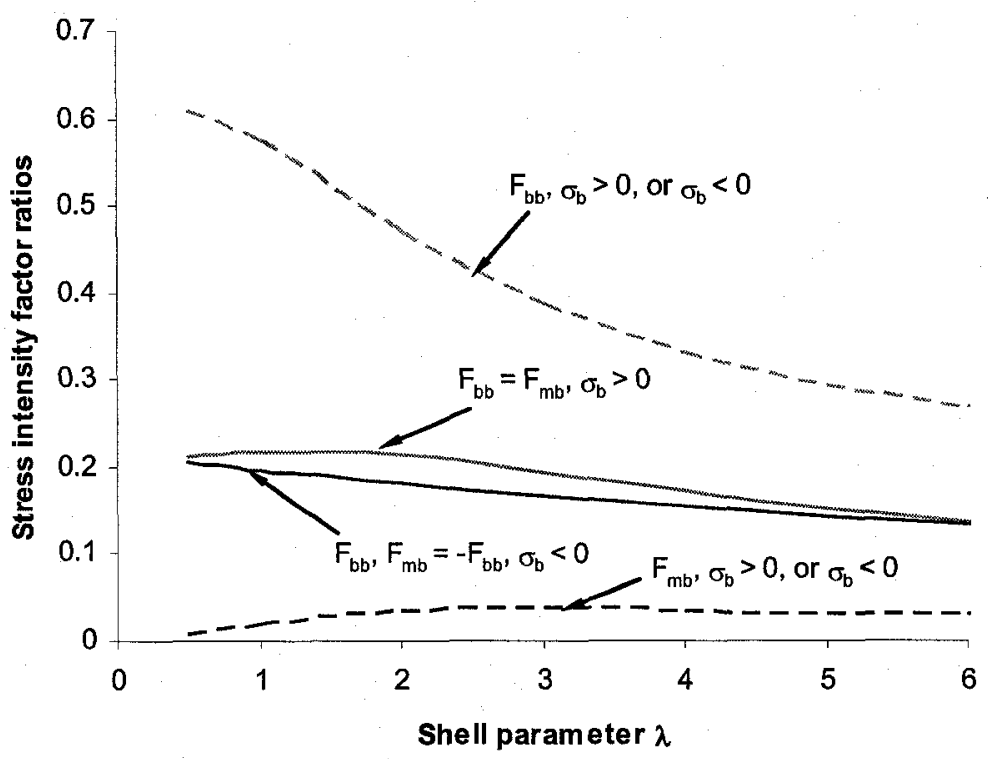

(b) 


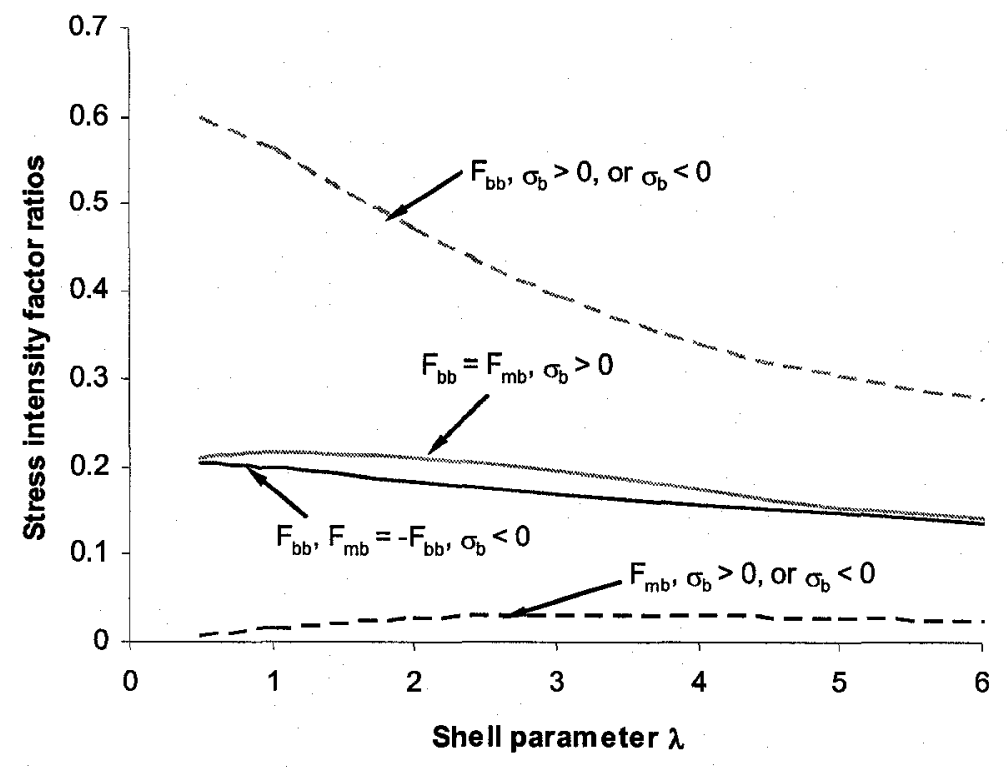

(c)

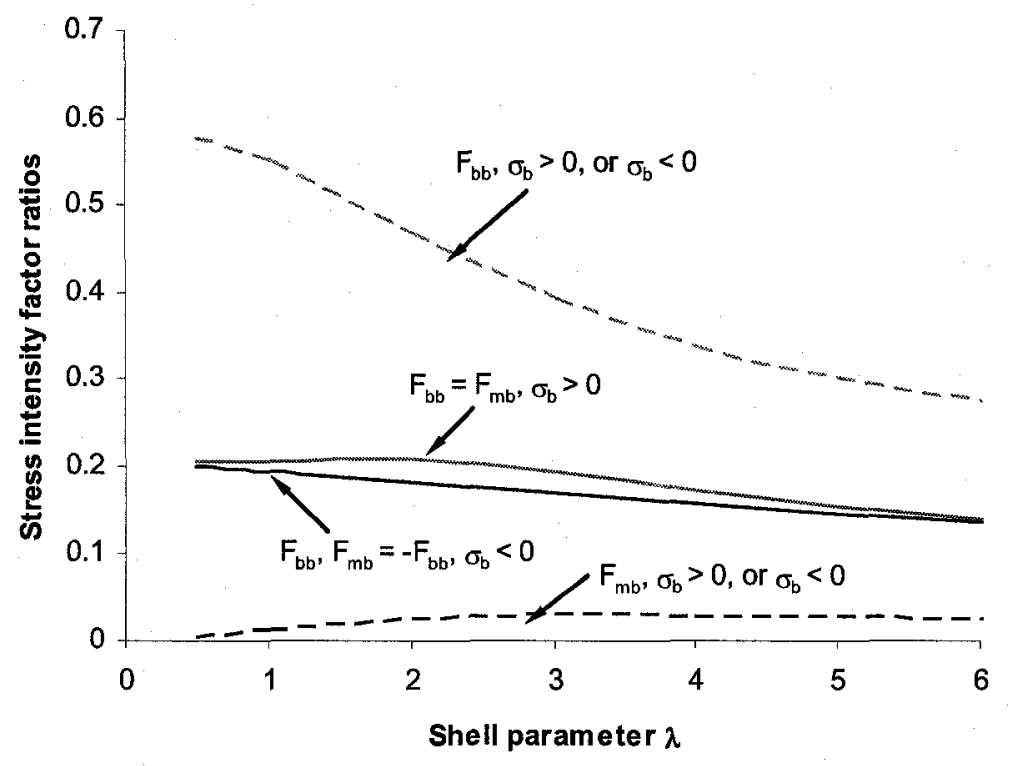

(d)

Figure 3.19 Comparison between closure and non-closure stress intensity factor ratios for a specially orthotropic cylindrical shell containing a circumferential crack with $a / h=10$ ( $\longrightarrow$ closure and -- - non-closure):

(a) $E_{1} / E_{2}=0.725 ;$ (b) $E_{1} / E_{2}=1.38 ;$ (c) $E_{1} / E_{2}=0.037$ and (d) $E_{1} / E_{2}=26.67$ 


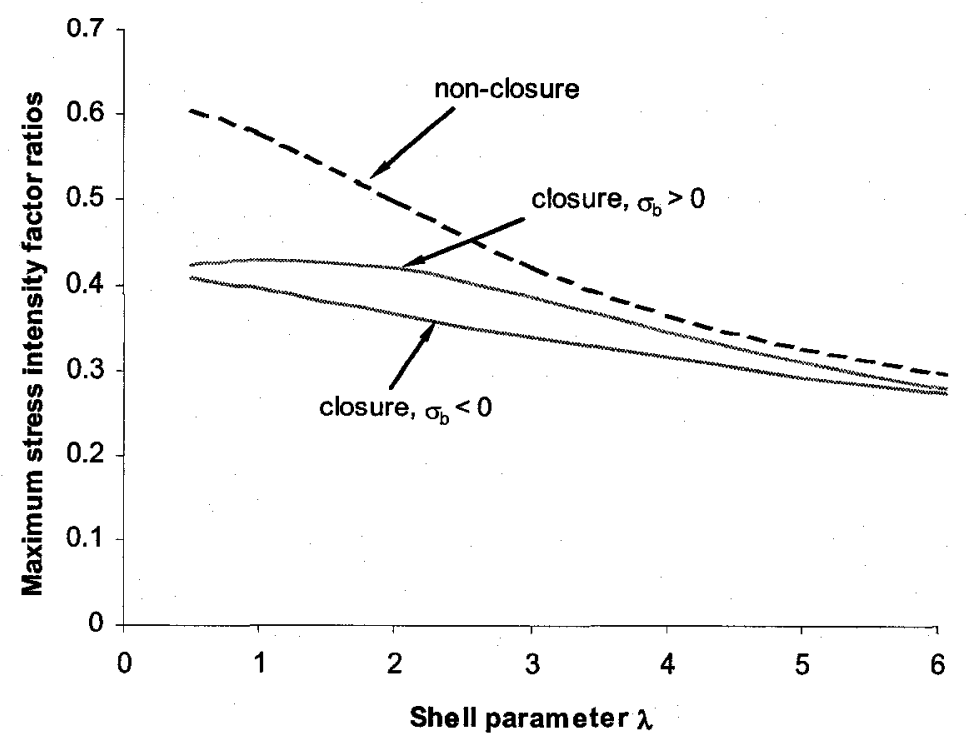

(a)

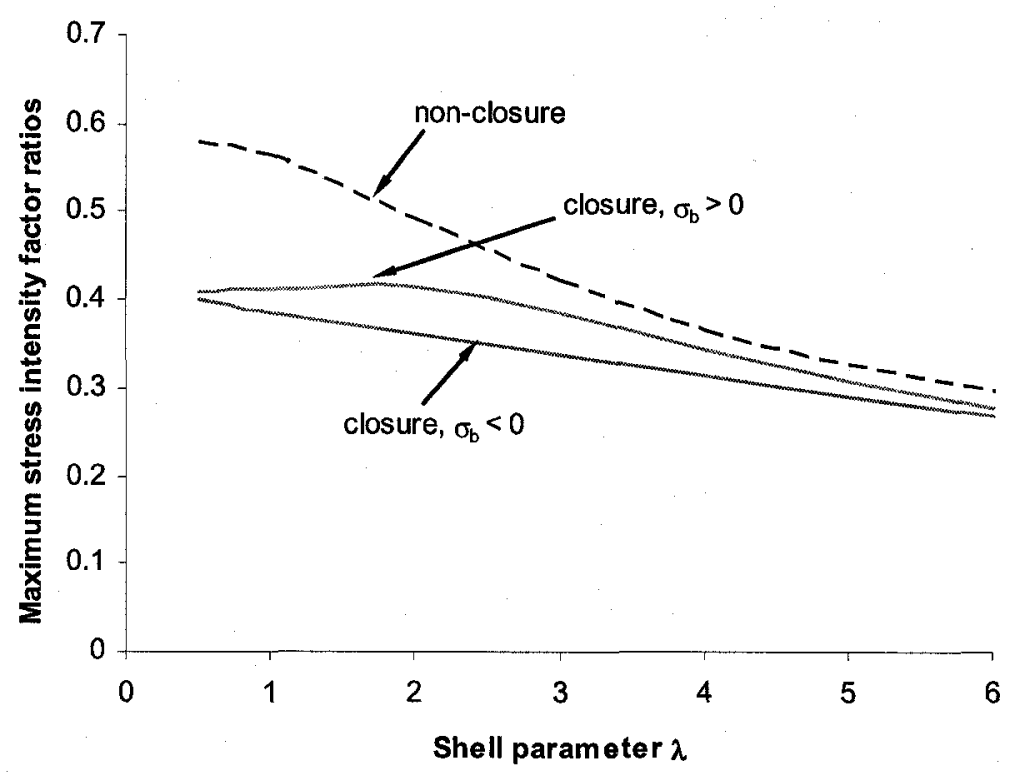

(b)

Figure 3.20 Comparison between closure solutions and non-closure solutions of the maximum stress intensity factor ratio for a specially orthotropic cylindrical shell containing a circumferential crack with $a / h=10$ :

(a) $E_{1} / E_{2}=0.037$ and (b) $E_{1} / E_{2}=26.67$ 
Regarding material orthotropy, for a circumferentially cracked cylindrical shell, it is hard to see the difference in the maximum stress intensity factor ratios between different $E_{1} / E_{2}$ ratios, see Figure 3.19, that is, the influence of material orthotropy on the SIFs is almost negligible for a circumferentially cracked cylindrical shell, except for very small $\lambda$ values. Figure 3.21 illustrates the reduction of the maximum stress intensity factor ratios due to the crack closure effect for the mildly orthotropic and the strongly orthotropic cylindrical shell containing a circumferential crack respectively. As seen in Figure 3.21, material orthotropy almost does not affect the reduction of the maximum SIF, that is, the reduction by the crack closure is nearly same for different $E_{1} / E_{2}$ ratios, which differs from axially cracked cylindrical shells. However, it is noticed that the reduction of the maximum SIF does vary with the $E_{1} / E_{2}$ ratios for very small $\lambda$ values. In the limit case, $\lambda$ approaching zero, the difference of the reduction between the $E_{1} / E_{2}$ ratios is the maximum.

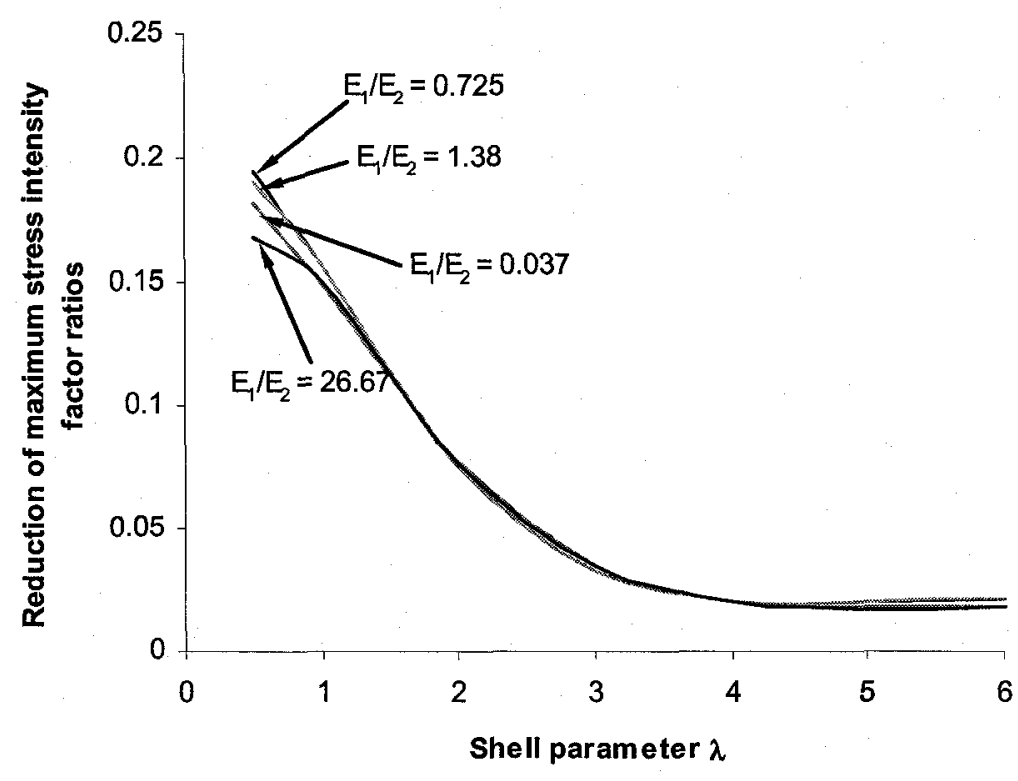

(a) 


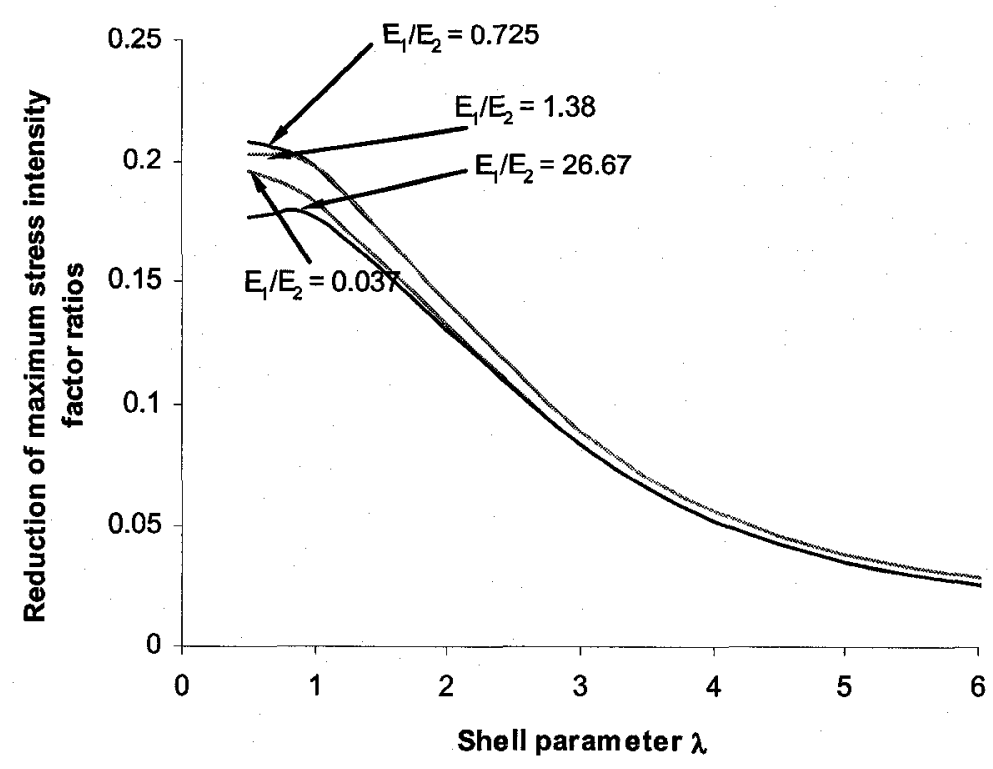

(b)

Figure 3.21 Reductions of the maximum stress intensity factor ratios due to the crack closure effect for a specially orthotropic cylindrical shell containing a circumferential crack with $a / h=10$ : (a) under positive bending and (b) under negative bending

\subsection{Specially Orthotropic Spherical Shell}

\subsubsection{Definition of a crack in a specially orthotropic spherical shell}

For the general case in Figure 3.1, consider another special case that the two curvatures, $R_{1}$ and $R_{2}$, are equal, that is, the shell has a uniform curvature, the problem then becomes a specially orthotropic spherical shell containing a crack. The configuration of the cracked shell in a Cartesian coordinate system is shown in Figure 3.22. The shell has a thickness $h$ and radius $R$, containing a crack of length $2 a$. The material properties $E_{1}, v_{1}$ and $E_{2}, v_{2}$ are in the directions along the crack and perpendicular to the crack respectively, see Figure 3.22. 


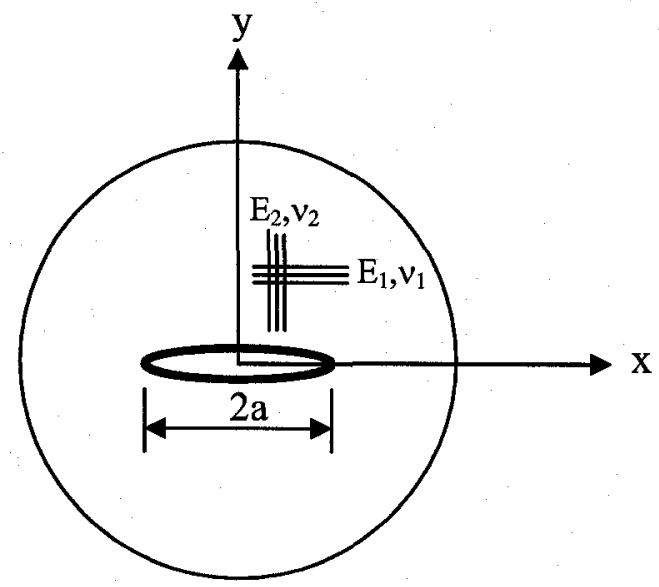

(a)

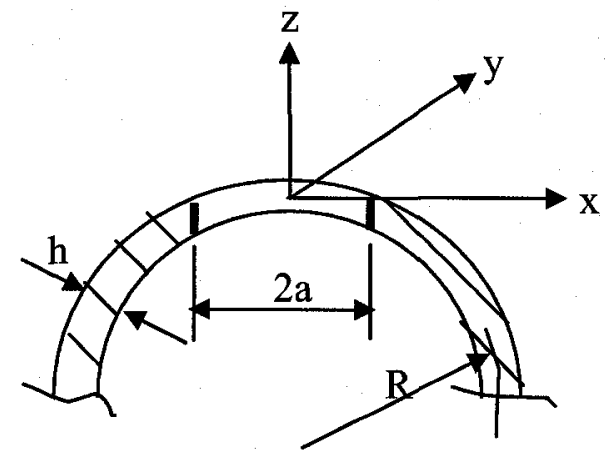

(b)

Figure 3.22 Geometry and coordinates for a through-the-thickness crack in a spherical shell: (a) top view and (b) side view

Within the coordinate system defined in Figure 3.22 the equilibrium equations for the spherical shell can be expressed as $[7,15]$,

$$
\nabla^{4} \nabla^{4} \phi+\nabla_{\lambda}^{2} \nabla_{\lambda}^{2}\left(1-\kappa \nabla^{2}\right) \phi=0
$$

where 


$$
\nabla_{\lambda}^{2}=\lambda_{1}^{2} \frac{\partial^{2}}{\partial x^{2}}+\lambda_{2}^{2} \frac{\partial^{2}}{\partial y^{2}}
$$

The expressions of $\lambda_{1}$ and $\lambda_{2}$ are given by eqns (3.24) and (3.25). The problems can be solved using Fourier transforms. The characteristic equations of the problems and their four roots, which appear in the expressions of the kernels $k_{i j}$ of the integral equations, are given in Appendix $\mathrm{A}$, where $\lambda_{1} \neq 0$ and $\lambda_{2} \neq 0$, and also $\lambda_{1} \neq \lambda_{2}$ due to the material orthotropy parameter, $c$, even though $R_{1}=R_{2}$.

\subsubsection{Crack closure in a specially orthotropic spherical shell}

Taking the two orthotropic materials, mildly orthotropic titanium and strongly orthotropic graphite-epoxy, as examples again, compute the contact force along the crack assuming full-length closure under positive bending. As demonstrated in Figure 3.23, similar to other shell geometries, the contact force distributes nonuniformly along the crack length. Furthermore, for small values of $\lambda$, where $\lambda=\left[12\left(1-v^{2}\right)\right]^{1 / 4} a / \sqrt{R h}$, which represents the curvature of the shell when the crack length, $a$, and shell thickness, $h$, are given, the contact force is positive over the entire crack length. However, with the increase of the $\lambda$ value the contact force becomes negative in the region around the center of the crack.

A negative bending condition was also analyzed. It is shown that entire-length crack closure always occurrs as long as $\lambda \leq 6$. At even higher $\lambda$ values, it can be expected that partial closure would occur over the central region of the crack. 


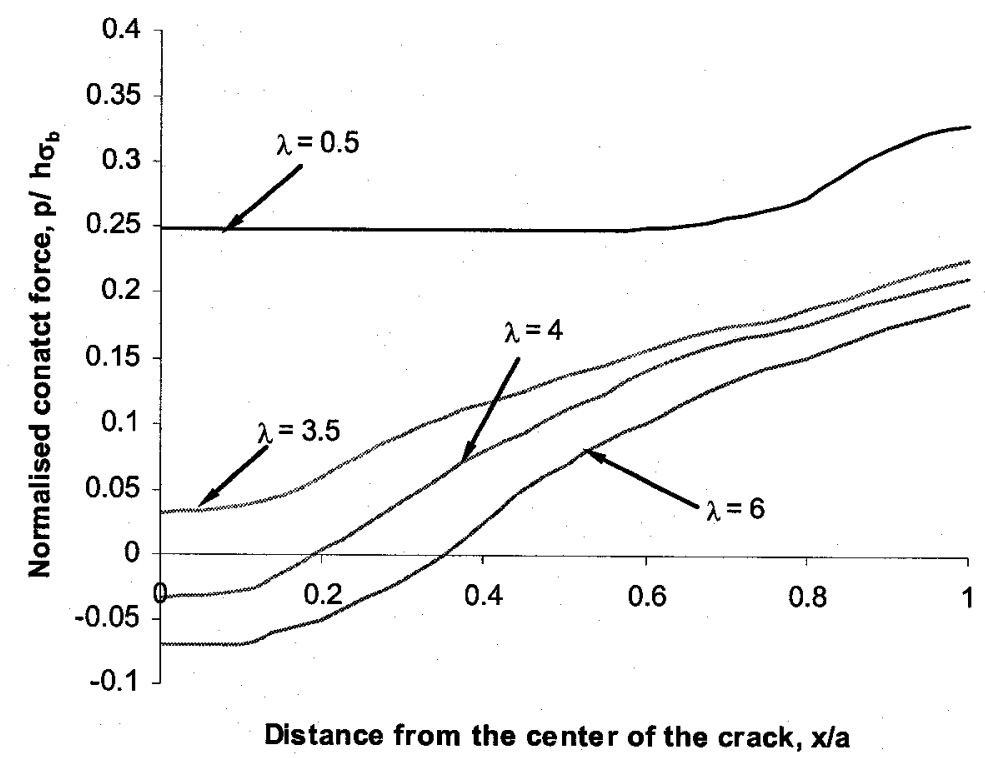

(a)

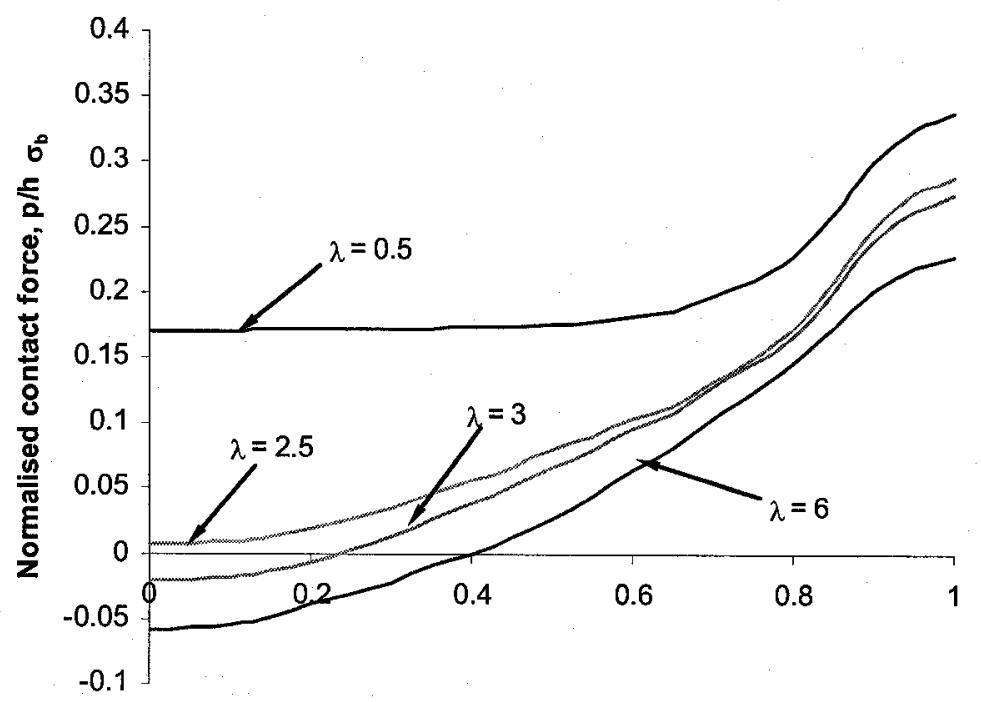

Distance from the center of the crack, $x / a$

(b) 


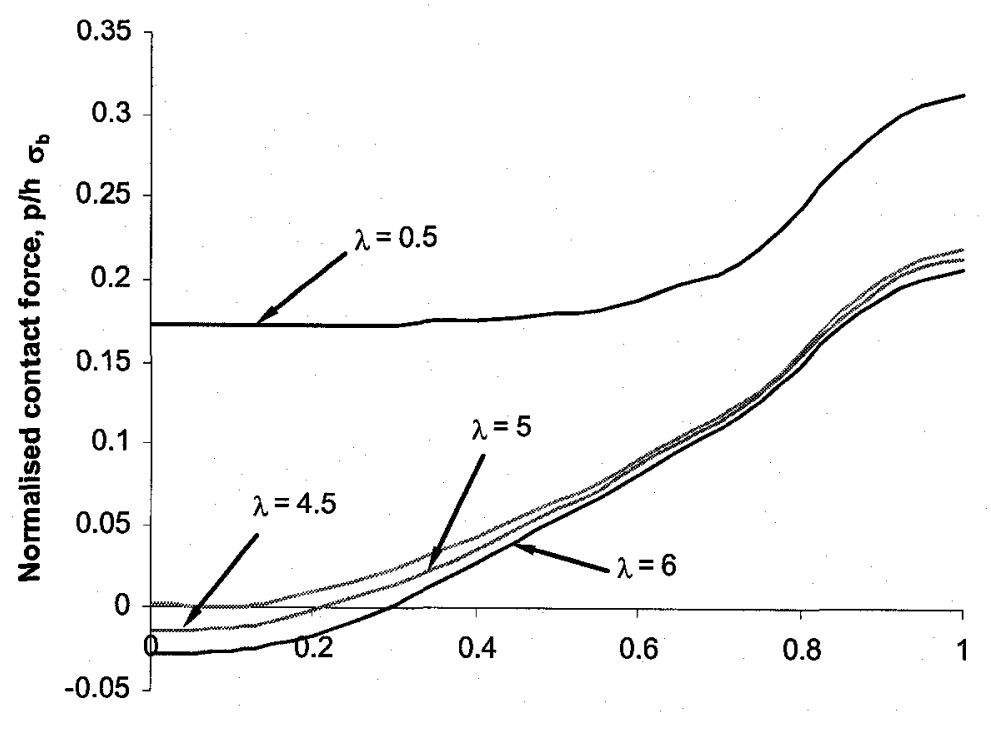

Distance from the center of the crack, $x / a$

(c)

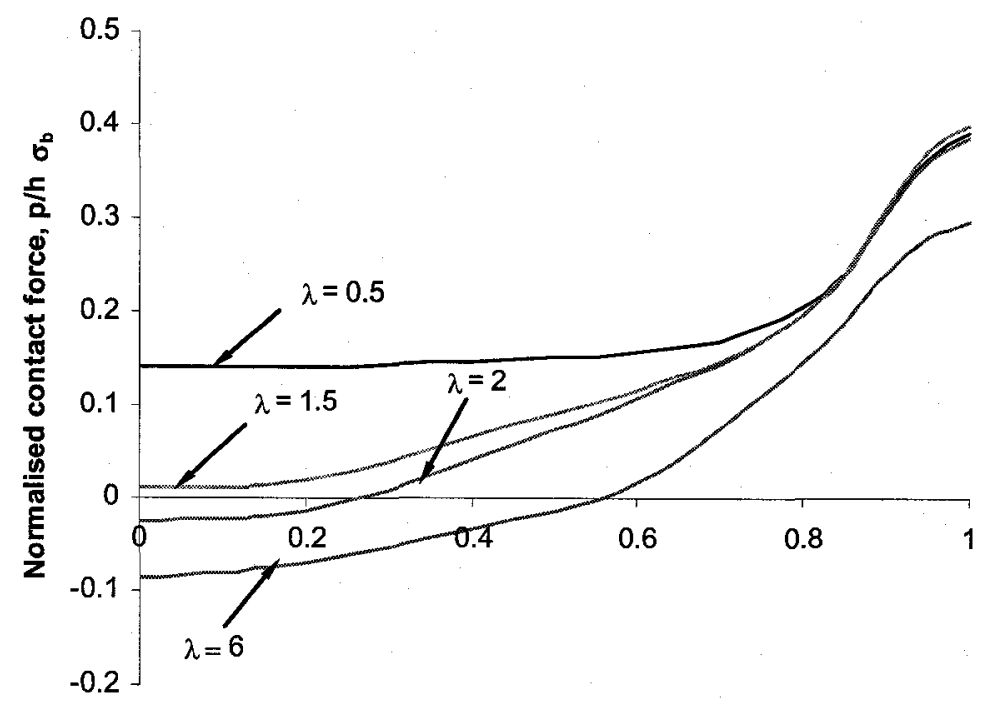

Distance from the center of the crack, $x / a$

(d)

Figure 3.23 Contact force distributions assuming full-length closure for a specially orthotropic spherical shell under positive bending with $a / h=10$ :

(a) $E_{1} / E_{2}=0.725 ;$ (b) $E_{1} / E_{2}=1.38 ;$ (c) $E_{1} / E_{2}=0.037$ and (d) $E_{1} / E_{2}=26.67$ 
As concerns the effect of material orthotropy on the crack closure, it is observed in Figure 3.23 that for the mildly orthotropic shell partial closure occurs when the $\lambda$ value is up to 4 for the ratio $E_{1} / E_{2}=0.725$ and 3 for $E_{1} / E_{2}=1.38$, while for the strongly orthotropic shell partial closure occurs when the $\lambda$ value reaches 5 for $E_{1} / E_{2}=0.037$ and 2 for $E_{1} / E_{2}=26.67$. This manifests that the curvature effect on the crack closure is enhanced with the increase of the $E_{1} / E_{2}$ ratio.

Comparing the SIFs and the maximum SIFs in the closure case with those in the non-closure case, as presented in Figure 3.24 and Figure 3.25 respectively, for different $E_{1} / E_{2}$ ratios, the same conclusions drawn for other shell geometries can be obtained for spherical shells.

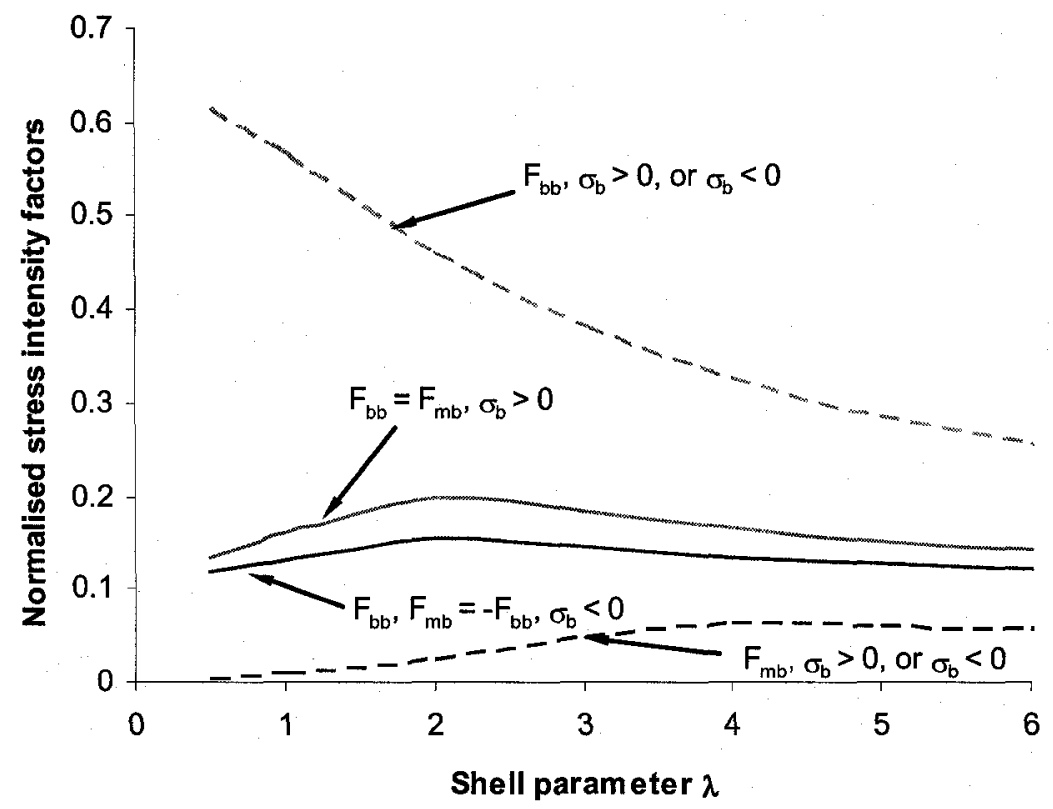

(a) 


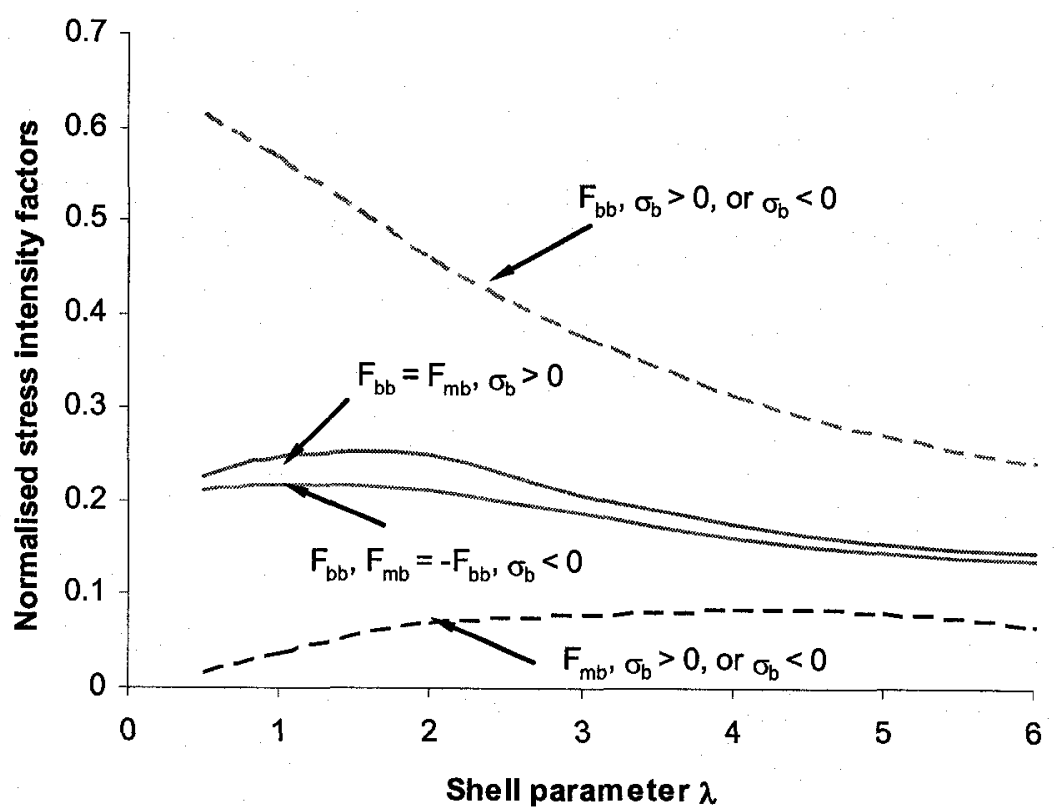

(b)

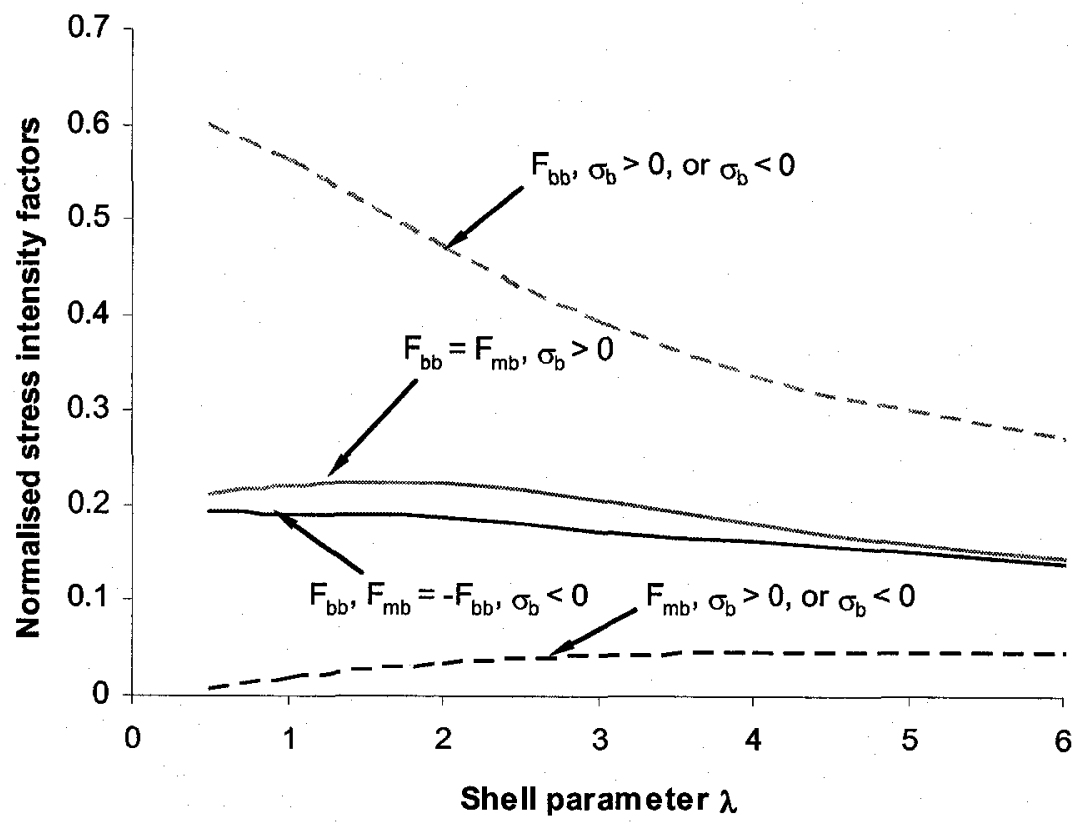

(c) 


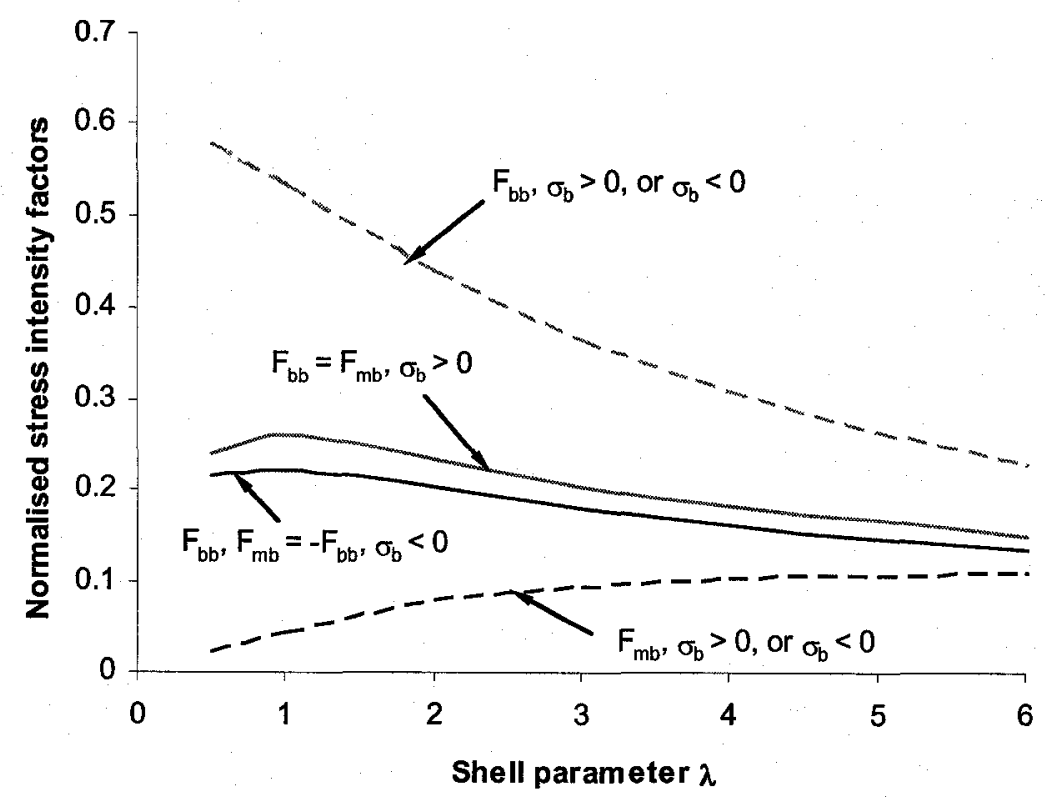

(d)

Figure 3.24 Comparison between closure and non-closure stress intensity factor ratios for a specially orthotropic spherical shell with $a / h=10$ ( — closure and ---- non-closure):

(a) $E_{1} / E_{2}=0.725$; (b) $E_{1} / E_{2}=1.38$; (c) $E_{1} / E_{2}=0.037$ and (d) $E_{1} / E_{2}=26.67$

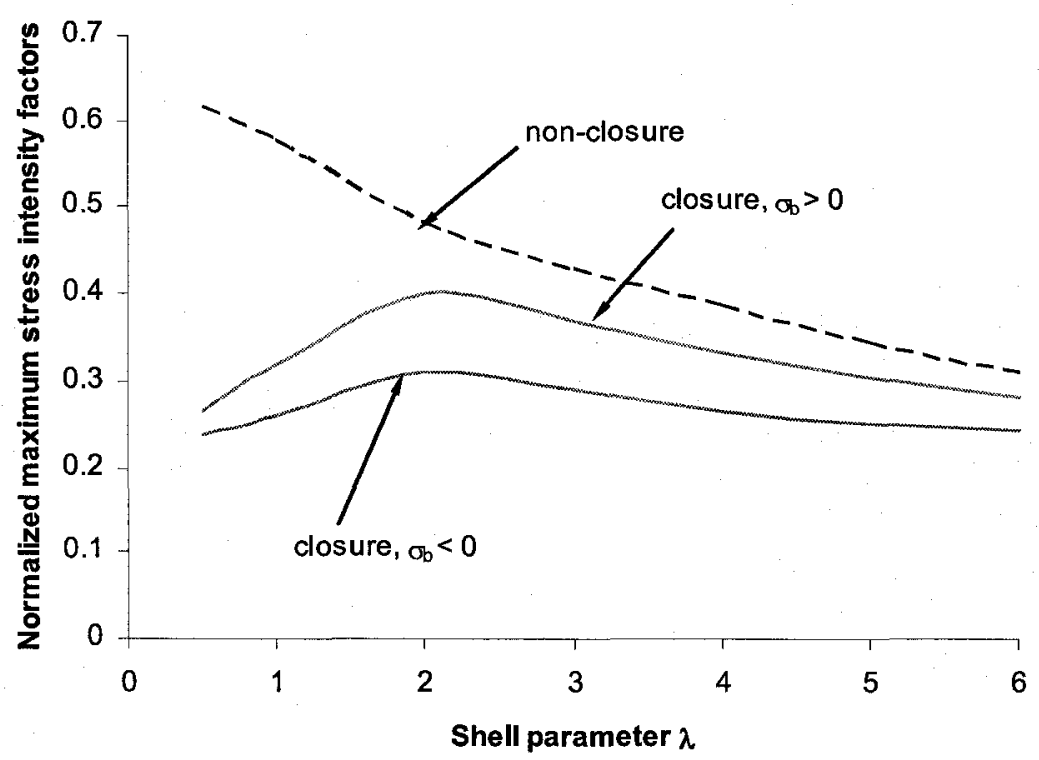

(a) 


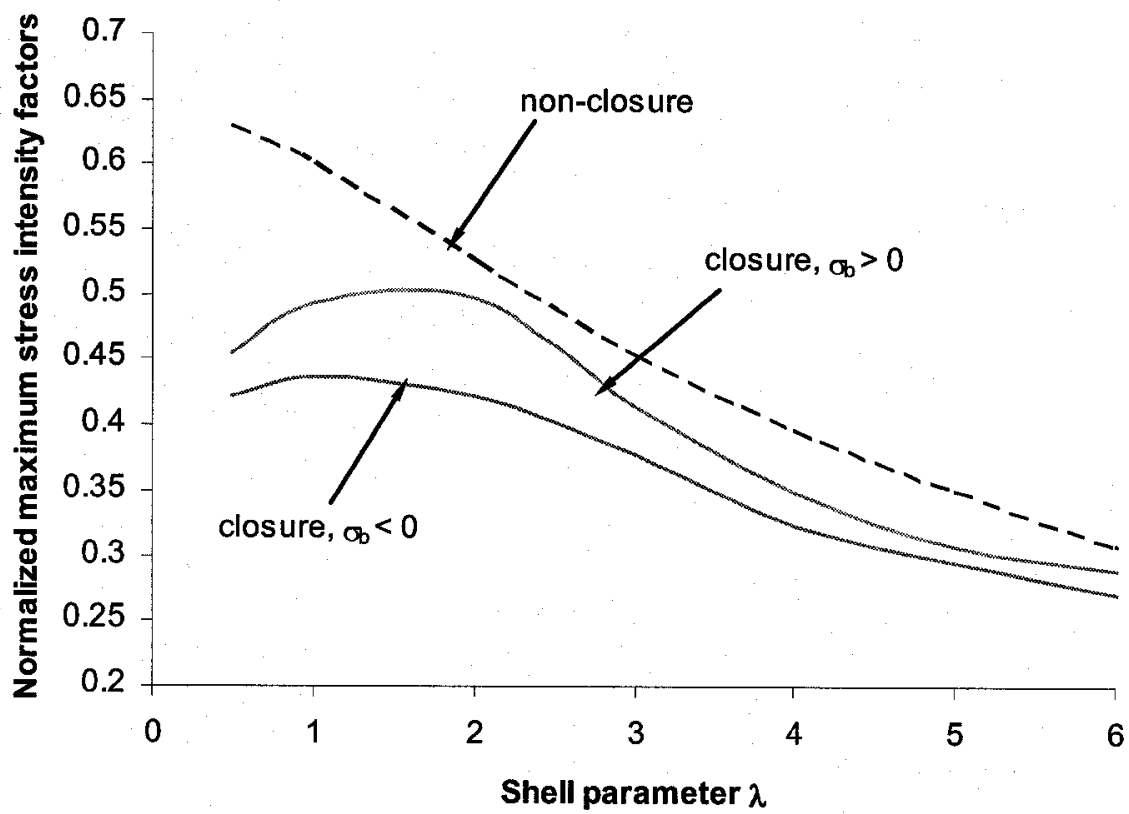

(b)

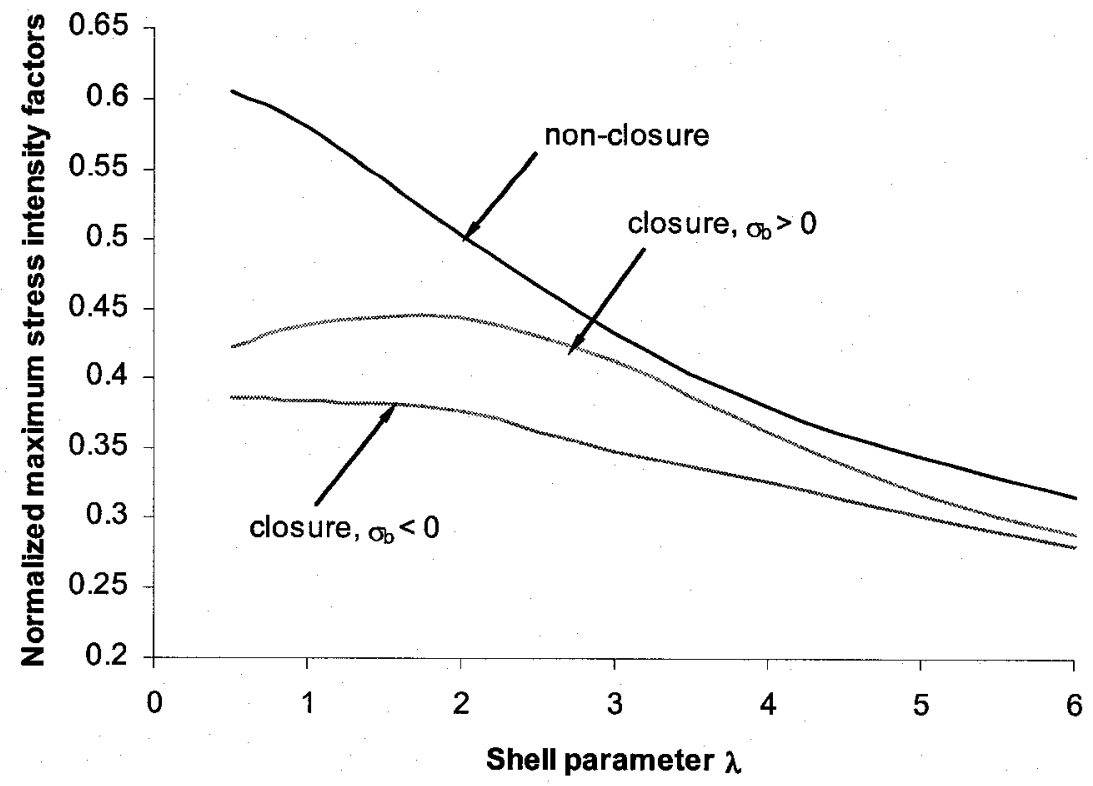

(c) 


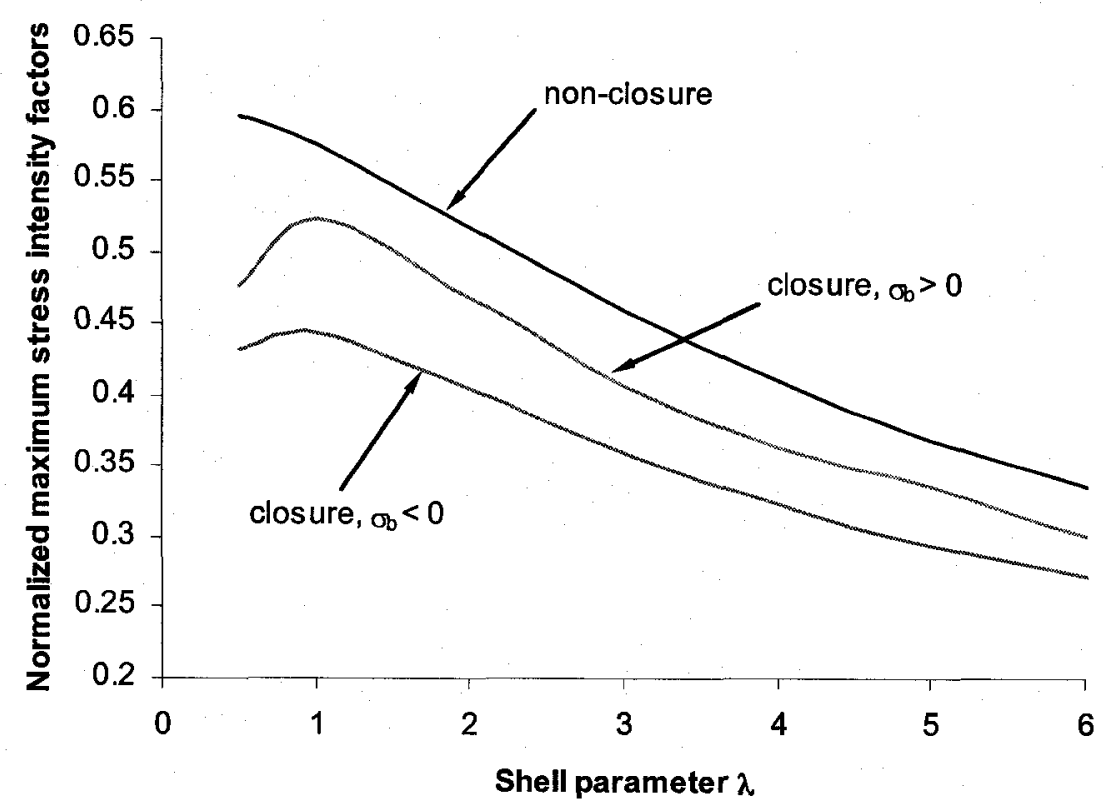

(d)

Figure 3.25 Comparison between closure solutions and non-closure solutions of the maximum stress intensity factor ratio for a specially orthotropic spherical shell with $a / h=10$ :

(a) $E_{1} / E_{2}=0.725 ;$ (b) $E_{1} / E_{2}=1.38 ;$ (c) $E_{1} / E_{2}=0.037$ and (d) $E_{1} / E_{2}=26.67$

Regarding material orthotropy, it is observed in Figure 3.25 that the effect of material orthotropy on the crack closure in spherical shells varies with the vale of $\lambda$. With the increase of the $\lambda$ value the material orthotropic effect decreases. For small curvature shells $(\lambda<2) E_{1} / E_{2}=0.725$ has the largest reduction of the maximum stress intensity factor ratios and $E_{1} / E_{2}=26.67$ has the least in both positive bending and negative bending cases. However, for large curvature shells $(\lambda>6)$ the material orthotropic effect can be negligible, especially for positive bending case. 
Figure 3.26 illustrates the influence of material orthotropy on the crack closure effect in a more obvious way. The difference of the stress intensity factor ratios between the closure solution and the non-closure solution is the maximum when $\lambda$ approaches zero for all $E_{1} / E_{2}$ ratios. As seen in Figure 3.26, when the $\lambda$ value is small (less than 2), the four curves which represent different $E_{1} / E_{2}$ ratios are very divergent but they become close with increasing the $\lambda$ value. This indicates that the influence of material orthotropy on the crack closure is stronger when the shell curvature is small, whereas it is weaker.

In summary, from the results of various shell structures discussed above, it can be concluded that the influence of material orthotropy on the crack closure is stronger in plates than in shells.

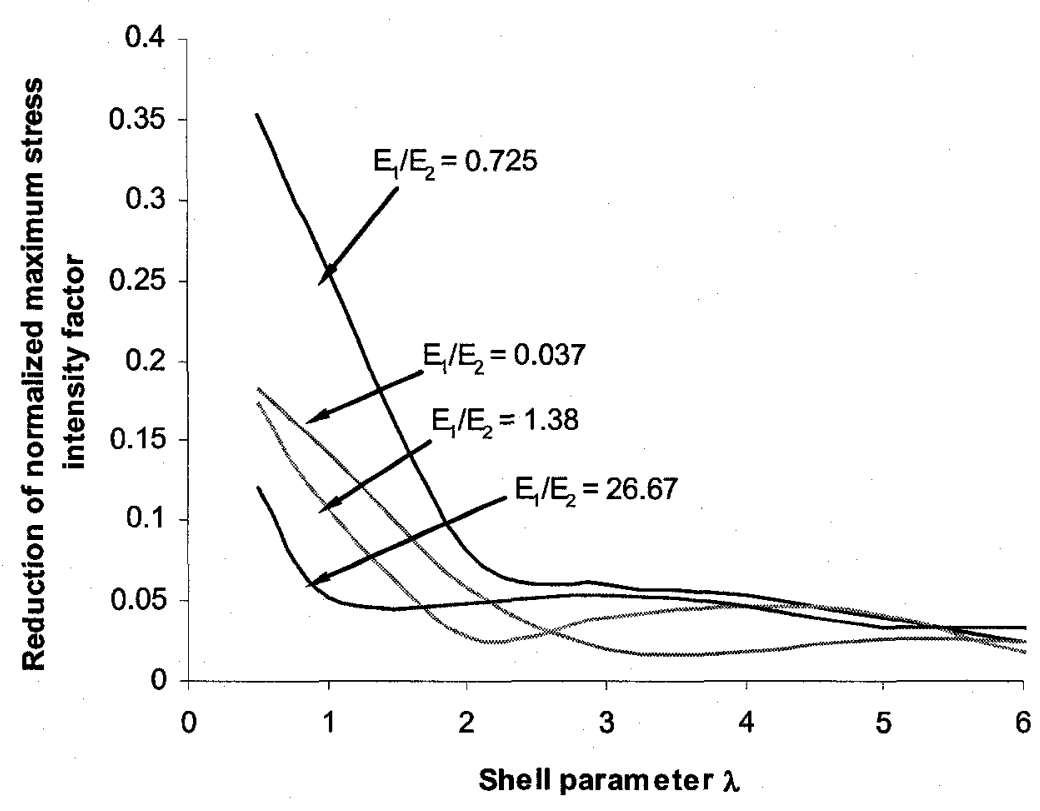

(a) 


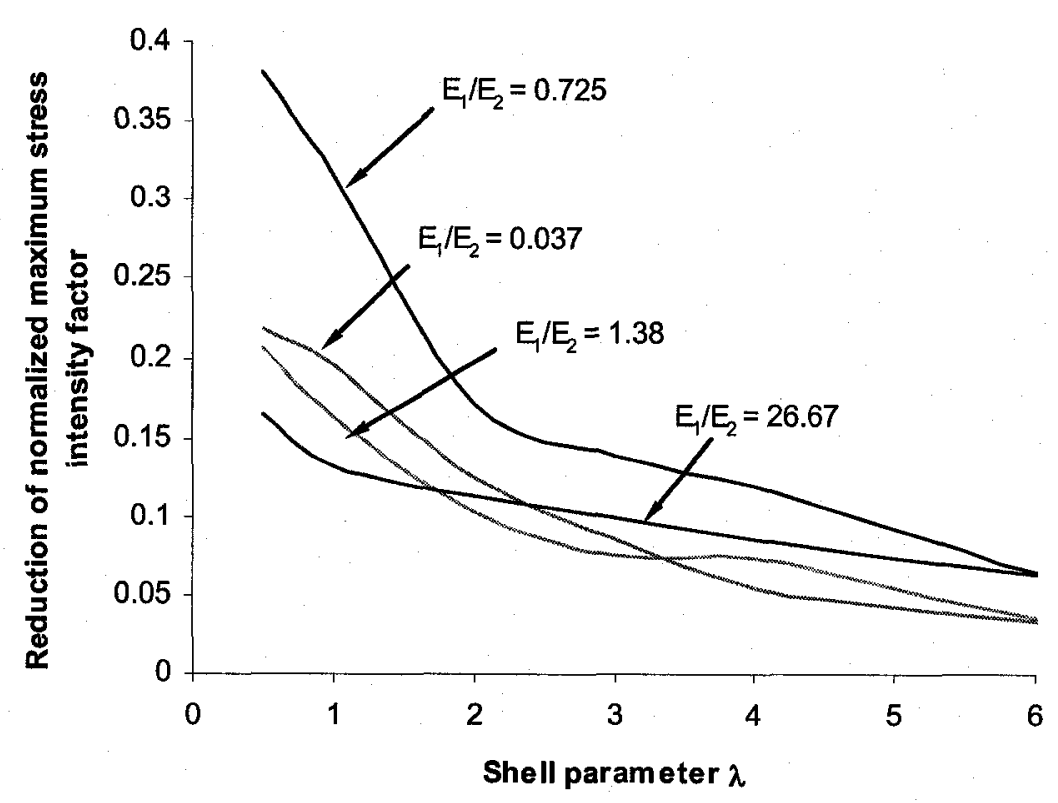

(b)

Figure 3.26 Reductions of the maximum stress intensity factor ratios due to the crack closure effect for a specially orthotropic spherical shell with $a / h=10$ :

(a) under positive bending and (b) under negative bending

\subsection{Isotropic Shells}

\subsubsection{Problem formulation}

For isotropic shells, the material properties $E_{1}=E_{2}=E$ and $v_{1}=v_{2}=v$, therefore the parameter $c$ in the coupled integral equations (3.1) becomes unity. Thus the integral equations for isotropic shells are written as

$$
\begin{aligned}
& \int_{-1}^{1} \frac{G_{1}(\tau)}{\tau-\eta} d \tau+\int_{-1}^{1}\left[k_{11}(\eta, \tau) G_{1}(\tau)+k_{12}(\eta, \tau) G_{2}(\tau)\right] d \tau=2 \pi F_{1}(\eta), \\
& \frac{1-v^{2}}{\lambda_{0}^{4}} \int_{-1}^{1} \frac{G_{2}(\tau)}{\tau-\eta} d \tau+\int_{-1}^{1}\left[k_{21}(\eta, \tau) G_{1}(\tau)+k_{22}(\eta, \tau) G_{2}(\tau)\right] d \tau=2 \pi \frac{h}{a} F_{2}(\eta),
\end{aligned}
$$




$$
\int_{-1}^{1} G_{i}(\tau) d \tau=0,(i=1,2)
$$

where $\eta=y / a,-a<y<a$. The stress functions for pure bending in closure and non-closure cases are then expressed as

$$
\begin{aligned}
F_{1}(\eta) & =0 \text { (non-closure) } \\
\text { and } F_{2}(\eta) & =-\sigma_{b} /(6 E) \text { (non-closure), } \\
F_{1}(\eta) & =-\frac{p(\eta) / h}{E} \text { (closure) } \\
\text { and } F_{2}(\eta) & =-\frac{\sigma_{b}(\eta) \pm 3 p(\eta) / h}{6 E} \text { (closure) }
\end{aligned}
$$

The shell parameters $\lambda_{1}$ and $\lambda_{2}$ are

$$
\begin{aligned}
& \lambda_{1}^{4}=12\left(1-v^{2}\right) \frac{a^{4}}{h^{2} R_{1}^{2}}, \\
& \lambda_{2}{ }^{4}=12\left(1-v^{2}\right) \frac{a^{4}}{h^{2} R_{2}^{2}} .
\end{aligned}
$$

In the closure region, the integral equations are

$$
\begin{aligned}
\pm \frac{h}{2 a} \int_{-1}^{1} \frac{G_{2}(\tau)}{\tau-\eta} d \tau+\int_{-1}^{1}\left[ \pm \frac{h}{2 a} k_{11}(\eta, \tau) G_{2}(\tau)-k_{12}(\eta, \tau) G_{2}(\tau)\right] d \tau=2 \pi \frac{p(\eta) / h}{E}, \\
-\frac{1-v^{2}}{\lambda_{0}^{4}} \int_{-1}^{1} \frac{G_{2}(\tau)}{\tau-\eta} d \tau+\int_{-1}^{1}\left[ \pm \frac{h}{2 a} k_{21}(\eta, \tau) G_{2}(\tau)-k_{22}(\eta, \tau) G_{2}(\tau)\right] d \tau=\pi \frac{h}{a}\left[\frac{\sigma_{b}(\eta) \pm 3 p(\eta) / h}{3 E}\right] .
\end{aligned}
$$

The equations for the solution $G_{2}$ and the contact force $p$ are

$$
\int_{-1}^{1}\left[-\left(\frac{1-v^{2}}{\lambda_{0}^{4}}+\frac{h^{2}}{4 a^{2}}\right) \frac{1}{\tau-\eta}-\frac{h^{2}}{4 a^{2}} k_{11} \pm \frac{h}{2 a}\left(k_{12}+k_{21}\right)-k_{22}\right] G_{2}(\tau) d \tau=\frac{\pi h \sigma_{b}}{3 a E},
$$




$$
p(\eta)= \pm \frac{E h^{2}}{4 \pi a} \int_{-1}^{1} \frac{G_{2}(\tau)}{\tau-\eta} d \tau+\frac{E h}{2 \pi} \int_{-1}^{1}\left[ \pm \frac{h}{2 a} k_{11}(\eta, \tau) G_{2}(\tau)-k_{12}(\eta, \tau) G_{2}(\tau)\right] d \tau
$$

The Gauss-Chebyshev integration formulas were employed to solve the singular integral equations for isotropic shells are given in Appendix C. The SIF at the crack tip is calculated by the following equation,

$$
k_{I}=-\frac{E \sqrt{a}}{2}\left[g_{1}(1)+\frac{z}{a} g_{2}(1)\right]
$$

The corresponding stress intensity factor ratios are expressed as

$$
\begin{gathered}
F_{m b}=-\frac{E}{2 \sigma_{b}} g_{1}(1) \\
\text { and } F_{b b}=-\frac{E}{2 \sigma_{b}} \frac{h}{2 a} g_{2}(1) .
\end{gathered}
$$

\subsubsection{Double-curvature shells}

Two curvature ratios, $R_{1} / R_{2}=0.25$ and $R_{1} / R_{2}=4$, are taken as examples to study the crack closure behavior in isotropic shells with two nonzero curvatures. As presented in Figure 3.27, for small values of $\lambda$, where take $\lambda=\lambda_{1}=\left[12\left(1-v^{2}\right)\right]^{1 / 4} a / \sqrt{R_{1} h}$ for simplicity, complete crack closure occurs. However, similar to orthotropic shells, partial closure occurs when the $\lambda$ value increases. The critical values for partial crack closure are 3.5 for $R_{1} / R_{2}=0.25$ and 3 for $R_{1} / R_{2}=4$. For spherical shells $\left(R_{1} / R_{2}=1\right)$, it is reported that when $\lambda \geq 3$, partial closure occurs [18]. It is evident that the curvature effect on the closure behavior is enhanced with the increase of the $R_{1} / R_{2}$ ratio. 


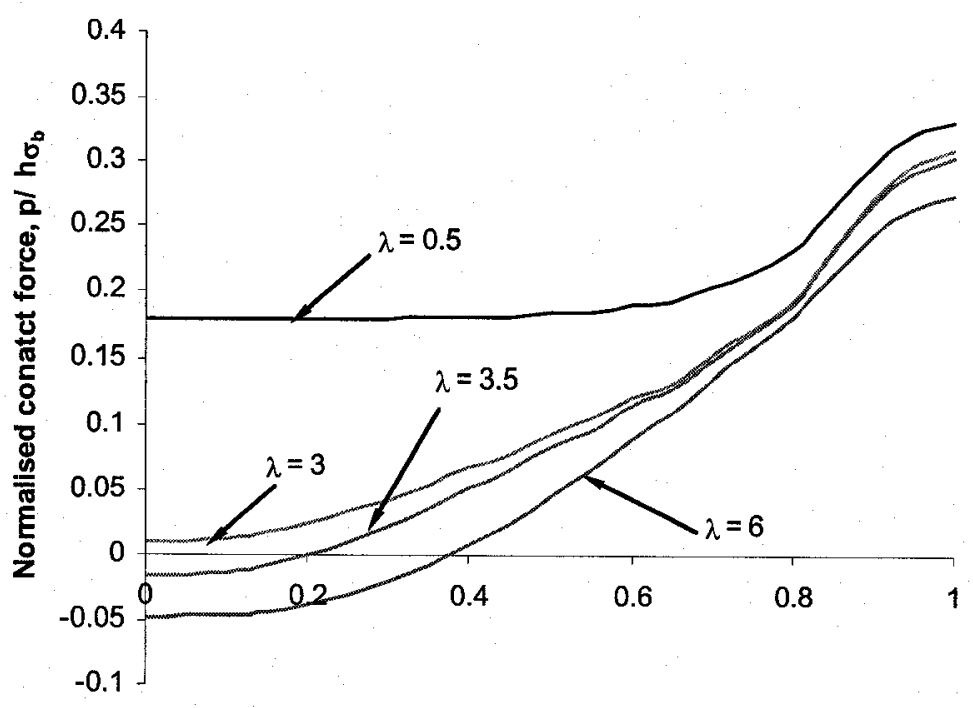

Distance from the center of the crack, y/a

(a)

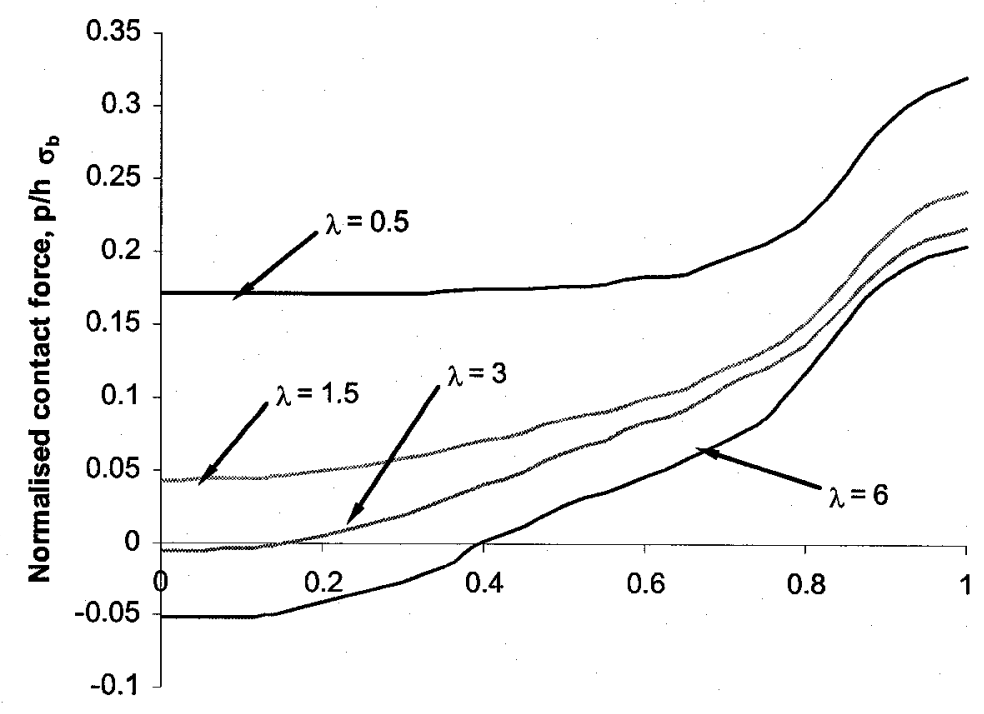

Distance from the center of the crack, y/a

(b)

Figure 3.27 Distributions of the contact force assuming full-length closure for an isotropic shell having double curvature with $a / h=10$ :

(a) $R_{1} / R_{2}=0.25$ and (b) $R_{1} / R_{2}=4$ 
For negative bending the results show that the contact force is positive over the whole crack length, that is, complete closure occurs, for all the $\lambda$ values being considered (up to $\lambda=6$ ), whether $R_{1} / R_{2}=0.25$ or $R_{1} / R_{2}=4$.

Comparison of the stress intensity factor ratios between the closure and non-closure cases for an isotropic shell having double curvature is given in Figure 3.28, for $R_{1} / R_{2}=0.25$ and $R_{1} / R_{2}=4$, respectively. The maximum stress intensity factor ratios are presented in Figure 3.39. It is found that the results are similar to those of orthotropic shells. It is also noticed that crack-face closure has stronger influence on the SIFs of the shell with $R_{1} / R_{2}=0.25$ than that of the shell with $R_{1} / R_{2}=4$ when comparing the reduction of the maximum SIF due to the crack closure between the two shells, see Figure 3.29. The closure SIFs for various ratios of $a$ to $h$ are presented with the variation of the $\lambda$ value in Figure 3.30 for the $R_{1} / R_{2}=0.25$ and $R_{1} / R_{2}=4$ shells, respectively.

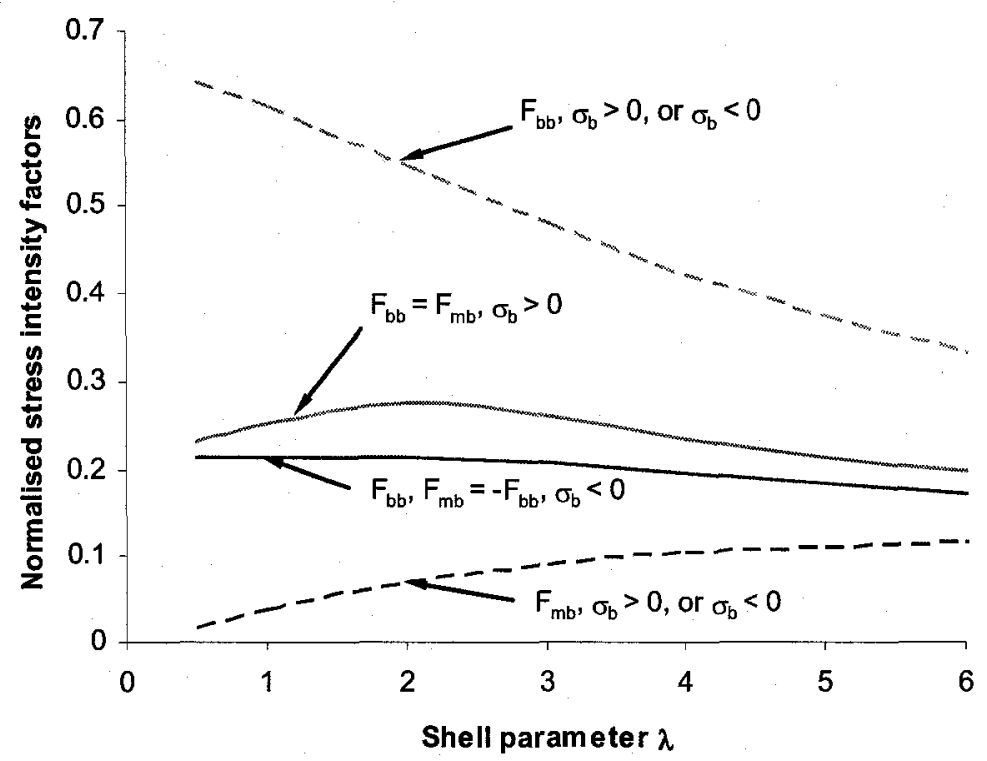

(a) 


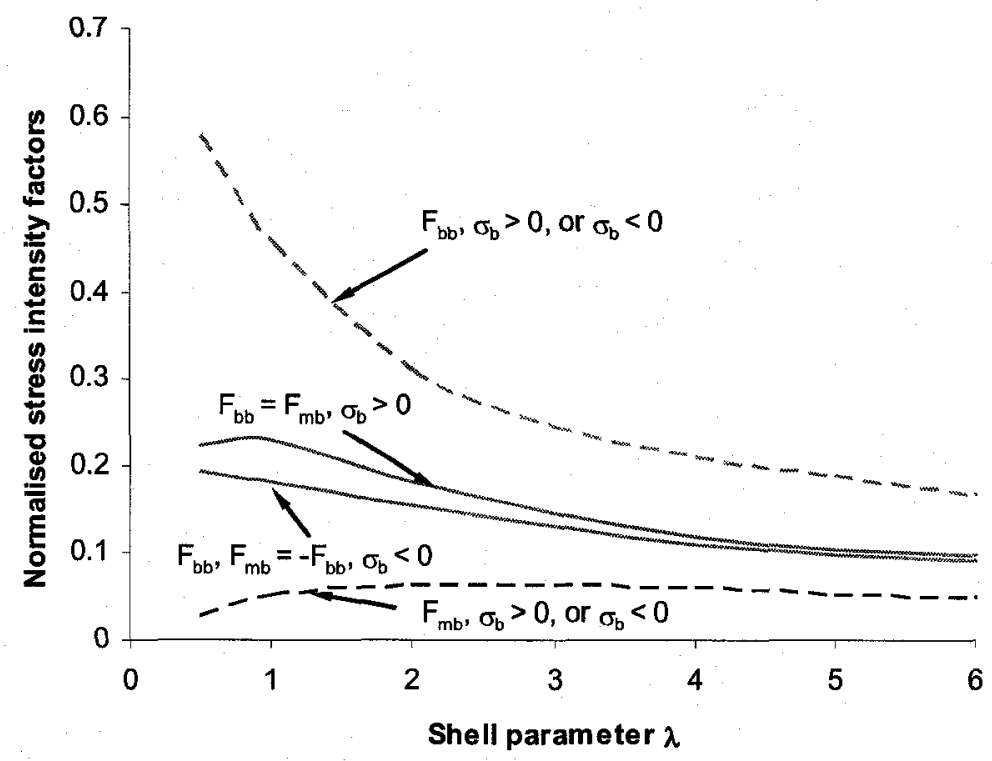

(b)

Figure 3.28 Comparison between closure and non-closure stress intensity factor ratios for an isotropic shell having double curvature with $a / h=10$ ( $\longrightarrow$ closure and - - - non-closure): (a) $R_{1} / R_{2}=0.25$ and (b) $R_{1} / R_{2}=4$

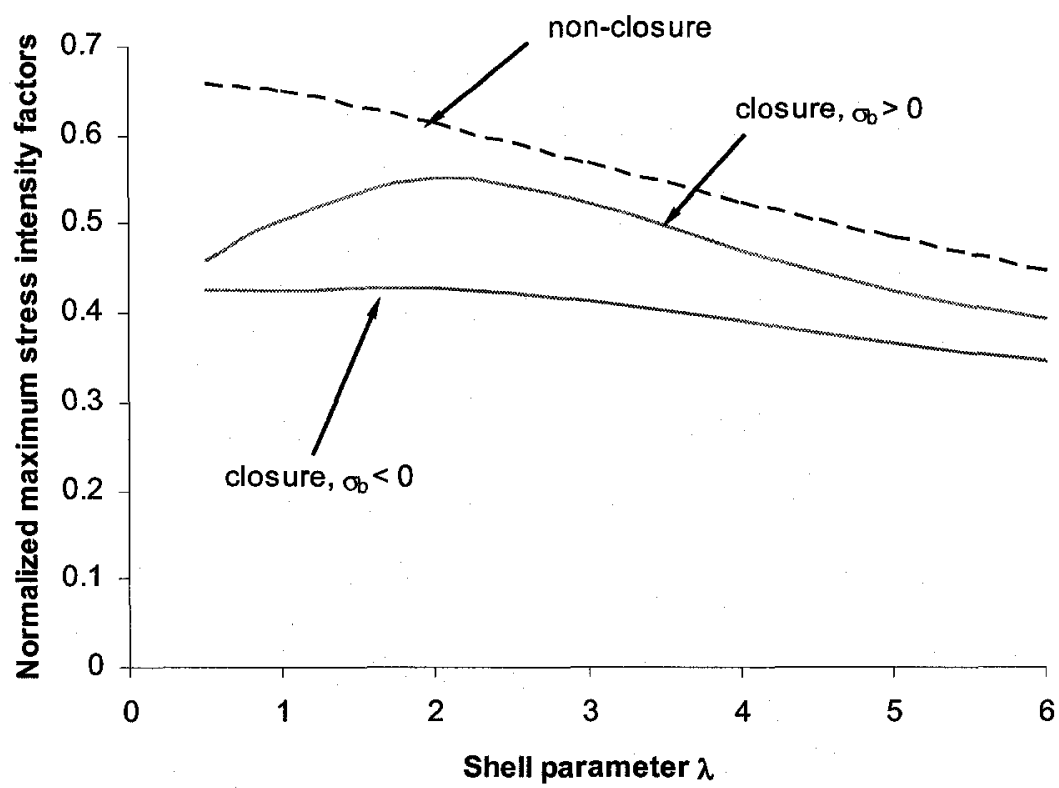

(a) 


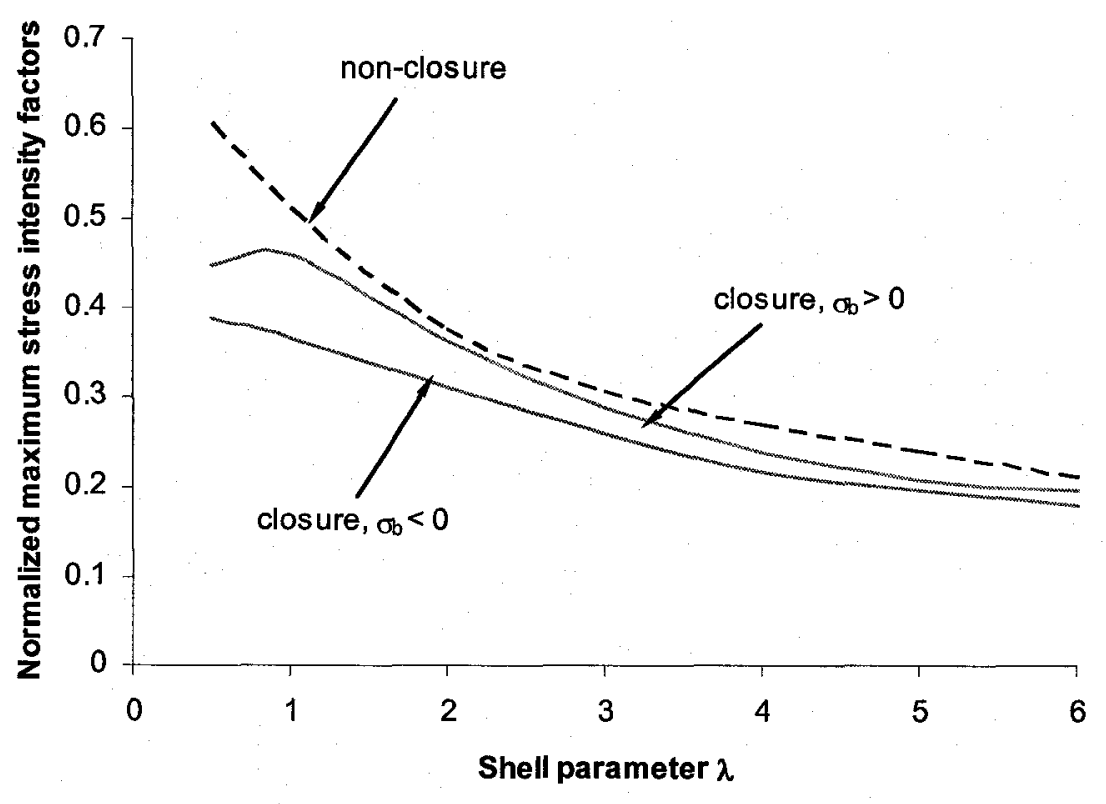

(b)

Figure 3.29 Comparison between closure solutions and non-closure solutions of the maximum stress intensity factor ratio for an isotropic shell having double curvature with $a / h=10:$ (a) $R_{1} / R_{2}=0.25$ and (b) $R_{1} / R_{2}=4$

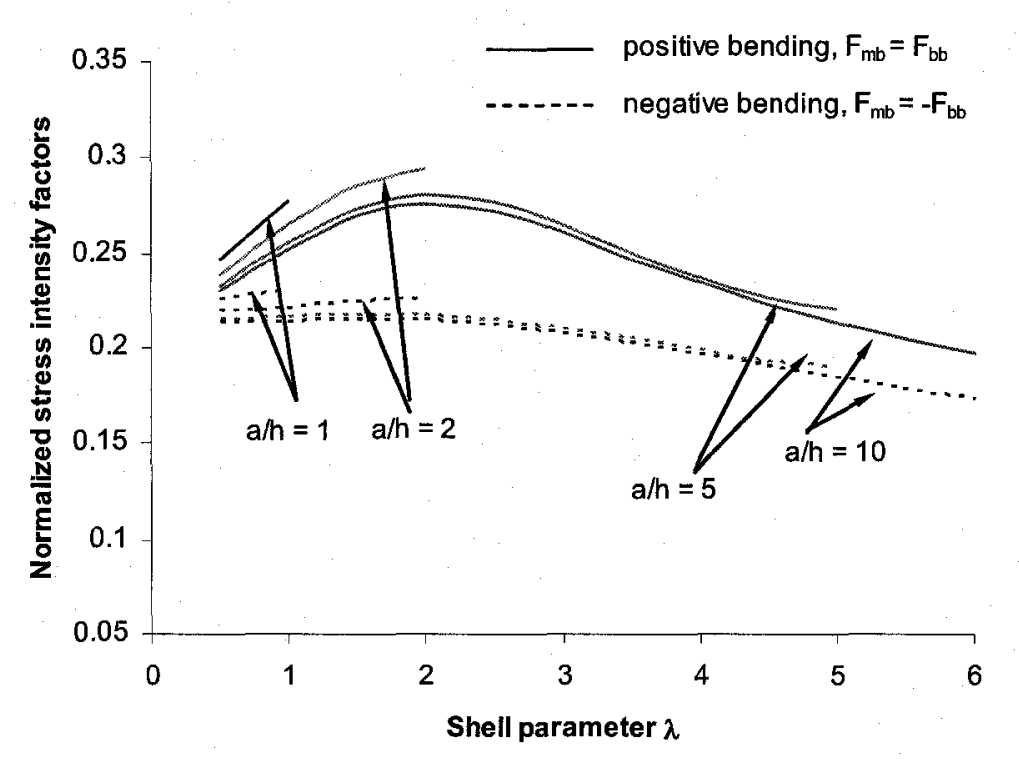

(a) 


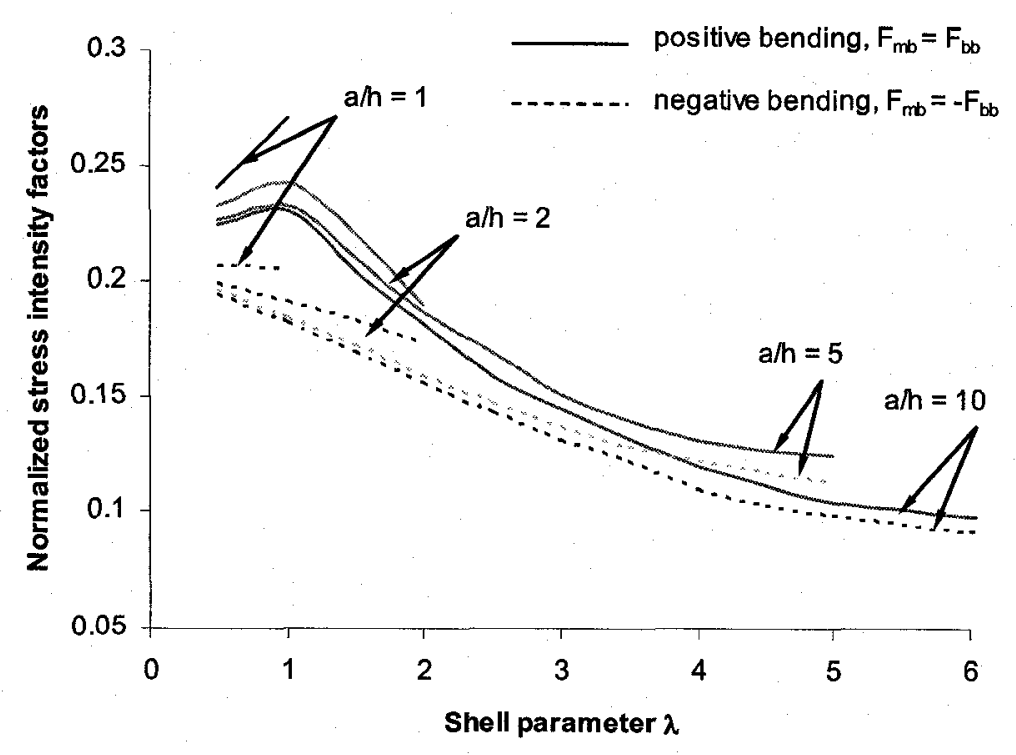

(b)

Figure 3.30 Closure solutions of the stress intensity factor ratios for an isotropic shell having double curvature: (a) $R_{1} / R_{2}=0.25$ and (b) $R_{1} / R_{2}=4$

\subsubsection{Cylindrical shells}

The crack closure behaviors in an isotropic cylindrical shell containing an axial crack or containing a circumferential crack can be demonstrated by the contact force distributions along the crack length, as shown in Figure 3.31 and Figure 3.32. It is found that the curvature effect is more obvious on the axial crack than on the circumferential crack because the contact force starts to become negative at $\lambda=4$ for the axial crack and at $\lambda=6.5$ for the circumferential crack, where $\lambda$ is defined same as above. For negative bending, the results show that the contact force is positive over the whole crack length for all the $\lambda$ values being considered (up to 6). The closure and non-closure stress intensity factor ratios and the maximum stress intensity factor ratios for an isotropic cylindrical shell containing an axial crack or containing a circumferential crack are presented in 
Figure 3.33 and Figure 3.34, respectively. The same conclusion made on orthotropic shells can also be drawn for isotropic shells.

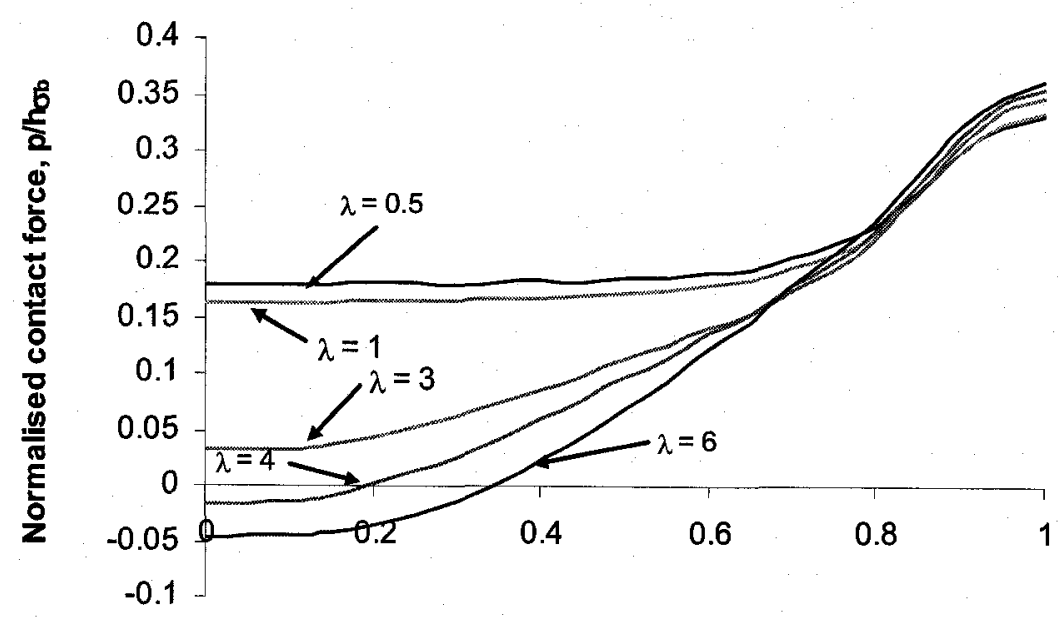

Distance from the centre of crack, $\eta=y / a$

(a)

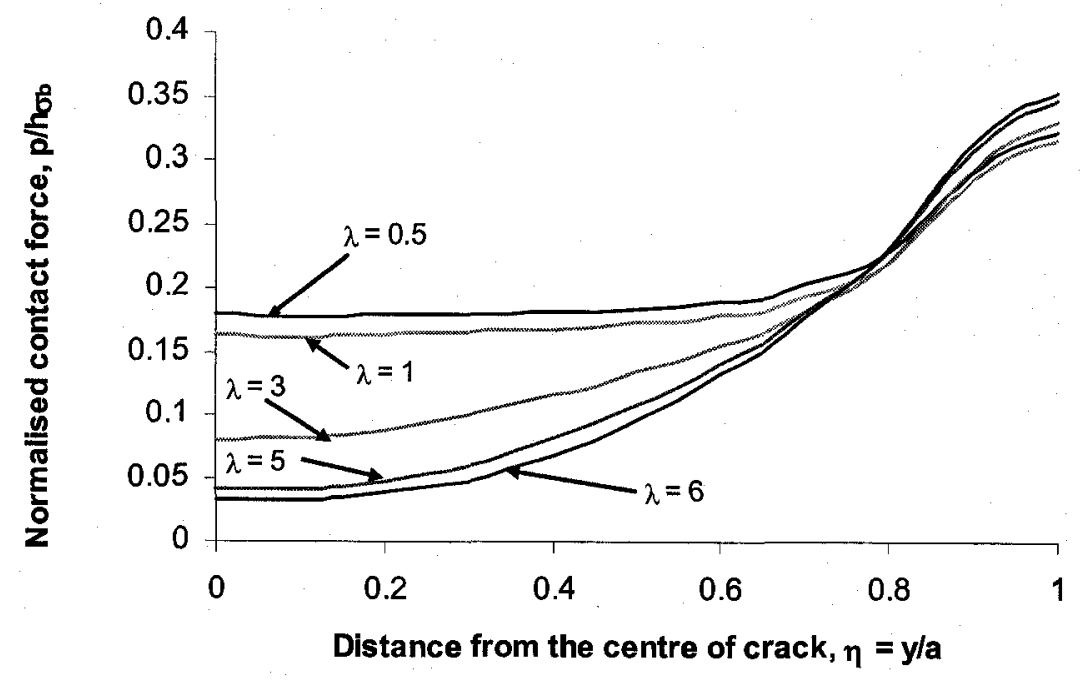

(b)

Figure 3.31 Distributions of the contact force assuming full-length closure for an isotropic cylindrical shell containing an axial crack with $a / h=10$ :

(a) under positive bending and (b) under negative bending 


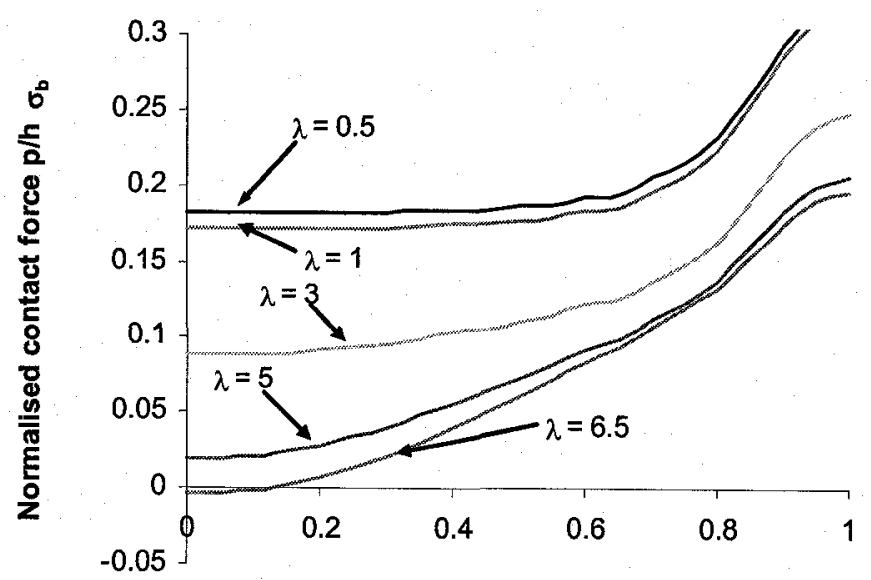

Distance from the centre of crack $\eta=y / a$

(a)

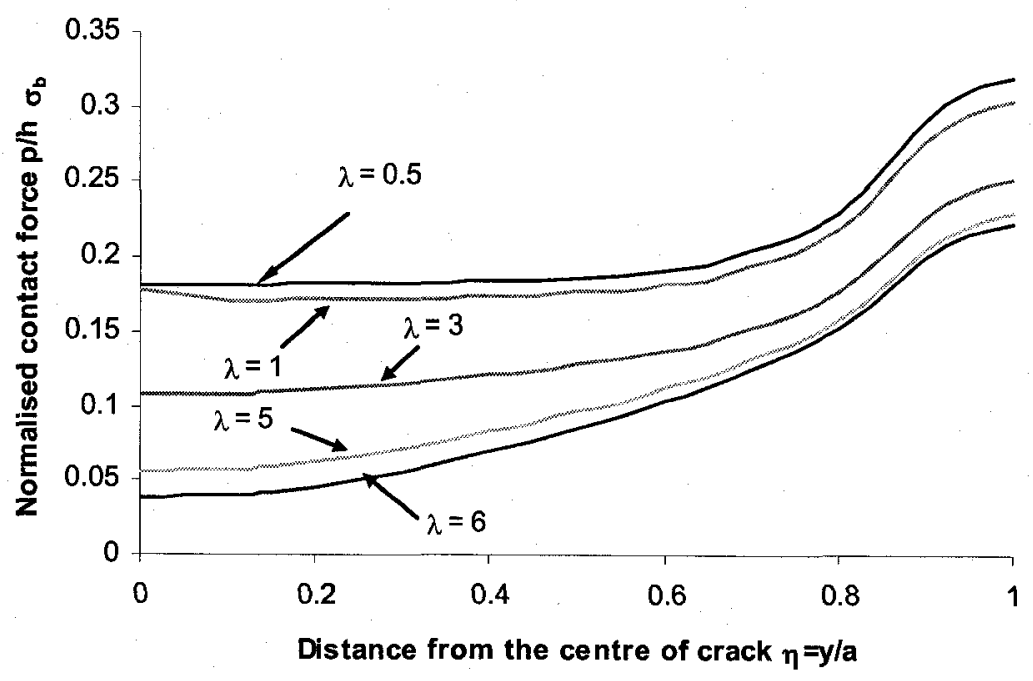

(b)

Figure 3.32 Distributions of the contact force assuming full-length closure for an isotropic cylindrical shell containing a circumferential crack with $a / h=10$ :

(a) under positive bending and (b) under negative bending 


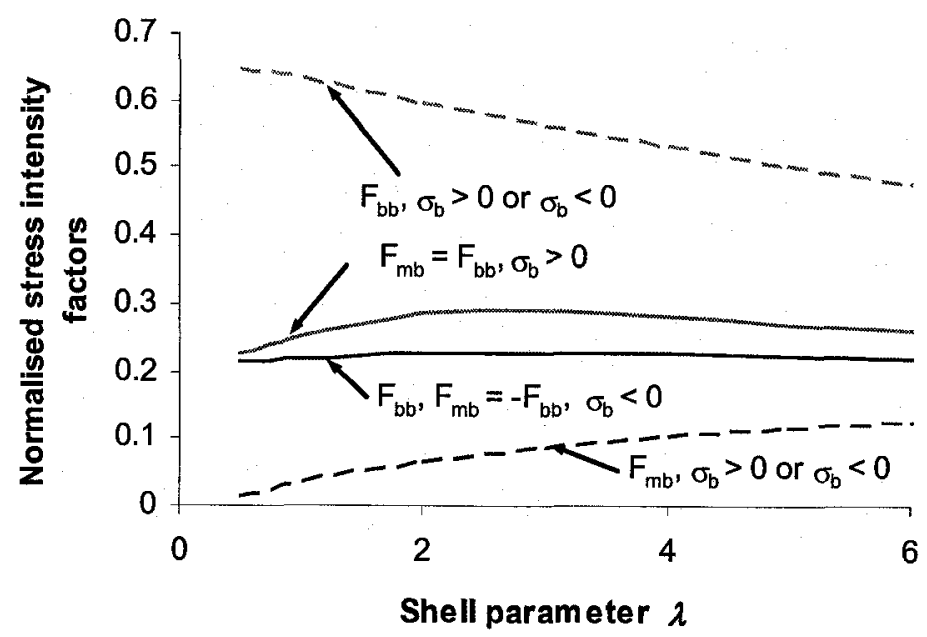

(a)

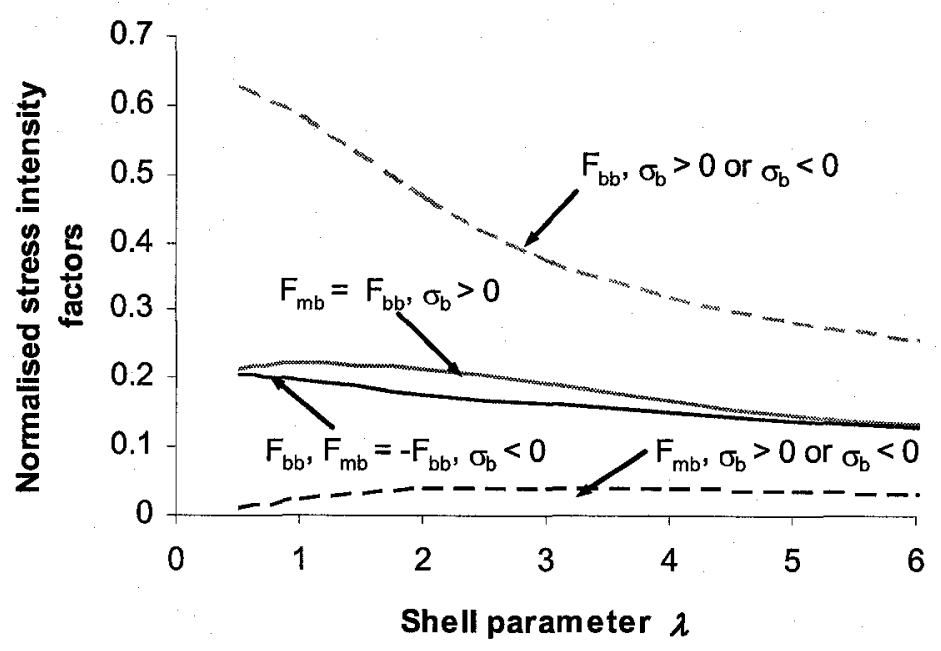

(b)

Figure 3.33 Comparison between closure and non-closure stress intensity factor ratios for an isotropic cylindrical shell with $a / h=10$ ( - closure and -- non-closure):

(a) containing an axial crack and (b) containing a circumferential crack 


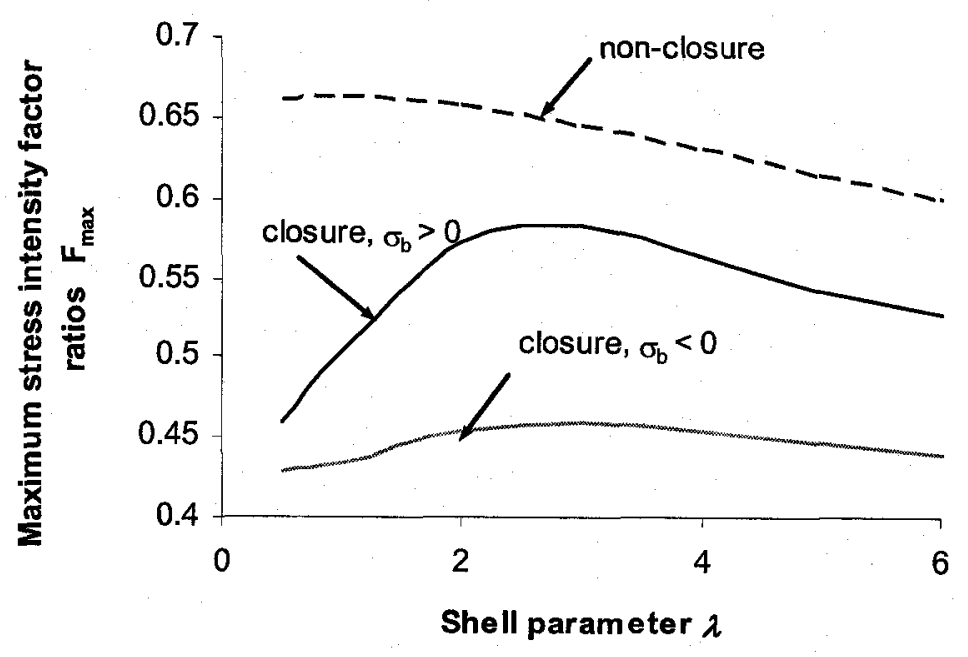

(a)

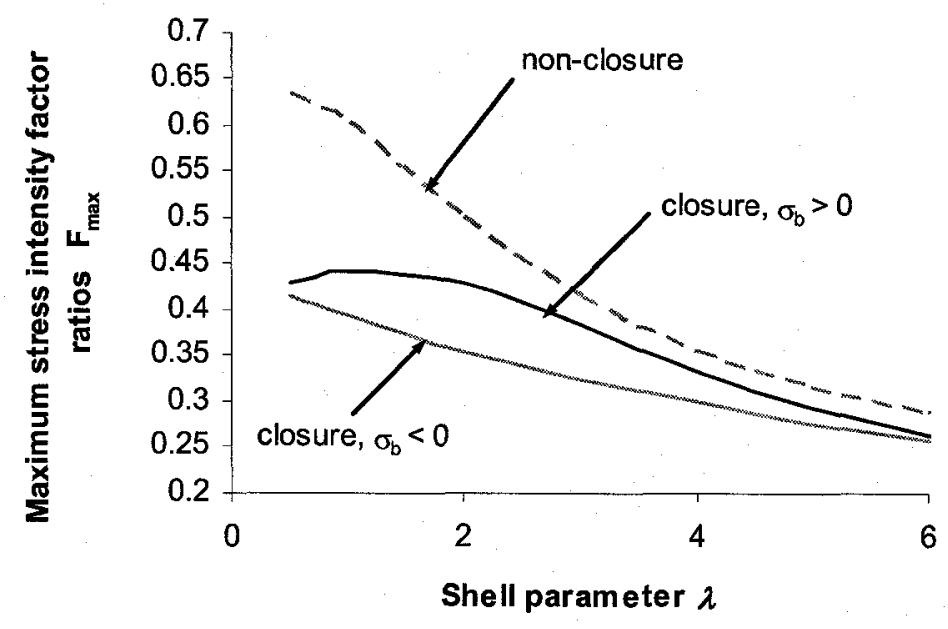

(b)

Figure 3.34 Comparison between closure solutions and non-closure solutions of the maximum stress intensity factor ratio for an isotropic cylindrical shell with $a / h=10:$

(a) containing an axial crack and (b) containing a circumferential crack 
The closure stress intensity factor ratios for various ratios of $a$ to $h$ are presented with the variation of the $\lambda$ value in Figure 3.35 and Figure 3.36 for an axially cracked cylindrical shell and for a circumferentially cracked cylindrical shell respectively.

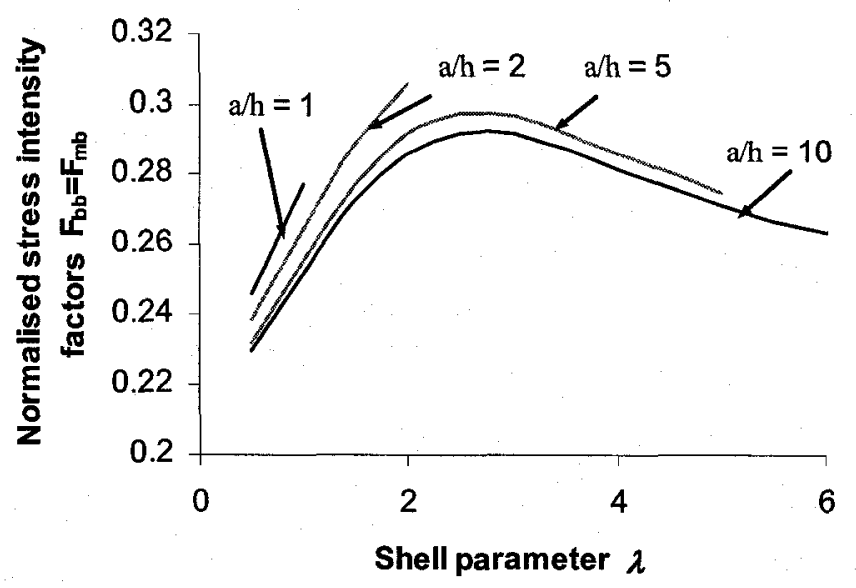

(a)

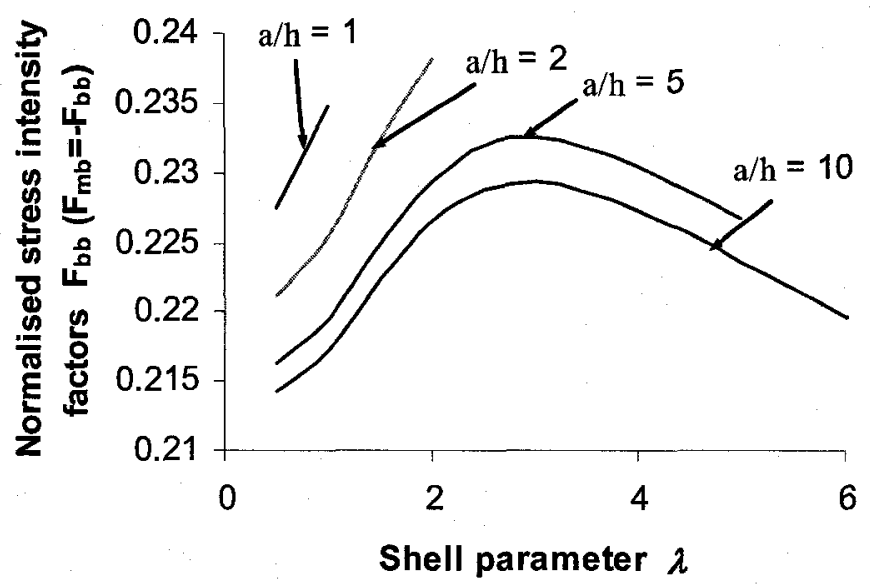

(b)

Figure 3.35 Closure solutions of the stress intensity factor ratios for an isotropic cylindrical shell containing an axial crack:

(a) under positive bending and (b) under negative bending 


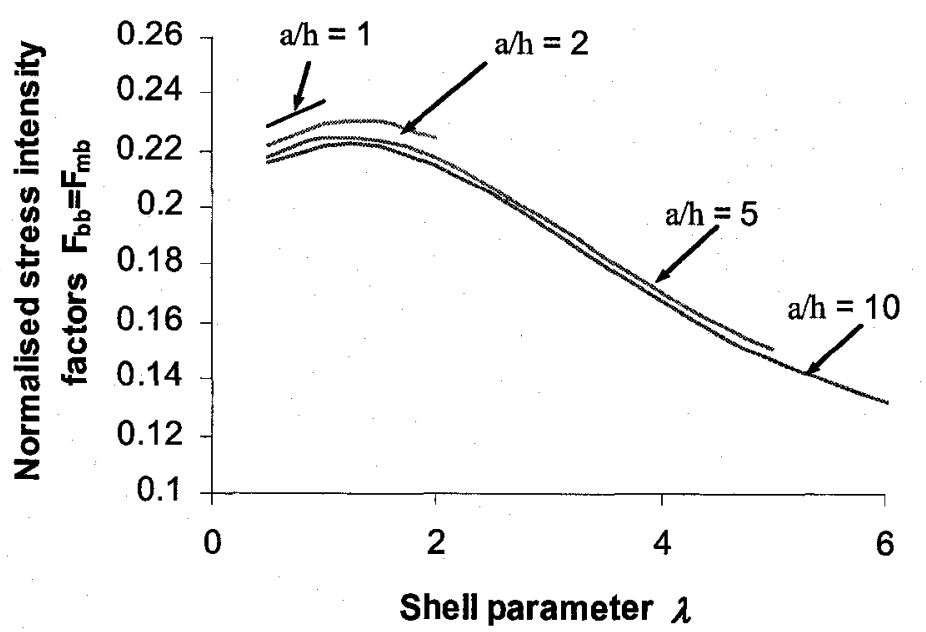

(a)

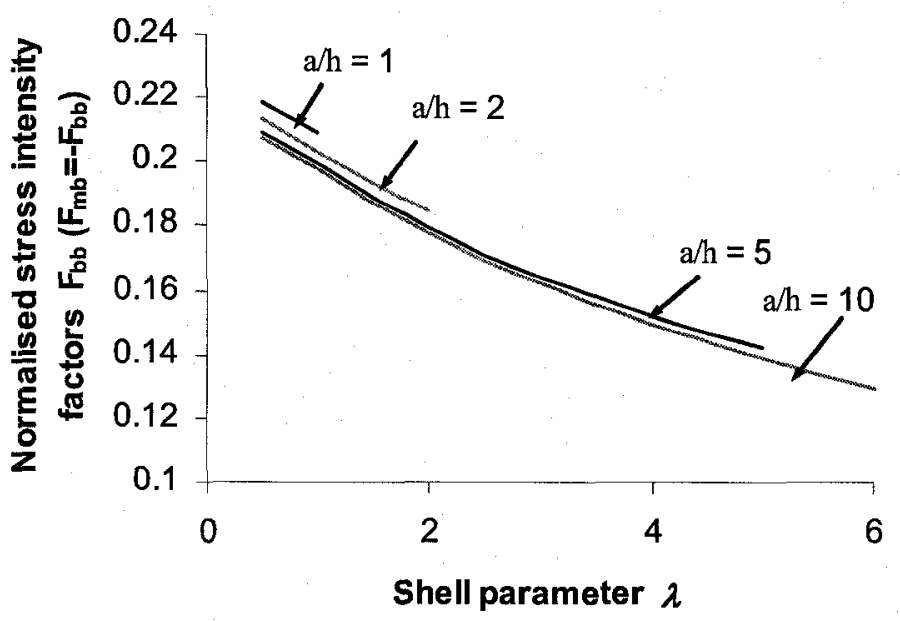

(b)

Figure 3.36 Closure solutions of the stress intensity factor ratios for an isotropic cylindrical shell containing a circumferential crack:

(a) under positive bending and (b) under negative bending 


\subsection{Summary of the Results}

\subsubsection{Crack closure behaviors}

To demonstrate systematically the effects of material orthotropy and shell geometry on the crack closure behavior of shells under bending, one may plot the variations of the critical $\lambda$ value for partial closure commence with the $E_{1} / E_{2}$ ratio for different $R_{1} / R_{2}$ ratios. As shown in Figure 3.37, for all shell geometries except for a cylindrical shell containing a circumferential crack, the critical $\lambda$ value decreases with the $E_{1} / E_{2}$ ratio. This indicates that the shell curvature effect on crack closure is enhanced with the increase of the $E_{1} / E_{2}$ ratio, that is, partial closure occurs at smaller values of $\lambda$ (larger shell curvature radii) for larger $E_{1} / E_{2}$ ratios. For a cylindrical shell containing an axial crack, the decrease in the critical $\lambda$ value is more obvious than other shell geometries when the $E_{1} / E_{2}$ ratio is less than unity. However, for a cylindrical shell containing a circumferential crack, the critical $\lambda$ value is constant for all $E_{1} / E_{2}$ ratios, that is, the shell curvature effect on crack closure is not influenced by material orthotropy. Also the critical $\lambda$ value for a cylindrical shell containing a circumferential crack is much higher than that for other shell geometries when the $E_{1} / E_{2}$ ratio is greater than unity, see Figure 3.37. This means that the shell curvature effect on the crack closure behavior in a cylindrical shell containing a circumferential crack is less than that in other shell geometries.

The critical $\lambda$ values for all the shell geometries with different $E_{1} / E_{2}$ ratios are presented in Table 3.2. 


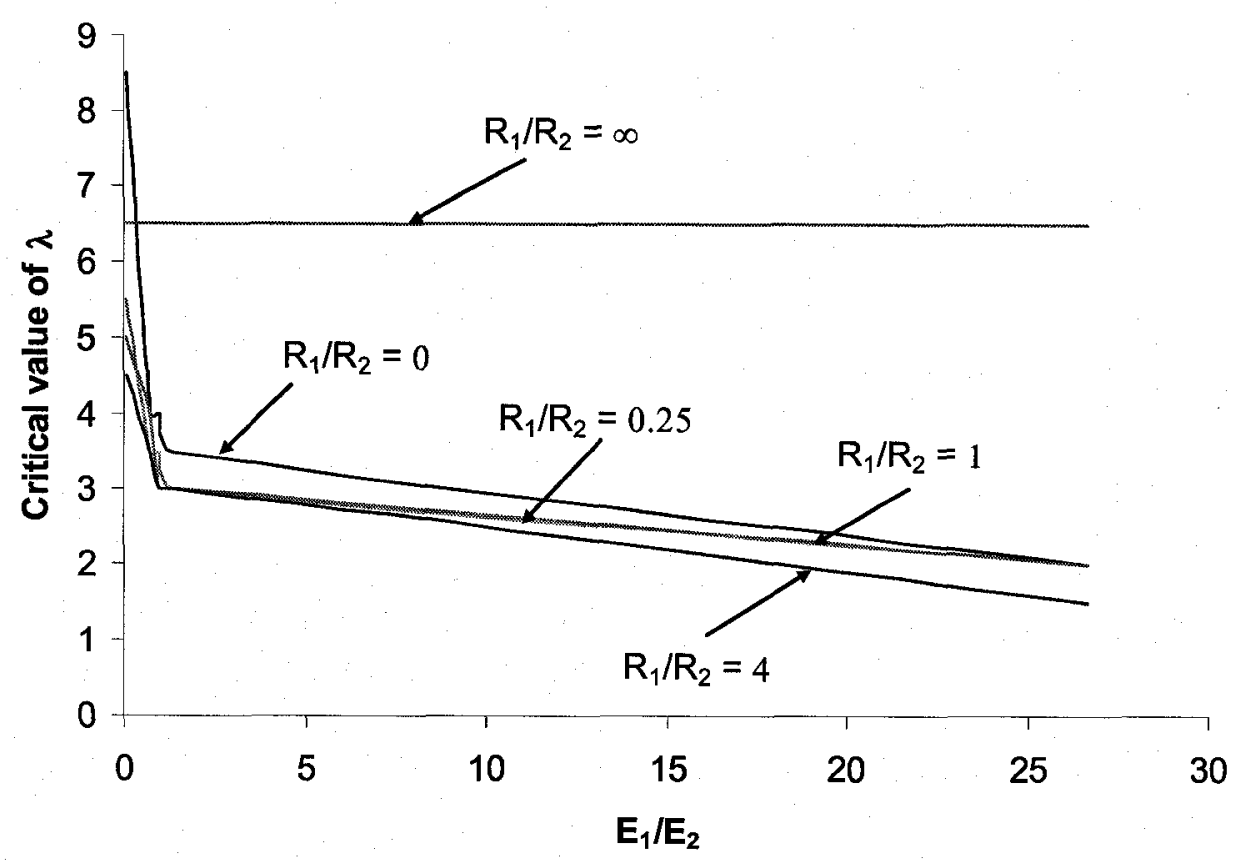

Figure 3.37 Effects of material orthotropy and shell geometry on the crack closure behavior of shells under positive bending

Table 3.2 Critical $\lambda$ values for partial closure under positive bending

\begin{tabular}{|c|c|c|c|c|c|}
\hline & $\varphi=0.037$ & $\varphi=0.725$ & $\varphi=1$ & $\varphi=1.38$ & $\varphi=26.67$ \\
\hline$\psi=0$ & 8.5 & 4 & 4 & 3.5 & 2 \\
\hline$\psi=0.25$ & 5.5 & 3.5 & 3.5 & 3 & 2 \\
\hline$\psi=1$ & 5 & 4 & 3 & 3 & 2 \\
\hline$\psi=4$ & 4.5 & 3.5 & 3 & 3 & 1.5 \\
\hline$\psi=\infty$ & 6.5 & 6.5 & 6.5 & 6.5 & 6.5 \\
\hline
\end{tabular}




\subsubsection{Stress intensity factors}

The closure stress intensity factor ratios for all the shell geometries under positive bending and negative bending respectively with different $E_{1} / E_{2}$ ratios and different $a / h$ ratios are tabulated, which are given with respect to the shell parameter $\lambda$ in Table 3.3 to Table 3.8. It is evident that stress intensity factor ratios vary with shell geometry $\left(R_{1} / R_{2}\right.$ ratio), material orthotropic property $\left(E_{1} / E_{2}\right.$ ratio), shell curvature $(\lambda)$, crack geometry $(a / h$ ratio) and the nature of bending load $\left(\sigma_{b}>0\right.$ or $\left.\sigma_{b}<0\right)$.

Firstly, for all the cases, that is, all the shell geometries under positive bending or negative bending, all the $E_{1} / E_{2}$ ratios and all the $a / h$ ratios, the membrane and bending components of SIF increase with the $\lambda$ value in the range of small $\lambda$ values (about $\lambda<2$ ); but they decrease for larger $\lambda$ values.

Secondly, for a given shell geometry and $E_{1} / E_{2}$ ratio, the stress intensity factor ratios decrease with the $a / h$ ratio for all the $\lambda$ values.

Thirdly, cylindrical shells with an axial crack have highest stress intensity factor ratios among all the shell geometries for all the $E_{1} / E_{2}$ and $a / h$ ratios. The shell with $R_{1} / R_{2}=0.25$ has the second higher stress intensity factor ratios. The other three geometries have similar values of stress intensity factor ratios except the spherical shell with $E_{1} / E_{2}=0.725$, which has much lower stress intensity factor ratios compared with the others. 
Table 3.3 Closure solutions of stress intensity factor ratios $\left(F_{m b}=F_{b b}\right)$ for the mildly orthotropic material (titanium), $\varphi=0.725$ and $\varphi=1.38, \sigma_{\mathrm{b}}>0$

\begin{tabular}{|c|c|c|c|c|c|c|c|c|c|c|c|c|c|c|c|}
\hline$\varphi$ & \multicolumn{5}{|c|}{$\mathbf{a} / \mathbf{h}=10$} & \multicolumn{5}{|c|}{$a / h=5$} & \multicolumn{5}{|c|}{$a / h=1$} \\
\hline 0.725 & \multicolumn{5}{|c|}{$\psi$} & \multicolumn{5}{|c|}{$\psi$} & \multicolumn{5}{|c|}{$\psi$} \\
\hline$\lambda$ & 0 & 0.25 & 1 & 4 & $\infty$ & 0 & 0.25 & 1 & 4 & $\infty$ & 0 & 0.25 & 1 & 4 & $\infty$ \\
\hline 0.5 & 0.22 & 0.23 & 0.13 & 0.21 & 0.21 & 0.23 & 0.23 & 0.12 & 0.22 & 0.22 & 0.24 & 0.24 & 0.12 & 0.23 & 0.23 \\
\hline 1 & 0.24 & 0.24 & 0.16 & 0.23 & 0.22 & 0.25 & 0.25 & 0.15 & 0.24 & 0.22 & 0.27 & 0.27 & 0.156 & 0.25 & 0.24 \\
\hline 2 & 0.27 & 0.26 & 0.2 & 0.22 & 0.21 & 0.29 & 0.27 & 0.19 & 0.22 & 0.22 & & & & & \\
\hline 3 & 0.29 & 0.24 & 0.18 & 0.17 & 0.19 & 0.30 & 0.24 & 0.23 & 0.18 & 0.20 & & & & & \\
\hline 4 & 0.28 & 0.21 & 0.17 & 0.15 & 0.17 & 0.29 & 0.21 & 0.18 & 0.15 & 0.17 & & & & & \\
\hline 5 & 0.27 & 0.18 & 0.15 & 0.13 & 0.15 & 0.28 & 0.19 & 0.15 & 0.14 & 0.15 & & & & & \\
\hline 6 & 0.27 & 0.17 & 0.14 & 0.11 & 0.14 & & & & & & & & & & \\
\hline$\varphi$ & & & $h=1$ & & & & & $\mathrm{~h}=$ & & & & & $/ \mathrm{h}=$ & & \\
\hline 1.38 & & & $\psi$ & & & & & $\psi$ & & & & & $\psi$ & & \\
\hline$\lambda$ & 0 & 0.25 & 1 & 4 & $\infty$ & 0 & 0.25 & 1 & 4 & $\infty$ & 0 & 0.25 & 1 & 4 & $\infty$ \\
\hline 0.5 & 0.23 & 0.23 & 0.23 & 0.20 & 0.21 & 0.23 & 0.23 & 0.23 & 0.20 & 0.22 & 0.25 & 0.25 & 0.25 & 0.21 & 0.23 \\
\hline 1 & 0.25 & 0.25 & 0.25 & 0.23 & 0.22 & 0.26 & 0.25 & 0.25 & 0.23 & 0.22 & 0.28 & 0.28 & 0.28 & 0.25 & 0.24 \\
\hline 2 & 0.28 & 0.26 & 0.25 & 0.21 & 0.21 & 0.29 & 0.27 & 0.25 & 0.22 & 0.22 & & & & & \\
\hline 3 & 0.29 & 0.24 & 0.21 & 0.17 & 0.19 & 0.29 & 0.24 & 0.21 & 0.17 & 0.19 & & & & & \\
\hline 4 & 0.28 & 0.21 & 0.18 & 0.15 & 0.17 & 0.28 & 0.21 & 0.17 & 0.15 & 0.17 & & & & & \\
\hline 5 & 0.27 & 0.19 & 0.15 & 0.13 & 0.15 & 0.27 & 0.19 & 0.14 & 0.12 & 0.15 & & & & & \\
\hline 6 & 0.26 & 0.18 & 0.14 & 0.12 & 0.14 & & & & & & & & & & \\
\hline
\end{tabular}


Table 3.4 Closure solutions of stress intensity factor ratios $\left(F_{b b}, F_{m b}=-F_{b b}\right)$ for the mildly orthotropic material (titanium), $\varphi=0.725$ and $\varphi=1.38, \sigma_{\mathrm{b}}<0$

\begin{tabular}{|c|c|c|c|c|c|c|c|c|c|c|c|c|c|c|c|}
\hline$\varphi$ & \multicolumn{5}{|c|}{$\mathrm{a} / \mathrm{h}=10$} & \multicolumn{5}{|c|}{$\mathrm{a} / \mathrm{h}=5$} & \multicolumn{5}{|c|}{$\mathrm{a} / \mathrm{h}=1$} \\
\hline 0.725 & \multicolumn{5}{|c|}{$\psi$} & \multicolumn{5}{|c|}{$\psi$} & \multicolumn{5}{|c|}{$\psi$} \\
\hline$\lambda$ & 0 & 0.25 & 1 & 4 & $\infty$ & 0 & 0.25 & 1 & 4 & $\infty$ & 0 & 0.25 & 1 & 4 & $\infty$ \\
\hline 0.5 & 0.21 & 0.21 & 0.12 & 0.20 & 0.21 & 0.22 & 0.21 & 0.12 & 0.20 & 0.21 & 0.23 & 0.23 & 0.12 & 0.21 & 0.22 \\
\hline 1 & 0.22 & 0.21 & $\begin{array}{c}0.14 \\
0\end{array}$ & 0.20 & 0.20 & 0.22 & $\overline{0.21}$ & 0.14 & 0.20 & 0.20 & 0.23 & 0.23 & 0.14 & 0.22 & 0.21 \\
\hline 2 & 0.23 & 0.21 & 0.17 & 0.18 & 0.18 & 0.23 & 0.21 & 0.17 & 0.18 & 0.18 & & & & & \\
\hline 3 & 0.23 & 0.20 & 0.19 & 0.16 & 0.17 & 0.23 & 0.20 & 0.20 & 0.16 & 0.16 & & & & & \\
\hline 4 & 0.23 & 0.18 & 0.16 & 0.14 & 0.15 & 0.23 & 0.19 & 0.16 & 0.14 & 0.15 & & & & & \\
\hline 5 & 0.23 & 0.17 & 0.14 & 0.12 & 0.14 & 0.23 & 0.17 & 0.15 & 0.13 & 0.14 & & & & & \\
\hline 6 & 0.23 & 0.16 & 0.14 & 0.10 & 0.13 & & & & & & & & & & \\
\hline$\varphi$ & \multicolumn{5}{|c|}{$\mathrm{a} / \mathrm{h}=10$} & \multicolumn{5}{|c|}{$\mathrm{a} / \mathrm{h}=5$} & \multicolumn{5}{|c|}{$\mathrm{a} / \mathrm{h}=1$} \\
\hline 1.38 & \multicolumn{5}{|c|}{$\psi$} & \multicolumn{5}{|c|}{$\psi$} & \multicolumn{5}{|c|}{$\psi$} \\
\hline$\lambda$ & 0 & 0.25 & 1 & 4 & $\infty$ & 0 & 0.25 & 1 & 4 & $\infty$ & 0 & 0.25 & 1 & 4 & $\infty$ \\
\hline 0.5 & 0.21 & 0.21 & 0.21 & 0.19 & 0.21 & 0.22 & 0.21 & 0.21 & 0.19 & 0.21 & 0.23 & 0.23 & 0.23 & 0.20 & 0.22 \\
\hline 1 & 0.22 & 0.21 & 0.21 & 0.19 & 0.20 & 0.22 & $\overline{0.22}$ & 0.21 & 0.20 & 0.20 & 0.24 & 0.23 & 0.23 & 0.20 & 0.21 \\
\hline 2 & 0.23 & 0.21 & $\overline{0.20}$ & 0.18 & 0.18 & 0.23 & 0.21 & 0.20 & 0.18 & 0.18 & & & & & \\
\hline 3 & 0.23 & 0.20 & 0.18 & 0.16 & 0.17 & 0.23 & 0.20 & 0.18 & 0.16 & 0.16 & & & & & \\
\hline 4 & 0.23 & 0.18 & $\begin{array}{c}0.16 \\
2\end{array}$ & 0.14 & 0.15 & 0.23 & 0.19 & 0.17 & 0.14 & 0.15 & & & & & \\
\hline 5 & 0.23 & 0.17 & 0.15 & 0.13 & 0.14 & 0.22 & 0.17 & 0.16 & 0.12 & 0.14 & & & & & \\
\hline 6 & 0.22 & 0.16 & 0.14 & 0.12 & 0.13 & & & & & & & & & & \\
\hline
\end{tabular}


Table 3.5 Closure solutions of stress intensity factor ratios $\left(F_{m b}=F_{b b}\right)$ for the strongly orthotropic material (graphite-epoxy), $\varphi=0.037$ and $\varphi=26.67, \sigma_{\mathrm{b}}>0$

\begin{tabular}{|c|c|c|c|c|c|c|c|c|c|c|c|c|c|c|c|}
\hline$\varphi$ & \multicolumn{5}{|c|}{$a / h=10$} & \multicolumn{5}{|c|}{$\mathrm{a} / \mathrm{h}=5$} & \multicolumn{5}{|c|}{$\mathrm{a} / \mathrm{h}=1$} \\
\hline 0.037 & \multicolumn{5}{|c|}{$\psi$} & \multicolumn{5}{|c|}{$\psi$} & \multicolumn{5}{|c|}{$\psi$} \\
\hline$\lambda$ & 0 & 0.25 & 1 & 4 & $\infty$ & 0 & 0.25 & 1 & $\overline{4}$ & $\infty$ & 0 & 0.25 & 1 & 4 & $\infty$ \\
\hline 0.5 & 0.21 & 0.21 & 0.21 & 0.21 & 0.21 & 0.22 & 0.21 & 0.21 & $\overline{0.22}$ & 0.22 & 0.24 & 0.23 & 0.23 & 0.23 & 0.24 \\
\hline 1 & 0.22 & 0.22 & 0.22 & 0.22 & 0.22 & 0.23 & 0.22 & 0.22 & 0.22 & 0.23 & 0.25 & 0.24 & 0.22 & 0.24 & 0.25 \\
\hline 2 & 0.23 & 0.23 & 0.22 & 0.21 & 0.21 & 0.25 & 0.23 & 0.23 & 0.21 & 0.22 & & & & & \\
\hline 3 & 0.25 & 0.23 & 0.21 & 0.18 & 0.19 & 0.27 & 0.23 & 0.21 & 0.18 & 0.20 & & & & & \\
\hline 4 & 0.26 & 0.21 & 0.18 & 0.17 & 0.17 & 0.29 & 0.22 & 0.19 & 0.17 & 0.17 & & & & & \\
\hline 5 & 0.27 & 0.19 & 0.16 & 0.15 & 0.16 & 0.30 & 0.20 & 0.20 & 0.17 & 0.16 & & & & & \\
\hline 6 & 0.28 & 0.18 & 0.15 & 0.14 & 0.14 & & & & & & & & & & \\
\hline$\varphi$ & & & $\mathbf{h}=$ & & & & & $/ \mathrm{h}=$ & & & & & $/ \mathrm{h}=$ & & \\
\hline 26.67 & & & $\psi$ & & & & & $\psi$ & & & & & $\psi$ & & \\
\hline$\lambda$ & 0 & 0.25 & 1 & 4 & $\infty$ & 0 & 0.25 & 1 & 4 & $\infty$ & 0 & 0.25 & 1 & 4 & $\infty$ \\
\hline 0.5 & 0.24 & 0.24 & 0.24 & 0.24 & 0.20 & 0.26 & 0.24 & 0.24 & 0.24 & 0.21 & 0.28 & 0.26 & 0.26 & 0.26 & 0.22 \\
\hline 1 & 0.27 & 0.26 & 0.26 & 0.25 & 0.21 & 0.29 & 0.27 & 0.27 & 0.26 & 0.21 & 0.32 & 0.29 & 0.29 & 0.28 & 0.22 \\
\hline 2 & 0.27 & 0.25 & 0.23 & 0.21 & 0.21 & 0.28 & 0.25 & 0.24 & 0.21 & 0.22 & & & & & \\
\hline 3 & 0.26 & 0.23 & 0.20 & 0.17 & 0.19 & 0.26 & 0.23 & 0.20 & 0.17 & 0.19 & & & & & \\
\hline 4 & 0.25 & 0.20 & 0.19 & 0.17 & 0.17 & 0.24 & 0.21 & 0.19 & 0.17 & 0.17 & & & & & \\
\hline 5 & 0.24 & 0.21 & 0.17 & 0.15 & 0.15 & 0.23 & 0.20 & 0.17 & 0.14 & 0.15 & & & & & \\
\hline 6 & 0.23 & 0.17 & 0.15 & 0.13 & 0.14 & & & & & & & & & & \\
\hline
\end{tabular}


Table 3.6 Closure solutions of stress intensity factor ratios $\left(F_{b b}, F_{m b}=-F_{b b}\right)$ for the strongly orthotropic material (graphite-epoxy), $\varphi=0.037$ and $\varphi=26.67$, $\sigma_{\mathrm{b}}<0$

\begin{tabular}{|c|c|c|c|c|c|c|c|c|c|c|c|c|c|c|c|}
\hline$\varphi$ & \multicolumn{5}{|c|}{$\mathrm{a} / \mathrm{h}=10$} & \multicolumn{5}{|c|}{$\mathrm{a} / \mathrm{h}=5$} & \multicolumn{5}{|c|}{$a / h=1$} \\
\hline 0.037 & \multicolumn{5}{|c|}{$\psi$} & \multicolumn{5}{|c|}{$\psi$} & \multicolumn{5}{|c|}{$\psi$} \\
\hline$\lambda$ & 0 & 0.25 & 1 & 4 & $\infty$ & 0 & 0.25 & 1 & 4 & $\infty$ & 0 & 0.25 & 1 & 4 & $\infty$ \\
\hline 0.5 & 0.21 & 0.20 & 0.20 & 0.20 & 0.20 & 0.22 & 0.21 & 0.21 & 0.20 & 0.21 & 0.23 & 0.22 & 0.22 & 0.22 & 0.22 \\
\hline 1 & 0.22 & 0.20 & 0.20 & 0.19 & 0.20 & 0.22 & 0.21 & 0.20 & 0.20 & 0.20 & 0.23 & 0.22 & 0.23 & 0.22 & 0.21 \\
\hline 2 & $\overline{0.22}$ & 0.20 & 0.19 & 0.18 & 0.18 & 0.22 & 0.20 & 0.19 & 0.18 & 0.18 & & & & & \\
\hline 3 & 0.22 & 0.19 & 0.18 & 0.16 & 0.17 & 0.23 & 0.20 & 0.18 & 0.17 & 0.17 & & & & & \\
\hline 4 & 0.23 & 0.18 & 0.17 & 0.15 & 0.16 & 0.23 & 0.19 & 0.17 & 0.16 & 0.16 & & & & & \\
\hline 5 & 0.23 & 0.17 & 0.16 & 0.14 & 0.15 & 0.23 & 0.18 & 0.16 & 0.15 & 0.15 & & & & & \\
\hline 6 & 0.22 & 0.16 & 0.14 & 0.13 & 0.14 & & & & & & & & & & \\
\hline$\varphi$ & \multicolumn{5}{|c|}{$\mathrm{a} / \mathrm{h}=10$} & \multicolumn{5}{|c|}{$\mathrm{a} / \mathrm{h}=5$} & \multicolumn{5}{|c|}{$\mathrm{a} / \mathrm{h}=1$} \\
\hline 26.67 & \multicolumn{5}{|c|}{$\psi$} & \multicolumn{5}{|c|}{$\psi$} & \multicolumn{5}{|c|}{$\psi$} \\
\hline$\lambda$ & 0 & 0.25 & 1 & 4 & $\infty$ & 0 & 0.25 & 1 & 4 & $\infty$ & 0 & 0.25 & 1 & 4 & $\infty$ \\
\hline 0.5 & 0.22 & 0.22 & 0.22 & 0.21 & 0.20 & 0.22 & 0.22 & 0.22 & 0.22 & 0.21 & 0.23 & 0.23 & 0.23 & 0.23 & $\overline{0.21}$ \\
\hline 1 & 0.23 & 0.23 & 0.22 & 0.21 & 0.19 & 0.23 & 0.23 & 0.22 & 0.20 & 0.20 & 0.25 & 0.25 & 0.24 & 0.23 & 0.20 \\
\hline 2 & 0.23 & 0.21 & 0.20 & 0.19 & 0.18 & 0.23 & 0.22 & 0.20 & 0.19 & 0.18 & & & & & \\
\hline 3 & 0.22 & 0.20 & 0.18 & 0.16 & 0.17 & 0.22 & 0.20 & 0.18 & 0.16 & 0.16 & & & & & \\
\hline 4 & 0.21 & 0.18 & 0.16 & 0.14 & 0.16 & 0.21 & 0.18 & 0.16 & 0.14 & 0.15 & & & & & \\
\hline 5 & 0.20 & 0.17 & 0.15 & 0.13 & 0.15 & 0.20 & 0.17 & 0.15 & 0.13 & 0.14 & & & & & \\
\hline 6 & 0.20 & 0.16 & 0.14 & 0.12 & 0.14 & & & & & & & & & & \\
\hline
\end{tabular}


Table 3.7 Closure solutions of stress intensity factor ratios $\left(F_{m b}=F_{b b}\right)$ for isotropic materials, $\varphi=1, \sigma_{\mathrm{b}}>0$

\begin{tabular}{|c|c|c|c|c|c|c|c|c|c|c|c|c|c|c|c|}
\hline$\varphi$ & \multicolumn{5}{|c|}{$\mathrm{a} / \mathrm{h}=10$} & \multicolumn{5}{c|}{$\mathrm{a} / \mathrm{h}=5$} & \multicolumn{5}{c|}{$\mathrm{a} / \mathrm{h}=1$} \\
\hline 1 & \multicolumn{3}{|c|}{$\psi$} & \multicolumn{3}{c|}{$\psi$} & \multicolumn{3}{c|}{$\psi$} \\
\hline$\lambda$ & 0 & 0.25 & 1 & 4 & $\infty$ & 0 & 0.25 & 1 & 4 & $\infty$ & 0 & 0.25 & 1 & 4 & $\infty$ \\
\hline 0.5 & 0.23 & 0.23 & 0.23 & 0.22 & 0.22 & 0.23 & 0.23 & 0.23 & 0.23 & 0.22 & 0.25 & 0.25 & 0.25 & 0.24 & 0.23 \\
\hline 1 & 0.25 & 0.25 & 0.25 & 0.23 & 0.22 & 0.26 & 0.26 & 0.25 & 0.23 & 0.22 & 0.28 & 0.28 & 0.28 & 0.27 & 0.24 \\
\hline 2 & 0.29 & 0.28 & 0.25 & 0.17 & 0.22 & 0.29 & 0.28 & 0.25 & 0.17 & 0.22 & & & & & \\
\hline 3 & 0.29 & 0.26 & 0.20 & 0.13 & 0.19 & 0.30 & 0.26 & 0.20 & 0.14 & 0.19 & & & & & \\
\hline 4 & 0.28 & 0.23 & 0.17 & 0.12 & 0.17 & 0.29 & 0.24 & 0.17 & 0.13 & 0.17 & & & & & \\
\hline 5 & 0.27 & 0.21 & 0.15 & 0.12 & 0.15 & 0.28 & 0.23 & 0.15 & 0.12 & 0.15 & & & & & \\
\hline 6 & 0.26 & 0.20 & 0.14 & 0.10 & 0.13 & & & & & & & & & & \\
\hline
\end{tabular}

Table 3.8 Closure solutions of stress intensity factor ratios $\left(F_{b b}, F_{m b}=-F_{b b}\right)$ for isotropic materials, $\varphi=1, \sigma_{b}>0$

\begin{tabular}{|c|c|c|c|c|c|c|c|c|c|c|c|c|c|c|c|}
\hline$\varphi$ & \multicolumn{5}{|c|}{$\mathrm{a} / \mathrm{h}=10$} & \multicolumn{5}{c|}{$\mathrm{a} / \mathrm{h}=5$} & \multicolumn{5}{c|}{$\mathrm{a} / \mathrm{h}=1$} \\
\hline 1 & \multicolumn{3}{|c|}{$\psi$} & \multicolumn{6}{c|}{$\psi$} & \multicolumn{3}{c|}{$\psi$} \\
\hline$\lambda$ & 0 & 0.25 & 1 & 4 & $\infty$ & 0 & 0.25 & 1 & 4 & $\infty$ & 0 & 0.25 & 1 & 4 & $\infty$ \\
\hline 0.5 & 0.21 & 0.21 & 0.21 & 0.19 & 0.21 & 0.22 & 0.21 & 0.21 & 0.20 & 0.21 & 0.23 & 0.23 & 0.22 & 0.21 & 0.22 \\
\hline 1 & 0.22 & 0.21 & 0.20 & 0.18 & 0.20 & 0.22 & 0.22 & 0.21 & 0.18 & 0.20 & 0.23 & 0.23 & 0.22 & 0.21 & 0.21 \\
\hline 2 & 0.23 & 0.21 & 0.19 & 0.16 & 0.18 & 0.23 & 0.22 & 0.19 & 0.16 & 0.18 & & & & & \\
\hline 3 & 0.23 & 0.21 & 0.17 & 0.13 & 0.16 & 0.23 & 0.21 & 0.18 & 0.14 & 0.16 & & & & & \\
\hline 4 & 0.23 & 0.20 & 0.16 & 0.11 & 0.15 & 0.23 & 0.20 & 0.16 & 0.12 & 0.15 & & & & & \\
\hline 5 & 0.22 & 0.18 & 0.14 & 0.11 & 0.14 & 0.23 & 0.20 & 0.15 & 0.11 & 0.14 & & & & & \\
\hline 6 & 0.22 & 0.17 & 0.13 & 0.10 & 0.13 & & & & & & & & & & \\
\hline
\end{tabular}




\section{Chapter 4 Case Study}

\subsection{A Pressurized Cylinder Containing an Axial Crack}

In this chapter, the developed crack closure theory will be applied to a case study, considering an axially cracked cylinder with isotropic material subjected to both internal and external pressure loads. The SIFs will be determined in terms of the conventional shell theory and the crack closure shell theory, respectively. The two solutions will be compared to demonstrate the significant effect of crack-face closure on the SIF values.

The circumferential stress varies through the wall thickness when a cylinder is subjected to internal or external pressure. Internal pressure will induce tensile circumferential stress in the wall while external pressure compressive stress, in terms of the thick-walled cylinder theory [105]. The resultant stress due to the synergetic contribution of internal and external pressure loads may be tensile at the inner surface and compressive at the outer surface or vice versa, depending on the load levels. The uneven circumferential stress through the wall thickness produces a bending moment on the crack face when the cylinder has an axial crack, which would lead to crack-face closure at the compressive edges.

\subsection{Determination of Bending Stress}

Consider an isotropic cylinder, made of stainless steel, with the inner radius, $r_{i}$, and outer radius, $r_{o}$, subjected to internal pressure, $P_{i}$, and external pressure, $P_{o}$, as shown 
in Figure 4.1. The cylinder has a uniform wall thickness, $h$, containing an axial crack of $2 a$ long.

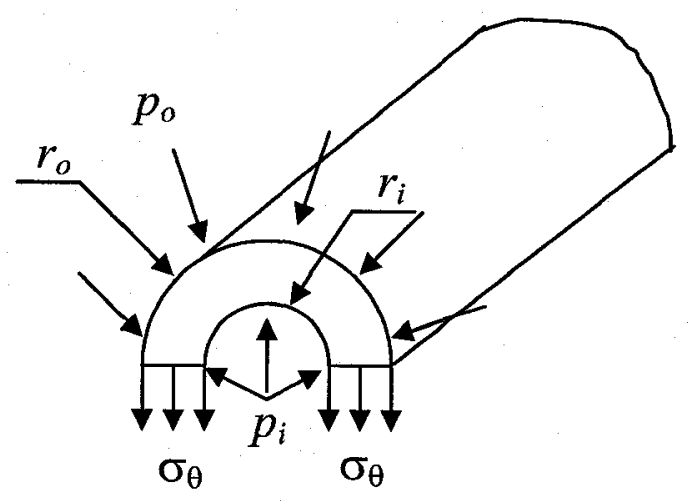

Figure 4.1 A thick-walled cylinder subjected to internal pressure and external pressure

In terms of the thick-walled cylinder theory [105], the circumferential stress, $\sigma_{\theta}$ induced in the cylinder wall can be determined by the following equations:

$$
\begin{aligned}
& \sigma_{\theta}=\frac{p_{i}}{\mathrm{~K}^{2}-1}\left(1+\frac{r_{o}^{2}}{r^{2}}\right)(\text { due to internal pressure }) \\
& \sigma_{\theta}=-\frac{p_{o}}{\mathrm{~K}^{2}-1}\left(\mathrm{~K}^{2}+\frac{r_{o}^{2}}{r^{2}}\right)(\text { due to external pressure })
\end{aligned}
$$

where $\mathrm{K}=\frac{r_{0}}{r_{i}}$ and $r$ is the radius along the wall thickness. It is clear that the circumferential stress varies nonlinearly through the wall thickness and internal pressure induces tensile stress and external pressure compressive stress. The resultant stress due to concurrent internal pressure and external pressure may lead to the stress in opposite sign at the inner surface and outer surface (depending on the levels of internal pressure and 
external pressure), which would produce a bending moment to close the crack at the compressive edge when the cylinder contains an axial crack. For example, a cylinder has the $r_{i}$ of $200 \mathrm{~mm}$ and the $r_{o}$ of $250 \mathrm{~mm}$, with a wall thickness of $50 \mathrm{~mm}$, subjected to an internal pressure of $100 \mathrm{MPa}$ and an external pressure of $80 \mathrm{MPa}$, the circumferential stresses due to the internal and external pressure are calculated in terms of eqn (4.1) and the stress distributions through the wall thickness are plotted in Figure 4.2. It is seen in Figure. 4.2(a) that both the maximum tensile stress due to the internal pressure and the maximum compressive stress due to the external pressure occur at the inner surface of the cylinder $\left(r=r_{i}=200 \mathrm{~mm}\right)$. The resultant stress by the concurrent internal pressure and external pressure varies linearly through the wall thickness, with approximately equal magnitude (10 $\mathrm{MPa})$ in tension at the inner surface and in compression at the outer surface, as illustrated in Figure 4.2(b). The resultant circumferential stress produces a uniform bending moment in the shell wall along the axial direction, which is termed bending stress $\left(\sigma_{\mathrm{b}}<0\right)$, and leads to nearly zero membrane stress, because the tensile stresses in the inner layer approximately equals the corresponding compressive stresses in the outer layer. Of course, it is not always the case in engineering problems that the stress at the inner surface always equals that at the outer surface in magnitude. If they are not equal, membrane stress exists simultaneously with bending stress. As discussed in Chapter 3, in this case, the stress functions in the integral equations should include the membrane stress. Since the crack closure issue studied in this thesis involves bending stress only, the example selected here is for demonstration only; therefore it is bending-stress predominant; membrane stress is trivial and can be negligible. 


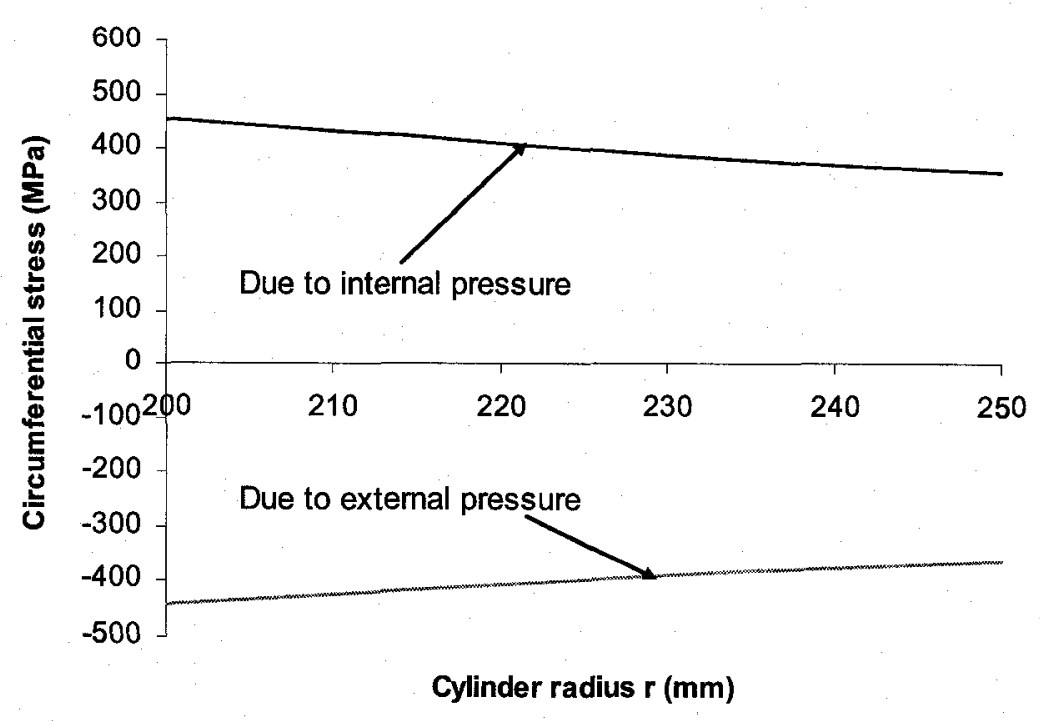

(a)

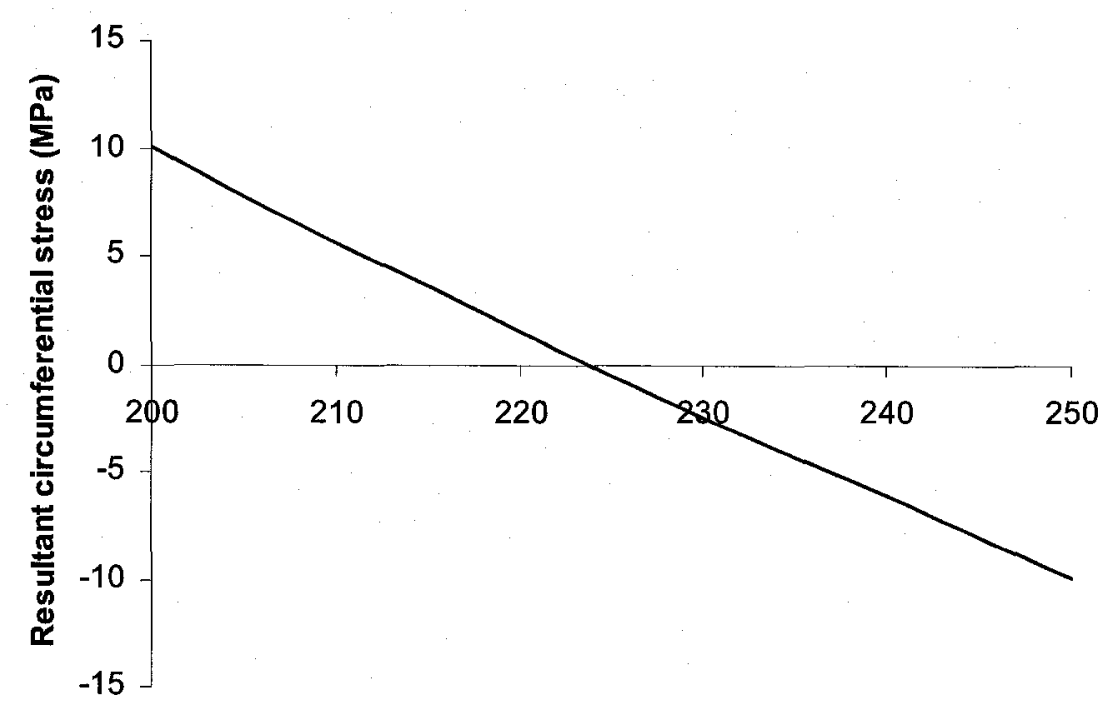

Cylinder radius $\mathrm{r}(\mathrm{mm})$

(b)

Figure 4.2 Circumferential stresses in a cylinder subject to internal pressure and external pressure: (a) stresses due to internal pressure and external pressure respectively and (b) resultant stress due to concurrent internal pressure and external pressure 


\subsection{Solution of Stress Intensity Factors}

\subsubsection{Determination of crack closure regions}

For this given cylinder geometry, the crack closure behavior will be studied for a range of crack length, $2 a=10 \sim 100 \mathrm{~mm}$. Thus for different crack lengths the contact forces at the compressive edge (outer surface) were computed assuming full-length closure. The results are presented in Figure 4.3.

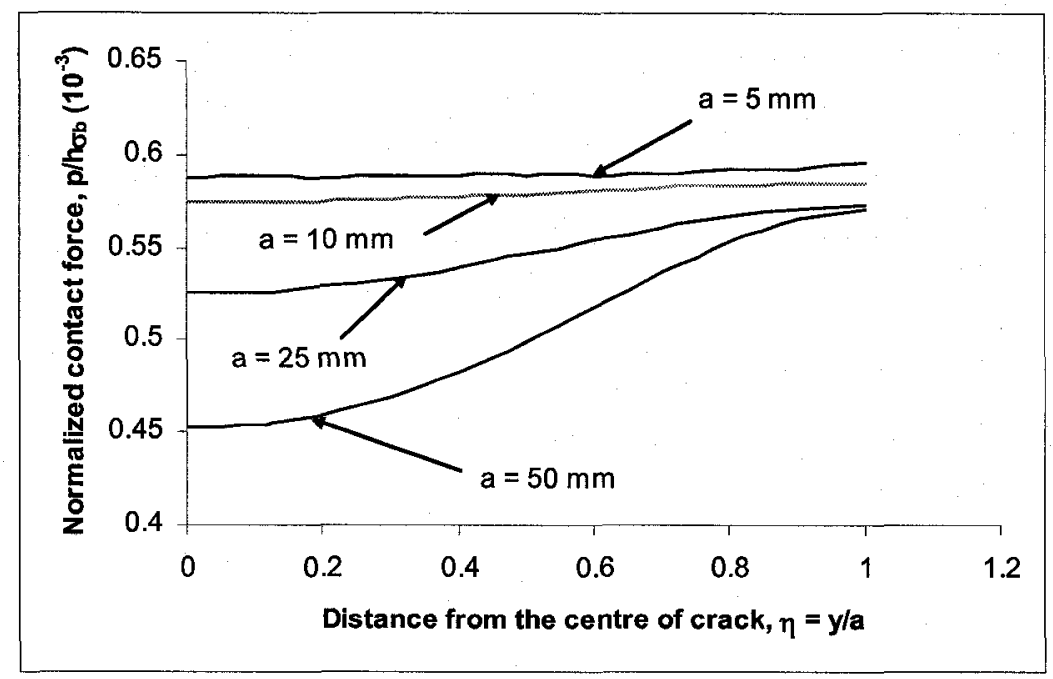

Figure 4.3 Contact force distributions along the crack length of the cylinder containing an axial crack under internal and external pressure

It is seen that the contact forces are positive everywhere along the crack so that full-length closure always occurs in the cylinder for the crack lengths being considered. Using eqn (3.31), one may calculate the shell parameter $\lambda$ corresponding to the crack lengths. For the crack length range, $2 a=10 \sim 100 \mathrm{~mm}$, it is calculated that $\lambda=0.085 \sim$ 0.85. From the analysis of Chapter 3, negative bending always results in complete closure as long as $\lambda \leq 6$. The $\lambda$ values with respect to the cylinder and crack geometries being 
discussed are in the range of $\lambda$ values for complete crack closure occurrence. Therefore, the computed contact forces are consistent with the theoretical analysis.

\subsubsection{Results of stress intensity factors}

Since full-length closure occurs in the cylinder being discussed for the given range of crack length, eqns (3.33) and (3.34) are used to obtain the solution $G_{2}$, and $G_{1}$ is then derived from eqn (3.15). The Gauss-Chebyshev integration formulas given in appendix $\mathrm{C}$ were employed to solve the equations. The stress intensity factor ratios and the combined SIFs due to the synergetic effect of membrane and bending components at the crack tip were calculated using eqns (3.37) and (3.38); the results are presented in Figures 4.4 and 4.5. The non-closure SIFs were also computed for comparison. The cylinder material is steel, with the Young's modulus $E=2.1 \times 10^{5} \mathrm{MPa}$ and the Poisson's ratio $v=0.3$.

It is evident that the crack-face closure influences significantly the SIF for a cylinder containing an axial crack. As shown in Figure 4.4, the closure decreases the bending component of the SIF and increases the membrane component in magnitude but changes the sign. Comparing the curves in Figure 4.5, without considering crack face closure, the SIF varies constantly from positive at the tensile edge (inner surface) to negative at the compressive edge (outer surface), and is zero at the middle surface of the wall. However, with the closure the SIF becomes zero at the compressive edge, and also the magnitude of the SIF at the tensile edge has been reduced about $33 \%$ (for $a=50 \mathrm{~mm}$ ) $\sim 49 \%$ (for $a=5 \mathrm{~mm}$ ) by the closure effect, that is, the effect of the crack closure is 
stronger for short cracks than for long cracks. In addition, the stress intensity factor ratios of the non-closure case and the closure case all decrease with the crack length, as seen in Figure 4.4 while the total SIFs increase with the crack length, as shown in Figure 4.5.

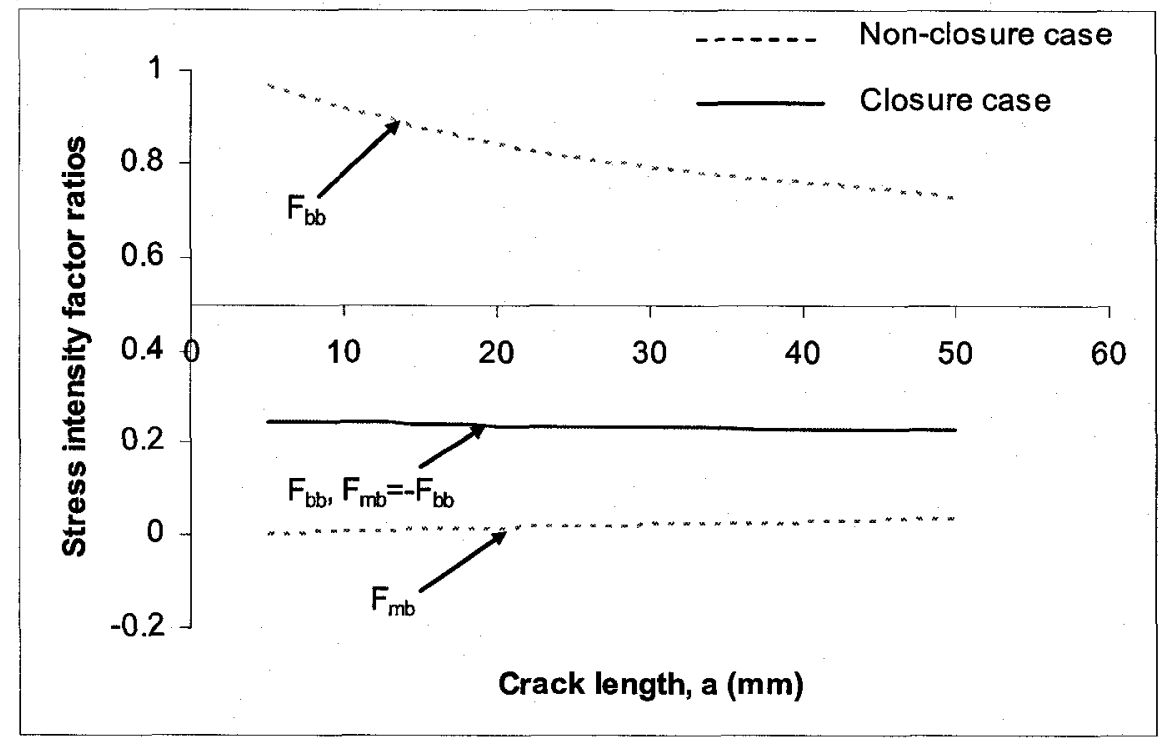

Figure 4.4 Stress intensity factor ratios for the axially cracked cylindrical shell subjected to internal and external pressure

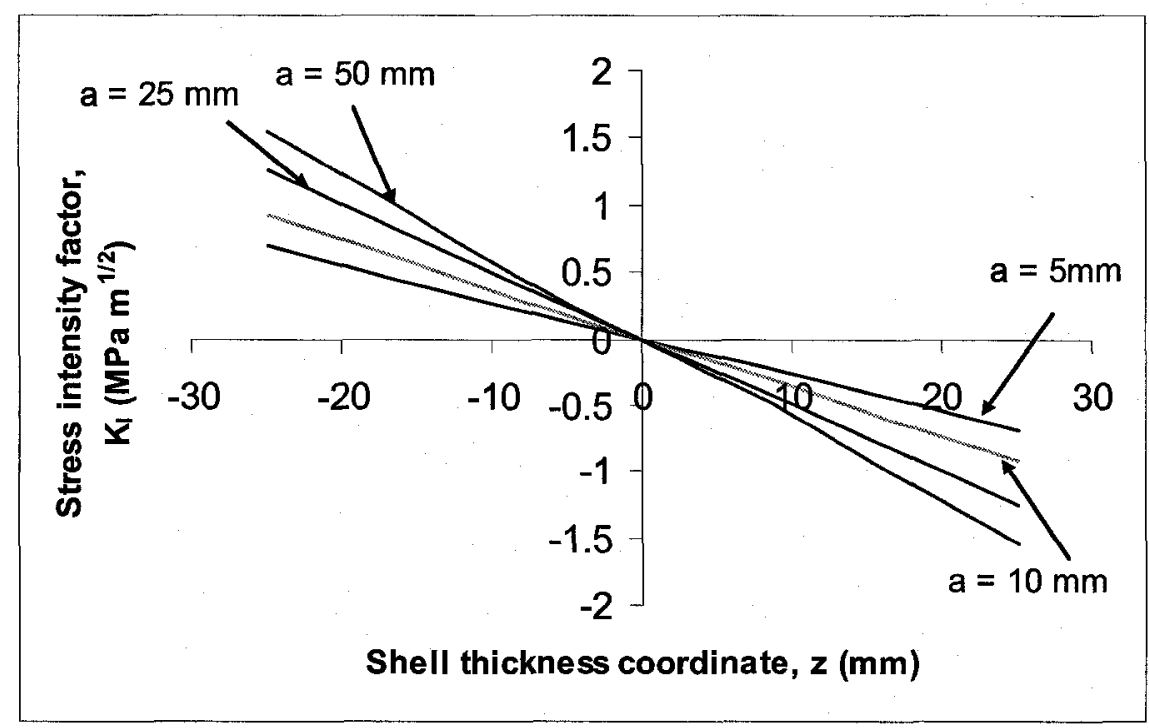

(a) 


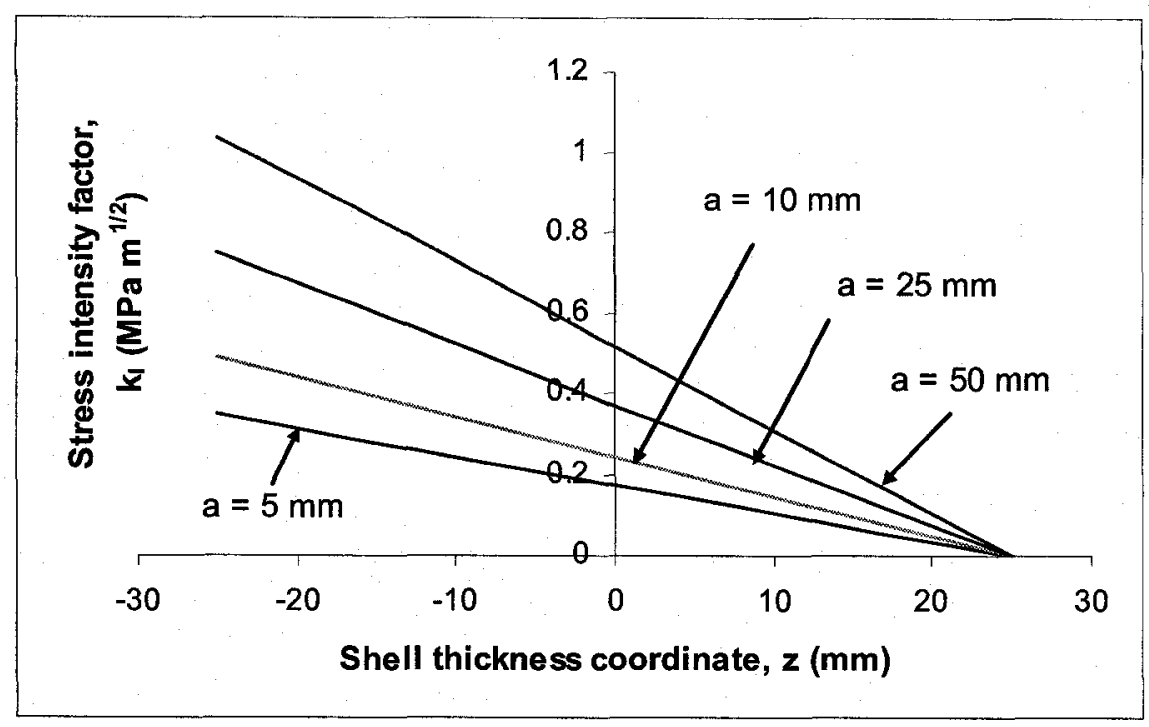

(b)

Figure 4.5 Stress intensity factors for the axially cracked cylindrical shell subjected to internal and external pressure: (a) in non-closure case and (b) in closure case

As the formulation of shell problems based on the conventional shell theory $[1,2]$ does not include the crack-face closure effect, it allows the material to penetrate into each other at the compressive edges. For the cylinder being discussed, it is subjected to bending stress only, which results in a pure rotation of the crack face about the middle surface of the shell wall, leading to negative SIFs on the compressive side of the crack face and same magnitudes of positive SIFs symmetrically on the tensile side of the crack face. However, when the crack-face closure is taken into account, the compressive edges are in contact with a reaction force being induced, therefore the crack faces neither penetrate into each other nor open at the compressive edges, leading to zero of the SIF. The crack face would open, however, at the tensile edge so that the SIF is positive. 


\section{Chapter 5 Finite Element Analysis}

\subsection{Significance and Objectives}

In the theoretical analysis, the simulation of crack-face closure is made based on the line-contact assumption at compressive edges of the crack surfaces when they are rotated by a bending load, in order to be consistent with the frame work of shell theories that plane sections remain plane in shell bending. However, in reality, due to the deformation of materials, the contact region of the crack surfaces may be an area, not a line. Considering the inherent relationship between SIFs and crack opening displacement (COD), omitting of the crack face deformation may result in inaccurate expression of the crack tip stress field in terms of SIFs, therefore, it is necessary to investigate the real contact area and to estimate the error due to the approximation in the assumption, thus validating the theoretical solutions.

In this thesis, besides the theoretical analysis, three-dimensional FEA is also conducted with a commercial software package, ABAQUS. To be consistent with the theoretical analysis, various shell geometries such as shell with two nonzero curvatures, cylindrical shell containing an axial crack or containing a circumferential crack and spherical shell, are considered. Material orthotropic properties are not included in this FEA due to the limitation of the material properties in the third direction, because for the orthotropic materials used for shell structures the material properties in thickness direction are commonly not important and therefore they are usually not available in literature, but it is required for three-dimensional FEA. In the theoretical analysis, the 
shell material is treated as two-dimensional, but in the FEA it is considered in three directions of the Cartesian coordinate unless shell elements are used. However, as discussed in Chapter 2, shell elements have limitations in solving the problems in which the stress and strain variations in the thickness direction are mainly concerned. Since the objectives of FEA in this research are to verify the validity of the theoretical solutions made on the line-contact assumption, the results for isotropic shells should also be suitable for orthotropic shells, that is, material orthotropy does not affect the validity of the theoretical solutions.

\subsection{Construction of the Models}

\subsubsection{Modeling crack problem in shells}

As well known, the crack face is stress-free in a loaded solid body. However, in order to solve crack problem in shells, the application of thin shell theory in fracture mechanics analysis requires linearity assumption. Under such an assumption, the solution of the crack tip stress field may be achieved from a local perturbation termed by G.C.Sih [1], in which surface tractions are applied to the crack face as the only external loads, as shown in Figure 5.1. The crack surface tractions are obtained by solving the problem of a shell structure under external loads by ignoring the existence of a crack first. Then the solved membrane and bending stress resultants at the proposed cracked location of the shell are applied on the crack surface, as described in $[1,7,15,106]$. Finally, the stress intensity factor for the crack may be solved based on such a local loading condition. The theoretical solution in this research follows this assumption and loading condition. 


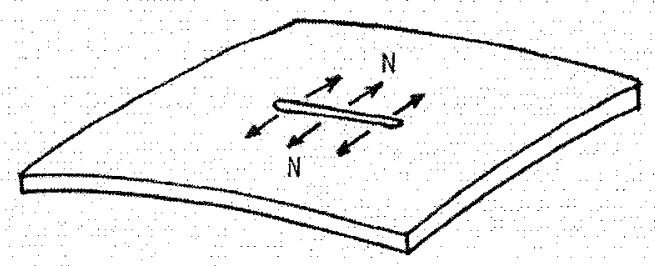

(a)

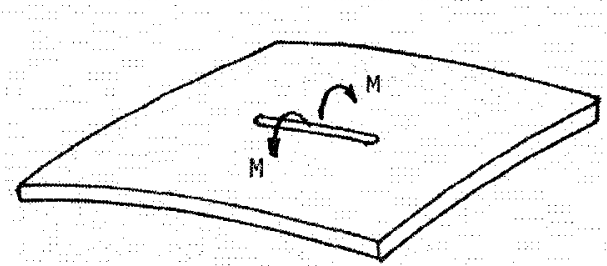

(b)

Figure 5.1 A cracked shell subjected to (a) membrane force and (b) bending moment

In order to be consistent with the theoretical frame work, in the present FEM modeling, a bending load is directly applied on the crack face, representing the local problem as perturbation. For example, as demonstrated in Figure 5.2, a pressurized cylinder contains a through-the-thickness axial crack. In reality, the crack face is stressfree, but this problem can be transferred from the general loading condition to a local problem on perturbation in which the only external loads are the bending moment and/or membrane force on the crack surface as shown in Figure 5.1. Since the crack closure issue discussed in this thesis is associated with bending load only, the membrane force is assumed to be zero in the present FEM modeling.

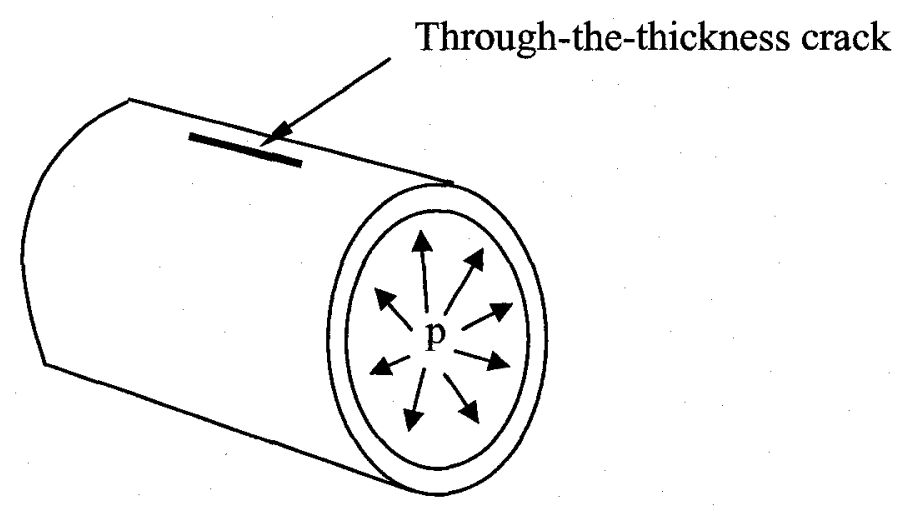

Figure 5.2 A pressurized cylinder contains a through-the-thickness axial crack 


\subsubsection{Simulation of crack-face closure}

In the FEA, various shell geometries containing a through-the-thickness crack are modeled in three dimensions. The crack-face closure requires that the two crack surfaces do not penetrate each other when they rotate under a bending load. To simulate this feature, a rigid plate is placed against the crack surface, which remains static when the crack face deforms under bending, as illustrated in Figure 5.3. The tensile side (outer surface) will open and the compressive side (inner surface) will remain static due to the constraint of the stationary rigid plate, in the positive bending condition.

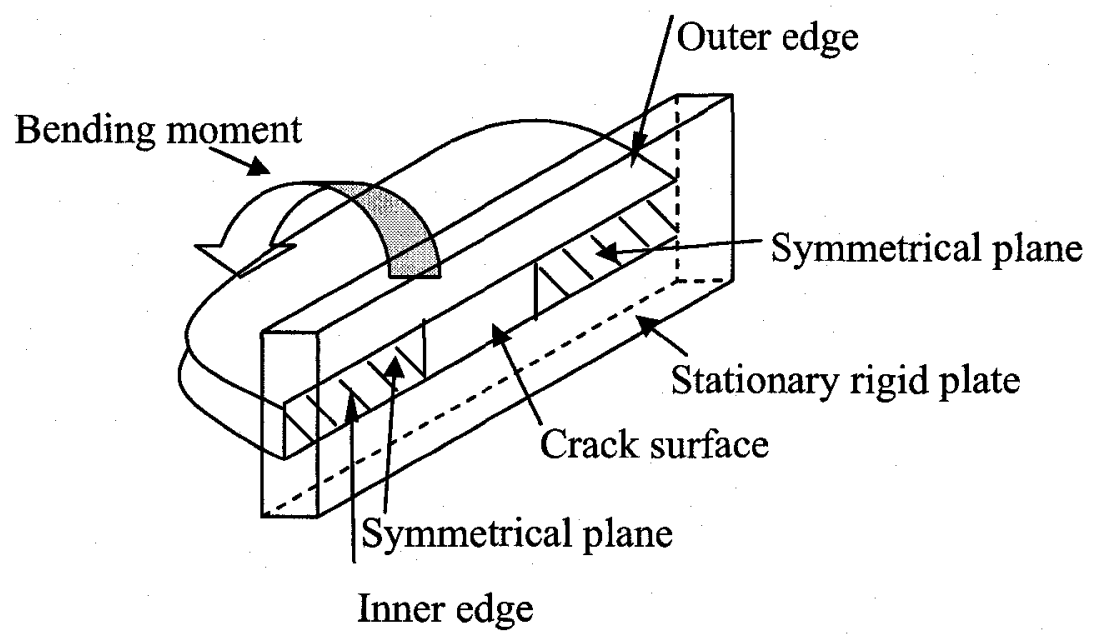

Figure 5.3 Crack closure modeling in FEA

The real contact area on the crack surface can be examined by checking the crack opening displacement or the reaction force distribution after the solution is completed. 'Surface contact' is treated as a non-linear problem in FEA and special facilities are employed to define contact surfaces in the FEA software. 


\subsubsection{Selection of shell parameters}

In accordance with the theoretical analysis, for all shell geometries the shell parameters are selected to satisfy $a / h=10$ with $a=250 \mathrm{~mm}$ and $h=25 \mathrm{~mm}$. For isotropic materials, steel is selected with Young's modulus $E=2.1 \times 10^{5} \mathrm{MPa}$ and Poisson's ratio $v=0.3$. For shells with double curvature, the two $R_{1} / R_{2}$ ratios, 0.25 and 4 are still selected to be studied. Similar to the theoretical analysis, the SIFs are determined with varying $\lambda$ values in the FEA. However, theoretically $\lambda$ can be small or large, but when building the FEA models, the $\lambda$ value is selected based on reasonable shell geometries. For example, when $\lambda$ is smaller than 1 , the wall of the shell is too thin compared with the radius, buckling behavior may dominate the destruction of the whole structure other than fracture. Also, there are two conditions, which restrict the curvature parameters, that have to be considered in the FEA modeling. One is when the value of $\lambda$ is equal to or greater than 6 for cylindrical or spherical shells, the radius of the shell is calculated to be less than $230 \mathrm{~mm}$; the other is for shells with double curvature, when $\lambda$ is equal to or greater than 3 and $R_{1} / R_{2}$ equals 4 , the radius in the second curvature plane, $R_{2}$, is equal to or less than $230 \mathrm{~mm}$. The physical unreality of these two conditions is that the half-crack length, $a=250 \mathrm{~mm}$, is beyond the shell body, which cannot be achieved in the FEA modeling, as illustrated in Figure 5.4. As a result, the $\lambda$ values are selected in a certain range, which coincide with geometrical feasibility of individual type of shells, in the current FEA.

All the shell parameters used in the current FEA are given in Tables 5.1 and 5.2. 


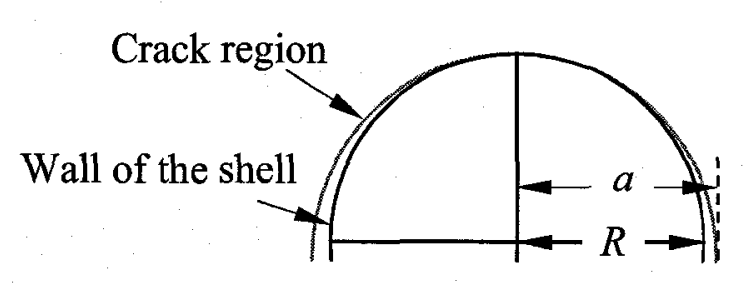

Figure 5.4 Physical unreality due to unreasonable $\lambda$ values

Table 5.1 Geometrical parameters of cylindrical and spherical shells

\begin{tabular}{|c|c|c|c|c|}
\hline$a$ & \multicolumn{5}{|c|}{$250 \mathrm{~mm}$} \\
\hline$h$ & \multicolumn{4}{|c|}{$25 \mathrm{~mm}$} \\
\hline$\lambda$ & 2 & 3 & 4 & 5 \\
\hline$R$ & $2065 \mathrm{~mm}$ & $918 \mathrm{~mm}$ & $516 \mathrm{~mm}$ & $330 \mathrm{~mm}$ \\
\hline
\end{tabular}

Table 5.2 Geometrical parameters of shells with double curvature

\begin{tabular}{|c|c|}
\hline$a$ & $250 \mathrm{~mm}$ \\
\hline$h$ & $25 \mathrm{~mm}$ \\
\hline$\lambda$ & 2 \\
\hline$R_{1}$ & $2065 \mathrm{~mm}$ \\
\hline$R_{2}\left(R_{1} / R_{2}=4\right)$ & $516 \mathrm{~mm}$ \\
\hline$R_{2}\left(R_{1} / R_{2}=0.25\right)$ & $8261 \mathrm{~mm}$ \\
\hline
\end{tabular}




\subsubsection{Boundary conditions of the FEA models}

The crack closure effect being discussed in the analytical solution is due to a pure bending load on the crack faces. In the FEA modeling, this loading condition is achieved by applying a linear pressure load through the wall thickness, with the pressure being zero at the middle of the wall and linearly increasing towards the inner surface and outer surface in opposite directions.

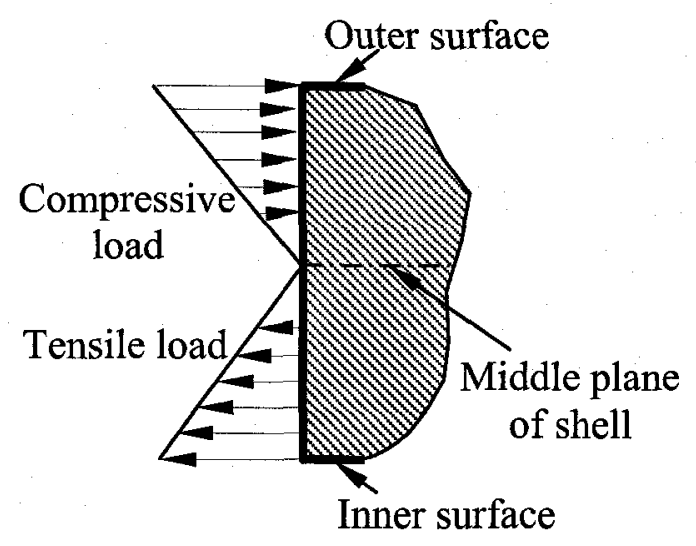

(a)

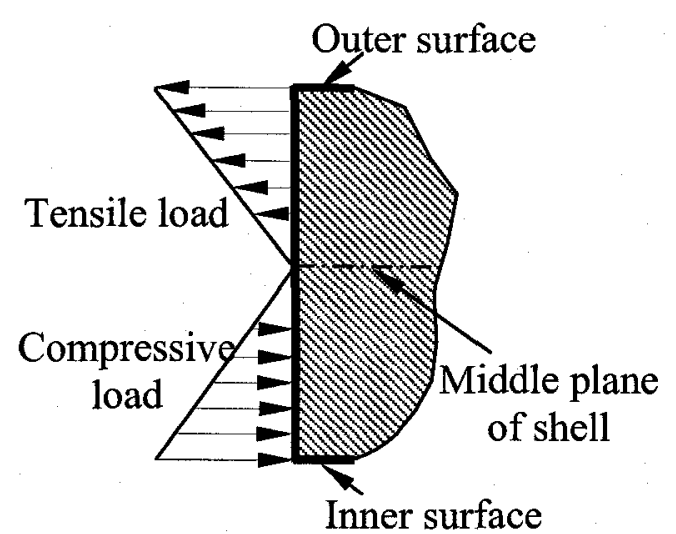

(b) 
Figure 5.5 Modeling of pure bending load on the crack surface:

(a) positive bending and (b) negative bending

Depending on positive bending or negative bending, the outer surface layer is subjected to a compressive pressure, which causes the crack open, and the inner surface layer is subjected a tensile pressure, which causes the crack close, or vice versa, as shown in Figure 5.5.

Such a Neumann boundary condition is defined in a local $(r, \theta, z)$ cylindrical coordinate system, which has the same origin with the principal curvature plane where crack is addressed, as demonstrated in Figure 5.6. Since the crack surface is subjected to a linear distributed pressure load, the corresponding Neumann traction $p(r)$ on the crack surface is expressed in equation 5.1, where $R$ and $h$ is the radius of the mid-plane and the thickness of the shell respectively, $p_{o}$ is the prescribed maximum value of the pressure load on the outer half crack surface. To be in consistent with the analytical method, $p_{o}$ has the same value as the bending stress $\sigma_{b}$ applied in analytical solution, where we define a compressive pressure to be positive. The coordinate $z$ equals to zero, and $\theta$ varies within the domain of the crack surface.

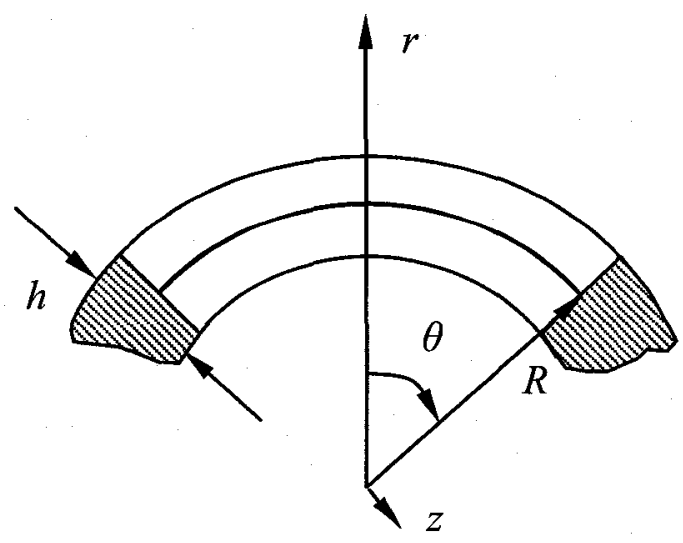

Figure 5.6 Crack surface under Neumann boundary condition 


$$
p(r, \theta)=\frac{2 p_{o}}{h}(r-R) \quad\left(R-\frac{h}{2} \leq r \leq R+\frac{h}{2}\right)
$$

It should be indicated that the resultant tensile force does not exactly equal the resultant compressive force on the crack face because the area of the crack face under tension is not equal to that of the crack face under compression due to the curvature effect, as shown in Figure 5.7. However, this error can be neglected with respect to the large ratio of shell radius to wall thickness. Therefore this loading model can be considered as a pure bending load.

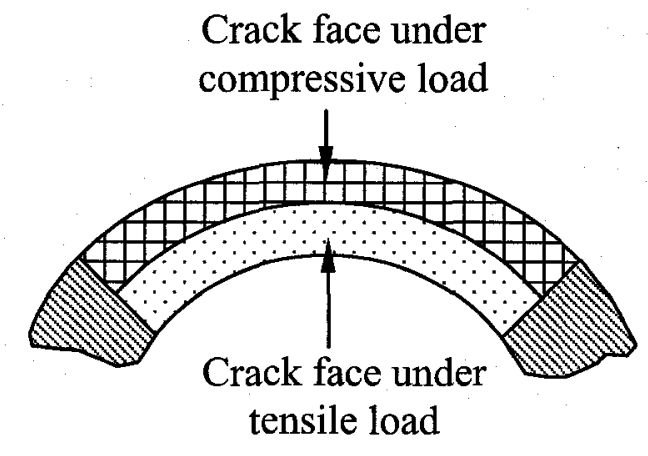

(a)

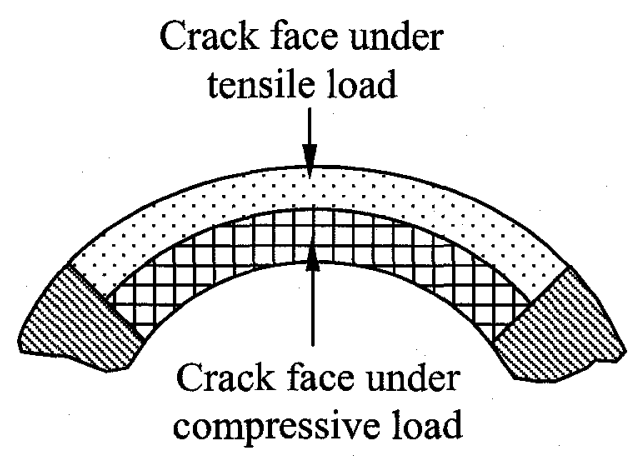

(b)

Figure 5.7 Resultant tensile force and resultant compressive force on the crack surface:

(a) under positive bending and (b) under negative bending 
Due to symmetry of both geometry and loading, only half of one crack surface is modeled in finite element analysis as shown in Figure 5.8. A Cartesian coordinate system is addressed with crack surface in the plane $x=0$, the mid-point of the crack at $y=0$, crack tip at $y=a$, and $z$ along thickness direction of the shell. By defining the displacement as $u, v, w$ corresponding to $x, y$ and $z$ Cartesian coordinates, the full boundary conditions of the shell model are described as below.

For $a \leq y$, the $x$-displacement is prescribed to zero, $u=0$; for $0 \leq y<a$, the crack face is subjected to the normal traction $p(r)$, as given in equation 5.1, and a simple Dirichlet boundary with the prescribed $x$-displacement $u=0$ is applied if the traction force is compressive. Such a non-linear boundary condition due to contact behavior is solved using an iterative method. The contact region is finally defined to be the area of of the crack face with zero $x$-displacement. On the symmetry plane $y=0$, the $y$-displacement $v$ is prescribed to zero. At the far end of the model an arbitrary point $A$ is constrained by applying $w=0$.

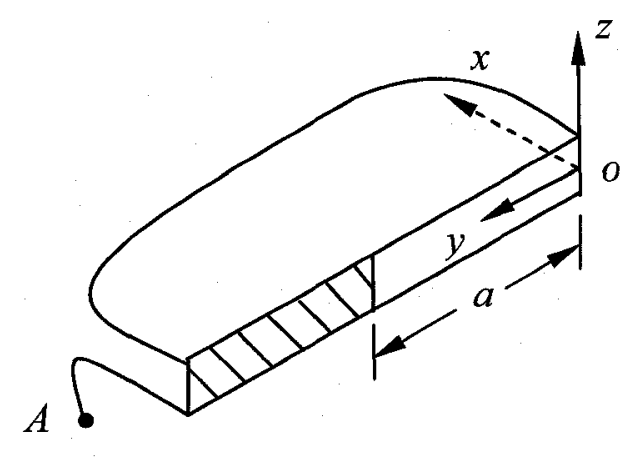

Figure 5.8 Definition of boundary conditions of FEA model 


\subsubsection{Meshing of the models}

Three-dimensional solid elements are employed in the current FEA. The final mesh is achieved by gradually increasing the fineness until the error of the SIFs between the last two computations is less than $1 \%$. As in common fracture mechanics FEA, the crack tip region is finely meshed and the other region is relatively coarsely meshed. The mesh patterns for various shell geometries are presented in Figure 5.9.

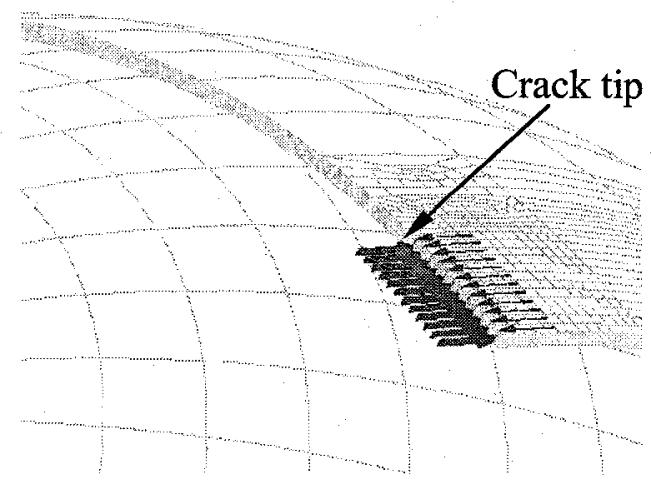

(a)

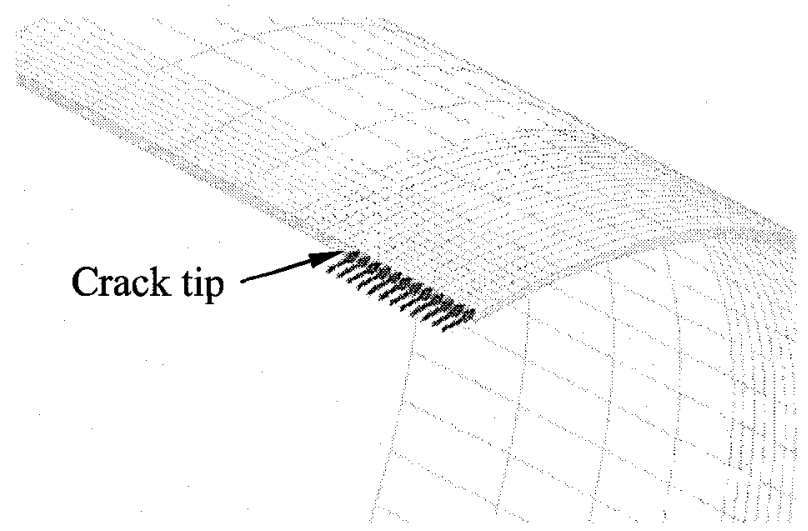


(b)

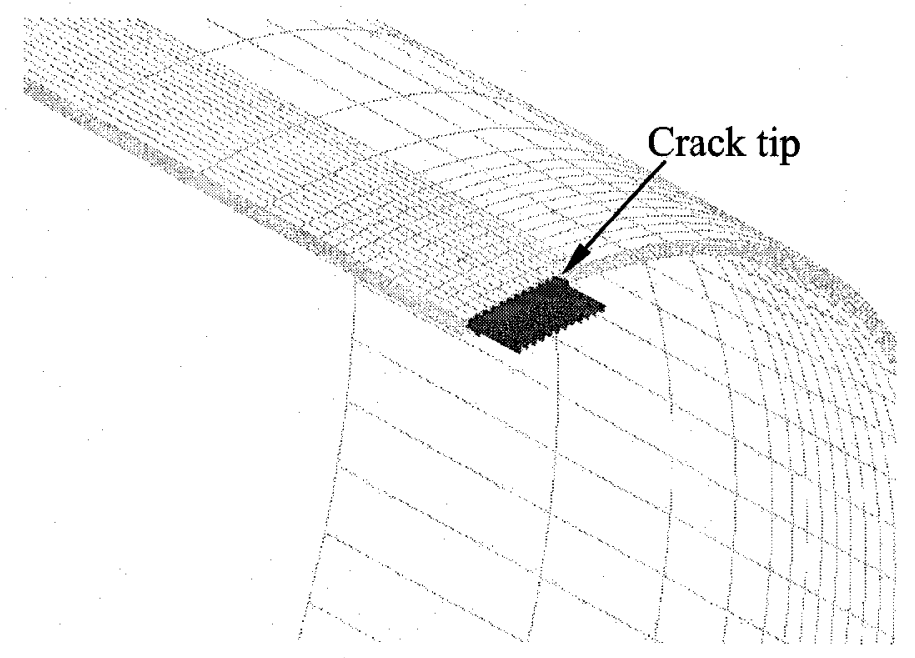

(c)

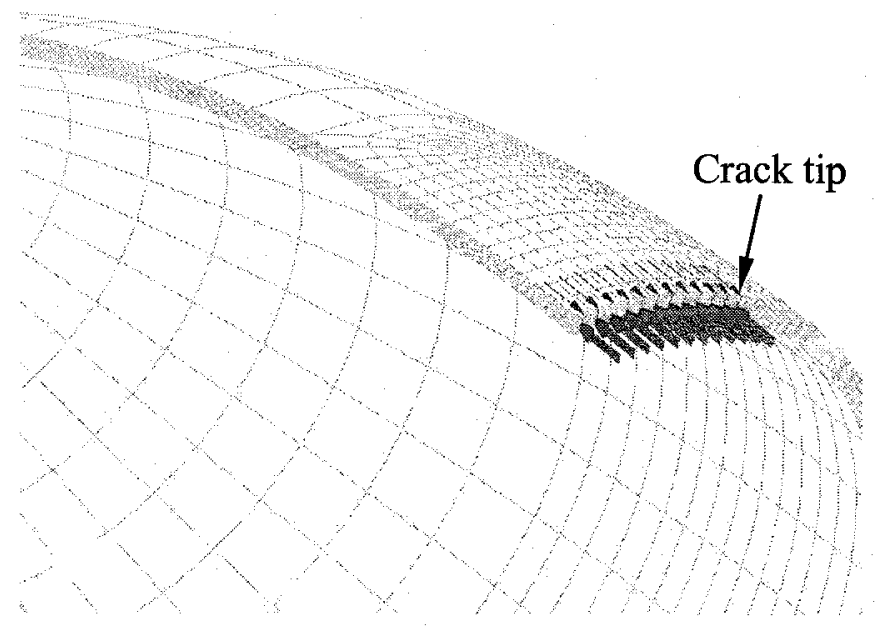

(d) 


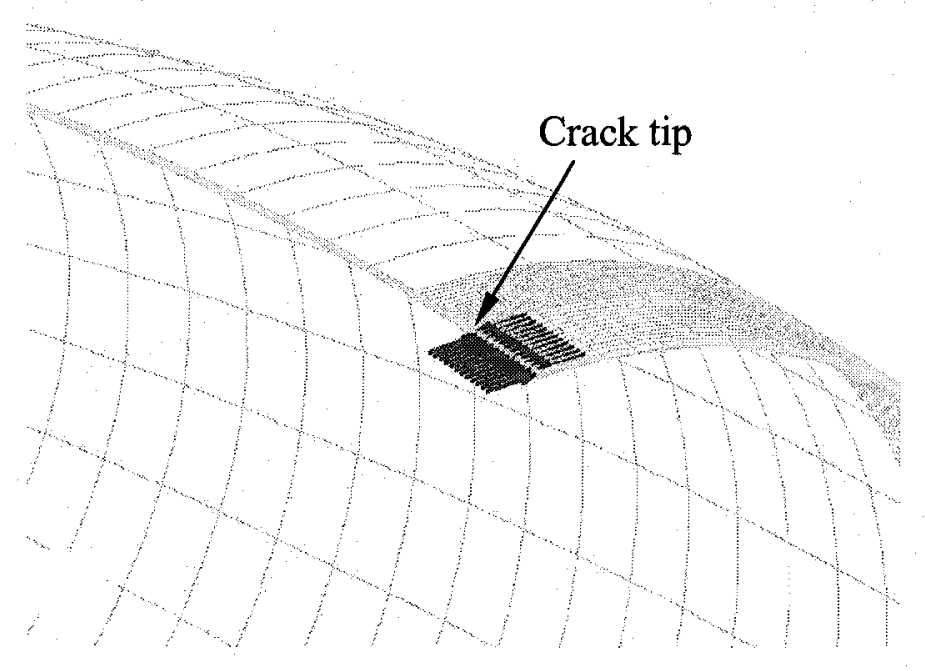

(e)

Figure 5.9 Mesh patterns of FEA models: (a) spherical shell; (b) cylindrical shell with an axial crack; (c) cylindrical shell with a circumferential crack; (d) shell with double curvature $R_{1} / R_{2}=4$ and (e) shell with double curvature $R_{1} / R_{2}=0.25$

\subsection{FEA Solutions}

\subsubsection{Data processing}

Two types of data are derived from the FEA, crack-face opening displacement (COD) and contact force on the crack face. The COD data are used to calculate the SIFs along the crack front in terms of eqn (2.13) and demonstrate the crack face rotation, as well as the crack closure behavior. The contact force distribution on the crack face is alternative way to examine the contact or closure region on the crack face. 
The results of the FEA for various shell geometries in terms of the shell parameters defined in Tables 5.1 and 5.2 have been obtained. The plot of the COD variation through the shell thickness at a distance of $a / 2$ from the crack tip, the contour of the COD on the crack face and the plot of SIFs against the shell thickness coordinate, for both positive and negative bending cases, are presented in the following sections for spherical shell, cylindrical shell containing an axial crack, cylindrical shell containing a circumferential crack and shell with double curvature, respectively. For spherical and cylindrical shells, only the results for $\lambda=2$ and 4 are presented as examples, but those for other valid $\lambda$ values in Tables 5.1 and 5.2 are also available.

\subsubsection{Spherical shell}

The plots in Figure 5.10 demonstrate the COD variations through the shell wall for the spherical shell with $\lambda=2$ under positive bending and negative bending respectively. It is seen that generally the COD varies linearly through the shell wall, but it is clear that in the crack closure region, inner surface for positive bending and outer surface for negative bending, non-linear variation exists, which implies that the real closure region in the crack face is an area due to the material deformation, not a line. This phenomenon can be further confirmed in the COD contours on the crack face shown in Figure 5.11. From red color to black color, the COD varies from large to small until zero. It is noticed that no partial closure is observed in the FEA for this geometrical case; this is because the selected $\lambda$ value $(\lambda=2)$ is small. According to the theoretical analysis, fulllength closure should occur for the $\lambda$ value. 
In the positive bending case, closure occurs at the inner surface so that the COD approaches zero, especially in the region close to the crack tip (block area); the contact area of the crack faces is the maximum with the COD is zero. It is apparent that the region in black color, that is, the crack closure region is an area, not a line. It is also observed that maximum COD occurs at the outer surface in the region of crack center and it becomes smaller and smaller towards the crack tip. In negative bending case, whereas the COD is zero at the outer surface due to the crack closure and linearly increases towards the inner surface. Comparing the CODs in positive bending case with negative bending case, see Figure 5.10, it is found that the maximum COD of positive bending is smaller that that of negative bending.

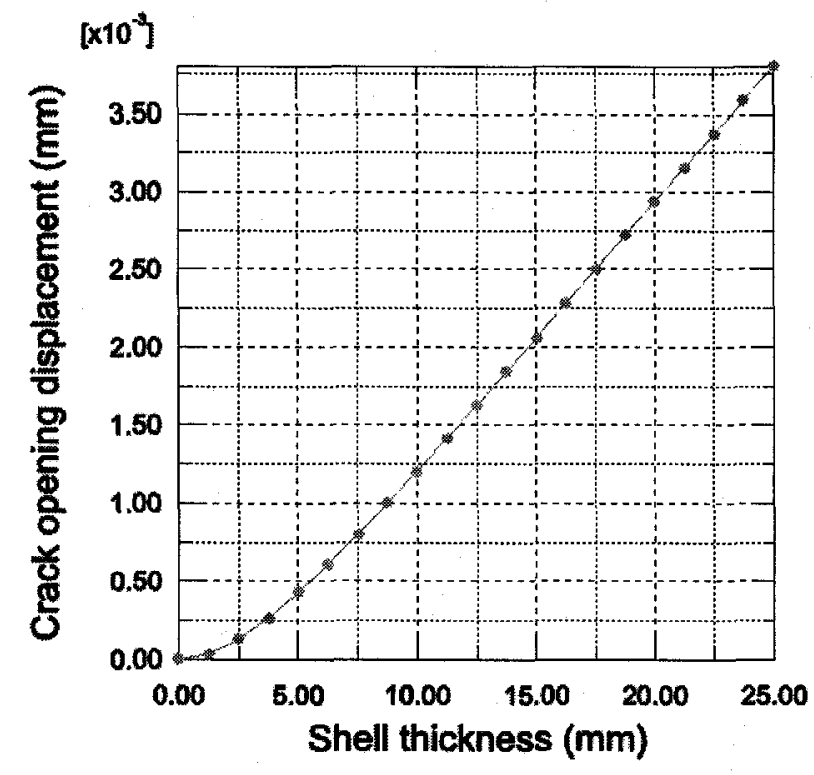

(a) 


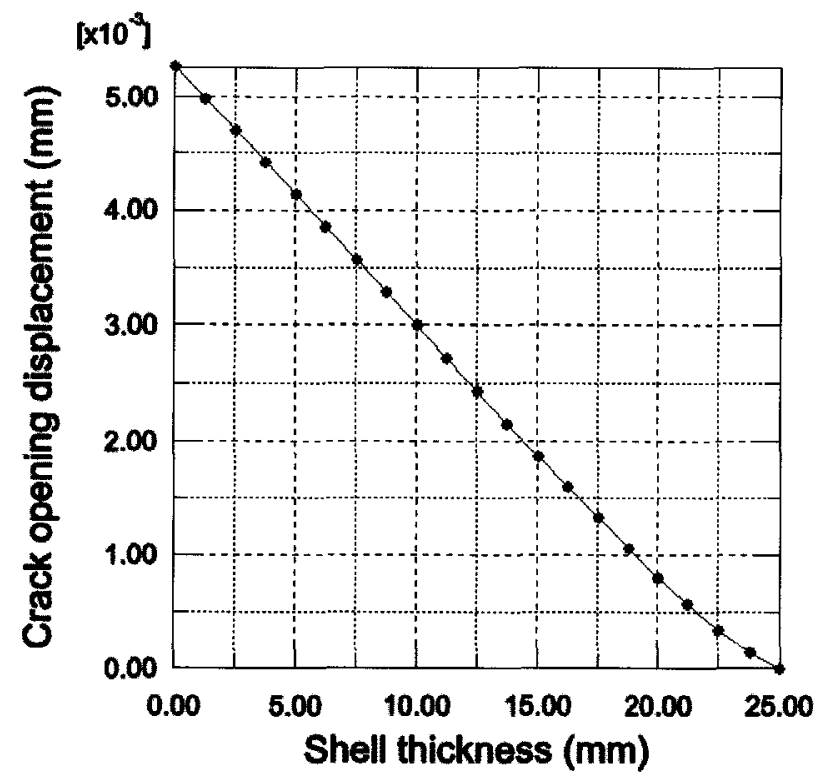

(b)

Figure 5.10 CODs of the spherical shell with $\lambda=2$ at a distance of $a / 2$ from the crack tip:

(a) positive bending and (b) negative bending

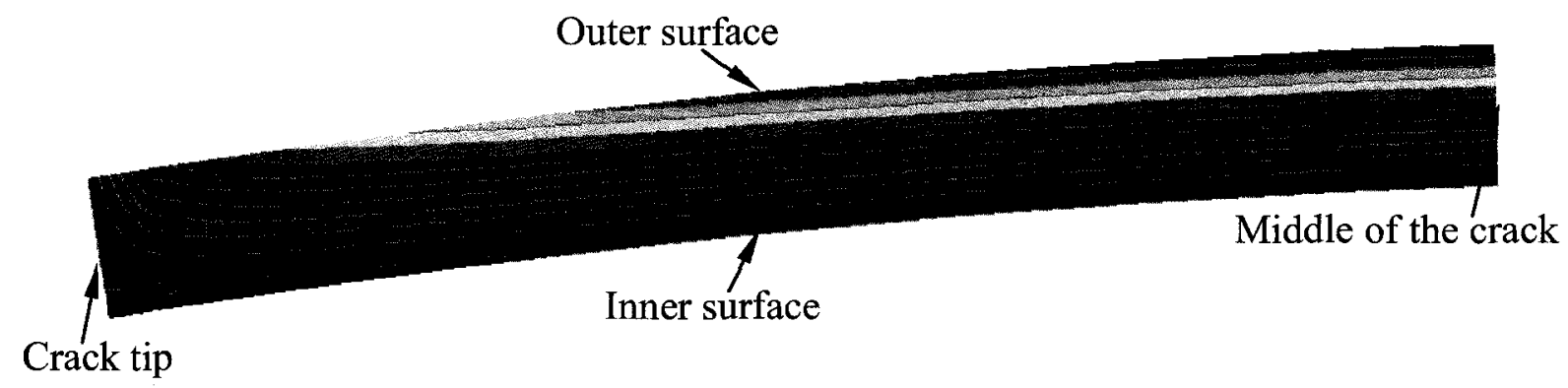

(a) 


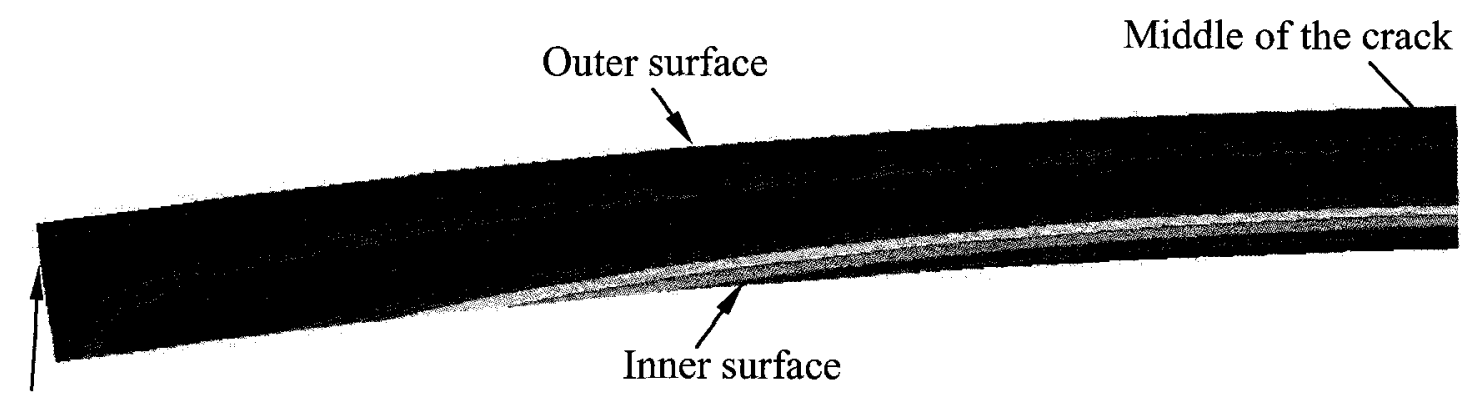

Crack tip

(b)

Figure 5.11 COD contours on the crack face of the spherical shell with $\lambda=2$ :

(a) positive bending and (b) negative bending

Figure 5.12 presents the FEA solution of the SIF, which varies with the wall thickness coordinate, for the spherical shell with $\lambda=2$ under positive bending and negative bending, respectively. The theoretical solution is also presented for comparison. Firstly, the FEA solution confirms the linear variation of the SIF through the wall thickness, which is derived in the theoretical solution. Secondly, due to crack closure, the SIF in the closure region (inner surface for positive bending and outer surface for negative bending) is zero, and it reaches the maximum at the open edge (outer surface for positive bending and inner surface for negative bending). Again, non-linear variation of the SIF is observed in the region close to inner surface for positive bending and in the region close to outer surface for negative bending; see Figure 5.12. This is due to the crack closure effect and implies that the closure region is an area rather than a line. Finally, it is demonstrated that generally the theoretical and FEA solutions of the SIF are 
in good agreement but there is an error between the two solutions. This error is likely caused by the line-contact assumption for crack-face closure simulation in the theoretical solution and the numerical approximation in the FEA.

For the spherical shell with $\lambda=2$, under positive bending the FEA solution of the SIF is lower than the theoretical solution, while under negative bending the relationship between the two solutions switches at the middle surface of the shell wall, see Figure 5.12. However, whether the FEA solution is higher or lower than the theoretical solution does not follow a definite rule, it may vary from case to case. In addition, the FEA results show that the SIF for positive bending is higher than that for negative bending, which is consistent with the theoretical solution.

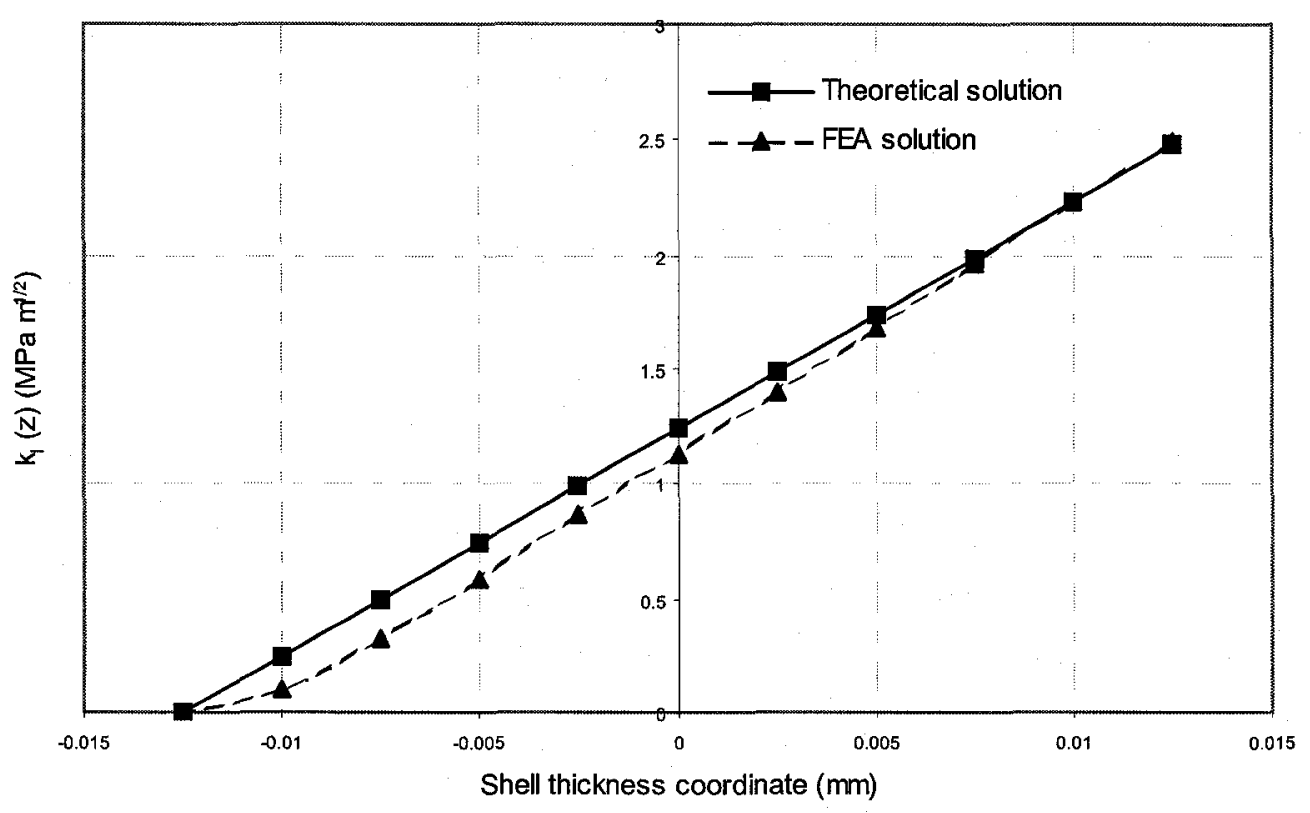

(a) 


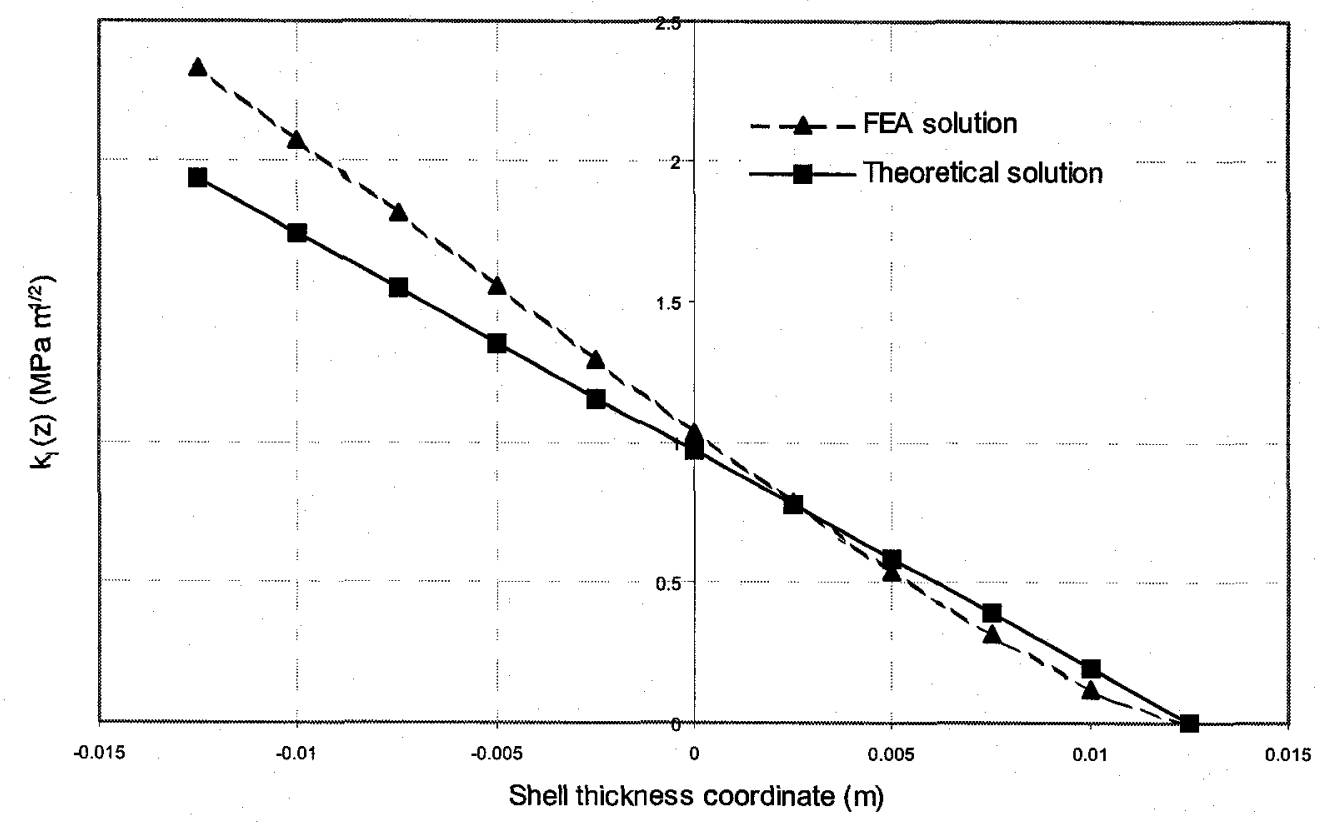

(b)

Figure 5.12 Variations of SIF through the shell wall of the spherical shell with $\lambda=2$ :

(a) positive bending and (b) negative bending

For another geometrical case, the spherical shell with $\lambda=4$, the FEA results are presented in Figures 5.13 to 5.15. Similar observations to $\lambda=2$ are obtained; the CODs for $\lambda=2$ and $\lambda=4$ in positive bending case are close, but in negative bending case the COD for $\lambda=4$ decreases compared with the COD for $\lambda=2$. Crack closure over an area not on a line is confirmed once again by the $\lambda=4$ results.

Comparing these two shell geometries, the shell radius is smaller for $\lambda=4$, that is, the shell curvature is larger. According to the theoretical analysis about the critical values 
of the shell parameter $\lambda$ for partial closure in various shell geometries, partial closure should occur in spherical shells under positive bending when $\lambda$ is equal to or greater than 3 , see Table 3.1 , that is, complete closure occurs for the spherical shell with $\lambda=2$ and partial closure occurs with $\lambda=4$. Consistent with the theoretical analysis, the FEA results show that for $\lambda=2$, the COD along the entire crack length at the inner surface is very close to zero (As well known, FEA numerical computation cannot give exact zero value), while for $\lambda=4$, the COD in the region of the crack center is not very close to zero although it is still small. It should be pointed out that this feature cannot be demonstrated on the COD contours due to the limitation of color scale, but it is confirmed by examining the COD data. Under negative bending complete closure occurs in spherical shells with $\lambda=2$ or $\lambda=4$. The FEA SIF and the theoretical SIF of the spherical shell with $\lambda=4$ are also in good agreement. The error is less than that of the $\lambda=2$ results, as shown in Figure 5.12.

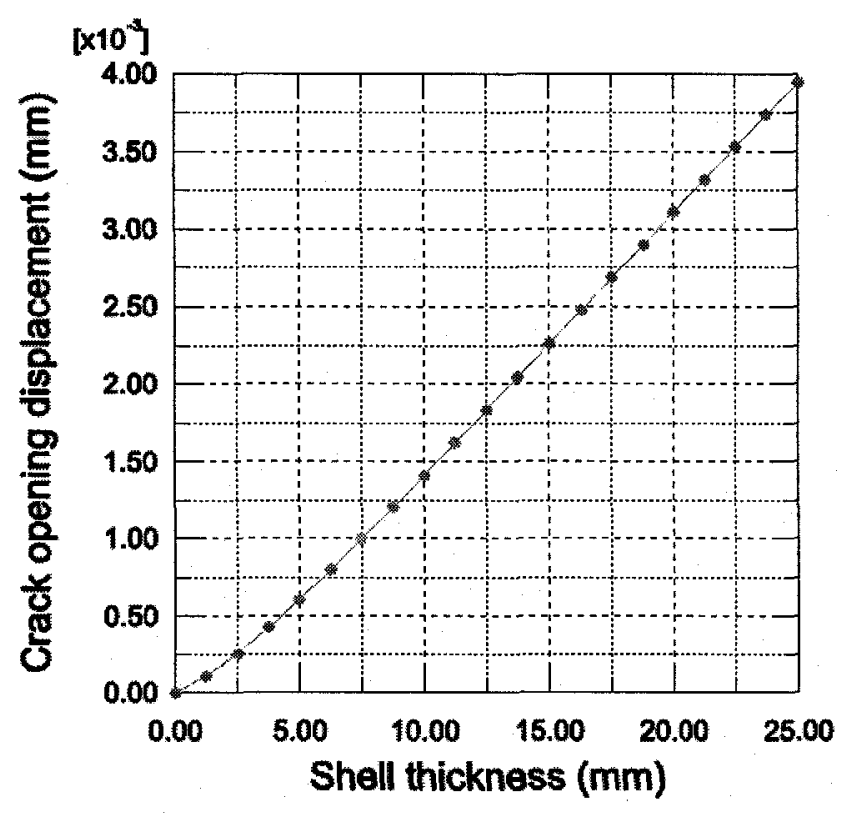


(a)

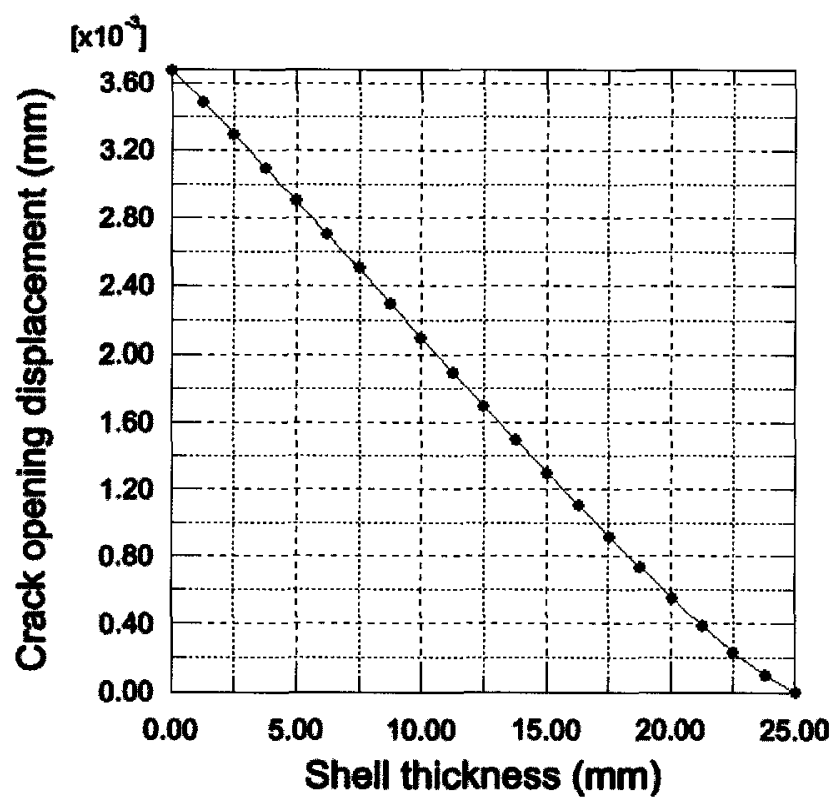

(b)

Figure 5.13 CODs of the spherical shell with $\lambda=4$ at a distance of $a / 2$ from the crack tip: (a) positive bending and (b) negative bending

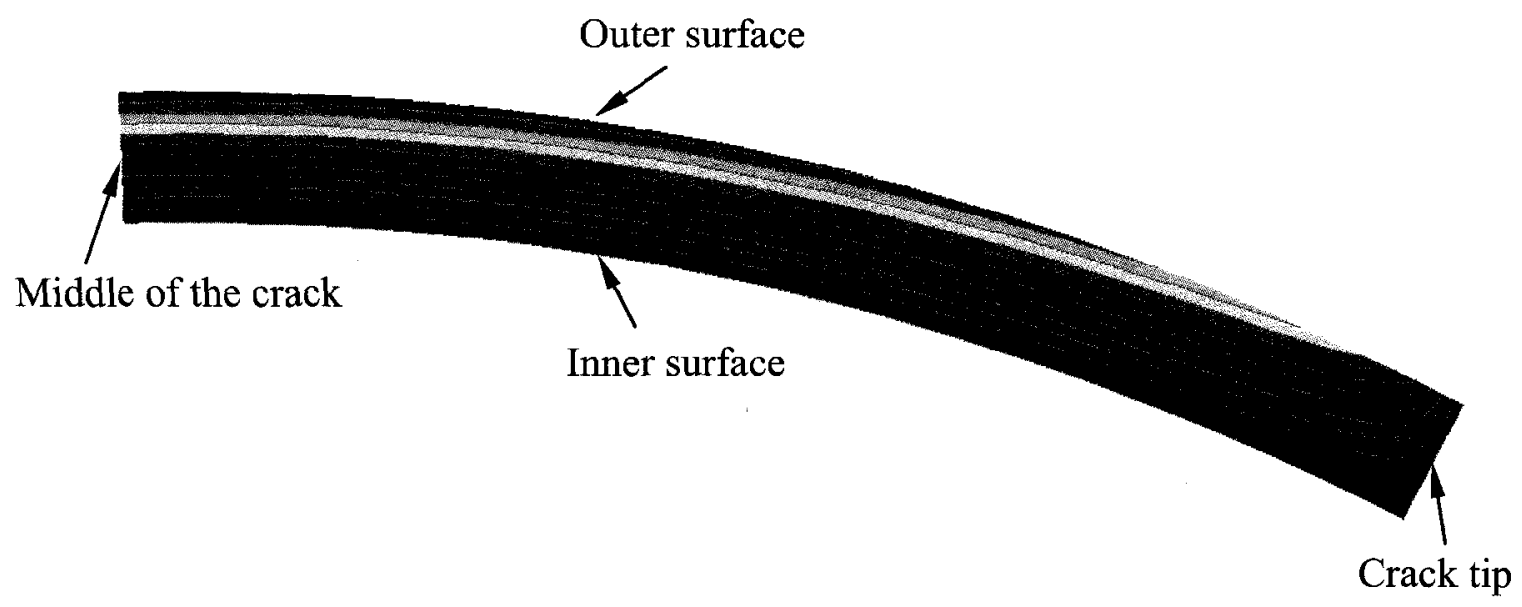

(a) 


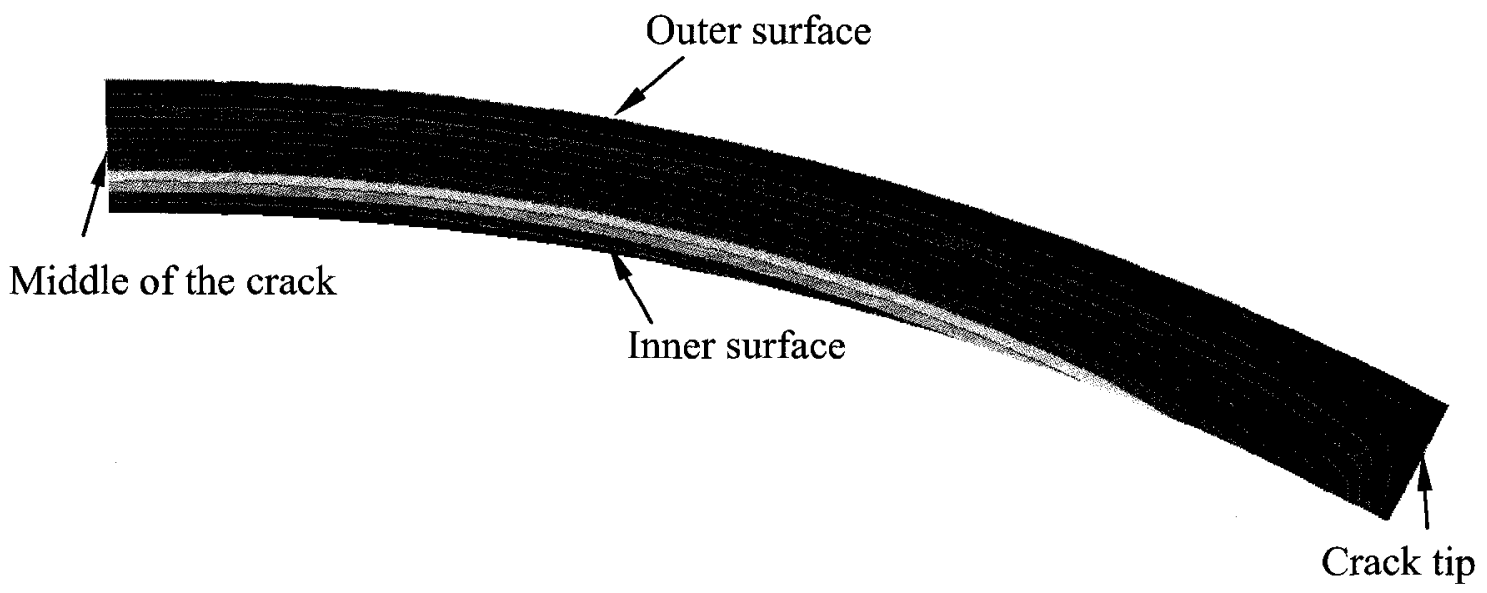

(b)

Figure 5.14 COD contours on the crack face of the spherical shell with $\lambda=4$ :

(a) positive bending and (b) negative bending

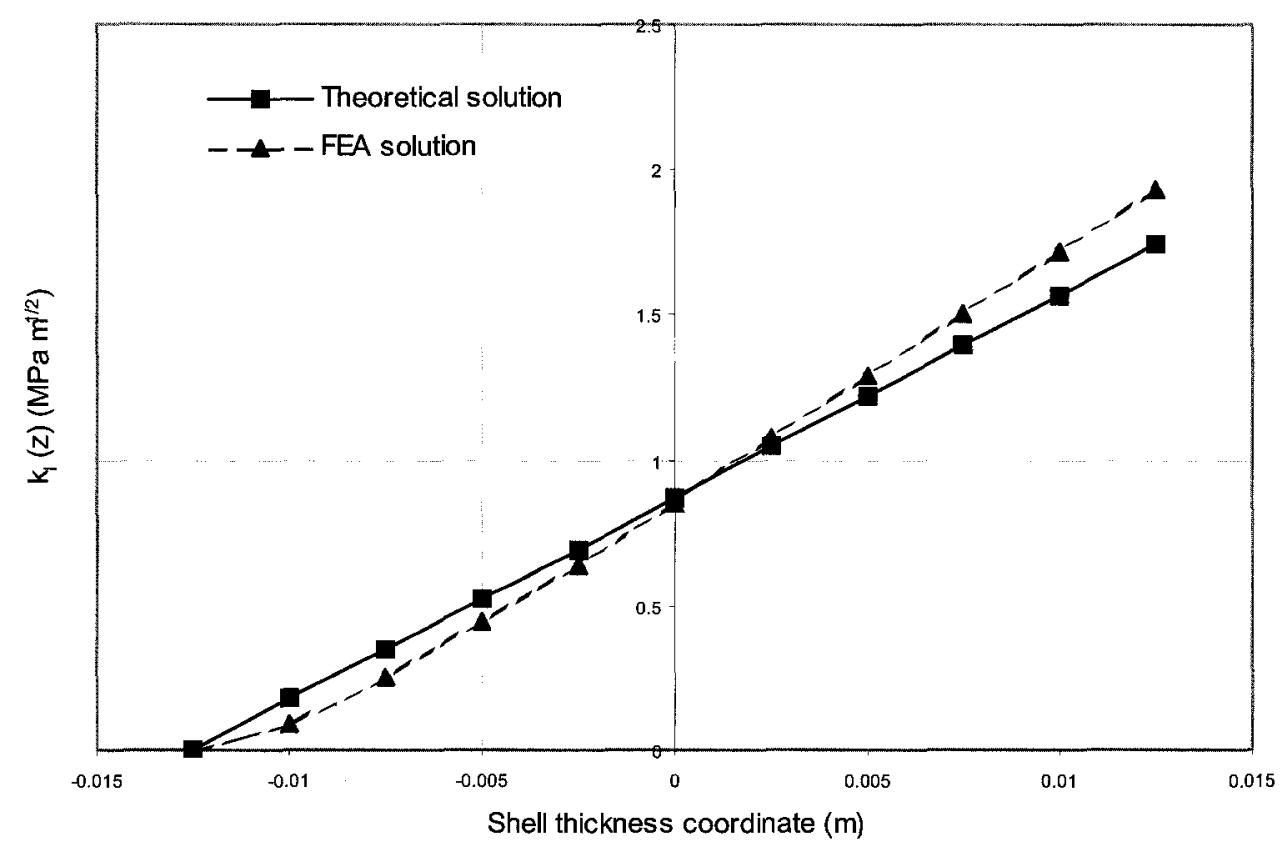

(a) 


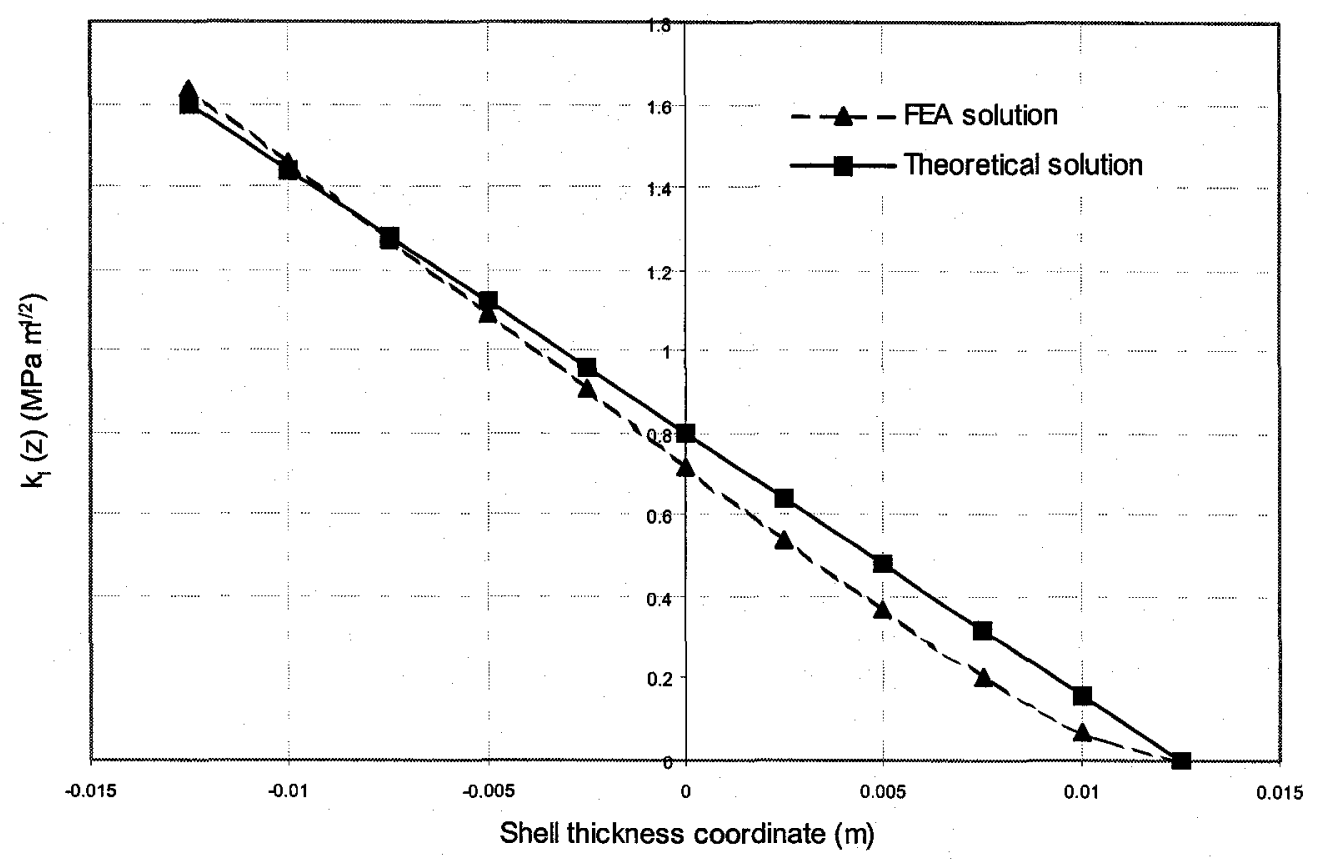

(b)

Figure 5.15 Variations of SIF through the shell wall of the spherical shell with $\lambda=4$ :

(a) positive bending and (b) negative bending

\subsubsection{Cylindrical shell containing an axial crack}

For cylindrical shells containing an axial crack, the FEA results for two values of the shell parameter, $\lambda=2$ and $\lambda=4$, are presented. As shown in Figures 5.16 to 5.21 , the COD and SIF results of cylindrical shells containing an axial crack have the basic features as those of spherical shells have, but the CODs of the former are smaller than those of the latter generally. In Table 3.1 the critical value for partial closure of cylindrical shells under positive bending is $\lambda=4$, accordingly, the FEA results show that for $\lambda=2$, the COD along the entire crack length at the inner surface is very close to zero, while for $\lambda=4$, the COD in a small region around the crack center is not very close to 
zero even though it is small. Under negative bending complete closure occurs in cylindrical shells containing an axial with $\lambda=2$ or $\lambda=4$. Again, the FEA SIF and the theoretical SIF of cylindrical shells containing an axial crack with $\lambda=2$ or $\lambda=4$ agree very well.

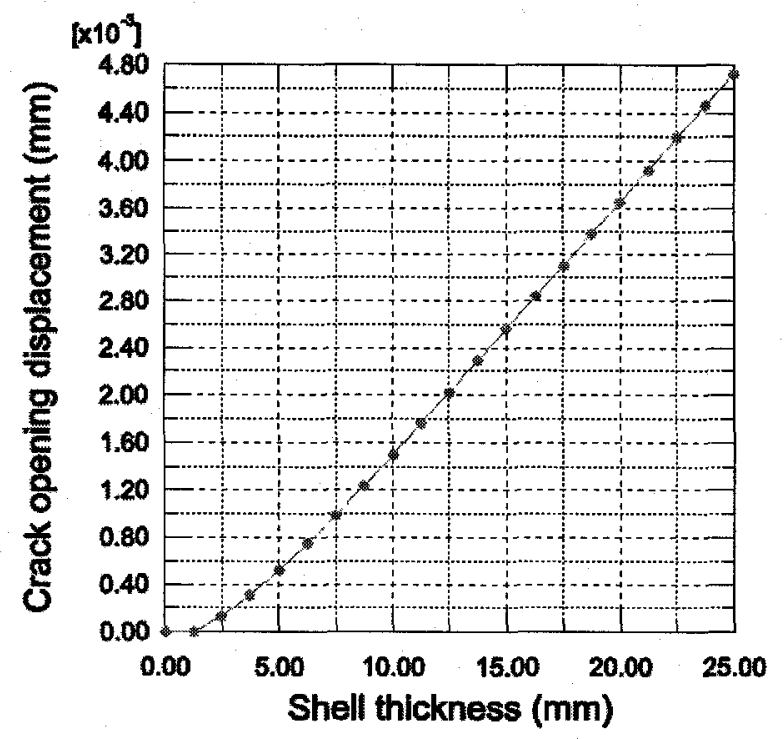

(a)

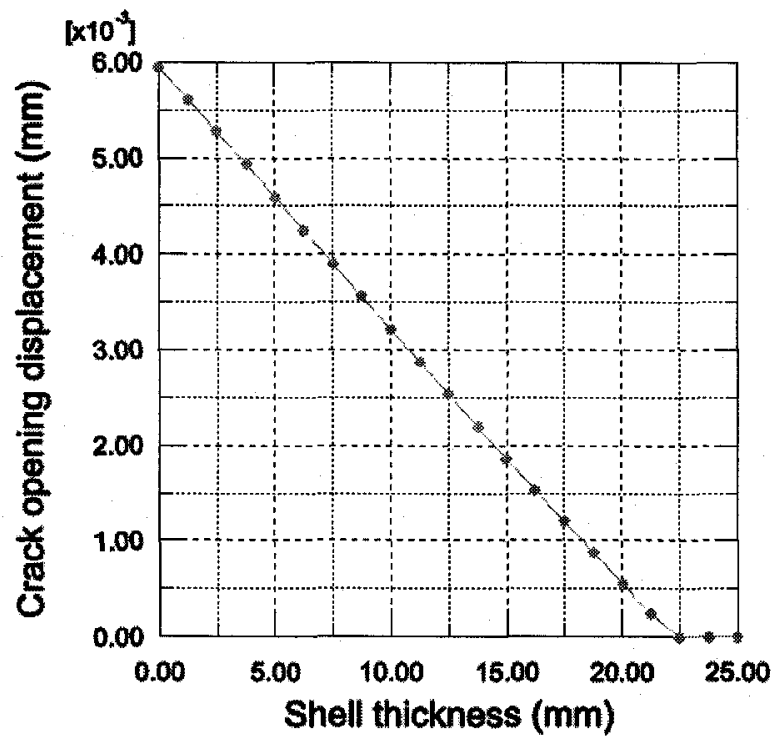

(b)

Figure 5.16 CODs of the axially cracked cylindrical shell with $\lambda=2$ at a distance of $a / 2$ from the crack tip: (a) positive bending and (b) negative bending 


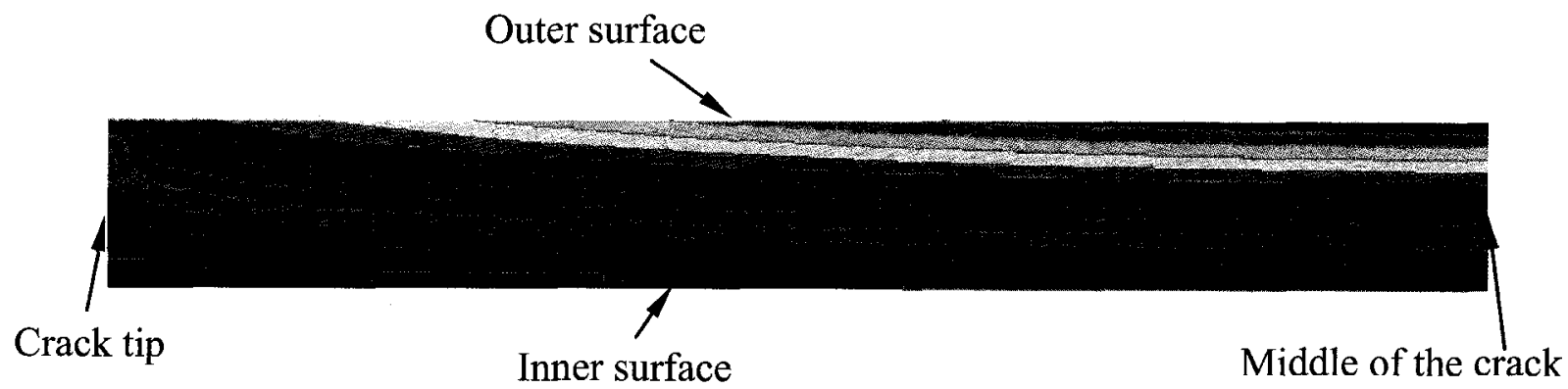

(a)

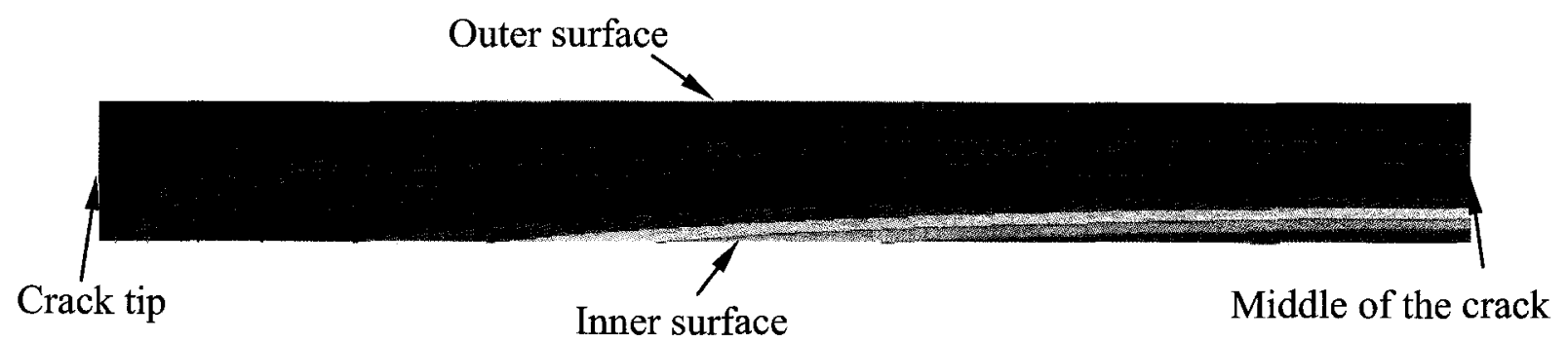

(b)

Figure 5.17 COD contours on the crack face of the axially cracked cylindrical shell with $\lambda=2$ : (a) positive bending and (b) negative bending 


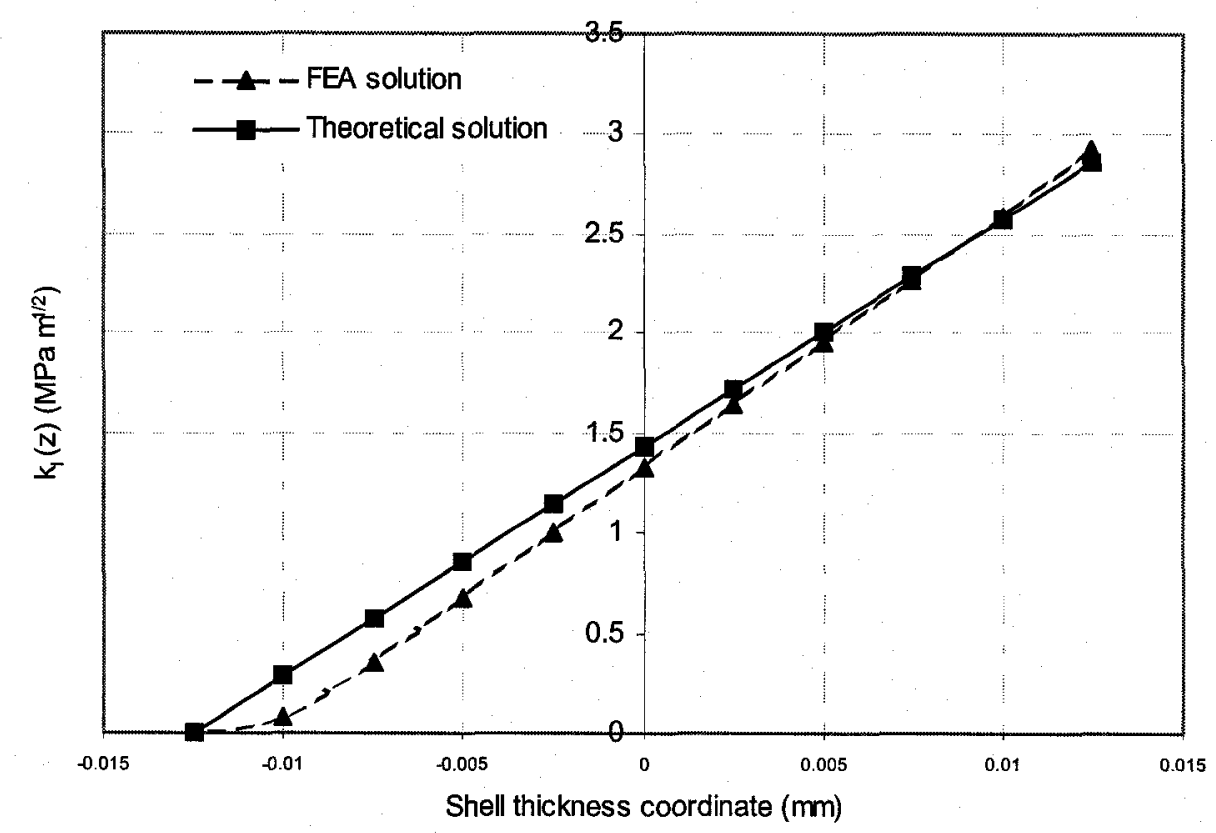

(a)

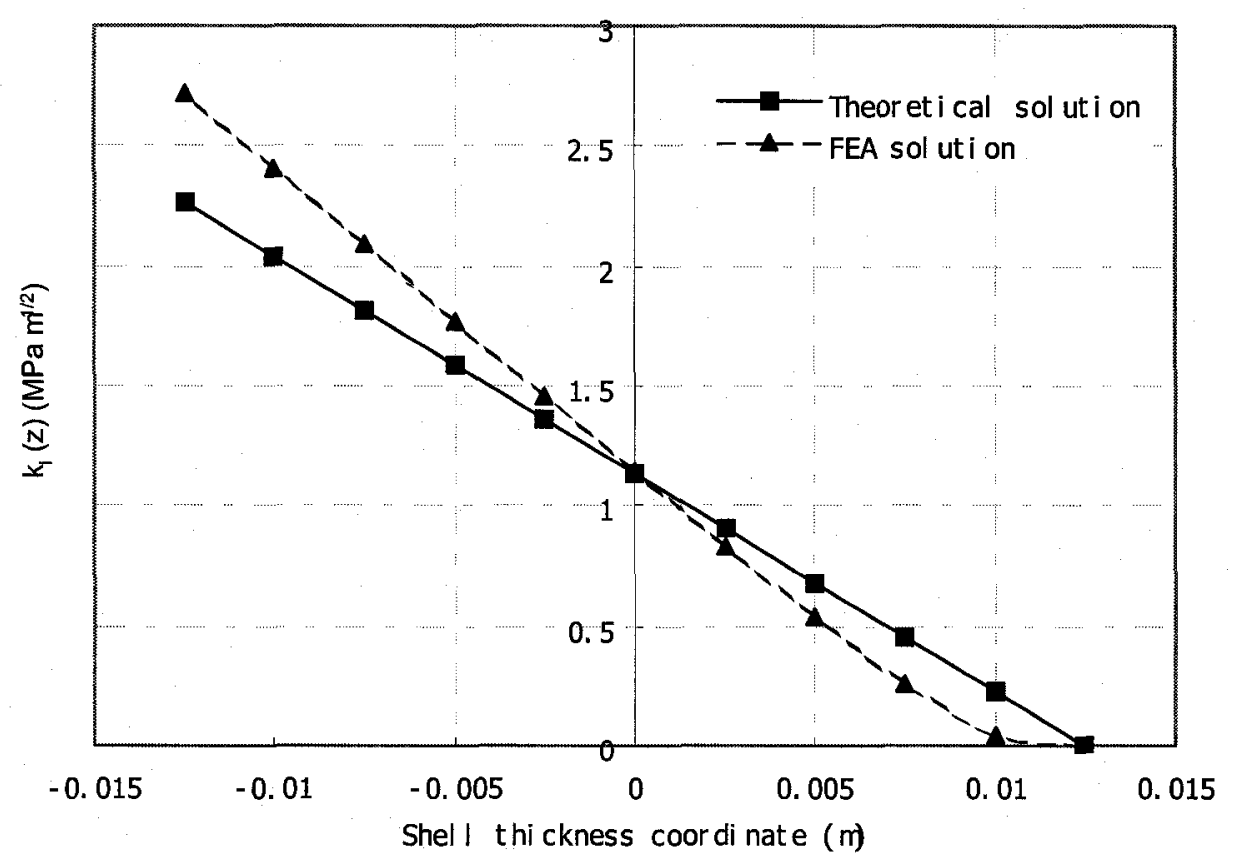

(b)

Figure 5.18 Variations of SIF through the shell wall of the axially cracked cylindrical shell with $\lambda=2$ : (a) positive bending and (b) negative bending 


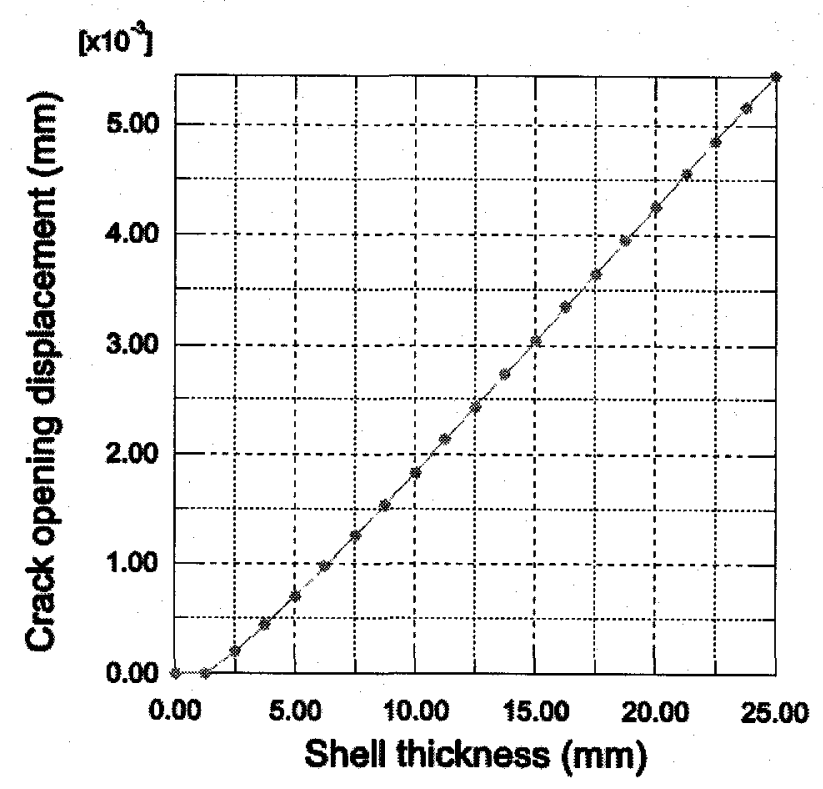

(a)

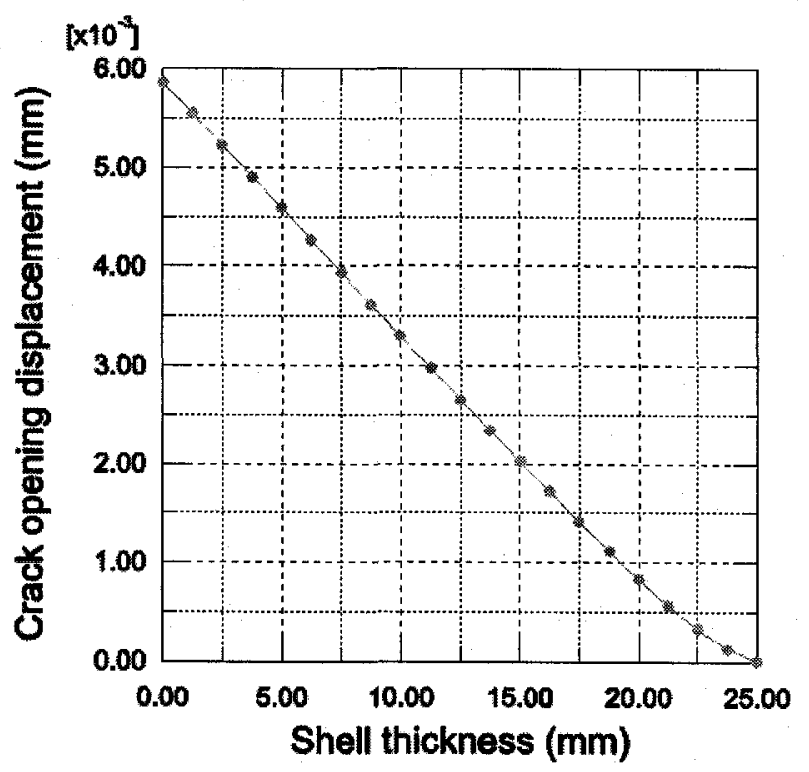

(b)

Figure 5.19 CODs of the axially cracked cylindrical shell with $\lambda=4$ at a distance of $a / 2$ from the crack tip: (a) positive bending and (b) negative bending 


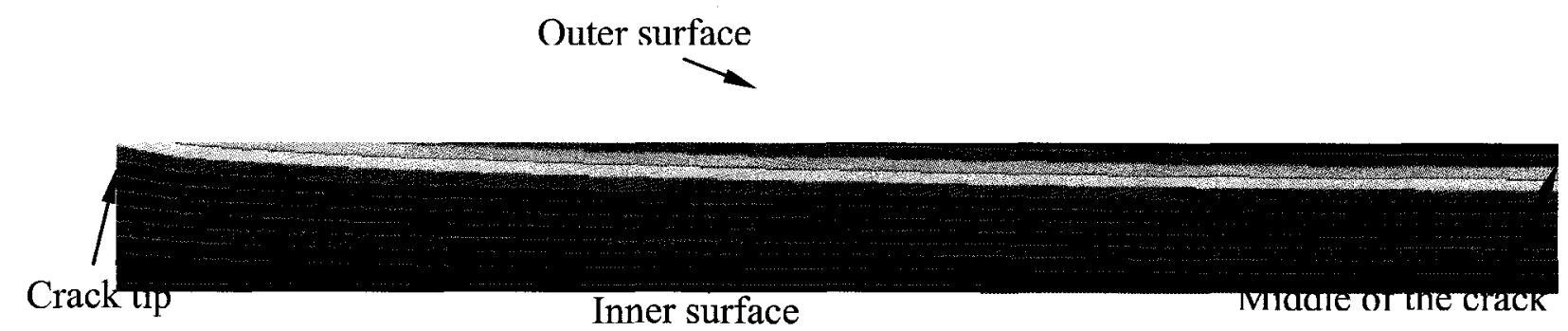

(a)

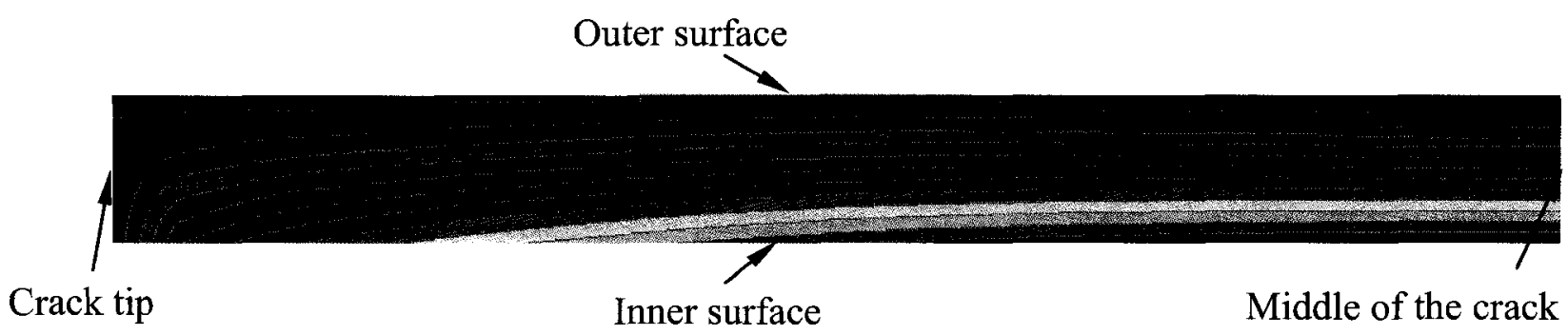

(b)

Figure 5.20 COD contours on the crack face of the axially cracked cylindrical shell with $\lambda=4:$ (a) positive bending and (b) negative bending 


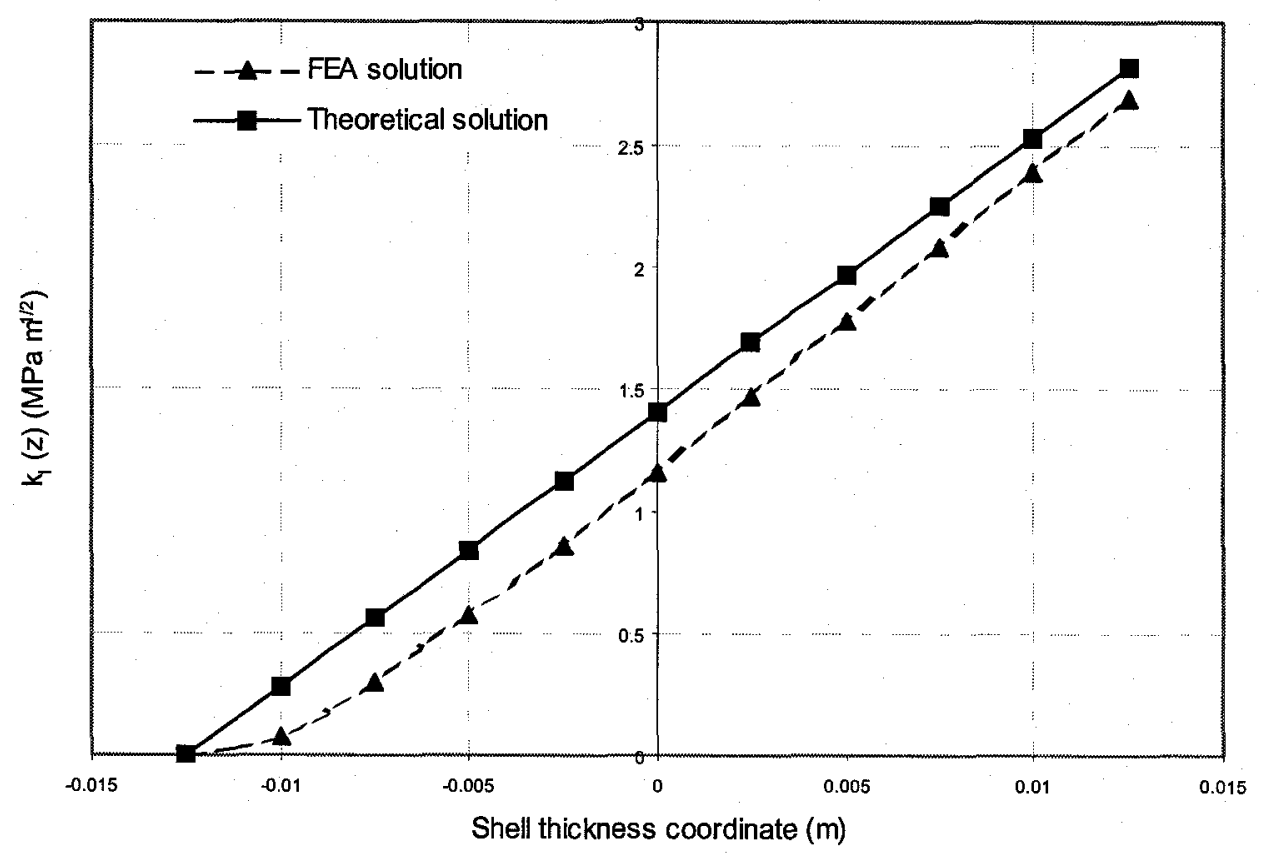

(a)

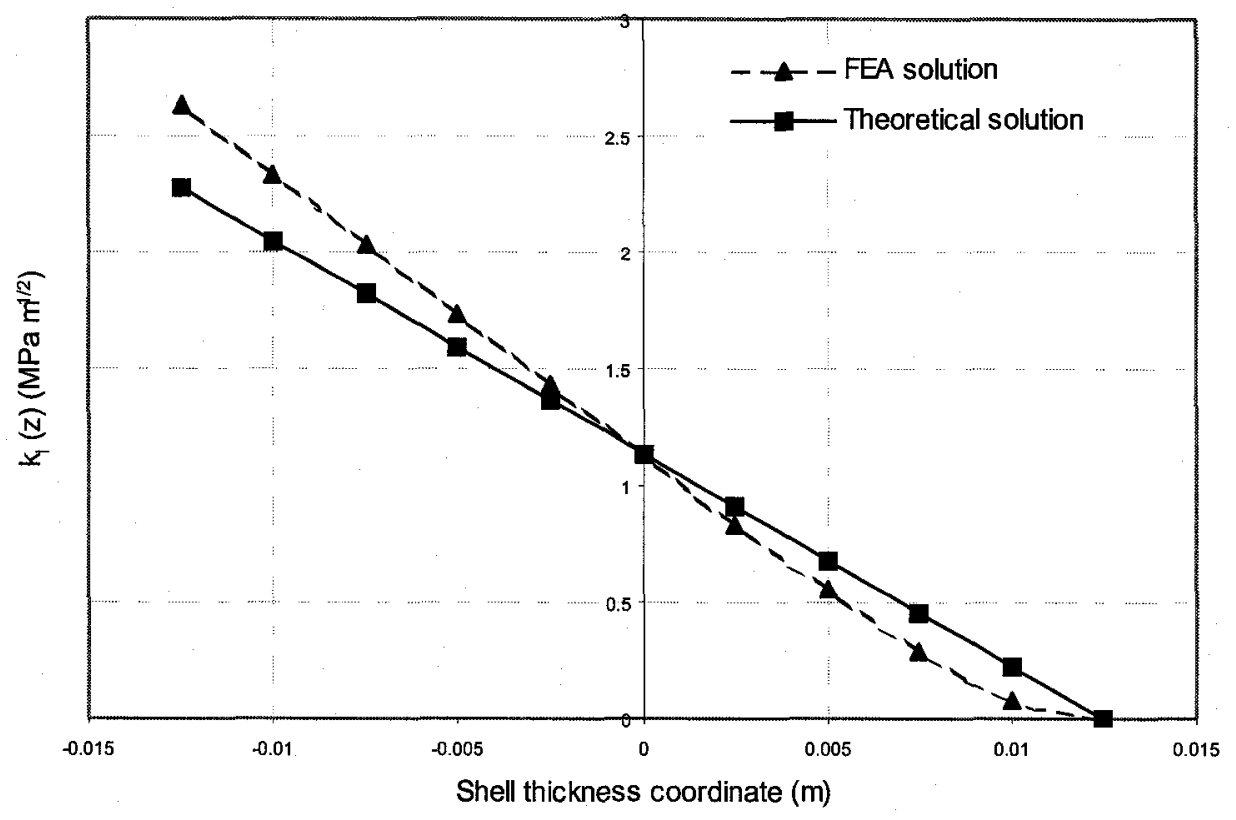

(b)

Figure 5.21 Variations of SIF through the shell wall of the axially cracked cylindrical shell with $\lambda=4$ : (a) positive bending and (b) negative bending 


\subsubsection{Cylindrical shell containing a circumferential crack}

Similar to cylindrical shells containing an axial crack, the FEA results of cylindrical shells containing a circumferential crack for $\lambda=2$ and $\lambda=4$ are presented, as shown in Figures 5.22 to 5.27. The basic features of the FEA results for spherical shells and cylindrical shells containing an axial crack apply for cylindrical shells containing a circumferential crack. It is known from the theoretical analysis that partial closure occurs in cylindrical shells containing a circumferential crack under positive bending only when $\lambda$ is equal to or greater than 6.5 , see Table 3.1 , therefore for $\lambda=2$ and $\lambda=4$ complete closure should occur in the shell. Examining the COD on the crack face finds that along the entire crack length at the inner surface the COD is nearly uniform and very close to zero for both $\lambda=2$ and $\lambda=4$. Complete closure occurs in cylindrical shells containing a circumferential crack with $\lambda=2$ or $\lambda=4$ under negative bending. Good agreement of the FEA SIF with the theoretical SIF can be seen in Figures 5.24 and 5.27.

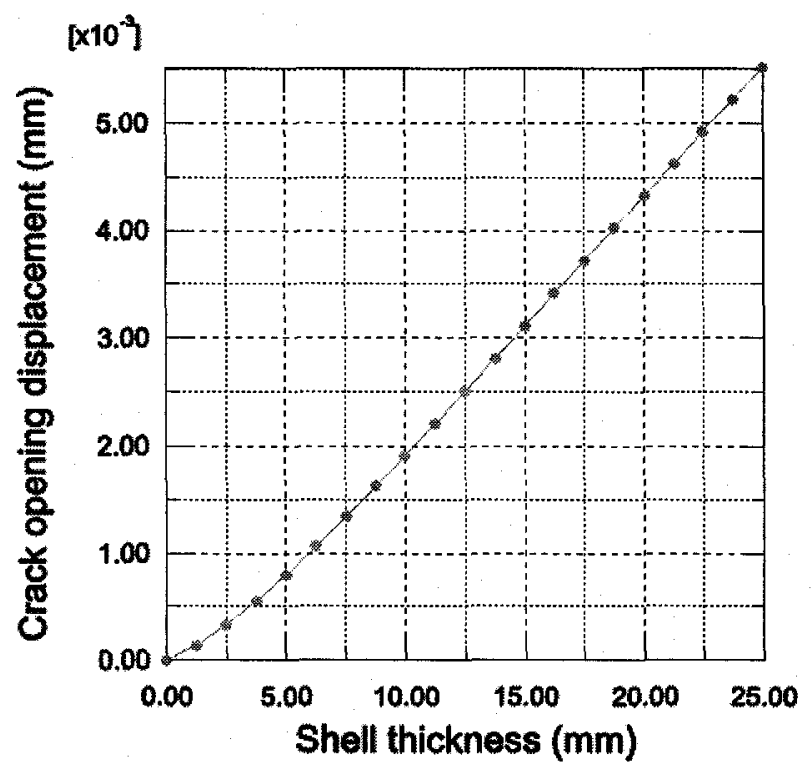


(a)

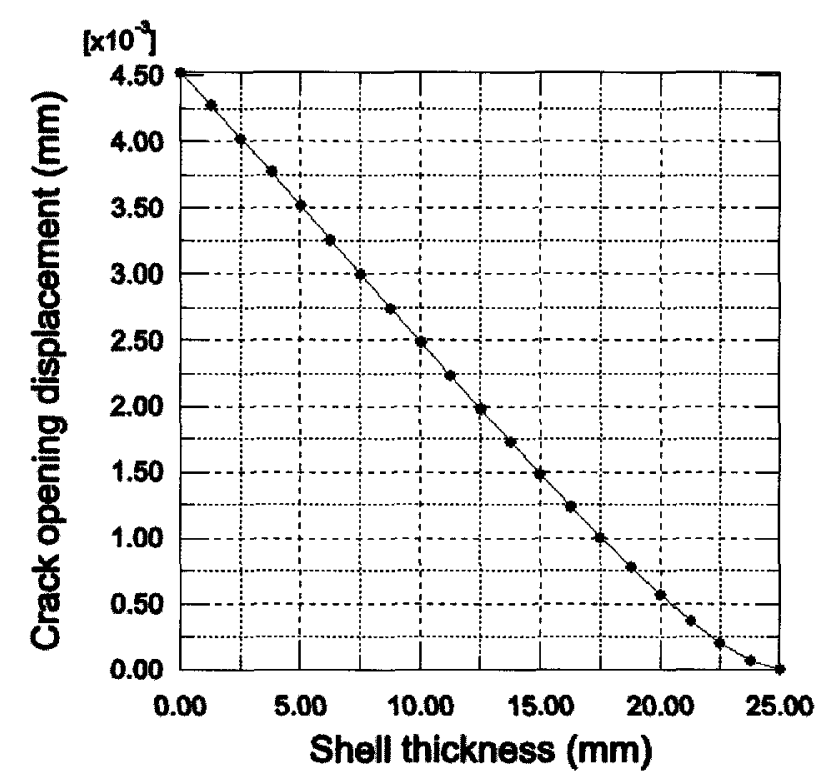

(b)

Figure 5.22 CODs of the circumferentially cracked cylindrical shell with $\lambda=2$ at a distance of $a / 2$ from the crack tip: (a) positive bending and (b) negative bending

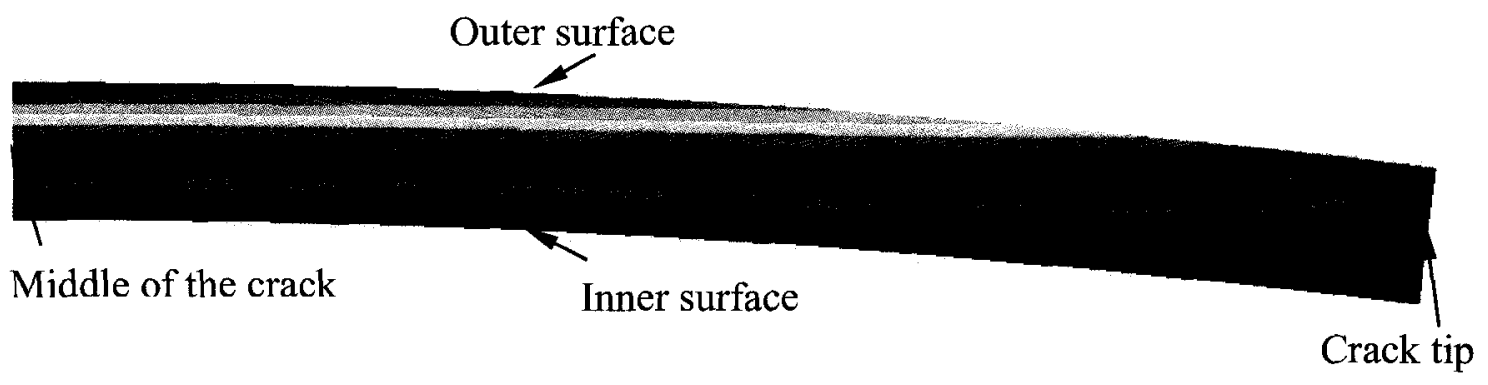

(a) 


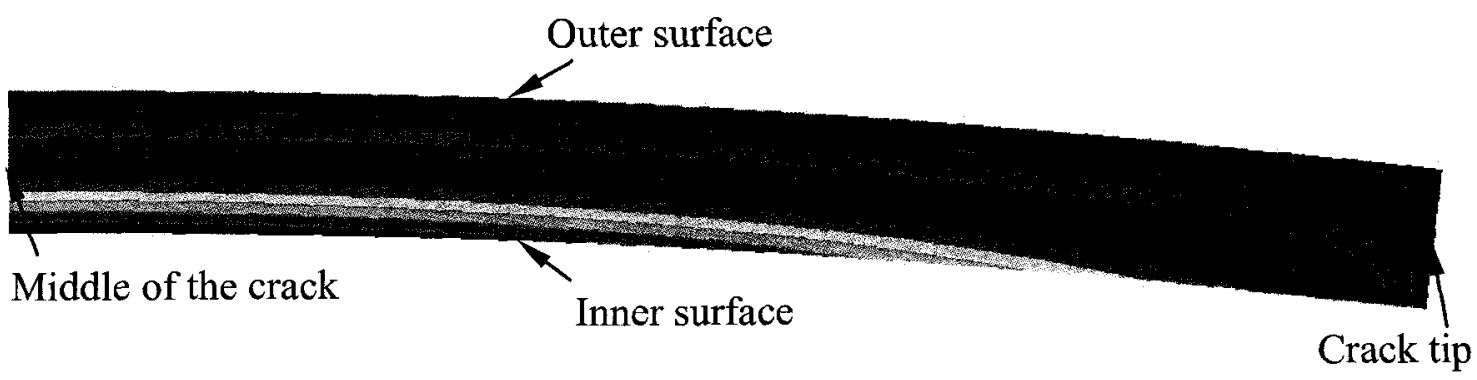

(b)

Figure 5.23 COD contours on the crack face of the circumferentially cracked cylindrical shell with $\lambda=2$ : (a) positive bending and (b) negative bending

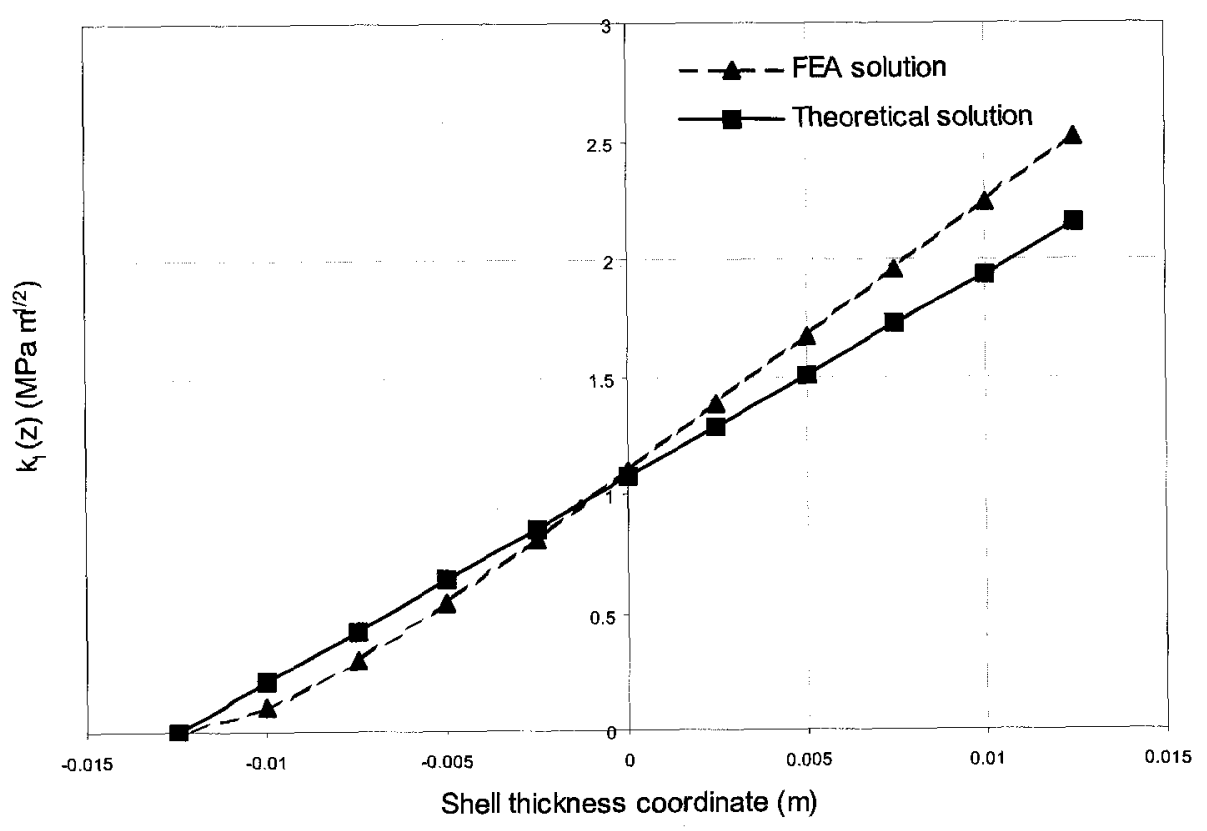

(a) 


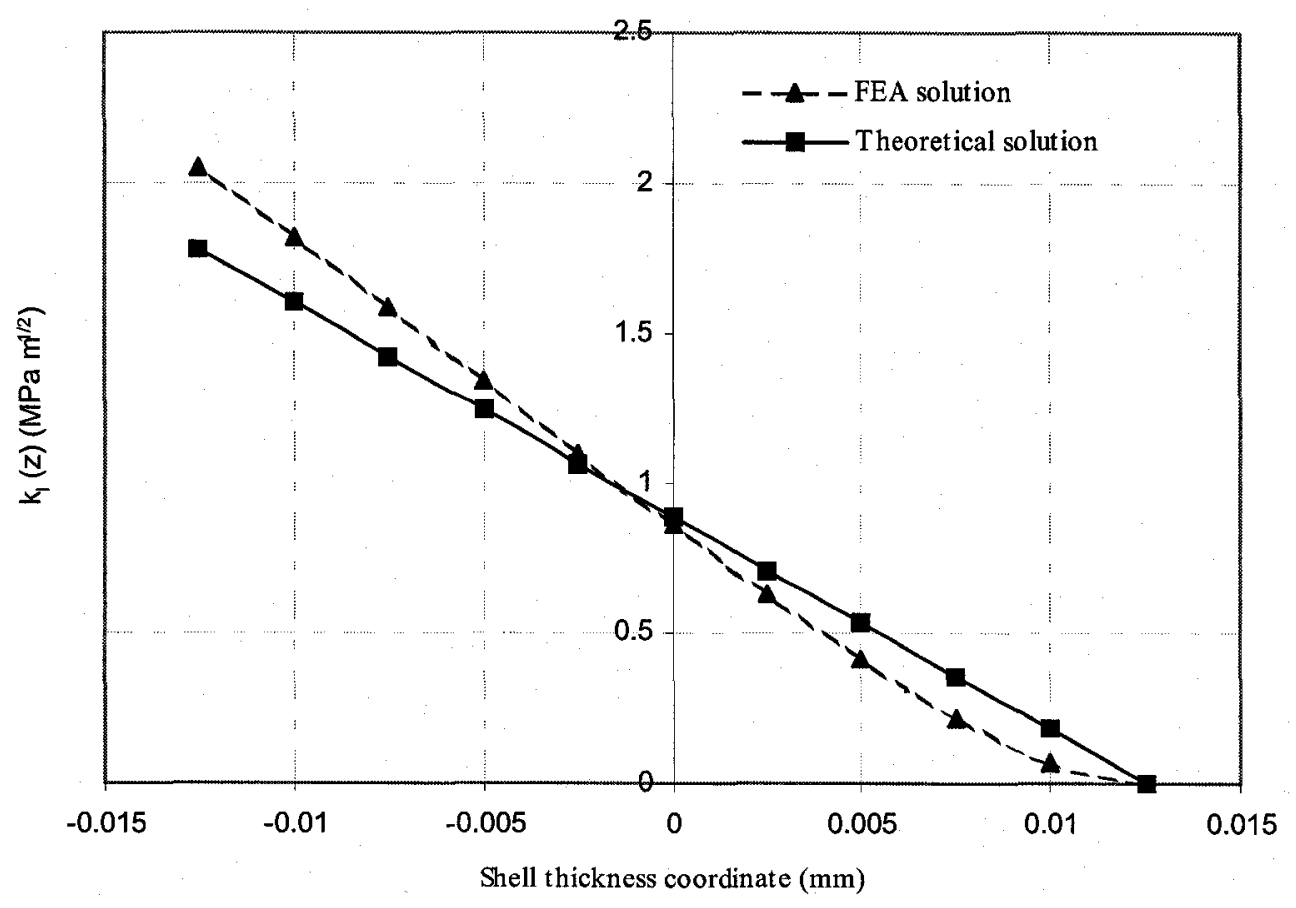

(b)

Figure 5.24 Variations of SIF through the shell wall of the circumferentially cracked cylindrical shell with $\lambda=2$ : (a) positive bending and (b) negative bending

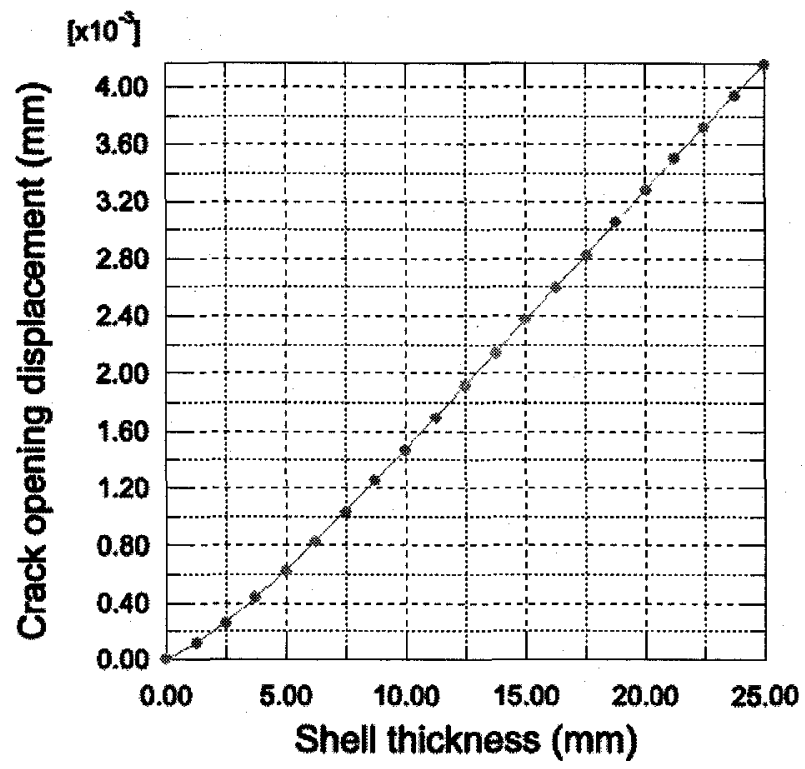


(a)

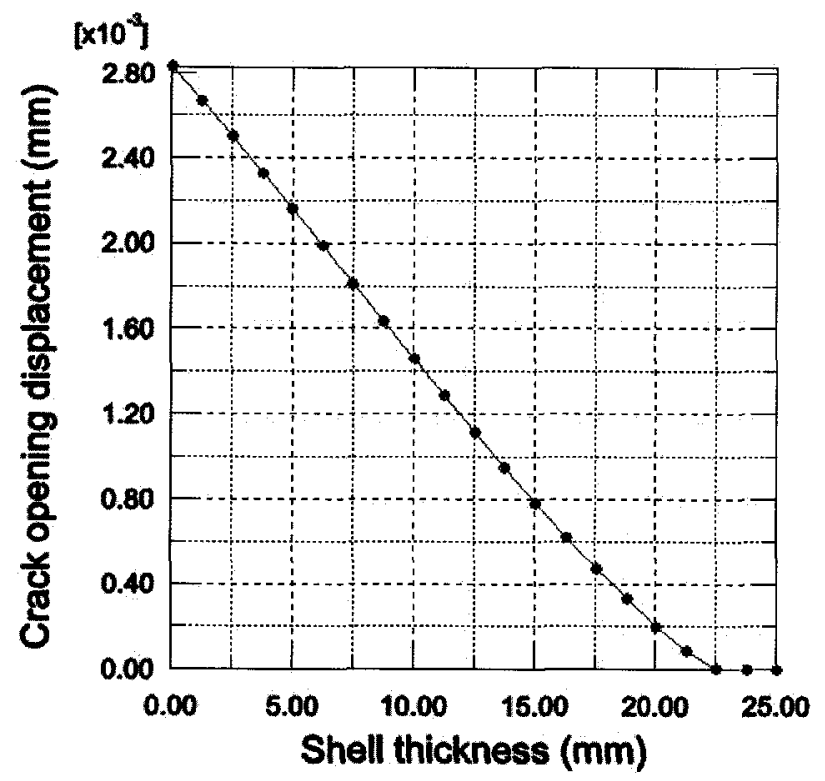

(b)

Figure 5.25 CODs of the circumferentially cracked cylindrical shell with $\lambda=4$ at a distance of $a / 2$ from the crack tip: (a) positive bending and (b) negative bending

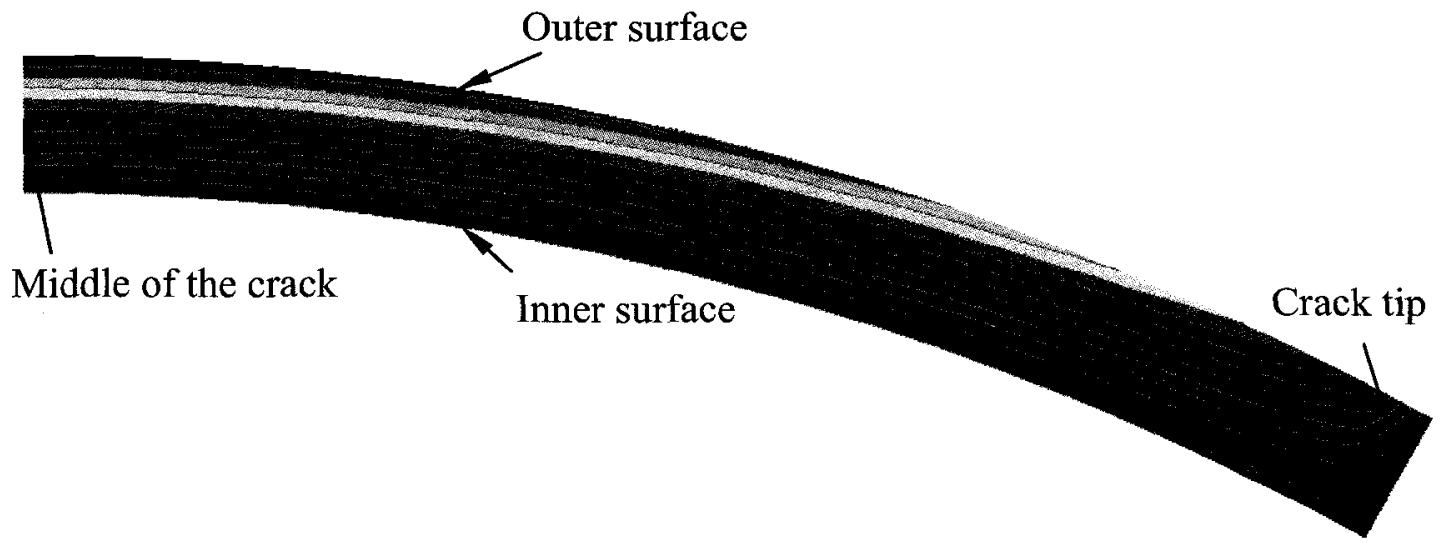

(a) 


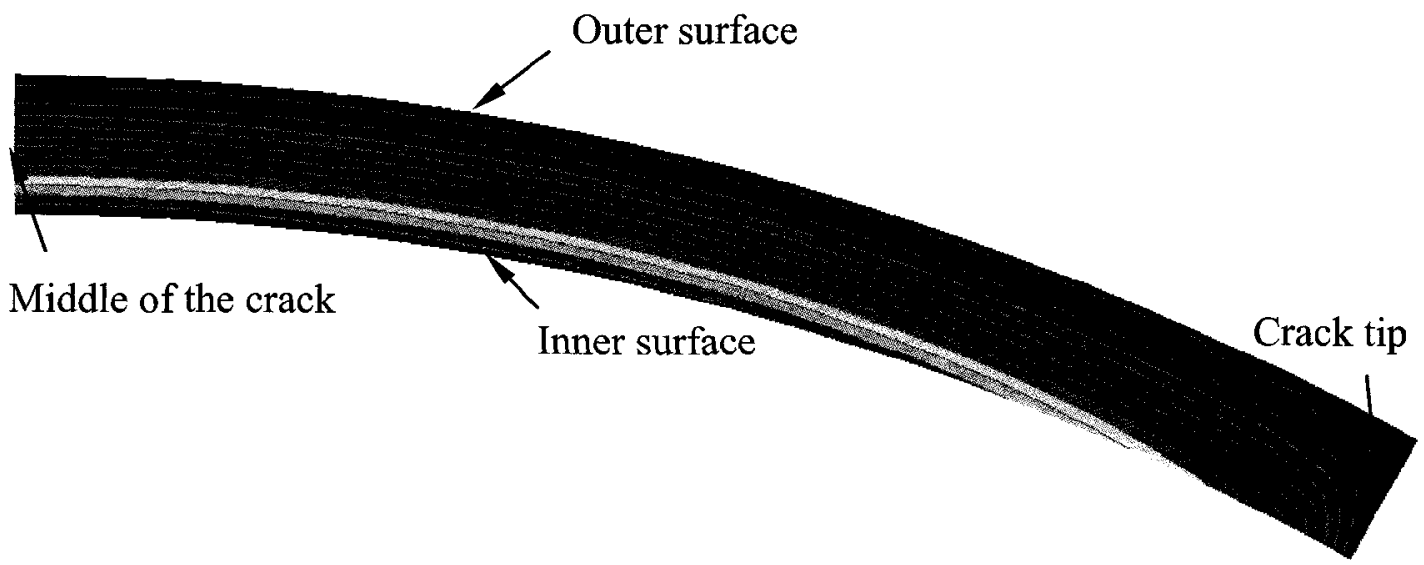

(b)

Figure 5.26 COD contours on the crack face of the circumferentially cracked cylindrical shell with $\lambda=4$ : (a) positive bending and (b) negative bending

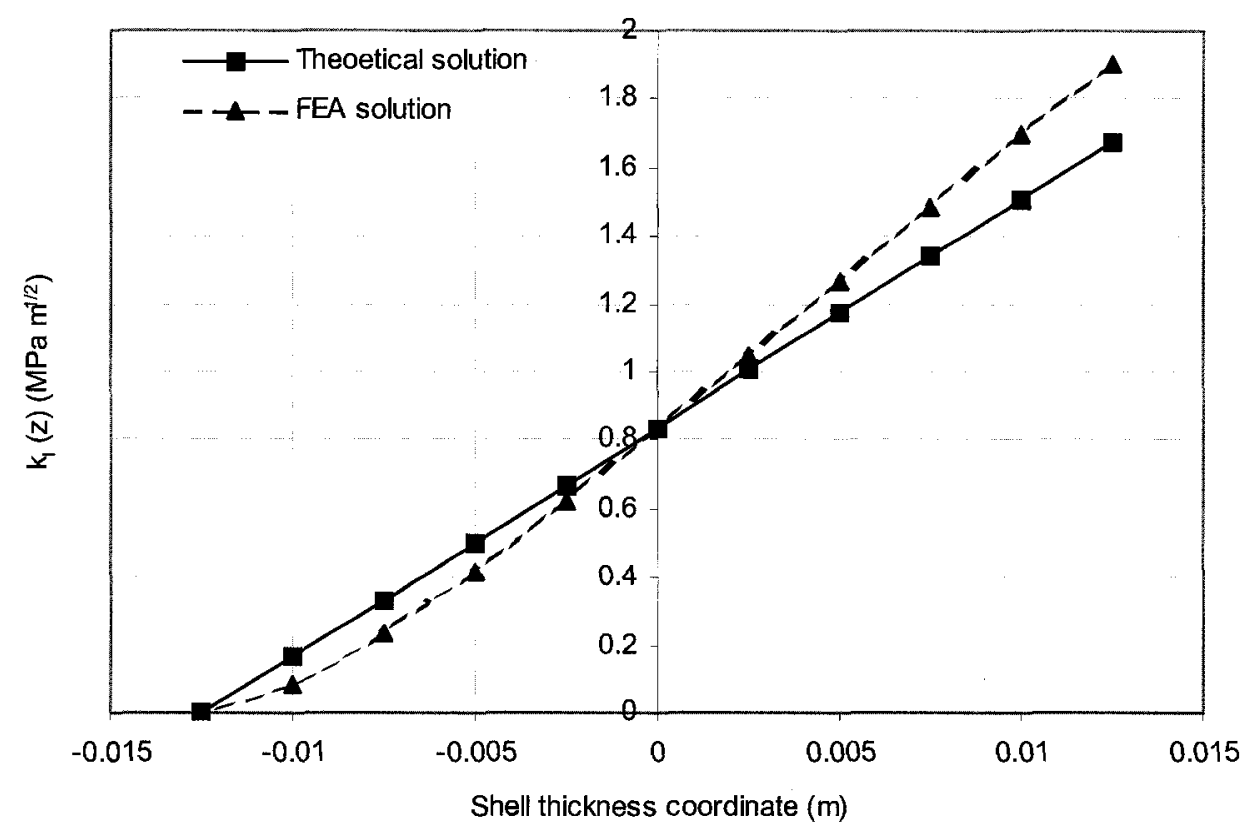

(a) 


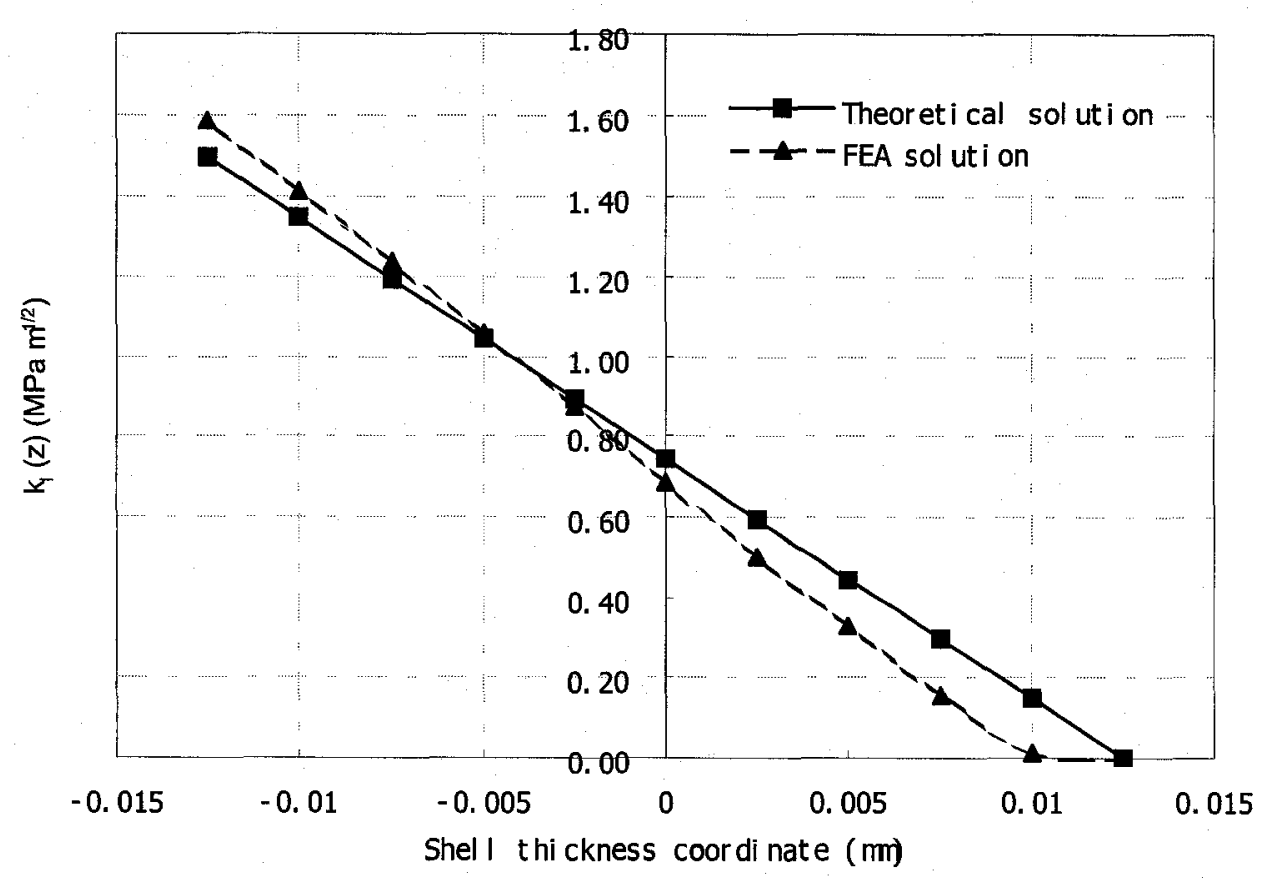

(b)

Figure 5.27 Variations of SIF through the shell wall of the circumferentially cracked cylindrical shell with $\lambda=4$ : (a) positive bending and (b) negative bending

\subsubsection{Shell with double curvature}

The FEA results of two shell geometries, $R_{1} / R_{2}=0.25$ and $R_{1} / R_{2}=4$ with $\lambda=2$, for shells with double curvature are presented in Figures 5.28 to 5.33. Similar features of the results to other shell geometries are found for shells with double curvature. Since the critical values for partial closure are $\lambda=3.5$ for $R_{1} / R_{2}=0.25$ and $\lambda=3$ for $R_{1} / R_{2}=4$ under positive bending according to the theoretical analysis, complete closure should occur in the two shell geometries with $\lambda=2$. The COD data have confirmed that closure occurs over the entire crack length at the inner surface. Under negative bending, again, 
complete closure occurs in the shells. Finally, good agreement of the FEA SIF with the theoretical SIF can also be found for shells with double curvature.

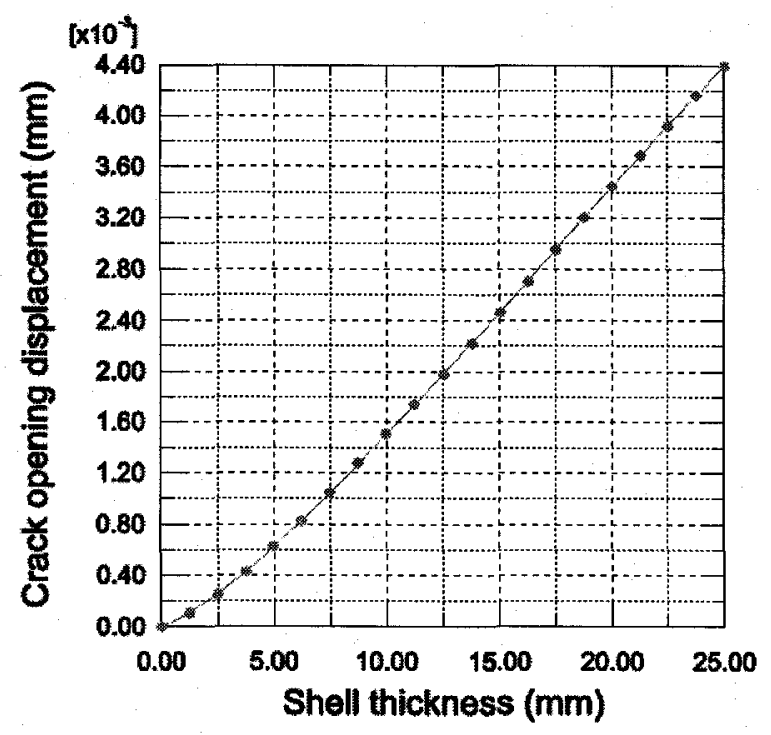

(a)

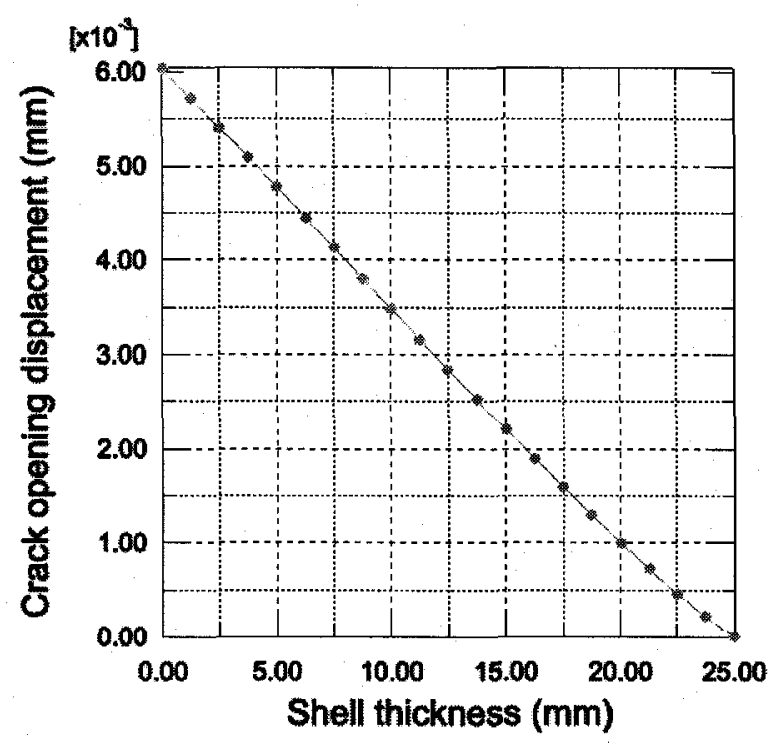

(b)

Figure 5.28 CODs of the shell with $R_{1} / R_{2}=0.25$ and $\lambda=2$ at a distance of $a / 2$ from the crack tip: (a) positive bending and (b) negative bending 


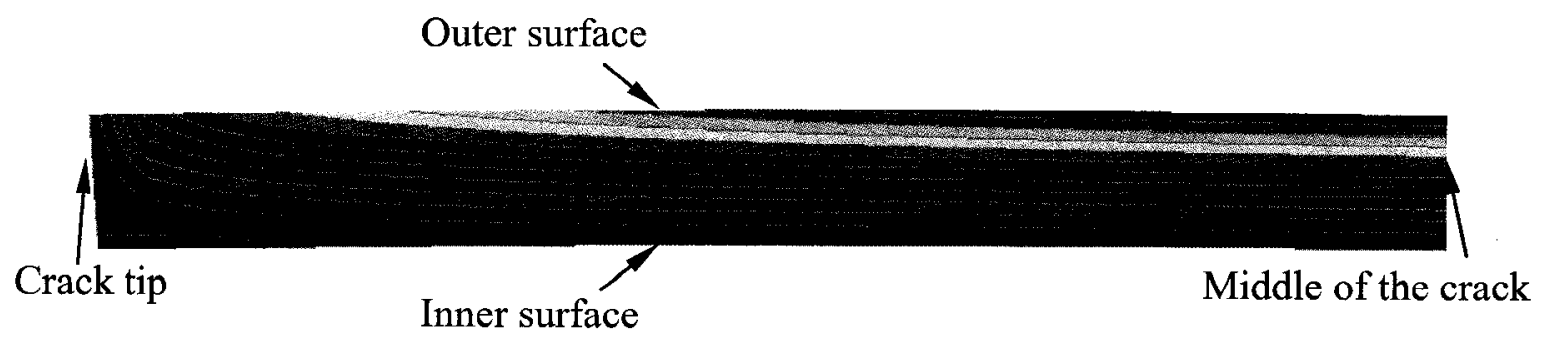

(a)

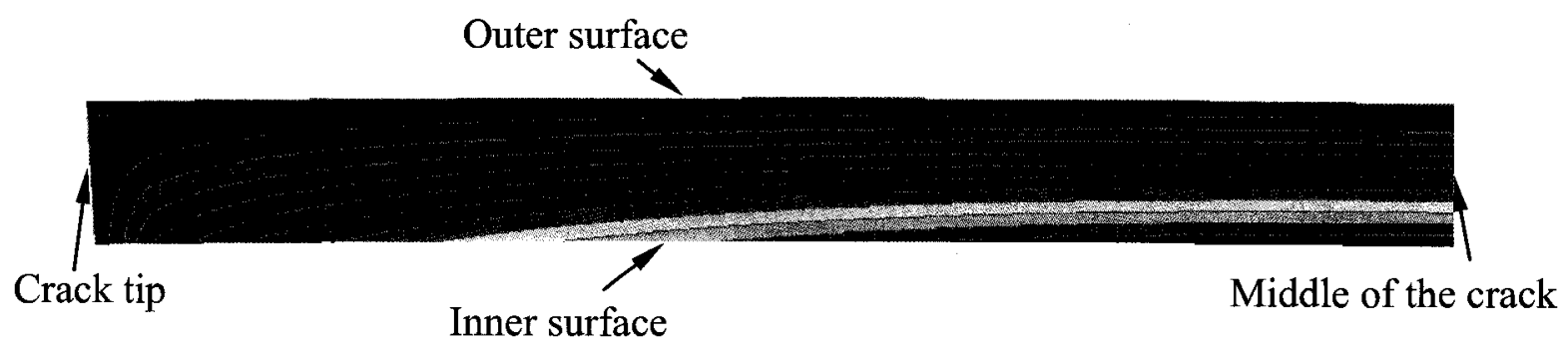

(b)

Figure 5.29 COD contours on the crack face of the shell with $R_{1} / R_{2}=0.25$ and $\lambda=2$ :

(a) positive bending and (b) negative bending 


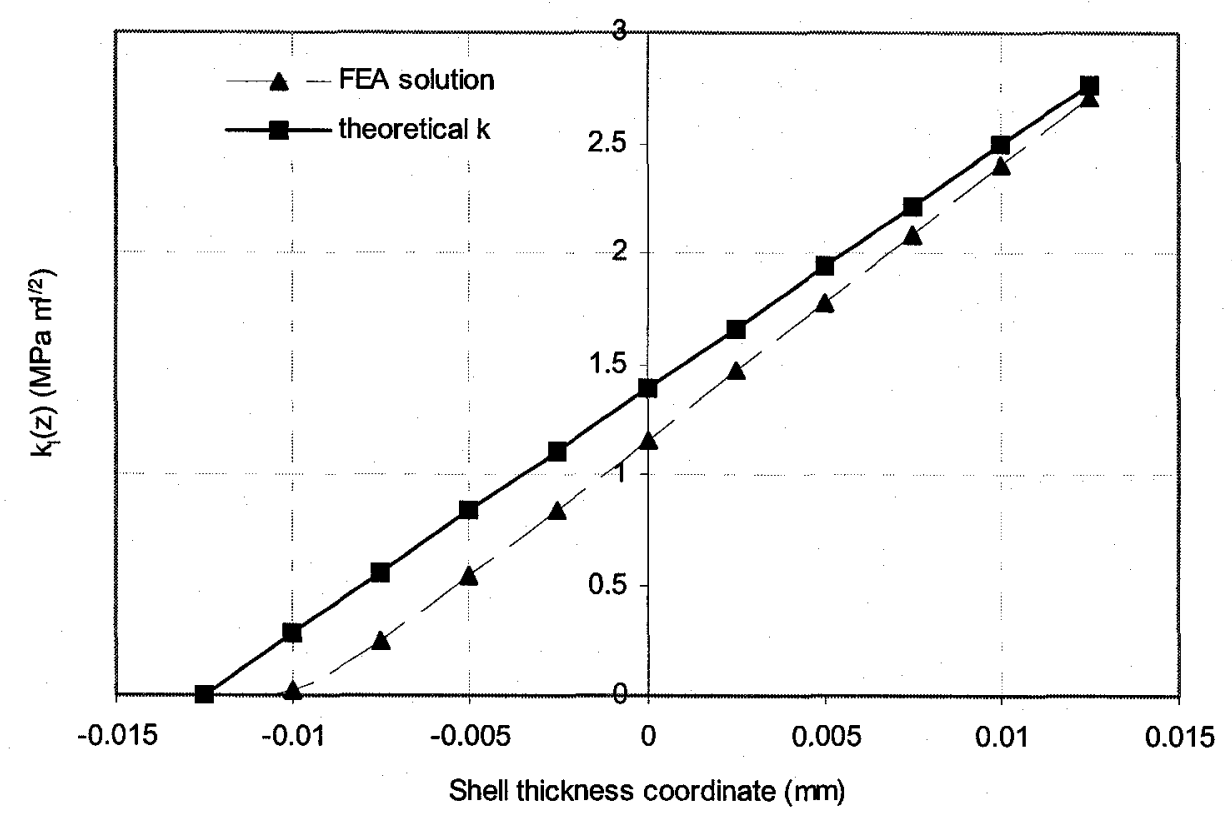

(a)

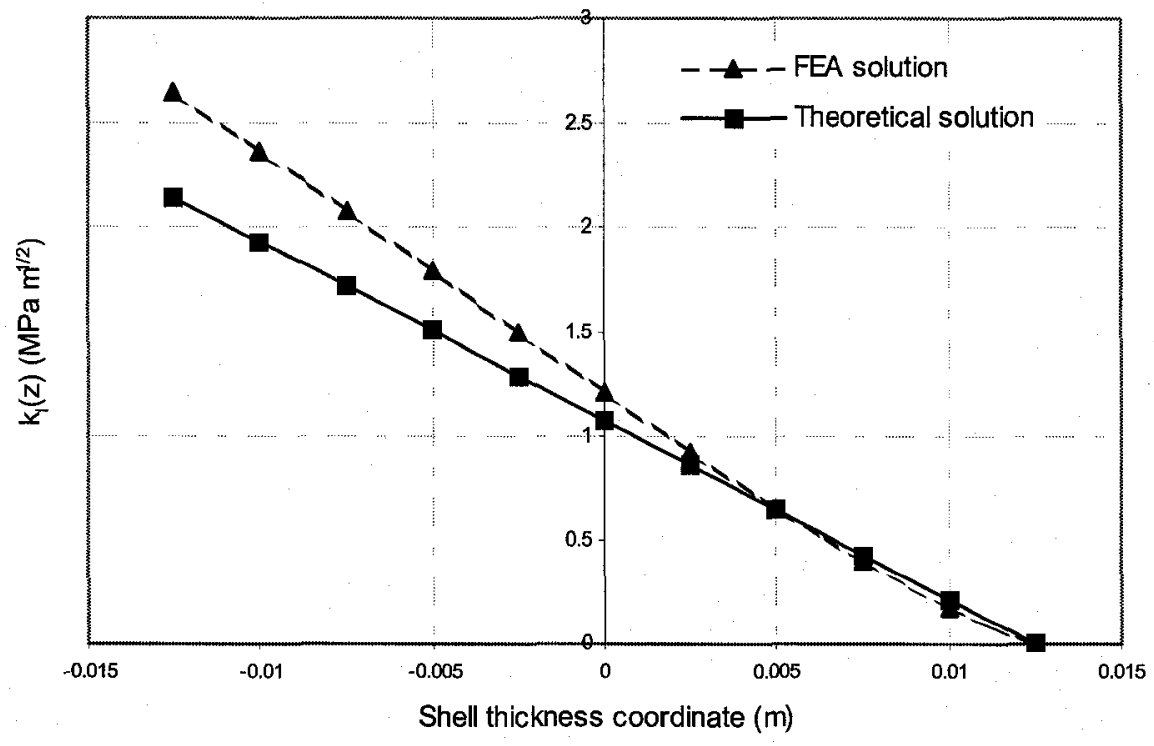

(b)

Figure 5.30 Variations of SIF through the shell wall of the shell with $R_{1} / R_{2}=0.25$ and $\lambda=2:$ (a) positive bending and (b) negative bending 


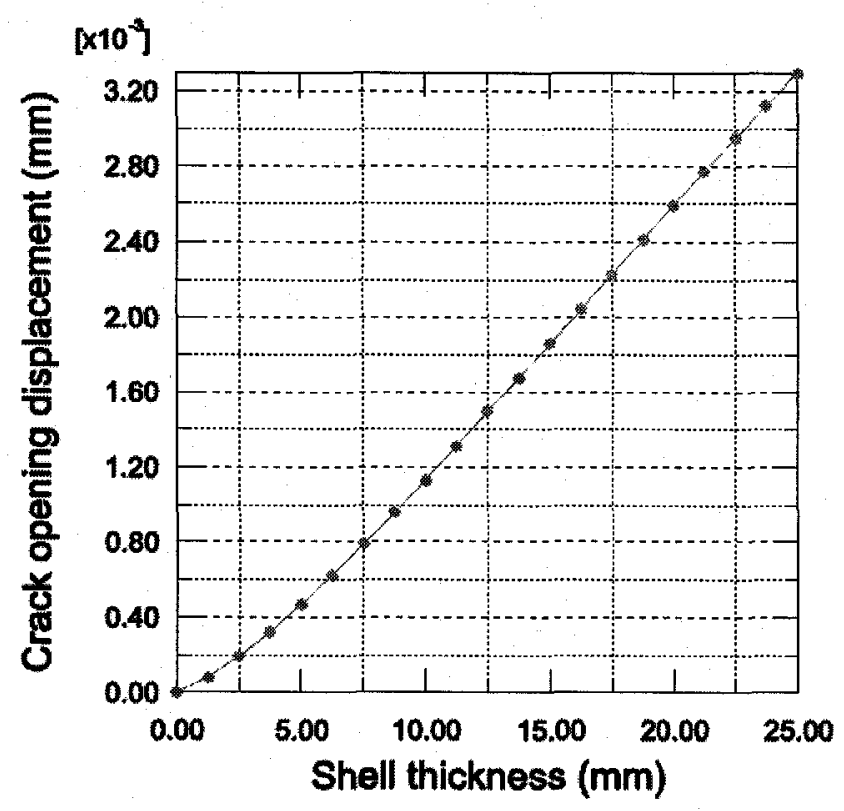

(a)

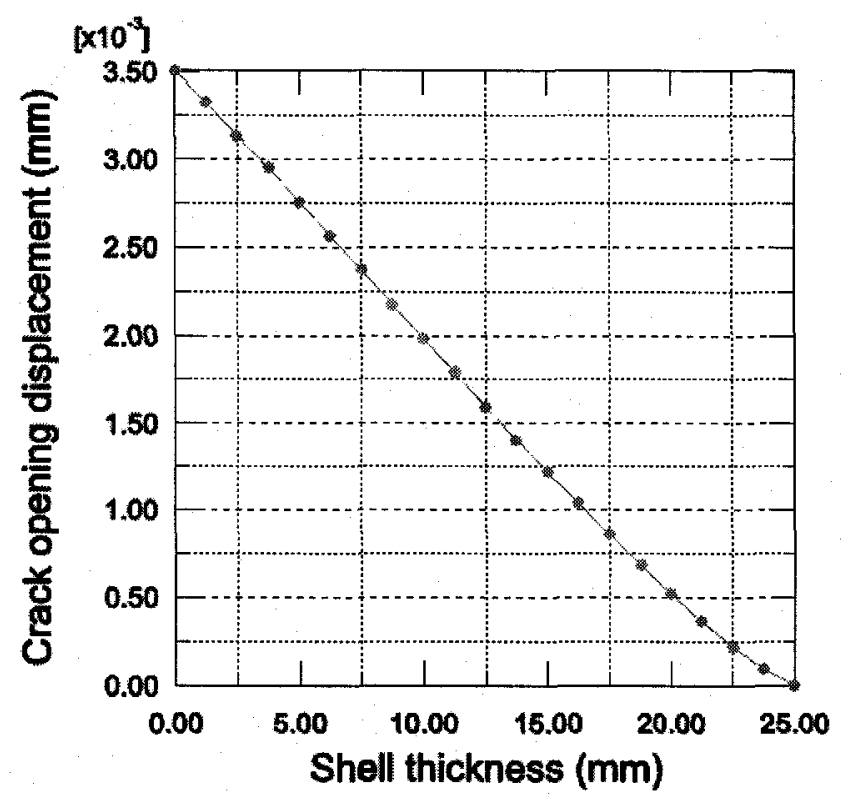

(b)

Figure 5.31 CODs of the shell with $R_{1} / R_{2}=4$ and $\lambda=2$ at a distance of $a / 2$ from the crack tip: (a) positive bending and (b) negative bending 


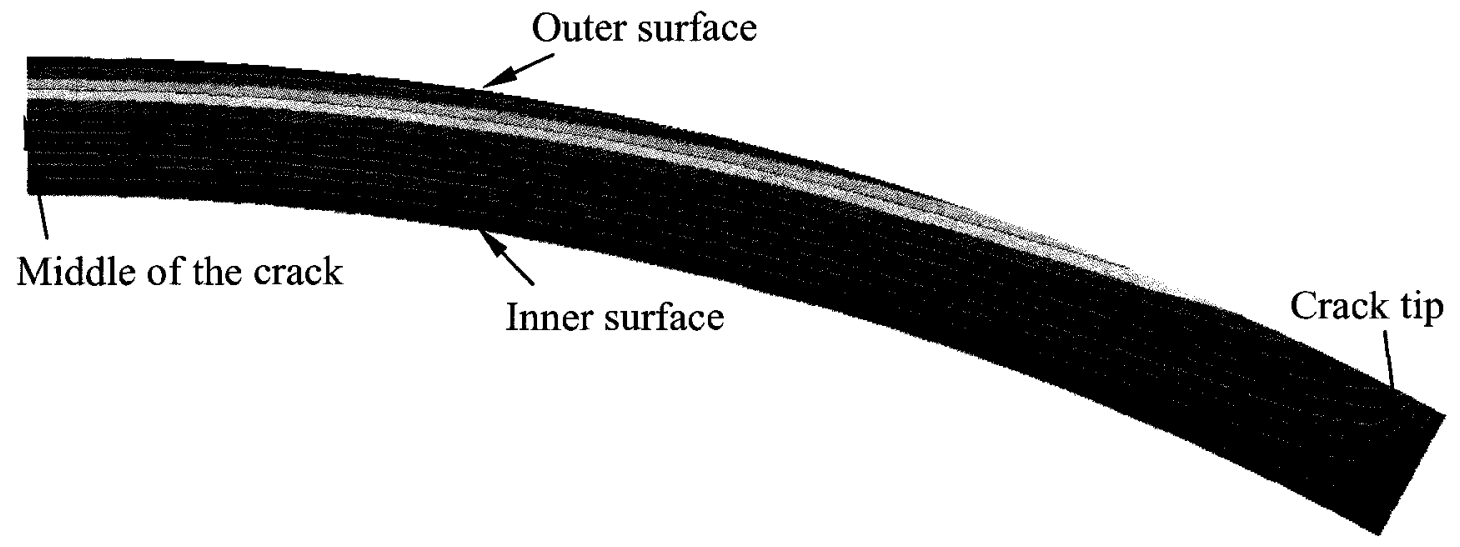

(a)

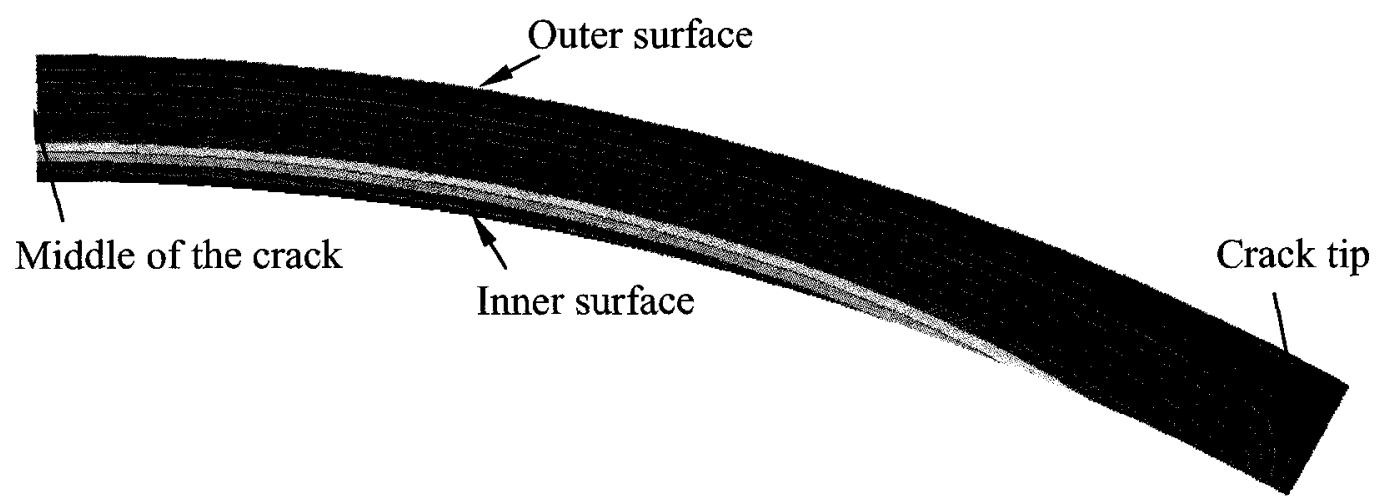

(b)

Figure 5.32 COD contours on the crack face of the shell with $R_{1} / R_{2}=4$ and $\lambda=2$ :

(a) positive bending and (b) negative bending 


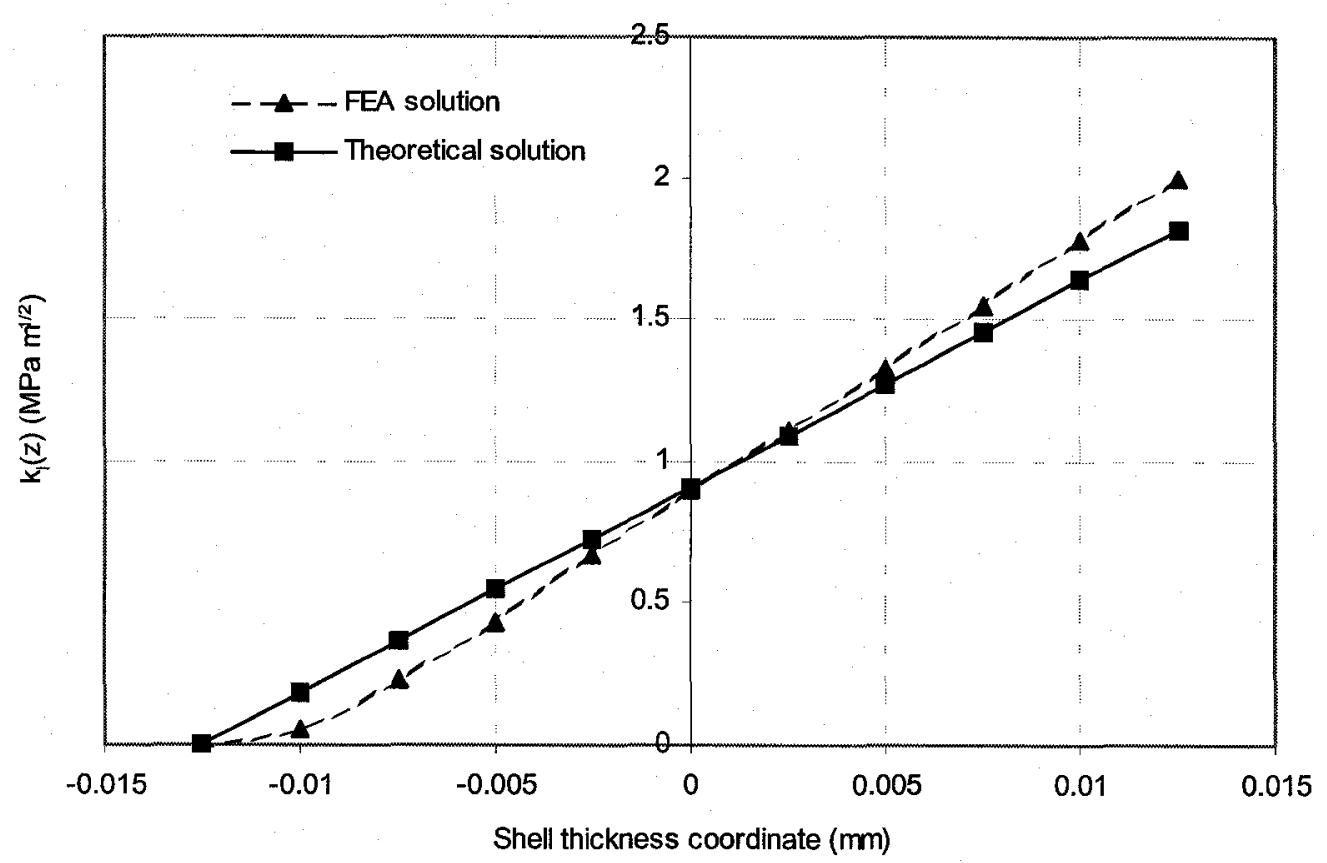

(a)

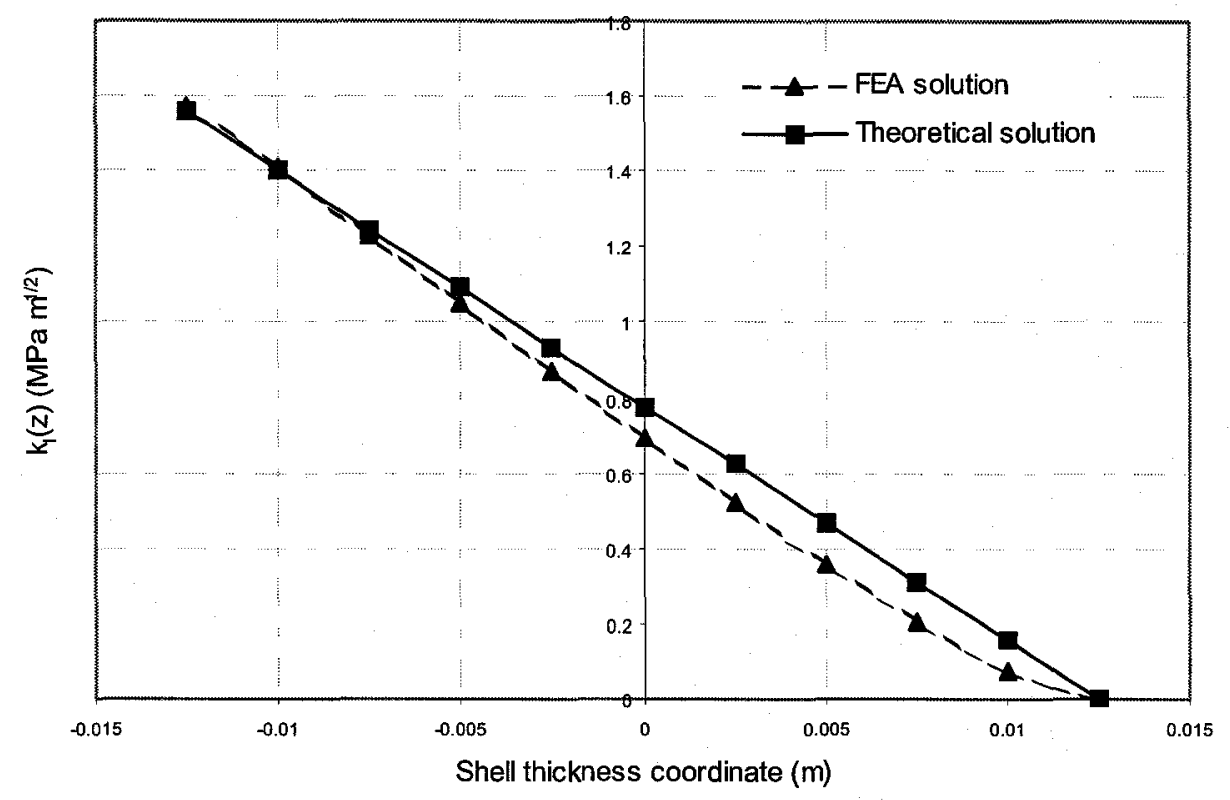

(b)

Figure 5.33 Variations of SIF through the shell wall of the shell with $R_{1} / R_{2}=4$ and $\lambda=2:$ (a) positive bending and (b) negative bending 


\section{Chapter 6 Discussion}

\subsection{Effects of Crack Closure on Stress Intensity Factors}

Crack-face closure occurs physically when a cracked plate or a shell is subjected to a bending load, which protects material penetration at the contacting edges of the crack surfaces. With the constraint of crack closure that the normal displacement at the compressive edge of the crack surface must be zero, the membrane component $F_{m b}$ and bending component $F_{b b}$ of the SIF are always equal when the closure occurs in the inner surface (positive bending, $\sigma_{\mathrm{b}}>0$ ) of the shell while the two components are equal in magnitude but opposite in sign when the closure is in the outer surface (negative bending, $\sigma_{\mathrm{b}}>0$ ), which is concluded from the theoretical analysis in Chapter 3 . The crack closure effect increases the membrane component but decreases the bending component for all shell geometries under either positive bending or negative bending. The membrane component and bending component in positive bending condition are not equal to those in negative bending condition when crack closure is concerned; they are greater in the former condition than in the latter. However, in the non-closure case, the membrane component is not equal to the bending component; and these two components do not vary with the direction of the bending load, that is, the membrane component and bending component in positive bending condition are identical to those in the negative bending condition. In other words, without crack closure considered, the sign of the bending load can be ignored in determining the SIFs. 
It has been noticed that the difference of the stress intensity factor ratios between the closure case and the non-closure case increases with the decrease of the $\lambda$ value, that is, the difference is the maximum when the shell curvature approaches zero. In other words, the crack closure effect on the SIF for plates is the most significant. Between different shell geometries, comparing the stress intensity factor ratios for various isotropic shells, double curvature shells in Figure 3.28, cylindrical shells containing an axial crack or containing a circumferential crack in Figure 3.33, and spherical shells in Figure 2.9 , it may be observed that for the shells with $R_{1} / R_{2}>1$, that is, for the shells with a crack lying along the larger curvature direction, the influence of the bending load sign on the SIFs is less, especially, when $\lambda$ is greater than 5 , the difference of the SIF in positive bending case and negative bending case is almost negligible. Also, the stress intensity factor ratios of the shells with $R_{1} / R_{2}<1$ for both closure case and non-closure case are higher than those of the shells with $R_{1} / R_{2}>1$. This indicates that a shell with a crack along the smaller curvature direction is more dangerous for fracture, for example, a cylinder with an axial crack is more likely to fail than one with a circumferential crack. Certainly, for all shell geometries, when $\lambda$ approaches zero, the SIFs should be identical, because all the geometries at $\lambda=0$ represent plates. This can be seen in Figures 3.28, 3.33 and 2.9. It is also observed that when $\lambda \rightarrow 0$, the closure stress intensity factor ratios in positive bending case and the negative bending case are identical, that is, for plates the effect of crack-face closure on the SIF is not influenced by the sign of the bending load. This is because the difference of the crack-face closure effect on the SIF between positive bending and negative bending is caused by the shell curvature, while the curvature of plates is zero. It is not hard to understand that positive bending and negative bending do 
not make any difference on plates due to the same behavior of closure at the inner surface and at the outer surface of plates.

Regarding the maximum SIF, it is found that for all shell geometries the non-closure maximum SIF always occurs at $\lambda \rightarrow 0$, but the closure one does not, it mostly occurs in the range of $\lambda<3$ except for a cylindrical shell with $E_{1} / E_{2}=0.037$ containing an axial crack, as shown in Figures 3.9, 3.16, 3.20, 3.25, 3.29 and 3.34. This means that without crack closure concerned, the influence of shell curvature on the SIF is in a simple manner, while it becomes complex when crack closure is concerned. It is also found that the closure and non-closure maximum SIFs become close with the increase of the $\lambda$ value except for a cylindrical shell containing an axial crack. This implies that the crack closure effect on the SIF is abated in the shells with large curvature (small curvature radius or large $\lambda$ value). As analyzed in Chapter 3 , for different shell geometries, when $\lambda$ reaches the critical value for individual shell geometry, the crack surfaces are not in complete contact at the compressive edges, that is, partial closure occurs. Furthermore, from the contact force distributions along the crack length, as demonstrated in Chapter 3 (Figures 3.5, 3.14, 3.18, 3.23, 3.27, 3.31 and 3.32), for a given shell geometry the larger the $\lambda$ value, the less contact of the crack surfaces, represented by the larger range with negative contact force along the crack length, therefore less effect of crack closure. It may be predicted that the crack surfaces would be totally free of contact when $\lambda$ is large up to a certain value, that is, crack closure no longer occurs. However, for cylindrical shells containing an axial crack, since curvature does not exist in the direction along the crack, although the shell curvature does affect the partial 
closure behavior, as shown in Figures 3.14 and 3.31, the partial closure effect on the SIF should be relatively weak compared with the curvature occurring in the direction along the crack. Certainly, the closure and non-closure maximum SIFs will become close for cylindrical shells containing an axial crack as those of other shell geometries do when the $\lambda$ value is very large. This tendency can be observed in Figures 3.16 and 3.34.

As concerns material orthotropy, it is evident that the influence of material orthotropy on the crack closure in shells varies with shell geometry, for example, the reduction of the maximum SIF due to crack-face closure varies significantly with material orthotropy for cylindrical shells containing an axial crack, see Figure 3.17, while it is almost not changed with different $E_{1} / E_{2}$ ratios for cylindrical shells containing a circumferential crack, as shown in Figure 3.21. For other shell geometries, the variations of the crack closure behavior with material orthotropy are more complex cylindrical shells, but it is clear that for the mildly orthotropic material, $E_{1} / E_{2}=0.725$ or $E_{1} / E_{2}=1.38$, the difference of the crack closure effect on the SIF between $E_{1} / E_{2}=0.725$ and $E_{1} / E_{2}=1.38$ is small, while for the strongly orthotropic material, $E_{1} / E_{2}=0.037$ or $E_{1} / E_{2}=26.67$, the difference of the crack closure effect on the SIF between $E_{1} / E_{2}=0.037$ and $E_{1} / E_{2}=26.67$ is large, as illustrated in Figure 3.10 and Figure 3.26. This means that material orthotropy does affect the crack closure behavior of shells, the stronger the orthotropic of the shell material, the larger the effect of material orthotropy on the closure behavior. 
It is also found that the influence of material orthotropy on the crack closure varies with the shell parameter $\lambda$ for a given shell geometry. From Figures $3.10,3.17$, 3.21 and 3.26 , it is clear that the material orthotropy effect on the crack closure is stronger for small values of $\lambda$, generally for $\lambda<1$. It can be inferred that the material orthotropy effect on the crack closure is strongest when $\lambda \rightarrow 0$, that is, the difference of the reduction of the maximum SIF due to the crack closure between the different $E_{1} / E_{2}$ ratios is the maximum for plates.

\subsection{Effects of Shell Curvature on Crack Closure Behavior}

It is demonstrated in Chapter 3 that unlike in plates crack closure behavior in shells is complex. Young and Sun $[16,17]$ reported that the contact force at the compressive edge of a flat plate under bending distributed uniformly along the crack length. However, for shells the results have shown that the contact force is non-uniform over the crack. The distribution of the contact force depends on the curvature of the shell. As seen in Figures 3.5, 3.14, 3.18, 3.23, 3.27, 3.31 and 3.32, when the $\lambda$ value is small, for instance, $\lambda<0.5$, the variation of the contact force along the crack is not very obvious, except in the region close to the crack tips. However, with the increase of the $\lambda$ value, the variation becomes large, especially in the region of the crack center negative contact force appears, which indicates that partial closure occurs. The critical $\lambda$ value for partial closure varies with shell geometry and is also influenced by material orthotropy, as illustrated in Figure 3.37. Among the shell geometries, the critical $\lambda$ value of cylindrical shells containing a circumferential crack is the largest, which means that the effect of shell curvature on the crack closure behavior in the shells is the least, that is, 
partial closure occurs only when the shell curvature radius is very small. Between cylindrical shells containing a circumferential crack and containing an axial crack, the crack closure behavior in the latter is more prone to be influenced by shell curvature. In the meanwhile, from the discussion of last section, for cylindrical shells containing an axial crack the difference of the maximum SIF between closure and non-closure solutions is larger than that for cylindrical shells containing a circumferential crack. Therefore, the curvature existing in the direction perpendicular to the crack length has stronger effect on the crack closure behavior; this may be due to the fact that the shell curvature is in the same direction with the bending load or the crack-face closure, which enhances the closure effect. The other shell geometries have similar crack closure behavior, that is, they have close critical $\lambda$ values, as seen in Figure 3.37.

It is also interesting that the partial closure behavior in cylindrical shells containing a circumferential crack is not affected by material orthotropy, see Figure 3.37; the critical $\lambda$ value is constant for both the mildly orthotropic material and the strongly orthotropic material. However, for other shell geometries, the critical $\lambda$ value decreases with the $E_{1} / E_{2}$ ratio and the reduction is the maximum for $E_{1} / E_{2}<1$.

Comparing positive bending with negative bending, as demonstrated in Chapter 3 , complete crack closure always occurs in all shell geometries under negative bending within the $\lambda$ range discussed $(\lambda=6)$. This is not hard to understand because the bending load is in the direction to reduce the shell curvature thus rebating its effect on the crack closure behavior. The SIFs of negative bending are always lower than those of positive 
bending for all shell geometries; therefore a cracked shell under positive bending is more dangerous than under negative bending. In addition, the reduction of the maximum SIF due to crack-face closure in negative bending case is larger than that in positive bending case, which implies that the former case causes stronger effect of crack closure in shells. This is consistent with the crack closure behavior analysis, since the crack surfaces are in complete contact at the compressive edges in negative bending condition, the crack closure effect should be stronger than the case of crack surfaces in partial contact. This issue can be further confirmed by comparing the maximum SIFs in closure and non-closure cases for small values of $\lambda$. As known, for all shell geometries complete closure occurs whether under positive bending or under negative bending when the $\lambda$ value is small, therefore the difference of the maximum SIF between closure and non-closure solutions is relatively large, as seen in the SIF results. When the $\lambda$ value is large, the difference gets less whenever partial crack closure occurs. The closure and non-closure maximum SIFs would become closer and closer with the increase of the $\lambda$ value, as seen in Figures 3.9, 3.16, 3.20, 3.25, 3.29 and 3.34, because the crack closure effect is reduced with partial contact of the crack surfaces.

\subsection{Comparison between Theoretical and FEA Solutions}

The FEA analysis conducted in this research simulates crack closure behavior in various shell geometries under a bending load using three-dimensional models, which represents the reality of the physical phenomenon. Comparing the FEA results with the theoretical solutions, it is found that they are in good agreement. Firstly, as demonstrated 
in Chapter 3, the theoretical COD is expressed as $u \pm \frac{z}{2 a} \beta$ (where "+" denotes closure at the inner surface and "-" at the outer surface), which varies linearly with the shell thickness coordinate $z$; in consistent with the theoretical analysis, the FEA results also show the linear variation of the COD through the shell thickness, except in the crack-face contact area the FEA results show the nonlinear variation. Secondly, the FEA SIFs and the theoretical SIFs are very close, although the line-contact assumption made for crack closure modeling in the theoretical deviates from the real behavior simulated in the FEA. Thirdly, the theoretical analysis reveals that crack closure may occur over entire length of the crack or only on some segments, depending on the shell curvature and the nature of the bending load; accordingly, the COD results of the FEA confirm the feature of crack closure in shells. It is found that when partial closure occurs, the CODs of the crack face in the region of the crack center are not zero, which implies the crack faces are not in contact in the region.

As emphasized in the theoretical analysis, in order to coincide with the frame work of formulating crack problems in shells, the crack closure can only occur at the compressive edge of the crack face, that is, the crack faces must in contact on a line, not over an area. However, due to the deformation of shell material under the bending load, the contact of the crack faces may occur in an area, which has been confirmed by the FEA. Although the line-contact assumption made in the theoretical analysis deviates from the real behavior of the contact of the crack faces, the theoretical SIFs and the FEA SIFs are in good agreement. This is because the contact area is very small compared with the 
shell thickness, as demonstrated in the COD plots; the nonlinear part of the curve is trivial.

The effect of shell curvature on the crack closure behavior in shells has been demonstrated by both the theoretical analysis and the FEA. The critical values of the shell parameter $\lambda$ for partial closure determined in the theoretical analysis match the FEA results. 


\section{Chapter 7 Conclusions}

From the theoretical solutions in Chapter 3 and the FEA solutions in Chapter 5 as well as the discussion in Chapter 6, the following conclusions can be drawn from this research:

1. Crack-face closure occurs physically when a cracked shell is subjected to a bending load. Traditional shell theories allow the crack surfaces to overlap at the compressive edges, which is not correct physically. The simulation of crack closure in a cracked shell under bending in this research describes correctly the physical behavior that the crack surfaces do not penetrate at the compressive edges when they are rotated due to the bending load. The obtained SIFs in the closure case are compared with those in the non-closure case. It is found that crack-face closure has a significant effect on the SIFs. The improved shell theory with consideration of crack-face closure will make a significant impact on traditional shell theories.

2. The formulation of the shell problem is based on shear deformation theory, incorporating with crack-face closure effect which is modeled as the crack surfaces are in contact at the compressive edges. Thus a line-contact force is induced at the compressive edge. The contact force can be translated to the mid-plane of the shell, accompanied with an additional distributed bending moment. 
3. Different from plates, due to curvature effect, crack closure behavior in shells is more complex because full-length closure does not always occur in shells, depending on the shell curvature and the nature (direction) of the bending load, partial closure may occur when the shell curvature is large, which is characterized by negative values of the contact force computed assuming full-line closure. Then the constraint condition of crack-face closure would be a mixed-boundary case, that is, along the crack length, either the normal displacement of the crack face at the compressive edges is equal to zero or the contact pressure is equal to zero.

4. The solution to the problem is to solve a pair of singular integral equations. When complete closure occurs, with the closure constraint the coupled integral equations are reduced to a single equation. The unknown contact force can be obtained from the solution. The membrane component and bending component of the SIF are always equal when the closure occurs along the inner surface (positive bending) of the shell while the two components are equal in magnitude but opposite in sign when the closure is along the outer surface (negative bending), which differs from the non-closure solution where the membrane component is not equal to the bending component.

5. The crack closure effect increases the membrane component but decreases the bending component for all shell geometries under either positive bending or negative bending. The membrane component and bending component in positive bending case are not equal to those in the negative bending case when crack closure 
is concerned; they are greater in the former than in the latter; unlike the non-closure condition, the membrane component and bending component do not vary with the nature of the bending load, that is, the membrane component and bending component in the positive bending condition are identical to those in the negative bending condition.

6. The critical value of the shell parameter $\lambda$, which represents the shell curvature, for partial closure varies with the shell geometry and the material orthotropic property. For all shell geometries except for a cylindrical shell containing a circumferential crack, the critical $\lambda$ value decreases with the $E_{1} / E_{2}$ ratio. The critical $\lambda$ value for a cylindrical shell containing a circumferential crack is constant for all $E_{1} / E_{2}$ ratios, which is much higher than that for other shell geometries when the $E_{1} / E_{2}$ ratio is greater than unity.

7. The curvature effect on the crack closure behavior of shells is more significant for positive bending than for negative bending because the critical $\lambda$ values for all shell geometries are higher in negative bending case than in positive bending case.

8. The influence of material orthotropy on the crack closure effect in shells varies with shell geometry. Reduction of the maximum SIF due to crack-face closure varies significantly with material orthotropy for cylindrical shells containing an axial crack, while it is almost constant for different $E_{1} / E_{2}$ ratios for cylindrical shells containing a circumferential crack. For a given shell geometry the material 
orthotropy effect on the crack closure varies with shell curvature, it is stronger for small values of $\lambda$, it is the strongest for plates.

9. Three-dimensional FEA has been conducted to simulate crack closure behavior in various shell geometries under a bending load, which represents the reality of the physical behavior. It is revealed that the contact of the crack faces occur in an area, not on a line, when crack closure occurs. However, since the contact area is very small compared with the shell thickness, the theoretical SIFs based on the linecontact assumption and the FEA SIFs derived from the area-contact simulation are in good agreement; the error can be neglected.

10. Partial closure behavior in shells is confirmed by the FEA. The critical $\lambda$ values for partial closure determined in the theoretical analysis for various geometries match the FEA results. 


\section{References}

1. Sih, G.C. Ed. (1977) Mechanics of Fracture3: Plates and Shells with Cracks. Noordhoff International, Netherlands.

2. Reissner, E. (1958) On some problems in shell theory. Proc. First Symposium on Naval Structural Mechanics, Structure Mechanics, 74-113.

3. Reissner, E. (1946) Stresses and small displacements of shallow spherical shells I. $J$. Math. Phys., 25, 80-85.

4. Reissner, E. (1946) Stresses and small displacements of shallow spherical shells II. J. Math. Phys., 25, 279-300.

5. Naghdi, P. M. (1956) Note on the equations of shallow elastic shells. Quart. Appl. Math., 14, 331-333.

6. Erdogan, F. and Kibler, J. J. (1969) Cylindrical and spherical shells with cracks. Int. J. Fract. Mech., 5, 229-237.

7. Delale, F. and Erdogan, F. (1979) Effect of transverse shear and material orthotropy in a cracked spherical cap. Int. J. Solids Struct., 15, 907-926.

8. Folias, E. S. (1965) An axial crack in a pressurized cylindrical shell. Int. J. Fract. Mech., 1, 104-113.

9. Copley, L. G. and Sanders, J. L. (1969) A longitudinal crack in a cylindrical shell under internal pressure. Int. J. Fract. Mech., 5, 113-131.

10. Folias, E. S. (1967) A circumferential crack in a pressurized cylindrical shell. Int. J. Fract. Mech., 3, 1-12.

11. F. Erdogan, F. and Ratwani, M. (1970) Fatigue and fracture of cylindrical shells containing a circumferential crack. Int. J. Fract. Mech., 6, 379-392.

12. Erdogan, F. and Ratwani, M. (1972) A circumferential crack in a cylindrical shell under torsion. Int. J. Fract. Mech., 8, 87-95.

13. Krenk, S. (1978) Influence of transverse shear on an axial crack in a cylindrical shell. Int. J. Fract., 14, 123-143.

14. Delale, F. and Erdogan, F. (1979) Transverse shear effect in a circumferentially cracked cylindrical shell. Quart. Appl. Math., 37, 239-258. 
15. Delale, F. and Erdogan, F. (1983) The crack problem in a specially orthotropic shell with double curvature. Eng. Fract. Mech., 18, 529-544.

16. Young, M. J. and Sun, C. T. (1992) Influence of crack closure on the stress intensity factor in bending plates - a classical plate solution. Int. J. Fract., 55, 81-93.

17. Young, M. J. and Sun, C. T. (1993) On the strain energy release rate for a cracked plate subjected to out-of-plane bending moment. Int. J. Fract., 60, 227-247.

18. Liu, R., Wang, C. H. and Bathgate, R. G. (1999) Crack closure in spherical shells. Int. J. Fract., 99, 307-323.

19. Liu, R., Wang, C.H. and Bathgate, R.G. (1999) Fracture analysis of cracked macadamia nutshells under contact load between two rigid plates. J. agric. Engng. Res., 74, 243-250.

20. Ugural, A.C. (1981) Stresses in Plates and Shells. McGraw-Hill, New York.

21. Kraus, H. (1967) Thin Elastic Shells. John Wiley \& Sons, Inc., New York.

22. Kirchhoff, G.(1850) Uber das Gleichgewicht und die Bewegung einer elastischen Scheibe, J. Reine Angew. Math., 40, 51-58.

23. Sayir, M. and Mitropoulos, C. (1980) On elementary theories of linear elastic beams, plates and shells. J. Appl. Math. Phys., 31, 1-55.

24. Maewal, A. (1984) An asymptotic method for construction of plate theories: cylindrical bending of a homogeneous plate. Comp. Meth. Appl. Mech. Eng., 43, 127-136.

25. Seide, P. (1975) Small elastic deformations of thin shells. Noordhoff International, Leyden.

26. Reismann, H. (1988) Elastic Plates: Theory and Applications. John Wiley and Sons, New York.

27. Love, A.E.H. (1944) A Treatise on the Mathematical Theory of Elasticity. 4th edition. Dover Publications Inc., New York.

28. Rissner, E., (1944) On the theory of bending of elastic plates. J. Math. and Phys., 23, 184-191.

29. Rissner, E., (1945) The effect of transverse shear deformations on the bending of elastic plates. J. Appl. Mech., 12, A69-A77. 
30. Lo, K.H. (1977) A high-order theory of plate deformation. J. Appl. Mech., 99, 663-676.

31. Tsui, Edward Y.W. (1968) Stresses in Shells of Revolution. Pacific Coast Publishers, Menlo Park.

32. Updike, D.P. and Kalnins, A. (1972) Contact pressure between an elastic spherical shell and a rigid plate. Trans. ASME, J.Appl. Mech. 39, 1110-1114.

33. Sih, G.c. and Hagendorf, H.C. (1974) 'A new theory of spherical shells with cracks'. In: Thin-shell Structures: Theory, Experiment, and Design. Eds: Y.C. Fung and E.E. Sechler. Prentice-Hall, New Jersey. pp. 519-545.

34. Ang, D.D., Folias, E.S. and Williams, M.L. (1964) The bending stress in a cracked plate on an elastic foundation. J.Appl. Mech. 30, 245-251.

35. Sih, G.C. and Setzer, D.E. (1964) Discussion of the bending stress in a cracked plate on an elastic foundation. J.Appl. Mech. 31, 365-367.

36. Folias, E.S. (1965) The stresses in a cracked spherical shell. J.Mathematics Physics 44, 164-176.

37. Knowles, J.K. and Wang, N.M. (1960) On the bending of an elastic plate containing a crack. J.Mathematical Physics 39, 223-236.

38. Wang, N.M. (1968) Effects of plate thickness on the bending of an elastic plate containing a crack. J.Mathematical Physics 47, 371-390.

39. Sih, G.C. and Dobreff, P.S. (1971) Crack-like imperfections in a spherical shell. Glasgow Mathematical Journal 12, 65-88.

40. Sih, G.C. (1971) A review of the three-dimensional stress problem for a cracked plate. Int. J.Fract. Mech. 7, 39-61.

41. Updike, D.P. and Kalnins, A. (1970) Axisymmetric behavior of an elastic spherical shell compressed between rigid Plates. Trans. ASME, J.Appl. Mech. 37, 635-640.

42. Jones, D.P.and Swedlow, J.L. (1975) The influence of crack closure and elasto-plastic flow on the bending of a cracked plate. Int. J.Fracture 11, 897-914.

43. Heming Jr., F.S. (1980) Sixth order analysis of crack closure in bending of an elastic plate. Int.J.Fracture, 16, 289-304. 
44. Alwar, R.S. and Ramachandran Nambissan, K.N. (1983) Influence of crack closure on the stress intensity factor for plates subjected to bending- a 3-D finite element analysis. Eng. Fract. Mech., 17, 323-333.

45. Smith, D.G. and Smith, C.W. (1970) A photoelastic evaluation of the influence of closure and other effects upon the local bending stresses in cracked plates. Int.J. Fract. Mech., 6, 305-317.

46. Rybicki, E.F. and Kanninen, M.F. (1977) A finite element calculation of stress intensity factors by a modified crack closure integral. Eng. Fract. Mech., 9, 931-938.

47. Byskov, E. (1970) The calculation of stress intensity factors using the finite-element method. Int. J. Fract. Mech., 6 (2), 159-167.

48. Benzley, S.E. (1974) Representation of singularities with isoparametric finite element. Int. J. Numer. Methods Engng., 8, 537-545.

49. Jordan, S., Padlog, J., Hopper, A.T., Rybicki, E.F., Hulbert, L.E. and Kanninen, M.F. (1973) Development and application of improved analytical techniques for fracture analysis using MAGIC III. Wright-Patterson AFB AFFDL-TR-73-61, Air Force Flight Dynamics Laboratory, Ohio.

50. Watwood, V.B. (1969) The finite-element method for prediction of crack behavior. Nucl. Engng Design, 11, 323-332.

51. Anderson, G.P., Ruggles, V.L. and Stibor, G.S. (1971) Use of finite-element computer programs in fracture mechanics. Int. J. Fract. Mech., 7, 63-76.

52. Dixon, J.R. and Pook, L.P. (1969) Stress intensity factors calculated generally by the finite-element technique. Nature 224, 166.

53. Mowbray, D.F. (1970) A note of the finite-element method in linear fracture mechanics. Eng. Fract. Mech., 2, 173.

54. Parks, D.M. (1973) A stiffness derivative finite-element technique for determination of elastic tip stress intensity factors. NASA NGL 40-002-080/13.

55. Hellen, T.K. (1973) The finite-element calculation of stress-intensity factors using energy techniques. 2nd Int. Conf. On Structural Mechanics in Reactor Technology, 10-14 Sept., Berlin, Germany paper G5/3.

56. Hellen, T.K. and Blackburn, W.S. (1975) The calculation of stress intensity factors in two-and three-dimensions using finite elements. Presented at the Second National Congress On pressure Vessels and Piping, 23-27 June, San Francisco, California. 
57. Rice, J.R. (1968) A path independent integral and the approximate analyusis of strain concentration by notches and cracks. Trans. ASME, J. Appl. Mech., 35, 379386.

58. G.De Roeck, A.Samartin Quiroga, M.Van Laethem and E.Backx, Eds. (1987) Shell and Spatial Structures: Computational Aspects, Springer-Verlag, Berlin.

59. Parisch, H. (1979) A critical survey of the 9-node degenerated shell element with special emphasis on thin shell application and reduced integration. Computer Methods in Applied Mechanics 20, 323-350.

60. Key, S.W. and Beisinger, Z.E. (1971) The analysis of thin shells by the finite element method, high speed computing of elastic structures. IUTAM Symposium Liege 1, 209-252.

61. Ahmad, S., Irons, B.M. and Zienkiewicz, O.C. (1970) Analysis of thick and thin shell structures by curved finite elements. Int. J. Numer. Methods Engng., 2, 419-451.

62. MacNeal, R. H. (1998) Perspective on finite elements for shell analysis. Finite Elem. Anal. Des., 30, 175-186.

63. Ahmad, S. (1969) Curved Finite Elements in the Analysis of Solid, Shell, and Plate Structures. PhD Thesis, University of Wales.

64. Zienkiewicz, O.C., Too, J. and Taylor, R.L. (1971) Reduced integration technique in general analysis of plates and shells. Int. J. Numer. Methods Eng. 3, 275-290.

65. Belytschko, T., Ong, J.S.J. and Liu, W.K. (1984) A constistent control of spurious singular modes in the 9-node Lagrange element for the Laplace and Mindlin Plate equations. Comp. Methods Appl. Mech. Eng. 44, 269-295.

66. Ausserer, M.F. and Lee, S.W. (1988) An eighteen-node solid element for thin shell analysis, Int. J. Numer. Methods Eng., 26, 1345-1364.

67. Chapelle, D. (2001) Some new results and current challenges in the finite element analysis of shells. Acta Numerica, 215-250.

68. Chapelle, D. and Bathe, K.J. (2003) The Finite Element Analysis of Shells- Fundamentals. Springer-Verlag, Berlin.

69. Arnold, D. N. and Brezzi, F. (1997) Locking-free finite element methods for shells. Math. Comput., 66 (217), 1-14. 
70. Kim, J.H., Kim, Y. H. and Lee, S. W. (2000) An assumed strain formulation of efficient solid triangular element for general shell analysis. Int. J. Numer. Methods Eng., 47, 1481-1497.

71. Chapelle, D. and Bathe, K. J. (1998) Fundamental considerations for the finite element analysis of shell structures. Comput. Struct., 66 (1), 19-36.

72. Szabo, B.A. and Sahrmann, G.j. (1988) Hierarchic plate and shell models based on p-extension. Int. J. Numer. Methods Eng. 26, 1858-1881.

73. Leino, Y. and Pitkaranta, J. (1992) On the Membrane Locking of H-P Finite Elements in a Cylindrical Shell Problem. Inst. Math. Res. Reports A 311, Helsinki Univ. of Technology.

74. Shimizu, T. and Sano, T. (1996) Penalty method contact-friction algorithm and expression of tools by B-spliine function-penalty method contact algorithm and rigid plastic FEM. Journal of JSTP 37, 225-230.

75. Tan, X.G. and Vu-Quoc, L. (2005) Efficient and accurate multiplayer solid-shell element: Non-linear materials at finite strain. Int. J. Numer. Methods Eng. 63, 2124-2170

76. Cho, J.W., Yang, D.Y. and Chung, W.J. (2002) A simplified approach for incorporating thickness stress in the analysis of sheet metal forming using shell elements. Int. J. Numer. Meth. Engng. 53 (10), 2311-2327.

77. Sze, K.Y. (2002) Three-dimensional continuum finite element models for plate/shell analysis. Prog. Struct. Engng Mater. 4, 400-407.

78. Parisch, H. (1995) A continuum-based shell theory for non-linear applications. Int. J. Numer. Methods Eng. 38, 1855-1883.

79. Hauptmann, R. and Schweizerhof, K. (1998) A systematic development of 'solid-shell' element formulations for linear and non-linear analyses employing only displacement degrees of freedom. Int. J. Numer. Methods Eng. 42, 49-69.

80. Zienkiewicz, O.C. (1977) The Finite Element Method. Third edition. McGraw-Hill, London.

81. Seweryn, A. (2002) Modeling of singular stress fields using finite element method. Int. J. Solids Struct. 39, 4787-4804.

82. Kanninen, M.F. and Popelar,C.H. (1985) Advanced Fracture Mechanics. Oxford University Press, New York. 
83. Broek, D. (1978) Determination of stress intensity factors, Elementary Engineering Fracture Mechanics, Alphen aan den Rijn, The Netherlands.

84. Ye, J.Q. (2003) Laminated Composite Plates and Shells. Springer, London.

85. Reddy, J.N.(2004) Mechanics of Laminated Composite Plates and Shells: Theory and Analysis. Second edition. CRC Press, Boca Raton.

86. Valot, E. and Vannucci, P. (2005) Some exact solutions for fully orthotropic laminates. Compos. Struct. 69, 157-166.

87. Ambartsumyan, S.A. (1953) Calculation of Laminated Anisotropic Shells, Izvestiia Akademiia Nauk Armenskoi SSR, Ser. Fiz. Mat. Est. Tekh. Nauk., 6(3), p.15

88. Ambartsumyan, S.A. (1956) On the theory of anisotropic shellow shells. NACA Tech. Memo. 1424.

89. Ambartsumyan, S.A. (1964) Theory of anisotropic shallow shells. NASA Tech. Transl. F-118.

90. Flugge, W. (1960) Stresses in Shells. Springer-Verlag, Berlin.

91. Dym, C.L. (1974) Introduction to the theory of shells. Pergamon, New York.

92. Librescu, L. (1975) Elastostatics and Kinetics of Anisotropic and Heterogeneous Shell-Type Structures. Noordhoff, Leyden.

93. Dong, S.B., Pister, K.S. and Taylor, R.L. (1962) On the theory of laminated anisotropic shells and plates. J. Aerosp. Sci. 29, 969-975.

94. Donnell, L.N.(1933) Stability of Thin Walled Tubes in Torsion. NASA Report.

95. Naghdi, P.M. (1963) Foundations of Elastic Shell Theory, Progress in Solid Mechanics, 4, Sneddon, I.N. and Hill, R. (Eds.), North-Holland, Amsterdam, The Netherlands.

96. Stavsky, Y. (1963) Thermoelasticity of heterogeneous aeolotropic plates. Journal of Engineering Mechanics Division EM2, 89-105.

97. Gulati, S.T. and Essenberg, F. (1967) Effects of anisotropy in axisymmetric cylindrical shells. J.Appl. Mechan., 34, 659-666.

98. Whitney, J.M. and Sun, C.T. (1974) A refined theory for laminated anisotropic, cylindrical shells. J.Appl. Mechan., 41, 471-476. 
99. Zhou, C.Q. and Qiu, Y.Y. (1985) Elastoplastic analysis of cylindrically orthotropic composite thick-walled tube. Appl. Math. and Mech. 6 (3), 241-248.

100. Verijenko, V. E., Adali, S. and Tabakov, P.Y. (1995) Stress distribution in continuously heterogeneous thick laminated pressure vessels. Composites for the Pressure Vessel Industry, PVP 302, ASME.

101. Kumosa, M. (1991) Bulging effects in circumferentially cracked orthotropic cylindrical shells. Eng. Fract. Mech., 38, n 4-5, 255-62.

102. Gulgec, M. and Yahsi, O. S. (1993) Cylindrical shell with a fixed end containing a circumferential through crack under the general loading, ASME, Pressure Vessels Piping Div. Publ. PVP, 269, Recent Advances in Structural Mechanics, 171-179.

103. Yahsi, O. S., and Erdogan, F. (1983) Cylindrical shell with an arbitrarily oriented crack. Int.J. Solids Struct., 19 (11), 955-972.

104. Theocaris, P.S. and Ioakimidis, N.I. (1977) Numerical integration methods for the solution of singular integral equations. Quart. Appl. Math., 35, 173-183.

105. Benham, P.P., Crawford, R.J. and Armstrong, C.G. (1996) Mechanics of Engineering Materials. Singapore, Longman Group Limited.

106. Murakami, Y., Ed. (1987) Cracks in shell. Stress Intensity Factors Handbook. Pergamon Press. 


\section{Appendices}

\section{Appendix A}

\section{Asymptotic Solutions of the Kernels $\boldsymbol{k}_{i j}$ in the Integral Equations}

The integration kernel functions $k_{i j}$, which are bounded in the interval $-\sqrt{c} \leq(\eta, \tau) \leq \sqrt{c}$, are expressed as:

$$
\begin{aligned}
k_{11}(\eta, \tau)= & \int_{0}^{\infty}\left[2 \alpha^{2} \sum_{j=1}^{4} Q_{j}(\alpha)-1\right] \sin \alpha(\tau-\eta) d \alpha, \\
k_{12}(\eta, \tau)= & \int_{0}^{\infty} 2 \alpha^{2} \sum_{j=1}^{4} N_{j}(\alpha) \sin \alpha(\tau-\eta) d \alpha, \\
k_{21}(\eta, \tau)= & -\frac{2}{\lambda_{0}^{2}} \int_{0}^{\infty} \sum_{j=1}^{4} \frac{p_{j}^{2}\left(m_{j}^{2}-v \alpha^{2}\right)}{\left(\kappa p_{j}-1\right)\left(\lambda_{2}^{2} m_{j}^{2}-\lambda_{1}^{2} \alpha^{2}\right)} Q_{j}(\alpha) \sin \alpha(\tau-\eta) d \alpha, \\
k_{22}(\eta, \tau)= & -\frac{2}{\lambda_{0}^{4}} \int_{j}^{\infty}\left[\lambda_{0}^{2} \sum_{j=1}^{4} \frac{p_{j}^{2}\left(m_{j}^{2}-v \alpha^{2}\right)}{\left(\kappa p_{j}-1\right)\left(\lambda_{2}^{2} m_{j}^{2}-\lambda_{1}^{2} \alpha^{2}\right)} N_{j}(\alpha)\right. \\
& \left.-\kappa(1-v)^{2} \alpha r_{1}+\frac{\left(1-v^{2}\right)}{2}\right] \sin \alpha(\tau-\eta) d \alpha,
\end{aligned}
$$

where $r_{1}=-\sqrt{\alpha^{2}+2 /(\kappa(1-v))}, m_{j}^{2}=p_{j}+\alpha^{2}, j=1,2,3$ and 4. $p_{j}$ is the root of the characteristic equation of the problem, expressed below

$$
p^{4}+a_{1} p^{3}+a_{2} p^{2}+a_{3} p+a_{4}=0,
$$

where $a_{1}=-\kappa \lambda_{2}^{4}, a_{2}=2 \kappa \lambda_{1}^{2} \lambda_{2}^{2} \alpha^{2}+\lambda_{2}^{4}\left(1-2 \kappa \alpha^{2}\right)$,

$$
\begin{aligned}
& a_{3}=-\kappa \alpha^{4}\left(\lambda_{2}^{2}-\lambda_{1}^{2}\right)^{2}+2 \lambda_{2}^{2} \alpha^{2}\left(\lambda_{2}^{2}-\lambda_{1}^{2}\right), \\
& a_{4}=\alpha^{4}\left(\lambda_{2}^{2}-\lambda_{1}^{2}\right)^{2} .
\end{aligned}
$$


The four roots are

$$
\begin{aligned}
& p_{1}=\frac{1}{4}\left\{-a_{1}-b_{1}+\left[\left(a_{1}+b_{1}\right)^{2}-8\left(a_{5}+b_{2}\right)\right]^{1 / 2}\right\}, \\
& p_{2}=\frac{1}{4}\left\{-a_{1}-b_{1}-\left[\left(a_{1}+b_{1}\right)^{2}-8\left(a_{5}+b_{2}\right)\right]^{1 / 2}\right\}, \\
& p_{3}=\frac{1}{4}\left\{-a_{1}+b_{1}+\left[\left(a_{1}-b_{1}\right)^{2}-8\left(a_{5}-b_{2}\right)\right]^{1 / 2}\right\}, \\
& p_{4}=\frac{1}{4}\left\{-a_{1}+b_{1}-\left[\left(a_{1}-b_{1}\right)^{2}-8\left(a_{5}-b_{2}\right)\right]^{1 / 2}\right\},
\end{aligned}
$$

where $b_{1}=\sqrt{a_{1}^{2}-4 a_{2}+4 a_{5}}, b_{2}=\sqrt{a_{5}^{2}-4 a_{4}}$,

$$
\begin{aligned}
& a_{5}=\left(-a_{6}+\sqrt{a_{6}^{2}+a_{7}^{3}}\right)^{1 / 3}+\left(-a_{6}-\sqrt{a_{6}^{2}+a_{7}^{3}}\right)^{1 / 3}+\frac{1}{3} a_{2}, \\
& a_{6}=-\frac{1}{27} a_{2}{ }^{3}+\frac{1}{6} a_{1} a_{2} a_{3}-\frac{1}{2} a_{1}{ }^{2} a_{4}+\frac{4}{3} a_{2} a_{4}-\frac{1}{2} a_{3}{ }^{2}, a_{7}=\frac{1}{3} a_{1} a_{3}-\frac{4}{3} a_{4}-\frac{1}{9} a_{2}{ }^{3} .
\end{aligned}
$$

The expressions of $N_{j}$ and $Q_{j}$ in the $k_{i j}$ equations are

$$
\begin{aligned}
N_{1}= & \frac{1}{D m_{1}}\left(\lambda_{2}^{2} p_{1}+A_{5}\right)\left(\kappa p_{1}-1\right)\left\{A_{1} \kappa^{2} p_{2} p_{3} p_{4}\left(p_{3}-p_{2}\right)\left(p_{4}-p_{2}\right)\left(p_{4}-p_{3}\right)\right. \\
& +A_{1}\left(p_{2}\left(\kappa p_{2}-1\right)\left(p_{4}^{3}-p_{3}^{3}\right)+p_{3}\left(\kappa p_{3}-1\right)\left(p_{2}^{3}-p_{4}^{3}\right)+p_{4}\left(\kappa p_{4}-1\right)\left(p_{3}^{3}-p_{2}^{3}\right)\right) \\
& \left.-A_{2}\left(p_{2}\left(\kappa p_{2}-1\right)\left(p_{4}^{2}-p_{3}^{2}\right)+p_{3}\left(\kappa p_{3}-1\right)\left(p_{2}^{2}-p_{4}^{2}\right)+p_{4}\left(\kappa p_{4}-1\right)\left(p_{3}^{2}-p_{2}^{2}\right)\right)\right\}, \\
Q_{1}= & \frac{1}{D m_{1}}\left(\lambda_{2}^{2} p_{1}+A_{5}\right)\left(\kappa p_{1}-1\right) A_{3}\left\{A_{4} p_{2} p_{3} p_{4}\left(p_{3}-p_{2}\right)\left(p_{4}-p_{2}\right)\left(p_{4}-p_{3}\right)\right. \\
& \left.-p_{2}^{2} p_{3}^{2}\left(p_{3}-p_{2}\right)-p_{3}^{2} p_{4}^{2}\left(p_{4}-p_{3}\right)-p_{2}^{2} p_{4}^{2}\left(p_{2}-p_{4}\right)\right\},
\end{aligned}
$$

where $\left.D=\left(p_{2}-p_{1}\right)\left(p_{3}-p_{1}\right) p_{4}-p_{1}\right)\left(p_{3}-p_{2}\right)\left(p_{4}-p_{2}\right)\left(p_{4}-p_{3}\right)$,

$$
\begin{aligned}
& A_{1}=\kappa(1-v)\left(\alpha^{2}+r_{1}^{2}\right) /\left(2 \alpha \lambda_{0}{ }^{2}\right), A_{2}=\alpha(1-v) / \lambda_{0}{ }^{2}, A_{3}=1 /\left(\alpha\left(\lambda_{1}^{2}-\lambda_{2}^{2}\right)\right), \\
& A_{4}=\kappa+\lambda_{2}^{2} /\left(\alpha^{2}\left(\lambda_{1}^{2}-\lambda_{2}^{2}\right)\right), A_{5}=\left(\lambda_{2}^{2}-\lambda_{1}^{2}\right) \alpha^{2} .
\end{aligned}
$$


Let $N_{1}=N_{1}(1,2,3,4)$ be the indices function of $m_{j}$ and $p_{j}$, then for $N_{2}, N_{3}$ and $N_{4}$, we have

$$
N_{2}=N_{1}(2,3,4,1), N_{3}=N_{2}(3,4,1,2), N_{4}=N_{3}(4,1,2,3)
$$

Similarly, the expressions of $Q_{2}, Q_{3}$ and $Q_{4}$ can be derived. 


\section{Appendix B}

Gauss-Chebyshev Integration Formulas for Specially Orthotropic Shells

$$
\begin{aligned}
& \sum_{j=1}^{n} w_{j}\left[\frac{g_{1}\left(t_{j}\right)}{\left(t_{j}-d_{i}\right)}+\sqrt{c} \sum_{m=1}^{2} k_{1 m}\left(\sqrt{c} d_{i}, \sqrt{c} t_{j}\right) g_{m}\left(t_{j}\right)\right]=2 \pi F_{1}\left(\sqrt{c} d_{i}\right), \quad i=1,2, \ldots, n-1, \\
& \sum_{j=1}^{n} w_{j}\left[\frac{1-v^{2}}{\lambda_{0}^{4}} \cdot \frac{g_{2}\left(t_{j}\right)}{t_{j}-d_{i}}+\sqrt{c} \sum_{m=1}^{2} k_{2 m}\left(\sqrt{c} d_{i}, \sqrt{c} t_{j}\right) g_{m}\left(t_{j}\right)\right]=2 \pi \frac{h}{a} F_{2}\left(\sqrt{c} d_{i}\right), \quad i=1,2, \ldots, n-
\end{aligned}
$$

1 ,

$\sum_{j=1}^{n} w_{j} g_{k}\left(t_{j}\right)=0, \quad k=1,2$,

$t_{j}=\cos \left(\pi \frac{j-1}{n-1}\right), \quad j=1,2, \ldots, n, d_{i}=\cos \left(\pi \frac{2 i-1}{2 n-2}\right), i=1,2, \ldots, n-1$,

$W_{1}=W_{n}=\frac{\pi}{2(n-1)}, w_{j}=\frac{\pi}{n-1}, \quad j=2,3, \ldots, n-1$. 


\section{Appendix C}

Gauss-Chebyshev Integration Formulas for Isotropic Shells

$$
\begin{gathered}
\sum_{j=1}^{n} W_{i}\left[\frac{g_{1}\left(\tau_{j}\right)}{\tau_{j}-\eta_{i}}+\sum_{m=1}^{2} k_{1 m}\left(\eta_{i}, \tau_{j}\right) g_{m}\left(\tau_{j}\right)\right]=2 \pi F_{1}\left(\eta_{i}\right), \quad i=1,2, \ldots, n-1, \\
\sum_{j=1}^{n} W_{j}\left[\frac{1-v^{2}}{\lambda_{0}^{4}} \cdot \frac{g_{2}\left(\tau_{j}\right)}{\tau_{j}-\eta_{i}}+\sum_{m=1}^{2} k_{2 m}\left(\eta_{i}, \tau_{j}\right) g_{m}\left(\tau_{j}\right)\right]=2 \pi \frac{h}{a} F_{2}\left(\eta_{i}\right), \quad i=1,2, \ldots, n-1, \\
\sum_{j=1}^{n} W_{j} g_{k}\left(\tau_{j}\right)=0, \quad k=1,2 \\
\tau_{j}=\cos \left(\pi \frac{j-1}{n-1}\right), \quad j=1,2, \ldots, n, \\
\eta_{i}=\cos \left(\pi \frac{2 i-1}{2 n-2}\right), \quad i=1,2, \ldots, n-1, \\
W_{1}=W_{n}=\frac{\pi}{2(n-1)}, \quad W_{j}=\frac{\pi}{n-1}, \quad j=2,3, \ldots, n-1 .
\end{gathered}
$$




\section{Appendix D}

\section{FORTRAN Codes for Numerical Solutions of Theoretical Analysis}

\section{Program 1 - Specially Orthotropic Shell with Double Curvature}

C----MAIN PROGRAM: CRACK CLOSURE ANALYSIS ON SPHERICAL SHELL WITH DIFFERENT E1 AND E2

PARAMETER $(\mathrm{N}=41, \mathrm{~T}=60.0)$

REAL LAM0,LAM1,LAM14, LAM, LAM4, LAM2, LAM24, KA, Km, Kb DOUBLE PRECISION REK11(N,N), REK12(N,N), REK21(N,N), REK22(N,N),

\& $\quad \mathrm{A}\left(2 * \mathrm{~N}, 2^{*} \mathrm{~N}\right), \mathrm{A} 1\left(2 * \mathrm{~N}, 2^{*} \mathrm{~N}\right), \mathrm{A} 2\left(2^{*} \mathrm{~N}, 2^{*} \mathrm{~N}\right), \mathrm{B}(2 * \mathrm{~N}), \mathrm{B} 1(2 * \mathrm{~N}), \mathrm{B} 2\left(2^{*} \mathrm{~N}\right)$,

\& $\quad \mathrm{G}\left(2^{*} \mathrm{~N}\right), \mathrm{W}(\mathrm{N}), \mathrm{TT}(\mathrm{N}), \mathrm{YY}(\mathrm{N}), \mathrm{P}(\mathrm{N})$

COMMON

DETA,CE,LAM1,LAM14,LAM,LAM4,LAM2,LAM24,KA,VV,AA,HH,F1,F2,A0

OPEN(UNIT=10, FILE='ELLIPE.DAT', STATUS='UNKNOWN',

\& FORM='FORMATTED', POSITION='APPEND', IOSTAT=ISTAT )

IF(ISTAT/=0) PRINT*, 'FILE OPEN FAIL: ISTAT=',ISTAT

$\mathrm{E} 12=26.67$

$\mathrm{CE}=\mathrm{E} 12 * *(1.0 / 4)$

$\operatorname{IF}(\mathrm{E} 12=0.725 . \mathrm{OR} \cdot \mathrm{E} 12==1.38)$ THEN

$\mathrm{V} 1=0.1966$

$\mathrm{V} 2=0.2174$

ELSE

$\mathrm{V} 1=0.0075$

$\mathrm{V} 2=0.2$

END IF

$\mathrm{VV}=\mathrm{SQRT}(\mathrm{V} 1 * \mathrm{~V} 2)$

$\mathrm{IF}(\mathrm{E} 12==1.0) \mathrm{VV}=1.0 / 3$

$\mathrm{R} 12=0.5$

$\mathrm{DETA}=\mathrm{SQRT}(\mathrm{R} 12) / \mathrm{CE}$
$\mathrm{AA}=1$
$\mathrm{HH}=1$
LAM4 $=12 *\left(1-\mathrm{VV}^{*} \mathrm{VV}\right) * \mathrm{AA} * \mathrm{AA} /(\mathrm{HH} * \mathrm{HH})$ 


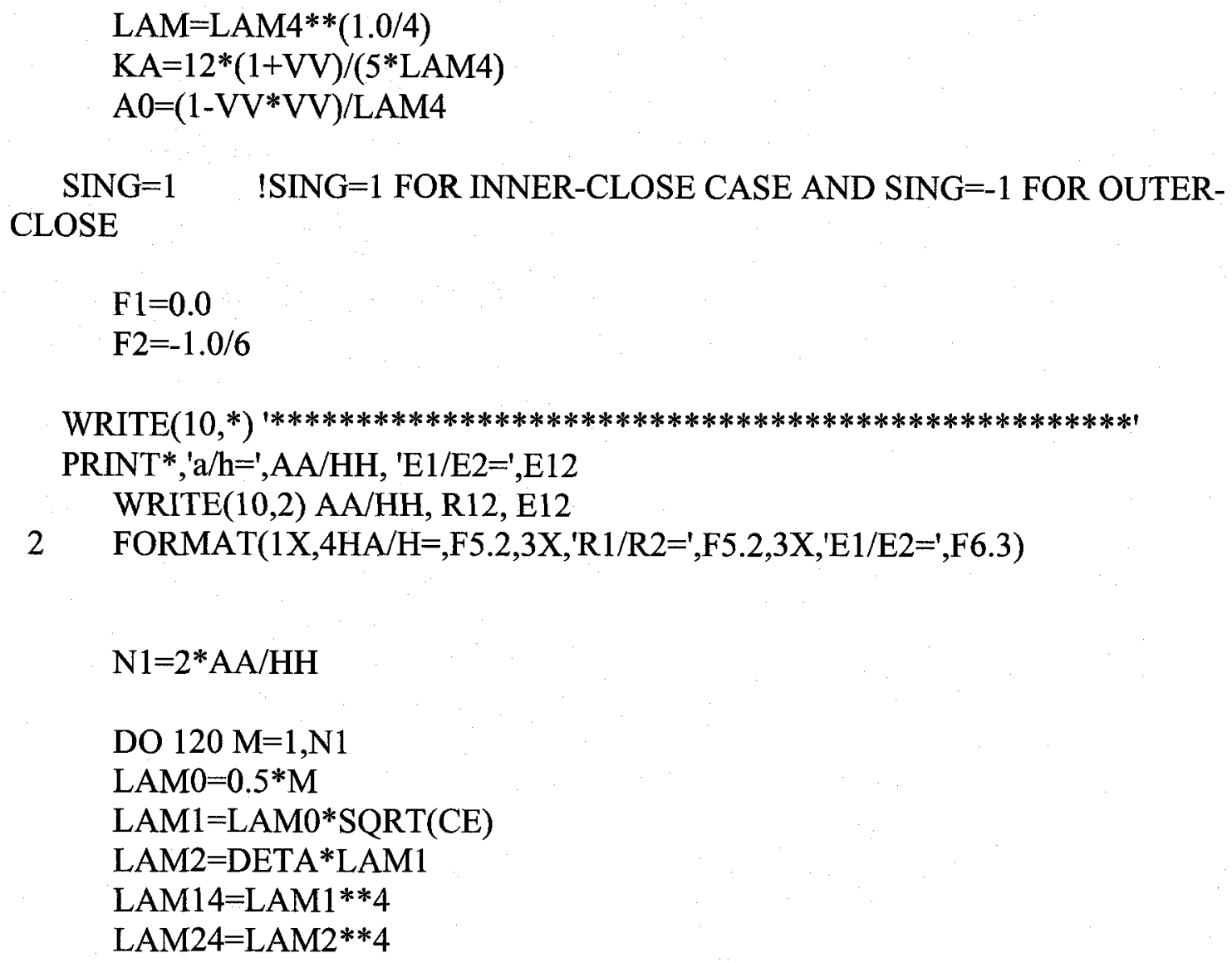

CALL KERLFUNC(N,T,REK11,REK12,REK21,REK22)

CALL OPENEQ(N,A1,B1,W,TT,YY,REK11,REK12,REK21,REK22)

IF(SING==1) THEN

CALL CLOSEEQINNER(N,A2,B2,W,TT,YY,REK11,REK12,REK21,REK22) ELSE

CALL CLOSEEQOUTER(N,A2,B2,W,TT,YY,REK11,REK12,REK21,REK22) END IF

C-----IF ONLY CACULATE OPEN CASE, PLEASE IMPLEMENT THESE TWO SENTANCES, OTHERWISE OMIT THEM 
CALL SOLVEEQ $(2 * \mathrm{~N}, \mathrm{~A} 1, \mathrm{~B} 1, \mathrm{G})$

GO TO 110

C----IF ONLY CACULATE OPEN CASE, PLEASE IMPLEMENT THESE TWO SENTANCES, OTHERWISE OMIT THEM

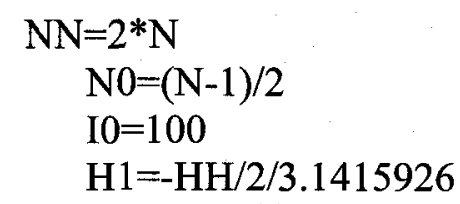

DO $80 \mathrm{~K}=1$, N0

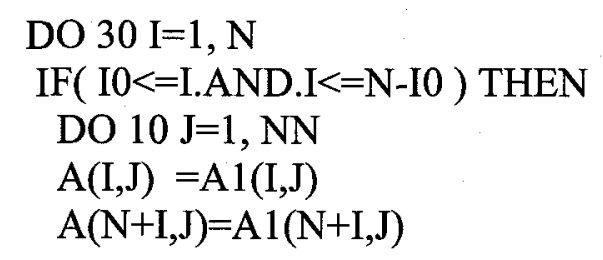

$\mathrm{B}(\mathrm{I})=\mathrm{B} 1(\mathrm{I})$

$\mathrm{B}(\mathrm{N}+\mathrm{I})=\mathrm{B} 1(\mathrm{~N}+\mathrm{I})$

ELSE

DO $20 \mathrm{~J}=1, \mathrm{NN}$

$\mathrm{A}(\mathrm{I}, \mathrm{J})=\mathrm{A} 2(\mathrm{I}, \mathrm{J})$

$20 \quad \mathrm{~A}(\mathrm{~N}+\mathrm{I}, \mathrm{J})=\mathrm{A} 2(\mathrm{~N}+\mathrm{I}, \mathrm{J})$

$\mathrm{B}(\mathrm{I})=\mathrm{B} 2(\mathrm{I})$

$\mathrm{B}(\mathrm{N}+\mathrm{I})=\mathrm{B} 2(\mathrm{~N}+\mathrm{I})$

END IF

30 CONTINUE

\section{CALL SOLVEEQ(NN,A,B,G)}

DO $50 \mathrm{I}=1, \mathrm{~N}-1$

$\mathrm{P}(\mathrm{I})=0.0$

DO $40 \mathrm{~J}=1, \mathrm{~N}$

$\&$

$\mathrm{P}(\mathrm{I})=\mathrm{P}(\mathrm{I})+\mathrm{SING} * \mathrm{H} 1 * \mathrm{~W}(\mathrm{~J}) *(\mathrm{G}(\mathrm{J}) /(\mathrm{TT}(\mathrm{J})-\mathrm{YY}(\mathrm{I}))+\mathrm{G}(\mathrm{J}) * \mathrm{REK} 11(\mathrm{I}, \mathrm{J})$ 
$\mathrm{PP}=\mathrm{SNGL}(\mathrm{P}(\mathrm{I}))$

!WRITE(10,4) K,I,PP,YY(I)

4 FORMAT(1X,'K=',I2,3X,'I=',I2,3X,'P=',F10.4,2X,'Y=',F7.4)

50 CONTINUE

!WRITE $\left(10,{ }^{*}\right)$

$\mathrm{I} 0=100$

DO $60 \mathrm{I}=1$, N0

$60 \mathrm{IF}(\mathrm{P}(\mathrm{I})<-1.0 / 10 * * 8 . \mathrm{AND} . \mathrm{I}<\mathrm{I} 0) \mathrm{I} 0=\mathrm{I}$

WRITE $(*, *)$ 'K=',K,'I0=',I0

$\mathrm{IF}(\mathrm{I} 0==100)$ GOTO 90

80 CONTINUE

90 IF $(\mathrm{K}<=\mathrm{N} 0)$ THEN

WRITE $(*, *)$ 'ITERATION CONVERGENCE, $\mathrm{K}=$ ', $\mathrm{K}$

ELSE

WRITE $(*, *)$ 'ITERATION FAIL, $\mathrm{K}=$ ',K

END IF

$\mathrm{J} 0=\mathrm{N} 0$

DO $100 \mathrm{I}=1, \mathrm{~N} 0$

$100 \operatorname{IF}(\operatorname{ABS}(\mathrm{P}(\mathrm{I}))<=1.0 / 10 * * 9 . \mathrm{AND} \cdot \mathrm{I}<\mathrm{J} 0) \mathrm{J} 0=\mathrm{I}$

$\mathrm{IF}(\mathrm{J} 0=-\mathrm{N} 0)$ THEN

PRINT*', 'CRACK IS CLOSED, J0=',J0

WRITE $\left(10,{ }^{*}\right)$ 'CRACK IS CLOSED, J0=',J0

ELSE

PRINT*, 'CRACK IS OPEN-CLOSE'

PRINT*, 'J0=',J0,'P(J0)=',P(J0)

WRITE $\left(10,{ }^{*}\right)$ 'CRACK IS OPEN-CLOSE'

WRITE $\left(10,{ }^{*}\right)$ 'J0=',J0, 'Y(J0)=',YY(J0)

END IF

$110 \mathrm{Km}=-0.5^{*} \mathrm{G}(1)$

$\mathrm{Kb}=-\mathrm{HH} /(4 * \mathrm{AA}) * \mathrm{G}(\mathrm{N}+1)$

PRINT*,'LAM0=',LAM0,'Km=',Km,'Kb=',Kb 


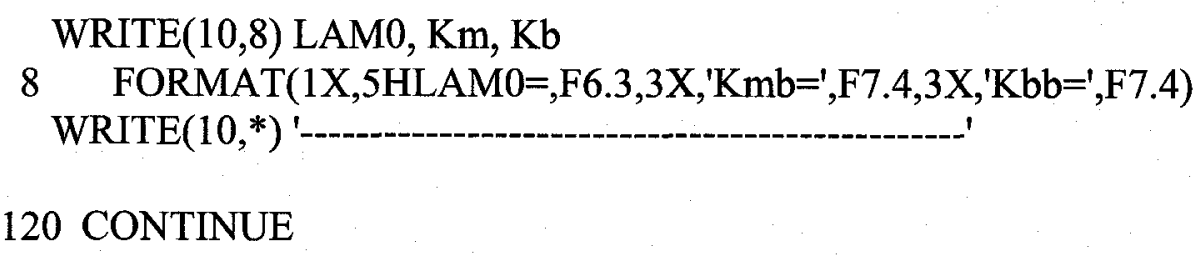

CLOSE(10)

STOP

END

C----- THIS IS THE SUBPROGRAM FOR SOLVING THE KERLFUNCTIONS

SUBROUTINE KERLFUNC(N,T,REK11,REK12,REK21,REK22)

REAL LAM1,LAM14, LAM, LAM4, LAM2, LAM24, KA, LAM0

DOUBLE PRECISION REK11(N,N),REK12(N,N), REK21(N,N),REK22(N,N),

\& FREK11(20000),FREK12(20000),FREK21(20000),FREK22(20000)

COMMON

DETA,CE,LAM1,LAM14,LAM,LAM4,LAM2,LAM24,KA,VV,AA,HH,F1,F2,A0

COMPLEX P1, P2, P3, P4, M1, M2, M3, M4, N1, N2, N3, N4,

\& $\quad \mathrm{Q} 1, \mathrm{Q} 2, \mathrm{Q} 3, \mathrm{Q} 4, \mathrm{CA}, \mathrm{CB}, \mathrm{CC}, \mathrm{CD}, \mathrm{CX}, \mathrm{CY}, \mathrm{P}, \mathrm{FP}$

DOUBLE PRECISION A, B, C, D, E, B0, B1, Y, D0,D1,PR,PI

$\mathrm{FP}(\mathrm{P})=\mathrm{P} * * 4+\mathrm{B}^{*} \mathrm{P} * * 3+\mathrm{C} * \mathrm{P} * * 2+\mathrm{D} * \mathrm{P}+\mathrm{E}$

$\mathrm{PAI}=3.141592653589798$

$\mathrm{DA}=0.01$

$\mathrm{M}=\mathrm{T} / \mathrm{DA}$

C----- FINDING THE ROOTS Pj AND Mj OF THE POLYNOMIAL EQUATIONS

$\mathrm{P} 1=\left(\mathrm{KA}^{*} \mathrm{LAM} 24+\mathrm{LAM} 2 * * 2 * \operatorname{CSQRT}\left(\mathrm{CMPLX}\left(\mathrm{KA}^{* *} 2 * \mathrm{LAM} 24-4,0.0\right)\right)\right) / 2$

$\mathrm{P} 2=\left(\mathrm{KA}^{*}\right.$ LAM24-LAM2 $* * 2 *$ CSQRT(CMPLX $\left(\mathrm{KA}^{* *} 2 *\right.$ LAM24-4,0.0)) $) / 2$

DO $8 \mathrm{~K}=1, \mathrm{M}$

$\mathrm{AL}=\mathrm{K} * \mathrm{DA}$
$\mathrm{B}=-\mathrm{KA} * \mathrm{LAM} 24$
$\mathrm{C}=2 * \mathrm{KA}^{*}(\mathrm{LAM} 1 * \mathrm{LAM} 2 * \mathrm{AL}) * * 2+\mathrm{LAM} 24 *\left(1.0-2 * \mathrm{KA}^{*} \mathrm{AL} * \mathrm{AL}\right)$ 
$\mathrm{LAM} 1 * * 2$ )

$\mathrm{E}=(\mathrm{LAM} 2 * * 2-\mathrm{LAM} 1 * * 2) * * 2 * \mathrm{AL}^{* * 4}$

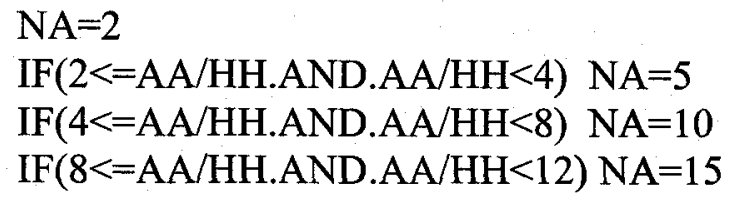

$\operatorname{IF}(\mathrm{DETA}<1.0) \mathrm{NA}=0$

$\mathrm{IF}(\mathrm{AL}<=\mathrm{NA}) \mathrm{THEN}$

$\mathrm{CC}=\mathrm{FP}(\mathrm{P} 1)$

IF (CABS $(C C)>0.01)$ THEN

$\mathrm{PR}=\mathrm{REAL}(\mathrm{P} 1)$

PI=AIMAG(P1)

CALL NEWTON(PR,PI,B,C,D,E)

P1=CMPLX(PR,PI)

END IF

$\mathrm{CC}=\mathrm{FP}(\mathrm{P} 2)$

IF(CABS $(C C)>0.01)$ THEN

$\mathrm{PR}=\mathrm{REAL}(\mathrm{P} 2)$

PI=AIMAG(P2)

CALL NEWTON(PR,PI,B,C,D,E)

$\mathrm{P} 2=\mathrm{CMPLX}(\mathrm{PR}, \mathrm{PI})$

END IF

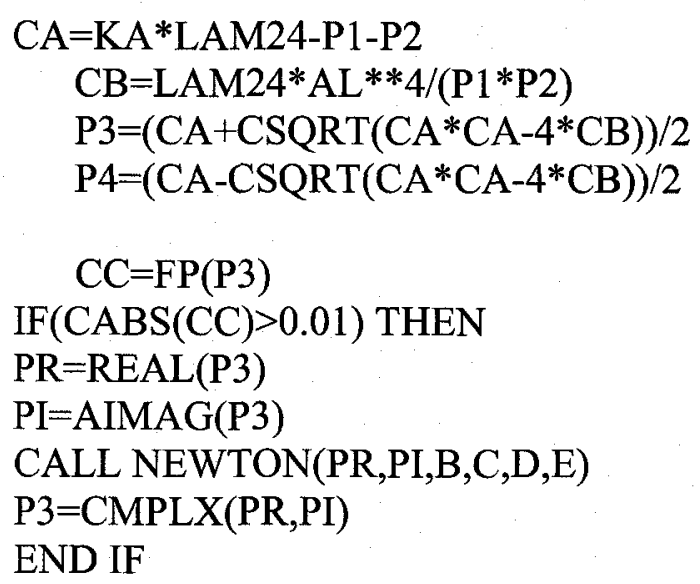


$\operatorname{IF}(\mathrm{CABS}(\mathrm{CC})>0.01)$ THEN

$\mathrm{PR}=\mathrm{REAL}(\mathrm{P} 4)$

$\mathrm{PI}=\mathrm{AIMAG}(\mathrm{P} 4)$

CALL NEWTON(PR,PI,B,C,D,E)

P4=CMPLX(PR,PI)

END IF

ELSE

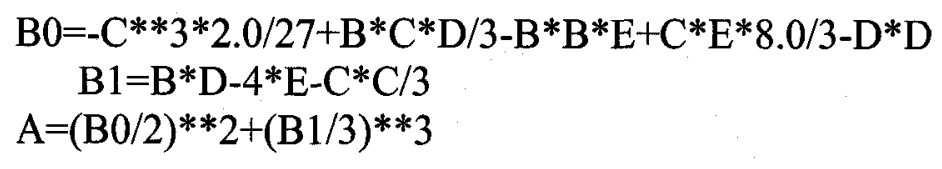

IF(A.LT.0.0) THEN

$\mathrm{CA}=\mathrm{CMPLX}(0.0, \mathrm{DSQRT}(-\mathrm{A}))$

$\mathrm{CB}=\mathrm{CMPLX}(\mathrm{B} 0,0.0)$

$\mathrm{CY}=\mathrm{CEXP}(\mathrm{CLOG}(-\mathrm{CB} / 2+\mathrm{CA}) / 3)+\mathrm{CEXP}(\mathrm{CLOG}(-\mathrm{CB} / 2-\mathrm{CA}) / 3)$

$\mathrm{Y}=\mathrm{REAL}(\mathrm{CY})$

ELSE

$\mathrm{A}=\mathrm{DSQRT}(\mathrm{A})$

IF(B0.LT.0.0) Y=DEXP(DLOG(-B0/2+A)/3)+DEXP(DLOG(-B0/2-A)/3)

IF(B0.GE.0.0) Y=-DEXP(DLOG(B0/2-A)/3)-DEXP(DLOG(B0/2+A)/3)

END IF

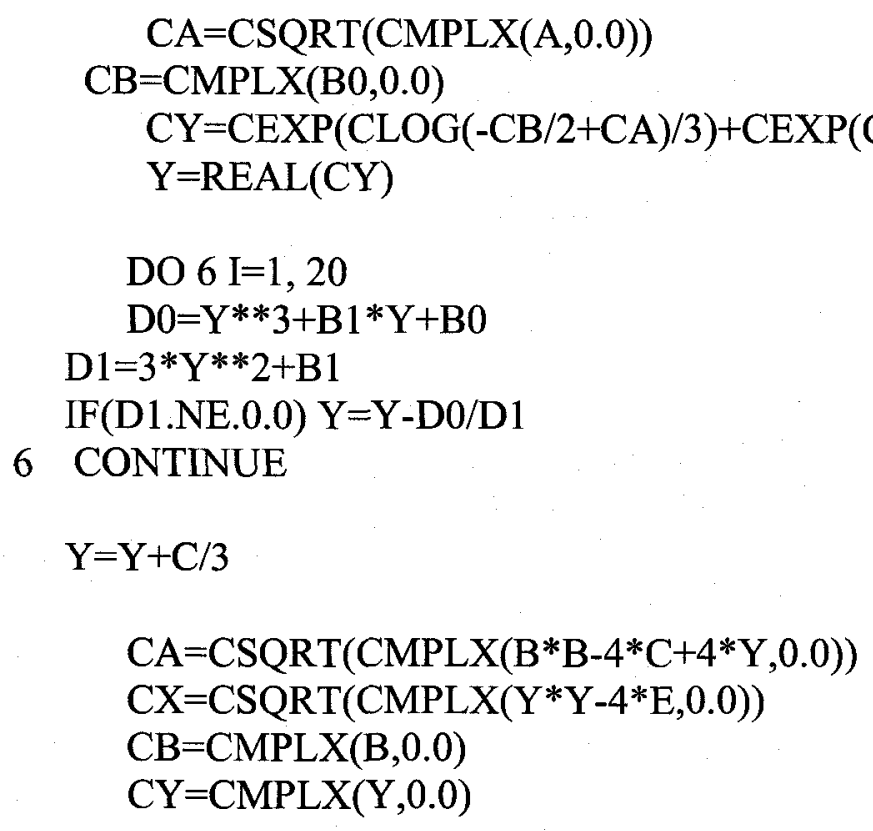


IF(CABS(KA*P3-1)<0.000000001) P3 $=1.0000000001 / \mathrm{KA}$ IF(CABS(KA*P4-1)<0.000000001) P4=1.0000000001/KA

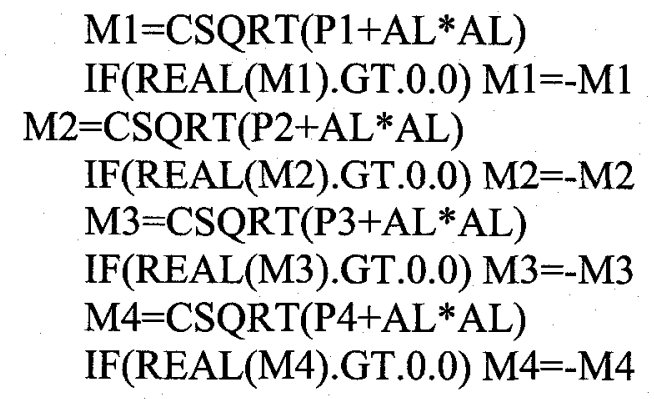

IF(CABS(M1) $<0.000000001) \mathrm{M} 1=-\mathrm{AL}$

$\mathrm{IF}(\mathrm{CABS}(\mathrm{M} 2)<0.000000001) \mathrm{M} 2=-\mathrm{AL}$

$\operatorname{IF}(\mathrm{CABS}(\mathrm{M} 3)<0.000000001) \mathrm{M} 3=-\mathrm{AL}$

$\mathrm{IF}(\mathrm{CABS}(\mathrm{M} 4)<0.000000001) \mathrm{M} 4=-\mathrm{AL}$

C----- CALCULATING THE VALUES OF Nj AND Qj FOR $\mathrm{j}=1-4$

$\mathrm{CD}=(\mathrm{P} 2-\mathrm{P} 1)^{*}(\mathrm{P} 3-\mathrm{P} 1)^{*}(\mathrm{P} 4-\mathrm{P} 1)^{*}(\mathrm{P} 3-\mathrm{P} 2) *(\mathrm{P} 4-\mathrm{P} 2) *(\mathrm{P} 4-\mathrm{P} 3)$

$\operatorname{IF}\left(\mathrm{CABS}(\mathrm{CD})<10.0^{* *}(-22)\right)$ GOTO 8

$\mathrm{CD}=1.0 / \mathrm{CD}$

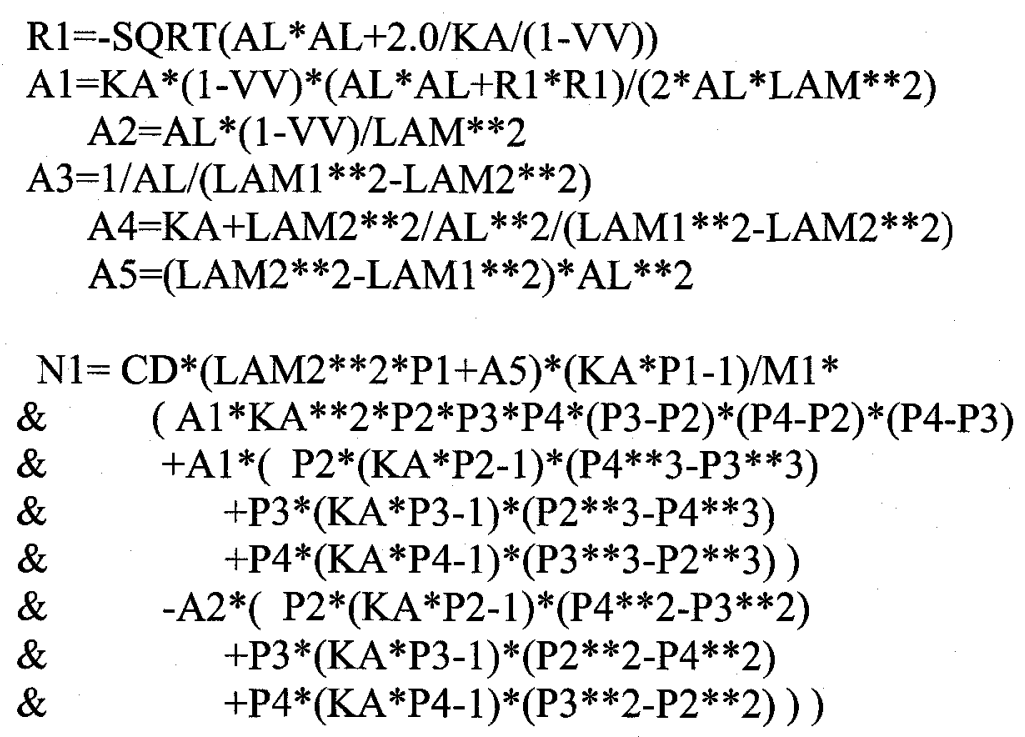



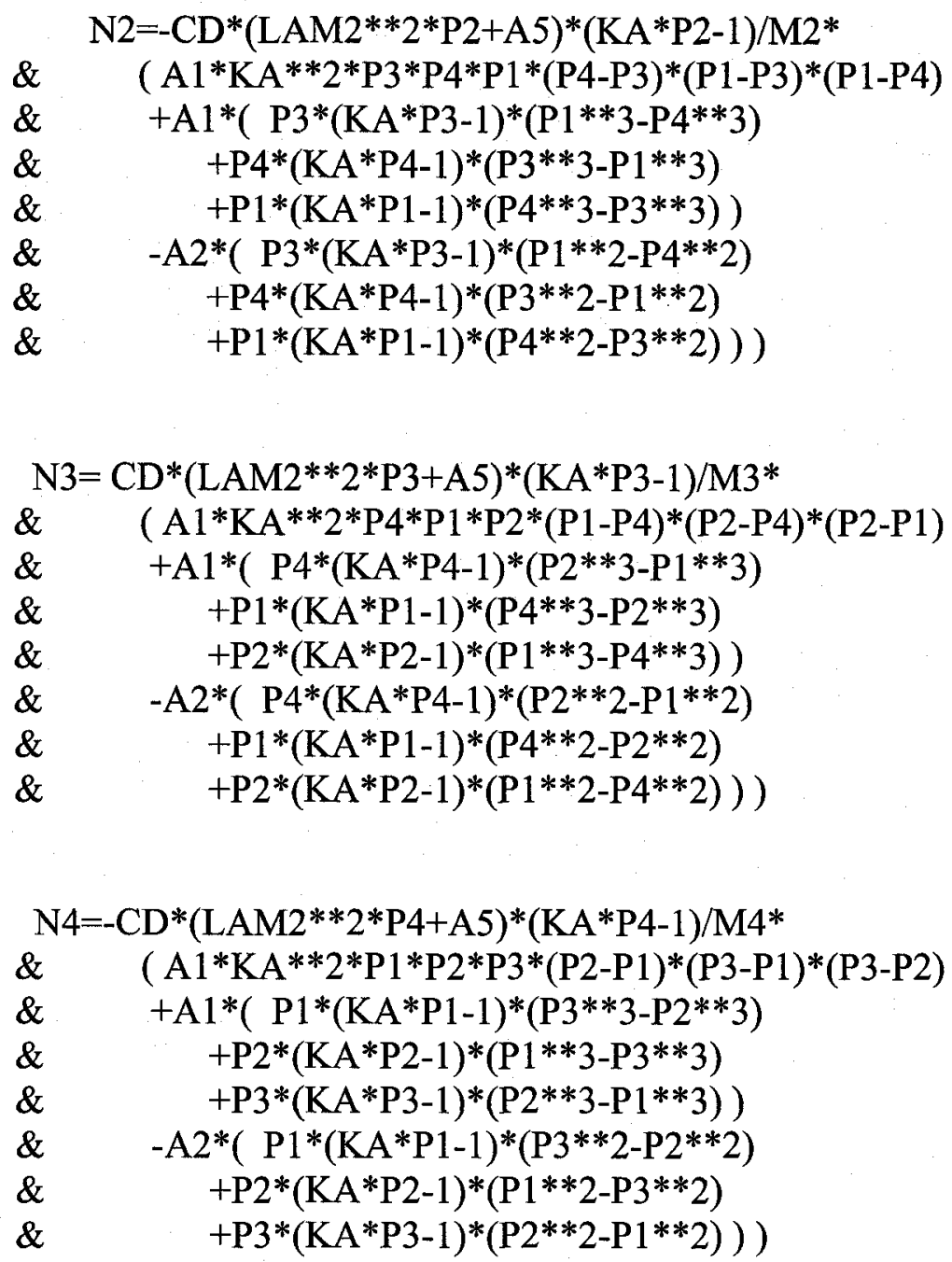

$\mathrm{Q} 1=\mathrm{CD} *(\mathrm{LAM} 2 * * 2 * \mathrm{P} 1+\mathrm{A} 5) *(\mathrm{KA} * \mathrm{P} 1-1) * \mathrm{~A} 3 / \mathrm{M} 1 *$

$\& \quad(\mathrm{~A} 4 * \mathrm{P} 2 * \mathrm{P} 3 * \mathrm{P} 4 *(\mathrm{P} 3-\mathrm{P} 2) *(\mathrm{P} 4-\mathrm{P} 2) *(\mathrm{P} 4-\mathrm{P} 3)$

$\& \quad-(\mathrm{P} 2 * \mathrm{P} 3) * * 2 *(\mathrm{P} 3-\mathrm{P} 2)-(\mathrm{P} 3 * \mathrm{P} 4) * * 2 *(\mathrm{P} 4-\mathrm{P} 3)-(\mathrm{P} 2 * \mathrm{P} 4) * * 2 *(\mathrm{P} 2-\mathrm{P} 4))$

$\mathrm{Q} 2=-\mathrm{CD} *(\mathrm{LAM} 2 * * 2 * \mathrm{P} 2+\mathrm{A} 5) *\left(\mathrm{KA}^{*} \mathrm{P} 2-1\right) * \mathrm{~A} 3 / \mathrm{M} 2 *$

$\& \quad(\mathrm{~A} 4 * \mathrm{P} 3 * \mathrm{P} 4 * \mathrm{P} 1 *(\mathrm{P} 4-\mathrm{P} 3) *(\mathrm{P} 1-\mathrm{P} 3) *(\mathrm{P} 1-\mathrm{P} 4)$

$\left.\& \quad-(\mathrm{P} 3 * \mathrm{P} 4) * * 2 *(\mathrm{P} 4-\mathrm{P} 3)-(\mathrm{P} 4 * \mathrm{P} 1)^{* *} 2 *(\mathrm{P} 1-\mathrm{P} 4)-(\mathrm{P} 3 * \mathrm{P} 1)^{* *} 2 *(\mathrm{P} 3-\mathrm{P} 1)\right)$

$\mathrm{Q} 3=\mathrm{CD} *(\mathrm{LAM} 2 * 2 * \mathrm{P} 3+\mathrm{A} 5) *(\mathrm{KA} * \mathrm{P} 3-1) * \mathrm{~A} 3 / \mathrm{M} 3 *$

$\& \quad(\mathrm{~A} 4 * \mathrm{P} 4 * \mathrm{P} 1 * \mathrm{P} 2 *(\mathrm{P} 1-\mathrm{P} 4) *(\mathrm{P} 2-\mathrm{P} 4) *(\mathrm{P} 2-\mathrm{P} 1)$

$\left.\& \quad-(\mathrm{P} 4 * \mathrm{P} 1)^{* *} 2 *(\mathrm{P} 1-\mathrm{P} 4)-(\mathrm{P} 1 * \mathrm{P} 2) * * 2 *(\mathrm{P} 2-\mathrm{P} 1)-(\mathrm{P} 4 * \mathrm{P} 2) * * 2 *(\mathrm{P} 4-\mathrm{P} 2)\right)$

$\mathrm{Q} 4=-\mathrm{CD} *(\mathrm{LAM} 2 * * 2 * \mathrm{P} 4+\mathrm{A} 5) *\left(\mathrm{KA}^{*} \mathrm{P} 4-1\right) * \mathrm{~A} 3 / \mathrm{M} 4 *$ 
$\& \quad(\mathrm{~A} 4 * \mathrm{P} 1 * \mathrm{P} 2 * \mathrm{P} 3 *(\mathrm{P} 2-\mathrm{P} 1) *(\mathrm{P} 3-\mathrm{P} 1) *(\mathrm{P} 3-\mathrm{P} 2)$

$\left.\& \quad-(\mathrm{P} 1 * \mathrm{P} 2) * * 2 *(\mathrm{P} 2-\mathrm{P} 1)-(\mathrm{P} 2 * \mathrm{P} 3) * * 2 *(\mathrm{P} 3-\mathrm{P} 2)-(\mathrm{P} 1 * \mathrm{P} 3)^{* *} 2 *(\mathrm{P} 1-\mathrm{P} 3)\right)$

$\mathrm{CC}=2 * \mathrm{AL} * \mathrm{AL} *(\mathrm{Q} 1+\mathrm{Q} 2+\mathrm{Q} 3+\mathrm{Q} 4)-1$

FREK $11(\mathrm{~K})=\mathrm{REAL}(\mathrm{CC})$

$\mathrm{CA}=2 * \mathrm{AL}^{*} \mathrm{AL} *(\mathrm{~N} 1+\mathrm{N} 2+\mathrm{N} 3+\mathrm{N} 4)$

FREK12(K)=REAL(CA)

$\mathrm{CB}=-2 / \mathrm{LAM}^{* *} 2 *$

$\&\left(\mathrm{P} 1 * * 2 *\left(\mathrm{M} 1 * * 2-\mathrm{VV}^{*} \mathrm{AL} * \mathrm{AL}\right) /(\mathrm{KA} * \mathrm{P} 1-1) /((\mathrm{LAM} 2 * \mathrm{M} 1) * * 2-(\mathrm{LAM} 1 * \mathrm{AL}) * * 2) * \mathrm{Q} 1\right.$

$\&+\mathrm{P} 2 * * 2 *\left(\mathrm{M} 2 * * 2-\mathrm{VV}^{*} \mathrm{AL} * \mathrm{AL}\right) /\left(\mathrm{KA}^{*} \mathrm{P} 2-1\right) /\left((\mathrm{LAM} 2 * \mathrm{M} 2)^{* *} 2-\right.$

$(\mathrm{LAM} 1 * \mathrm{AL}) * * 2) * \mathrm{Q} 2$

$\&+\mathrm{P} 3 * * 2 *\left(\mathrm{M} 3 * * 2-\mathrm{VV}^{*} \mathrm{AL} * \mathrm{AL}\right) /\left(\mathrm{KA}^{*} \mathrm{P} 3-1\right) /\left((\mathrm{LAM} 2 * \mathrm{M} 3)^{* *} 2-\right.$

$(\mathrm{LAM} 1 * \mathrm{AL}) * * 2) * \mathrm{Q} 3$

$\&+\mathrm{P} 4 * * 2 *\left(\mathrm{M} 4 * * 2-\mathrm{VV}^{*} \mathrm{AL} * \mathrm{AL}\right) /\left(\mathrm{KA}^{*} \mathrm{P} 4-1\right) /((\mathrm{LAM} 2 * \mathrm{M} 4) * * 2-$

$(\mathrm{LAM} 1 * \mathrm{AL}) * * 2) * \mathrm{Q} 4)$

FREK21(K)=REAL $(\mathrm{CA})$

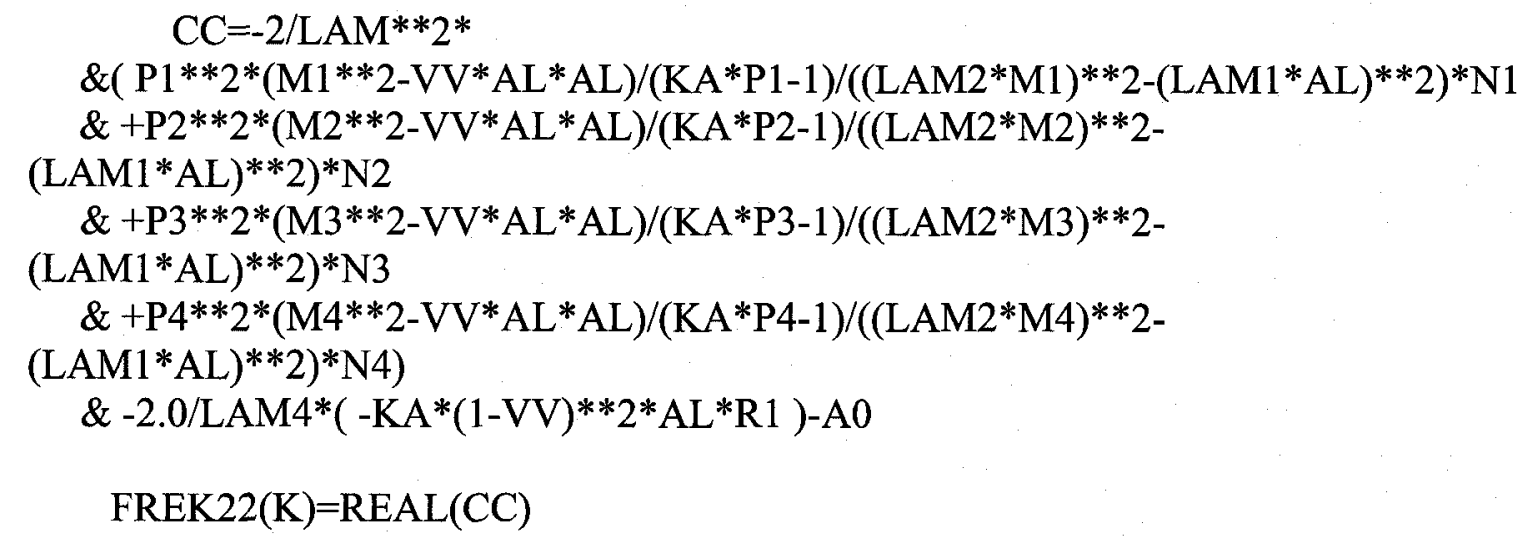

8 CONTINUE

C---- CALCULATING THE KERNEL FUNCTIONS OF THE INTEGRAL

$\mathrm{C} 1=\mathrm{SQRT}(\mathrm{CE})$

DO $20 \mathrm{I}=1, \mathrm{~N}$

DO $20 \mathrm{~J}=1, \mathrm{~N}$

$\operatorname{REK} 11(\mathrm{I}, \mathrm{J})=0.0$

$\operatorname{REK} 12(\mathrm{I}, \mathrm{J})=0.0$ 


\author{
$\operatorname{REK} 21(\mathrm{I}, \mathrm{J})=0.0$ \\ $\operatorname{REK} 22(\mathrm{I}, \mathrm{J})=0.0$ \\ $\mathrm{YI}=\mathrm{COS}\left(\mathrm{PAI}{ }^{*}(\mathrm{I}-0.5) /(\mathrm{N}-1)\right)$ \\ $\mathrm{TJ}=\operatorname{COS}\left(\mathrm{PAI}^{*}(\mathrm{~J}-1.0) /(\mathrm{N}-1)\right)$ \\ DO $10 \mathrm{~K}=1, \mathrm{M}$ \\ $\mathrm{AL}=\mathrm{K} * \mathrm{DA}$ \\ $\mathrm{SINK}=\mathrm{SIN}\left(\mathrm{C} 1 * A \mathrm{AL}^{*}(\mathrm{TJ}-\mathrm{YI})\right)$
}

REK11(I,J) $=$ REK 11(I,J)+C1*FREK11(K)*SINK*DA

REK12(I,J)=REK12(I,J)+C1*FREK12(K)*SINK*DA

REK21(I,J) $=$ REK21(I,J)+C1*FREK21(K)*SINK *DA

REK22(I,J) $=$ REK22(I,J)+C1*FREK22(K)*SINK*DA

10 CONTINUE

20 CONTINUE

RETURN

END

C------THIS IS THE NEWTON ITERATIVE METHOD FOR FINDING THE ROOTS OF NONLINEAR EQAUTIONS

SUBROUTINE NEWTON(PR,PI,B,C,D,E)

DOUBLE PRECISION PR,PI,B,C,D,E,

\& $\mathrm{P} 1, \mathrm{P} 2, \mathrm{DD}, \mathrm{D} 1, \mathrm{D} 2, \mathrm{~F} 1, \mathrm{~F} 2, \mathrm{~F} 11, \mathrm{~F} 12, \mathrm{~F} 21, \mathrm{~F} 22$

$$
\begin{aligned}
& \mathrm{F} 1(\mathrm{P} 1, \mathrm{P} 2)=\mathrm{P} 1 * * 4-6 * \mathrm{P} 1 * * 2 * \mathrm{P} 2 * * 2+\mathrm{P} 2 * * 4+\mathrm{B} *(\mathrm{P} 1 * * 3-3 * \mathrm{P} 1 * \mathrm{P} 2 * * 2) \\
& \text { \& } \quad+\mathrm{C} *(\mathrm{P} 1 * * 2-\mathrm{P} 2 * * 2)+\mathrm{D} * \mathrm{P} 1+\mathrm{E} \\
& \mathrm{F} 2(\mathrm{P} 1, \mathrm{P} 2)=4 * \mathrm{P} 1 * * 3 * \mathrm{P} 2-4 * \mathrm{P} 1 * \mathrm{P} 2 * * 3+\mathrm{B} *(3 * \mathrm{P} 1 * * 2 * \mathrm{P} 2-\mathrm{P} 2 * * 3) \\
& \& \quad+2 * \mathrm{C} * \mathrm{P} 1 * \mathrm{P} 2+\mathrm{D} * \mathrm{P} 2
\end{aligned}
$$

$\mathrm{F} 11(\mathrm{P} 1, \mathrm{P} 2)=4 * \mathrm{P} 1 * * 3-12 * \mathrm{P} 1 * \mathrm{P} 2 * * 2+\mathrm{B} *(3 * \mathrm{P} 1 * * 2-3 * \mathrm{P} 2 * * 2)+2 * \mathrm{C} * \mathrm{P} 1+\mathrm{D}$

$\mathrm{F} 12(\mathrm{P} 1, \mathrm{P} 2)=-12 * \mathrm{P} 1 * * 2 * \mathrm{P} 2+4 * \mathrm{P} 2 * * 3-6 * \mathrm{~B} * \mathrm{P} 1 * \mathrm{P} 2-2 * \mathrm{C} * \mathrm{P} 2$

$\mathrm{F} 21(\mathrm{P} 1, \mathrm{P} 2)=12 * \mathrm{P} 1 * * 2 * \mathrm{P} 2-4 * \mathrm{P} 2 * * 3+6 * \mathrm{~B} * \mathrm{P} 1 * \mathrm{P} 2+2 * \mathrm{C} * \mathrm{P} 2$

$\mathrm{F} 22(\mathrm{P} 1, \mathrm{P} 2)=4 * \mathrm{P} 1 * * 3-12 * \mathrm{P} 1 * \mathrm{P} 2 * * 2+\mathrm{B} *(3 * \mathrm{P} 1 * * 2-3 * \mathrm{P} 2 * * 2)+2 * \mathrm{C} * \mathrm{P} 1+\mathrm{D}$ 
DO $2 \mathrm{I}=1,30$

$\mathrm{DD}=\mathrm{F} 11(\mathrm{PR}, \mathrm{PI}) * \mathrm{~F} 22(\mathrm{PR}, \mathrm{PI})-\mathrm{F} 12(\mathrm{PR}, \mathrm{PI}){ }^{*} \mathrm{~F} 21(\mathrm{PR}, \mathrm{PI})$

IF(DD.NE.0.0) THEN

$\mathrm{D} 1=\mathrm{F} 2(\mathrm{PR}, \mathrm{PI}) * \mathrm{~F} 12(\mathrm{PR}, \mathrm{PI})-\mathrm{F} 1(\mathrm{PR}, \mathrm{PI}) * \mathrm{~F} 22(\mathrm{PR}, \mathrm{PI})$

$\mathrm{D} 2=\mathrm{F} 1(\mathrm{PR}, \mathrm{PI})^{*} \mathrm{~F} 21(\mathrm{PR}, \mathrm{PI})-\mathrm{F} 11(\mathrm{PR}, \mathrm{PI}) * \mathrm{~F} 2(\mathrm{PR}, \mathrm{PI})$

$\mathrm{P} 1=\mathrm{PR}+\mathrm{D} 1 / \mathrm{DD}$

$\mathrm{P} 2=\mathrm{PI}+\mathrm{D} 2 / \mathrm{DD}$

IF(DABS(F1(P1,P2))>DABS(F1(PR,PI))+1) RETURN

$\mathrm{PR}=\mathrm{P} 1 ; \mathrm{PI}=\mathrm{P} 2$

END IF

2 CONTINUE

RETURN

END

C---- THIS IS THE SUBPROGRAM FOR CALCULATING THE DATA OF THE OPEN DISCRET EQUATIONS

SUBROUTINE OPENEQ(N,A,F,W,T,Y,REK11,REK12,REK21,REK22)

DOUBLE PRECISION REK11(N,N), REK12(N,N), REK21(N,N),REK22(N,N)

DOUBLE PRECISION A $(2 * \mathrm{~N}, 2 * \mathrm{~N}), \mathrm{F}(2 * \mathrm{~N}), \mathrm{W}(\mathrm{N}), \mathrm{Y}(\mathrm{N}), \mathrm{T}(\mathrm{N}), \mathrm{PAI}, \mathrm{H}$

COMMON

DETA,CE,LAM1,LAM14,LAM,LAM4,LAM2,LAM24,KA,VV,AA,HH,F1,F2,A0

PAI $=3.141592653589798$

$\mathrm{H}=\mathrm{PAI} /(\mathrm{N}-1)$

DO $2 \mathrm{I}=2, \mathrm{~N}-1$

$2 \mathrm{~W}(\mathrm{I})=\mathrm{H}$

$\mathrm{W}(1)=\mathrm{H} / 2 ; \mathrm{W}(\mathrm{N})=\mathrm{H} / 2$

DO $4 \mathrm{I}=1, \mathrm{~N}$

$\mathrm{T}(\mathrm{I})=\operatorname{COS}\left(\mathrm{H}^{*}(\mathrm{I}-1.0)\right)$

$\mathrm{Y}(\mathrm{I})=\operatorname{COS}\left(\mathrm{H}^{*}(\mathrm{I}-0.5)\right)$

$\mathrm{F}(\mathrm{I})=2 * \mathrm{PAI}^{*} \mathrm{~F} 1$

$\mathrm{F}(\mathrm{N}+\mathrm{I})=2 * \mathrm{PAI} * \mathrm{HH} / \mathrm{AA}^{*} \mathrm{~F} 2$

4 CONTINUE

$\mathrm{F}(\mathrm{N})=0.0 ; \mathrm{F}\left(2^{*} \mathrm{~N}\right)=0.0$

DO $6 \mathrm{I}=1, \mathrm{~N}-1$

DO $6 \mathrm{~J}=1, \mathrm{~N}$

$\mathrm{A}(\mathrm{I}, \mathrm{J})=\mathrm{W}(\mathrm{J})^{*}(1.0 /(\mathrm{T}(\mathrm{J})-\mathrm{Y}(\mathrm{I}))+\mathrm{REK} 11(\mathrm{I}, \mathrm{J}))$ 


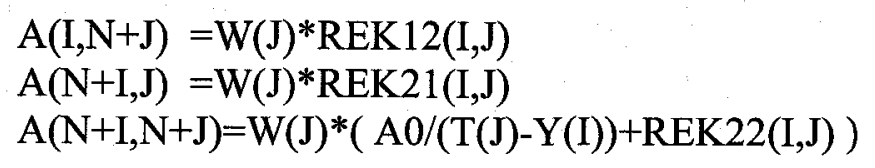

6 CONTINUE

DO $8 \mathrm{~J}=1, \mathrm{~N}$

$\mathrm{A}(\mathrm{N}, \mathrm{J})=\mathrm{W}(\mathrm{J})$

$\mathrm{A}(\mathrm{N}, \mathrm{N}+\mathrm{J})=0.0$

$\mathrm{A}\left(2^{*} \mathrm{~N}, \mathrm{~J}\right)=0.0$

$\mathrm{A}(2 * \mathrm{~N}, \mathrm{~N}+\mathrm{J})=\mathrm{W}(\mathrm{J})$

8 CONTINUE

\section{RETURN \\ END}

C----- THIS IS THE SUBPROGRAM FOR CALCULATING THE DATA OF THE CLOSE-INNER DISCRET EQUATIONS

SUBROUTINE CLOSEEQINNER(N,A,F,W,T,Y,REK11,REK12,REK21,REK22)

DOUBLE PRECISION REK11(N,N), REK12(N,N), REK21(N,N),REK22(N,N)

DOUBLE PRECISION A $\left(2 * \mathrm{~N}, 2^{*} \mathrm{~N}\right), \mathrm{F}(2 * \mathrm{~N}), \mathrm{W}(\mathrm{N}), \mathrm{Y}(\mathrm{N}), \mathrm{T}(\mathrm{N}), \mathrm{PAI}, \mathrm{H} 1$

COMMON

DETA,CE,LAM1,LAM14,LAM,LAM4,LAM2,LAM24,KA,VV,AA,HH,F1,F2,A0

$\mathrm{PAI}=3.141592653589798$

$\mathrm{H} 1=\mathrm{HH} / \mathrm{AA} / 2$

DO $2 \mathrm{I}=1, \mathrm{~N}-1$

$\mathrm{F}(\mathrm{I})=2 * \mathrm{PAI} * \mathrm{~F} 1$

$\mathrm{F}(\mathrm{N}+\mathrm{I})=2 * \mathrm{PAI} * \mathrm{HH} / \mathrm{AA}^{*} \mathrm{~F} 2$

2 CONTINUE

$\mathrm{F}(\mathrm{N})=0.0 ; \mathrm{F}\left(2^{*} \mathrm{~N}\right)=0.0$

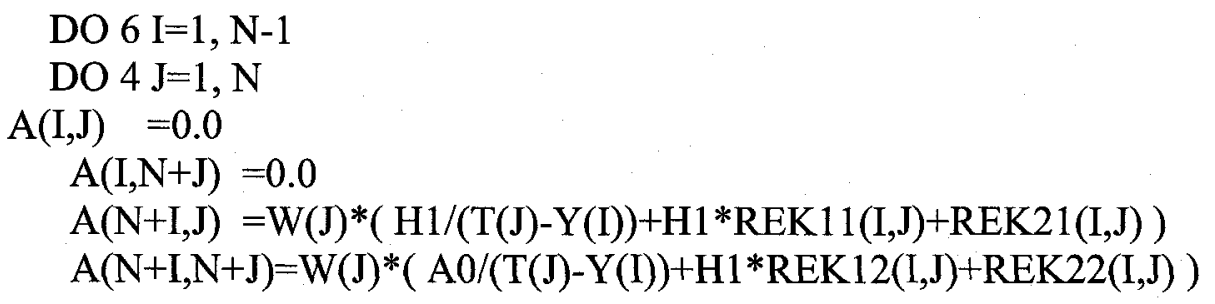


4 CONTINUE

$\mathrm{A}(\mathrm{I}, \mathrm{I})=1.0$

$\mathrm{A}(\mathrm{I}, \mathrm{N}+\mathrm{I})=-\mathrm{H} 1$

6 CONTINUE

DO $8 \mathrm{~J}=1, \mathrm{~N}$

$\mathrm{A}(\mathrm{N}, \mathrm{J})=\mathrm{W}(\mathrm{J})$

$\mathrm{A}(\mathrm{N}, \mathrm{N}+\mathrm{J})=0.0$

$\mathrm{A}(2 * \mathrm{~N}, \mathrm{~J})=0.0$

$\mathrm{A}\left(2^{*} \mathrm{~N}, \mathrm{~N}+\mathrm{J}\right)=\mathrm{W}(\mathrm{J})$

8 CONTINUE

\section{RETURN}

END

C----- THIS IS THE SUBPROGRAM FOR CALCULATING THE DATA OF THE CLOSE-OUTER DISCRET EQUATIONS

SUBROUTINE CLOSEEQOUTER(N,A,F,W,T,Y,REK11,REK12,REK21,REK22)

DOUBLE PRECISION REK11(N,N), REK12(N,N), REK21(N,N),REK22(N,N)

DOUBLE PRECISION A(2*N,2*N),F(2*N),W(N),Y(N),T(N),PAI,H1

COMMON

DETA,CE,LAM1,LAM14,LAM,LAM4,LAM2,LAM24,KA,VV,AA,HH,F1,F2,A0

$\mathrm{PAI}=3.141592653589798$

$\mathrm{H} 1=\mathrm{HH} / \mathrm{AA} / 2$

DO $2 \mathrm{I}=1, \mathrm{~N}-1$

$\mathrm{F}(\mathrm{I})=2 * \mathrm{PAI} * \mathrm{~F} 1$

$\mathrm{F}(\mathrm{N}+\mathrm{I})=-2 * \mathrm{PAI}^{*} \mathrm{HH} / \mathrm{AA}^{*} \mathrm{~F} 2$

2 CONTINUE

$\mathrm{F}(\mathrm{N})=0.0 ; \mathrm{F}(2 * \mathrm{~N})=0.0$

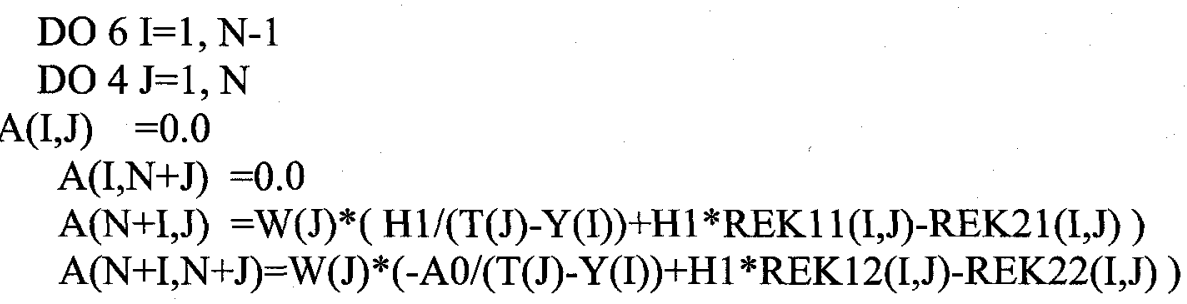


4 CONTINUE

$\mathrm{A}(\mathrm{I}, \mathrm{I})=1.0$

$\mathrm{A}(\mathrm{I}, \mathrm{N}+\mathrm{I})=\mathrm{H} 1$

6 CONTINUE

DO $8 \mathrm{~J}=1, \mathrm{~N}$

$\mathrm{A}(\mathrm{N}, \mathrm{J})=\mathrm{W}(\mathrm{J})$

$\mathrm{A}(\mathrm{N}, \mathrm{N}+\mathrm{J})=0.0$

$\mathrm{A}\left(2^{*} \mathrm{~N}, \mathrm{~J}\right)=0.0$

$\mathrm{A}\left(2^{*} \mathrm{~N}, \mathrm{~N}+\mathrm{J}\right)=\mathrm{W}(\mathrm{J})$

8 CONTINUE

RETURN

END

C---- THIS IS THE SUBPROGRAM FOR SOLVING THE LINEAR EQUATIONS BY GAUSS ELIMINATE METHOD

SUBROUTINE SOLVEEQ(N,A,B,X)

DOUBLE PRECISION A(N,N), B(N), X(N), C, T, S

DO $12 \mathrm{~K}=1, \mathrm{~N}-1$

$\mathrm{L}=\mathrm{K}$

DO $2 \mathrm{I}=\mathrm{K}+1, \mathrm{~N}$

IF(DABS(A(I,K)).GT.DABS(A(L,K))) L=I

2 CONTINUE

$\operatorname{IF}(\mathrm{A}(\mathrm{L}, \mathrm{K})==0.0) \mathrm{THEN}$

PRINT*, 'NO UNIQUE SOLUTION'

STOP

END IF

IF(L.NE.K) THEN

$\mathrm{T}=\mathrm{B}(\mathrm{K})$

$\mathrm{B}(\mathrm{K})=\mathrm{B}(\mathrm{L})$

$\mathrm{B}(\mathrm{L})=\mathrm{T}$

DO $4 \mathrm{~J}=\mathrm{K}, \mathrm{N}$

$\mathrm{T}=\mathrm{A}(\mathrm{K}, \mathrm{J})$

$\mathrm{A}(\mathrm{K}, \mathrm{J})=\mathrm{A}(\mathrm{L}, \mathrm{J})$

$\mathrm{A}(\mathrm{L}, \mathrm{J})=\mathrm{T}$

4 CONTINUE

END IF

DO $8 \mathrm{I}=\mathrm{K}+1, \mathrm{~N}$ 


\author{
$\mathrm{C}=\mathrm{A}(\mathrm{I}, \mathrm{K}) / \mathrm{A}(\mathrm{K}, \mathrm{K})$ \\ $\mathrm{B}(\mathrm{I})=\mathrm{B}(\mathrm{I})-\mathrm{B}(\mathrm{K}) * \mathrm{C}$ \\ DO $6 \mathrm{~J}=\mathrm{K}+1, \mathrm{~N}$ \\ $6 \quad \mathrm{~A}(\mathrm{I}, \mathrm{J})=\mathrm{A}(\mathrm{I}, \mathrm{J})-\mathrm{A}(\mathrm{K}, \mathrm{J}){ }^{*} \mathrm{C}$ \\ 8 CONTINUE \\ 12 CONTINUE \\ $\mathrm{X}(\mathrm{N})=\mathrm{B}(\mathrm{N}) / \mathrm{A}(\mathrm{N}, \mathrm{N})$ \\ DO $16 \mathrm{M}=1, \mathrm{~N}-1$ \\ $\mathrm{I}=\mathrm{N}-\mathrm{M}$ \\ $S=0.0$ \\ DO $14 \mathrm{~J}=\mathrm{I}+1, \mathrm{~N}$ \\ $14 \quad \mathrm{~S}=\mathrm{S}+\mathrm{A}(\mathrm{I}, \mathrm{J}) * \mathrm{X}(\mathrm{J})$ \\ $\mathrm{X}(\mathrm{I})=(\mathrm{B}(\mathrm{I})-\mathrm{S}) / \mathrm{A}(\mathrm{I}, \mathrm{I})$ \\ 16 CONTINUE \\ RETURN \\ END
}




\section{Program 2 - Specially Orthotropic Cylindrical Shells}

C-----MAIN PROGRAM: CRACK CLOSURE ANALYSIS ON CYLINDER SHELL WITH DIFFERENT E1 AND E2

PARAMETER ( $\mathrm{N}=41, \mathrm{~T}=60.0)$

REAL LAM0,LAM1,LAM14, LAM, LAM4, LAM2, LAM24, KA, Km, Kb

DOUBLE PRECISION REK11(N,N), REK12(N,N), REK21(N,N), REK22(N,N),

\& $\quad \mathrm{A}(2 * \mathrm{~N}, 2 * \mathrm{~N}), \mathrm{Al}(2 * \mathrm{~N}, 2 * \mathrm{~N}), \mathrm{A} 2(2 * \mathrm{~N}, 2 * \mathrm{~N}), \mathrm{B}(2 * \mathrm{~N}), \mathrm{B} 1(2 * \mathrm{~N}), \mathrm{B} 2(2 * \mathrm{~N})$,

$\& \quad \mathrm{G}(2 * \mathrm{~N}), \mathrm{W}(\mathrm{N}), \mathrm{TT}(\mathrm{N}), \mathrm{YY}(\mathrm{N}), \mathrm{P}(\mathrm{N})$

COMMON CE,LAM1,LAM14,LAM,LAM4,LAM2,LAM24, KA,VV, $\mathrm{AA}, \mathrm{HH}, \mathrm{F} 1, \mathrm{~F} 2, \mathrm{~A} 0$

OPEN(UNIT=10, FILE='test.DAT', STATUS='UNKNOWN',

\& FORM='FORMATTED', POSITION='APPEND', IOSTAT=ISTAT )

IF(ISTAT/=0) PRINT*, 'FILE OPEN FAIL: ISTAT=',ISTAT

$\mathrm{E} 12=1$

$\mathrm{CE}=\mathrm{E} 12^{* *}(1.0 / 4)$

$\operatorname{IF}(\mathrm{E} 12==0.725 . \mathrm{OR} \cdot \mathrm{E} 12==1.38) \mathrm{THEN}$

$\mathrm{V} 1=0.1966$

$\mathrm{V} 2=0.2174$

ELSE

$\mathrm{V} 1=0.0075$

$\mathrm{V} 2=0.2$

END IF

$\mathrm{VV}=\mathrm{SQRT}(\mathrm{V} 1 * \mathrm{~V} 2)$

$\mathrm{IF}(\mathrm{E} 12==1.0) \mathrm{VV}=1.0 / 3$

PRINT*, V1,V2,VV

$\mathrm{AA}=10$

$\mathrm{HH}=1$

LAM4 $=12 *\left(1-\mathrm{VV}^{*} \mathrm{VV}\right) * \mathrm{AA} * \mathrm{AA} /\left(\mathrm{HH}^{*} \mathrm{HH}\right)$

$\mathrm{LAM}=\mathrm{LAM} 4 * *(1.0 / 4)$

$\mathrm{KA}=12 *(1+\mathrm{VV}) /\left(5^{*} \mathrm{LAM} 4\right)$

$\mathrm{A} 0=\left(1-\mathrm{VV}^{*} \mathrm{VV}\right) / \mathrm{LAM} 4$

$\mathrm{SING}=1 \quad$ !SING=1 FOR INNER-CLOSE CASE AND SING=-1 FOR OUTERCLOSE

$\mathrm{F} 1=0.0$ 
$\mathrm{F} 2=-1.0 / 6$

WRITE $(10, *)^{\prime * * * * * * * * * * * * * * * * * * * * * * * * * * * * * * * * * * * * * * * * * * * * * * * * * * * * * * * 1}$

PRINT*,'a/h=',AA/HH, 'E1/E2=',E12

WRITE(10,2) AA/HH, E12

2 FORMAT(1X,4HA/H=,F5.2,3X,'E1/E2=',F6.3)

$\mathrm{N} 1=2 * \mathrm{AA} / \mathrm{HH}$

DO $120 \mathrm{M}=1, \mathrm{~N} 1$

LAM0 $=0.5^{*} \mathrm{M}$

$\mathrm{LAM} 1=\mathrm{LAM} 0 * \mathrm{SQRT}(\mathrm{CE})$

LAM2 $=0.0$ !LAM0/SQRT(CE)

LAM14=LAM $1 * * 4$

LAM24=0.0 !LAM2**4

CALL KERLFUNC(N,T,REK11,REK12,REK21,REK22)

CALL OPENEQ(N,A1,B1,W,TT,YY,REK11,REK12,REK21,REK22)

$\mathrm{IF}(\mathrm{SING}==1) \mathrm{THEN}$

CALL CLOSEEQINNER(N,A2,B2,W,TT,YY,REK11,REK12,REK21,REK22)

ELSE

CALL CLOSEEQOUTER(N,A2,B2,W,TT,YY,REK11,REK12,REK21,REK22)

END IF

C-----IF ONLY CACULATE OPEN CASE, PLEASE IMPLEMENT THESE TWO SENTANCES, OTHERWISE OMIT THEM

!CALL SOLVEEQ $(2 * \mathrm{~N}, \mathrm{~A} 1, \mathrm{~B} 1, \mathrm{G})$

!GO TO 110

C-----IF ONLY CACULATE OPEN CASE, PLEASE IMPLEMENT THESE TWO SENTANCES, OTHERWISE OMIT THEM

$\mathrm{NN}=2 * \mathrm{~N}$ 
$\mathrm{N} 0=(\mathrm{N}-1) / 2$

$\mathrm{I} 0=100$

$\mathrm{H} 1=-\mathrm{HH} / 2 / 3.1415926$

DO $80 \mathrm{~K}=1$, N0

DO $30 \mathrm{I}=1, \mathrm{~N}$

IF ( I $<=$ I.AND.I $<=\mathrm{N}-\mathrm{I0})$ THEN

DO $10 \mathrm{~J}=1, \mathrm{NN}$

$\mathrm{A}(\mathrm{I}, \mathrm{J})=\mathrm{A} 1(\mathrm{I}, \mathrm{J})$

10

$\mathrm{A}(\mathrm{N}+\mathrm{I}, \mathrm{J})=\mathrm{A} 1(\mathrm{~N}+\mathrm{I}, \mathrm{J})$

$\mathrm{B}(\mathrm{I})=\mathrm{B} 1(\mathrm{I})$

$\mathrm{B}(\mathrm{N}+\mathrm{I})=\mathrm{B} 1(\mathrm{~N}+\mathrm{I})$

ELSE

DO $20 \mathrm{~J}=1, \mathrm{NN}$

$\mathrm{A}(\mathrm{I}, \mathrm{J})=\mathrm{A} 2(\mathrm{I}, \mathrm{J})$

20

$\mathrm{A}(\mathrm{N}+\mathrm{I}, \mathrm{J})=\mathrm{A} 2(\mathrm{~N}+\mathrm{I}, \mathrm{J})$

$\mathrm{B}(\mathrm{I})=\mathrm{B} 2(\mathrm{I})$

$\mathrm{B}(\mathrm{N}+\mathrm{I})=\mathrm{B} 2(\mathrm{~N}+\mathrm{I})$

END IF

30 CONTINUE

CALL SOLVEEQ(NN,A,B,G)

DO $50 \mathrm{I}=1, \mathrm{~N}-1$

$\mathrm{P}(\mathrm{I})=0.0$

DO $40 \mathrm{~J}=1, \mathrm{~N}$

$\mathrm{P}(\mathrm{I})=\mathrm{P}(\mathrm{I})+\mathrm{SING} * \mathrm{H} 1 * \mathrm{~W}(\mathrm{~J}) *(\mathrm{G}(\mathrm{J}) /(\mathrm{TT}(\mathrm{J})-\mathrm{YY}(\mathrm{I}))+\mathrm{G}(\mathrm{J}) * \mathrm{REK} 11(\mathrm{I}, \mathrm{J})$

$\&$ $+\mathrm{G}(\mathrm{N}+\mathrm{J}) * \mathrm{REK} 12(\mathrm{I}, \mathrm{J}))$

40 CONTINUE

$\mathrm{PP}=\mathrm{SNGL}(\mathrm{P}(\mathrm{I}))$

!WRITE(10,4) K,I,PP,YY(I)

4 FORMAT(1X,'K=',I2,3X,'I=',I2,3X,'P=',F10.4,2X,'Y=',F7.4) 
50 CONTINUE

!WRITE $\left(10,{ }^{*}\right)$

$\mathrm{I} 0=100$

DO $60 \mathrm{I}=1$, N0

$60 \operatorname{IF}(\mathrm{P}(\mathrm{I})<-1.0 / 10 * * 8 . \mathrm{AND} . \mathrm{I}<\mathrm{I} 0) \mathrm{I} 0=\mathrm{I}$

WRITE $\left(*,{ }^{*}\right)$ 'K=',K,'I0=',I0

$\mathrm{IF}(\mathrm{I} 0==100)$ GOTO 90

80 CONTINUE

$90 \operatorname{IF}(\mathrm{K}<=\mathrm{N} 0)$ THEN

WRITE $(*, *)$ 'ITERATION CONVERGENCE, $\mathrm{K}=$ ', K

ELSE

WRITE $(*, *)$ 'ITERATION FAIL,K=',K

END IF

$\mathrm{J} 0=\mathrm{N} 0$

DO $100 \mathrm{I}=1$, N0

$100 \operatorname{IF}(\operatorname{ABS}(\mathrm{P}(\mathrm{I}))<=1.0 / 10 * * 9 . \mathrm{AND} . \mathrm{I}<\mathrm{J} 0) \mathrm{J} 0=\mathrm{I}$

$\mathrm{IF}(\mathrm{J} 0==\mathrm{N} 0)$ THEN

PRINT*, 'CRACK IS CLOSED, J0=',J0

WRITE $\left(10,{ }^{*}\right)$ 'CRACK IS CLOSED, J0=',J0

ELSE

PRINT*, 'CRACK IS OPEN-CLOSE'

PRINT*, 'J0=',J0,'P(J0)=',P(J0)

WRITE $(10, *)$ 'CRACK IS OPEN-CLOSE'

WRITE $\left(10,{ }^{*}\right)$ 'J0=',J0, 'Y(J0)=', YY(J0)

END IF

$110 \quad \mathrm{Km}=-0.5 * \mathrm{G}(1)$

$\mathrm{Kb}=-\mathrm{HH} /(4 * \mathrm{AA}) * \mathrm{G}(\mathrm{N}+1)$

PRINT*,'LAM0=',LAM0,'Km=',Km,'Kb=',Kb

WRITE $(10,8)$ LAM0, Km, Kb

8 FORMAT(1X,5HLAM0=,F6.3,3X,'Kmb=',F7.4,3X,'Kbb=',F7.4)

WRITE $\left(10,{ }^{*}\right)$

120 CONTINUE

CLOSE(10) 
STOP

END

C----- THIS IS THE SUBPROGRAM FOR SOLVING THE KERLFUNCTIONS

SUBROUTINE KERLFUNC(N,T,REK11,REK12,REK21,REK22)

REAL LAM1,LAM14, LAM, LAM4, LAM2, LAM24, KA

DOUBLE PRECISION REK11(N,N),REK12(N,N), REK21(N,N),REK22(N,N),

\& FREK11(20000),FREK12(20000),FREK21(20000),FREK22(20000)

COMMON CE,LAM1,LAM14,LAM,LAM4,LAM2,LAM24, KA,VV,

$\mathrm{AA}, \mathrm{HH}, \mathrm{F} 1, \mathrm{~F} 2, \mathrm{~A} 0$

COMPLEX P1, P2, P3, P4, M1, M2, M3, M4, N1, N2, N3, N4,

\& $\quad \mathrm{Q} 1, \mathrm{Q} 2, \mathrm{Q} 3, \mathrm{Q} 4, \mathrm{CA}, \mathrm{CB}, \mathrm{CC}, \mathrm{CD}, \mathrm{CX}, \mathrm{CY}, \mathrm{P}, \mathrm{FP}$

DOUBLE PRECISION A, B, C, D, E, B0, B1, Y, D0,D1,PR,PI

$\mathrm{FP}(\mathrm{P})=\mathrm{P} * * 4+\mathrm{B} * \mathrm{P} * * 3+\mathrm{C} * \mathrm{P} * * 2+\mathrm{D} * \mathrm{P}+\mathrm{E}$

$\mathrm{PAI}=3.141592653589798$

$\mathrm{DA}=0.01$

$\mathrm{M}=\mathrm{T} / \mathrm{DA}$

C------ FINDING THE ROOTS Pj AND Mj OF THE POLYNOMIAL EQUATIONS

$\mathrm{P} 1=\left(\mathrm{KA}^{*} \mathrm{LAM} 24+\mathrm{LAM} 2 * * 2 * \operatorname{CSQRT}\left(\mathrm{CMPLX}\left(\mathrm{KA}^{* *} 2 * \mathrm{LAM} 24-4,0.0\right)\right)\right) / 2$

$\mathrm{P} 2=\left(\mathrm{KA}^{*}\right.$ LAM24-LAM2 $* * 2 *$ CSQRT$\left(\mathrm{CMPLX}\left(\mathrm{KA}^{* *} 2 *\right.\right.$ LAM $\left.\left.\left.24-4,0.0\right)\right)\right) / 2$

DO $8 \mathrm{~K}=1, \mathrm{M}$

$\mathrm{AL}=\mathrm{K} * \mathrm{DA}$

$\operatorname{IF}(\mathrm{LAM} 1=0.0)$ GOTO 4

$\mathrm{B}=0.0$

$\mathrm{C}=0.0$

$\mathrm{D}=-\mathrm{KA} * \mathrm{LAM} 14 * \mathrm{AL}^{* * 4}$

$\mathrm{E}=\mathrm{LAM} 14 * \mathrm{AL} * 4$

$\mathrm{A}=\left(\mathrm{D}^{*} \mathrm{D} / 2\right)^{* * 2}-(4 * \mathrm{E} / 3)^{* * 3}$ 


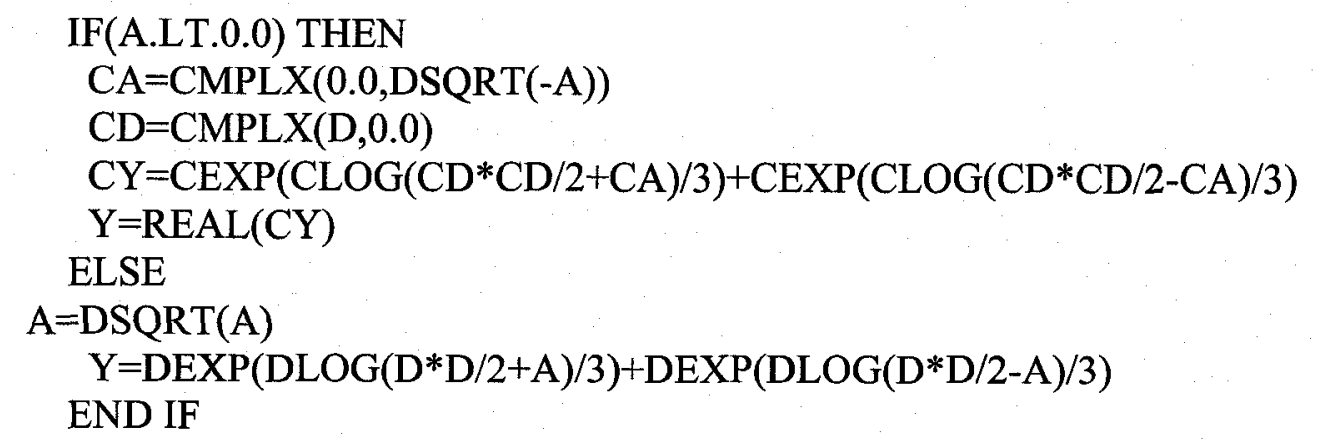

DO $2 \mathrm{I}=1,20$

$\mathrm{D} 0=\mathrm{Y}^{* *} 3-4^{*} \mathrm{E}^{*} \mathrm{Y}-\mathrm{D} * \mathrm{D}$

$\mathrm{D} 1=3 * \mathrm{Y}^{* *} 2-4 * \mathrm{E}$

IF(D1.NE.0.0) $Y=Y-D 0 / D 1$

2 CONTINUE

$$
\begin{aligned}
& \mathrm{CX}=\mathrm{CSQRT}\left(\mathrm{CMPLX}\left(\mathrm{Y}^{*} \mathrm{Y}-4 * \mathrm{E}, 0.0\right)\right) \\
& \mathrm{CY}=\mathrm{CMPLX}(\mathrm{Y}, 0.0) \\
& \mathrm{P} 1=\left(-\mathrm{DSQRT}(\mathrm{Y})+\mathrm{CSQRT}\left(-\mathrm{CY}-2^{*} \mathrm{CX}\right)\right) / 2.0 \\
& \mathrm{P} 2=\left(-\mathrm{DSQRT}(\mathrm{Y})-\mathrm{CSQRT}\left(-\mathrm{CY}-2^{*} \mathrm{CX}\right)\right) / 2.0 \\
& \mathrm{P} 3=\left(\mathrm{DSQRT}(\mathrm{Y})+\mathrm{CSQRT}\left(-\mathrm{CY}+2^{*} \mathrm{CX}\right)\right) / 2.0 \\
& \mathrm{P} 4=\left(\mathrm{DSQRT}(\mathrm{Y})-\mathrm{CSQRT}\left(-\mathrm{CY}+2^{*} \mathrm{CX}\right)\right) / 2.0
\end{aligned}
$$

$\mathrm{CC}=\mathrm{FP}(\mathrm{P} 1)$

\author{
IF $(\mathrm{CABS}(\mathrm{CC})>0.01)$ THEN \\ $\mathrm{PR}=\mathrm{REAL}(\mathrm{P} 1)$ \\ $\mathrm{PI}=\mathrm{AIMAG}(\mathrm{P} 1)$ \\ CALL NEWTON(PR,PI,B,C,D,E) \\ $\mathrm{P} 1=\mathrm{CMPLX}$ (PR,PI) \\ END IF

$$
\mathrm{CC}=\mathrm{FP}(\mathrm{P} 2)
$$ \\ $\mathrm{IF}(\mathrm{CABS}(\mathrm{CC})>0.01) \mathrm{THEN}$ \\ $\mathrm{PR}=\mathrm{REAL}(\mathrm{P} 2)$ \\ $\mathrm{PI}=\mathrm{AIMAG}(\mathrm{P} 2)$ \\ CALL NEWTON(PR,PI,B,C,D,E) \\ $\mathrm{P} 2=\mathrm{CMPLX}(\mathrm{PR}, \mathrm{PI})$ \\ END IF \\ $\mathrm{CC}=\mathrm{FP}(\mathrm{P} 3)$ \\ $\mathrm{IF}(\mathrm{CABS}(\mathrm{CC})>0.01)$ THEN \\ $\mathrm{PR}=\mathrm{REAL}(\mathrm{P} 3)$
}


PI=AIMAG(P3)

CALL NEWTON(PR,PI,B,C,D,E)

P3=CMPLX(PR,PI)

END IF

$\mathrm{CC}=\mathrm{FP}(\mathrm{P} 4)$

$\mathrm{IF}(\mathrm{CABS}(\mathrm{CC})>0.01)$ THEN

$\mathrm{PR}=\mathrm{REAL}(\mathrm{P} 4)$

$\mathrm{PI}=\mathrm{AIMAG}(\mathrm{P} 4)$

CALL NEWTON(PR,PI,B,C,D,E)

P4=CMPLX(PR,PI)

END IF

GOTO 12

$4 \quad \mathrm{~B}=-\mathrm{KA}^{*} \mathrm{LAM} 24$

$\mathrm{C}=2 * \mathrm{KA} *(\mathrm{LAM} 1 * \mathrm{LAM} 2 * \mathrm{AL}) * * 2+\mathrm{LAM} 24 *(1.0-2 * \mathrm{KA} * \mathrm{AL} * \mathrm{AL})$

$\mathrm{D}=-\mathrm{KA}^{*} \mathrm{AL} * * 4 *(\mathrm{LAM} 2 * * 2-\mathrm{LAM} 1 * * 2) * * 2+2 *(\mathrm{LAM} 2 * \mathrm{AL}) * * 2 *(\mathrm{LAM} 2 * * 2-$

LAM1**2)

$\mathrm{E}=(\mathrm{LAM} 2 * * 2-\mathrm{LAM} 1 * * 2) * * 2 * \mathrm{AL}^{* * 4}$

$\mathrm{NA}=2$

$\mathrm{IF}(2<=\mathrm{AA} / \mathrm{HH} . \mathrm{AND} . \mathrm{AA} / \mathrm{HH}<4) \mathrm{NA}=5$

IF $(4<=\mathrm{AA} / \mathrm{HH} . \mathrm{AND} . \mathrm{AA} / \mathrm{HH}<8) \mathrm{NA}=10$

$\mathrm{IF}(8<=\mathrm{AA} / \mathrm{HH} . \mathrm{AND} . \mathrm{AA} / \mathrm{HH}<12) \mathrm{NA}=15$

$\mathrm{IF}(\mathrm{AL}<=\mathrm{NA})$ THEN

$\mathrm{CC}=\mathrm{FP}(\mathrm{P} 1)$

IF $(\mathrm{CABS}(\mathrm{CC})>0.01)$ THEN

$\mathrm{PR}=\mathrm{REAL}(\mathrm{P} 1)$

$\mathrm{PI}=\mathrm{AIMAG}(\mathrm{P} 1)$

CALL NEWTON(PR,PI,B,C,D,E)

P1=CMPLX(PR,PI)

END IF

$\mathrm{CC}=\mathrm{FP}(\mathrm{P} 2)$

IF $(\mathrm{CABS}(\mathrm{CC})>0.01)$ THEN

$\mathrm{PR}=\mathrm{REAL}(\mathrm{P} 2)$

$\mathrm{PI}=\mathrm{AIMAG}(\mathrm{P} 2)$ 


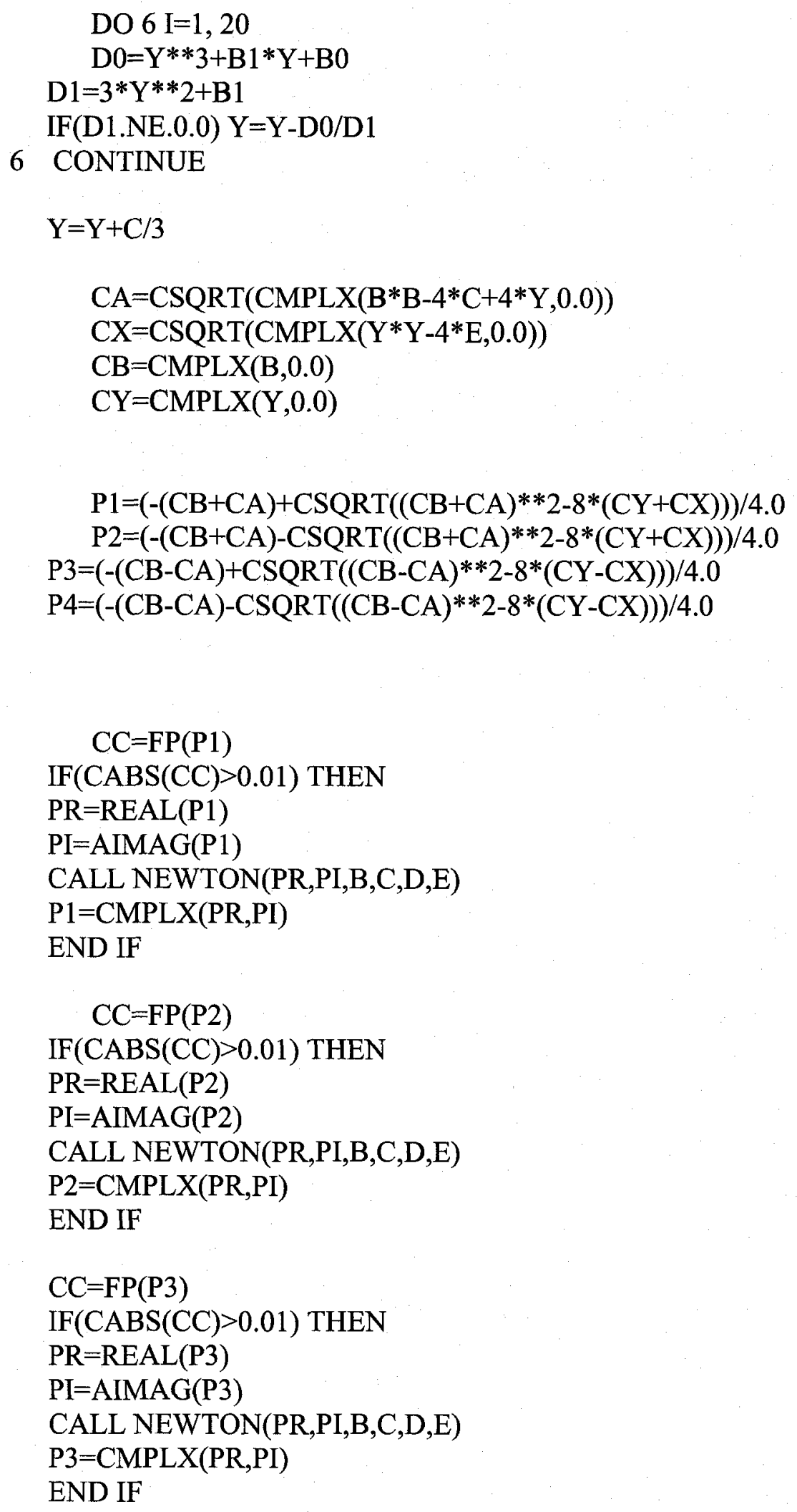


$\mathrm{CC}=\mathrm{FP}(\mathrm{P} 4)$

$\operatorname{IF}(\mathrm{CABS}(\mathrm{CC})>0.01)$ THEN

$\mathrm{PR}=\mathrm{REAL}(\mathrm{P} 4)$

$\mathrm{PI}=\mathrm{AIMAG}(\mathrm{P} 4)$

CALL NEWTON(PR,PI,B,C,D,E)

P4=CMPLX(PR,PI)

END IF

END IF

12 IF(CABS(KA*P1-1)<0.000000001) P1 $=1.0000000001 / \mathrm{KA}$

IF $\left(\mathrm{CABS}\left(\mathrm{KA}^{* \mathrm{P} 2-1)<0.000000001)} \mathrm{P} 2=1.0000000001 / \mathrm{KA}\right.\right.$

IF(CABS(KA $*$ P3-1) $<0.000000001) P 3=1.0000000001 / K A$

IF(CABS(KA*P4-1)<0.000000001) P4 $=1.0000000001 / \mathrm{KA}$

\footnotetext{
$\mathrm{M} 1=\mathrm{CSQRT}(\mathrm{P} 1+\mathrm{AL} * \mathrm{AL})$

IF(REAL(M1).GT.0.0) M1=-M1

$\mathrm{M} 2=\mathrm{CSQRT}(\mathrm{P} 2+\mathrm{AL} * \mathrm{AL})$

IF(REAL(M2).GT.0.0) M2=-M2

$\mathrm{M} 3=\mathrm{CSQRT}(\mathrm{P} 3+\mathrm{AL} * \mathrm{AL})$

IF(REAL(M3).GT.0.0) M3=-M3

$\mathrm{M} 4=\mathrm{CSQRT}(\mathrm{P} 4+\mathrm{AL} * \mathrm{AL})$

IF(REAL(M4).GT.0.0) M4=-M4
}

$\mathrm{IF}(\mathrm{CABS}(\mathrm{M} 1)<0.000000001) \mathrm{M} 1=-\mathrm{AL}$

$\operatorname{IF}(\mathrm{CABS}(\mathrm{M} 2)<0.000000001) \mathrm{M} 2=-\mathrm{AL}$

IF(CABS(M3)<0.000000001) M3 $=-\mathrm{AL}$

$\mathrm{IF}(\mathrm{CABS}(\mathrm{M} 4)<0.000000001) \mathrm{M} 4=-\mathrm{AL}$

C------ CALCULATING THE VALUES OF Nj AND Qj FOR j=1-4

$\mathrm{CD}=(\mathrm{P} 2-\mathrm{P} 1)^{*}(\mathrm{P} 3-\mathrm{P} 1)^{*}(\mathrm{P} 4-\mathrm{P} 1)^{*}(\mathrm{P} 3-\mathrm{P} 2) *(\mathrm{P} 4-\mathrm{P} 2)^{*}(\mathrm{P} 4-\mathrm{P} 3)$

$\operatorname{IF}(\mathrm{CABS}(\mathrm{CD})<10.0 * *(-20))$ GOTO 8

$\mathrm{CD}=1.0 / \mathrm{CD}$

$\mathrm{R} 1=-\mathrm{SQRT}(\mathrm{AL} * \mathrm{AL}+2.0 / \mathrm{KA} /(1-\mathrm{VV}))$ 

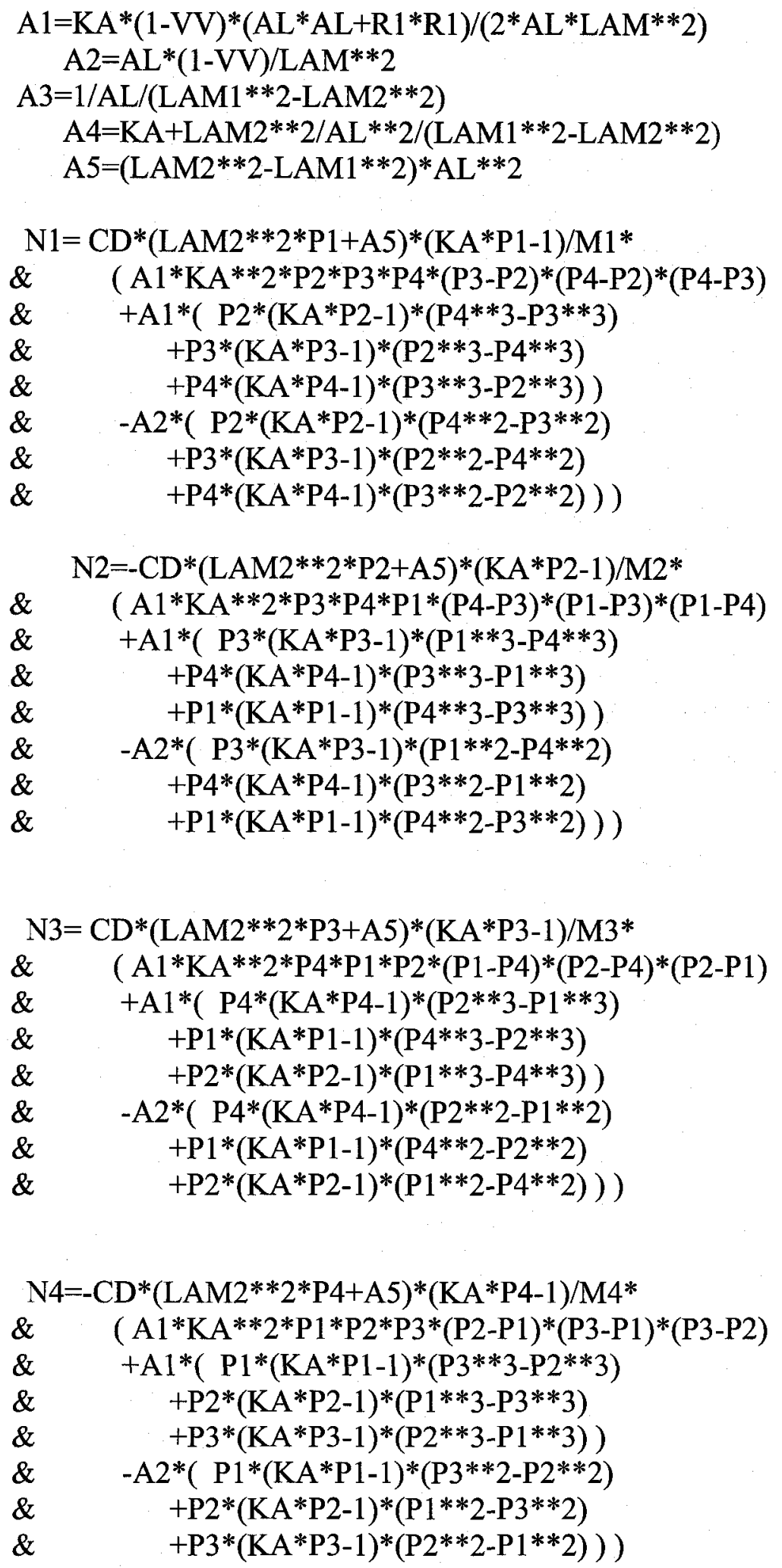

$\mathrm{Q} 1=\mathrm{CD} *(\mathrm{LAM} 2 * * 2 * \mathrm{P} 1+\mathrm{A} 5) *(\mathrm{KA} * \mathrm{P} 1-1) * \mathrm{~A} 3 / \mathrm{M} 1 *$ 

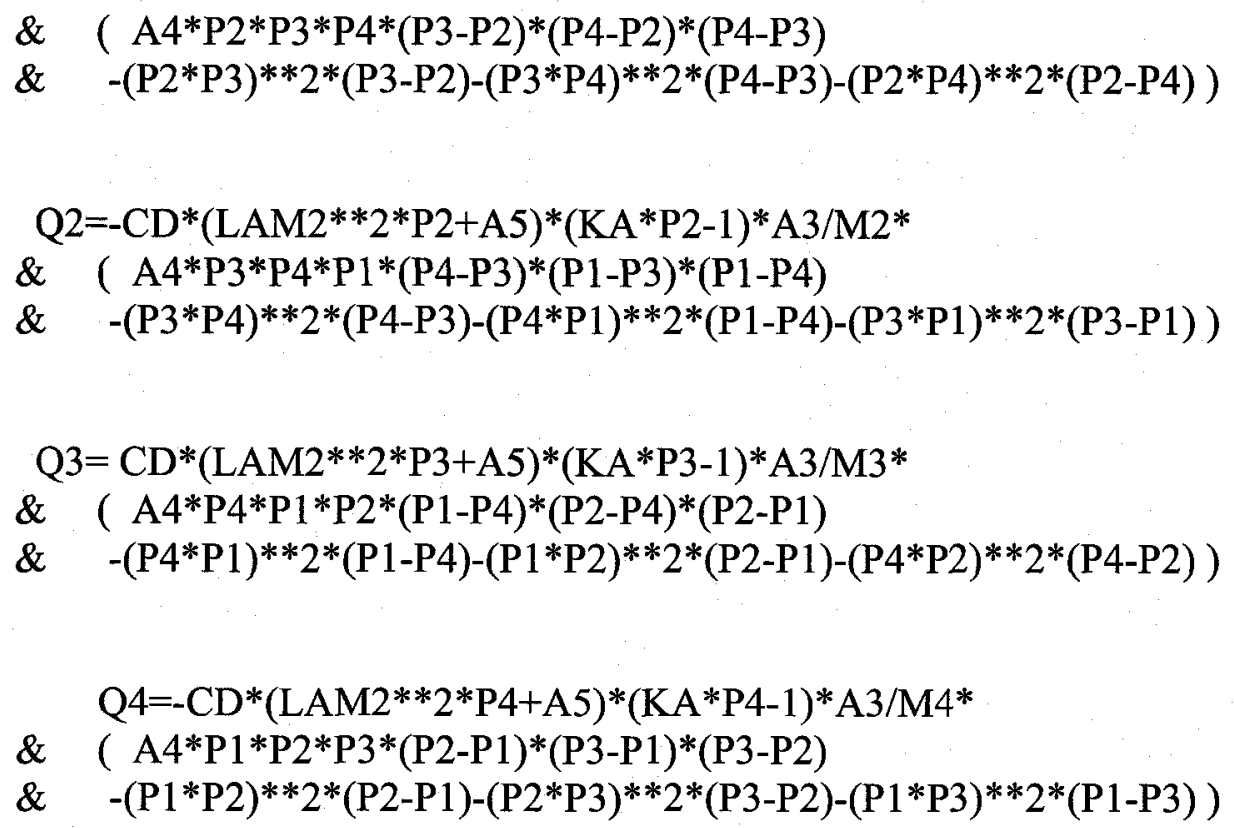

$\mathrm{CC}=2 * \mathrm{AL}^{*} \mathrm{AL} *(\mathrm{Q} 1+\mathrm{Q} 2+\mathrm{Q} 3+\mathrm{Q} 4)-1$

FREK11(K)=REAL(CC)

$$
\mathrm{CA}=2 * A L * A L *(\mathrm{~N} 1+\mathrm{N} 2+\mathrm{N} 3+\mathrm{N} 4)
$$

FREK12(K)=REAL(CA)

$$
\mathrm{CB}=-2 / \mathrm{LAM}^{* *} 2 *
$$

$\&\left(\mathrm{P} 1 * * 2 *\left(\mathrm{M} 1 * * 2-\mathrm{VV}^{*} \mathrm{AL} * \mathrm{AL}\right) /\left(\mathrm{KA}^{*} \mathrm{P} 1-1\right) /((\mathrm{LAM} 2 * \mathrm{M} 1) * * 2-(\mathrm{LAM} 1 * \mathrm{AL}) * * 2) * \mathrm{Q} 1\right.$ $\&+\mathrm{P} 2 * * 2 *\left(\mathrm{M} 2 * * 2-\mathrm{VV}^{*} \mathrm{AL} * \mathrm{AL}\right) /(\mathrm{KA} * \mathrm{P} 2-1) /((\mathrm{LAM} 2 * \mathrm{M} 2) * * 2-$

$(\mathrm{LAM} 1 * \mathrm{AL}) * * 2) * \mathrm{Q} 2$

$\&+\mathrm{P} 3 * * 2 *\left(\mathrm{M} 3 * * 2-\mathrm{VV}^{*} \mathrm{AL} * \mathrm{AL}\right) /\left(\mathrm{KA}^{*} \mathrm{P} 3-1\right) /\left((\mathrm{LAM} 2 * \mathrm{M} 3)^{* * 2-}\right.$

$(\mathrm{LAM} 1 * \mathrm{AL}) * * 2) * \mathrm{Q} 3$

$\&+\mathrm{P} 4 * * 2 *(\mathrm{M} 4 * * 2-\mathrm{VV} * \mathrm{AL} * \mathrm{AL}) /\left(\mathrm{KA}^{*} \mathrm{P} 4-1\right) /((\mathrm{LAM} 2 * \mathrm{M} 4) * * 2-$

$(\mathrm{LAM} 1 * \mathrm{AL}) * * 2) * \mathrm{Q} 4)$

$\operatorname{FREK} 21(\mathrm{~K})=\mathrm{REAL}(\mathrm{CA})$

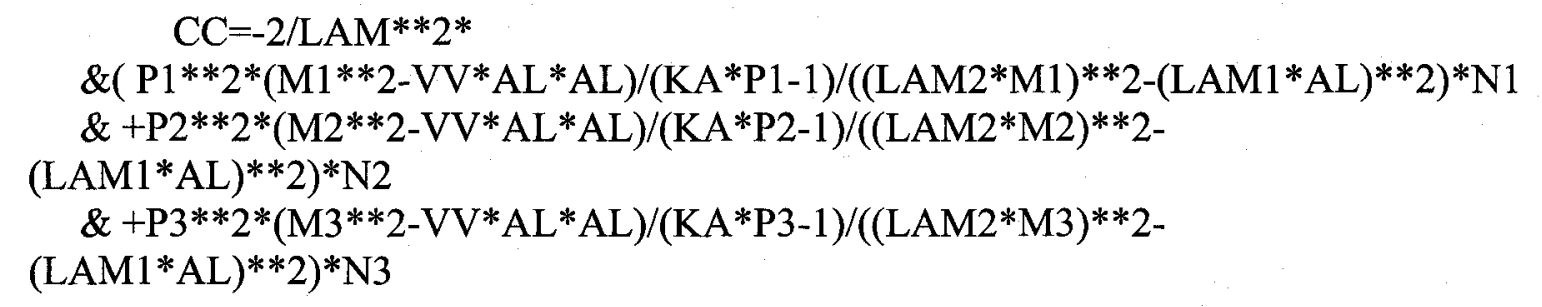


$\&+\mathrm{P} 4 * * 2 *\left(\mathrm{M} 4 * * 2-\mathrm{VV}^{*} \mathrm{AL} * \mathrm{AL}\right) /\left(\mathrm{KA}^{*} \mathrm{P} 4-1\right) /\left((\mathrm{LAM} 2 * \mathrm{M} 4)^{* *} 2-\right.$

$(\mathrm{LAM} 1 * \mathrm{AL}) * * 2) * \mathrm{~N} 4)$

\& $-2.0 / \mathrm{LAM} 4 *(-\mathrm{KA} *(1-\mathrm{VV}) * * 2 * \mathrm{AL} * \mathrm{R} 1)-\mathrm{A} 0$

FREK22(K)=REAL $(\mathrm{CC})$

8 CONTINUE

C----- CALCULATING THE KERNEL FUNCTIONS OF THE INTEGRAL

$\mathrm{C} 1=\mathrm{SQRT}(\mathrm{CE})$

DO $20 \mathrm{I}=1, \mathrm{~N}$

DO $20 \mathrm{~J}=1, \mathrm{~N}$

REK $11(\mathrm{I}, \mathrm{J})=0.0$

REK $12(\mathrm{I}, \mathrm{J})=0.0$

$\operatorname{REK} 21(\mathrm{I}, \mathrm{J})=0.0$

$\operatorname{REK} 22(\mathrm{I}, \mathrm{J})=0.0$

$\mathrm{YI}=\mathrm{COS}(\mathrm{PAI} *(\mathrm{I}-0.5) /(\mathrm{N}-1))$

$\mathrm{TJ}=\operatorname{COS}(\mathrm{PAI} *(\mathrm{~J}-1.0) /(\mathrm{N}-1))$

DO $10 \mathrm{~K}=1, \mathrm{M}$

$\mathrm{AL}=\mathrm{K} * \mathrm{DA}$

$\mathrm{SINK}=\mathrm{SIN}\left(\mathrm{C} 1 * A L^{*}(\mathrm{TJ}-\mathrm{YI})\right)$

REK11(I,J)=REK11(I,J)+C1*FREK11(K)*SINK*DA

REK12(I,J)=REK12(I,J)+C1 *FREK12(K)*SINK *DA

REK21 $(\mathrm{I}, \mathrm{J})=\mathrm{REK} 21(\mathrm{I}, \mathrm{J})+\mathrm{C} 1 *$ FREK $21(\mathrm{~K}) * \mathrm{SINK} * \mathrm{DA}$

REK22(I,J) $=$ REK22(I,J)+C1*FREK22(K)*SINK*DA

10 CONTINUE

20 CONTINUE

RETURN

END 
C-----THIS IS THE NEWTON ITERATIVE METHOD FOR FINDING THE ROOTS OF NONLINEAR EQAUTIONS

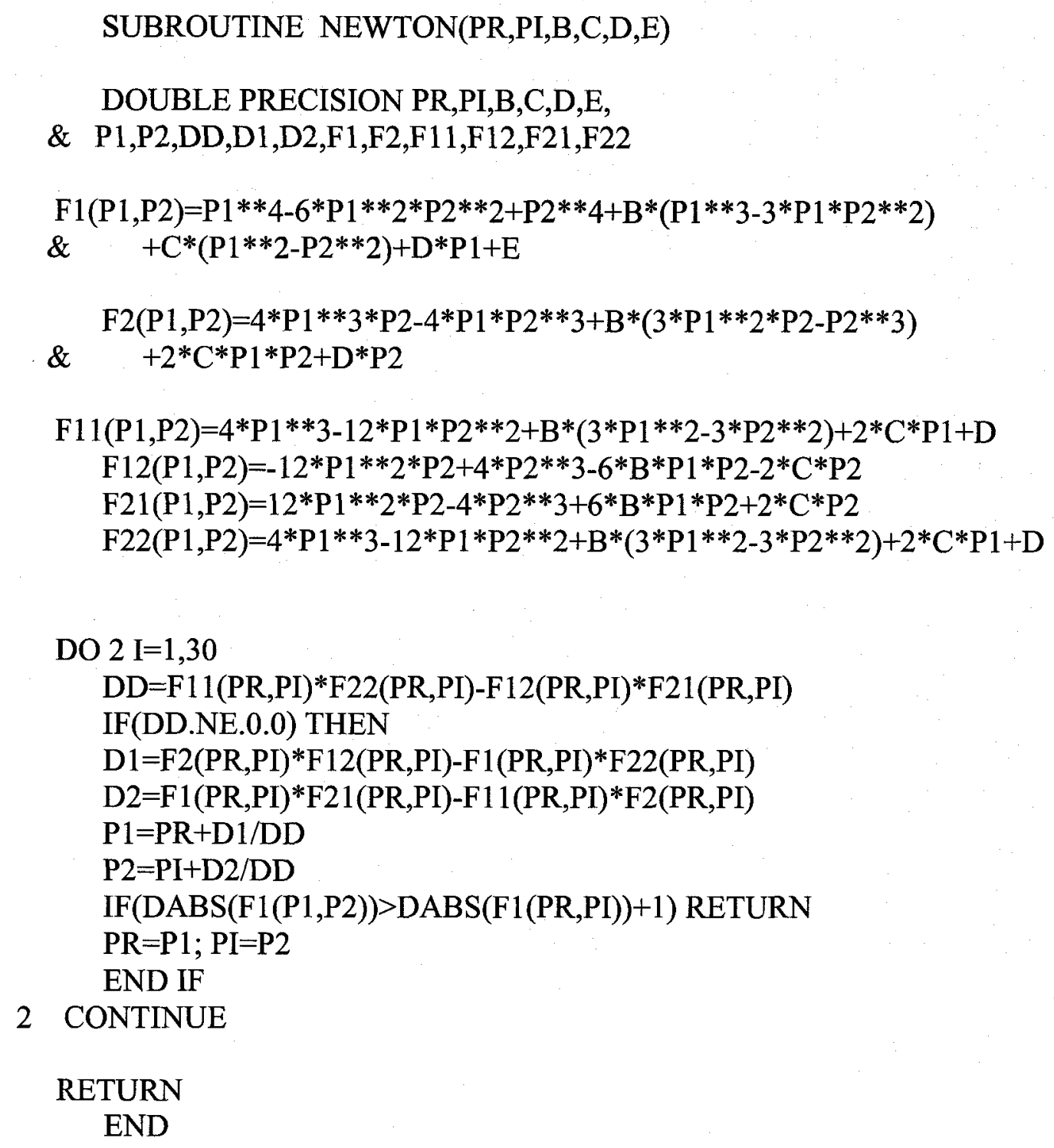

C---- THIS IS THE SUBPROGRAM FOR CALCULATING THE DATA OF THE OPEN DISCRET EQUATIONS

SUBROUTINE OPENEQ(N,A,F,W,T,Y,REK11,REK12,REK21,REK22)

DOUBLE PRECISION REK11(N,N), REK12(N,N), REK21(N,N),REK22(N,N)

DOUBLE PRECISION A $(2 * \mathrm{~N}, 2 * \mathrm{~N}), \mathrm{F}(2 * \mathrm{~N}), \mathrm{W}(\mathrm{N}), \mathrm{Y}(\mathrm{N}), \mathrm{T}(\mathrm{N}), \mathrm{PAI}, \mathrm{H}$

COMMON CE,LAM1,LAM14,LAM,LAM4,LAM2,LAM24, KA,VV, $\mathrm{AA}, \mathrm{HH}, \mathrm{F} 1, \mathrm{~F} 2, \mathrm{~A} 0$ 
$\mathrm{PAI}=3.141592653589798$

$\mathrm{H}=\mathrm{PAI} /(\mathrm{N}-1)$

DO $2 \mathrm{I}=2, \mathrm{~N}-1$

$2 \mathrm{~W}(\mathrm{I})=\mathrm{H}$

$\mathrm{W}(1)=\mathrm{H} / 2 ; \mathrm{W}(\mathrm{N})=\mathrm{H} / 2$

DO $4 \mathrm{I}=1, \mathrm{~N}$

$\mathrm{T}(\mathrm{I})=\operatorname{COS}\left(\mathrm{H}^{*}(\mathrm{I}-1.0)\right)$

$\mathrm{Y}(\mathrm{I})=\mathrm{COS}\left(\mathrm{H}^{*}(\mathrm{I}-0.5)\right)$

$\mathrm{F}(\mathrm{I})=2 * \mathrm{PAI} * \mathrm{~F} 1$

$\mathrm{F}(\mathrm{N}+\mathrm{I})=2 * \mathrm{PAI} * \mathrm{HH} / \mathrm{AA}^{*} \mathrm{~F} 2$

4 CONTINUE

$\mathrm{F}(\mathrm{N})=0.0 ; \mathrm{F}(2 * \mathrm{~N})=0.0$

DO $6 \mathrm{I}=1, \mathrm{~N}-1$

DO $6 \mathrm{~J}=1, \mathrm{~N}$

$\mathrm{A}(\mathrm{I}, \mathrm{J})=\mathrm{W}(\mathrm{J}) *(1.0 /(\mathrm{T}(\mathrm{J})-\mathrm{Y}(\mathrm{I}))+\mathrm{REK} 11(\mathrm{I}, \mathrm{J}))$

$\mathrm{A}(\mathrm{I}, \mathrm{N}+\mathrm{J})=\mathrm{W}(\mathrm{J}) * \mathrm{REK} 12(\mathrm{I}, \mathrm{J})$

$\mathrm{A}(\mathrm{N}+\mathrm{I}, \mathrm{J})=\mathrm{W}(\mathrm{J}) * \mathrm{REK} 21(\mathrm{I}, \mathrm{J})$

$\mathrm{A}(\mathrm{N}+\mathrm{I}, \mathrm{N}+\mathrm{J})=\mathrm{W}(\mathrm{J}) *(\mathrm{~A} 0 /(\mathrm{T}(\mathrm{J})-\mathrm{Y}(\mathrm{I}))+\mathrm{REK} 22(\mathrm{I}, \mathrm{J}))$

6 CONTINUE

DO $8 \mathrm{~J}=1, \mathrm{~N}$

$\mathrm{A}(\mathrm{N}, \mathrm{J}) \quad=\mathrm{W}(\mathrm{J})$

$\mathrm{A}(\mathrm{N}, \mathrm{N}+\mathrm{J})=0.0$

$\mathrm{A}(2 * \mathrm{~N}, \mathrm{~J})=0.0$

$\mathrm{A}(2 * \mathrm{~N}, \mathrm{~N}+\mathrm{J})=\mathrm{W}(\mathrm{J})$

8 CONTINUE

RETURN

END

C----- THIS IS THE SUBPROGRAM FOR CALCULATING THE DATA OF THE CLOSE-INNER DISCRET EQUATIONS

SUBROUTINE CLOSEEQINNER(N,A,F,W,T,Y,REK11,REK12,REK21,REK22)

DOUBLE PRECISION REK11(N,N), REK12(N,N), REK21(N,N),REK22(N,N) 

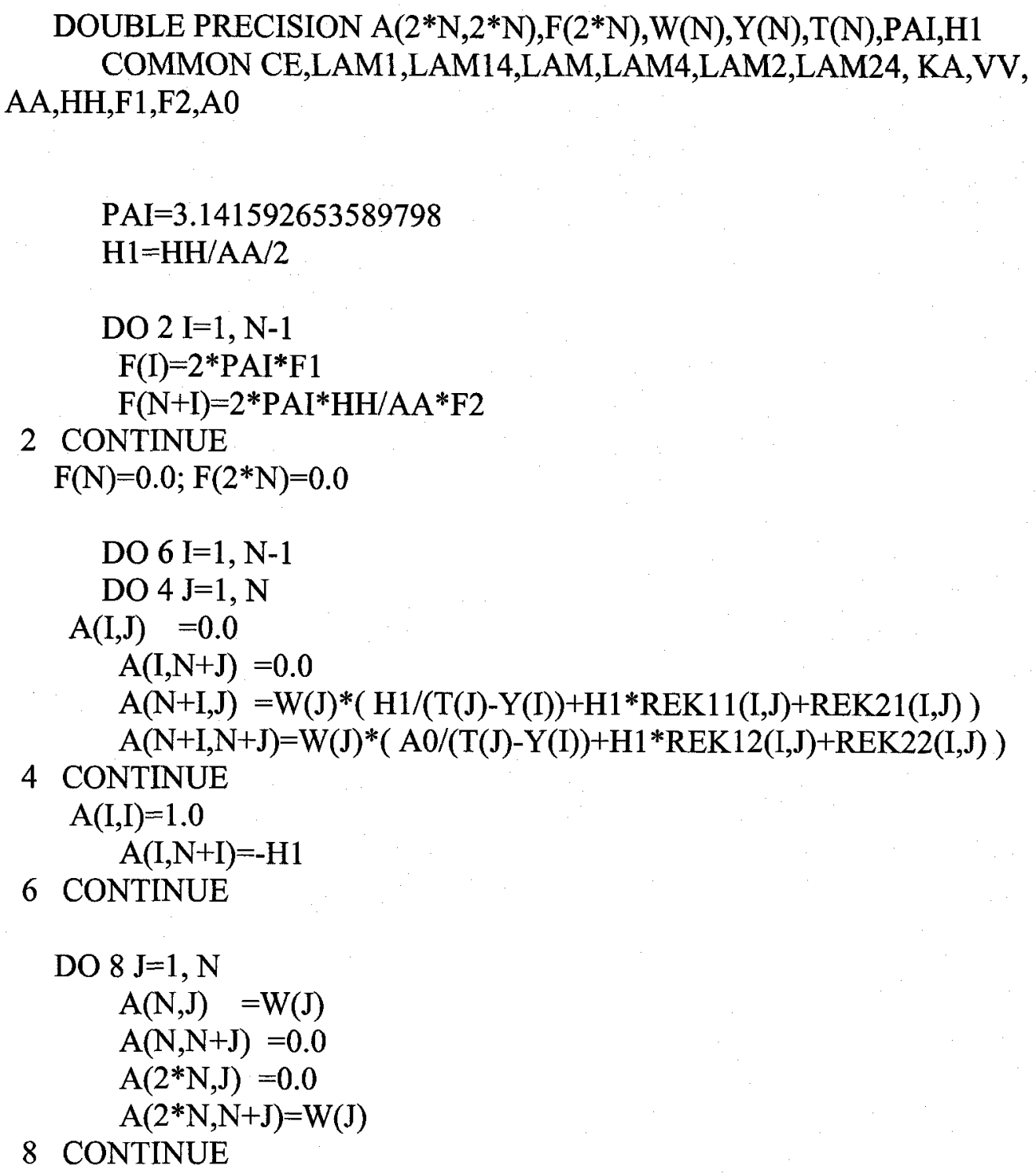

\section{RETURN}

END

C---- THIS IS THE SUBPROGRAM FOR CALCULATING THE DATA OF THE CLOSE-OUTER DISCRET EQUATIONS

SUBROUTINE CLOSEEQOUTER(N,A,F,W,T,Y,REK11,REK12,REK21,REK22)

DOUBLE PRECISION REK11(N,N), REK12(N,N), REK21(N,N),REK22(N,N) DOUBLE PRECISION A $\left(2^{*} \mathrm{~N}, 2^{*} \mathrm{~N}\right), \mathrm{F}\left(2^{*} \mathrm{~N}\right), \mathrm{W}(\mathrm{N}), \mathrm{Y}(\mathrm{N}), \mathrm{T}(\mathrm{N}), \mathrm{PAI}, \mathrm{H} 1$ 


\section{COMMON CE,LAM1,LAM14,LAM,LAM4,LAM2,LAM24, KA,VV, $\mathrm{AA}, \mathrm{HH}, \mathrm{F} 1, \mathrm{~F} 2, \mathrm{~A} 0$}

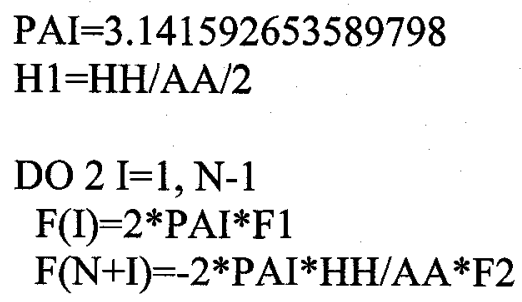

2 CONTINUE

$\mathrm{F}(\mathrm{N})=0.0 ; \mathrm{F}(2 * \mathrm{~N})=0.0$

DO $6 \mathrm{I}=1, \mathrm{~N}-1$

DO $4 \mathrm{~J}=1, \mathrm{~N}$

$\mathrm{A}(\mathrm{I}, \mathrm{J})=0.0$

$\mathrm{A}(\mathrm{I}, \mathrm{N}+\mathrm{J})=0.0$

$\mathrm{A}(\mathrm{N}+\mathrm{I}, \mathrm{J})=\mathrm{W}(\mathrm{J}) *(\mathrm{H} 1 /(\mathrm{T}(\mathrm{J})-\mathrm{Y}(\mathrm{I}))+\mathrm{H} 1 * \mathrm{REK} 11(\mathrm{I}, \mathrm{J})-\mathrm{REK} 21(\mathrm{I}, \mathrm{J}))$

$\mathrm{A}(\mathrm{N}+\mathrm{I}, \mathrm{N}+\mathrm{J})=\mathrm{W}(\mathrm{J}) *(-\mathrm{A} 0 /(\mathrm{T}(\mathrm{J})-\mathrm{Y}(\mathrm{I}))+\mathrm{H} 1 *$ REK12(I,J)-REK22(I,J) )

4 CONTINUE

$\mathrm{A}(\mathrm{I}, \mathrm{I})=1.0$

$\mathrm{A}(\mathrm{I}, \mathrm{N}+\mathrm{I})=\mathrm{H} 1$

6 CONTINUE

DO $8 \mathrm{~J}=1, \mathrm{~N}$

$\mathrm{A}(\mathrm{N}, \mathrm{J})=\mathrm{W}(\mathrm{J})$

$\mathrm{A}(\mathrm{N}, \mathrm{N}+\mathrm{J})=0.0$

$\mathrm{A}(2 * \mathrm{~N}, \mathrm{~J})=0.0$

$\mathrm{A}(2 * \mathrm{~N}, \mathrm{~N}+\mathrm{J})=\mathrm{W}(\mathrm{J})$

8 CONTINUE

RETURN

END

C---- THIS IS THE SUBPROGRAM FOR SOLVING THE LINEAR EQUATIONS BY GAUSS ELIMINATE METHOD

SUBROUTINE SOLVEEQ(N,A,B,X)

DOUBLE PRECISION A(N,N), B(N), X(N), C, T, S

DO $12 \mathrm{~K}=1, \mathrm{~N}-1$ 
$\mathrm{L}=\mathrm{K}$

DO $2 \mathrm{I}=\mathrm{K}+1, \mathrm{~N}$

IF(DABS(A(I,K)).GT.DABS(A(L,K))) L=I

2 CONTINUE

$\mathrm{IF}(\mathrm{A}(\mathrm{L}, \mathrm{K})==0.0) \mathrm{THEN}$

PRINT*, 'NO UNIQUE SOLUTION'

STOP

END IF

IF(L.NE.K) THEN

$\mathrm{T}=\mathrm{B}(\mathrm{K})$

$\mathrm{B}(\mathrm{K})=\mathrm{B}(\mathrm{L})$

$\mathrm{B}(\mathrm{L})=\mathrm{T}$

DO $4 \mathrm{~J}=\mathrm{K}, \mathrm{N}$

$\mathrm{T}=\mathrm{A}(\mathrm{K}, \mathrm{J})$

$A(K, J)=A(L, J)$

$\mathrm{A}(\mathrm{L}, \mathrm{J})=\mathrm{T}$

4 CONTINUE

END IF

DO $8 \mathrm{I}=\mathrm{K}+1, \mathrm{~N}$

$\mathrm{C}=\mathrm{A}(\mathrm{I}, \mathrm{K}) / \mathrm{A}(\mathrm{K}, \mathrm{K})$

$\mathrm{B}(\mathrm{I})=\mathrm{B}(\mathrm{I})-\mathrm{B}(\mathrm{K}) * \mathrm{C}$

DO $6 \mathrm{~J}=\mathrm{K}+1, \mathrm{~N}$

$6 \quad \mathrm{~A}(\mathrm{I}, \mathrm{J})=\mathrm{A}(\mathrm{I}, \mathrm{J})-\mathrm{A}(\mathrm{K}, \mathrm{J}) * \mathrm{C}$

8 CONTINUE

12 CONTINUE

$\mathrm{X}(\mathrm{N})=\mathrm{B}(\mathrm{N}) / \mathrm{A}(\mathrm{N}, \mathrm{N})$

DO $16 \mathrm{M}=1, \mathrm{~N}-1$

$\mathrm{I}=\mathrm{N}-\mathrm{M}$

$\mathrm{S}=0.0$

DO $14 \mathrm{~J}=\mathrm{I}+1, \mathrm{~N}$

$14 \mathrm{~S}=\mathrm{S}+\mathrm{A}(\mathrm{I}, \mathrm{J}) * \mathrm{X}(\mathrm{J})$

$X(I)=(B(I)-S) / A(I, I)$

16 CONTINUE

RETURN

END 


\section{Program 3-Specially Orthotropic Spherical Shell}

C----MAIN PROGRAM: CRACK CLOSURE ANALYSIS ON SPHERICAL SHELL WITH DIFFERENT E1 AND E2

PARAMETER ( $\mathrm{N}=41, \mathrm{~T}=60.0)$

REAL LAM0,LAM1,LAM14, LAM, LAM4, LAM2, LAM24, KA, Km, Kb

DOUBLE PRECISION REK11(N,N), REK12(N,N), REK21(N,N), REK22(N,N),

$\& \quad \mathrm{~A}\left(2 * \mathrm{~N}, 2^{*} \mathrm{~N}\right), \mathrm{A} 1(2 * \mathrm{~N}, 2 * \mathrm{~N}), \mathrm{A} 2(2 * \mathrm{~N}, 2 * \mathrm{~N}), \mathrm{B}(2 * \mathrm{~N}), \mathrm{B} 1(2 * \mathrm{~N}), \mathrm{B} 2(2 * \mathrm{~N})$,

\& $\quad \mathrm{G}(2 * \mathrm{~N}), \mathrm{W}(\mathrm{N}), \mathrm{TT}(\mathrm{N}), \mathrm{YY}(\mathrm{N}), \mathrm{P}(\mathrm{N})$

COMMON

CE,LAM1,LAM14,LAM,LAM4,LAM2,LAM24,KA,VV,AA,HH,F1,F2,A0

OPEN(UNIT=10, FILE='TEST3.DAT', STATUS='UNKNOWN',

\& FORM='FORMATTED', POSITION='APPEND', IOSTAT=ISTAT )

IF(ISTAT/=0) PRINT*, 'FILE OPEN FAIL: ISTAT=','ISTAT

$\mathrm{E} 12=26.67$

$\mathrm{CE}=\mathrm{E} 12 * *(1.0 / 4)$

$\operatorname{IF}(\mathrm{E} 12=0.725 . \mathrm{OR} \cdot \mathrm{E} 12==1.38)$ THEN

$\mathrm{V} 1=0.1966$

$\mathrm{V} 2=0.2174$

ELSE

$\mathrm{V} 1=0.0075$

$\mathrm{V} 2=0.2$

END IF

$\mathrm{VV}=\mathrm{SQRT}(\mathrm{V} 1 * \mathrm{~V} 2)$

$\mathrm{IF}(\mathrm{E} 12=1.0) \mathrm{VV}=1.0 / 3$

$\mathrm{AA}=10$

$\mathrm{HH}=1$

LAM4 $=12 *\left(1-\mathrm{VV}^{*} \mathrm{VV}\right) * \mathrm{AA} * \mathrm{AA} /(\mathrm{HH} * \mathrm{HH})$

LAM $=$ LAM $4 * *(1.0 / 4)$

$\mathrm{KA}=12 *(1+\mathrm{VV}) /(5 * \mathrm{LAM} 4)$

$\mathrm{A} 0=(1-\mathrm{VV} * \mathrm{VV}) / \mathrm{LAM} 4$

SING $=-1 \quad$ !SING=1 FOR INNER-CLOSE CASE AND SING=-1 FOR OUTERCLOSE

$\mathrm{F} 1=0.0$

$\mathrm{F} 2=-1.0 / 6$ 
$\operatorname{WRITE}(10, *))^{\prime * * * * * * * * * * * * * * * * * * * * * * * * * * * * * * * * * * * * * * * * * * * * * * * * * * * *}$

PRINT*','a/h=',AA/HH, 'E1/E2=',E12

WRITE(10,2) AA/HH, E12

2 FORMAT(1X,4HA/H=,F5.2,3X,'E1/E2=',F6.3)

$\mathrm{N} 1=2 * \mathrm{AA} / \mathrm{HH}$

DO $120 \mathrm{M}=1, \mathrm{~N} 1$

LAM $0=0.5^{*} \mathrm{M}$

LAM $1=L A M 0 * S Q R T(C E)$

LAM2 $=$ LAM0/SQRT(CE)

LAM14=LAM1**4

$\mathrm{LAM} 24=\mathrm{LAM} 2 * * 4$

CALL KERLFUNC(N,T,REK11,REK12,REK21,REK22)

CALL OPENEQ(N,A1,B1,W,TT,YY,REK11,REK12,REK21,REK22)

$\mathrm{IF}(\mathrm{SING}==1) \mathrm{THEN}$

CALL CLOSEEQINNER(N,A2,B2,W,TT,YY,REK11,REK12,REK21,REK22)

ELSE

CALL CLOSEEQOUTER(N,A2,B2,W,TT,YY,REK11,REK12,REK21,REK22) END IF

C-----IF ONLY CACULATE OPEN CASE, PLEASE IMPLEMENT THESE TWO SENTANCES, OTHERWISE OMIT THEM

!CALL SOLVEEQ $(2 * \mathrm{~N}, \mathrm{~A} 1, \mathrm{~B} 1, \mathrm{G})$

!GO TO 110

C-----IF ONLY CACULATE OPEN CASE, PLEASE IMPLEMENT THESE TWO SENTANCES, OTHERWISE OMIT THEM

$$
\begin{aligned}
& \mathrm{NN}=2 * \mathrm{~N} \\
& \mathrm{~N} 0=(\mathrm{N}-1) / 2
\end{aligned}
$$


$\mathrm{I} 0=100$

$\mathrm{H} 1=-\mathrm{HH} / 2 / 3.1415926$

DO $80 \mathrm{~K}=1$, N0

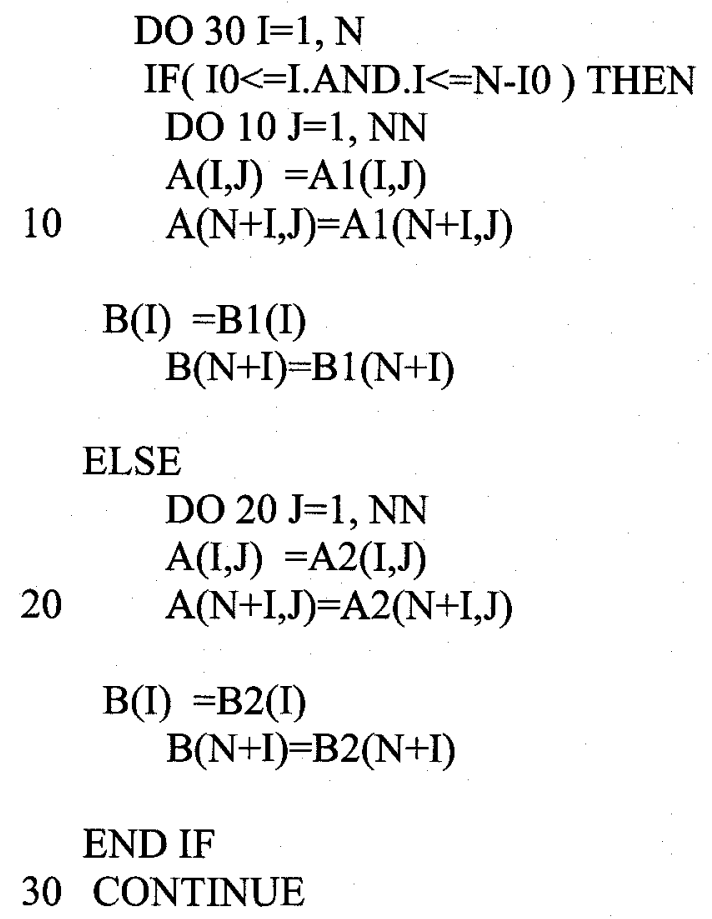

CALL SOLVEEQ(NN,A,B,G)

DO $50 \mathrm{I}=1, \mathrm{~N}-1$

$\mathrm{P}(\mathrm{I})=0.0$

DO $40 \mathrm{~J}=1, \mathrm{~N}$

$\mathrm{P}(\mathrm{I})=\mathrm{P}(\mathrm{I})+\mathrm{SING} \mathrm{H}^{*} 1^{*} \mathrm{~W}(\mathrm{~J}) *(\mathrm{G}(\mathrm{J}) /(\mathrm{TT}(\mathrm{J})-\mathrm{YY}(\mathrm{I}))+\mathrm{G}(\mathrm{J}) * \mathrm{REK} 11(\mathrm{I}, \mathrm{J})$

$\&$ $+\mathrm{G}(\mathrm{N}+\mathrm{J}) * \mathrm{REK} 12(\mathrm{I}, \mathrm{J}))$

40 CONTINUE

$\mathrm{PP}=\mathrm{SNGL}(\mathrm{P}(\mathrm{I}))$

WRITE(10,4) K,I,PP,YY(I)

4 FORMAT(1X,'K=',I2,3X,'I=',I2,3X,'P=',F10.4,2X,'Y=',F7.4)

50 CONTINUE 


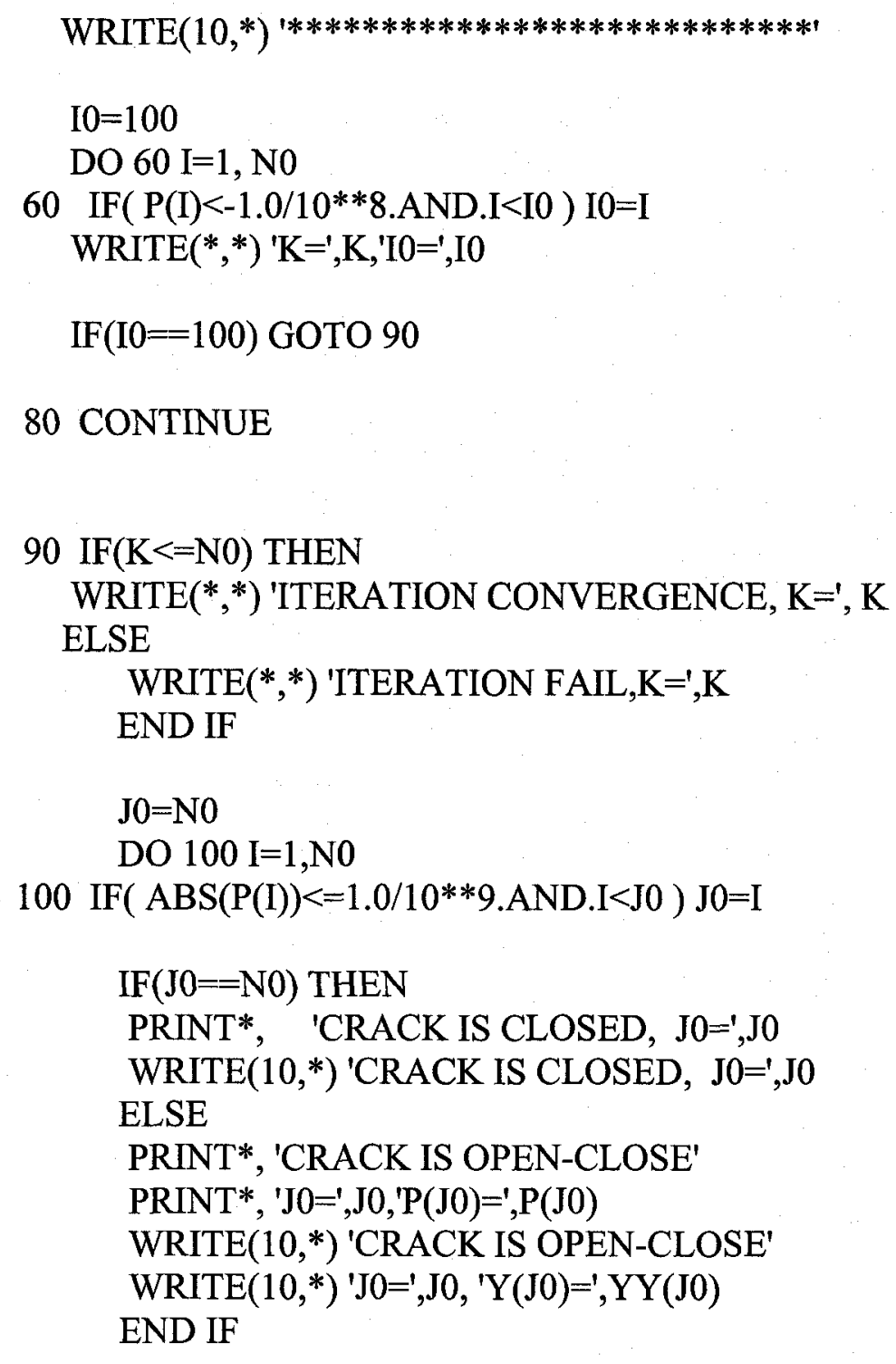

$110 \quad \mathrm{Km}=-0.5 * \mathrm{G}(1)$

$\mathrm{Kb}=-\mathrm{HH} /(4 * \mathrm{AA}) * \mathrm{G}(\mathrm{N}+1)$

PRINT*,'LAM0=',LAM0,'Km=',Km,'Kb=',Kb

WRITE $(10,8)$ LAM0, Km, Kb

8 FORMAT(1X,5HLAM0=,F6.3,3X,'Kmb=',F7.4,3X,'Kbb=',F7.4)

$\operatorname{WRITE}(10, *)$

120 CONTINUE

CLOSE(10)

STOP 
END

C----- THIS IS THE SUBPROGRAM FOR SOLVING THE KERLFUNCTIONS

SUBROUTINE KERLFUNC(N,T,REK11,REK12,REK21,REK22)

REAL LAM1,LAM14, LAM, LAM4, LAM2, LAM24, KA, LAM0

DOUBLE PRECISION REK11(N,N),REK12(N,N), REK21(N,N),REK22(N,N),

\& FREK11(20000),FREK12(20000),FREK21(20000),FREK22(20000)

COMMON

CE,LAM1,LAM14,LAM,LAM4,LAM2,LAM24,KA,VV,AA,HH,F1,F2,A0

COMPLEX P1, P2, P3, P4, M1, M2, M3, M4, N1, N2, N3, N4,

\& $\quad \mathrm{Q} 1, \mathrm{Q} 2, \mathrm{Q} 3, \mathrm{Q} 4, \mathrm{CA}, \mathrm{CB}, \mathrm{CC}, \mathrm{CD}, \mathrm{CX}, \mathrm{CY}, \mathrm{P}, \mathrm{FP}$

DOUBLE PRECISION A, B, C, D, E, B0, B1, Y, D0,D1,PR,PI

$\mathrm{FP}(\mathrm{P})=\mathrm{P} * * 4+\mathrm{B} * \mathrm{P} * * 3+\mathrm{C} * \mathrm{P} * * 2+\mathrm{D} * \mathrm{P}+\mathrm{E}$

$\mathrm{PAI}=3.141592653589798$

$\mathrm{DA}=0.01$

$\mathrm{M}=\mathrm{T} / \mathrm{DA}$

C------ FINDING THE ROOTS Pj AND Mj OF THE POLYNOMIAL EQUATIONS

$\mathrm{P} 1=\left(\mathrm{KA} *\right.$ LAM $24+\mathrm{LAM} 2 * * 2 *$ CSQRT $\left(\right.$ CMPLX $\left(\mathrm{KA}^{* *} 2 *\right.$ LAM $\left.\left.\left.24-4,0.0\right)\right)\right) / 2$

$\mathrm{P} 2=\left(\mathrm{KA}^{*}\right.$ LAM24-LAM $2 * * 2 *$ CSQRT $\left(\right.$ CMPLX $\left(\mathrm{KA}^{* *} 2 *\right.$ LAM24-4,0.0) $\left.)\right) / 2$

DO $8 \mathrm{~K}=1, \mathrm{M}$

\author{
$\mathrm{AL}=\mathrm{K} * \mathrm{DA}$ \\ $\mathrm{B}=-\mathrm{KA} * \mathrm{LAM} 24$ \\ $\mathrm{C}=2 * \mathrm{KA}^{*}(\mathrm{LAM} 1 * \mathrm{LAM} 2 * \mathrm{AL}) * * 2+\mathrm{LAM} 24 *(1.0-2 * \mathrm{KA} * \mathrm{AL} * \mathrm{AL})$ \\ $\mathrm{D}=-\mathrm{KA}^{*} \mathrm{AL} * * 4 *\left(\mathrm{LAM}^{*} * 2-\mathrm{LAM} 1 * * 2\right) * * 2+2 *(\mathrm{LAM} 2 * \mathrm{AL}) * * 2 *(\mathrm{LAM} 2 * * 2-$ \\ LAM1**2) \\ $\mathrm{E}=(\mathrm{LAM} 2 * * 2-\mathrm{LAM} 1 * * 2) * * 2 * \mathrm{AL}^{* *} 4$ \\ $\mathrm{NA}=2$ \\ IF $(2<=\mathrm{AA} / \mathrm{HH} . \mathrm{AND} . \mathrm{AA} / \mathrm{HH}<4) \mathrm{NA}=5$ \\ $\mathrm{IF}(4<=\mathrm{AA} / \mathrm{HH} . \mathrm{AND} . \mathrm{AA} / \mathrm{HH}<8) \mathrm{NA}=10$ \\ $\operatorname{IF}(8<=\mathrm{AA} / \mathrm{HH} . \mathrm{AND} \cdot \mathrm{AA} / \mathrm{HH}<12) \mathrm{NA}=15$
}


$\operatorname{IF}(\mathrm{CE}>1.0) \mathrm{NA}=0$

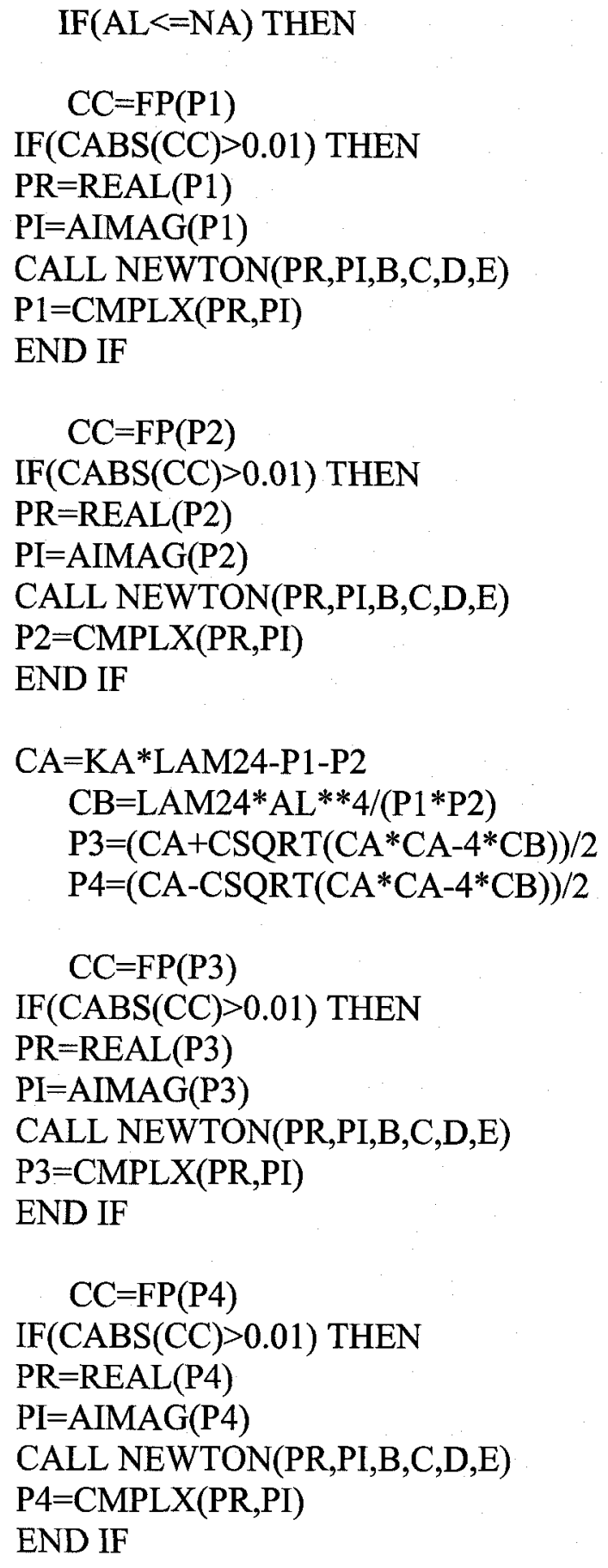

ELSE 


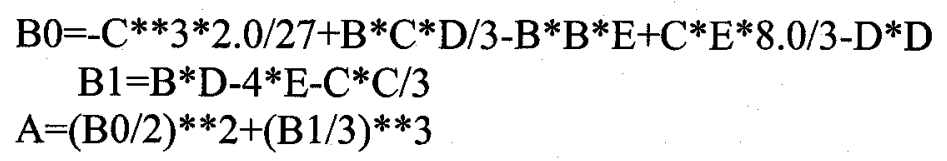

IF(A.LT.0.0) THEN

$\mathrm{CA}=\mathrm{CMPLX}(0.0, \mathrm{DSQRT}(-\mathrm{A}))$

$\mathrm{CB}=\mathrm{CMPLX}(\mathrm{B} 0,0.0)$

$\mathrm{CY}=\mathrm{CEXP}(\mathrm{CLOG}(-\mathrm{CB} / 2+\mathrm{CA}) / 3)+\mathrm{CEXP}(\mathrm{CLOG}(-\mathrm{CB} / 2-\mathrm{CA}) / 3)$

$\mathrm{Y}=\mathrm{REAL}(\mathrm{CY})$

ELSE

$\mathrm{A}=\mathrm{DSQRT}(\mathrm{A})$

$\operatorname{IF}(B 0 . L T .0 .0) \mathrm{Y}=\mathrm{DEXP}(\mathrm{DLOG}(-\mathrm{B} 0 / 2+\mathrm{A}) / 3)+\mathrm{DEXP}(\mathrm{DLOG}(-\mathrm{B} 0 / 2-\mathrm{A}) / 3)$

$\mathrm{IF}(\mathrm{B} 0 . \mathrm{GE} .0 .0) \mathrm{Y}=-\mathrm{DEXP}(\mathrm{DLOG}(\mathrm{B} 0 / 2-\mathrm{A}) / 3)-\mathrm{DEXP}(\mathrm{DLOG}(\mathrm{B} 0 / 2+\mathrm{A}) / 3)$

END IF

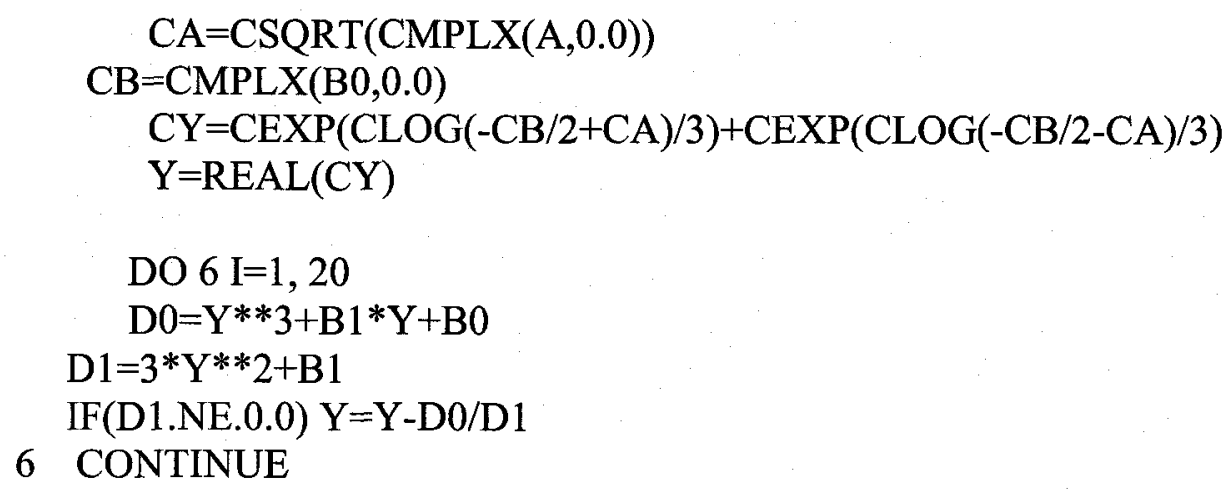




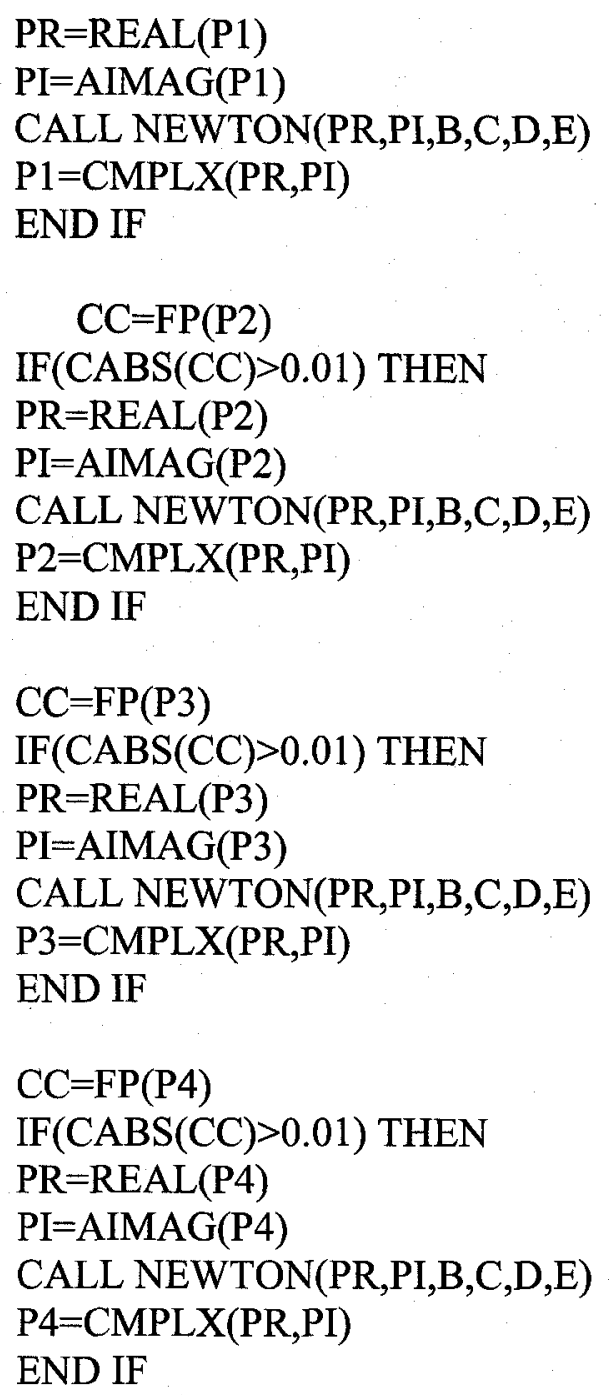

END IF

$12 \quad \mathrm{IF}(\mathrm{CABS}(\mathrm{KA} * \mathrm{P} 1-1)<0.000000001) \mathrm{P} 1=1.0000000001 / \mathrm{KA}$ IF(CABS(KA*P2-1)<0.000000001) P2 $=1.0000000001 / \mathrm{KA}$ IF(CABS(KA*P3-1)<0.000000001) P3 $=1.0000000001 / \mathrm{KA}$ $\mathrm{IF}(\mathrm{CABS}(\mathrm{KA} * \mathrm{P} 4-1)<0.000000001) \mathrm{P} 4=1.0000000001 / \mathrm{KA}$

\footnotetext{
$\mathrm{M} 1=\mathrm{CSQRT}(\mathrm{P} 1+\mathrm{AL} * \mathrm{AL})$

IF(REAL(M1).GT.0.0) M1=-M1

$\mathrm{M} 2=\mathrm{CSQRT}(\mathrm{P} 2+\mathrm{AL} * \mathrm{AL})$

IF(REAL(M2).GT.0.0) M2=-M2

$\mathrm{M} 3=\mathrm{CSQRT}(\mathrm{P} 3+\mathrm{AL} * \mathrm{AL})$
} 
IF(REAL(M3).GT.0.0) M3=-M3

$\mathrm{M} 4=\mathrm{CSQRT}(\mathrm{P} 4+\mathrm{AL} * \mathrm{AL})$

IF(REAL(M4).GT.0.0) M4=-M4

$\operatorname{IF}(\mathrm{CABS}(\mathrm{M} 1)<0.000000001) \mathrm{M} 1=-\mathrm{AL}$

$\mathrm{IF}(\mathrm{CABS}(\mathrm{M} 2)<0.000000001) \mathrm{M} 2=-\mathrm{AL}$

$\operatorname{IF}(\mathrm{CABS}(\mathrm{M} 3)<0.000000001) \mathrm{M} 3=-\mathrm{AL}$

$\operatorname{IF}(\mathrm{CABS}(\mathrm{M} 4)<0.000000001) \mathrm{M} 4=-\mathrm{AL}$

C----- CALCULATING THE VALUES OF Nj AND Qj FOR $\mathrm{j}=1-4$

$\mathrm{CD}=(\mathrm{P} 2-\mathrm{P} 1)^{*}(\mathrm{P} 3-\mathrm{P} 1)^{*}(\mathrm{P} 4-\mathrm{P} 1)^{*}(\mathrm{P} 3-\mathrm{P} 2)^{*}(\mathrm{P} 4-\mathrm{P} 2) *(\mathrm{P} 4-\mathrm{P} 3)$

$\operatorname{IF}\left(\mathrm{CABS}(\mathrm{CD})<10.0^{* *}(-22)\right)$ GOTO 8

$\mathrm{CD}=1.0 / \mathrm{CD}$

$\mathrm{R} 1=-\mathrm{SQRT}(\mathrm{AL} * \mathrm{AL}+2.0 / \mathrm{KA} /(1-\mathrm{VV}))$

$\mathrm{A} 1=\mathrm{KA} *(1-\mathrm{VV}) *(\mathrm{AL} * \mathrm{AL}+\mathrm{R} 1 * \mathrm{R} 1) /\left(2 * \mathrm{AL}^{*} \mathrm{LAM}^{* *} 2\right)$

$\mathrm{A} 2=\mathrm{AL} *(1-\mathrm{VV}) / \mathrm{LAM}^{* * 2}$

A3 $=1 /$ AL $/($ LAM $1 * * 2-$ LAM $2 * * 2)$

A4=KA+LAM2 $* * 2 / \mathrm{AL}^{* *} 2 /\left(\mathrm{LAM}^{*} * 2-\mathrm{LAM}^{2} * 2\right)$

A5 $=\left(\mathrm{LAM}_{2} * * 2-\mathrm{LAM} 1 * * 2\right) * \mathrm{AL} * * 2$

$\mathrm{N} 1=\mathrm{CD} *(\mathrm{LAM} 2 * * 2 * \mathrm{P} 1+\mathrm{A} 5) *(\mathrm{KA} * \mathrm{P} 1-1) / \mathrm{M} 1 *$

\& $\quad\left(\mathrm{A} 1{ }^{*} \mathrm{KA}^{* *} 2^{*}{ }^{*} 2{ }^{*} \mathrm{P} 3 * \mathrm{P} 4 *(\mathrm{P} 3-\mathrm{P} 2) *(\mathrm{P} 4-\mathrm{P} 2) *(\mathrm{P} 4-\mathrm{P} 3)\right.$

$\& \quad+\mathrm{A} 1 *\left(\mathrm{P} 2 *\left(\mathrm{KA}^{*} \mathrm{P} 2-1\right) *(\mathrm{P} 4 * * 3-\mathrm{P} 3 * * 3)\right.$

$\& \quad+\mathrm{P} 3 *\left(\mathrm{KA}^{*} \mathrm{P} 3-1\right) *(\mathrm{P} 2 * * 3-\mathrm{P} 4 * * 3)$

$\left.\& \quad+\mathrm{P} 4 *\left(\mathrm{KA}^{*} \mathrm{P} 4-1\right) *(\mathrm{P} 3 * * 3-\mathrm{P} 2 * * 3)\right)$

$\& \quad-\mathrm{A} 2 *\left(\mathrm{P} 2 *\left(\mathrm{KA}^{*} \mathrm{P} 2-1\right) *(\mathrm{P} 4 * * 2-\mathrm{P} 3 * * 2)\right.$

$\& \quad+\mathrm{P} 3 *\left(\mathrm{KA}^{*} \mathrm{P} 3-1\right) *(\mathrm{P} 2 * * 2-\mathrm{P} 4 * * 2)$

$\left.\left.\& \quad+\mathrm{P} 4 *\left(\mathrm{KA}^{*} \mathrm{P} 4-1\right) *(\mathrm{P} 3 * * 2-\mathrm{P} 2 * * 2)\right)\right)$

\begin{tabular}{|c|c|}
\hline & $2=-\mathrm{CD} *(\mathrm{LAM} 2 * 2 * \mathrm{P} 2+\mathrm{A} 5) *(\mathrm{KA} * \mathrm{P} 2-1) / \mathrm{M} 2 *$ \\
\hline \& & $\left(\mathrm{A} 1 * \mathrm{KA}^{*} * 2 * \mathrm{P} 3 * \mathrm{P} 4 * \mathrm{P} 1 *(\mathrm{P} 4-\mathrm{P} 3) *(\mathrm{P} 1-\mathrm{P} 3) *(\mathrm{P} 1-\mathrm{P} 4)\right.$ \\
\hline$\&$ & $+\mathrm{A} 1 *\left(\mathrm{P} 3 *\left(\mathrm{KA}^{*} \mathrm{P} 3-1\right) *(\mathrm{P} 1 * * 3-\mathrm{P} 4 * * 3)\right.$ \\
\hline & $+\mathrm{P} 4 *\left(\mathrm{KA}^{*} \mathrm{P} 4-1\right) *(\mathrm{P} 3 * * 3-\mathrm{P} 1 * * 3)$ \\
\hline & $+\mathrm{P} 1 *(\mathrm{KA} * \mathrm{P} 1-1) *(\mathrm{P} 4 * * 3-\mathrm{P} 3 * * 3))$ \\
\hline$\&$ & $-\mathrm{A} 2 *\left(\mathrm{P} 3 *\left(\mathrm{KA}^{*} \mathrm{P} 3-1\right) *(\mathrm{P} 1 * * 2-\mathrm{P} 4 * * 2)\right.$ \\
\hline & $+\mathrm{P} 4 *(\mathrm{KA} * \mathrm{P} 4-1) *(\mathrm{P} 3 * * 2-\mathrm{P} 1 * * 2)$ \\
\hline & $+\mathrm{P} 1 *(\mathrm{KA} * \mathrm{P} 1-1) *(\mathrm{P} 4 * * 2-\mathrm{P} 3 * * 2)))$ \\
\hline
\end{tabular}




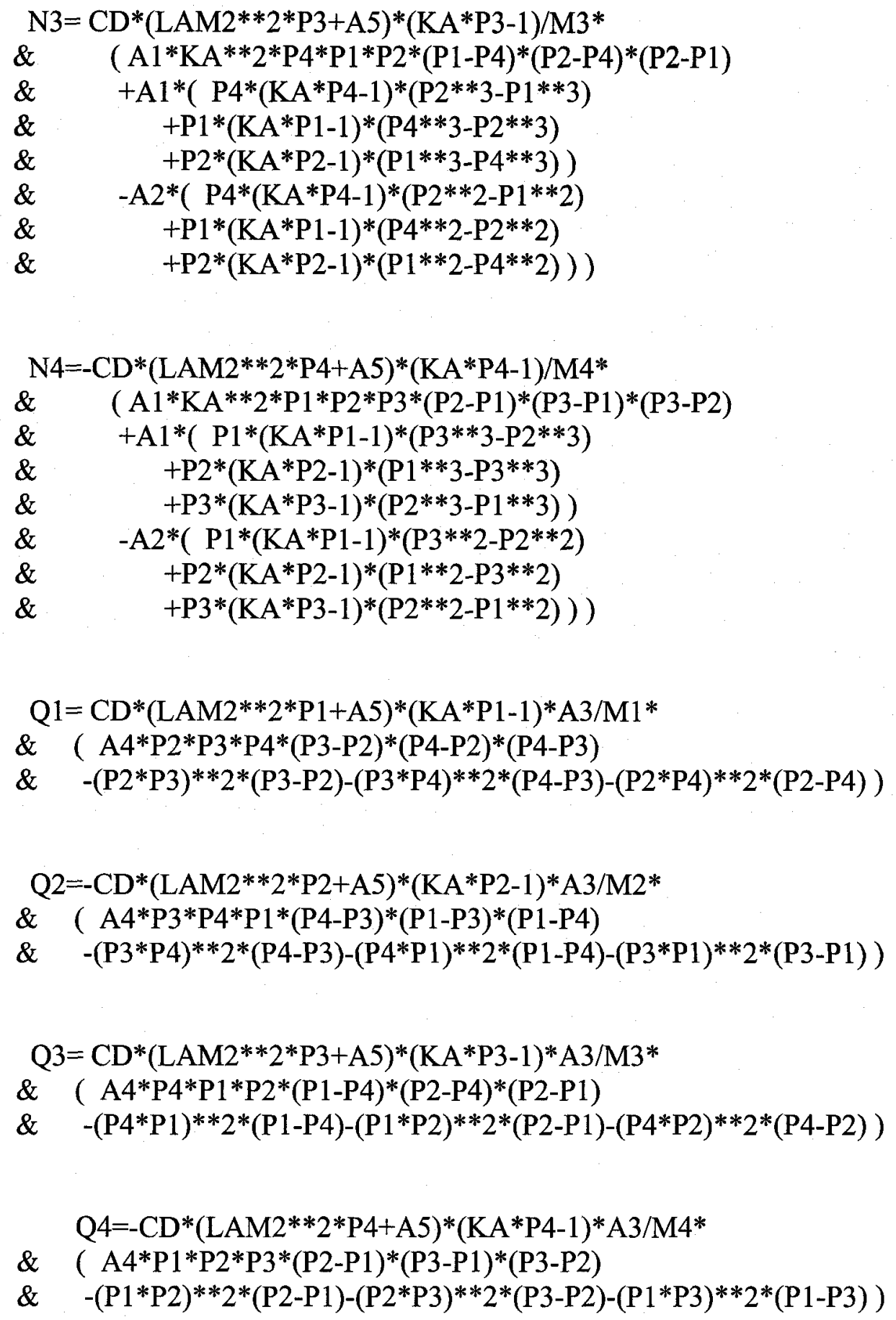

$\mathrm{CC}=2 * \mathrm{AL}^{*} \mathrm{AL} *(\mathrm{Q} 1+\mathrm{Q} 2+\mathrm{Q} 3+\mathrm{Q} 4)-1$

FREK11(K)=REAL $(\mathrm{CC})$

$\mathrm{CA}=2 * \mathrm{AL}^{*} \mathrm{AL} *(\mathrm{~N} 1+\mathrm{N} 2+\mathrm{N} 3+\mathrm{N} 4)$ 


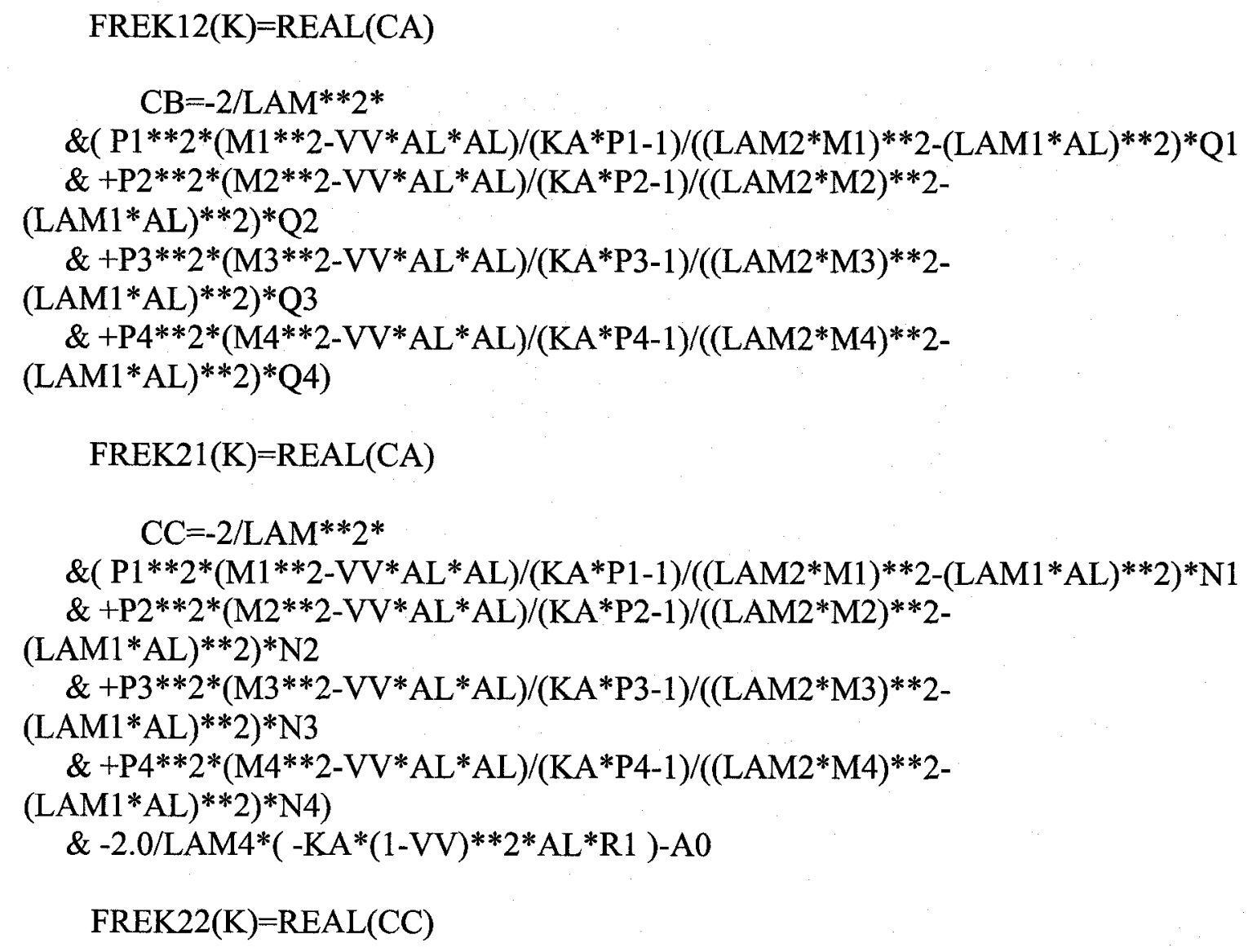

8 CONTINUE

C--.- CALCULATING THE KERNEL FUNCTIONS OF THE INTEGRAL

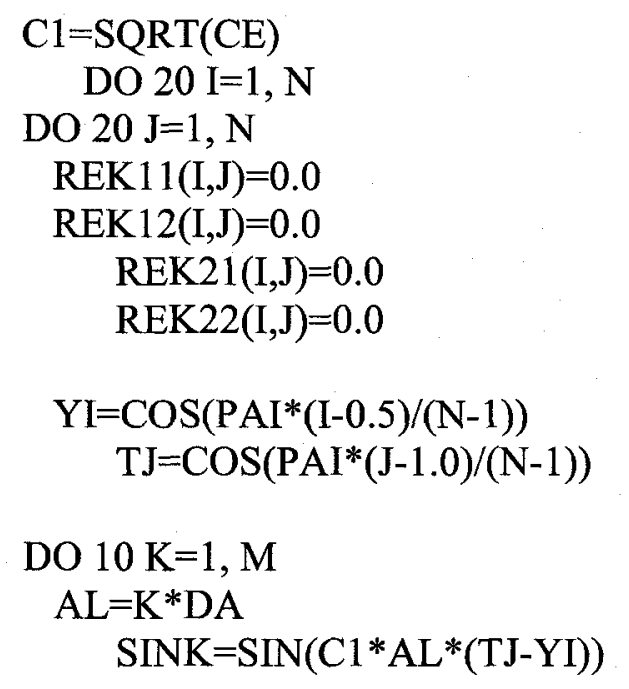


REK11(I,J)=REK11(I,J)+C1*FREK11(K)*SINK *DA

REK12(I,J)=REK12(I,J)+C1*FREK12(K)*SINK *DA

REK21(I,J)=REK21(I,J)+C1*FREK21(K)*SINK *DA

REK22(I,J)=REK22(I,J)+C1*FREK22(K)*SINK *DA

10 CONTINUE

20 CONTINUE

RETURN

END

C------THIS IS THE NEWTON ITERATIVE METHOD FOR FINDING THE ROOTS OF NONLINEAR EQAUTIONS

SUBROUTINE NEWTON(PR,PI,B,C,D,E)

DOUBLE PRECISION PR,PI,B,C,D,E,

\& P1,P2,DD,D1,D2,F1,F2,F11,F12,F21,F22

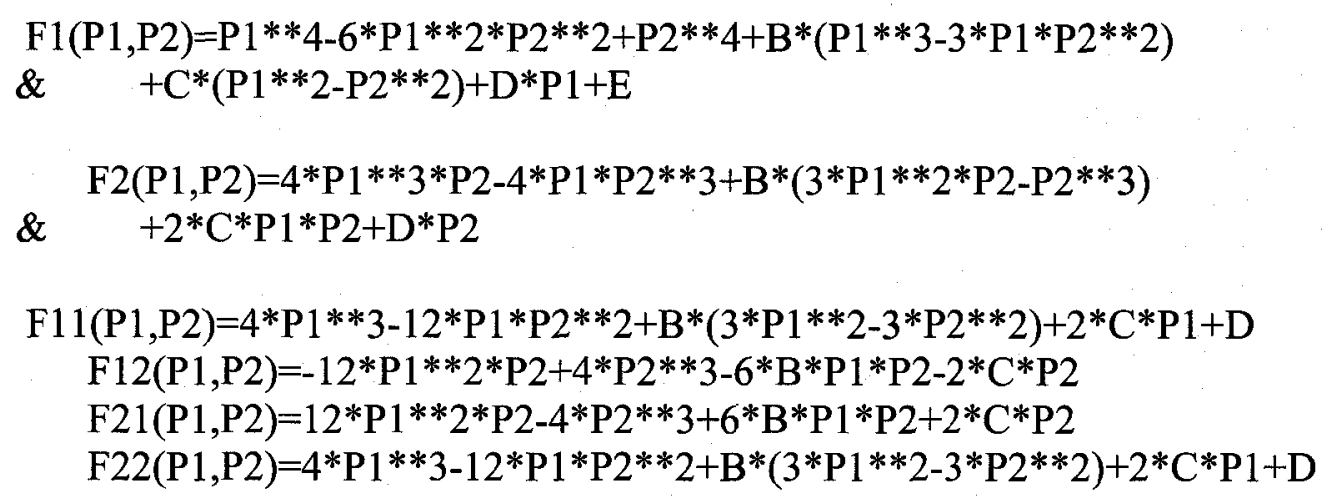

DO $2 \mathrm{I}=1,30$

$\mathrm{DD}=\mathrm{F} 11(\mathrm{PR}, \mathrm{PI}) * \mathrm{~F} 22(\mathrm{PR}, \mathrm{PI})-\mathrm{F} 12(\mathrm{PR}, \mathrm{PI}) * \mathrm{~F} 21(\mathrm{PR}, \mathrm{PI})$

IF(DD.NE.0.0) THEN

$\mathrm{D} 1=\mathrm{F} 2(\mathrm{PR}, \mathrm{PI})^{*} \mathrm{~F} 12(\mathrm{PR}, \mathrm{PI})-\mathrm{F} 1(\mathrm{PR}, \mathrm{PI}) * \mathrm{~F} 22(\mathrm{PR}, \mathrm{PI})$

$\mathrm{D} 2=\mathrm{F} 1(\mathrm{PR}, \mathrm{PI}) * \mathrm{~F} 21(\mathrm{PR}, \mathrm{PI})-\mathrm{F} 11(\mathrm{PR}, \mathrm{PI})^{*} \mathrm{~F} 2(\mathrm{PR}, \mathrm{PI})$

$\mathrm{P} 1=\mathrm{PR}+\mathrm{D} 1 / \mathrm{DD}$

$\mathrm{P} 2=\mathrm{PI}+\mathrm{D} 2 / \mathrm{DD}$

IF(DABS(F1(P1,P2))>DABS(F1(PR,PI))+1) RETURN

$\mathrm{PR}=\mathrm{P} 1 ; \mathrm{PI}=\mathrm{P} 2$

END IF 
2 CONTINUE

RETURN

END

C----- THIS IS THE SUBPROGRAM FOR CALCULATING THE DATA OF THE OPEN DISCRET EQUATIONS

SUBROUTINE OPENEQ(N,A,F,W,T,Y,REK11,REK12,REK21,REK22)

DOUBLE PRECISION REK11(N,N), REK12(N,N), REK21(N,N),REK22(N,N)

DOUBLE PRECISION A $(2 * \mathrm{~N}, 2 * \mathrm{~N}), \mathrm{F}(2 * \mathrm{~N}), \mathrm{W}(\mathrm{N}), \mathrm{Y}(\mathrm{N}), \mathrm{T}(\mathrm{N}), \mathrm{PAI}, \mathrm{H}$

COMMON CE,LAM1,LAM14,LAM,LAM4,LAM2,LAM24, KA,VV, $\mathrm{AA}, \mathrm{HH}, \mathrm{F} 1, \mathrm{~F} 2, \mathrm{~A} 0$

$\mathrm{PAI}=3.141592653589798$

$\mathrm{H}=\mathrm{PAI} /(\mathrm{N}-1)$

DO $2 \mathrm{I}=2, \mathrm{~N}-1$

$2 \mathrm{~W}(\mathrm{I})=\mathrm{H}$

$\mathrm{W}(1)=\mathrm{H} / 2 ; \mathrm{W}(\mathrm{N})=\mathrm{H} / 2$

DO $4 \mathrm{I}=1, \mathrm{~N}$

$\mathrm{T}(\mathrm{I})=\operatorname{COS}\left(\mathrm{H}^{*}(\mathrm{I}-1.0)\right)$

$\mathrm{Y}(\mathrm{I})=\operatorname{COS}\left(\mathrm{H}^{*}(\mathrm{I}-0.5)\right)$

$\mathrm{F}(\mathrm{I})=2 * \mathrm{PAI}^{*} \mathrm{~F} 1$

$\mathrm{F}(\mathrm{N}+\mathrm{I})=2 * \mathrm{PAI} * \mathrm{HH} / \mathrm{AA} * \mathrm{~F} 2$

4 CONTINUE

$\mathrm{F}(\mathrm{N})=0.0 ; \mathrm{F}(2 * \mathrm{~N})=0.0$

DO $6 \mathrm{I}=1, \mathrm{~N}-1$

DO $6 \mathrm{~J}=1, \mathrm{~N}$

$\mathrm{A}(\mathrm{I}, \mathrm{J})=\mathrm{W}(\mathrm{J}) *(1.0 /(\mathrm{T}(\mathrm{J})-\mathrm{Y}(\mathrm{I}))+\mathrm{REK} 11(\mathrm{I}, \mathrm{J}))$

$\mathrm{A}(\mathrm{I}, \mathrm{N}+\mathrm{J})=\mathrm{W}(\mathrm{J}) * \mathrm{REK} 12(\mathrm{I}, \mathrm{J})$

$\mathrm{A}(\mathrm{N}+\mathrm{I}, \mathrm{J})=\mathrm{W}(\mathrm{J}) * \mathrm{REK} 21(\mathrm{I}, \mathrm{J})$

$\mathrm{A}(\mathrm{N}+\mathrm{I}, \mathrm{N}+\mathrm{J})=\mathrm{W}(\mathrm{J}) *(\mathrm{~A} 0 /(\mathrm{T}(\mathrm{J})-\mathrm{Y}(\mathrm{I}))+\mathrm{REK} 22(\mathrm{I}, \mathrm{J}))$

6 CONTINUE

DO $8 \mathrm{~J}=1, \mathrm{~N}$

$\mathrm{A}(\mathrm{N}, \mathrm{J})=\mathrm{W}(\mathrm{J})$

$\mathrm{A}(\mathrm{N}, \mathrm{N}+\mathrm{J})=0.0$

$\mathrm{A}\left(2^{*} \mathrm{~N}, \mathrm{~J}\right)=0.0$ 


\section{$\mathrm{A}(2 * \mathrm{~N}, \mathrm{~N}+\mathrm{J})=\mathrm{W}(\mathrm{J})$}

8 CONTINUE

\section{RETURN}

END

C----- THIS IS THE SUBPROGRAM FOR CALCULATING THE DATA OF THE CLOSE-INNER DISCRET EQUATIONS

SUBROUTINE CLOSEEQINNER(N,A,F,W,T,Y,REK11,REK12,REK21,REK22)

DOUBLE PRECISION REK11(N,N), REK12(N,N), REK21(N,N),REK22(N,N)

DOUBLE PRECISION A $(2 * \mathrm{~N}, 2 * \mathrm{~N}), \mathrm{F}(2 * \mathrm{~N}), \mathrm{W}(\mathrm{N}), \mathrm{Y}(\mathrm{N}), \mathrm{T}(\mathrm{N}), \mathrm{PAI}, \mathrm{H} 1$

COMMON CE,LAM1,LAM14,LAM,LAM4,LAM2,LAM24, KA,VV, $\mathrm{AA}, \mathrm{HH}, \mathrm{F} 1, \mathrm{~F} 2, \mathrm{~A} 0$

$\mathrm{PAI}=3.141592653589798$

$\mathrm{H} 1=\mathrm{HH} / \mathrm{AA} / 2$

DO $2 \mathrm{I}=1, \mathrm{~N}-1$

$\mathrm{F}(\mathrm{I})=2 * \mathrm{PAI} * \mathrm{~F} 1$

$\mathrm{F}(\mathrm{N}+\mathrm{I})=2 * \mathrm{PAI} * \mathrm{HH} / \mathrm{AA} * \mathrm{~F} 2$

2 CONTINUE

$\mathrm{F}(\mathrm{N})=0.0 ; \mathrm{F}(2 * \mathrm{~N})=0.0$

DO $6 \mathrm{I}=1, \mathrm{~N}-1$

DO $4 \mathrm{~J}=1, \mathrm{~N}$

$\mathrm{A}(\mathrm{I}, \mathrm{J})=0.0$

$\mathrm{A}(\mathrm{I}, \mathrm{N}+\mathrm{J})=0.0$

$\mathrm{A}(\mathrm{N}+\mathrm{I}, \mathrm{J})=\mathrm{W}(\mathrm{J}) *(\mathrm{H} 1 /(\mathrm{T}(\mathrm{J})-\mathrm{Y}(\mathrm{I}))+\mathrm{H} 1 * \mathrm{REK} 11(\mathrm{I}, \mathrm{J})+\mathrm{REK} 21(\mathrm{I}, \mathrm{J}))$

4 CONTINUE

$\mathrm{A}(\mathrm{N}+\mathrm{I}, \mathrm{N}+\mathrm{J})=\mathrm{W}(\mathrm{J}) *(\mathrm{~A} 0 /(\mathrm{T}(\mathrm{J})-\mathrm{Y}(\mathrm{I}))+\mathrm{H} 1 * \mathrm{REK} 12(\mathrm{I}, \mathrm{J})+\mathrm{REK} 22(\mathrm{I}, \mathrm{J}))$

$\mathrm{A}(\mathrm{I}, \mathrm{I})=1.0$

$\mathrm{A}(\mathrm{I}, \mathrm{N}+\mathrm{I})=-\mathrm{H} 1$

6 CONTINUE

DO $8 \mathrm{~J}=1, \mathrm{~N}$

$\mathrm{A}(\mathrm{N}, \mathrm{J})=\mathrm{W}(\mathrm{J})$

$\mathrm{A}(\mathrm{N}, \mathrm{N}+\mathrm{J})=0.0$

$\mathrm{A}(2 * \mathrm{~N}, \mathrm{~J})=0.0$

$\mathrm{A}(2 * \mathrm{~N}, \mathrm{~N}+\mathrm{J})=\mathrm{W}(\mathrm{J})$ 
8 CONTINUE

RETURN

END

C----- THIS IS THE SUBPROGRAM FOR CALCULATING THE DATA OF THE CLOSE-OUTER DISCRET EQUATIONS

SUBROUTINE CLOSEEQOUTER(N,A,F,W,T,Y,REK11,REK12,REK21,REK22)

DOUBLE PRECISION REK11(N,N), REK12(N,N), REK21(N,N),REK22(N,N)

DOUBLE PRECISION A $(2 * \mathrm{~N}, 2 * \mathrm{~N}), \mathrm{F}(2 * \mathrm{~N}), \mathrm{W}(\mathrm{N}), \mathrm{Y}(\mathrm{N}), \mathrm{T}(\mathrm{N}), \mathrm{PAI}, \mathrm{H} 1$

COMMON CE,LAM1,LAM14,LAM,LAM4,LAM2,LAM24, KA,VV, $\mathrm{AA}, \mathrm{HH}, \mathrm{F} 1, \mathrm{~F} 2, \mathrm{~A} 0$

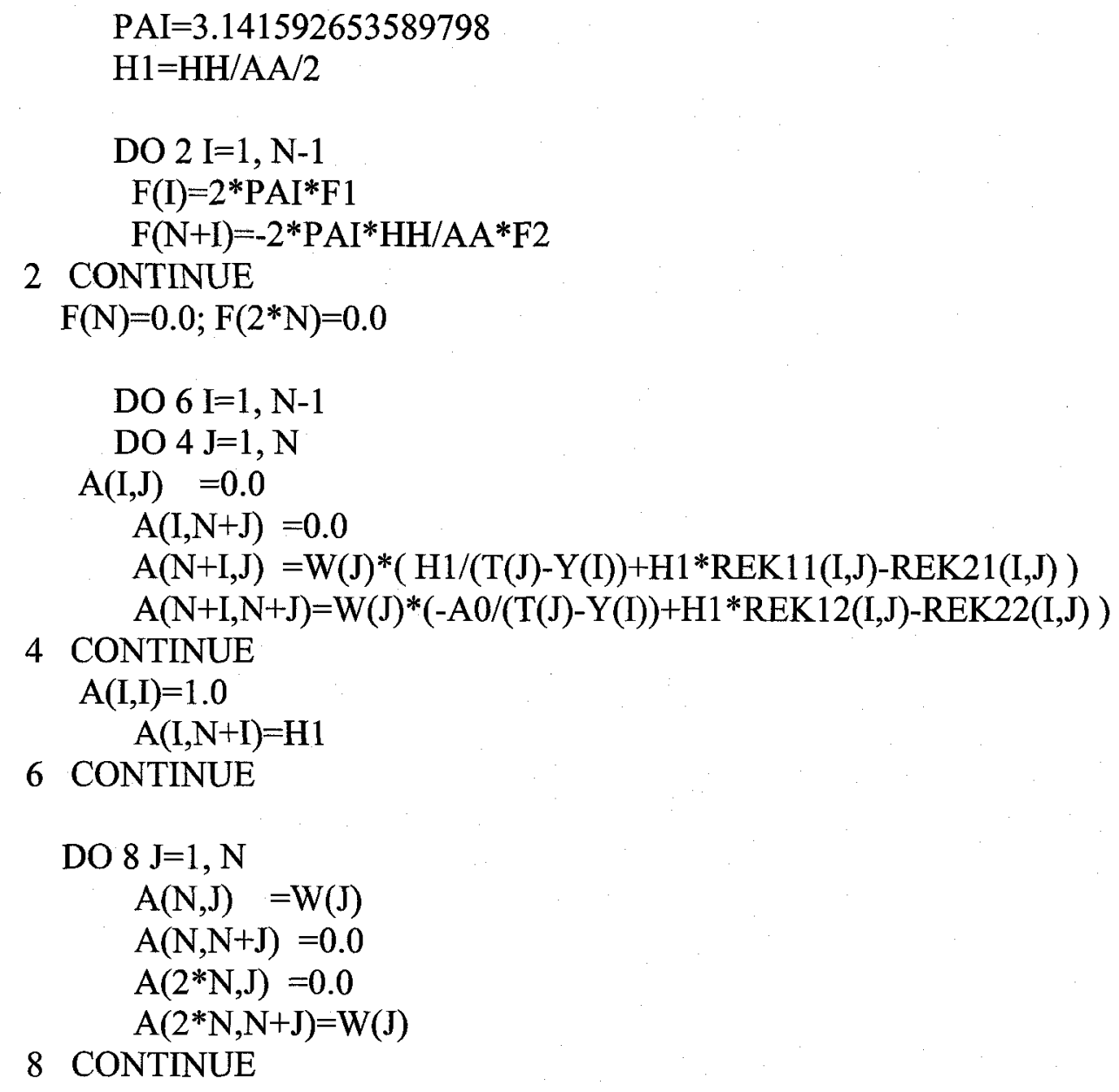




\section{RETURN}

END

C---- THIS IS THE SUBPROGRAM FOR SOLVING THE LINEAR EQUATIONS BY GAUSS ELIMINATE METHOD

SUBROUTINE SOLVEEQ(N,A,B,X)

DOUBLE PRECISION A(N,N), B(N), X(N), C, T, S

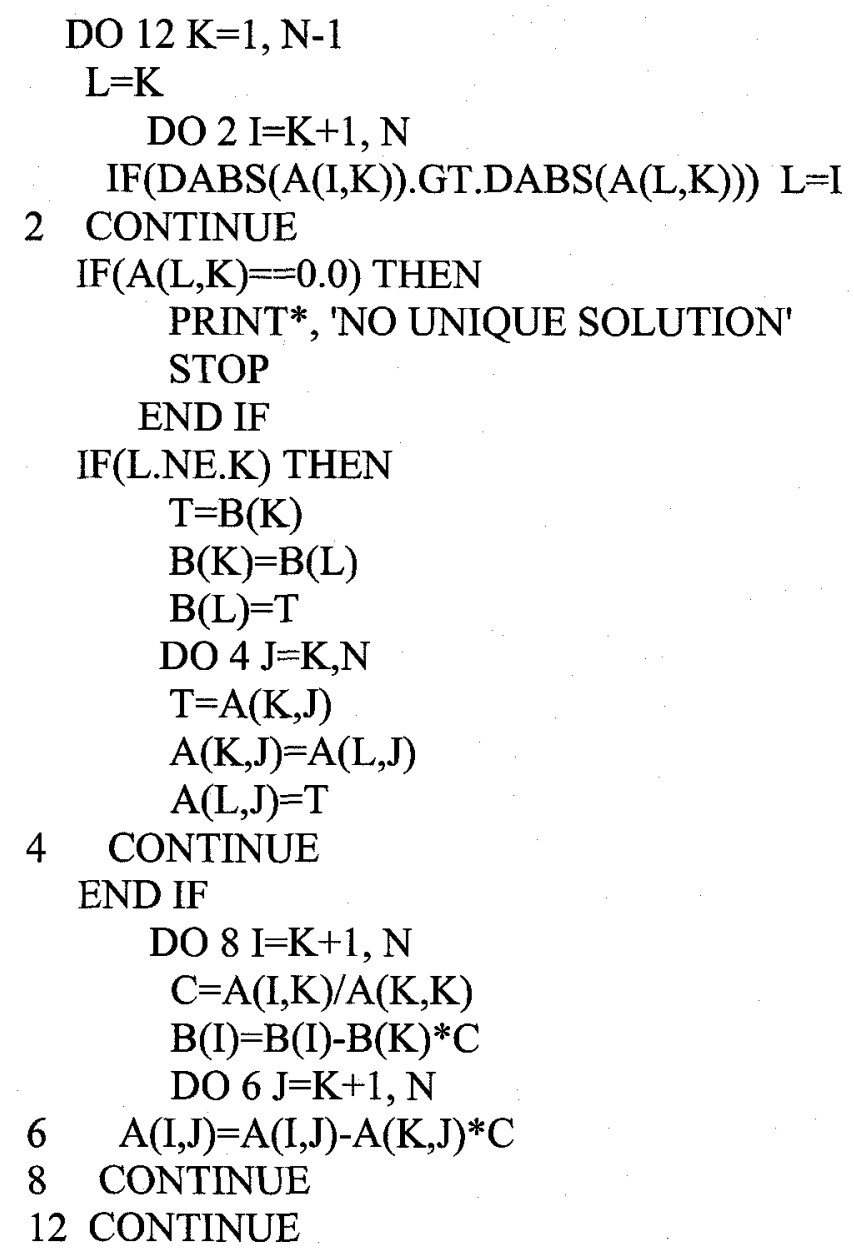




\section{DO $14 \mathrm{~J}=\mathrm{I}+1, \mathrm{~N}$ \\ $14 \mathrm{~S}=\mathrm{S}+\mathrm{A}(\mathrm{I}, \mathrm{J}) * \mathrm{X}(\mathrm{J})$ \\ $\mathrm{X}(\mathrm{I})=(\mathrm{B}(\mathrm{I})-\mathrm{S}) / \mathrm{A}(\mathrm{I}, \mathrm{I})$}

\section{CONTINUE}

\section{RETURN}

END 


\section{Program 4-Isotropic Cylindrical Shells}

C----- MAIN PROGRAM: CRACK CLOSURE ANALYSIS ON AXIAL CRACK IN CYLINDER SHELL

PARAMETER ( $\mathrm{N}=41, \mathrm{~T}=60.0)$

REAL LAM, LAM4, LAM1, LAM14, KA, Km, Kb

DOUBLE PRECISION REK11(N,N), REK12(N,N), REK21(N,N), REK22(N,N),

$\& \quad \mathrm{~A}(2 * \mathrm{~N}, 2 * \mathrm{~N}), \mathrm{A} 1(2 * \mathrm{~N}, 2 * \mathrm{~N}), \mathrm{A} 2(2 * \mathrm{~N}, 2 * \mathrm{~N}), \mathrm{B}(2 * \mathrm{~N}), \mathrm{B} 1(2 * \mathrm{~N}), \mathrm{B} 2(2 * \mathrm{~N})$,

\& $\quad \mathrm{G}\left(2^{*} \mathrm{~N}\right), \mathrm{W}(\mathrm{N}), \mathrm{TT}(\mathrm{N}), \mathrm{YY}(\mathrm{N}), \mathrm{P}(\mathrm{N})$

COMMON LAM, LAM4, LAM1, LAM14, KA,VV, AA,HH,F1,F2,A0

OPEN(UNIT=10, FILE='TEST2.DAT', STATUS='UNKNOWN',

\& FORM='FORMATTED', POSITION='REWIND', IOSTAT=ISTAT )

IF(ISTAT/=0) PRINT*, 'FILE OPEN FAIL: ISTAT=',ISTAT

$\mathrm{VV}=1.0 / 3$

$\mathrm{AA}=8 ! 10$

$\mathrm{HH}=1$

LAM4 $=12 *\left(1-V^{*} \mathrm{VV}\right) * \mathrm{AA}^{*} \mathrm{AA} /\left(\mathrm{HH}^{*} \mathrm{HH}\right)$

LAM $=$ LAM $4 * *(1.0 / 4)$

$\mathrm{KA}=12 *(1+\mathrm{VV}) /(5 * \mathrm{LAM} 4)$

$\mathrm{A} 0=(1-\mathrm{VV} * \mathrm{VV}) / \mathrm{LAM} 4$

SING $=1 \quad$ !SING=1 FOR INNER-CLOSE CASE AND SING=-1 FOR OUTERCLOSE

!label FORMAT('First Descriptor')

$$
\begin{aligned}
& F 1=0.0 \\
& F 2=-1.0 / 6
\end{aligned}
$$

\section{WRITE $(10, *)$ \\ PRINT*','a/h=',AA/HH \\ WRITE(10,2) AA/HH \\ 2 FORMAT(1X,4HA/H=,F5.2)}

$\mathrm{N} 1=2 * \mathrm{AA} / \mathrm{HH}$

DO $120 \mathrm{M}=1, \mathrm{~N} 1$

$\mathrm{LAM} 1=0.5 * \mathrm{M}$

LAM14=LAM $1 * * 4$

CALL KERLFUNC(N,T,REK11,REK12,REK21,REK22) 
CALL OPENEQ(N,A1,B1,W,TT,YY,REK11,REK12,REK21,REK22)

IF(SING==1) THEN

CALL CLOSEEQINNER(N,A2,B2,W,TT,YY,REK11,REK12,REK21,REK22) ELSE

CALL CLOSEEQOUTER(N,A2,B2,W,TT,YY,REK11,REK12,REK21,REK22) END IF

C-----IF ONLY CACULATE OPEN CASE, PLEASE IMPLEMENT THESE TWO SENTANCES, OTHERWISE OMIT THEM

$! \quad$ CALL SOLVEEQ $(2 * \mathrm{~N}, \mathrm{~A} 1, \mathrm{~B} 1, \mathrm{G})$

! GO TO 110

C-----IF ONLY CACULATE OPEN CASE, PLEASE IMPLEMENT THESE TWO SENTANCES, OTHERWISE OMIT THEM

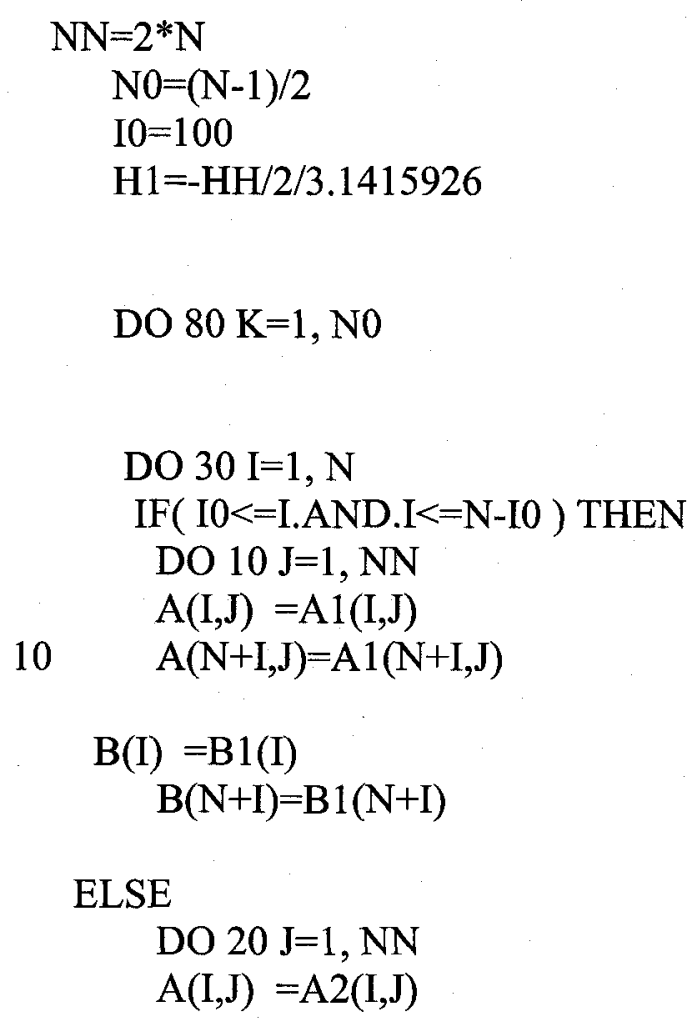

ELSE

DO $20 \mathrm{~J}=1, \mathrm{NN}$

$\mathrm{A}(\mathrm{I}, \mathrm{J})=\mathrm{A} 2(\mathrm{I}, \mathrm{J})$ 
$20 \quad \mathrm{~A}(\mathrm{~N}+\mathrm{I}, \mathrm{J})=\mathrm{A} 2(\mathrm{~N}+\mathrm{I}, \mathrm{J})$

$$
\begin{aligned}
& \mathrm{B}(\mathrm{I})=\mathrm{B} 2(\mathrm{I}) \\
& \mathrm{B}(\mathrm{N}+\mathrm{I})=\mathrm{B} 2(\mathrm{~N}+\mathrm{I})
\end{aligned}
$$

END IF

30 CONTINUE

\section{CALL SOLVEEQ(NN,A,B,G)}

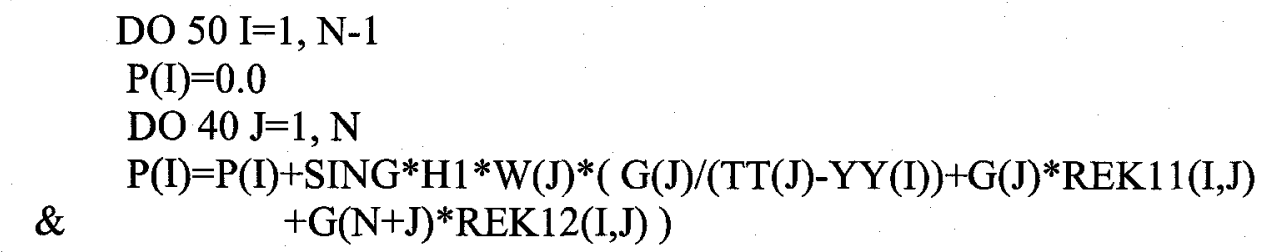

\section{CONTINUE}

$\mathrm{PP}=\mathrm{SNGL}(\mathrm{P}(\mathrm{I}))$

WRITE $\left(10,{ }^{*}\right) \mathrm{K}, \mathrm{I}, \mathrm{PP}, \mathrm{YY}(\mathrm{I})$

4 FORMAT(1X,'K=',I2,3X,'I=',I2,3X,'P=',F7.4,2X,'Y=',F7.4)

50 CONTINUE

WRITE $(10, *)$

$\mathrm{I} 0=100$

DO $60 \mathrm{I}=1$, N0

$60 \operatorname{IF}(\mathrm{P}(\mathrm{I})<-0.00000000001 . A N D . \mathrm{I}<\mathrm{I} 0) \mathrm{I} 0=\mathrm{I}$

WRITE $\left({ }^{*},{ }^{*}\right) ' \mathrm{~K}={ }^{\prime}, \mathrm{K},{ }^{\prime} \mathrm{I} 0={ }^{\prime}, \mathrm{I} 0$

IF $(10==100)$ GOTO 90

80 CONTINUE

90 IF $(\mathrm{K}<=\mathrm{N} 0)$ THEN

WRITE $(*, *)$ 'ITERATION CONVERGENCE, $\mathrm{K}=$ ', $\mathrm{K}$ ELSE WRITE $(*, *)$ 'ITERATION FAIL, $\mathrm{K}=$ ', $\mathrm{K}$ END IF

$\mathrm{J} 0=\mathrm{N} 0$

DO $100 \mathrm{I}=1, \mathrm{~N} 0$ 
$100 \operatorname{IF}(\operatorname{ABS}(\mathrm{P}(\mathrm{I}))<=1.0 / 10 * * 9 . \mathrm{AND} . \mathrm{I}<\mathrm{J} 0) \mathrm{J} 0=\mathrm{I}$

$\mathrm{IF}(\mathrm{J} 0==\mathrm{N} 0)$ THEN

PRINT*, 'CRACK IS CLOSED, J0=',J0

WRITE $\left(10,{ }^{*}\right)$ 'CRACK IS CLOSED, J0 $=$ ',J0

ELSE

PRINT*, 'CRACK IS OPEN-CLOSE'

PRINT*, 'J0=',J0,'P(J0)=',P(J0)

WRITE $\left(10,{ }^{*}\right)$ 'CRACK IS OPEN-CLOSE'

WRITE $\left(10,{ }^{*}\right)$ 'J0 $=$ ',J0, 'Y(J0)=',YY(J0)

END IF

$110 \quad \mathrm{Km}=-0.5 * \mathrm{G}(1)$

$\mathrm{Kb}=-\mathrm{HH} /(4 * \mathrm{AA}) * \mathrm{G}(\mathrm{N}+1)$

PRINT*,'LAM1=',LAM1,'Km=','Km,'Kb=',Kb

WRITE $(10,8)$ LAM1, Km, Kb

8 FORMAT(1X,5HLAM1=,F6.3,3X,3HKm=,F7.4,3X,3HKb=,F7.4)

WRITE $(10, *)^{1 * * * * * * * * * * * * * * * * * * * * * * * * * * * * * * * * * * * * * * * * * * * * * * 1}$

120 CONTINUE

CLOSE(10)

STOP

END

C----- THIS IS THE SUBPROGRAM FOR SOLVING THE KERLFUNCTIONS

SUBROUTINE KERLFUNC(N,T,REK11,REK12,REK21,REK22)

REAL LAM, LAM4, LAM1, LAM14, KA

DOUBLE PRECISION REK11(N,N),REK12(N,N), REK21(N,N),REK22(N,N),

\& FREK11(20000),FREK12(20000),FREK21(20000),FREK22(20000)

COMMON LAM, LAM4, LAM1, LAM14, KA,VV, AA,HH,F1,F2,A0

COMPLEX P1, P2, P3, P4, M1, M2, M3, M4, N1, N2, N3, N4,

\& $\quad \mathrm{Q} 1, \mathrm{Q} 2, \mathrm{Q} 3, \mathrm{Q} 4, \mathrm{CA}, \mathrm{CB}, \mathrm{CC}, \mathrm{CD}, \mathrm{CX}, \mathrm{CY}, \mathrm{P}, \mathrm{FP}$

DOUBLE PRECISION A, B, C, D, E, Y, D0,D1,PR,PI

$\mathrm{FP}(\mathrm{P})=\mathrm{P} * * 4+\mathrm{D} * \mathrm{P}+\mathrm{E}$

PAI $=3.141592653589798$

$\mathrm{DA}=0.01$

$\mathrm{M}=\mathrm{T} / \mathrm{DA}$ 
C----- FINDING THE ROOTS Pj AND Mj OF THE POLYNOMIAL EQUATIONS

DO $8 \mathrm{~K}=1, \mathrm{M}$

$\mathrm{AL}=\mathrm{K} * \mathrm{DA}$

$\mathrm{D}=-\mathrm{KA} * \mathrm{LAM} 14 * \mathrm{AL} * * 4$

$\mathrm{E}=\mathrm{LAM} 14^{*} \mathrm{AL} * * 4$

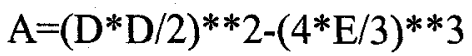

IF(A.LT.0.0) THEN

$\mathrm{CA}=\mathrm{CMPLX}(0.0, \mathrm{DSQRT}(-\mathrm{A}))$

$\mathrm{CD}=\mathrm{CMPLX}(\mathrm{D}, 0.0)$

$\mathrm{CY}=\mathrm{CEXP}(\mathrm{CLOG}(\mathrm{CD} * \mathrm{CD} / 2+\mathrm{CA}) / 3)+\mathrm{CEXP}(\mathrm{CLOG}(\mathrm{CD} * \mathrm{CD} / 2-\mathrm{CA}) / 3)$

$\mathrm{Y}=\mathrm{REAL}(\mathrm{CY})$

ELSE

$\mathrm{A}=\mathrm{DSQRT}(\mathrm{A})$

$\mathrm{Y}=\mathrm{DEXP}\left(\mathrm{DLOG}\left(\mathrm{D}^{*} \mathrm{D} / 2+\mathrm{A}\right) / 3\right)+\mathrm{DEXP}\left(\mathrm{DLOG}\left(\mathrm{D}^{*} \mathrm{D} / 2-\mathrm{A}\right) / 3\right)$

END IF

DO $6 \mathrm{I}=1,20$

$\mathrm{D} 0=\mathrm{Y} * * 3-4 * \mathrm{E}^{*} \mathrm{Y}-\mathrm{D} * \mathrm{D}$

$\mathrm{D} 1=3 * \mathrm{Y} * 2-4 * \mathrm{E}$

IF(D1.NE.0.0) Y=Y-D0/D1

6 CONTINUE

$\mathrm{CX}=\mathrm{CSQRT}(\mathrm{CMPLX}(\mathrm{Y} * \mathrm{Y}-4 * \mathrm{E}, 0.0))$

$\mathrm{CY}=\mathrm{CMPLX}(\mathrm{Y}, 0.0)$

$\mathrm{P} 1=(-\mathrm{DSQRT}(\mathrm{Y})+\mathrm{CSQRT}(-\mathrm{CY}-2 * \mathrm{CX})) / 2.0$

$\mathrm{P} 2=(-\mathrm{DSQRT}(\mathrm{Y})-\mathrm{CSQRT}(-\mathrm{CY}-2 * \mathrm{CX})) / 2.0$

$\mathrm{P} 3=(\mathrm{DSQRT}(\mathrm{Y})+\mathrm{CSQRT}(-\mathrm{CY}+2 * \mathrm{CX})) / 2.0$

$\mathrm{P} 4=(\mathrm{DSQRT}(\mathrm{Y})-\mathrm{CSQRT}(-\mathrm{CY}+2 * \mathrm{CX})) / 2.0$

$\mathrm{CC}=\mathrm{FP}(\mathrm{P} 1)$

IF(CABS(CC)>0.01) THEN

$\mathrm{PR}=\mathrm{REAL}(\mathrm{P} 1)$

$\mathrm{PI}=\mathrm{AIMAG}(\mathrm{P} 1)$ 


\author{
CALL NEWTON(PR,PI,D,E) \\ $\mathrm{P} 1=\mathrm{CMPLX}(\mathrm{PR}, \mathrm{PI})$ \\ END IF \\ $\mathrm{CC}=\mathrm{FP}(\mathrm{P} 2)$ \\ IF (CABS $(\mathrm{CC})>0.01)$ THEN \\ $\mathrm{PR}=\mathrm{REAL}(\mathrm{P} 2)$ \\ $\mathrm{PI}=\mathrm{AIMAG}(\mathrm{P} 2)$ \\ CALL NEWTON(PR,PI,D,E) \\ P2 =CMPLX(PR,PI) \\ END IF \\ $\mathrm{CC}=\mathrm{FP}(\mathrm{P} 3)$ \\ IF (CABS(CC)>0.01) THEN \\ $\mathrm{PR}=\mathrm{REAL}(\mathrm{P} 3)$ \\ $\mathrm{PI}=\mathrm{AIMAG}(\mathrm{P} 3)$ \\ CALL NEWTON(PR,PI,D,E) \\ P3 $=$ CMPLX $(P R, P I)$ \\ END IF \\ $\mathrm{CC}=\mathrm{FP}(\mathrm{P} 4)$ \\ IF $(\mathrm{CABS}(\mathrm{CC})>0.01)$ THEN \\ $\mathrm{PR}=\mathrm{REAL}(\mathrm{P} 4)$ \\ $\mathrm{PI}=\mathrm{AIMAG}(\mathrm{P} 4)$ \\ CALL NEWTON(PR,PI,D,E) \\ P4=CMPLX(PR,PI) \\ END IF
}

IF(CABS(P1) $<0.0000000001) \mathrm{P} 1=\mathrm{P} 1+0.00000000001$

$\operatorname{IF}(\mathrm{CABS}(\mathrm{P} 2)<0.0000000001) \mathrm{P} 2=\mathrm{P} 2+0.00000000001$

$\mathrm{IF}(\mathrm{CABS}(\mathrm{P} 3)<0.0000000001) \mathrm{P} 3=\mathrm{P} 3+0.00000000001$

IF(CABS(P4)<0.0000000001) P4=P4+0.00000000001

IF(CABS(KA $*$ P1-1) $<0.000000001) P 1=1.0000000001 / \mathrm{KA}$

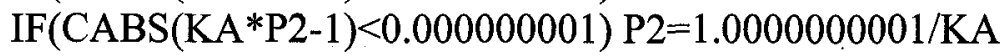

IF(CABS(KA*P3-1)<0.000000001) P3 $=1.0000000001 / \mathrm{KA}$

IF(CABS(KA*P4-1)<0.000000001) P4 $=1.0000000001 / \mathrm{KA}$

$\mathrm{M} 1=\mathrm{CSQRT}(\mathrm{P} 1+\mathrm{AL} * \mathrm{AL})$

IF(REAL(M1).GT.0.0) M1=-M1

$\mathrm{M} 2=\mathrm{CSQRT}(\mathrm{P} 2+\mathrm{AL} * \mathrm{AL})$

IF(REAL(M2).GT.0.0) M2=-M2

$\mathrm{M} 3=\mathrm{CSQRT}(\mathrm{P} 3+\mathrm{AL} * \mathrm{AL})$ 
IF(REAL(M3).GT.0.0) M3=-M3

$\mathrm{M} 4=\mathrm{CSQRT}(\mathrm{P} 4+\mathrm{AL} * \mathrm{AL})$

IF(REAL(M4).GT.0.0) M4=-M4

$\operatorname{IF}(\mathrm{CABS}(\mathrm{M} 1)<0.000000001) \mathrm{M} 1=-\mathrm{AL}$

$\operatorname{IF}(\mathrm{CABS}(\mathrm{M} 2)<0.000000001) \mathrm{M} 2=-\mathrm{AL}$

$\operatorname{IF}(\mathrm{CABS}(\mathrm{M} 3)<0.000000001) \mathrm{M} 3=-\mathrm{AL}$

IF(CABS(M4)<0.000000001) M4=-AL

C----- CALCULATING THE VALUES OF Nj AND Qj FOR $\mathrm{j}=1-4$

$\mathrm{CD}=(\mathrm{P} 2-\mathrm{P} 1) *(\mathrm{P} 4-\mathrm{P} 3) *\left((\mathrm{P} 3 * \mathrm{P} 4) * * 2 /\left(\mathrm{KA}^{*} \mathrm{P} 3-1\right) /\left(\mathrm{KA}^{*} \mathrm{P} 4-1\right)\right.$

$\left.\& \quad+(\mathrm{P} 1 * \mathrm{P} 2)^{* *} 2 /\left(\mathrm{KA}^{*} \mathrm{P} 1-1\right) /(\mathrm{KA} * \mathrm{P} 2-1)\right)$

$\&+(\mathrm{P} 3-\mathrm{P} 1) *(\mathrm{P} 2-\mathrm{P} 4) *\left(\left(\mathrm{P} 1{ }^{*} \mathrm{P} 3\right)^{* *} 2 /(\mathrm{KA} * \mathrm{P} 1-1) /(\mathrm{KA} * \mathrm{P} 3-1)\right.$

$\left.\& \quad+(\mathrm{P} 2 * \mathrm{P} 4) * * 2 /\left(\mathrm{KA}^{*} \mathrm{P} 2-1\right) /(\mathrm{KA} * \mathrm{P} 4-1)\right)$

$\&+(\mathrm{P} 4-\mathrm{P} 1) *(\mathrm{P} 3-\mathrm{P} 2) *\left((\mathrm{P} 1 * \mathrm{P} 4)^{* *} 2 /\left(\mathrm{KA}^{*} \mathrm{P} 1-1\right) /\left(\mathrm{KA}^{*} \mathrm{P} 4-1\right)\right.$

$\&$ $\left.+(\mathrm{P} 2 * \mathrm{P} 3) * * 2 /\left(\mathrm{KA}^{*} \mathrm{P} 2-1\right) /(\mathrm{KA} * \mathrm{P} 3-1)\right)$

$\mathrm{CD}=1.0 / \mathrm{CD}$

$\mathrm{R} 1=-\mathrm{SQRT}(\mathrm{AL} * \mathrm{AL}+2.0 / \mathrm{KA} /(1-\mathrm{VV}))$

$\mathrm{A} 1=(\mathrm{LAM} 1 / \mathrm{LAM}) * * 2 *(1-\mathrm{VV}) * \mathrm{AL}^{* *} 3$

$\mathrm{A} 2=-0.5 *(\mathrm{LAM} 1 / \mathrm{LAM}) * * 2 * \mathrm{KA}^{*}(1-\mathrm{VV}) * \mathrm{AL} *(\mathrm{AL} * \mathrm{AL}+\mathrm{R} 1 * \mathrm{R} 1)$

$\mathrm{N} 1=\mathrm{CD} / \mathrm{M} 1 *$

$\& \quad\left(\mathrm{~A} 1 *\left((\mathrm{P} 2-\mathrm{P} 4) * \mathrm{P} 3 * * 2 /\left(\mathrm{KA}^{*} \mathrm{P} 3-1\right)\right.\right.$

$\& \quad+(\mathrm{P} 4-\mathrm{P} 3) * \mathrm{P} 2 * * 2 /\left(\mathrm{KA}^{*} \mathrm{P} 2-1\right)$

$\left.\& \quad+(\mathrm{P} 3-\mathrm{P} 2) * \mathrm{P} 4 * * 2 /\left(\mathrm{KA}^{*} \mathrm{P} 4-1\right)\right)$

$\& \quad+\mathrm{A} 2 *\left((\mathrm{P} 2-\mathrm{P} 4) * \mathrm{P} 3 * * 3 /\left(\mathrm{KA}^{*} \mathrm{P} 3-1\right)\right.$

$\& \quad+(\mathrm{P} 4-\mathrm{P} 3)^{*} \mathrm{P} 2 * * 3 /\left(\mathrm{KA}^{*} \mathrm{P} 2-1\right)$

$\left.\left.\& \quad+(\mathrm{P} 3-\mathrm{P} 2) * \mathrm{P} 4 * * 3 /\left(\mathrm{KA}^{*} \mathrm{P} 4-1\right)\right)\right)$

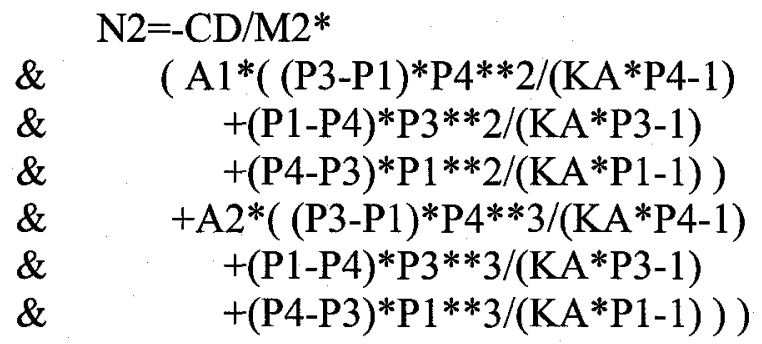

$$
\begin{array}{lc}
\mathrm{N} 3=\mathrm{CD} / \mathrm{M} 3 \\
\& & (\mathrm{~A} 1 * \\
\& & +(\mathrm{P} 4-\mathrm{P} 2)^{*} \mathrm{P} 1 * * 2 /\left(\mathrm{KA}^{*} \mathrm{P} 1-1\right) \\
\& & +(\mathrm{P} 2-\mathrm{P} 1) * \mathrm{P} 4 * * 2 /\left(\mathrm{KA}^{*} \mathrm{P} 4-1\right) \\
\& & \left.+(\mathrm{P} 1-\mathrm{P} 4) * \mathrm{P} 2 * * 2 /\left(\mathrm{KA}^{*} \mathrm{P} 2-1\right)\right)
\end{array}
$$



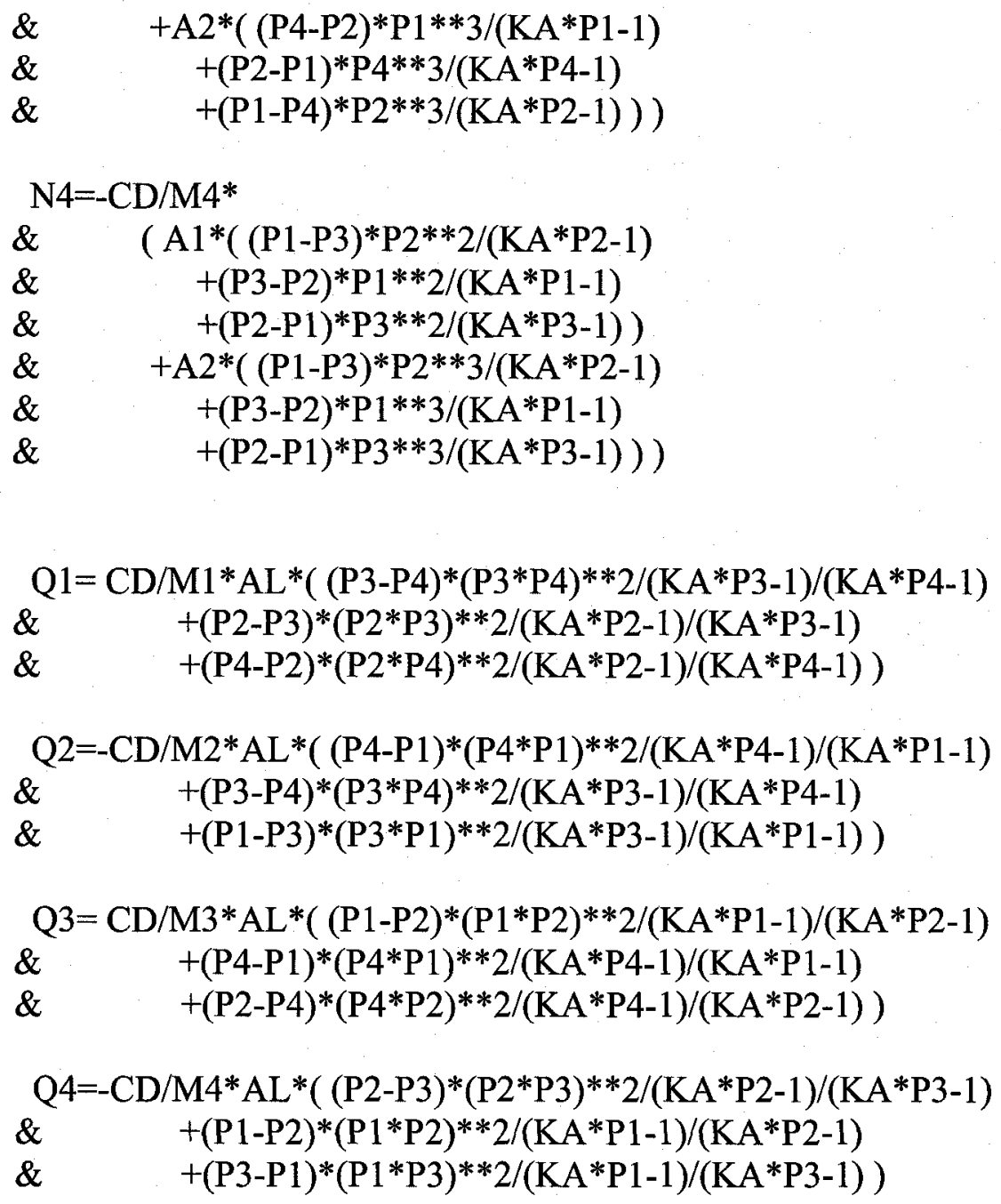

$\mathrm{CC}=2 * \mathrm{AL}^{*} \mathrm{AL} *(\mathrm{Q} 1+\mathrm{Q} 2+\mathrm{Q} 3+\mathrm{Q} 4)-1.0$

FREK11(K) $=$ REAL(CC)

$\mathrm{CA}=2 * A L^{*} A L *(\mathrm{~N} 1+\mathrm{N} 2+\mathrm{N} 3+\mathrm{N} 4)$

FREK12(K)=REAL(CA)

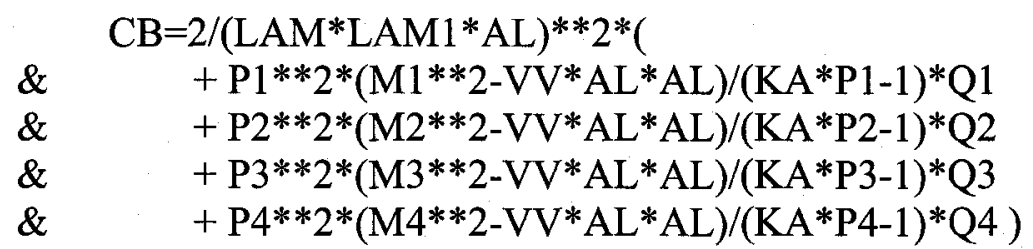

$\operatorname{FREK} 21(\mathrm{~K})=\operatorname{REAL}(\mathrm{CA})$

$\mathrm{CC}=2 /\left(\mathrm{LAM}^{*} \mathrm{LAM} 1 * \mathrm{AL}\right) * * 2 *($ 


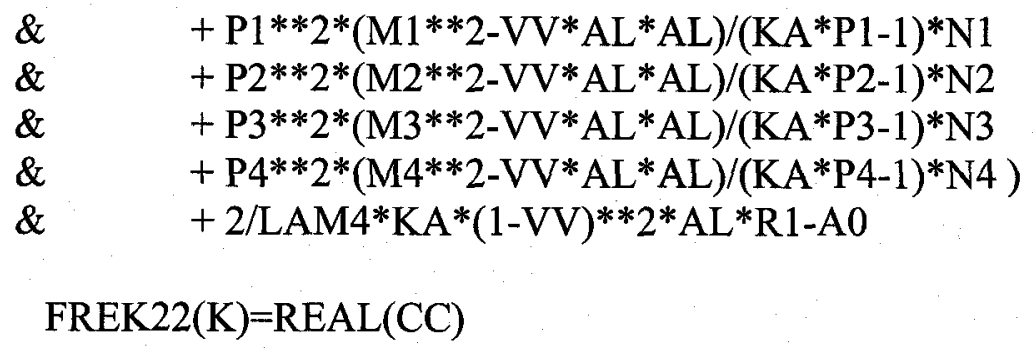

\section{CONTINUE}

C---- CALCULATING THE KERNEL FUNCTIONS OF THE INTEGRAL

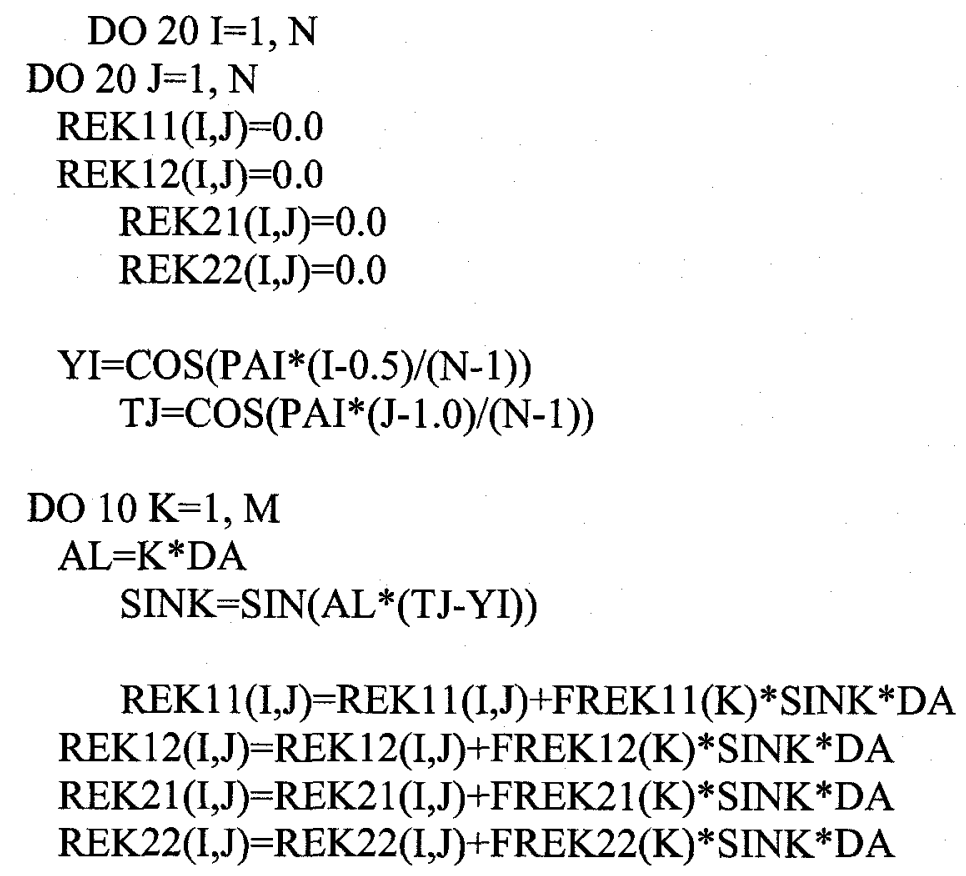

10 CONTINUE

20 CONTINUE

RETURN

END

C------THIS IS THE NEWTON ITERATIVE METHOD FOR FINDING THE ROOTS OF NONLINEAR EQAUTIONS

SUBROUTINE NEWTON(PR,PI,D,E)

DOUBLE PRECISION PR,PI,D,E, 
\& $\quad \mathrm{P} 1, \mathrm{P} 2, \mathrm{DD}, \mathrm{D} 1, \mathrm{D} 2, \mathrm{~F} 1, \mathrm{~F} 2, \mathrm{~F} 11, \mathrm{~F} 12, \mathrm{~F} 21, \mathrm{~F} 22$

$\mathrm{F} 1(\mathrm{P} 1, \mathrm{P} 2)=\mathrm{P} 1 * * 4-6 * \mathrm{P} 1 * * 2 * \mathrm{P} 2 * * 2+\mathrm{P} 2 * * 4+\mathrm{D} * \mathrm{P} 1+\mathrm{E}$

$\mathrm{F} 2(\mathrm{P} 1, \mathrm{P} 2)=4 * \mathrm{P} 1 * * 3 * \mathrm{P} 2-4 * \mathrm{P} 1 * \mathrm{P} 2 * * 3+\mathrm{D} * \mathrm{P} 2$

$\mathrm{F} 11(\mathrm{P} 1, \mathrm{P} 2)=4 * \mathrm{P} 1 * * 3-12 * \mathrm{P} 1 * \mathrm{P} 2 * * 2+\mathrm{D}$

$\mathrm{F} 12(\mathrm{P} 1, \mathrm{P} 2)=-12 * \mathrm{P} 1 * * 2 * \mathrm{P} 2+4 * \mathrm{P} 2 * * 3$

$\mathrm{F} 21(\mathrm{P} 1, \mathrm{P} 2)=12 * \mathrm{P} 1 * * 2 * \mathrm{P} 2-4 * \mathrm{P} 2 * * 3$

$\mathrm{F} 22(\mathrm{P} 1, \mathrm{P} 2)=4 * \mathrm{P} 1 * * 3-12 * \mathrm{P} 1 * \mathrm{P} 2 * * 2+\mathrm{D}$

DO $2 \mathrm{I}=1,30$

$\mathrm{DD}=\mathrm{F} 11(\mathrm{PR}, \mathrm{PI}) * \mathrm{~F} 22(\mathrm{PR}, \mathrm{PI})-\mathrm{F} 12(\mathrm{PR}, \mathrm{PI}) * \mathrm{~F} 21(\mathrm{PR}, \mathrm{PI})$

IF(DD.NE.0.0) THEN

D1=F2(PR,PI)*F12(PR,PI)-F1(PR,PI)*F22(PR,PI)

D2=F1(PR,PI)*F21(PR,PI)-F11(PR,PI)*F2(PR,PI)

$\mathrm{P} 1=\mathrm{PR}+\mathrm{D} 1 / \mathrm{DD}$

$\mathrm{P} 2=\mathrm{PI}+\mathrm{D} 2 / \mathrm{DD}$

IF(DABS(F1(P1,P2))>DABS(F1(PR,PI))+1) RETURN

$\mathrm{PR}=\mathrm{P} 1 ; \mathrm{PI}=\mathrm{P} 2$

END IF

2 CONTINUE

RETURN

END

C----- THIS IS THE SUBPROGRAM FOR CALCULATING THE DATA OF THE OPEN DISCRET EQUATIONS

SUBROUTINE OPENEQ(N,A,F,W,T,Y,REK11,REK12,REK21,REK22)

DOUBLE PRECISION REK11(N,N), REK12(N,N), REK21(N,N),REK22(N,N)

DOUBLE PRECISION A $(2 * \mathrm{~N}, 2 * \mathrm{~N}), \mathrm{F}(2 * \mathrm{~N}), \mathrm{W}(\mathrm{N}), \mathrm{Y}(\mathrm{N}), \mathrm{T}(\mathrm{N}), \mathrm{PAI}, \mathrm{H}$

COMMON LAM, LAM4, LAM1, LAM14, KA,VV, AA,HH,F1,F2,A0

PAI $=3.141592653589798$

$\mathrm{H}=\mathrm{PAI} /(\mathrm{N}-1)$

DO $2 \mathrm{I}=2, \mathrm{~N}-1$

$2 \mathrm{~W}(\mathrm{I})=\mathrm{H}$

$\mathrm{W}(1)=\mathrm{H} / 2 ; \mathrm{W}(\mathrm{N})=\mathrm{H} / 2$

DO $4 \mathrm{I}=1, \mathrm{~N}$ 


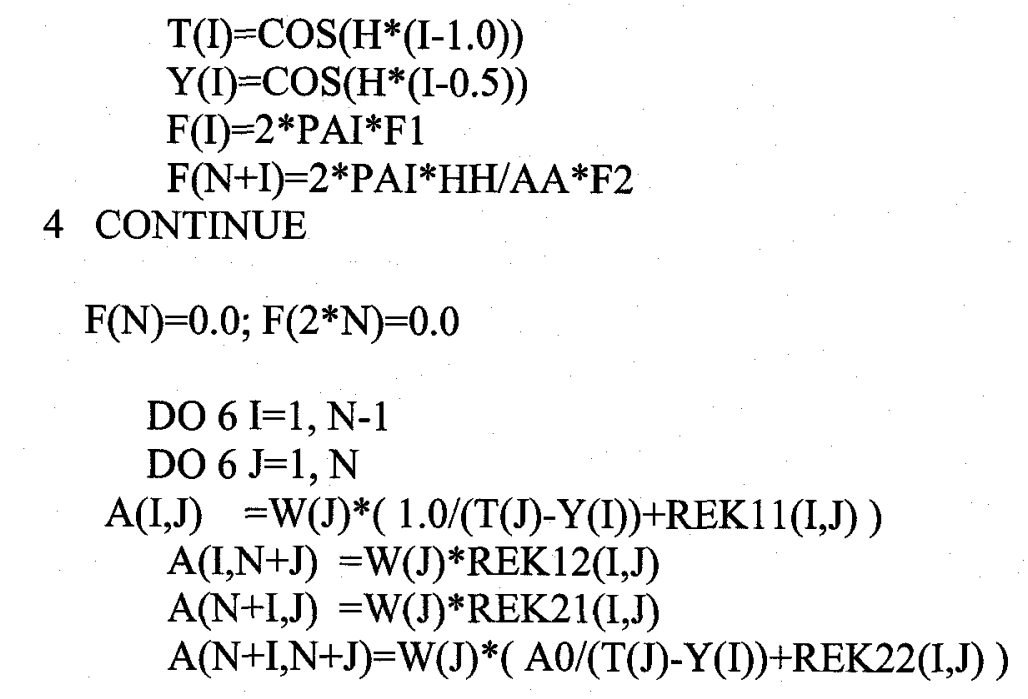

6 CONTINUE

DO $8 \mathrm{~J}=1, \mathrm{~N}$

$\mathrm{A}(\mathrm{N}, \mathrm{J})=\mathrm{W}(\mathrm{J})$

$\mathrm{A}(\mathrm{N}, \mathrm{N}+\mathrm{J})=0.0$

$\mathrm{A}\left(2^{*} \mathrm{~N}, \mathrm{~J}\right)=0.0$

$\mathrm{A}(2 * \mathrm{~N}, \mathrm{~N}+\mathrm{J})=\mathrm{W}(\mathrm{J})$

8 CONTINUE

RETURN
END

C----- THIS IS THE SUBPROGRAM FOR CALCULATING THE DATA OF THE CLOSE-INNER DISCRET EQUATIONS

SUBROUTINE CLOSEEQINNER(N,A,F,W,T,Y,REK11,REK12,REK21,REK22)

DOUBLE PRECISION REK11(N,N), REK12(N,N), REK21(N,N),REK22(N,N)

DOUBLE PRECISION A $\left(2 * \mathrm{~N}, 2^{*} \mathrm{~N}\right), \mathrm{F}\left(2^{*} \mathrm{~N}\right), \mathrm{W}(\mathrm{N}), \mathrm{Y}(\mathrm{N}), \mathrm{T}(\mathrm{N}), \mathrm{PAI}, \mathrm{H} 1$

COMMON LAM, LAM4, LAM1, LAM14, KA,VV, AA,HH,F1,F2,A0

\author{
PAI $=3.141592653589798$ \\ $\mathrm{H} 1=\mathrm{HH} / \mathrm{AA} / 2$ \\ DO $2 \mathrm{I}=1, \mathrm{~N}-1$ \\ $\mathrm{F}(\mathrm{I})=2 * \mathrm{PAI} * \mathrm{~F} 1$
}




\title{
$\mathrm{F}(\mathrm{N}+\mathrm{I})=2 * \mathrm{PAI} * \mathrm{HH} / \mathrm{AA} * \mathrm{~F} 2$
}

2 CONTINUE

$\mathrm{F}(\mathrm{N})=0.0 ; \mathrm{F}(2 * \mathrm{~N})=0.0$

DO $6 \mathrm{I}=1, \mathrm{~N}-1$

DO $4 \mathrm{~J}=1, \mathrm{~N}$

$\mathrm{A}(\mathrm{I}, \mathrm{J})=0.0$

$\mathrm{A}(\mathrm{I}, \mathrm{N}+\mathrm{J})=0.0$

$\mathrm{A}(\mathrm{N}+\mathrm{I}, \mathrm{J})=\mathrm{W}(\mathrm{J}) *(\mathrm{H} 1 /(\mathrm{T}(\mathrm{J})-\mathrm{Y}(\mathrm{I}))+\mathrm{H} 1 * \mathrm{REK} 11(\mathrm{I}, \mathrm{J})+\mathrm{REK} 21(\mathrm{I}, \mathrm{J}))$

$\mathrm{A}(\mathrm{N}+\mathrm{I}, \mathrm{N}+\mathrm{J})=\mathrm{W}(\mathrm{J}) *(\mathrm{~A} 0 /(\mathrm{T}(\mathrm{J})-\mathrm{Y}(\mathrm{I}))+\mathrm{H} 1 * \mathrm{REK} 12(\mathrm{I}, \mathrm{J})+\mathrm{REK} 22(\mathrm{I}, \mathrm{J}))$

4 CONTINUE

$\mathrm{A}(\mathrm{I}, \mathrm{I})=1.0$

$$
\mathrm{A}(\mathrm{I}, \mathrm{N}+\mathrm{I})=-\mathrm{H} 1
$$

6 CONTINUE

DO $8 \mathrm{~J}=1, \mathrm{~N}$

$\mathrm{A}(\mathrm{N}, \mathrm{J})=\mathrm{W}(\mathrm{J})$

$\mathrm{A}(\mathrm{N}, \mathrm{N}+\mathrm{J})=0.0$

$\mathrm{A}\left(2^{*} \mathrm{~N}, \mathrm{~J}\right)=0.0$

$\mathrm{A}\left(2^{*} \mathrm{~N}, \mathrm{~N}+\mathrm{J}\right)=\mathrm{W}(\mathrm{J})$

8 CONTINUE

\section{RETURN}

END

C---- THIS IS THE SUBPROGRAM FOR CALCULATING THE DATA OF THE CLOSE-OUTER DISCRET EQUATIONS

SUBROUTINE CLOSEEQOUTER(N,A,F,W,T,Y,REK11,REK12,REK21,REK22)

DOUBLE PRECISION REK11(N,N), REK12(N,N), REK21(N,N),REK22(N,N)

DOUBLE PRECISION A $(2 * \mathrm{~N}, 2 * \mathrm{~N}), \mathrm{F}(2 * \mathrm{~N}), \mathrm{W}(\mathrm{N}), \mathrm{Y}(\mathrm{N}), \mathrm{T}(\mathrm{N}), \mathrm{PAI}, \mathrm{H} 1$

COMMON LAM, LAM4, LAM1, LAM14, KA,VV, AA,HH,F1,F2,A0

\author{
PAI $=3.141592653589798$ \\ $\mathrm{H} 1=\mathrm{HH} / \mathrm{AA} / 2$ \\ DO $2 \mathrm{I}=1, \mathrm{~N}-1$ \\ $\mathrm{F}(\mathrm{I})=2 * \mathrm{PAI}^{*} \mathrm{~F} 1$ \\ $\mathrm{F}(\mathrm{N}+\mathrm{I})=-2 * \mathrm{PAI} * \mathrm{HH} / \mathrm{AA}^{*} \mathrm{~F} 2$
}


2 CONTINUE

$\mathrm{F}(\mathrm{N})=0.0 ; \mathrm{F}(2 * \mathrm{~N})=0.0$

DO $6 \mathrm{I}=1, \mathrm{~N}-1$

DO $4 \mathrm{~J}=1, \mathrm{~N}$

$\mathrm{A}(\mathrm{I}, \mathrm{J})=0.0$

$\mathrm{A}(\mathrm{I}, \mathrm{N}+\mathrm{J})=0.0$

$\mathrm{A}(\mathrm{N}+\mathrm{I}, \mathrm{J})=\mathrm{W}(\mathrm{J}) *(\mathrm{H} 1 /(\mathrm{T}(\mathrm{J})-\mathrm{Y}(\mathrm{I}))+\mathrm{H} 1 * \mathrm{REK} 11(\mathrm{I}, \mathrm{J})-\mathrm{REK} 21(\mathrm{I}, \mathrm{J}))$

$\mathrm{A}(\mathrm{N}+\mathrm{I}, \mathrm{N}+\mathrm{J})=\mathrm{W}(\mathrm{J}) *(-\mathrm{A} 0 /(\mathrm{T}(\mathrm{J})-\mathrm{Y}(\mathrm{I}))+\mathrm{H} 1 * \mathrm{REK} 12(\mathrm{I}, \mathrm{J})-\mathrm{REK} 22(\mathrm{I}, \mathrm{J}))$

4 CONTINUE

$\mathrm{A}(\mathrm{I}, \mathrm{I})=1.0$

$\mathrm{A}(\mathrm{I}, \mathrm{N}+\mathrm{I})=\mathrm{H} 1$

6 CONTINUE

DO $8 \mathrm{~J}=1, \mathrm{~N}$

$\mathrm{A}(\mathrm{N}, \mathrm{J})=\mathrm{W}(\mathrm{J})$

$\mathrm{A}(\mathrm{N}, \mathrm{N}+\mathrm{J})=0.0$

$\mathrm{A}(2 * \mathrm{~N}, \mathrm{~J})=0.0$

$\mathrm{A}(2 * \mathrm{~N}, \mathrm{~N}+\mathrm{J})=\mathrm{W}(\mathrm{J})$

8 CONTINUE

RETURN

END

C---- THIS IS THE SUBPROGRAM FOR SOLVING THE LINEAR EQUATIONS BY GAUSS ELIMINATE METHOD

SUBROUTINE SOLVEEQ(N,A,B,X)

DOUBLE PRECISION A(N,N), B(N), X(N), C, T, S

DO $12 \mathrm{~K}=1, \mathrm{~N}-1$

$\mathrm{L}=\mathrm{K}$

DO $2 \mathrm{I}=\mathrm{K}+1, \mathrm{~N}$

$\operatorname{IF}(\operatorname{DABS}(\mathrm{A}(\mathrm{I}, \mathrm{K})) \cdot \mathrm{GT} \cdot \mathrm{DABS}(\mathrm{A}(\mathrm{L}, \mathrm{K}))) \mathrm{L}=\mathrm{I}$

2 CONTINUE

$\operatorname{IF}(\mathrm{A}(\mathrm{L}, \mathrm{K})=0.0)$ THEN

PRINT*, 'NO UNIQUE SOLUTION'

STOP

END IF

IF(L.NE.K) THEN

$\mathrm{T}=\mathrm{B}(\mathrm{K})$ 

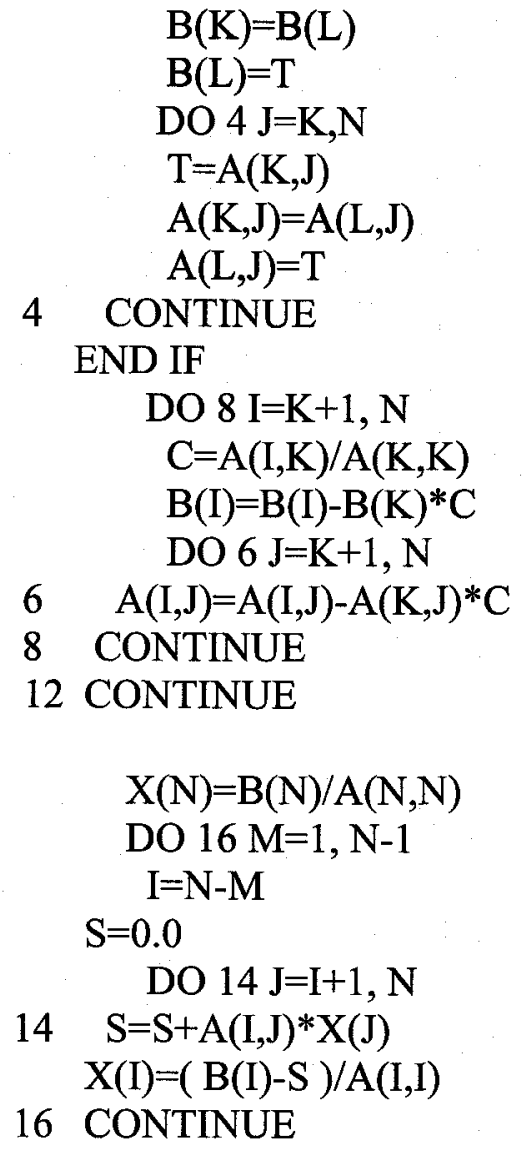

RETURN

END 